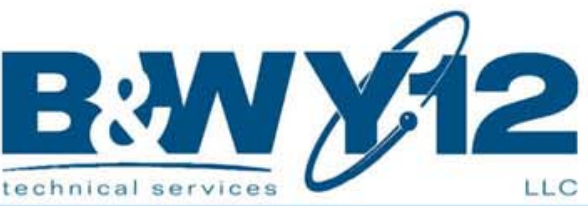

Y-12

NATIONAL SECURITY COMPLEX
UPDATED SUBSURFACE DATA BASE FOR BEAR CREEK VALLEY, CHESTNUT RIDGE, AND PARTS OF BETHEL VALLEY ON THE U.S. DEPARTMENT OF ENERGY OAK RIDGE RESERVATION

Revision 6

December 2013

Prepared by the

Environmental Compliance Department, Environment, Safety, and Health Division Y-12 National Security Complex Oak Ridge, Tennessee 37831

\title{
Managed by
}

Babcock \& Wilcox Technical Services Y-12, L.L.C.

For the U.S. DEPARTMENT OF ENERGY Under contract DE-AC0-00OR22800 


\section{Disclaimer}

This report was prepared as an account of work sponsored by an agency of the United States Government. Neither the United States Government nor any agency thereof, nor any of their employees, makes any warranty, express or implied, or assumes any legal liability or responsibility for the accuracy, completeness, or usefulness of any information, apparatus, product, or process disclosed, or represents that its use would not infringe privately owned rights. Reference herein to any specific commercial product, process, or service by trade name, trademark, manufacturer, or otherwise, does not necessarily constitute or imply its endorsement, recommendation, or favoring by the United States Government or any agency thereof. The views and opinions of authors expressed herein do not necessarily state or reflect those of the United States Government or any agency thereof. 
UPDATED SUBSURFACE DATA BASE FOR BEAR CREEK VALLEY, CHESTNUT RIDGE, AND PARTS OF BETHEL VALLEY ON THE U.S. DEPARTMENT OF ENERGY OAK RIDGE RESERVATION

Revision 6

December 2013

Prepared by the

Environmental Compliance Department Environment, Safety \& Health Division Y-12 National Security Complex

Oak Ridge, Tennessee 37831

Managed by

Babcock \& Wilcox Technical Services Y-12, LLC For the U.S. DEPARTMENT OF ENERGY Under contract DE-AC05-00OR22800 


\section{CONTENTS}

$\underline{\text { Page }}$

LIST OF FIGURES AND MAPS $\quad \ldots \ldots \ldots \ldots \ldots \ldots \ldots \ldots \ldots \ldots$ iii

EXECUTIVE SUMMARY $\ldots \ldots \ldots \ldots \ldots \ldots \ldots \ldots \ldots \ldots \ldots \ldots$

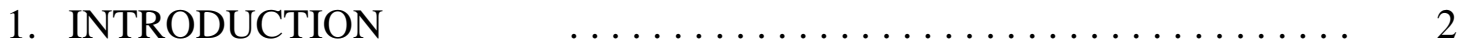

2. WELL AND BOREHOLE IDENTIFICATION $\quad \ldots \ldots \ldots \ldots \ldots \ldots \ldots$.

3. DATA BASE ORGANIZATION $\quad \ldots \ldots \ldots \ldots \ldots \ldots \ldots \ldots \ldots$

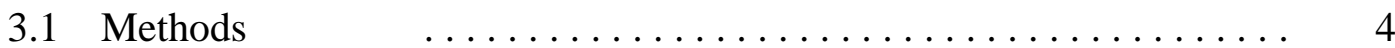

3.2 Borehole Location Maps $\quad \ldots \ldots \ldots \ldots \ldots \ldots \ldots \ldots \ldots \ldots$

3.3 Tabulation of Construction and Hydrological Data $\ldots \ldots \ldots \ldots \ldots$

3.4 Explanation of Data Base Entries $\quad \ldots \ldots \ldots \ldots \ldots \ldots \ldots \ldots$

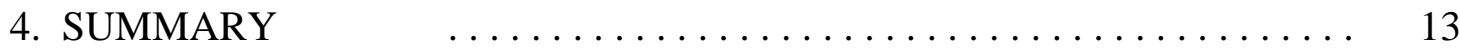

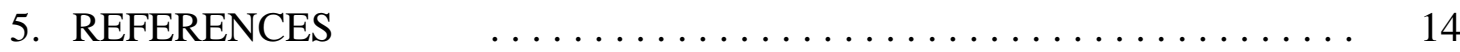

APPENDIX A: Borehole and Well Location Summary $\quad \ldots \ldots \ldots \ldots \ldots \ldots$ A-1

APPENDIX B: Borehole and Well Nomenclature, Construction Type, and Status Summary $\quad \ldots \ldots \ldots \ldots \ldots \ldots \ldots \ldots \ldots \ldots \ldots$

APPENDIX C: Borehole and Well Reference and Comments Summary . . . . . C-1

APPENDIX D: Screened Well Construction Summary $\quad \ldots \ldots \ldots \ldots \ldots$ D-1

APPENDIX E: Open Well Construction Summary $\quad \ldots \ldots \ldots \ldots \ldots \ldots \ldots$ E-1

APPENDIX F: $\quad$ Borehole and Well Hydrogeological Data Summary . . . . . . F-1

APPENDIX G: Borehole and Well Geological Data Summary . . . . . . . G G-1

APPENDIX H: Westbay Multiport Instrumented Corehole Data Summary . . H-1

APPENDIX I: $\quad$ Surface Water Location Summary $\ldots \ldots \ldots \ldots \ldots \ldots \ldots$ I-1

APPENDIX J: Data Correction Form $\quad \ldots \ldots \ldots \ldots \ldots \ldots \ldots \ldots \ldots . . .1$

APPENDIX K: Borehole Location Maps - Existing Boreholes and Wells. ... . K-1

APPENDIX L: $\quad$ Borehole Location Maps - Inactive Boreholes and Wells. . . . . L-1 


\section{LIST OF FIGURES AND MAPS}

$\underline{\text { Page }}$

Fig. 1 Generalized site map of the U.S. Department of Energy

Oak Ridge Reservation

5

Map 1 Existing borehole and well location map for area No. 1 in Fig. 1.

Appendix K

Map 2 Existing borehole and well location map for area No. 2 in Fig. 1. Appendix K

Map Insert A Appendix K

Map 3 Existing borehole and well location map for area No. 3 in Fig. 1. Appendix K

Map 4 Existing borehole and well location map for area No. 4 in Fig. 1. Appendix K

Map 5 Existing borehole and well location map for area No. 5 in Fig. 1. Appendix K

Map 6 Existing borehole and well location map for area No. 6 in Fig. 1. Appendix K

Map 7 Outlying Surface Water Monitoring Locations ........... Appendix K

Map 1 Abandoned or destroyed borehole and well location map for area No. 1 in Fig. 1

Appendix L

Map 2 Abandoned or destroyed borehole and well location map for area No. 2 in Fig. 1. Appendix L

Map 3 Abandoned or destroyed borehole and well location map for area No. 3 in Fig. 1. Appendix L

Map 4 Abandoned or destroyed borehole and well location map for area No. 4 in Fig. 1.

Appendix L

Map 5 Abandoned or destroyed borehole and well location map for area No. 5 in Fig. 1. Appendix L

Map 6 Abandoned or destroyed borehole and well location map for area No. 6 in Fig. 1. Appendix L

Map 7 Abandoned or destroyed borehole and well location map for area No. 7 in Fig. 1. Appendix L 


\section{EXECUTIVE SUMMARY}

This document represents a compilation of location, construction, and hydrologic information relating to boreholes, groundwater monitoring wells, and surface water locations that have been installed/established at the Y-12 National Security Complex (Y-12) through August 2013. To date, a total of 1422 boreholes and wells have been installed in and around the Y-12 area. Of those, 835 existing boreholes and wells continue to be utilized for groundwater monitoring programs, research, remedial investigations, plume characterization and delineation studies, and various other hydrogeologic endeavors. In addition, 215 surface water locations, such as rivers, streams, seeps, springs, lakes, ponds, and building sumps are included in this database. General data about boreholes and wells included in the database are survey coordinates, survey system, elevations, alternative names and well status. Surface water location information (Appendix I) includes name, alias, functional area, northing and easting coordinates, survey system, map number and sampling history. Tabulated construction data include total depth, completion method, borehole diameter, casing and screen materials, casing and screen diameters, casing and screen depths, filter pack depths, open-hole intervals, and open-hole diameters. Hydrogeological data summarized in this document include the aquifer monitored by the completion interval, depth to weathered and fresh bedrock, formations penetrated, well sampling history, and whether rock core and geophysical logs were obtained.

This document (which is the sixth revision to Y/TS-881 and the ninth overall update of a previous compilation) is published on a regular basis by the Y-12 Groundwater Protection Program (GWPP), which serves as custodian of drilling records and well construction data for the network of wells and other groundwater monitoring stations at Y-12. The tabulations in this database are arranged in appendices of like information. An example application of the data compiled in this document would be the evaluation of the suitability of wells for continued use in groundwater investigations and monitoring studies. Other uses may include: the siting of hydrogeological characterization activities, background evaluations of sites prior to initiation of new drilling activities, and hydrogeological review of selected sites prior to the initiation of remedial actions or new construction activity.

Existing boreholes, wells, and surface water locations are presented on a series of maps (Appendix K). Boreholes and wells that have been plugged and abandoned, destroyed, could not be located, or otherwise are of unknown status/condition appear on a second series of maps of identical coverage (Appendix L). 


\section{INTRODUCTION}

This document represents the ninth overall revision (sixth revision since being assigned the Y/TS-881 document number). This version of the Subsurface Data Base incorporates information on a total of 1637 sampling locations of interest (1422 boreholes and wells, 215 other types of monitoring stations) to the Y-12 Groundwater Protection Program (GWPP). These include 835 existing boreholes/monitoring wells, and 215 surface water locations. Of the wells listed in the Subsurface Data Base, 587 locations have a status (or condition) of plugged and abandoned (P\&A), Destroyed, Could not locate, or Unknown. This revision incorporates information gained from activities performed between October 2002 and August 2013. Information on previous revisions of the Subsurface Data Base can be found in BWXT (2003).

Geotechnical investigations, site geological and hydrological studies, and groundwater monitoring programs have been conducted at the U.S. Department of Energy Y-12 National Security Complex (Y-12) and in its vicinity for the past 70 years. Drilling records and construction details for boreholes and wells drilled in conjunction with such programs represent important information for ongoing site characterization. Such information is essential to the interpretation of historical data obtained from wells in the vicinity of the Y12 Complex. It also is essential to the evaluation of the integrity and suitability of such wells for continued use in site hydrogeological investigations and groundwater monitoring programs. Finally, drilling records and construction information frequently contain important geological and hydrological data that are of use to ongoing site investigations.

Data pertaining to existing boreholes and wells at Y-12 and surrounding vicinity are contained in various published and unpublished Union Carbide Corporation - Nuclear Division, Martin Marietta Energy Systems, Inc. [Lockheed Martin Energy Systems, Inc.], BWXT Y-12, and B\&W Y-12 reports, internal memoranda, engineering drawings, and field notebooks. Data on boreholes and wells were also obtained from published and unpublished consulting subcontractor reports, and from unpublished drilling subcontractor reports and field notebooks. The amount and quality of information pertaining to specific wells or boreholes varies and is frequently divided among several sources.

Surface water location information, found in Appendix I of this document, was first included in the eighth revision of the Subsurface Data Base (fifth revision of Y/TS-881). Appendix I contains the name, alias, type of location, functional area, northing and easting coordinates, survey system, map number, and sampling history of the surface water locations of interest to the GWPP. 


\section{WELL AND BOREHOLE IDENTIFICATION}

Boreholes and wells are identified in several different ways. Historically, Y-12 boreholes and wells have been identified by some type of alpha-numeric system (e.g., 001-L, 1001, 38-DC, BC-01, CH-001, CO-1, FP-001, FT-001, GW-001, J-001, K-020, LL/HAZ-01, M-04, OR-2, RB-001). Presently, the officially adopted alphanumeric identification of monitoring wells and piezometers by the GWPP is the GW-Series. The GWPP maintains and controls the assignment of GW-Series well identification numbers. All wells or piezometers installed at Y-12 are assigned a GW-Series identification number. Any organization involved in well installation projects may request a block of GW-Series numbers, as they are needed. In the past this method of operation has worked well; however, there are some gaps in the GW-Series due to unused portions of block assignments to other organizations. The following is a tabulation of a total of $78 \mathrm{GW}$-Series numbers that have not been assigned to existing wells or piezometers.

$\begin{array}{llll}\text { GW-351 } & \text { GW-391 } & \text { GW-524 } & \text { GW-590 } \\ \text { GW-352 } & \text { GW-392 } & \text { GW-525 } & \text { GW-591 } \\ \text { GW-353 } & \text { GW-393 } & \text { GW-529 } & \text { GW-592 } \\ \text { GW-354 } & \text { GW-394 } & \text { GW-530 } & \text { GW-593 } \\ \text { GW-355 } & \text { GW-395 } & \text { GW-547 } & \text { GW-594 } \\ \text { GW-356 } & \text { GW-396 } & \text { GW-548 } & \text { GW-595 } \\ \text { GW-357 } & \text { GW-397 } & \text { GW-549 } & \text { GW-596 } \\ \text { GW-358 } & \text { GW-398 } & \text { GW-550 } & \text { GW-597 } \\ \text { GW-359 } & \text { GW-399 } & \text { GW-577 } & \text { GW-598 } \\ \text { GW-360 } & \text { GW-500 } & \text { GW-578 } & \text { GW-599 } \\ \text { GW-361 } & \text { GW-501 } & \text { GW-579 } & \text { GW-600 } \\ \text { GW-362 } & \text { GW-503 } & \text { GW-581 } & \text { GW-650 } \\ \text { GW-377 } & \text { GW-504 } & \text { GW-582 } & \text { GW-716 } \\ \text { GW-378 } & \text { GW-510 } & \text { GW-583 } & \text { GW-717 } \\ \text { GW-379 } & \text { GW-515 } & \text { GW-584 } & \text { GW-718 } \\ \text { GW-386 } & \text { GW-516 } & \text { GW-585 } & \text { GW-719 } \\ \text { GW-387 } & \text { GW-517 } & \text { GW-586 } & \text { GW-720 } \\ \text { GW-388 } & \text { GW-518 } & \text { GW-587 } & \text { GW-741 } \\ \text { GW-389 } & \text { GW-519 } & \text { GW-588 } & \text { GW-962 } \\ \text { GW-390 } & \text { GW-523 } & \text { GW-589 } & \text { GW-963 }\end{array}$

Well numbers GW-574 and GW-575 were assigned to boreholes located on Chestnut Ridge in the vicinity of Landfills V and VII, and were completed uncased. The two boreholes do not appear in this database. In addition, well numbers GW-962 and GW-963 were assigned as "place-holders" for wells intended as replacements for GW-773 and GW-774 which were located within the footprint of a future construction project at Y-12 and scheduled for plugging and abandonment. The construction project was subsequently cancelled. The GWSeries identification system implies a time frame or a location of installation, therefore the above numbers will not be assigned to boreholes or wells in the future.

Well identification systems used by other organizations may be included in this database; however, all new wells will be assigned a GW-series identification number. 


\section{DATA BASE ORGANIZATION}

The tabulation of boreholes, wells, and surface water locations in the Subsurface Data Base is not a complete listing of all sample locations at $\mathrm{Y}-12$ and its surrounding environs. In reviewing available documents and information, an attempt was made to identify boreholes, wells, and surface water locations of greatest importance to hydrogeological research and groundwater monitoring applications relevant to Y-12. For this reason, this Subsurface Data Base does not include numerous borings drilled for soil sampling and engineering purposes throughout the Y-12 area.

Locations of existing boreholes, wells, and surface water locations contained in the database are plotted on a seven-map series (Appendix K) which extends from the Clinch River at the west end of Bear Creek Valley to beyond Illinois Avenue east of Y-12, and includes a portion of the University of Tennessee Arboretum (Figure 1.). Map Insert A is included due to a high concentration of wells in one location in Bear Creek Valley, located on maps two and three of this series. Map seven in Appendix K depicts surface water monitoring locations in outlying areas not covered by maps 1-6. Boreholes and wells that have been plugged and abandoned, destroyed, could not be located, or had an unknown status are plotted separately on an additional seven-map series (Appendix L). Note that the area covered by each map in Appendix $L$ is identical to the area covered by each corresponding map in Appendix K. Also included in the map coverage area are portions of Chestnut Ridge, Pine Ridge, Bethel Valley and the southern area of the City of Oak Ridge adjacent to Y-12 and associated facilities.

\subsection{METHODS}

The information summarized in this report was obtained by reviewing available documents dealing with drilling and monitoring well installation activities and well plugging and abandonment activities performed at Y-12. These documents are referenced in Appendix C. When possible, information for wells was cross-correlated among several sources. If necessary, the information was field checked to resolve ambiguities or to obtain information where none could be located in available documents.

\subsection{BOREHOLE LOCATION MAPS}

The base for all sampling location maps used in this report is the latest digital version of the Oak Ridge Reservation map (digital map data obtained from the Oak Ridge Environmental Information System (OREIS). The maps were plotted using the AutoCAD ${ }^{\circledR}$ software application. Scales of the completed borehole location maps are $400 \mathrm{ft}$ to $1 \mathrm{in}$. The grid lines illustrated on the maps are in the $\mathrm{Y}-12$ coordinate system.

Boreholes, wells, and surface water locations were plotted on the borehole location maps only if survey coordinates were available at the time of final map editing (August 2013). For this reason, there are several instances in which data for a borehole or well is tabulated in the appendices, but the well is not plotted on the corresponding borehole location map. 


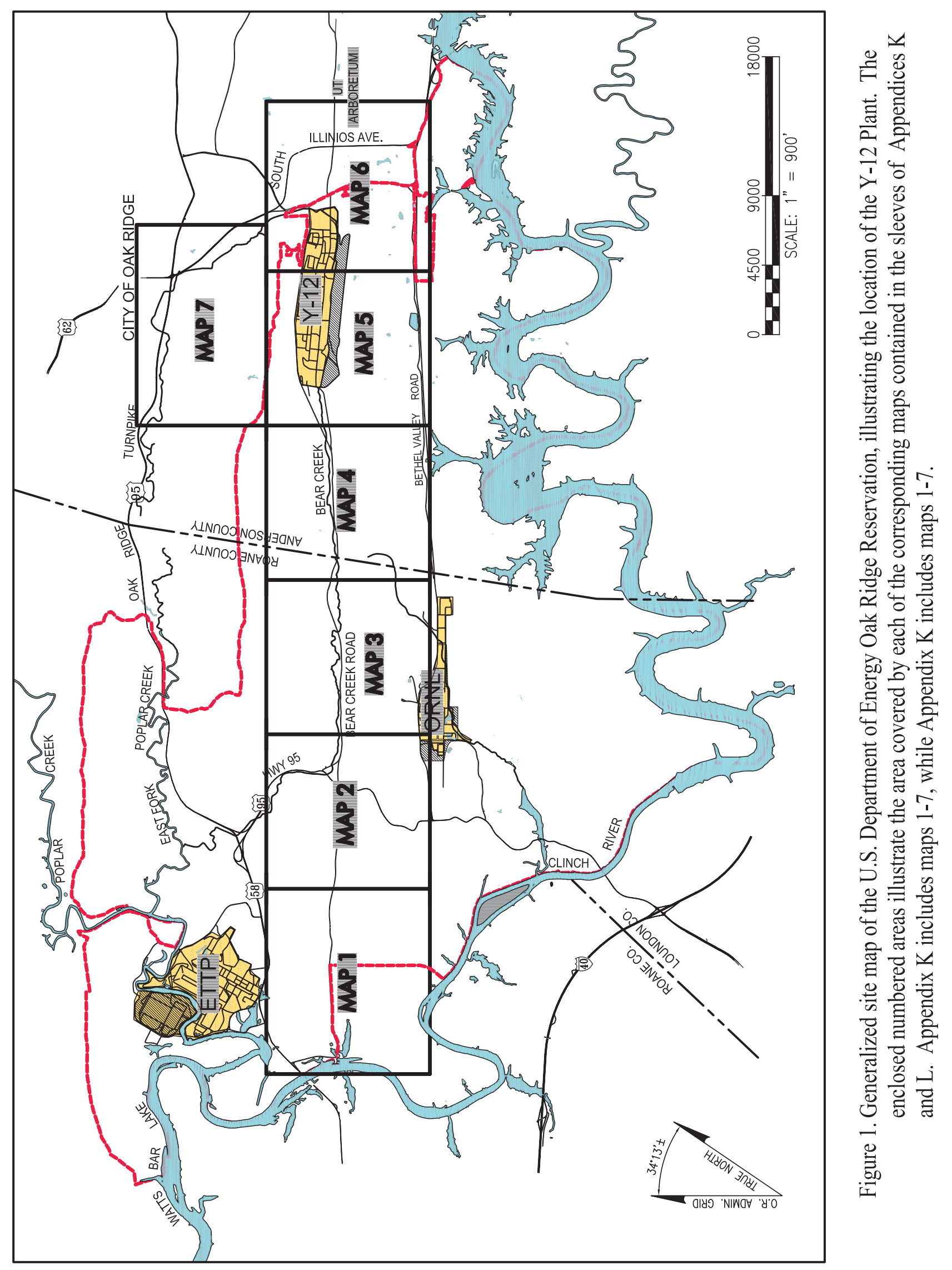




\subsection{TABULATION OF CONSTRUCTION AND HYDROLOGICAL DATA}

Data on boreholes and wells at Y-12 and vicinity are presented in nine appendices.

- Appendix A contains information on borehole and well locations (Functional Area and location coordinates, map number, elevations, and total constructed depth).

- Appendix B summarizes information on borehole and well nomenclature, construction methods, current status (or condition), and water elevation minimum, mean, and maximum values.

- Appendix C lists references for construction and hydrogeological data for all installed boreholes and wells in the database as of August 2013.

- Appendix D summarizes construction data for wells completed using screens.

- Appendix E contains construction data for boreholes and wells completed with an open interval, i.e., without screens.

- Appendix F contains depth-to-bedrock information, hydrological data, and well sampling history.

- Appendix G summarizes geological information and includes the name(s) of the rock formation(s) penetrated by the drilling, whether rock core was obtained during borehole drilling, whether geophysical logging was performed in the borehole, and if $\boldsymbol{8}$ which geophysical logs were run in the borehole.

- Appendix $\mathrm{H}$ is a summary of $\mathrm{Westbay}^{\mathrm{TM}}$, Solinst ${ }^{\circledR}$, and BarCad $^{\circledR}$ multi-port monitoring well data. This appendix incorporates multi-port instrumentation information of 14 coreholes, and includes: current well name (number), other name (parent name), packer intervals, measurement port depth, references, and applicable comments. Coreholes GW-131, GW-132, GW-133, GW-134, GW-135, GW-722, GW-726, GW-727, GW-729, GW-730, GW-790, and GW-934 have been instrumented with Westbay ${ }^{\mathrm{TM}}$ multi-port monitoring systems. Coreholes GW-821, GW-822, and GW-823 have had Solinst ${ }^{\circledR}$ multiple-interval monitoring systems installed. Coreholes GW-954 and GW-956 have each been completed with a $\mathrm{BarCad}^{\circledR}$ system. Each monitoring zone within each of these wells has a unique identification number which incorporates both the well number and zone identification (e.g., GW-131-01). The well number alone (e.g., GW-131) is the "Parent" name. Zone identification numbers increase from the deepest to the shallowest in each Westbay ${ }^{\mathrm{TM}}$, Solinst ${ }^{\circledR}$, and BarCad $^{\circledR}$ well.

- Appendix I contains surface water location information including location name, alias, location type, functional area, northing and easting coordinates, survey system, map number, and sampling history.

- Appendix $\mathrm{J}$ is a data correction form to be used to identify erroneous or inaccurate Subsurface Data Base entries.

- Appendix K includes location maps for existing boreholes, wells, and surface water monitoring locations.

- Appendix L includes borehole location maps for inactive boreholes and wells (i.e., destroyed or plugged and abandoned). 


\subsection{EXPLANATION OF DATA BASE ENTRIES}

A brief summary of the data base entries that appear in Appendices A through I is provided in this section. This summary defines each entry and explains the derivation and meaning of the information in each entry, as well as identifying in which Appendix the entry appears.

- Aquifer - Indicates in which of three generalized aquifer types the screened/packed interval or open interval of a borehole or well is completed. The aquifer designations are not tied to specific hydrostratigraphic units; rather, they are related to the depth of overburden and weathering (Geraghty and Miller 1985). The letter $\underline{\mathrm{U}}$ designates unconsolidated overburden; $\underline{\mathrm{B}}$, weathered bedrock; and $\underline{\mathrm{B}}$, unweathered bedrock. Appendix F.

- Casing Depth - The depth, below ground surface, to which the casing extends in the borehole. In open-interval wells, the casing depth is the depth of the bottom of the casing, including the floatshoe, if present. In screened wells, the casing depth is the depth to the top of the screened interval. Appendix E.

- Casing I.D. - The inside diameter of the casing material, measured in inches. Appendices D and E.

- Casing O.D. - The outside diameter of the casing material, measured in inches. Appendices D and E.

- Casing Type - The casing material is presented in format $X / Y$, where $X$ refers to the specific casing material and $\mathrm{Y}$ refers to the size or grade of the casing material. Materials include: polyvinyl chloride (PVC), steel (STL), and stainless steel (SS). Sizes or grades of casing materials include: \#40 (schedule 40), \#80 (schedule 80), F25 (API Steel casing grade F25), \#304 (type \#304 stainless steel), gal (galvanized steel), and J55 (API Steel casing grade J55). SLS represents stainless steel casing of no known specific type or grade. Appendices D and E.

- Comments - Comments listed in Appendix C include general information about a borehole or well. See System for an explanation regarding TORC or TORC'ed. Comments presented in Appendix $\mathrm{H}$ reports the zone number and the depth, below ground surface, of the measurement port and pumping port (if present) in each zone of a Westbay $^{\mathrm{TM}}$ Multi-port monitoring well.

- Core? - Indicates whether drill core was obtained during the drilling of the borehole or well. Appendix G.

- Current Name - The preferred name or identification for the borehole or well. Appendix A.

- Easting - East coordinate for the location of the borehole, well, or surface water location, measured in feet. Appendices A and I.

- Elevation - Generally refers to the ground surface elevation of a monitoring well or borehole. The surveyed point is typically located immediately adjacent to the well casing or borehole. Elevation is reported in feet above mean sea level. Appendix A.

- Fractures, Cavities, Water Breaks - Downhole depths below ground surface of fractures or fracture zones (FRAC), cavities (CAV), and water breaks (WB) identified during drilling operations. Information within the parentheses indicates the type and amount of water associated with a particular feature: (contains DNAPL) - dense non- 
aqueous phase liquids are present; (foam) - foamy or frothy water; (gas) - natural gas; (m) $-<5.0$ gpm very muddy water; (mw) $-<5.0$ gpm slightly muddy water; (mW) $->5.0$ gpm slightly muddy water; (MW) - >5.0 gpm very muddy water; (o) - open, lacks mud or water; (oil) - strong petroliferous odor but no visible petroleum; (sulfur) - sulfurous odor associated with the water; (suspected DNAPL) - dense non-aqueous phase liquids are suspected; (w) - <5.0 gpm clear water; (W) - >5.0 gpm clear water. For wells in which ground elevations have been modified subsequent to well installation, downhole depths of the identified features have been adjusted accordingly. Appendix F.

- Functional Area - Geographical or operational region, facility, or site in which the borehole, well, or surface water location is located. Appendices A and I.

- Hole Depth - Constructed depth of the borehole of the specified diameter, in which the casing is installed, measured in feet below ground surface. Hole depth may be less than Total Depth (T.D.) due to backfilling or caving of borehole prior to well completion. Appendices D and E.

- Hole Dia. - Diameter of the borehole in which the casing is installed, measured in inches. Appendices D and E.

- Loc Type - The type of surface water location is listed. Abbreviations are:

Abbreviation $\quad$ Surface Water Location

$\begin{array}{ll}\text { A } & \text { Alias: former name of location (ex. CBS-1 is now SCR4.3SP) } \\ \text { CB } & \text { Catch Basin } \\ \text { CH } & \text { Channel/Ditch } \\ \text { FL } & \text { Flume } \\ \text { HP } & \text { Holding Pond/Lagoon } \\ \text { LK } & \text { Lake/Pond } \\ \text { PP } & \text { Pipe } \\ \text { RV } & \text { River } \\ \text { SE } & \text { Seep } \\ \text { SL } & \text { Surface Location } \\ \text { SP } & \text { Spring } \\ \text { SU } & \text { Sump } \\ \text { TF } & \text { Treatment Facility }\end{array}$

Appendix I.

- Logs? - Indicates whether geophysical logs have been obtained from the borehole or well. Appendix G.

- Map - Number of the particular map on which the borehole, well, or surface water location is plotted. Because adjacent maps overlap, some wells and surface water locations plot on two maps, as indicated by dual entries (such as "3 \& 4"). Absence of an entry indicates that a well was not plotted on a map. Location maps for existing boreholes, wells, and surface water monitoring locations are included in Appendix K. Location maps for inactive boreholes and wells (i.e., destroyed and/or plugged and abandoned) are included in Appendix L.

- Measurement Port Depth - Depth in feet below ground surface to the measurement port of the Westbay ${ }^{\mathrm{TM}}$, Barcad ${ }^{\circledR}$, or Solinst ${ }^{\circledR}$ multi-port monitoring well. Appendix $\mathrm{H}$. 
- Northing - North coordinate for the location of the borehole, well, or surface water location, measured in feet. Appendices A and I.

- Open - Indicates whether the well was finished with an open interval, without a screen or a perforated casing. Appendix B.

- Open Interval - Depths of the top and bottom of the open, uncased or unscreened interval in boreholes and wells completed without screens or perforated casing, measured in feet below ground surface. Appendix E.

- Open Interval Dia. - Diameter of the uncased and unscreened portion of open boreholes and open-interval wells, measured in inches. Appendix E.

- Other Name - Alternative or additional names used in records and reports to refer to the borehole, well, or surface water location. Use of any of these names is discouraged in order to promote consistent identification and to minimize confusion. In the case of the Westbay ${ }^{\mathrm{TM}}$ and $\mathrm{BarCad}{ }^{\circledR}$ wells, the other name is the parent name (well number without the interval designation). Appendices $\mathrm{B}, \mathrm{H}$, and I.

- Packed Interval - Depths of the top and bottom of the filter pack material in screened wells, measured in feet below ground surface. Appendix E.

- Packer Interval - Depths in feet below ground surface to the top and bottom of the zone isolated from other zones of the corehole using water inflated packers along Westbay ${ }^{\mathrm{TM}}$ multi-port casing. Appendix H.

- $\quad$ Reference - Primary document reference of construction and hydrogeological data. References for this entry are listed at the end of Appendix C.

- Rock Formation(s) - Rock unit(s) penetrated by the borehole or well. Where indicated, depths are in feet below ground surface and represent the interpreted top of the formation specified. Abbreviations are Chickamauga - Chickamauga Group; Conasauga Conasauga Group; Knox - Knox Group; and Rome - Rome Formation. For boreholes or wells penetrating the Chickamauga Group, the specific unit(s) penetrated [i.e., units A through $\mathrm{H}$ as defined by Stockdale (1951)] are indicated. For boreholes or wells penetrating the Conasauga Group, the specific formation(s) penetrated (i.e., Maynardville Limestone, Nolichucky Shale, Maryville Limestone, Rogersville Shale, Rutledge Limestone, or Pumpkin Valley Shale) are indicated. For boreholes or wells penetrating the Knox Group, the specific formation(s) penetrated (i.e., Mascot Dolomite, Kingsport Formation, Longview Dolomite, Chepultepec Dolomite, or Copper Ridge Dolomite). Appendix G.

- Sampling History - The quarterly dates of water quality sampling of surface water monitoring locations. The format in this field is the quarter and the year of the sampling event (Q\#)/(\#\#), where Q3/02 would indicate third quarter (July through September) of the year 2002. Appendix I.

- Screened - Indicates whether the well is finished with a screen or some type of perforated casing. Appendix B.

- Screened Interval - Depths to the top and bottom of the screened interval, measured in feet below ground surface. Appendix D.

- Screen Type - The screen type is presented in format $\mathrm{X} / \mathrm{Y} / \mathrm{Z}$, where $\mathrm{X}$ refers to the 
specific construction material, Y refers to the type of screen, and $\mathrm{Z}$ gives the size of the screen opening, measured in inches. Screen materials include polyvinyl chloride (PVC), steel (STL), and stainless steel (SS), typically type \#304 stainless steel. Screen types include slotted (SL), spiral wound (SW), perforated (PER), and pre-packed (PPCK). Pre-packed screens consist of an inner and outer spiral wound screen. The annular space between the screens is prepacked with silica sand. SLS represents stainless steel screen of no known specific type or grade. UNKN is listed if neither the screen material nor the type of screen is known. Appendix D.

- Status - The current condition of the well is indicated. Wells that have been destroyed (Destroyed), that could not be physically located in the field (Could not locate), or plugged and abandoned (P\&A) are noted. Wells in which the status is unknown and indeterminable are listed as "Unknown". Existing and currently accessible boreholes, core holes, and wells are listed as "Open". All existing and currently accessible borehole, coreholes, and wells (and surface water locations) are plotted on maps 1 through 7 in Appendix K of this report. The locations of boreholes core holes, and wells that have been destroyed or plugged and abandoned are plotted on Maps 1 through 7 in Appendix L of this report. Wells that have become buried under materials are listed as Covered. Appendix B.

- Status Date - The date on which the well was completed, if known, is indicated for Open wells. The date on which the well was destroyed or plugged and abandoned, if known, is indicated for Destroyed or P\&A wells. Appendix B.

- System - The coordinate system in which the northing and easting coordinates of a borehole, well, or surface water sampling location is reported. Typically, the location coordinates of a specific location are reported in the Y-12 Grid, but locations may also be reported in the Administrative Grid. The absence of a survey entry indicates that the borehole, well, or surface water location has not been surveyed or that no coordinates have been determined. A program developed by ORNL, Transform Oak Ridge Coordinate (TORC), has been used toconvert northing and easting coordinates from one grid system to another (i.e. Administrative grid to Y-12 grid) if required. Locations where coordinates were "TORCed" were converted from one grid system to another. Appendices A and I.

- T.D. - The total drilled depth of the borehole or well, measured in feet below ground surface. Appendix A.

- T.O.C. - Elevation of the top of the well casing. The surveyed point is typically located at the measurement point marked at the top of the well casing. Top of Casing elevation is reported in feet above mean sea level. Appendix A.

- T.O.F.R. - Top of fresh bedrock. Depth at which essentially unweathered bedrock material was encountered during drilling, measured in feet below ground surface. Corresponds approximately to the base of appreciable weathering. Appendix F.

- T.O.W.R. - Top of weathered bedrock. Depth at which bedrock was encountered during drilling, measured in feet below ground surface. Corresponds to the first encounter with in situ bedrock. If bedrock at a site lacks significant weathering, T.O.W.R. corresponds to T.O.F.R. Appendix F.

- $\quad$ T.O.W.W. - Elevation of the top of the Well Wizard $®$ sleeve/cap (dedicated sampling equipment). The TOWW may be a surveyed point which is typically located at the measurement point marked at the top of the Well Wizard® sleeve/cap or it may be derived by adding the height of the Well Wizard ${ }^{\circledR}$ sleeve/cap to the surveyed TOC 
figure. TOWW elevation is reported in feet above mean sea level. Appendix A.

- Water Levels - Minimum (MIN), mean (MEAN), and maximum (MAX) values were calculated for those wells with a water elevation measurement history. Sources of information for these values may include; well inspections, groundwater sampling events, annual water level campaigns, and special studies. The elevation is referenced from mean sea level. Appendix B.

- Well Sample History - Well sampling is performed quarterly and is based on a calendar year. The format of information in this field is Quarter of sampling (Q\#)/year (\#\#). Therefore, Q1/90-Q2/91: Q3/92-Q4/92 indicates that a well was sampled from the first quarter (January through March) of 1990 to the second quarter (April through June) of 1991 and then again in the third quarter (July through September) of 1992 to the fourth quarter (October through December) of 1992. This field only applies to well sampling that occurred after 1986. Information prior to 1986 is not easily accessible and is reported in formats inconsistent with post-1986 data. No analyte information is conveyed. This history includes locations sampled by the GWPP and DOE-EM Contractor (Bechtel Jacobs Company/URS CH2M Oak Ridge) Water Resources Restoration Program (WRRP) and may not be comprehensive, other organizations may have performed some degree of sampling within wells listed within the database. These events may not be included due to differing data quality objectives from those utilized by the GWPP or the WRRP. Appendix F.

- Which Ones? - Indicates which geophysical logs were obtained for a borehole or well. Abbreviations are:

Abbreviation Geophysical Well Log

\begin{tabular}{ll}
\hline & \\
\cline { 2 - 2 } 4PiD & 4pi density \\
BHTV & acoustic borehole televiewer log \\
CAV & compensated acoustic velocity log \\
CDL & compensated density log \\
CL & caliper log \\
DEV & borehole deviation log \\
DHC & down hole camera \\
EMFL & electromagnetic flow log \\
FCL & borehole fluid conductivity log \\
FRL & fluid resistivity log \\
FWS & full waveform sonic \\
GRL & gamma ray log \\
IFL & impeller flow meter \\
LSN & long- and short-normal resistivity log \\
mRL & microresistivity log \\
SNL & side-walled neutron log \\
SP & spontaneous potential log \\
SPR & single point resistance log \\
TEM & temperature log \\
UAV & uncompensated acoustic velocity log \\
UNL & uncompensated neutron log \\
VDL & acoustic variable density log
\end{tabular}


Appendix G. 


\section{SUMMARY}

This document presents a comprehensive database that contains location and construction information on boreholes, wells, and surface water locations at Y-12 and the surrounding vicinity. The information summarized is a tabulation of all available data through August 2013 for newly installed wells and surface water locations and plugged and abandoned wells of interest to the GWPP. The data have been reviewed for accuracy, and as many inconsistencies as possible have been resolved. Errors may still exist in the data. In keeping with the review objectives of this document, however, any errors noted by readers should be brought to the attention of the Y-12 GWPP Program Manager using the form in Appendix J. 


\section{REFERENCES}

BWXT Y-12, L.L.C. 2003. Updated Subsurface Data Base for Bear Creek Valley, Chestnut Ridge, and parts of Bethel Valley on the U.S. Department of energy Oak Ridge Reservation. Y/TS-881/R5.

ERCE 1990. Documentation Report for the 1988 Monitor Well Plugging and Abandonment Program, Oak Ridge, Y-12 Plant. Y/SUB/90B-99005V/1.

ERCE 1991. Documentation Report for the 1989 Monitor Well Plugging and Abandonment Program, Oak Ridge, Y-12 Plant. Y/SUB/90B-99005V/2.

Geraghty and Miller, Inc. 1985. Remedial Alternatives for the Bear Creek Valley Waste Disposal Area. Y/SUB/85B-00206C/3.

Haase, C. S., G. A. Gillis, and H. L. King 1987. Subsurface Data Base for Bear Creek Valley, Chestnut Ridge, and parts of Bethel Valley on the U. S. Department of Energy Oak Ridge Reservation. ORNL/TM-10000.

Jones, S. B., S. M. Field, and B. K. Harrington, 1992. Updated Subsurface Data Base for Bear Creek Valley, Chestnut Ridge, and parts of Bethel Valley on the U. S. Department of Energy Oak Ridge Reservation. Y/TS-881.

Jones, S. B., S. M. Field, and B. K. Thompson, 1993. Updated Subsurface Data Base for Bear Creek Valley, Chestnut Ridge, and parts of Bethel Valley on the U. S. Department of Energy Oak Ridge Reservation. Y/TS-881/R1.

Jones, S. B., S. M. Field, and B. K. Thompson, 1994. Updated Subsurface Data Base for Bear Creek Valley, Chestnut Ridge, and parts of Bethel Valley on the U. S. Department of Energy Oak Ridge Reservation. Y/TS-881/R2.

Jones, S. B., S. M. Field, and B. K. Thompson, 1995. Updated Subsurface Data Base for Bear Creek Valley, Chestnut Ridge, and parts of Bethel Valley on the U. S. Department of Energy Oak Ridge Reservation. Y/TS-881/R3.

King, H. L., D. L. Schaeffer, and C. S. Haase 1989. Updated Subsurface Data Base for Bear Creek Valley, Chestnut Ridge, and parts of Bethel Valley on the U. S. Department of Energy Oak Ridge Reservation. Y/SUB/89B-E4371V/1.

King, H. L., C. S. Haase, and B. K. Harrington, 1991. Updated Subsurface Data Base for Bear Creek Valley, Chestnut Ridge, and parts of Bethel Valley on the U. S. Department of Energy Oak Ridge Reservation. Y/TS-735.

Lee, R. R., and R. H. Ketelle 1989. Geology of the west Bear Creek Valley site, Oak Ridge, Tennessee. ORNL/TM-10887.

Lockheed Martin Energy Systems 1998. Updated Subsurface Data Base for Bear Creek Valley, Chestnut Ridge, and parts of Bethel Valley on the U. S. Department of Energy Oak Ridge Reservation. Y/TS-881/R4

Stockdale, P.B. 1951. Geological conditions at the Oak Ridge National Laboratory (X-10) area relevant to the disposal of radioactive wastes. OR0-58. U.S. Atomic Energy Commission, Oak Ridge Operations, Oak Ridge, Tennessee.

Tennessee Valley Authority. 1987. Oak Ridge Area S-16A Map 
APPENDIX A

Borehole and Well Location Summary 
This Page Intentionally Left Blank 


$\begin{array}{ll}\begin{array}{c}\text { CURRENT } \\ \text { NAME }\end{array} & \text { FUNCTIONAL AR } \\ \text { 001-L } & \text { Y-12 Plant Site } \\ \text { 002-L } & \text { Y-12 Plant Site } \\ \text { 003-L } & \text { Y-12 Plant Site } \\ \text { 004-L } & \text { Y-12 Plant Site } \\ \text { 005-L } & \text { Y-12 Plant Site } \\ \text { 006-L } & \text { Y-12 Plant Site } \\ \text { 007-L } & \text { Y-12 Plant Site } \\ \text { 008-L } & \text { Y-12 Plant Site } \\ \text { 009-L } & \text { Y-12 Plant Site } \\ \text { 010-L } & \text { Y-12 Plant Site } \\ \text { 011-L } & \text { Y-12 Plant Site } \\ \text { 012-L } & \text { Y-12 Plant Site } \\ \text { 013-L } & \text { Y-12 Plant Site } \\ \text { 014-L } & \text { Y-12 Plant Site } \\ \text { 015-L } & \text { Y-12 Plant Site } \\ \text { 016-L } & \text { Y-12 Plant Site } \\ \text { 017-L } & \text { Y-12 Plant Site } \\ \text { 018-L } & \text { Y-12 Plant Site } \\ \text { 019-L } & \text { Y-12 Plant Site } \\ \text { 020-L } & \text { Y-12 Plant Site } \\ \text { 021-L } & \text { Y-12 Plant Site } \\ \text { 022-L } & \text { Y-12 Plant Site } \\ \text { 023-L } & \text { Y-12 Plant Site } \\ \text { 024-L } & \text { Y-12 Plant Site } \\ \text { 025-L } & \text { Y-12 Plant Site } \\ \text { 026-L } & \text { Y-12 Plant Site } \\ \text { 027-L } & \text { Y-12 Plant Site }\end{array}$

\begin{tabular}{|c|c|c|c|c|c|c|}
\hline MAP & NORTHING & EASTING & SYSTEM & ELEVATION & T.O.W.W. & T.D. \\
\hline 5 & 30500.00 & 57500.00 & Y-12 & 990.20 & & 30.00 \\
\hline 5 & 30000.00 & 57500.00 & Y-12 & 962.40 & & 45.00 \\
\hline 5 & 30500.00 & 57000.00 & Y-12 & 972.60 & & 35.00 \\
\hline 5 & 30000.00 & 57000.00 & Y-12 & 960.50 & & 33.00 \\
\hline 5 & 29500.00 & 57000.00 & Y-12 & 942.90 & & 43.00 \\
\hline 5 & 30500.00 & 56500.00 & $Y-12$ & 992.00 & & 35.00 \\
\hline 5 & 30000.00 & 56500.00 & Y-12 & 964.10 & & 32.00 \\
\hline 5 & 29500.00 & 56500.00 & Y-12 & 947.30 & & 50.00 \\
\hline 5 & 30500.00 & 56000.00 & Y-12 & 985.20 & & 38.00 \\
\hline 5 & 30000.00 & 56000.00 & Y-12 & 978.10 & & 55.00 \\
\hline 5 & 29500.00 & 56000.00 & Y-12 & 950.60 & & 50.00 \\
\hline 5 & 30500.00 & 55500.00 & Y-12 & 990.00 & & 30.00 \\
\hline 5 & 30000.00 & 55500.00 & Y-12 & 981.20 & & 35.00 \\
\hline 5 & 29500.00 & 55500.00 & Y-12 & 956.30 & & 46.00 \\
\hline 5 & 30500.00 & 55000.00 & Y-12 & 991.00 & & 35.00 \\
\hline 5 & 30000.00 & 55000.00 & Y-12 & 971.20 & & 35.00 \\
\hline 5 & 29500.00 & 55000.00 & Y-12 & 966.10 & & 36.00 \\
\hline 5 & 30500.00 & 54500.00 & Y-12 & 999.50 & & 60.00 \\
\hline 5 & 30000.00 & 54500.00 & Y-12 & 985.10 & & 60.00 \\
\hline 5 & 29500.00 & 54500.00 & Y-12 & 977.30 & & 60.00 \\
\hline 5 & 30500.00 & 54000.00 & Y-12 & 1003.30 & & 51.00 \\
\hline 5 & 30000.00 & 54000.00 & Y-12 & 985.30 & & 60.00 \\
\hline 5 & 30250.00 & 57250.00 & Y-12 & 973.60 & & 55.00 \\
\hline 5 & 29750.00 & 57250.00 & $Y-12$ & 962.40 & & 60.00 \\
\hline 5 & 30250.00 & 56750.00 & Y-12 & 975.30 & & 60.00 \\
\hline 5 & 29800.00 & 56750.00 & Y-12 & 959.20 & & 60.00 \\
\hline 5 & 30250.00 & 56250.00 & Y-12 & 974.90 & & 60.00 \\
\hline
\end{tabular}




$\begin{array}{ll}\begin{array}{c}\text { CURRENT } \\ \text { NAME }\end{array} & \\ \text { 028-L } & \text { FUNCTIONAL A } \\ \text { 029-L } & \text { Y-12 Plant Site } \\ \text { 030-L } & \text { Y-12 Plant Site } \\ \text { 031-L } & \text { Y-12 Plant Site } \\ \text { 032-L } & \text { Y-12 Plant Site } \\ 033-\text { L } & \text { Y-12 Plant Site } \\ 034-\text { L } & \text { Y-12 Plant Site } \\ 035-\text { L } & \text { Y-12 Plant Site } \\ 1001 & \text { S-3 Ponds } \\ 1002 & \text { S-3 Ponds } \\ 1003 & \text { S-3 Ponds } \\ 1004 & \text { S-3 Ponds } \\ 1005 & \text { S-3 Ponds } \\ 1006 & \text { S-3 Ponds } \\ 1007 & \text { S-3 Ponds } \\ 1008 & \text { S-3 Ponds } \\ 1009 & \text { S-3 Ponds } \\ 1010 & \text { S-3 Ponds } \\ 1011 & \text { S-3 Ponds } \\ 1012 & \text { S-3 Ponds } \\ 1013 & \text { S-3 Ponds } \\ 1014 & \text { S-3 Ponds } \\ 1015 & \text { S-3 Ponds } \\ 1016 & \text { S-3 Ponds } \\ 1017 & \text { S-3 Ponds } \\ 1018 & \text { S-3 Ponds } \\ 1019 & \text { S-3 Ponds } \\ & \end{array}$

MAP NORTHING

EASTING

SYSTEM

ELEVATION

T.O.C.

T.O.W.W.

T.D.

$\begin{array}{rrrrrr}5 & 29750.00 & 56250.00 & \mathrm{Y}-12 & 958.00 & \\ 5 & 30250.00 & 55750.00 & \mathrm{Y}-12 & 988.30 & \\ 5 & 29750.00 & 55750.00 & \mathrm{Y}-12 & 971.50 & \\ 5 & 30250.00 & 55250.00 & \mathrm{Y}-12 & 971.40 & \\ 5 & 29750.00 & 55250.00 & \mathrm{Y}-12 & 962.50 & \\ 5 & 30250.00 & 54750.00 & \mathrm{Y}-12 & 981.60 & \\ 5 & 29750.00 & 54750.00 & \mathrm{Y}-12 & 970.30 & \\ 5 & 30250.00 & 54250.00 & \mathrm{Y}-12 & 988.70 & \\ 5 & 31740.00 & 51930.00 & \mathrm{Y}-12 & 1112.00 & 1115.00 \\ 5 & 30916.00 & 52541.00 & \mathrm{Y}-12 & 1044.50 & 1045.40 \\ 5 & 29939.00 & 51595.00 & \mathrm{Y}-12 & 1000.90 & 1003.90 \\ 4 \& 5 & 29732.00 & 50456.00 & \mathrm{Y}-12 & 981.12 & 983.44 \\ 5 & 29579.00 & 51064.00 & \mathrm{Y}-12 & 986.00 & 990.11 \\ 5 & 30056.00 & 52202.00 & \mathrm{Y}-12 & 1007.10 & 1009.10 \\ 5 & 29995.00 & 52310.00 & \mathrm{Y}-12 & 1005.60 & 1007.80 \\ 5 & 29933.00 & 52128.00 & \mathrm{Y}-12 & 1004.50 & 1006.10 \\ 5 & 30096.00 & 52418.00 & \mathrm{Y}-12 & 1007.90 & 1010.20 \\ 5 & 30556.00 & 52206.00 & \mathrm{Y}-12 & 1019.20 & 1020.90 \\ 5 & 29981.00 & 52016.00 & \mathrm{Y}-12 & 1006.50 & 1010.60 \\ 5 & 31114.00 & 51185.00 & \mathrm{Y}-12 & 1085.00 & 1088.00 \\ 5 & 31611.00 & 51496.00 & \mathrm{Y}-12 & 1067.10 & 1070.10 \\ 5 & 31759.00 & 51522.00 & \mathrm{Y}-12 & 1102.20 & 1105.20 \\ 5 & 31587.00 & 51811.00 & \mathrm{Y}-12 & 1071.90 & 1074.90 \\ 5 & 31278.00 & 51451.00 & \mathrm{Y}-12 & 1060.20 & 1063.20 \\ 5 & 31683.00 & 51399.00 & \mathrm{Y}-12 & 1078.90 & 1081.90 \\ 5 & 31207.00 & 51345.00 & \mathrm{Y}-12 & 1077.90 & 1080.90 \\ 5 & 31149.00 & 51346.00 & \mathrm{Y}-12 & 1084.00 & 1087.00\end{array}$

60.00

60.00

60.00

52.00

60.00

60.00

60.00

60.00

56.00

48.00

48.00

29.00

24.00

20.50

18.00

20.00

22.50

20.00

22.50

70.00

42.00

46.40

37.00

63.00

44.40

47.20

43.50 


\section{CURRENT}

NAME

1020
1021
1022
1023
1024
1025
$1025 A$
1026
1027
1028
1029
1030
1031
1032
1033
1034
1035
1036
1037
1038
1040
1041
1043
1044
1045
1046
1047

\section{FUNCTIONAL AREA}

S-3 Ponds

S-3 Ponds

S-3 Ponds

S-3 Ponds

S-3 Ponds

S-3 Ponds

S-3 Ponds

S-3 Ponds

Bear Creek Burial Grounds WMA

Bear Creek Burial Grounds WMA

Bear Creek Burial Grounds WMA

Bear Creek Burial Grounds WMA

Bear Creek Burial Grounds WMA

Bear Creek Burial Grounds WMA

Bear Creek Burial Grounds WMA

Bear Creek Burial Grounds WMA

Bear Creek Burial Grounds WMA

Bear Creek Burial Grounds WMA

Bear Creek Burial Grounds WMA

Bear Creek Burial Grounds WMA

Bear Creek Burial Grounds WMA

Bear Creek Burial Grounds WMA

Bear Creek Burial Grounds WMA

Bear Creek Burial Grounds WMA

Bear Creek Burial Grounds WMA

Bear Creek Burial Grounds WMA

Bear Creek Road
MAP NORTHING

EASTING

SYSTEM

ELEVATION

T.O.C.

T.O.W.W

T.D.

\begin{tabular}{|c|c|c|c|c|}
\hline 31336.00 & 51453.00 & $Y-12$ & 1048.20 & 1051.20 \\
\hline 31342.00 & 51508.00 & Y-12 & 1046.00 & 1049.00 \\
\hline 31280.00 & 51512.00 & $Y-12$ & 1056.10 & 1059.10 \\
\hline 31452.00 & 51467.00 & $\mathrm{Y}-12$ & 1043.10 & 1046.10 \\
\hline 31681.00 & 51703.00 & $Y-12$ & 1060.70 & 1063.70 \\
\hline 31581.00 & 51554.00 & Y-12 & 1060.10 & 1063.10 \\
\hline 30860.00 & 51059.00 & Y-12 & 1071.00 & 1074.00 \\
\hline 30663.00 & 44309.00 & Y-12 & 1023.00 & 1024.10 \\
\hline 29427.00 & 45511.00 & $Y-12$ & 923.84 & 926.96 \\
\hline 29502.00 & 44102.00 & Y-12 & 927.92 & 929.11 \\
\hline 29495.00 & 43369.00 & Y-12 & 920.65 & 921.33 \\
\hline 29677.00 & 42869.00 & Y-12 & 942.96 & 945.40 \\
\hline 29243.00 & 43748.00 & $\mathrm{Y}-12$ & 917.74 & 920.49 \\
\hline 30016.00 & 42899.00 & $Y-12$ & 948.28 & 950.19 \\
\hline 29953.00 & 42436.00 & Y-12 & 933.93 & 937.35 \\
\hline 31011.00 & 42430.00 & Y-12 & 1001.60 & 1003.80 \\
\hline 30197.00 & 42173.00 & $Y-12$ & 944.30 & 945.86 \\
\hline 29731.00 & 41185.00 & $Y-12$ & 914.40 & 917.70 \\
\hline 31111.00 & 42810.00 & $\mathrm{Y}-12$ & 1008.20 & 1010.60 \\
\hline 30766.00 & 42331.00 & $Y-12$ & 966.69 & 968.18 \\
\hline 30313.25 & 42184.66 & $Y-12$ & 955.50 & 958.97 \\
\hline 30434.00 & 42887.00 & Y-12 & 1009.10 & 1011.60 \\
\hline 29586.00 & 42905.00 & Y-12 & 940.42 & 942.10 \\
\hline 29175.00 & 42912.00 & $\mathrm{Y}-12$ & 909.68 & 912.25 \\
\hline 28774.00 & 42923.00 & $Y-12$ & 905.44 & 907.28 \\
\hline 28535.00 & 38325.00 & Y-12 & 862.00 & \\
\hline
\end{tabular}

40.00

27.50

48.00

36.00

40.00

50.00

41.60

70.00

100.00

29.00

18.50

47.00

47.00

57.00

160.00

45.00

100.00

100.00

45.00

26.00

23.00

29.50

150.00

160.00

155.00

75.00

23.20 


\section{CURRENT \\ NAME}

1047A

1048

1049

1050

1051

1052

1055

1056

1057

1058

1059

1060

1061

1062

1063

1070

1071

1072

1073

1080

1081

1082

1083

1084

1085

1086

1087

\section{FUNCTIONAL AREA}

Bear Creek Road

Oil Landfarm WMA

Oil Landfarm WMA

Oil Landfarm WMA

Oil Landfarm WMA

Oil Landfarm WMA

Oil Landfarm WMA

Oil Landfarm WMA

Oil Landfarm WMA

Oil Landfarm WMA

Oil Landfarm WMA

Oil Landfarm WMA

Oil Landfarm WMA

Oil Landfarm WMA

Oil Landfarm WMA

Bear Creek Burial Grounds WMA

Bear Creek Burial Grounds WMA

Bear Creek Burial Grounds WMA

Oil Landfarm WMA

Chestnut Ridge Security Pits

Oak Ridge Sludge Farm

Oak Ridge Sludge Farm

Oak Ridge Sludge Farm

Oak Ridge Sludge Farm

Industrial Landfill II

Industrial Landfill II

Industrial Landfill II
MAP NORTHING EASTING

SYSTEM

ELEVATION

T.O.C.

T.O.W.W

$\underline{\text { T.D. }}$

$\begin{array}{ll}28795.00 & 38555.00 \\ 30288.00 & 47771.00 \\ 29883.00 & 47387.00 \\ 29370.00 & 46969.00 \\ 29797.00 & 47777.00 \\ 29836.00 & 46990.00 \\ 29968.00 & 45400.00 \\ 29956.00 & 45375.00 \\ 29984.00 & 45349.00 \\ 29986.00 & 45380.00 \\ 29991.00 & 45419.00 \\ 30000.00 & 45477.00 \\ 30020.00 & 45423.00 \\ 30029.00 & 45396.00 \\ 29988.00 & 45399.00 \\ 30122.00 & 44388.00 \\ 30961.00 & 42826.00 \\ 30540.00 & 44198.00 \\ 29472.00 & 48394.00 \\ 28392.00 & 57782.00 \\ 23392.00 & 55966.00 \\ 23395.00 & 54418.00 \\ 23309.00 & 52550.00 \\ 25008.00 & 53599.00 \\ 26097.00 & 52997.00 \\ 26923.00 & 52316.00 \\ 25817.00 & 52289.00\end{array}$

$\mathrm{Y}-12$

$\mathrm{Y}-12$

981.65

983.54

170.00

$\mathrm{Y}-12$

954.49

956.12

100.00

20.00

100.00

28.00

22.00

70.00

70.00

70.00

70.00

70.00

70.00

70.00

70.00

70.00

41.40

20.00

20.00

21.00

181.00

38.00

50.00

58.50

144.50

122.00

156.00

145.00 
FUNCTIONAL AREA

Oil Landfarm WMA

United Nuclear Corporation Site

United Nuclear Corporation Site

United Nuclear Corporation Site

Chestnut Ridge Sediment

Disposal Basin

Chestnut Ridge Sediment

Disposal Basin

S-3 Ponds

Filled Coal Ash Pond

Filled Coal Ash Pond

Filled Coal Ash Pond

Filled Coal Ash Pond

Filled Coal Ash Pond

Filled Coal Ash Pond

Filled Coal Ash Pond

Filled Coal Ash Pond

Filled Coal Ash Pond

Filled Coal Ash Pond

Filled Coal Ash Pond

Filled Coal Ash Pond

Filled Coal Ash Pond

SPAD Project, south side

SPAD Project, south side

SPAD Project, south side

SPAD Project, south side

SPAD Project, south side

Industrial Landfill II

\section{MAP NORTHING}

EASTING

SYSTEM

ELEVATION

T.O.C.

T.O.W.W.

T.D.

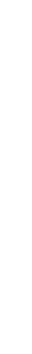

$\begin{array}{ccc}4 \& 5 & 29342.00 & \\ 5 & 28718.02 & 538 \\ 5 & 28551.32 & 54 \\ 5 & 28047.00 & 530 \\ 6 & 28088.00 & 630\end{array}$

$\begin{array}{ll}49162.00 & \text { Y-12 } \\ 53853.02 & \text { Y-12 } \\ 54406.26 & \text { Y-12 } \\ 53980.00 & \text { Y-12 } \\ 63601.00 & \text { Y-12 }\end{array}$

960.93

961.87

$\begin{array}{rr}117.70 \\ 1104.48 & 96.70 \\ & 102.20\end{array}$

1024.50

1002.60

1103.88

1123.34

1026.80

1004.70

118.00

$1045.90 \quad 1047.70$

68.00

1081.00

1084.00

40.00

31151.00
26365.00

51300.00

57018.00

57154.00

954.53

954.45

26317.00

57069.00

57056.00

57061.00

.

26348.00

26265.00

57054.00

57071.00

57055.00

26184.00

26185.00

26399.00

26489.00

26878.00

27225.00

26313.64

26833.79

27242.84

27423.24

26813.67

57048.00

57148.00

57173.00

57227.00

57235.00

59971.87

60035.50

60134.39

59147.36

58819.09

$\mathrm{Y}-12$

956.83

957.45

956.95

956.72

956.97

924.24

924.57

903.37

903.62

956.44

955.26

956.61

955.53

949.89

972.31

986.85

1062.34

1095.20

27.00

28.90

75.10

25.00

54.30

56.40

34.50

32.20

9.00

41.80

8.00

8.00

8.00

23.50

30.00

30.00

24.00

190.00

44.40 


\section{CURRENT}

21

$2 S$

38-DC

39-DC

3D

31

35

40-DC

41-DC

42-DC

44-DC

45-DC

4D

41

$4 S$

53-1A

54-1A

54-2A

55-1A

55-1B

55-1C

55-2A

55-2B

55-2C

55-3A

55-3B

55-3C
Industrial Landfill II

Industrial Landfill II

Gum Branch Road

Gum Branch Road

Industrial Landfill II

Industrial Landfill II

Industrial Landfill II

Gum Branch Road

Gum Branch Road

Gum Branch Road

Gum Branch Road

Gum Branch Road

Industrial Landfill II

Industrial Landfill II

Industrial Landfill II

Y-12 Plant Site

Y-12 Plant Site

$\mathrm{Y}-12$ Plant Site

Y-12 Grid Well B2

$\mathrm{Y}-12$ Plant Site

Y-12 Grid Well B2

Y-12 Plant Site

Y-12 Grid Well B3

Y-12 Grid Well B3

Y-12 Plant Site

Y-12 Plant Site

Y-12 Plant Site

\section{MAP}

$\begin{array}{ll}2 \& 3 & 30267.73 \\ 2 \& 3 & 30327.50\end{array}$

$\begin{array}{cc}2 \text { \& } 3 & 30680.60 \\ 2 \text { \& } 3 & 30307.30 \\ 3 & 30509.80 \\ 3 & 29806.00 \\ 2 \text { \& } 3 & 29737.00\end{array}$

30176.21
30010.02
30009.19
30469.88
30469.23
30466.74
30085.32
30084.88
30085.20
29959.34
29958.73
29958.59

29493.37

$\mathrm{Y}-12$

873.03

30927.13

$\mathrm{Y}-12$

868.96

30400.66

30442.55

32063.80

32584.00

29838.00

$\mathrm{Y}-12$

$\mathrm{Y}-12$

$\mathrm{Y}-12$

$\mathrm{Y}-12$

$\mathrm{Y}-12$

876.29

931.82

934.05

875.47

867.93

938.10

53814.62

$\mathrm{Y}-12$

990.67

981.71

981.07

986.20

986.05

985.85

976.17

976.17

976.07

971.59

971.57

971.76

34.00

29.60

44.10

41.10

42.80

32.00

27.20

36.60

102.00

98.30

42.40

30.00

46.20

36.40

31.20

22.10

23.00

26.30

19.30

38.80

75.70

14.10

27.60

75.90

14.30

55695.12
55698.77

55702.65

$\mathrm{Y}-12$

$\mathrm{Y}-12$

972.46

977.02

972.46

$\begin{array}{lll}972.46 & 972.46 & 14.30 \\ 973.32 & 973.32 & 38.10\end{array}$ 


\begin{tabular}{|c|c|}
\hline \multicolumn{2}{|c|}{ CURRENT } \\
\hline NAME & FUNCTIONAL AREA \\
\hline $55-4 B$ & Y-12 Plant Site \\
\hline $55-4 \mathrm{C}$ & Y-12 Plant Site \\
\hline $55-5 A$ & Y-12 Plant Site \\
\hline $55-6 \mathrm{~A}$ & Y-12 Grid Well C2 \\
\hline $55-7 \mathrm{~A}$ & Y-12 Coal Pile \\
\hline $55-8 \mathrm{~A}$ & Y-12 Coal Pile \\
\hline $55-8 B$ & Y-12 Coal Pile \\
\hline $55-9 A$ & Y-12 Coal Pile \\
\hline $56-1 \mathrm{~A}$ & Y-12 Plant Site \\
\hline $56-1 C$ & Y-12 Plant Site \\
\hline $56-2 A$ & Y-12 Grid Well C3 \\
\hline $56-2 B$ & Y-12 Grid Well C3 \\
\hline $56-2 C$ & Y-12 Grid Well C3 \\
\hline $56-3 A$ & Y-12 Plant Site \\
\hline $56-3 B$ & Y-12 Plant Site \\
\hline $56-3 C$ & Y-12 Plant Site \\
\hline $56-4 \mathrm{~A}$ & Y-12 Plant Site \\
\hline $56-4 B$ & Y-12 Plant Site \\
\hline $56-4 C$ & Y-12 Grid Well C3 \\
\hline $56-5 A$ & Y-12 Plant Site \\
\hline $56-5 B$ & Y-12 Plant Site \\
\hline $56-5 C$ & Y-12 Plant Site \\
\hline $56-6 \mathrm{~A}$ & Y-12 Plant Site \\
\hline $56-7 A$ & Y-12 Plant Site \\
\hline $56-8 \mathrm{~A}$ & Y-12 Plant Site \\
\hline $56-9 \mathrm{~A}$ & Y-12 Plant Site \\
\hline $58-1 \mathrm{~A}$ & Y-12 Plant Site \\
\hline
\end{tabular}

\begin{tabular}{|c|c|c|c|c|c|c|c|}
\hline MAP & NORTHING & EASTING & SYSTEM & ELEVATION & T.O.C. & T.O.W.W. & T.D. \\
\hline 5 & 30762.67 & 55694.55 & Y-12 & 1003.92 & 1004.68 & & 25.50 \\
\hline 5 & 30762.77 & 55698.10 & Y-12 & 1003.95 & 1004.86 & & 72.60 \\
\hline 5 & 30697.60 & 55556.53 & Y-12 & 986.67 & 988.58 & & 10.90 \\
\hline 5 & 30667.34 & 55906.66 & Y-12 & 986.82 & 989.04 & 989.29 & 12.90 \\
\hline 5 & 29736.00 & 55894.00 & Y-12 & & & & 22.30 \\
\hline 5 & 29755.00 & 55934.00 & Y-12 & & & & 22.00 \\
\hline 5 & 29739.00 & 55902.00 & Y-12 & & & & 45.00 \\
\hline 5 & 29747.00 & 55809.00 & Y-12 & & & & 18.50 \\
\hline 5 & 30350.69 & 56079.34 & Y-12 & 968.72 & 969.25 & 969.25 & 19.00 \\
\hline 5 & 30355.03 & 56077.03 & Y-12 & 968.89 & 969.49 & 969.69 & 75.30 \\
\hline 5 & 29880.85 & 56228.72 & Y-12 & 962.52 & 963.30 & 963.53 & 15.10 \\
\hline 5 & 29883.61 & 56225.60 & Y-12 & 962.21 & 962.28 & 962.45 & 38.80 \\
\hline 5 & 29884.63 & 56230.51 & Y-12 & 962.44 & 964.94 & 965.11 & 77.30 \\
\hline 5 & 29866.66 & 56452.76 & Y-12 & 962.35 & 963.03 & 963.03 & 17.80 \\
\hline 5 & 29865.55 & 56477.66 & Y-12 & 962.74 & 964.33 & 964.33 & 33.40 \\
\hline 5 & 29859.16 & 56449.10 & Y-12 & 962.36 & 962.86 & 962.86 & 55.50 \\
\hline 5 & 29819.59 & 56802.01 & Y-12 & 960.10 & 962.07 & 962.07 & 12.10 \\
\hline 5 & 29815.13 & 56799.28 & Y-12 & 960.56 & 961.38 & & 32.60 \\
\hline 5 & 29815.06 & 56804.51 & Y-12 & 959.05 & 962.62 & & 76.30 \\
\hline 5 & 29380.21 & 56823.85 & Y-12 & 965.90 & 966.69 & & 12.60 \\
\hline 5 & 29382.47 & 56827.82 & Y-12 & 965.87 & 966.72 & & 35.10 \\
\hline 5 & 29375.47 & 56818.57 & Y-12 & 966.34 & 967.30 & & 71.60 \\
\hline 5 & 29783.24 & 56915.30 & Y-12 & 958.12 & 960.28 & 960.28 & 21.00 \\
\hline 5 & 29449.16 & 56814.30 & $Y-12$ & 956.62 & 958.45 & & 21.10 \\
\hline 5 & 29465.51 & 56455.71 & Y-12 & 959.15 & 962.46 & 962.46 & 25.60 \\
\hline 5 & 29532.83 & 56043.39 & Y-12 & 963.81 & 965.86 & & 17.30 \\
\hline 5 & 29647.52 & 58777.80 & Y-12 & 947.20 & 947.84 & & 20.70 \\
\hline
\end{tabular}




\section{CURRENT}

NAME

$\begin{array}{ll}58-2 A & \text { Y-12 Plant Site } \\ 59-1 A & \text { Building } 9202 \\ 59-1 B & \text { Building 9202 } \\ 59-1 C & \text { Building 9202 } \\ 5 \text { D } & \text { Industrial Landfill II } \\ 51 & \text { Industrial Landfill II } \\ 5 \text { S } & \text { Industrial Landfill II } \\ 60-1 \text { A } & \text { Y-12 Plant Site } \\ 60-1 B & \text { Y-12 Plant Site } \\ 60-1 C & \text { Y-12 Plant Site } \\ 60-2 A & \text { Y-12 Plant Site } \\ \text { AN:D-1 } & \text { East Fork Poplar Creek } \\ \text { AN:D-10 } & \text { East Fork Poplar Creek } \\ \text { AN:D-11 } & \text { East Fork Poplar Creek } \\ \text { AN:D-2 } & \text { East Fork Poplar Creek } \\ \text { AN:D-3 } & \text { East Fork Poplar Creek } \\ \text { AN:D-4 } & \text { East Fork Poplar Creek } \\ \text { AN:D-5 } & \text { East Fork Poplar Creek } \\ \text { AN:D-6 } & \text { East Fork Poplar Creek } \\ \text { AN:D-7 } & \text { East Fork Poplar Creek } \\ \text { AN:D-8 } & \text { East Fork Poplar Creek } \\ \text { AN:D-9 } & \text { East Fork Poplar Creek } \\ \text { AN:D-UNKNOWN } & \text { East Fork Poplar Creek } \\ \text { B-1 } & \text { Urea Pile } \\ \text { B-3 } & \text { Urea Pile } \\ \text { BC-01 } & \text { Exxon Nuclear Site } \\ \text { BC-03 } & \text { Exxon Nuclear Site }\end{array}$

MAP NORTHING EAS

$\begin{array}{cc}5 & 29500.33 \\ 5 \& 6 & 29830.55 \\ 5 \& 6 & 29835.10 \\ 5 \& 6 & 29834.00\end{array}$

$58772.32 \quad \mathrm{Y}-12$

$59884.79 \quad \mathrm{Y}-12$

$59885.47 \quad Y-12$

$59881.56 \quad Y-12$

ELEVATION

T.O.C.

T.O.W.W

T.D.

$\left(\frac{10}{2}\right.$

$\begin{array}{cc}5 \text { \& } 6 & 29225.67 \\ 5 \text { \& } 6 & 29225.44 \\ 5 \text { \& } 6 & 29227.00 \\ 5 \text { \& } 6 & 29096.86 \\ 7 & 34088.00 \\ 7 & 40191.00 \\ 7 & 40015.00 \\ 7 & 33990.00 \\ 7 & 33900.00 \\ 7 & 33810.00 \\ 7 & 33760.00 \\ 7 & 33680.00 \\ 7 & 40355.00 \\ 7 & 40295.00 \\ 7 & 40242.00 \\ 6 & 32502.00 \\ 6 & 28499.00 \\ 6 & 28519.00 \\ 2 & 28775.44 \\ 2 & 29333.20\end{array}$

60199.87
60203.55
60210.00
60118.44
62440.00
53237.00
53061.00
62402.00
62358.00
62321.00
62265.00
62197.00
53400.00
53360.00
53284.00
63738.00
61702.00
61760.00
22018.23
22032.82

\subsection{3}

945.26

945.07

945.12

Y-12

$\mathrm{Y}-12$

$\mathrm{Y}-12$

$\mathrm{Y}-12$

$\mathrm{Y}-12$

$\mathrm{Y}-12$

$\mathrm{Y}-12$

$\mathrm{Y}-12$

$\mathrm{Y}-12$

$\mathrm{Y}-12$

$\mathrm{Y}-12$

$\mathrm{Y}-12$

$\mathrm{Y}-12$

$\mathrm{Y}-12$

$\mathrm{Y}-12$

$\mathrm{Y}-12$

$\mathrm{Y}-12$

$\mathrm{Y}-12$

$\mathrm{Y}-12$

$\mathrm{Y}-12$
938.16

$945.75 \quad 945.95$

$945.69 \quad 945.94$

$945.87 \quad 946.12$

9.90

13.20

36.90

73.90

41.80

30.80

26.10

23.20

29.20

74.00

13.50

4.90

9.30

8.40

8.20

9.70

4.90

6.10

3.80

8.00

8.40

10.00

18.00

53.20

36.10

250.30

349.10 


$\begin{array}{ll}\begin{array}{c}\text { CURRENT } \\ \text { NAME }\end{array} & \text { FUNCTIONAL AREA } \\ \text { BC-06 } & \text { Exxon Nuclear Site } \\ \text { BC-07 } & \text { Exxon Nuclear Site } \\ \text { BC-08 } & \text { Exxon Nuclear Site } \\ \text { BC-09 } & \text { Exxon Nuclear Site } \\ \text { BC-10 } & \text { Exxon Nuclear Site } \\ \text { BC-11 } & \text { Exxon Nuclear Site } \\ \text { BC-14 } & \text { Exxon Nuclear Site } \\ \text { BC-15 } & \text { Exxon Nuclear Site } \\ \text { BC-16 } & \text { Exxon Nuclear Site } \\ \text { BC-17 } & \text { Exxon Nuclear Site } \\ \text { BC-18 } & \text { Exxon Nuclear Site } \\ \text { BC-19 } & \text { Exxon Nuclear Site } \\ \text { BC-21 } & \text { Exxon Nuclear Site } \\ \text { BC-22 } & \text { Exxon Nuclear Site } \\ \text { BC-23 } & \text { Exxon Nuclear Site } \\ \text { BC-25 } & \text { Exxon Nuclear Site } \\ \text { BC-30 } & \text { Exxon Nuclear Site } \\ \text { BC-31 } & \text { Exxon Nuclear Site } \\ \text { BC-32 } & \text { Exxon Nuclear Site } \\ \text { BC-33 } & \text { Exxon Nuclear Site } \\ \text { BC-34 } & \text { Exxon Nuclear Site } \\ \text { BC-35 } & \text { Exxon Nuclear Site } \\ \text { BC-36 } & \text { Exxon Nuclear Site } \\ \text { BC-37 } & \text { Exxon Nuclear Site } \\ \text { BC-38 } & \text { Exxon Nuclear Site } \\ \text { BC-39 } & \text { Exxon Nuclear Site } \\ \text { BC-40 } & \text { Exxon Nuclear Site }\end{array}$

\begin{tabular}{|c|c|c|c|c|c|c|}
\hline MAP & NORTHING & EASTING & SYSTEM & ELEVATION & T.O.W.W. & T.D. \\
\hline 2 & 29990.31 & 22039.64 & Y-12 & 970.47 & & 352.50 \\
\hline 2 & 30188.47 & 22037.38 & Y-12 & 983.01 & & 351.90 \\
\hline 2 & 30376.45 & 22015.22 & Y-12 & 936.60 & & 239.00 \\
\hline 2 & 30589.18 & 22038.75 & Y-12 & 921.17 & & 343.00 \\
\hline 2 & 30755.16 & 22024.95 & Y-12 & 955.66 & & 349.00 \\
\hline 2 & 30902.91 & 22003.74 & Y-12 & 955.31 & & 350.00 \\
\hline 2 & 30585.07 & 21311.81 & Y-12 & 899.36 & & 102.00 \\
\hline 2 & 30583.51 & 21601.39 & Y-12 & 904.29 & & 100.20 \\
\hline 2 & 30602.20 & 22616.06 & Y-12 & 900.20 & & 100.40 \\
\hline $1 \& 2$ & 30187.06 & 20407.90 & Y-12 & 917.53 & & 100.40 \\
\hline $1 \& 2$ & 30186.11 & 20799.48 & Y-12 & 925.88 & & 100.20 \\
\hline 2 & 30183.55 & 21199.97 & $Y-12$ & 873.25 & & 151.80 \\
\hline $1 \& 2$ & 29216.07 & 20780.89 & Y-12 & 849.40 & & 100.90 \\
\hline $1 \& 2$ & 30474.97 & 20247.46 & Y-12 & 888.61 & & 100.20 \\
\hline $1 \& 2$ & 30523.63 & 20763.44 & $Y-12$ & 900.32 & & 56.00 \\
\hline 2 & 30361.12 & 23438.16 & Y-12 & 889.80 & & 104.00 \\
\hline $1 \& 2$ & 29840.07 & 20548.99 & Y-12 & 880.27 & & 104.00 \\
\hline $1 \& 2$ & 30182.37 & 19620.18 & Y-12 & 860.50 & & 101.00 \\
\hline $1 \& 2$ & 30182.75 & 19080.26 & Y-12 & 862.54 & & 100.20 \\
\hline $1 \& 2$ & 30294.05 & 19601.61 & Y-12 & 867.78 & & 145.30 \\
\hline $1 \& 2$ & 30538.28 & 19600.26 & Y-12 & 890.95 & & 126.30 \\
\hline $1 \& 2$ & 30054.11 & 19795.72 & Y-12 & 922.12 & & 325.00 \\
\hline $1 \& 2$ & 30321.02 & 19795.05 & Y-12 & 862.70 & & 350.00 \\
\hline $1 \& 2$ & 30664.65 & 19820.31 & $Y-12$ & 885.30 & & 300.00 \\
\hline $1 \& 2$ & 30531.70 & 19809.82 & Y-12 & 885.60 & & 300.00 \\
\hline $1 \& 2$ & 30727.96 & 19792.60 & Y-12 & 896.57 & & 157.80 \\
\hline $1 \& 2$ & 30961.75 & 19878.21 & Y-12 & 964.90 & & 135.50 \\
\hline
\end{tabular}




$\begin{array}{ll}\begin{array}{ll}\text { CURRENT } \\ \text { NAME }\end{array} & \text { FUNCTIONAL AREA } \\ \text { BC-41 } & \text { Exxon Nuclear Site } \\ \text { BC-42 } & \text { Exxon Nuclear Site } \\ \text { BC-43 } & \text { Exxon Nuclear Site } \\ \text { BC-45 } & \text { Exxon Nuclear Site } \\ \text { BC-47 } & \text { Exxon Nuclear Site } \\ \text { BC-49 } & \text { Exxon Nuclear Site } \\ \text { BC-50 } & \text { Exxon Nuclear Site } \\ \text { BC-51 } & \text { Exxon Nuclear Site } \\ \text { BC-52 } & \text { Exxon Nuclear Site } \\ \text { BC-53 } & \text { Exxon Nuclear Site } \\ \text { BC-54 } & \text { Exxon Nuclear Site } \\ \text { BC-57 } & \text { Exxon Nuclear Site } \\ \text { BC-59 } & \text { Exxon Nuclear Site } \\ \text { BC-61 } & \text { Exxon Nuclear Site } \\ \text { BC-62 } & \text { Exxon Nuclear Site } \\ \text { BC-63 } & \text { Exxon Nuclear Site } \\ \text { BC-64 } & \text { Exxon Nuclear Site } \\ \text { BCU-01 } & \text { Exxon Nuclear Site } \\ \text { BCU-02 } & \text { Exxon Nuclear Site } \\ \text { BCU-03 } & \text { Exxon Nuclear Site } \\ \text { BCU-04 } & \text { Exxon Nuclear Site } \\ \text { BCU-05 } & \text { Exxon Nuclear Site } \\ \text { BCU-06 } & \text { Exxon Nuclear Site } \\ \text { BCU-07 } & \text { Exxon Nuclear Site } \\ \text { BCU-08 } & \text { Exxon Nuclear Site } \\ \text { BCU-09 } & \text { Exxon Nuclear Site } \\ \text { BCU-10 } & \text { Exxon Nuclear Site } \\ & \end{array}$

\section{MAP NORTHING EAS}

\begin{tabular}{|c|c|c|c|c|}
\hline $1 \& 2$ & 30537.78 & 20079.27 & Y-12 & 897.15 \\
\hline $1 \& 2$ & 30728.02 & 20279.59 & Y-12 & 900.77 \\
\hline $1 \& 2$ & 30918.63 & 20093.26 & $Y-12$ & 983.10 \\
\hline $1 \& 2$ & 30962.37 & 20430.43 & $Y-12$ & 985.10 \\
\hline 2 & 30327.25 & 21174.90 & Y-12 & 883.45 \\
\hline $1 \& 2$ & 28089.94 & 19747.68 & Y-12 & 858.60 \\
\hline $1 \& 2$ & 28303.75 & 19798.48 & Y-12 & 829.00 \\
\hline $1 \& 2$ & 28494.79 & 19787.20 & $Y-12$ & 830.00 \\
\hline $1 \& 2$ & 28698.81 & 19782.96 & $Y-12$ & 838.80 \\
\hline $1 \& 2$ & 28846.78 & 19789.51 & $Y-12$ & 850.00 \\
\hline $1 \& 2$ & 29086.79 & 19788.41 & Y-12 & 845.00 \\
\hline 1 & 28453.80 & 18717.05 & $Y-12$ & 817.50 \\
\hline 1 & 28783.50 & 18796.28 & Y-12 & 827.70 \\
\hline $1 \& 2$ & 30274.45 & 19608.86 & Y-12 & 864.20 \\
\hline $1 \& 2$ & 30494.92 & 19598.90 & $Y-12$ & 887.18 \\
\hline $1 \& 2$ & 29941.28 & 19793.72 & Y-12 & 942.58 \\
\hline 1 & 31727.61 & 17693.30 & $Y-12$ & 913.60 \\
\hline $1 \& 2$ & 30404.31 & 20459.75 & Y-12 & 884.78 \\
\hline $1 \& 2$ & 30260.75 & 20279.59 & Y-12 & 900.77 \\
\hline $1 \& 2$ & 30503.89 & 20131.08 & Y-12 & 889.23 \\
\hline $1 \& 2$ & 30503.85 & 20121.36 & Y-12 & 889.70 \\
\hline $1 \& 2$ & 30504.23 & 20116.69 & Y-12 & 889.82 \\
\hline $1 \& 2$ & 30504.31 & 20108.66 & Y-12 & 890.26 \\
\hline $1 \& 2$ & 30504.43 & 20100.66 & Y-12 & 890.99 \\
\hline $1 \& 2$ & 30497.00 & 20108.58 & Y-12 & 889.28 \\
\hline $1 \& 2$ & 30494.28 & 20121.05 & Y-12 & 888.38 \\
\hline $1 \& 2$ & 30524.18 & 20120.99 & Y-12 & 892.53 \\
\hline
\end{tabular}

T.D.

276.00

203.00

178.50

125.00

50.00

100.00

100.00

100.00

109.50

110.00

100.00

100.00

100.00

54.00

56.30

283.70

224.00

135.50

178.00

57.60

60.20

61.00

62.00

61.00

61.70

61.00

55.80 


$\begin{array}{ll}\begin{array}{l}\text { CURRENT } \\ \text { NAME }\end{array} & \text { FUNCTIONAL AREA } \\ \text { BCU-11 } & \text { Exxon Nuclear Site } \\ \text { BCU-12 } & \text { Exxon Nuclear Site } \\ \text { BCU-13 } & \text { Exxon Nuclear Site } \\ \text { BCU-14 } & \text { Exxon Nuclear Site } \\ \text { BCU-15 } & \text { Exxon Nuclear Site } \\ \text { BCU-16 } & \text { Exxon Nuclear Site } \\ \text { BCU-17 } & \text { Exxon Nuclear Site } \\ \text { BCU-18 } & \text { Exxon Nuclear Site } \\ \text { BCU-19 } & \text { Exxon Nuclear Site } \\ \text { BCU-20 } & \text { Exxon Nuclear Site } \\ \text { BCU-21 } & \text { Exxon Nuclear Site } \\ \text { BCU-22 } & \text { Exxon Nuclear Site } \\ \text { BCU-23 } & \text { Exxon Nuclear Site } \\ \text { BCU-24 } & \text { Exxon Nuclear Site } \\ \text { BCU-25 } & \text { Exxon Nuclear Site } \\ \text { BCU-26 } & \text { Exxon Nuclear Site } \\ \text { BCU-27 } & \text { Exxon Nuclear Site } \\ \text { BCU-28 } & \text { Exxon Nuclear Site } \\ \text { BCU-29 } & \text { Exxon Nuclear Site } \\ \text { BY-10 } & \text { Boneyard/Burnyard } \\ \text { BY-11 } & \text { Boneyard/Burnyard } \\ \text { BY-12 } & \text { Boneyard/Burnyard } \\ \text { BY-13 } & \text { Boneyard/Burnyard } \\ \text { BY-14 } & \text { Boneyard/Burnyard } \\ \text { BY-15 } & \text { Boneyard/Burnyard } \\ \text { BY-16 } & \text { Boneyard/Burnyard } \\ \text { BY-17 } & \text { Boneyard/Burnyard } \\ & \end{array}$

\section{MAP NORTHING}

EASTING

SYSTEM

ELEVATION

T.O.C.

T.O.W.W.

T.D.

$\begin{array}{ccccc}1 \text { \& } 2 & 30519.52 & 20107.38 & \mathrm{Y}-12 & 890.82 \\ 1 \text { \& } 2 & 30381.68 & 20102.51 & \mathrm{Y}-12 & 878.02 \\ 1 \text { \& } 2 & 30537.51 & 20054.32 & \mathrm{Y}-12 & 895.91 \\ 1 \text { \& } 2 & 30266.10 & 19795.93 & \mathrm{Y}-12 & 861.92 \\ 1 \text { \& } & 30281.48 & 19795.25 & \mathrm{Y}-12 & 864.48 \\ 1 \text { \& } 2 & 30058.21 & 19813.34 & \mathrm{Y}-12 & 923.06 \\ 1 \text { \& } 2 & 30179.52 & 19641.40 & \mathrm{Y}-12 & 859.52 \\ 1 \text { \& } & 30149.15 & 19621.93 & \mathrm{Y}-12 & 858.44 \\ 1 \text { \& } & 30180.77 & 19599.59 & \mathrm{Y}-12 & 861.26 \\ 1 \text { \& } & 29739.14 & 19734.18 & \mathrm{Y}-12 & 934.80 \\ 1 \text { \& } 2 & 30392.28 & 19790.92 & \mathrm{Y}-12 & 884.76 \\ 1 \text { \& } & 30670.67 & 19805.54 & \mathrm{Y}-12 & 890.10 \\ 1 \text { \&2 } & 30643.48 & 19828.10 & \mathrm{Y}-12 & 896.20 \\ 1 \text { \& } & 30646.89 & 19840.22 & \mathrm{Y}-12 & 898.13 \\ 1 \text { \& } 2 & 30643.30 & 19844.67 & \mathrm{Y}-12 & 898.04 \\ 1 \text { \& } & 30645.10 & 19844.41 & \mathrm{Y}-12 & 897.98 \\ 1 \text { \& } & 30646.73 & 19844.19 & \mathrm{Y}-12 & 898.18 \\ 1 \text { \& } & 30648.47 & 19844.00 & \mathrm{Y}-12 & 898.08 \\ 1 \text { \& } & 30649.92 & 19843.56 & \mathrm{Y}-12 & 898.04 \\ 4 & 29707.25 & 48300.80 & \mathrm{Y}-12 & 962.15 \\ 4 & 29756.49 & 48303.76 & \mathrm{Y}-12 & 964.02 \\ 4 & 29805.80 & 48306.54 & \mathrm{Y}-12 & 967.28 \\ 4 & 29857.11 & 48308.15 & \mathrm{Y}-12 & 965.99 \\ 4 & 29908.12 & 48313.50 & \mathrm{Y}-12 & 965.99 \\ 4 & 29955.65 & 48314.30 & \mathrm{Y}-12 & 967.74 \\ 4 & 30005.65 & 48312.95 & \mathrm{Y}-12 & 969.04 \\ 4 & 29862.86 & 48215.43 & \mathrm{Y}-12 & 963.71\end{array}$

45.00

201.00

25.10

15.00

17.00

146.30

10.00

51.00

34.00

76.60

242.00

10.00

58.30

59.80

58.50

59.10

58.30

58.30

59.20

14.00

16.00

18.00

16.00

15.00

18.00

17.00

14.00 


$\begin{array}{ll}\begin{array}{c}\text { CURRENT } \\ \text { NAME }\end{array} & \text { FUNCTIONAL AREA } \\ \text { BY-18 } & \text { Boneyard/Burnyard } \\ \text { BY-19 } & \text { Boneyard/Burnyard } \\ \text { BY-20 } & \text { Boneyard/Burnyard } \\ \text { BY-21 } & \text { Boneyard/Burnyard } \\ \text { BY-22 } & \text { Boneyard/Burnyard } \\ \text { BY-23 } & \text { Boneyard/Burnyard } \\ \text { BY-24 } & \text { Boneyard/Burnyard } \\ \text { BY-25 } & \text { Boneyard/Burnyard } \\ \text { BY-26 } & \text { Boneyard/Burnyard } \\ \text { BY-27 } & \text { Boneyard/Burnyard } \\ \text { BY-28 } & \text { Boneyard/Burnyard } \\ \text { BY-29 } & \text { Boneyard/Burnyard } \\ \text { BY-30 } & \text { Boneyard/Burnyard } \\ \text { BY-31 } & \text { Boneyard/Burnyard } \\ \text { BY-32 } & \text { Boneyard/Burnyard } \\ \text { BY-33 } & \text { Boneyard/Burnyard } \\ \text { BY-34 } & \text { Boneyard/Burnyard } \\ \text { BY-35 } & \text { Boneyard/Burnyard } \\ \text { BY-36 } & \text { Boneyard/Burnyard } \\ \text { BY-37 } & \text { Boneyard/Burnyard } \\ \text { BY-38 } & \text { Boneyard/Burnyard } \\ \text { BY-39 } & \text { Boneyard/Burnyard } \\ \text { BY-40 } & \text { Boneyard/Burnyard } \\ \text { BY-41 } & \text { Boneyard/Burnyard } \\ \text { BY-43 } & \text { Boneyard/Burnyard } \\ \text { BY-44 } & \text { Boneyard/Burnyard } \\ \text { BY-45 } & \text { Boneyard/Burnyard }\end{array}$

\begin{tabular}{|c|c|c|c|c|c|c|}
\hline MAP & NORTHING & EASTING & SYSTEM & ELEVATION & T.O.W.W. & T.D. \\
\hline 4 & 29859.30 & 48272.24 & Y-12 & 964.16 & & 16.00 \\
\hline 4 & 29850.36 & 48358.34 & Y-12 & 966.90 & & 20.00 \\
\hline 4 & 29848.80 & 48407.92 & Y-12 & 966.45 & & 17.00 \\
\hline 4 & 29871.97 & 48502.49 & $\mathrm{Y}-12$ & 965.36 & & 12.00 \\
\hline 4 & 29778.67 & 48152.66 & Y-12 & 959.02 & & 12.00 \\
\hline 4 & 29968.28 & 48241.63 & Y-12 & 964.93 & & 11.00 \\
\hline 4 & 30137.39 & 48442.43 & Y-12 & 969.36 & & 15.00 \\
\hline 4 & 29700.82 & 48415.39 & $\mathrm{Y}-12$ & 969.32 & & 15.00 \\
\hline 4 & 29739.70 & 48598.95 & Y-12 & 972.41 & & 15.50 \\
\hline 4 & 29601.46 & 48431.25 & Y-12 & 967.21 & & 18.00 \\
\hline 4 & 29643.73 & 48605.22 & Y-12 & 969.95 & & 18.00 \\
\hline 4 & 29899.66 & 48407.27 & Y-12 & 966.71 & & 12.00 \\
\hline 4 & 29954.12 & 48383.19 & $\mathrm{Y}-12$ & 967.22 & & 16.00 \\
\hline 4 & 29550.84 & 48187.38 & Y-12 & 954.55 & & 12.00 \\
\hline 4 & 29551.37 & 48290.64 & Y-12 & 956.93 & & 12.00 \\
\hline 4 & 29532.89 & 48406.54 & $Y-12$ & 964.92 & & 13.50 \\
\hline 4 & 29542.13 & 48502.03 & Y-12 & 966.62 & & 16.00 \\
\hline 4 & 29551.56 & 48600.94 & $\mathrm{Y}-12$ & 966.21 & & 18.00 \\
\hline 4 & 30284.73 & 48469.67 & $\mathrm{Y}-12$ & 974.37 & & 16.00 \\
\hline 4 & 30316.65 & 48549.35 & Y-12 & 977.12 & & 16.00 \\
\hline 4 & 30393.17 & 48520.07 & Y-12 & 977.92 & & 7.00 \\
\hline 4 & 30364.83 & 48466.59 & $Y-12$ & 976.38 & & 14.00 \\
\hline 4 & 29429.03 & 48243.13 & Y-12 & 953.59 & & 12.00 \\
\hline 4 & 29471.49 & 48364.80 & $\mathrm{Y}-12$ & 957.93 & & 8.00 \\
\hline 4 & 29990.75 & 48439.09 & Y-12 & 966.17 & & 15.00 \\
\hline 4 & 29910.78 & 48447.63 & Y-12 & 966.13 & & 14.00 \\
\hline 4 & 29775.52 & 48049.28 & Y-12 & 960.61 & & 12.00 \\
\hline
\end{tabular}




\begin{tabular}{|c|c|c|c|c|c|c|c|c|c|}
\hline \multicolumn{10}{|l|}{ CURRENT } \\
\hline NAME & FUNCTIONAL AREA & $\underline{\text { MAP }}$ & $\underline{\text { NORTHING }}$ & EASTING & SYSTEM & ELEVATION & T.O.C. & T.O.W.W. & $\underline{\text { T.D. }}$ \\
\hline$B Y-46$ & Boneyard/Burnyard & 4 & 29766.93 & 48239.62 & $\mathrm{Y}-12$ & 961.94 & & & 16.00 \\
\hline BY-47 & Boneyard/Burnyard & 4 & 29684.92 & 48231.20 & $\mathrm{Y}-12$ & 959.40 & & & 16.00 \\
\hline BY-48 & Boneyard/Burnyard & 4 & 29521.15 & 48237.15 & $\mathrm{Y}-12$ & 955.87 & & & 16.00 \\
\hline BY-49 & Boneyard/Burnyard & 4 & 30048.83 & 48399.88 & $\mathrm{Y}-12$ & 968.81 & & & 17.00 \\
\hline BY-50 & Boneyard/Burnyard & 4 & 29671.18 & 48502.17 & $\mathrm{Y}-12$ & 969.80 & & & 25.50 \\
\hline C-1 & EMWMF & 4 & 31269.00 & 46118.00 & $\mathrm{Y}-12$ & 1036.20 & & & 42.00 \\
\hline C-19 & EMWMF & 4 & 30939.00 & 46959.00 & $\mathrm{Y}-12$ & 1030.80 & & & 25.20 \\
\hline$C-21$ & EMWMF & 4 & 31263.00 & 47119.00 & $\mathrm{Y}-12$ & 1040.90 & & & 44.00 \\
\hline C-25 & EMWMF & 4 & 31384.00 & 47312.00 & $\mathrm{Y}-12$ & 1061.80 & & & 75.00 \\
\hline$C-40$ & EMWMF & 4 & 30744.00 & 47978.00 & $Y-12$ & 1045.80 & & & 40.10 \\
\hline C-45 & EMWMF & 4 & 31545.00 & 46895.00 & $\mathrm{Y}-12$ & 1119.90 & & & 93.00 \\
\hline$C-48$ & EMWMF & 4 & 31583.00 & 47293.00 & $\mathrm{Y}-12$ & 1136.90 & & & 115.00 \\
\hline$C-50$ & EMWMF & 4 & 31734.00 & 47890.00 & $\mathrm{Y}-12$ & 1146.70 & & & 36.00 \\
\hline$C-51$ & EMWMF & 4 & 31601.00 & 47874.00 & $\mathrm{Y}-12$ & 1092.50 & & & 80.00 \\
\hline $\mathrm{CH}-001$ & Y-12 Plant Site & 5 & 29537.00 & 53859.00 & $Y-12$ & 996.60 & & & \\
\hline $\mathrm{CH}-002$ & S-3 Ponds & 4 & 29091.00 & 48528.00 & $\mathrm{Y}-12$ & 961.60 & & & \\
\hline $\mathrm{CH}-143$ & Kerr Hollow Quarry & 6 & 24254.00 & 63512.00 & $\mathrm{Y}-12$ & 910.72 & 910.92 & & 600.00 \\
\hline $\mathrm{CH}-157$ & $\begin{array}{l}\text { Chestnut Ridge Sediment } \\
\text { Disposal Basin }\end{array}$ & 6 & 27447.00 & 64069.00 & $\mathrm{Y}-12$ & 1031.90 & 1032.30 & & 540.00 \\
\hline $\mathrm{CH}-185$ & Rogers Quarry & 5 & 23391.00 & 56923.00 & $\mathrm{Y}-12$ & 828.89 & 829.85 & & 841.70 \\
\hline $\mathrm{CH}-189$ & Rogers Quarry & 5 & 24112.27 & 56788.23 & $\mathrm{Y}-12$ & 877.45 & 877.18 & & 763.00 \\
\hline $\mathrm{CO}-2$ & Bear Creek Road & 3 & 30086.32 & 38510.25 & $\mathrm{Y}-12$ & 985.30 & 989.90 & & 107.20 \\
\hline $\mathrm{CO}-4$ & Bear Creek Road & 3 & 29410.66 & 38525.22 & $\mathrm{Y}-12$ & 908.10 & 908.69 & & 57.00 \\
\hline DC WELL & Bear Creek Burial Grounds WMA & 3 & 29447.76 & 38945.32 & $\mathrm{Y}-12$ & 904.33 & 904.33 & & \\
\hline ED/Y-12 No. 2 & Y-12 Plant Site & & & & & & & & 250.00 \\
\hline EMW-VWUNDRDR & EMWMF & 4 & 30331.50 & 47316.50 & $\mathrm{Y}-12$ & & & & \\
\hline FP-001 & Y-12 Water Plant & 6 & 31204.00 & 62482.00 & $\mathrm{Y}-12$ & 1138.90 & & & 33.50 \\
\hline
\end{tabular}




\begin{tabular}{ll}
$\begin{array}{c}\text { CURRENT } \\
\text { NAME }\end{array}$ & FUNCTIONAL AREA \\
FP-002 & Y-12 Water Plant \\
FP-003 & Y-12 Water Plant \\
FP-004 & Y-12 Water Plant \\
FP-005 & Y-12 Water Plant \\
FP-006 & Y-12 Water Plant \\
FP-007 & Y-12 Water Plant \\
FP-008A & Y-12 Water Plant \\
FP-008B & Y-12 Water Plant \\
FP-009 & Y-12 Water Plant \\
FP-010 & Y-12 Water Plant \\
FP-011 & Y-12 Water Plant \\
FT-001 & Y-12 Water Storage Tanks \\
FT-002 & Y-12 Water Storage Tanks \\
FT-003 & Y-12 Water Storage Tanks \\
FW001 & S-3 Site \\
FW002 & S-3 Site \\
FW003 & S-3 Site \\
FW004 & S-3 Site \\
FW005 & S-3 Site \\
GW-001 & Oil Landfarm WMA \\
GW-002 & Oil Landfarm WMA \\
GW-003 & Oil Landfarm WMA \\
GW-004 & Oil Landfarm WMA \\
GW-005 & Oil Landfarm WMA \\
GW-006 & Oil Landfarm WMA \\
GW-007 & Oil Landfarm WMA \\
GW-008 & Oil Landfarm WMA \\
& \\
\hline
\end{tabular}

MAP NORTHING

EASTING

SYSTEM

ELEVATION

T.O.C. T.O.W.W.

T.D.

$\begin{array}{ll}31210.00 & 62501.00 \\ 31166.00 & 62494.00 \\ 31172.00 & 62513.00 \\ 31290.00 & 62755.00 \\ 31295.00 & 62771.00 \\ 31252.00 & 62764.00 \\ 31247.00 & 62787.00 \\ 31273.00 & 62774.00 \\ 31276.00 & 62757.00 \\ 31320.00 & 62822.00 \\ 31382.00 & 62792.00 \\ 28671.00 & 55522.00 \\ 28685.00 & 55398.00 \\ 28699.00 & 55275.00 \\ 29889.08 & 51061.38 \\ 29894.31 & 51103.46 \\ 29914.72 & 51169.05 \\ 29949.43 & 51315.10 \\ 30260.70 & 51953.04 \\ 30297.30 & 47528.80 \\ 30298.00 & 47538.00 \\ 30151.00 & 48148.00 \\ 29940.20 & 48235.70 \\ 29923.47 & 48240.90 \\ 29818.00 & 47988.20 \\ 29817.20 & 47974.50 \\ 29783.05 & 47595.92\end{array}$

Y-12
Y-12
Y-12
Y-12
Y-12
Y-12
Y-12
Y-12
Y-12
Y-12
Y-12
Y-12
Y-12
Y-12
Admin
Admin
Admin
Admin
Admin
Y-12
Y-12
Y-12
Y-12
Y-12
Y-12
Y-12
Y-12

1139.90

1132.80

1133.90

1137.20

1135.20

1132.70

1118.00

1135.40

1132.70

1130.60

1132.60

1168.30

1168.60

1165.20

995.44

995.93

997.45

999.45

1009.02

978.70

979.20

970.70

962.60

963.72

959.90

960.30

962.11
24.50

34.00

32.00

30.00

30.00

30.00

21.40

34.50

30.00

35.00

30.00

56.20

61.50

60.00

22.27

22.96

23.38

22.63

17.42

45.00

60.80

35.20

58.40

12.50

46.80

16.50

25.50 


\section{CURRENT}

NAME

GW-009

GW-010

GW-011

GW-012

GW-013

GW-014

GW-015

GW-016

GW-017

GW-018

GW-019

GW-020

GW-021

GW-022

GW-023

GW-024

GW-025

GW-025A

GW-026

GW-027

GW-028

GW-029

GW-030

GW-031

GW-032

GW-033

GW-034

\section{FUNCTIONAL AREA}

Oil Landfarm WMA

Oil Landfarm WMA

Oil Landfarm WMA

Oil Landfarm WMA

Oil Landfarm WMA

Bear Creek Burial Grounds WMA

Bear Creek Burial Grounds WMA

Bear Creek Burial Grounds WMA

Bear Creek Burial Grounds WMA

Bear Creek Burial Grounds WMA

Bear Creek Burial Grounds WMA

Bear Creek Burial Grounds WMA

Bear Creek Burial Grounds WMA

Bear Creek Burial Grounds WMA

Bear Creek Burial Grounds WMA

Bear Creek Burial Grounds WMA

Bear Creek Burial Grounds WMA

Bear Creek Burial Grounds WMA

Bear Creek Burial Grounds WMA

Bear Creek Burial Grounds WMA

Bear Creek Burial Grounds WMA

Bear Creek Burial Grounds WMA

Bear Creek Burial Grounds WMA

Bear Creek Burial Grounds WMA

Bear Creek Burial Grounds WMA

Bear Creek Burial Grounds WMA

Bear Creek Burial Grounds WMA

\section{MAP NORTHING}

EASTING

SYSTEM

ELEVATION

T.o.C.

T.O.W.W

$\underline{\text { T.D. }}$

$\begin{array}{rrrrr}29957.10 & 47564.70 & \text { Y-12 } & 960.72 & 962.09 \\ 29763.70 & 47214.00 & \text { Y-12 } & 950.10 & 952.70 \\ 29871.10 & 47164.00 & \text { Y-12 } & 948.90 & 953.00 \\ 29869.29 & 47187.88 & \text { Y-12 } & 952.77 & 954.97 \\ 30177.10 & 47334.10 & \text { Y-12 } & 962.29 & 964.52 \\ 29848.00 & 44308.00 & \text { Y-12 } & 931.50 & 933.90 \\ 29659.00 & 44331.00 & \text { Y-12 } & 922.00 & 925.60 \\ 29534.00 & 44198.00 & \text { Y-12 } & 926.47 & 928.20 \\ 29499.00 & 43939.00 & \text { Y-12 } & 926.40 & 930.30 \\ 29497.00 & 43615.00 & \text { Y-12 } & 921.59 & 924.49 \\ 29569.00 & 43273.00 & \text { Y-12 } & 917.40 & 919.24 \\ 29736.00 & 43332.00 & \text { Y-12 } & 919.70 & 922.10 \\ 29902.00 & 44356.00 & \text { Y-12 } & 920.70 & 924.00 \\ 30005.00 & 43323.00 & \text { Y-12 } & 923.11 & 926.15 \\ 30075.00 & 44097.00 & \text { Y-12 } & 951.80 & 954.60 \\ 30320.00 & 43757.00 & \text { Y-12 } & 955.20 & 957.80 \\ 30318.00 & 43754.00 & \text { Y-12 } & 955.00 & 957.28 \\ 30315.00 & 43761.00 & \text { Y-12 } & 955.09 & 957.80 \\ 30389.00 & 43760.00 & \text { Y-12 } & 961.50 & 964.10 \\ 30454.00 & 43741.00 & \text { Y-12 } & 961.60 & 965.30 \\ 30536.00 & 43899.00 & \text { Y-12 } & 979.10 & 981.80 \\ 30431.00 & 44501.00 & \text { Y-12 } & 984.70 & 968.10 \\ 30247.00 & 43110.00 & \text { Y-12 } & 970.20 & 973.60 \\ 30242.00 & 43109.00 & \text { Y-12 } & 969.90 & 973.60 \\ 30394.00 & 42850.00 & \text { Y-12 } & 999.00 & 1001.10 \\ 30540.00 & 42946.00 & \text { Y-12 } & 995.70 & 997.30 \\ 30601.00 & 43138.00 & \text { Y-12 } & 995.30 & 998.10\end{array}$

63.80

15.00

$953.58 \quad 60.80$

$955.57 \quad 15.50$

$965.14 \quad 33.80$

$934.50 \quad 13.20$

92881

61.50

20.80

62.20

66.10

15.00

52.40

55.00

75.70

61.70

54.00

20.20

30.50

21.40

22.40

40.00

70.00

62.50

38.00

45.50 


\begin{tabular}{|c|c|c|c|c|c|c|c|c|c|}
\hline \multicolumn{10}{|c|}{ CURRENT } \\
\hline$\underline{\text { NAME }}$ & FUNCTIONAL AREA & $\underline{\text { MAP }}$ & $\underline{\text { NORTHING }}$ & EASTING & SYSTEM & ELEVATION & T.O.C. & T.O.W.W. & $\underline{\text { T.D. }}$ \\
\hline GW-035 & Bear Creek Burial Grounds WMA & 4 & 30719.00 & 43178.00 & $\mathrm{Y}-12$ & 994.20 & 996.50 & & 62.00 \\
\hline GW-036 & Bear Creek Burial Grounds WMA & 4 & 30787.00 & 43257.00 & $\mathrm{Y}-12$ & 991.90 & 995.30 & & 39.10 \\
\hline GW-037 & Bear Creek Burial Grounds WMA & 4 & 30695.00 & 43342.00 & $\mathrm{Y}-12$ & 1002.30 & 1005.60 & & 70.00 \\
\hline GW-038 & Bear Creek Burial Grounds WMA & 4 & 30543.00 & 43371.00 & $\mathrm{Y}-12$ & 1004.50 & 1007.20 & & 52.50 \\
\hline GW-039 & Bear Creek Burial Grounds WMA & 4 & 30359.00 & 44584.00 & $\mathrm{Y}-12$ & 955.70 & 958.40 & & 22.50 \\
\hline GW-040 & Bear Creek Burial Grounds WMA & 4 & 31116.00 & 43355.00 & $\mathrm{Y}-12$ & 1003.80 & 1008.00 & 1008.55 & 35.00 \\
\hline GW-041 & Bear Creek Burial Grounds WMA & 4 & 31100.00 & 43736.00 & $\mathrm{Y}-12$ & 1004.30 & 1007.50 & 1008.10 & 40.00 \\
\hline GW-042 & Bear Creek Burial Grounds WMA & 4 & 31142.00 & 43935.00 & $\mathrm{Y}-12$ & 1000.00 & 1003.30 & 1003.91 & 30.00 \\
\hline GW-043 & Oil Landfarm WMA & 4 & 30523.33 & 47929.65 & $\mathrm{Y}-12$ & 1010.43 & 1013.44 & 1014.04 & 40.00 \\
\hline GW-044 & Oil Landfarm WMA & 4 & 30533.00 & 47917.00 & $\mathrm{Y}-12$ & 1010.40 & 1013.60 & 1014.20 & 70.00 \\
\hline GW-045 & Bear Creek Burial Grounds WMA & 4 & 29146.00 & 43353.00 & $\mathrm{Y}-12$ & 907.40 & 909.70 & 910.29 & 15.20 \\
\hline GW-046 & Bear Creek Burial Grounds WMA & 4 & 29562.34 & 43283.53 & $\mathrm{Y}-12$ & 918.13 & 920.57 & 921.17 & 20.50 \\
\hline GW-047 & Bear Creek Burial Grounds WMA & 4 & 29614.00 & 44499.00 & $\mathrm{Y}-12$ & 925.90 & 928.40 & 929.00 & 25.50 \\
\hline GW-048 & Bear Creek Burial Grounds WMA & 4 & 29390.00 & 44236.00 & $\mathrm{Y}-12$ & 915.00 & 918.20 & & 9.00 \\
\hline GW-049 & Bear Creek Burial Grounds WMA & 4 & 29249.00 & 44125.00 & $\mathrm{Y}-12$ & 917.70 & 920.60 & & 20.50 \\
\hline GW-050 & Bear Creek Burial Grounds WMA & 4 & 29078.00 & 44097.00 & $\mathrm{Y}-12$ & 912.20 & 914.70 & & 15.00 \\
\hline GW-051 & Bear Creek Burial Grounds WMA & 4 & 29056.00 & 43740.00 & $\mathrm{Y}-12$ & 906.60 & 910.20 & & 11.20 \\
\hline GW-052 & Bear Creek Burial Grounds WMA & 4 & 29052.00 & 43478.00 & $\mathrm{Y}-12$ & 903.40 & 905.70 & 905.70 & 19.50 \\
\hline GW-053 & Bear Creek Burial Grounds WMA & 4 & 29066.00 & 43086.00 & $\mathrm{Y}-12$ & 900.50 & 902.80 & 903.42 & 39.70 \\
\hline GW-054 & Bear Creek Burial Grounds WMA & 4 & 28823.00 & 41280.00 & $\mathrm{Y}-12$ & 890.00 & 893.50 & 894.10 & 40.00 \\
\hline GW-055 & Bear Creek Burial Grounds WMA & 4 & 28811.00 & 41283.00 & $\mathrm{Y}-12$ & 889.60 & 892.70 & & 20.60 \\
\hline GW-056 & Exit Pathway - Traverse A & 4 & 28708.00 & 41382.00 & $\mathrm{Y}-12$ & 886.80 & 890.90 & 891.67 & 55.20 \\
\hline GW-057 & Exit Pathway - Traverse A & 4 & 28699.00 & 41379.00 & $\mathrm{Y}-12$ & 887.10 & 889.60 & 890.20 & 25.00 \\
\hline GW-058 & Bear Creek Burial Grounds WMA & 4 & 28714.00 & 43211.00 & $\mathrm{Y}-12$ & 910.00 & 913.30 & 913.30 & 45.20 \\
\hline GW-059 & Bear Creek Burial Grounds WMA & 4 & 28702.00 & 43215.00 & $\mathrm{Y}-12$ & 910.00 & 912.70 & & 27.00 \\
\hline GW-060 & Bear Creek Burial Grounds WMA & 4 & 28927.00 & 43053.00 & $\mathrm{Y}-12$ & 900.80 & 904.60 & 905.20 & 50.00 \\
\hline GW-061 & Bear Creek Burial Grounds WMA & 4 & 28916.00 & 43049.00 & $\mathrm{Y}-12$ & 901.00 & 904.60 & 904.60 & 25.00 \\
\hline
\end{tabular}




\begin{tabular}{|c|c|c|c|c|c|c|c|c|c|}
\hline \multicolumn{10}{|c|}{ CURRENT } \\
\hline NAME & FUNCTIONAL AREA & $\underline{\text { MAP }}$ & NORTHING & EASTING & SYSTEM & ELEVATION & $\underline{\text { T.O.C. }}$ & T.O.W.W. & T.D. \\
\hline GW-062 & Oil Landfarm WMA & 4 & 29005.00 & 48258.00 & $\mathrm{Y}-12$ & 959.80 & 962.40 & 963.10 & 56.70 \\
\hline GW-063 & Oil Landfarm WMA & 4 & 29016.00 & 48257.00 & $\mathrm{Y}-12$ & 959.00 & 962.10 & & 35.00 \\
\hline GW-064 & Oil Landfarm WMA & $4 \& 5$ & 29195.00 & 49169.00 & Y-12 & 979.30 & 981.70 & 982.41 & 57.00 \\
\hline GW-065 & Oil Landfarm WMA & $4 \& 5$ & 29185.00 & 49167.00 & $\mathrm{Y}-12$ & 979.70 & 982.50 & & 35.00 \\
\hline GW-066 & Oil Landfarm WMA & 4 & 29513.36 & 48676.82 & $\mathrm{Y}-12$ & 959.58 & 961.63 & 961.63 & 55.80 \\
\hline GW-067 & Oil Landfarm WMA & 4 & 29504.00 & 48679.00 & $\mathrm{Y}-12$ & 957.10 & 961.60 & & 16.50 \\
\hline GW-068 & Bear Creek Burial Grounds WMA & 4 & 29500.00 & 43377.00 & $\mathrm{Y}-12$ & 921.20 & 924.00 & 924.61 & 85.00 \\
\hline GW-069 & Bear Creek Burial Grounds WMA & 4 & 29489.00 & 43802.00 & $\mathrm{Y}-12$ & 924.20 & 927.00 & 927.60 & 100.00 \\
\hline GW-070 & Bear Creek Burial Grounds WMA & 4 & 29491.00 & 43787.00 & $\mathrm{Y}-12$ & 924.00 & 926.40 & 926.98 & 148.00 \\
\hline GW-071 & Bear Creek Burial Grounds WMA & 4 & 29495.00 & 44191.00 & Y-12 & 925.40 & 928.30 & 928.90 & 220.60 \\
\hline GW-072 & Bear Creek Burial Grounds WMA & 4 & 29502.00 & 44159.00 & Y-12 & 926.30 & 929.90 & 930.51 & 101.40 \\
\hline GW-073 & Oil Landfarm WMA & 4 & 29753.00 & 46940.00 & $\mathrm{Y}-12$ & 948.40 & 951.10 & 951.81 & 81.00 \\
\hline GW-074 & Oil Landfarm WMA & 4 & 29870.40 & 47227.56 & $\mathrm{Y}-12$ & 956.03 & 958.32 & & 202.00 \\
\hline GW-075 & Oil Landfarm WMA & 4 & 29795.00 & 47988.00 & $\mathrm{Y}-12$ & 963.50 & 966.34 & 966.94 & 200.00 \\
\hline GW-076 & Oil Landfarm WMA & 4 & 29909.00 & 48238.00 & $\mathrm{Y}-12$ & 964.01 & 967.06 & & 81.00 \\
\hline GW-077 & Bear Creek Burial Grounds WMA & 4 & 29729.00 & 41234.00 & Y-12 & 914.70 & 918.70 & 919.30 & 100.50 \\
\hline GW-078 & Bear Creek Burial Grounds WMA & 4 & 29730.00 & 41209.00 & Y-12 & 914.50 & 917.50 & 918.10 & 21.10 \\
\hline GW-079 & Bear Creek Burial Grounds WMA & 4 & 30630.00 & 41616.00 & $\mathrm{Y}-12$ & 977.20 & 980.60 & 981.20 & 65.00 \\
\hline GW-080 & Bear Creek Burial Grounds WMA & 4 & 30622.00 & 41621.00 & Y-12 & 977.10 & 980.40 & 981.00 & 30.00 \\
\hline GW-081 & Bear Creek Burial Grounds WMA & 4 & 31001.00 & 43144.00 & Y-12 & 976.80 & 979.00 & 979.82 & 18.80 \\
\hline GW-082 & Bear Creek Burial Grounds WMA & 4 & 30433.80 & 42090.25 & $\mathrm{Y}-12$ & 960.52 & 963.40 & 964.00 & 35.00 \\
\hline GW-083 & Bear Creek Burial Grounds WMA & 4 & 29944.00 & 42436.00 & Y-12 & 934.30 & 938.00 & 938.60 & 30.00 \\
\hline GW-084 & Oil Landfarm WMA & 4 & 30456.00 & 48769.00 & $\mathrm{Y}-12$ & 993.70 & 996.60 & 997.18 & 34.00 \\
\hline GW-085 & Oil Landfarm WMA & $4 \& 5$ & 30003.00 & 49058.00 & $\mathrm{Y}-12$ & 979.80 & 982.90 & 983.57 & 62.00 \\
\hline GW-086 & Oil Landfarm WMA & $4 \& 5$ & 29994.00 & 49044.00 & $\mathrm{Y}-12$ & 979.50 & 982.80 & & 33.50 \\
\hline GW-087 & Oil Landfarm WMA & 4 & 29534.00 & 48313.00 & Y-12 & 957.30 & 960.60 & 961.18 & 19.00 \\
\hline GW-088 & Bear Creek Burial Grounds WMA & 4 & 30578.00 & 43768.00 & Y-12 & 956.66 & 959.47 & & 30.00 \\
\hline
\end{tabular}




\begin{tabular}{|c|c|c|c|c|c|c|c|c|c|}
\hline \multicolumn{10}{|c|}{ CURRENT } \\
\hline NAME & FUNCTIONAL AREA & $\underline{\text { MAP }}$ & $\underline{\text { NORTHING }}$ & EASTING & SYSTEM & ELEVATION & T.O.C. & T.O.W.W. & T.D. \\
\hline GW-089 & Bear Creek Burial Grounds WMA & 4 & 30924.00 & 43406.00 & $\mathrm{Y}-12$ & 962.48 & 964.36 & 964.99 & 25.00 \\
\hline GW-090 & Bear Creek Burial Grounds WMA & 4 & 30907.00 & 43868.00 & $\mathrm{Y}-12$ & 959.02 & 961.88 & & 15.00 \\
\hline GW-091 & Bear Creek Burial Grounds WMA & 4 & 30620.94 & 43620.64 & Y-12 & 949.82 & 952.62 & 952.62 & 25.00 \\
\hline GW-092 & Bear Creek Burial Grounds WMA & 4 & 30762.00 & 44139.00 & $\mathrm{Y}-12$ & 984.78 & 987.87 & & 25.00 \\
\hline GW-093 & Bear Creek Burial Grounds WMA & 4 & 29597.00 & 42904.00 & Y-12 & 941.10 & 943.70 & & 49.00 \\
\hline GW-093A & Bear Creek Burial Grounds WMA & 4 & 29593.00 & 42906.00 & Y-12 & 940.72 & 943.60 & & 49.00 \\
\hline GW-094 & Bear Creek Burial Grounds WMA & 4 & 29167.00 & 42912.00 & Y-12 & 909.40 & 913.30 & 913.55 & 131.00 \\
\hline GW-095 & Bear Creek Burial Grounds WMA & 4 & 29142.00 & 43364.00 & Y-12 & 908.20 & 911.30 & 911.54 & 156.00 \\
\hline GW-096 & Bear Creek Burial Grounds WMA & 4 & 29249.00 & 44123.00 & $\mathrm{Y}-12$ & 919.00 & 921.20 & 921.80 & 61.00 \\
\hline GW-096A & Bear Creek Burial Grounds WMA & 4 & 29254.00 & 44116.00 & $\mathrm{Y}-12$ & 918.92 & 921.41 & & 61.00 \\
\hline GW-097 & Oil Landfarm WMA & 4 & 29459.00 & 46959.00 & Y-12 & 941.90 & 944.70 & 945.41 & 19.20 \\
\hline GW-097A & Oil Landfarm WMA & 4 & 29462.00 & 46959.00 & $\mathrm{Y}-12$ & 941.79 & 944.79 & & 19.00 \\
\hline GW-098 & Oil Landfarm WMA & 4 & 29452.00 & 46959.00 & Y-12 & 942.40 & 945.70 & 945.95 & 104.00 \\
\hline GW-099 & Industrial Landfill IV & 5 & 28495.16 & 52073.50 & $\mathrm{Y}-12$ & 1178.67 & 1181.15 & & 127.00 \\
\hline GW-100 & S-3 Site & $4 \& 5$ & 29759.00 & 50957.00 & $\mathrm{Y}-12$ & 984.60 & 986.80 & 987.40 & 20.70 \\
\hline GW-101 & S-3 Site & 5 & 30241.00 & 51844.00 & Y-12 & 1005.10 & 1007.40 & 1007.40 & 17.50 \\
\hline GW-102 & S-3 Ponds & 5 & 29852.00 & 51810.00 & $\mathrm{Y}-12$ & 1003.00 & 1006.60 & & 24.00 \\
\hline GW-103 & S-3 Site & 5 & 30058.00 & 52291.00 & $\mathrm{Y}-12$ & 1008.20 & 1011.80 & & 25.00 \\
\hline GW-104 & S-3 Site & 5 & 30059.00 & 52303.00 & Y-12 & 1008.50 & 1012.00 & & 74.00 \\
\hline GW-105 & S-3 Site & 5 & 30417.00 & 52833.00 & $Y-12$ & 1014.30 & 1017.60 & 1018.20 & 17.00 \\
\hline GW-106 & S-3 Site & 5 & 30418.00 & 52843.00 & $Y-12$ & 1014.50 & 1016.70 & 1016.92 & 75.00 \\
\hline GW-107 & S-3 Site & 5 & 30080.00 & 53206.00 & $\mathrm{Y}-12$ & 995.90 & 998.40 & 999.00 & 14.20 \\
\hline GW-108 & S-3 Site & 5 & 30070.00 & 53207.00 & $\mathrm{Y}-12$ & 995.80 & 998.80 & 999.00 & 58.60 \\
\hline GW-109 & S-3 Site & 5 & 30056.00 & 53207.00 & $Y-12$ & 995.30 & 997.60 & 997.82 & 147.60 \\
\hline GW-110 & Grassy Creek & 2 & 28717.50 & 21987.30 & $\mathrm{Y}-12$ & 863.30 & 863.50 & & 40.00 \\
\hline GW-111 & Grassy Creek & & & & & & & & 300.00 \\
\hline GW-112 & Grassy Creek & 2 & 28651.91 & 22027.43 & $Y-12$ & 872.83 & 877.48 & & 245.00 \\
\hline
\end{tabular}




\section{CURRENT \\ NAME}

GW-113

GW-114

GW-115

GW-116

GW-117

GW-118

GW-119

GW-120

GW-121

GW-122

GW-123

GW-124

GW-125

GW-126

GW-127

GW-128

GW-129

GW-130

GW-131

GW-132

GW-133

GW-134

GW-135

GW-136

GW-137

GW-138

GW-139

\section{FUNCTIONAL AREA}

Grassy Creek

Bear Creek Road

S-3 Site

Bear Creek Burial Grounds WMA

Bear Creek Burial Grounds WMA

Bear Creek Burial Grounds WMA

Bear Creek Burial Grounds WMA

Oil Landfarm WMA

Oil Landfarm WMA

S-3 Site

S-3 Site

S-3 Site

S-3 Site

Bear Creek Burial Grounds WMA

S-3 Site

Scarboro Road

Scarboro Road

Scarboro Road

Scarboro Road

S-3 Site

S-3 Site

S-3 Site

S-3 Site

Gum Branch Road

Gum Branch Road

Gum Branch Road

Exxon Nuclear Site
MAP NORTHING EASTING

SYSTEM

ELEVATION

T.o.C.

T.O.W.W

T.D.

$\begin{array}{rrrrr}28658.52 & 22035.23 & \mathrm{Y}-12 & 872.87 & 874.79 \\ 28575.10 & 28100.10 & \mathrm{Y}-12 & 824.36 & 828.27 \\ 31073.48 & 52684.99 & \mathrm{Y}-12 & 1051.92 & 1054.41 \\ 29762.20 & 42912.90 & \mathrm{Y}-12 & 942.05 & 945.07 \\ 29183.30 & 42918.20 & \mathrm{Y}-12 & 909.10 & 912.16 \\ 29147.40 & 43404.50 & \mathrm{Y}-12 & 909.37 & 912.39 \\ 29253.80 & 44097.70 & \mathrm{Y}-12 & 918.92 & 921.91 \\ 29455.50 & 46942.50 & \mathrm{Y}-12 & 944.19 & 947.27 \\ 29798.90 & 47963.60 & \mathrm{Y}-12 & 963.46 & 966.57 \\ 29741.00 & 51806.70 & \mathrm{Y}-12 & 1004.15 & 1007.20 \\ 29741.70 & 51794.20 & \mathrm{Y}-12 & 1004.43 & 1007.45 \\ 29655.70 & 52223.20 & \mathrm{Y}-12 & 1003.98 & 1006.85 \\ 29646.10 & 52207.60 & \mathrm{Y}-12 & 1003.51 & 1006.78 \\ 29361.50 & 42678.70 & \mathrm{Y}-12 & 929.02 & 932.06 \\ 29850.00 & 51828.00 & \mathrm{Y}-12 & 1003.67 & 1005.90 \\ 30086.00 & 64422.00 & \mathrm{Y}-12 & 905.62 & 908.73 \\ 29455.00 & 64844.00 & \mathrm{Y}-12 & 921.66 & 924.73 \\ 28661.00 & 65090.00 & \mathrm{Y}-12 & 931.68 & 934.76 \\ 27989.00 & 65059.00 & \mathrm{Y}-12 & 1008.40 & 1011.30 \\ 31210.00 & 52805.00 & \mathrm{Y}-12 & 1051.00 & 1053.92 \\ 30659.00 & 52637.00 & \mathrm{Y}-12 & 1022.60 & 1025.86 \\ 29741.00 & 52533.00 & \mathrm{Y}-12 & 1002.50 & 1005.63 \\ 28731.00 & 53053.00 & \mathrm{Y}-12 & 1175.40 & 1177.78 \\ 29147.48 & 32120.20 & \mathrm{Y}-12 & 855.81 & 858.53 \\ 28712.00 & 32796.00 & \mathrm{Y}-12 & 832.88 & 836.11 \\ 27959.00 & 30825.00 & \mathrm{Y}-12 & 838.78 & 842.03 \\ 29076.42 & 22825.35 & \mathrm{Y}-12 & 891.56 & 894.82\end{array}$

160.50

120.00

$1055.01 \quad 53.00$

285.00

530.00

575.00

510.00

180.00

600.00

142.00

572.00

150.00

552.00

$\begin{array}{rr}932.22 & 155.00 \\ 1005.90 & 24.00\end{array}$

914.00

953.50

1088.50

1096.50

759.50

599.00

842.00

1275.00

800.00

1000.00

1100.00

981.00 


\begin{tabular}{|c|c|c|c|c|c|c|c|c|c|}
\hline \multicolumn{10}{|c|}{ CURRENT } \\
\hline NAME & FUNCTIONAL AREA & $\underline{\text { MAP }}$ & NORTHING & EASTING & SYSTEM & ELEVATION & T.O.C. & T.O.W.W. & T.D. \\
\hline GW-140 & Exxon Nuclear Site & 2 & 27870.50 & 22806.84 & $\mathrm{Y}-12$ & 1039.20 & 1042.40 & & 1200.00 \\
\hline GW-141 & Industrial Landfill IV & 5 & 28754.79 & 52463.45 & Y-12 & 1183.45 & 1186.06 & 1186.23 & 156.00 \\
\hline GW-142 & Kerr Hollow Quarry & 6 & 24524.00 & 64030.00 & $Y-12$ & 968.29 & 970.35 & 971.15 & 295.00 \\
\hline GW-143 & Kerr Hollow Quarry & 6 & 24257.00 & 63522.00 & $\mathrm{Y}-12$ & 911.04 & 913.18 & 913.98 & 253.00 \\
\hline GW-144 & Kerr Hollow Quarry & 6 & 24255.00 & 63502.00 & $\mathrm{Y}-12$ & 910.48 & 913.34 & 913.54 & 195.00 \\
\hline GW-145 & Kerr Hollow Quarry & 6 & 24441.00 & 63266.00 & $\mathrm{Y}-12$ & 837.29 & 840.04 & 840.24 & 110.00 \\
\hline GW-146 & Kerr Hollow Quarry & 6 & 24432.00 & 63272.00 & $\mathrm{Y}-12$ & 837.00 & 838.16 & & 220.00 \\
\hline GW-147 & Kerr Hollow Quarry & 6 & 24731.00 & 63428.00 & $\mathrm{Y}-12$ & 848.41 & 851.62 & & 69.00 \\
\hline GW-148 & New Hope Pond & 6 & 29202.00 & 63817.00 & $\mathrm{Y}-12$ & 904.53 & 907.36 & 907.76 & 11.10 \\
\hline GW-149 & New Hope Pond & 6 & 29201.00 & 63824.00 & $\mathrm{Y}-12$ & 904.76 & 907.72 & & 50.50 \\
\hline GW-150 & New Hope Pond & 6 & 28959.00 & 64222.00 & $Y-12$ & 912.88 & 915.56 & & 11.70 \\
\hline GW-151 & New Hope Pond & 6 & 28958.00 & 64232.00 & $\mathrm{Y}-12$ & 913.06 & 915.97 & 916.17 & 96.50 \\
\hline GW-152 & New Hope Pond & 6 & 28614.00 & 63718.00 & $\mathrm{Y}-12$ & 918.36 & 921.18 & & 17.30 \\
\hline GW-153 & New Hope Pond & 6 & 28613.00 & 63728.00 & $Y-12$ & 918.53 & 921.44 & 921.68 & 60.00 \\
\hline GW-154 & New Hope Pond & 6 & 28987.00 & 63346.00 & $Y-12$ & 908.60 & 911.50 & 911.70 & 11.20 \\
\hline GW-155 & $\begin{array}{l}\text { Chestnut Ridge Sediment } \\
\text { Disposal Basin }\end{array}$ & 6 & 27722.00 & 64333.00 & $Y-12$ & 1058.88 & 1060.80 & & 177.00 \\
\hline GW-156 & $\begin{array}{l}\text { Chestnut Ridge Sediment } \\
\text { Disposal Basin }\end{array}$ & 6 & 27626.00 & 64020.00 & $Y-12$ & 1046.94 & 1049.10 & 1049.28 & 157.60 \\
\hline GW-157 & $\begin{array}{l}\text { Chestnut Ridge Sediment } \\
\text { Disposal Basin }\end{array}$ & 6 & 27477.00 & 63892.00 & $\mathrm{Y}-12$ & 1044.85 & 1047.00 & & 145.00 \\
\hline GW-158 & $\begin{array}{l}\text { Chestnut Ridge Sediment } \\
\text { Disposal Basin }\end{array}$ & 6 & 27069.00 & 63643.00 & $\mathrm{Y}-12$ & 981.24 & 983.05 & & 441.00 \\
\hline GW-159 & $\begin{array}{l}\text { Chestnut Ridge Sediment } \\
\text { Disposal Basin }\end{array}$ & 6 & 27764.00 & 63496.00 & $\mathrm{Y}-12$ & 1048.79 & 1051.20 & 1051.38 & 157.00 \\
\hline GW-160 & $\begin{array}{l}\text { Chestnut Ridge Borrow Area } \\
\text { Waste Pile }\end{array}$ & 6 & 27803.25 & 62165.00 & $\mathrm{Y}-12$ & 1090.66 & 1093.09 & & 235.00 \\
\hline GW-161 & $\begin{array}{l}\text { Chestnut Ridge Borrow Area } \\
\text { Waste Pile }\end{array}$ & 6 & 27804.89 & 62146.45 & $\mathrm{Y}-12$ & 1090.91 & 1093.54 & & 400.00 \\
\hline
\end{tabular}




\begin{tabular}{|c|c|c|c|c|c|c|c|c|c|}
\hline \multicolumn{10}{|c|}{ CURRENT } \\
\hline NAME & FUNCTIONAL AREA & $\underline{\text { MAP }}$ & $\underline{\text { NORTHING }}$ & EASTING & SYSTEM & ELEVATION & T.O.C. & T.O.W.W. & T.D. \\
\hline GW-162 & Bear Creek Burial Grounds WMA & 4 & 31330.75 & 44480.91 & $\mathrm{Y}-12$ & 1038.06 & 1040.44 & 1041.21 & 125.00 \\
\hline GW-163 & Bear Creek Burial Grounds WMA & 4 & 31327.96 & 44470.26 & $\mathrm{Y}-12$ & 1038.31 & 1040.44 & & 225.00 \\
\hline GW-164 & Bear Creek Burial Grounds WMA & 4 & 31338.79 & 44476.62 & $Y-12$ & 1038.74 & 1040.23 & & 405.00 \\
\hline GW-165 & Chestnut Ridge/Deer Trap \#10 & 4 & 27807.00 & 44547.00 & $\mathrm{Y}-12$ & 1090.39 & 1091.40 & & 325.00 \\
\hline GW-166 & Chestnut Ridge/Deer Trap \#10 & 4 & 27835.00 & 44531.00 & $\mathrm{Y}-12$ & 1092.01 & 1093.30 & & 385.00 \\
\hline GW-167 & Scarboro Road & 6 & 28703.00 & 65146.00 & $\mathrm{Y}-12$ & 929.67 & 931.38 & 931.95 & 30.10 \\
\hline GW-168 & Scarboro Road & 6 & 28698.45 & 65167.20 & Y-12 & 929.30 & 932.21 & & 135.50 \\
\hline GW-169 & Union Valley - Exit Pathway & 6 & 28545.00 & 66854.00 & $\mathrm{Y}-12$ & 929.95 & 931.50 & 932.12 & 34.80 \\
\hline GW-170 & Union Valley - Exit Pathway & 6 & 28545.00 & 66843.00 & $\mathrm{Y}-12$ & 930.70 & 932.44 & 932.64 & 156.90 \\
\hline GW-171 & Union Valley - Exit Pathway & 6 & 28403.00 & 69654.00 & $\mathrm{Y}-12$ & 918.55 & 920.10 & 920.72 & 31.20 \\
\hline GW-172 & Union Valley - Exit Pathway & 6 & 28358.45 & 69578.94 & Y-12 & 922.85 & 926.37 & 926.69 & 133.90 \\
\hline GW-173 & Chestnut Ridge Security Pits & $5 \& 6$ & 28271.00 & 59472.00 & $\mathrm{Y}-12$ & 1112.97 & 1115.00 & 1115.00 & 165.00 \\
\hline GW-174 & Chestnut Ridge Security Pits & $5 \& 6$ & 28205.00 & 59215.00 & $\mathrm{Y}-12$ & 1114.06 & 1116.50 & 1116.66 & 145.00 \\
\hline GW-175 & Chestnut Ridge Security Pits & 5 & 28676.73 & 58686.23 & $\mathrm{Y}-12$ & 1081.89 & 1084.00 & 1084.19 & 166.70 \\
\hline GW-176 & Chestnut Ridge Security Pits & 5 & 28294.00 & 58450.00 & $Y-12$ & 1122.13 & 1125.30 & 1125.30 & 145.00 \\
\hline GW-177 & Chestnut Ridge Security Pits & 5 & 28483.00 & 57497.00 & Y-12 & 1155.52 & 1158.00 & 1158.20 & 145.00 \\
\hline GW-178 & Chestnut Ridge Security Pits & 5 & 28551.80 & 57807.73 & $\mathrm{Y}-12$ & 1141.06 & 1143.49 & 1143.49 & 133.00 \\
\hline GW-179 & Chestnut Ridge Security Pits & 5 & 28522.00 & 58569.00 & $\mathrm{Y}-12$ & 1124.33 & 1128.00 & 1128.00 & 117.00 \\
\hline GW-180 & Chestnut Ridge Security Pits & $5 \& 6$ & 28494.41 & 59219.61 & $Y-12$ & 1101.43 & 1103.97 & 1104.14 & 144.00 \\
\hline GW-181 & Chestnut Ridge Security Pits & 5 & 28047.66 & 57736.43 & $\mathrm{Y}-12$ & 1090.55 & 1093.03 & & 168.00 \\
\hline GW-182 & Chestnut Ridge Security Pits & 5 & 28373.34 & 57662.78 & Y-12 & 1147.20 & 1147.35 & & 402.10 \\
\hline GW-183 & Y-12 Fuel Station & 6 & 29658.59 & 61954.52 & $\mathrm{Y}-12$ & 943.01 & 946.02 & & 30.60 \\
\hline GW-184 & Rogers Quarry & 5 & 23915.00 & 57476.00 & $\mathrm{Y}-12$ & 924.65 & 927.63 & & 130.00 \\
\hline GW-185 & Rogers Quarry & 5 & 23392.00 & 56924.00 & $Y-12$ & 829.63 & 831.13 & & 471.50 \\
\hline GW-186 & Rogers Quarry & 5 & 23388.00 & 56862.00 & $\mathrm{Y}-12$ & 828.31 & 831.32 & & 171.00 \\
\hline GW-187 & Rogers Quarry & 5 & 23393.00 & 55980.00 & $\mathrm{Y}-12$ & 831.59 & 834.28 & & 162.00 \\
\hline GW-188 & Rogers Quarry & 5 & 23663.00 & 56174.00 & $Y-12$ & 834.33 & 837.09 & & 68.00 \\
\hline
\end{tabular}




\section{CURRENT}

NAME

GW-189

GW-190

GW-191

GW-192

GW-193

GW-194

GW-195

GW-196

GW-197

GW-198

GW-199

GW-200

GW-201

GW-202

GW-203

GW-204

GW-205

GW-206

GW-207

GW-208

GW-209

GW-210

GW-211

GW-212

GW-213

\section{FUNCTIONAL AREA}

Rogers Quarry

Y-12 Plant Site

Beta-4 Security Pits

Beta-4 Security Pits

Y-12 Plant Site

Beta-4 Security Pits

Beta-4 Security Pits

Beta-4 Security Pits

Beta-4 Security Pits

Ravine Disposal Site

Y-12 Grid Well I1

Ravine Disposal Site

Ravine Disposal Site

Ravine Disposal Site

United Nuclear Corporation Site

Y-12 Plant Site

United Nuclear Corporation Site

Exit Pathway Scarboro Road/Pine Ridge

Exit Pathway Scarboro Road/Pine Ridge

Exit Pathway Scarboro Road/Pine Ridge

U.S. Geological Survey Sites

U.S. Geological Survey Sites

U.S. Geological Survey Sites

U.S. Geological Survey Sites

U.S. Geological Survey Sites

\section{MAP NORTHING}

EASTING

SYSTEM

ELEVATION

T.O.C.

T.O.W.W

$\underline{\text { T.D. }}$

\begin{tabular}{|c|c|c|c|c|c|c|c|}
\hline 5 & 23389.00 & 56886.00 & Y-12 & 828.39 & 831.53 & & 210.00 \\
\hline 5 & 30776.49 & 53254.42 & Y-12 & 1030.19 & 1033.69 & 1033.69 & 26.20 \\
\hline 5 & 30780.00 & 54279.00 & Y-12 & 1007.10 & 1011.10 & 1011.27 & 60.50 \\
\hline 5 & 30772.00 & 54277.00 & $\mathrm{Y}-12$ & 1006.04 & 1008.60 & 1008.83 & 17.50 \\
\hline $5 \& 6$ & 29343.93 & 59536.17 & Y-12 & 931.11 & 934.00 & 934.17 & 18.45 \\
\hline 5 & 30583.00 & 54218.00 & $\mathrm{Y}-12$ & 992.47 & 995.36 & & 12.50 \\
\hline 5 & 30573.00 & 54089.00 & Y-12 & 1000.20 & 1002.90 & & 23.00 \\
\hline 5 & 30829.00 & 53906.00 & Y-12 & 1034.44 & 1037.20 & & 27.00 \\
\hline 5 & 30734.00 & 54087.00 & Y-12 & 1001.72 & 1004.60 & & 17.00 \\
\hline 6 & 30455.00 & 62466.00 & $\mathrm{Y}-12$ & 961.54 & 964.52 & & 26.50 \\
\hline 6 & 30283.00 & 62464.00 & $\mathrm{Y}-12$ & 958.06 & 961.08 & & 22.50 \\
\hline 6 & 30373.00 & 61906.00 & Y-12 & 966.09 & 969.09 & & 57.50 \\
\hline 6 & 30378.00 & 61914.00 & $\mathrm{Y}-12$ & 965.87 & 968.89 & & 21.00 \\
\hline 6 & 30597.00 & 61987.00 & $\mathrm{Y}-12$ & 964.22 & 968.02 & & 20.10 \\
\hline 5 & 28355.82 & 54190.48 & $\mathrm{Y}-12$ & 1102.34 & 1105.26 & 1105.45 & 156.00 \\
\hline 5 & 29955.91 & 57410.93 & $\mathrm{Y}-12$ & 955.47 & 958.57 & 958.74 & 17.50 \\
\hline 5 & 28362.98 & 54008.30 & $\mathrm{Y}-12$ & 1101.46 & 1103.97 & 1104.14 & 164.00 \\
\hline 6 & 31603.00 & 64019.00 & Y-12 & 894.48 & 894.56 & & 16.90 \\
\hline 6 & 31596.45 & 64023.39 & $\mathrm{Y}-12$ & 894.38 & 899.40 & 899.40 & 109.60 \\
\hline 6 & 31612.65 & 64007.55 & Y-12 & 894.55 & 898.05 & 898.05 & 412.80 \\
\hline 2 & 31775.00 & 27699.00 & $\mathrm{Y}-12$ & 792.96 & 799.43 & & 48.10 \\
\hline 2 & 31765.00 & 27700.00 & $\mathrm{Y}-12$ & 792.87 & 799.10 & & 124.00 \\
\hline 2 & 31800.00 & 27699.00 & $\mathrm{Y}-12$ & 793.14 & 796.26 & & 410.00 \\
\hline 1 & 27759.81 & 9095.51 & $\mathrm{Y}-12$ & 750.95 & 751.11 & & 13.80 \\
\hline 1 & 27717.81 & 9094.35 & $\mathrm{Y}-12$ & 751.03 & 751.75 & & 128.00 \\
\hline
\end{tabular}




\begin{tabular}{|c|c|c|c|c|c|c|c|c|c|}
\hline \multicolumn{10}{|c|}{ CURRENT } \\
\hline$\underline{\text { NAME }}$ & FUNCTIONAL AREA & $\underline{\text { MAP }}$ & NORTHING & EASTING & $\underline{\text { SYSTEM }}$ & ELEVATION & T.O.C. & T.O.W.W. & T.D. \\
\hline GW-214 & U.S. Geological Survey Sites & 1 & 27774.81 & 9094.56 & Y-12 & 750.78 & 753.87 & & 430.00 \\
\hline GW-215 & U.S. Geological Survey Sites & 1 & 29442.49 & 9178.80 & $\mathrm{Y}-12$ & 754.46 & 754.78 & & 15.20 \\
\hline GW-216 & U.S. Geological Survey Sites & 1 & 29457.48 & 9179.86 & $\mathrm{Y}-12$ & 754.79 & 755.12 & & 10.70 \\
\hline GW-217 & Industrial Landfill IV & 5 & 28757.85 & 53019.91 & $\mathrm{Y}-12$ & 1174.29 & 1176.86 & 1177.03 & 180.00 \\
\hline GW-218 & Uranium Oxide Vault & 5 & 29136.16 & 58877.64 & $\mathrm{Y}-12$ & 932.77 & 935.81 & 935.98 & 27.50 \\
\hline GW-219 & Uranium Oxide Vault & 5 & 29163.24 & 58928.95 & $\mathrm{Y}-12$ & 931.27 & 935.64 & 935.83 & 11.30 \\
\hline GW-220 & New Hope Pond & 6 & 28949.00 & 64225.00 & $\mathrm{Y}-12$ & 912.74 & 915.64 & 915.64 & 45.20 \\
\hline GW-221 & United Nuclear Corporation Site & 5 & 28359.44 & 54388.57 & $\mathrm{Y}-12$ & 1103.36 & 1106.00 & 1106.16 & 158.00 \\
\hline GW-222 & New Hope Pond & 6 & 28954.00 & 63324.00 & $\mathrm{Y}-12$ & 908.82 & 911.82 & 911.82 & 25.00 \\
\hline GW-223 & New Hope Pond & 6 & 28938.00 & 63311.00 & $\mathrm{Y}-12$ & 908.97 & 911.62 & 911.62 & 90.50 \\
\hline GW-224 & Rogers Quarry & 5 & 23392.00 & 55959.00 & $\mathrm{Y}-12$ & 832.47 & 835.04 & & 126.00 \\
\hline GW-225 & Oil Landfarm WMA & 4 & 29155.00 & 47461.00 & $\mathrm{Y}-12$ & 940.21 & 943.11 & 943.11 & 200.00 \\
\hline GW-226 & Oil Landfarm WMA & 4 & 29156.00 & 47473.00 & $\mathrm{Y}-12$ & 940.56 & 943.40 & 943.57 & 55.00 \\
\hline GW-227 & Oil Landfarm WMA & 4 & 29172.00 & 47802.00 & $\mathrm{Y}-12$ & 943.91 & 946.46 & 946.46 & 40.00 \\
\hline GW-228 & Oil Landfarm WMA & 4 & 29171.00 & 47791.00 & $\mathrm{Y}-12$ & 943.85 & 946.47 & 946.47 & 100.00 \\
\hline GW-229 & Oil Landfarm WMA & 4 & 29256.00 & 47017.00 & $\mathrm{Y}-12$ & 945.71 & 949.00 & 949.00 & 55.00 \\
\hline GW-230 & Union Valley - Exit Pathway & 6 & 28388.20 & 69616.86 & $\mathrm{Y}-12$ & 919.57 & 922.86 & 923.11 & 406.40 \\
\hline GW-231 & Kerr Hollow Quarry & 6 & 24725.00 & 63410.00 & $\mathrm{Y}-12$ & 846.90 & 849.47 & 849.67 & 35.00 \\
\hline GW-232 & Union Valley - Exit Pathway & 6 & 28546.00 & 66863.00 & $\mathrm{Y}-12$ & 929.52 & 931.22 & 931.39 & 411.70 \\
\hline GW-233 & Industrial Landfill IV & 5 & 28415.05 & 52595.86 & $\mathrm{Y}-12$ & 1179.44 & 1181.84 & & 170.00 \\
\hline GW-234 & Bear Creek Burial Grounds WMA & 4 & 30620.00 & 43621.00 & $\mathrm{Y}-12$ & 947.36 & 950.83 & & 16.50 \\
\hline GW-235 & U.S. Geological Survey Sites & 6 & 28416.00 & 69712.00 & $\mathrm{Y}-12$ & 919.99 & & & 68.00 \\
\hline GW-236 & S-3 Site & $4 \& 5$ & 29712.00 & 50453.00 & $\mathrm{Y}-12$ & 980.39 & 983.21 & 983.21 & 18.50 \\
\hline GW-237 & Bear Creek Burial Grounds WMA & 4 & 29244.02 & 44125.11 & $\mathrm{Y}-12$ & 918.16 & 921.02 & 921.02 & 13.70 \\
\hline GW-238 & U.S. Geological Survey Sites & 1 & 27810.82 & 9091.70 & $\mathrm{Y}-12$ & 750.97 & 752.06 & & 129.00 \\
\hline GW-239 & Scarboro Road & 6 & 28714.85 & 65088.59 & $\mathrm{Y}-12$ & 928.51 & 931.50 & & 433.30 \\
\hline GW-240 & New Hope Pond & 6 & 28604.00 & 63726.00 & $\mathrm{Y}-12$ & 919.50 & 922.90 & 922.90 & 29.50 \\
\hline
\end{tabular}




\begin{tabular}{|c|c|c|c|c|c|c|c|c|c|}
\hline \multicolumn{10}{|c|}{ CURRENT } \\
\hline$\underline{\text { NAME }}$ & FUNCTIONAL AREA & $\underline{\text { MAP }}$ & $\underline{\text { NORTHING }}$ & EASTING & $\underline{\text { SYSTEM }}$ & ELEVATION & T.O.C. & T.O.W.W. & T.D. \\
\hline GW-241 & $\begin{array}{l}\text { Chestnut Ridge Sediment } \\
\text { Disposal Basin }\end{array}$ & 6 & 27069.00 & 63659.00 & Y-12 & 980.80 & 982.64 & 982.84 & 103.00 \\
\hline GW-242 & Bear Creek Burial Grounds WMA & 4 & 31004.00 & 43144.00 & Y-12 & 974.78 & 978.12 & 978.69 & 17.00 \\
\hline GW-243 & S-3 Site & 5 & 30154.79 & 51990.37 & $\mathrm{Y}-12$ & 1008.58 & 1011.55 & 1012.22 & 77.00 \\
\hline GW-244 & S-3 Site & 5 & 30060.35 & 51974.16 & $\mathrm{Y}-12$ & 1006.88 & 1009.24 & 1009.24 & 77.00 \\
\hline GW-245 & S-3 Site & 5 & 29977.06 & 51992.00 & $\mathrm{Y}-12$ & 1006.06 & 1009.08 & 1009.08 & 76.00 \\
\hline GW-246 & S-3 Site & 5 & 29992.24 & 52098.35 & $\mathrm{Y}-12$ & 1006.07 & 1009.19 & 1009.19 & 76.00 \\
\hline GW-247 & S-3 Site & 5 & 30004.72 & 52181.21 & Y-12 & 1006.73 & 1009.61 & 1009.61 & 78.00 \\
\hline GW-248 & Bear Creek Burial Grounds WMA & 4 & 30142.73 & 43245.71 & Y-12 & 958.82 & 961.25 & & 62.00 \\
\hline GW-249 & Bear Creek Burial Grounds WMA & 4 & 30354.63 & 42747.63 & $\mathrm{Y}-12$ & 988.62 & 991.15 & & 35.10 \\
\hline GW-250 & Bear Creek Burial Grounds WMA & 4 & 30367.81 & 42737.56 & $\mathrm{Y}-12$ & 988.40 & 991.25 & & 61.70 \\
\hline GW-251 & S-2 Site & 5 & 29467.00 & 53843.00 & $\mathrm{Y}-12$ & 1001.60 & 1003.60 & 1003.80 & 51.00 \\
\hline GW-252 & S-2 Site & 5 & 29326.00 & 53920.00 & $\mathrm{Y}-12$ & 1022.66 & 1024.21 & & 49.00 \\
\hline GW-253 & S-2 Site & 5 & 29404.00 & 54057.00 & Y-12 & 1001.60 & 1003.99 & 1004.24 & 50.00 \\
\hline GW-254 & S-2 Site & 5 & 29546.00 & 54132.00 & $\mathrm{Y}-12$ & 994.19 & 996.70 & & 58.00 \\
\hline GW-255 & S-2 Site & 5 & 29330.00 & 53906.00 & $\mathrm{Y}-12$ & 1024.27 & 1027.13 & & 81.50 \\
\hline GW-256 & S-2 Site & 5 & 29542.00 & 54116.00 & Y-12 & 994.33 & 996.12 & 996.12 & 18.50 \\
\hline GW-257 & Bear Creek Burial Grounds WMA & 4 & 30147.60 & 43229.71 & $\mathrm{Y}-12$ & 959.21 & 961.68 & 961.68 & 33.70 \\
\hline GW-258 & Bear Creek Burial Grounds WMA & 4 & 30021.98 & 43141.47 & Y-12 & 941.19 & 943.66 & & 50.00 \\
\hline GW-259 & Bear Creek Burial Grounds WMA & 4 & 30014.68 & 43128.61 & $\mathrm{Y}-12$ & 941.48 & 943.80 & & 33.50 \\
\hline GW-260 & U.S. Geological Survey Sites & 1 & 27744.80 & 9096.45 & $\mathrm{Y}-12$ & 750.86 & 751.23 & & 134.00 \\
\hline GW-261 & Y-12 Grid Well A1 & 5 & 30913.89 & 53093.69 & $\mathrm{Y}-12$ & 1047.57 & 1049.99 & & 23.70 \\
\hline GW-262 & Y-12 Grid Well A1 & 5 & 30912.76 & 53106.09 & $\mathrm{Y}-12$ & 1047.32 & 1049.67 & & 70.60 \\
\hline GW-263 & Y-12 Grid Well A2 & 5 & 30885.30 & 53673.59 & Y-12 & 1055.13 & 1057.73 & & 30.80 \\
\hline GW-264 & Y-12 Grid Well A2 & 5 & 30871.59 & 53669.75 & $\mathrm{Y}-12$ & 1055.50 & 1058.30 & & 71.00 \\
\hline GW-265 & Y-12 Salvage Yard & 5 & 30762.58 & 53307.80 & $\mathrm{Y}-12$ & 1030.85 & 1032.68 & 1032.68 & 23.10 \\
\hline GW-266 & Y-12 Salvage Yard & 5 & 30699.09 & 53391.76 & $\mathrm{Y}-12$ & 1028.87 & 1031.50 & & 25.00 \\
\hline
\end{tabular}




\section{CURRENT \\ NAME}

GW-267

GW-268

GW-269

GW-270

GW-271

GW-272

GW-273

GW-274

GW-275

GW-276

GW-277

GW-278

GW-279

GW-280

GW-281

GW-282

GW-283

GW-284

GW-285

GW-286

GW-287

GW-288

GW-289

GW-290

GW-291

GW-292

GW-293

\section{FUNCTIONAL AREA}

Y-12 Salvage Yard

Y-12 Salvage Yard

Y-12 Salvage Yard

Y-12 Salvage Yard

Y-12 Salvage Yard

Y-12 Salvage Yard

Y-12 Salvage Yard

Y-12 Salvage Yard

Y-12 Salvage Yard

S-3 Site

S-3 Site

S-2 Site

S-2 Site

S-2 Site

Y-12 Fuel Station

Y-12 Fuel Station

Y-12 Fuel Station

$Y$-12 Fuel Station

Y-12 Fuel Station

Bear Creek Burial Grounds WMA

Bear Creek Burial Grounds WMA

Bear Creek Burial Grounds WMA

Bear Creek Burial Grounds WMA

Bear Creek Burial Grounds WMA

Bear Creek Burial Grounds WMA

East Chestnut Ridge Waste Pile

East Chestnut Ridge Waste Pile
MAP NORTHING

EASTING

SYSTEM

ELEVATION

T.O.C.

T.O.W.W.

T.D.

$\begin{array}{rrrrrrr}30700.21 & 53464.23 & \text { Y-12 } & 1028.81 & 1031.52 & & 27.00 \\ 30616.18 & 53607.92 & \text { Y-12 } & 1024.13 & 1026.47 & & 35.00 \\ 30649.34 & 53778.81 & \text { Y-12 } & 1025.38 & 1027.81 & 1027.81 & 30.00 \\ 30423.60 & 53236.32 & \text { Y-12 } & 1006.35 & 1008.96 & 1008.96 & 18.50 \\ 30434.56 & 53234.33 & \text { Y-12 } & 1006.49 & 1009.01 & 1009.01 & 56.30 \\ 30485.04 & 53736.95 & \text { Y-12 } & 1006.62 & 1009.16 & 1009.16 & 16.20 \\ 30201.24 & 53261.34 & \text { Y-12 } & 1001.34 & 1003.52 & 1003.52 & 33.10 \\ 30151.84 & 53672.75 & \text { Y-12 } & 992.94 & 995.43 & 995.60 & 35.00 \\ 30151.36 & 53687.52 & \text { Y-12 } & 993.08 & 995.36 & 995.53 & 65.50 \\ 29925.61 & 52557.47 & \text { Y-12 } & 998.70 & 1001.27 & 1001.57 & 18.50 \\ 29937.47 & 52564.63 & \text { Y-12 } & 999.05 & 1001.76 & 1001.76 & 77.40 \\ 29648.70 & 53593.47 & \text { Y-12 } & 994.66 & 997.13 & & 21.10 \\ 29638.53 & 53590.73 & \text { Y-12 } & 995.30 & 998.15 & & 76.40 \\ 29630.09 & 53589.11 & \text { Y-12 } & 995.42 & 997.81 & & 154.00 \\ 29771.37 & 61907.13 & \text { Y-12 } & 946.53 & 946.10 & & 17.50 \\ 29747.00 & 62002.00 & \text { Y-12 } & 944.69 & 943.94 & & 16.00 \\ 29570.00 & 61990.00 & \text { Y-12 } & 939.22 & 938.62 & & 20.00 \\ 29590.00 & 61925.00 & \text { Y-12 } & 942.30 & 941.61 & 941.21 & 18.00 \\ 29595.00 & 61856.00 & \text { Y-12 } & 942.88 & 942.46 & & 21.00 \\ 29993.36 & 42287.07 & \text { Y-12 } & 924.52 & 926.83 & 927.05 & 32.30 \\ 29988.80 & 42288.43 & \text { Y-12 } & 924.60 & 926.87 & 927.04 & 12.50 \\ 29975.41 & 42874.40 & \text { Y-12 } & 946.07 & 948.36 & 948.36 & 60.00 \\ 29981.56 & 42874.66 & \text { Y-12 } & 946.32 & 948.47 & 948.73 & 40.80 \\ 30444.80 & 42580.23 & \text { Y-12 } & 945.39 & 948.46 & & 32.50 \\ 30450.41 & 42582.81 & \text { Y-12 } & 944.53 & 948.46 & 948.66 & 14.20 \\ 28140.54 & 62146.29 & \text { Y-12 } & 1070.11 & 1073.00 & & 186.00 \\ 28111.53 & 62321.31 & \text { Y-12 } & 1061.70 & 1063.90 & & 214.00\end{array}$

30.00

5.50

6.20

3.10

(5.00

77.40

1.10

54.00

16.00

.

21.00

32.30

0.80

. 50

214.00 


\begin{tabular}{|c|c|c|c|c|c|c|c|c|c|}
\hline \multicolumn{10}{|c|}{ CURRENT } \\
\hline NAME & FUNCTIONAL AREA & $\underline{\text { MAP }}$ & $\underline{\text { NORTHING }}$ & EASTING & SYSTEM & ELEVATION & T.O.C. & T.O.W.W. & T.D. \\
\hline GW-294 & East Chestnut Ridge Waste Pile & 6 & 27957.97 & 62482.79 & Y-12 & 1083.67 & 1083.60 & & 128.00 \\
\hline GW-295 & $\begin{array}{l}\text { Chestnut Ridge Borrow Area } \\
\text { Waste Pile }\end{array}$ & 6 & 27801.75 & 62183.52 & Y-12 & 1090.42 & 1092.71 & & 146.00 \\
\hline GW-296 & East Chestnut Ridge Waste Pile & 6 & 27993.62 & 62023.27 & $\mathrm{Y}-12$ & 1088.29 & 1090.99 & & 147.00 \\
\hline GW-297 & East Chestnut Ridge Waste Pile & 6 & 27884.63 & 62056.93 & Y-12 & 1098.88 & 1101.30 & & 120.00 \\
\hline GW-298 & $\begin{array}{l}\text { Chestnut Ridge Borrow Area } \\
\text { Waste Pile }\end{array}$ & 6 & 27494.72 & 62444.64 & Y-12 & 1046.40 & 1049.01 & & 190.00 \\
\hline GW-299 & $\begin{array}{l}\text { Chestnut Ridge Borrow Area } \\
\text { Waste Pile }\end{array}$ & 6 & 27392.44 & 62319.28 & $\mathrm{Y}-12$ & 1051.33 & 1053.86 & & 168.00 \\
\hline GW-300 & $\begin{array}{l}\text { Chestnut Ridge Borrow Area } \\
\text { Waste Pile }\end{array}$ & 6 & 27487.18 & 62041.13 & Y-12 & 1070.69 & 1073.12 & 1073.12 & 147.00 \\
\hline GW-301 & $\begin{array}{l}\text { Chestnut Ridge Borrow Area } \\
\text { Waste Pile }\end{array}$ & 6 & 27661.71 & 61963.77 & $\mathrm{Y}-12$ & 1083.94 & 1086.38 & 1086.55 & 182.00 \\
\hline GW-302 & United Nuclear Corporation Site & 5 & 28693.55 & 54353.40 & $\mathrm{Y}-12$ & 1139.59 & 1141.67 & 1141.84 & 136.00 \\
\hline GW-303 & $\begin{array}{l}\text { Chestnut Ridge Sediment } \\
\text { Disposal Basin }\end{array}$ & 6 & 28099.09 & 63488.41 & $\mathrm{Y}-12$ & 1004.44 & 1007.16 & & 321.00 \\
\hline GW-304 & $\begin{array}{l}\text { Chestnut Ridge Sediment } \\
\text { Disposal Basin }\end{array}$ & 6 & 27545.33 & 63425.33 & $\mathrm{Y}-12$ & 1042.82 & 1045.49 & & 167.00 \\
\hline GW-305 & Industrial Landfill IV & 5 & 28547.52 & 52961.67 & Y-12 & 1181.07 & 1183.55 & 1183.72 & 179.60 \\
\hline GW-306 & Rust Spoil Area & $4 \& 5$ & 29250.41 & 49520.99 & Y-12 & 989.44 & 991.78 & 992.00 & 58.50 \\
\hline GW-307 & Rust Spoil Area & $4 \& 5$ & 29345.98 & 49655.17 & Y-12 & 991.01 & 993.14 & 993.14 & 41.60 \\
\hline GW-308 & Rust Spoil Area & $4 \& 5$ & 29398.90 & 49795.17 & $\mathrm{Y}-12$ & 991.12 & 993.80 & & 37.70 \\
\hline GW-309 & Rust Spoil Area & $4 \& 5$ & 29529.87 & 50176.33 & $\mathrm{Y}-12$ & 985.77 & 988.17 & 988.46 & 38.00 \\
\hline GW-310 & Rust Spoil Area & $4 \& 5$ & 29437.19 & 50497.34 & $\mathrm{Y}-12$ & 992.40 & 995.35 & 995.52 & 27.10 \\
\hline GW-311 & Rust Spoil Area & $4 \& 5$ & 29266.88 & 50125.50 & Y-12 & 996.43 & 999.35 & 999.52 & 40.30 \\
\hline GW-312 & Rust Spoil Area & $4 \& 5$ & 29215.52 & 49777.77 & Y-12 & 994.13 & 996.49 & 996.70 & 41.00 \\
\hline GW-313 & Spoil Area I & 5 & 29350.83 & 52015.69 & $\mathrm{Y}-12$ & 1056.60 & 1059.74 & 1059.74 & 113.00 \\
\hline GW-314 & Spoil Area I & 5 & 29418.94 & 52125.43 & Y-12 & 1047.20 & 1049.81 & & 115.00 \\
\hline GW-315 & Spoil Area I & 5 & 29454.61 & 52268.13 & $\mathrm{Y}-12$ & 1044.84 & 1047.28 & 1047.45 & 104.00 \\
\hline
\end{tabular}




\section{CURRENT}

NAME

GW-316

GW-317

GW-318

GW-319

GW-320

GW-321

GW-322

GW-323

GW-324

GW-325

GW-326

GW-327

GW-328

GW-329

GW-330

GW-331

GW-332

GW-333

GW-334

GW-335

GW-336

GW-337

GW-338

GW-339

GW-340

GW-341

GW-342

\section{FUNCTIONAL AREA}

Spoil Area I

Spoil Area I

Rogers Quarry

Rogers Quarry

Filled Coal Ash Pond

Filled Coal Ash Pond

Chestnut Ridge Security Pits

Spoil Area I

S-3 Site

S-3 Site

Bear Creek Burial Grounds WMA

Bear Creek Burial Grounds WMA

Bear Creek Burial Grounds WMA

Bear Creek Burial Grounds WMA

Bear Creek Burial Grounds WMA

Waste Coolant Processing Facility

Waste Coolant Processing Facility

Waste Coolant Processing Facility

Waste Coolant Processing Facility

Waste Coolant Processing Facility

Waste Coolant Processing Facility

Waste Coolant Processing Facility

Waste Coolant Processing Facility

United Nuclear Corporation Site

Bear Creek Burial Grounds WMA

Bear Creek Burial Grounds WMA

Bear Creek Burial Grounds WMA
MAP NORTHING

EASTING

SYSTEM

ELEVATION

T.O.C.

T.O.W.W.

T.D.

\begin{tabular}{|c|c|c|c|}
\hline 29335.65 & 52411.91 & Y-12 & 1044.73 \\
\hline 29284.67 & 52191.99 & Y-12 & 1060.80 \\
\hline 23413.00 & 56298.00 & $Y-12$ & 820.76 \\
\hline 23410.00 & 56328.00 & $Y-12$ & 819.96 \\
\hline 26253.00 & 57084.00 & $Y-12$ & 922.41 \\
\hline 26275.00 & 57026.00 & Y-12 & 923.10 \\
\hline 28240.69 & 58912.05 & Y-12 & 1131.81 \\
\hline 28985.00 & 52105.99 & $\mathrm{Y}-12$ & 1127.41 \\
\hline 30307.02 & 50700.49 & Y-12 & 1001.44 \\
\hline 30306.19 & 50688.80 & $Y-12$ & 1000.53 \\
\hline 30464.00 & 43674.00 & Y-12 & 942.30 \\
\hline 30432.00 & 43700.00 & $Y-12$ & 947.70 \\
\hline 30400.00 & 43674.00 & Y-12 & 941.30 \\
\hline 29674.00 & 43319.00 & $\mathrm{Y}-12$ & 919.60 \\
\hline 29711.00 & 43313.00 & $Y-12$ & 919.10 \\
\hline 30053.71 & 54827.43 & $Y-12$ & 980.79 \\
\hline 30058.41 & 54882.34 & Y-12 & 979.55 \\
\hline 30089.74 & 54878.05 & $Y-12$ & 980.12 \\
\hline 30116.84 & 54829.27 & $Y-12$ & 980.23 \\
\hline 30123.07 & 54692.10 & $Y-12$ & 981.10 \\
\hline 30056.93 & 54694.44 & $Y-12$ & 981.56 \\
\hline 30057.37 & 54518.63 & $Y-12$ & 984.12 \\
\hline 30133.96 & 54429.78 & Y-12 & 984.02 \\
\hline 28658.72 & 54146.52 & Y-12 & 1122.18 \\
\hline 29808.00 & 43348.00 & $Y-12$ & 920.50 \\
\hline 29934.00 & 43833.00 & $Y-12$ & 942.10 \\
\hline 31326.90 & 42608.87 & Y-12 & 1084.49 \\
\hline
\end{tabular}

80.00

1062.98

1063.18

132.00

823.90

823.03

925.32

925.58

1134.25

1130.11

1003.83

1003.00

944.60

950.11

944.84

922.46

921.07

981.50

981.07

983.83

983.73

981.88

985.92

987.31

987.27

1124.59

922.84

944.46

1087.10

1134.98

193.00

108.00

80.00

17.90

7.90

7.90

16.80

26.80

27.00

30.10

$981.24 \quad 24.10$

25.10

27.20

15.70

$985.92 \quad 21.40$

$987.48 \quad 22.10$

$987.44 \quad 17.60$

$1124.83 \quad 114.00$

12.80

18.00

75.00 


\begin{tabular}{|c|c|c|c|c|c|c|c|c|c|}
\hline \multicolumn{10}{|c|}{ CURRENT } \\
\hline NAME & FUNCTIONAL AREA & $\underline{\text { MAP }}$ & $\underline{\text { NORTHING }}$ & EASTING & SYSTEM & ELEVATION & T.O.C. & T.O.W.W. & T.D. \\
\hline GW-343 & Bear Creek Burial Grounds WMA & 4 & 31320.53 & 42597.28 & $\mathrm{Y}-12$ & 1083.73 & 1087.03 & & 185.00 \\
\hline GW-344 & Bear Creek Burial Grounds WMA & 4 & 31313.16 & 42583.85 & Y-12 & 1082.91 & 1084.83 & & 316.00 \\
\hline GW-345 & S-3 Site & $4 \& 5$ & 30029.09 & 50699.37 & Y-12 & 994.93 & 999.46 & 999.63 & 26.30 \\
\hline GW-346 & S-3 Site & $4 \& 5$ & 30029.99 & 50703.01 & $\mathrm{Y}-12$ & 995.08 & 995.65 & 995.82 & 65.30 \\
\hline GW-347 & S-3 Site & $4 \& 5$ & 29292.00 & 50750.11 & Y-12 & 997.76 & 1001.05 & 1001.25 & 27.80 \\
\hline GW-348 & S-3 Site & $4 \& 5$ & 29293.70 & 50763.42 & Y-12 & 998.00 & 1000.12 & 1000.32 & 80.90 \\
\hline GW-349 & S-2 Site & 5 & 29765.62 & 53587.96 & $\mathrm{Y}-12$ & 990.98 & 993.50 & 993.50 & 25.50 \\
\hline GW-350 & S-2 Site & 5 & 29763.86 & 53595.10 & $\mathrm{Y}-12$ & 991.00 & 993.51 & 993.51 & 46.00 \\
\hline GW-363 & Oil Landfarm WMA & 4 & 29961.35 & 46871.80 & $\mathrm{Y}-12$ & 955.41 & 957.91 & 957.91 & 75.00 \\
\hline GW-364 & Industrial Landfill I & 4 & 29151.79 & 46507.65 & $\mathrm{Y}-12$ & 933.39 & 935.95 & 936.16 & 60.30 \\
\hline GW-365 & Industrial Landfill I & 4 & 29149.64 & 46490.30 & $\mathrm{Y}-12$ & 933.03 & 935.58 & 935.58 & 150.00 \\
\hline GW-366 & Industrial Landfill I & 4 & 28885.99 & 46707.72 & $\mathrm{Y}-12$ & 985.66 & 988.79 & & 102.50 \\
\hline GW-367 & Industrial Landfill I & 4 & 28884.20 & 46695.35 & $Y-12$ & 986.21 & 988.16 & & 151.40 \\
\hline GW-368 & Industrial Landfill I & 4 & 28912.85 & 47617.59 & $\mathrm{Y}-12$ & 998.63 & 1000.53 & 1000.53 & 245.00 \\
\hline GW-369 & Industrial Landfill I & 4 & 28920.62 & 47628.84 & $Y-12$ & 997.86 & 999.72 & 1000.36 & 150.20 \\
\hline GW-370 & Bear Creek Burial Grounds WMA & 4 & 30240.77 & 44988.50 & Y-12 & 957.94 & 960.62 & 960.81 & 33.10 \\
\hline GW-371 & Bear Creek Burial Grounds WMA & 4 & 30245.39 & 45035.53 & $\mathrm{Y}-12$ & 958.13 & 960.22 & & 125.00 \\
\hline GW-372 & Bear Creek Burial Grounds WMA & 4 & 30672.53 & 45256.97 & Y-12 & 979.87 & 983.00 & 983.16 & 51.60 \\
\hline GW-373 & Bear Creek Burial Grounds WMA & 4 & 30679.17 & 45271.21 & $\mathrm{Y}-12$ & 980.75 & 982.80 & & 158.00 \\
\hline GW-374 & Bear Creek Burial Grounds WMA & 4 & 29619.08 & 44485.89 & $\mathrm{Y}-12$ & 925.29 & 926.94 & & 150.10 \\
\hline GW-375 & Bear Creek Burial Grounds WMA & 4 & 29278.09 & 44135.70 & $\mathrm{Y}-12$ & 919.43 & 920.83 & & 161.50 \\
\hline GW-376 & Lysimeter Demo & $4 \& 5$ & 30467.09 & 49491.29 & $\mathrm{Y}-12$ & 998.35 & 1002.27 & & 218.00 \\
\hline GW-380 & New Hope Pond & 6 & 28714.28 & 62938.21 & $\mathrm{Y}-12$ & 913.66 & 913.55 & 913.55 & 15.50 \\
\hline GW-381 & New Hope Pond & 6 & 28715.04 & 62947.70 & $Y-12$ & 913.44 & 913.36 & 913.36 & 60.40 \\
\hline GW-382 & New Hope Pond & 6 & 28715.79 & 62956.12 & $\mathrm{Y}-12$ & 913.16 & 913.17 & & 173.00 \\
\hline GW-383 & New Hope Pond & 6 & 29201.44 & 63522.39 & $\mathrm{Y}-12$ & 906.00 & 908.50 & 908.77 & 24.10 \\
\hline GW-384 & New Hope Pond & 6 & 29215.69 & 63529.54 & $\mathrm{Y}-12$ & 905.76 & 908.38 & 909.23 & 55.70 \\
\hline
\end{tabular}




$\begin{array}{cl}\begin{array}{c}\text { CURRENT } \\ \text { NAME }\end{array} & \text { FUNCTIONAL AREA } \\ \text { GW-385 } & \text { New Hope Pond } \\ \text { GW-400 } & \text { Gum Branch Road } \\ \text { GW-401 } & \text { Gum Branch Road } \\ \text { GW-402 } & \text { Gum Branch Road } \\ \text { GW-403 } & \text { Gum Branch Road } \\ \text { GW-404 } & \text { Gum Branch Road } \\ \text { GW-405 } & \text { Gum Branch Road } \\ \text { GW-406 } & \text { Gum Branch Road } \\ \text { GW-407 } & \text { Gum Branch Road } \\ \text { GW-408 } & \text { Gum Branch Road } \\ \text { GW-409 } & \text { Gum Branch Road } \\ \text { GW-410 } & \text { Gum Branch Road } \\ \text { GW-411 } & \text { Gum Branch Road } \\ \text { GW-412 } & \text { Gum Branch Road } \\ \text { GW-413 } & \text { Gum Branch Road } \\ \text { GW-414 } & \text { Gum Branch Road } \\ \text { GW-415 } & \text { Gum Branch Road } \\ \text { GW-416 } & \text { Gum Branch Road } \\ \text { GW-417 } & \text { Gum Branch Road } \\ \text { GW-418 } & \text { Gum Branch Road } \\ \text { GW-419 } & \text { Gum Branch Road } \\ \text { GW-420 } & \text { Gum Branch Road } \\ \text { GW-421 } & \text { Gum Branch Road } \\ \text { GW-422 } & \text { Gum Branch Road } \\ \text { GW-423 } & \text { Gum Branch Road } \\ \text { GW-424 } & \text { Gum Branch Road } \\ \text { GW-425 } & \text { Gum Branch Road }\end{array}$

\begin{tabular}{|c|c|c|c|c|c|c|c|}
\hline MAP & NORTHING & EASTING & SYSTEM & ELEVATION & T.O.C. & T.O.W.W. & T.D. \\
\hline 6 & 29208.16 & 63525.77 & Y-12 & 905.87 & 908.24 & 908.98 & 178.70 \\
\hline 2 & 29926.99 & 28651.66 & $Y-12$ & 838.77 & 844.59 & & 1251.00 \\
\hline 2 & 29438.48 & 28709.57 & Y-12 & 834.80 & 836.24 & & 862.60 \\
\hline 2 & 28869.60 & 28805.33 & $Y-12$ & 807.96 & 811.06 & & 600.00 \\
\hline $2 \& 3$ & 29520.96 & 30479.75 & Y-12 & 835.43 & 839.22 & & 613.00 \\
\hline $2 \& 3$ & 29870.29 & 30636.05 & Y-12 & 852.30 & 852.81 & & 200.00 \\
\hline 2 & 30323.58 & 28253.06 & Y-12 & 813.79 & 815.51 & & 35.00 \\
\hline 2 & 30330.43 & 28247.33 & Y-12 & 813.90 & 815.94 & & 6.80 \\
\hline 2 & 30434.65 & 28828.24 & $Y-12$ & 832.17 & 834.22 & & 40.60 \\
\hline 2 & 30442.79 & 28837.92 & Y-12 & 832.33 & 834.50 & & 6.50 \\
\hline 2 & 30289.30 & 28916.54 & $Y-12$ & 875.49 & 877.74 & & 58.20 \\
\hline 2 & 30283.26 & 28916.09 & Y-12 & 875.49 & 877.75 & & 16.80 \\
\hline $2 \& 3$ & 30637.33 & 29868.24 & $Y-12$ & 862.18 & 864.25 & & 8.70 \\
\hline $2 \& 3$ & 30629.78 & 29868.86 & $Y-12$ & 862.00 & 864.05 & & 40.10 \\
\hline $2 \& 3$ & 30211.02 & 29578.32 & $Y-12$ & 891.22 & 893.15 & & 15.00 \\
\hline $2 \& 3$ & 30199.96 & 29585.74 & $Y-12$ & 891.72 & 893.53 & & 55.80 \\
\hline $2 \& 3$ & 30099.66 & 30018.45 & Y-12 & 894.39 & 896.59 & & 27.80 \\
\hline $2 \& 3$ & 30096.84 & 30022.85 & $Y-12$ & 894.64 & 896.66 & & 61.20 \\
\hline $2 \& 3$ & 29758.11 & 29855.75 & $Y-12$ & 867.33 & 869.16 & & 49.10 \\
\hline $2 \& 3$ & 29752.14 & 29862.24 & Y-12 & 866.98 & 868.69 & & 21.20 \\
\hline $2 \& 3$ & 29473.36 & 29425.25 & $Y-12$ & 834.66 & 836.29 & & 50.00 \\
\hline $2 \& 3$ & 29473.57 & 29439.80 & $Y-12$ & 835.07 & 837.11 & & 14.00 \\
\hline 2 & 28850.04 & 28843.60 & $Y-12$ & 808.32 & 809.77 & & 39.00 \\
\hline 2 & 28849.53 & 28855.92 & Y-12 & 807.42 & 809.09 & & 7.70 \\
\hline $2 \& 3$ & 28762.24 & 29314.19 & $Y-12$ & 814.27 & 816.39 & & 39.00 \\
\hline $2 \& 3$ & 28773.84 & 29299.44 & $Y-12$ & 814.33 & 816.28 & & 4.50 \\
\hline $2 \& 3$ & 29037.15 & 29867.41 & Y-12 & 841.27 & 842.98 & & 60.00 \\
\hline
\end{tabular}




$\begin{array}{cc}\begin{array}{c}\text { CURRENT } \\ \text { NAME }\end{array} & \text { FUNCTIONAL AREA } \\ \text { GW-426 } & \text { Gum Branch Road } \\ \text { GW-427 } & \text { Gum Branch Road } \\ \text { GW-428 } & \text { Gum Branch Road } \\ \text { GW-429 } & \text { Gum Branch Road } \\ \text { GW-430 } & \text { Gum Branch Road } \\ \text { GW-431 } & \text { Gum Branch Road } \\ \text { GW-432 } & \text { Gum Branch Road } \\ \text { GW-433 } & \text { Gum Branch Road } \\ \text { GW-434 } & \text { Gum Branch Road } \\ \text { GW-435 } & \text { Gum Branch Road } \\ \text { GW-436 } & \text { Gum Branch Road } \\ \text { GW-437 } & \text { Gum Branch Road } \\ \text { GW-438 } & \text { Gum Branch Road } \\ \text { GW-439 } & \text { Gum Branch Road } \\ \text { GW-440 } & \text { Gum Branch Road } \\ \text { GW-441 } & \text { Gum Branch Road } \\ \text { GW-442 } & \text { Gum Branch Road } \\ \text { GW-443 } & \text { Gum Branch Road } \\ \text { GW-445 } & \text { Gum Branch Road } \\ \text { GW-447 } & \text { Gum Branch Road } \\ \text { GW-448 } & \text { Gum Branch Road } \\ \text { GW-449 } & \text { Gum Branch Road } \\ \text { GW-450 } & \text { Gum Branch Road } \\ \text { GW-451 } & \text { Gum Branch Road } \\ \text { GW-452 } & \text { Gum Branch Road } \\ \text { GW-453 } & \text { Gum Branch Road } \\ \text { GW-455 } & \text { Gum Branch Road } \\ & \end{array}$

\begin{tabular}{|c|c|c|c|c|c|c|c|}
\hline MAP & NORTHING & EASTING & SYSTEM & ELEVATION & T.O.C. & T.O.W.W. & T.D. \\
\hline $2 \& 3$ & 29042.83 & 29872.19 & Y-12 & 841.47 & 843.22 & & 31.30 \\
\hline $2 \& 3$ & 28682.11 & 30210.83 & Y-12 & 815.96 & 817.79 & & 48.00 \\
\hline $2 \& 3$ & 28678.78 & 30217.99 & Y-12 & 815.97 & 817.84 & & 14.00 \\
\hline $2 \& 3$ & 29521.94 & 30471.44 & Y-12 & 835.40 & 837.16 & & 5.90 \\
\hline $2 \& 3$ & 29522.24 & 30463.95 & Y-12 & 834.87 & 836.87 & & 38.20 \\
\hline $2 \& 3$ & 30289.41 & 30828.81 & Y-12 & 862.78 & 864.86 & & 9.00 \\
\hline $2 \& 3$ & 30283.78 & 30821.16 & Y-12 & 862.08 & 864.43 & & 43.80 \\
\hline 3 & 30389.77 & 31192.07 & Y-12 & 869.58 & 870.84 & & 13.74 \\
\hline 3 & 30397.54 & 31195.83 & Y-12 & 869.49 & 871.43 & & 40.30 \\
\hline 3 & 30252.31 & 31298.41 & Y-12 & 902.56 & 904.36 & & 9.00 \\
\hline 3 & 30250.71 & 31291.04 & Y-12 & 902.23 & 904.03 & & 45.50 \\
\hline 3 & 29960.52 & 31457.62 & Y-12 & 891.09 & 892.99 & & 63.20 \\
\hline 3 & 29967.91 & 31456.01 & Y-12 & 891.53 & 893.25 & & 22.95 \\
\hline 3 & 29582.44 & 31447.29 & Y-12 & 868.28 & 870.05 & & 60.00 \\
\hline 3 & 29580.31 & 31453.12 & Y-12 & 868.18 & 870.13 & & 26.70 \\
\hline 3 & 28967.71 & 31214.63 & Y-12 & 836.54 & 838.09 & & 55.00 \\
\hline 3 & 28966.43 & 31206.25 & Y-12 & 837.10 & 839.32 & & 14.80 \\
\hline 3 & 28712.83 & 31880.66 & Y-12 & 828.11 & 830.89 & & 50.00 \\
\hline 3 & 29249.45 & 31805.39 & Y-12 & 843.57 & 846.00 & & 47.00 \\
\hline 3 & 29893.72 & 31744.64 & Y-12 & 872.64 & 874.25 & & 8.55 \\
\hline 3 & 29885.05 & 31738.31 & Y-12 & 872.23 & 873.86 & & 44.50 \\
\hline 3 & 30422.63 & 31713.87 & Y-12 & 934.21 & 935.82 & & 12.20 \\
\hline 3 & 30430.33 & 31726.04 & Y-12 & 933.84 & 935.47 & & 56.00 \\
\hline 3 & 30919.40 & 32478.64 & Y-12 & 928.77 & 931.46 & & 300.00 \\
\hline 3 & 29767.95 & 32590.72 & Y-12 & 872.73 & 874.79 & & 19.00 \\
\hline $2 \& 3$ & 30298.35 & 30814.34 & Y-12 & 862.27 & 863.59 & & 810.80 \\
\hline $2 \& 3$ & 29765.95 & 30676.25 & Y-12 & 853.44 & 855.75 & & 293.20 \\
\hline
\end{tabular}




$\begin{array}{cc}\begin{array}{c}\text { CURRENT } \\ \text { NAME }\end{array} & \text { FUNCTIONAL AREA } \\ \text { GW-456 } & \text { Gum Branch Road } \\ \text { GW-457 } & \text { Gum Branch Road } \\ \text { GW-458 } & \text { Gum Branch Road } \\ \text { GW-459 } & \text { Gum Branch Road } \\ \text { GW-460 } & \text { Gum Branch Road } \\ \text { GW-461 } & \text { Gum Branch Road } \\ \text { GW-462 } & \text { Gum Branch Road } \\ \text { GW-463 } & \text { Gum Branch Road } \\ \text { GW-464 } & \text { Gum Branch Road } \\ \text { GW-465 } & \text { Gum Branch Road } \\ \text { GW-466 } & \text { Gum Branch Road } \\ \text { GW-467 } & \text { Gum Branch Road } \\ \text { GW-468 } & \text { Gum Branch Road } \\ \text { GW-469 } & \text { Gum Branch Road } \\ \text { GW-470 } & \text { Gum Branch Road } \\ \text { GW-471 } & \text { Gum Branch Road } \\ \text { GW-472 } & \text { Gum Branch Road } \\ \text { GW-473 } & \text { Gum Branch Road } \\ \text { GW-474 } & \text { Gum Branch Road } \\ \text { GW-475A } & \text { Gum Branch Road } \\ \text { GW-475B } & \text { Gum Branch Road } \\ \text { GW-475C } & \text { Gum Branch Road } \\ \text { GW-476A } & \text { Gum Branch Road } \\ \text { GW-476B } & \text { Gum Branch Road } \\ \text { GW-476C } & \text { Gum Branch Road } \\ \text { GW-477A } & \text { Gum Branch Road } \\ \text { GW-477B } & \text { Gum Branch Road }\end{array}$

MAP NORTHING E

$\begin{array}{cccccc}2 \text { \& } 3 & 29621.30 & 29259.87 & \mathrm{Y}-12 & 842.73 & 844.88 \\ 2 \text { \& 3 } & 29621.30 & 29259.87 & \mathrm{Y}-12 & 842.73 & 845.36 \\ 2 \text { \& 3 } & 29581.22 & 29261.56 & \mathrm{Y}-12 & 842.72 & 844.68 \\ 2 \text { \& 3 } & 29581.22 & 29261.56 & \mathrm{Y}-12 & 842.72 & 845.18 \\ 2 \text { \& 3 } & 29601.91 & 29210.49 & \mathrm{Y}-12 & 840.22 & 842.15 \\ 2 \text { \& 3 } & 29601.91 & 29210.49 & \mathrm{Y}-12 & 840.22 & 842.55 \\ 2 \text { \& 3 } & 29601.15 & 29260.50 & \mathrm{Y}-12 & 842.46 & 843.32 \\ 2 \text { \& 3 } & 28679.66 & 30111.11 & \mathrm{Y}-12 & 815.19 & 816.87 \\ 2 \text { \& 3 } & 28688.53 & 30111.26 & \mathrm{Y}-12 & 815.80 & 817.32 \\ 2 \text { \& 3 } & 28708.36 & 30154.55 & \mathrm{Y}-12 & 816.50 & 817.74 \\ 2 \text { \& 3 } & 28650.90 & 30161.30 & \mathrm{Y}-12 & 816.63 & 817.84 \\ 2 \text { \& 3 } & 28678.88 & 30159.43 & \mathrm{Y}-12 & 816.36 & 818.09 \\ 2 \text { \& 3 } & 29040.14 & 30210.77 & \mathrm{Y}-12 & 826.19 & 829.12 \\ 2 & 29440.48 & 28678.32 & \mathrm{Y}-12 & 833.76 & 834.48 \\ 2 & 28935.46 & 28802.90 & \mathrm{Y}-12 & 808.63 & 810.97 \\ 2 \text { \& 3 } & 29875.29 & 30721.63 & \mathrm{Y}-12 & 864.14 & 865.46 \\ 3 & 29145.47 & 32157.27 & \mathrm{Y}-12 & 856.69 & 859.25 \\ 2 \text { \& 3 } & 29900.16 & 30737.60 & \mathrm{Y}-12 & 868.60 & 868.93 \\ 2 \text { \& 3 } & 29900.64 & 30743.32 & \mathrm{Y}-12 & 868.87 & 870.03 \\ 2 \text { \& 3 } & 29880.60 & 30737.01 & \mathrm{Y}-12 & 868.63 & 870.20 \\ 2 \text { \& 3 } & 29880.60 & 30737.01 & \mathrm{Y}-12 & 868.63 & 870.70 \\ 2 \text { \& 3 } & 29880.60 & 30737.01 & \mathrm{Y}-12 & 868.63 & 870.83 \\ 2 \text { \& 3 } & 29899.50 & 30761.82 & \mathrm{Y}-12 & 869.91 & 871.86 \\ 2 \text { \& 3 } & 29899.50 & 30761.82 & \mathrm{Y}-12 & 869.91 & 872.72 \\ 2 \text { \& 3 } & 29899.50 & 30761.82 & \mathrm{Y}-12 & 869.91 & 873.45 \\ 2 \text { \& 3 } & 29920.10 & 30738.54 & \mathrm{Y}-12 & 868.33 & 870.13 \\ 2 \text { \& 3 } & 29920.10 & 30738.54 & \mathrm{Y}-12 & 868.33 & 870.75\end{array}$

T.D.

70.00

70.00

70.00

70.00

70.00

70.00

70.00

56.50

22.50

50.00

48.00

63.00

500.50

470.00

267.00

104.70

313.00

94.00

44.50

99.40

99.40

99.40

82.00

82.00

82.00

67.00

67.00 


$\begin{array}{ll}\begin{array}{c}\text { CURRENT } \\ \text { NAME }\end{array} & \text { FUNCTIONAL AREA } \\ \text { GW-477C } & \text { Gum Branch Road } \\ \text { GW-478A } & \text { Gum Branch Road } \\ \text { GW-478B } & \text { Gum Branch Road } \\ \text { GW-478C } & \text { Gum Branch Road } \\ \text { GW-479 } & \text { Gum Branch Road } \\ \text { GW-480A } & \text { Gum Branch Road } \\ \text { GW-480B } & \text { Gum Branch Road } \\ \text { GW-480C } & \text { Gum Branch Road } \\ \text { GW-481A } & \text { Gum Branch Road } \\ \text { GW-481B } & \text { Gum Branch Road } \\ \text { GW-481C } & \text { Gum Branch Road } \\ \text { GW-482A } & \text { Gum Branch Road } \\ \text { GW-482B } & \text { Gum Branch Road } \\ \text { GW-482C } & \text { Gum Branch Road } \\ \text { GW-483 } & \text { Gum Branch Road } \\ \text { GW-484 } & \text { Gum Branch Road } \\ \text { GW-485 } & \text { Gum Branch Road } \\ \text { GW-486 } & \text { Gum Branch Road } \\ \text { GW-487 } & \text { Gum Branch Road } \\ \text { GW-488 } & \text { Gum Branch Road } \\ \text { GW-489 } & \text { Gum Branch Road } \\ \text { GW-490 } & \text { Gum Branch Road } \\ \text { GW-491 } & \text { Gum Branch Road } \\ \text { GW-492 } & \text { Gum Branch Road } \\ \text { GW-493 } & \text { Gum Branch Road } \\ \text { GW-494 } & \text { Gum Branch Road } \\ \text { GW-495 } & \text { Gum Branch Road }\end{array}$

MAP NORTHING EASTING

\begin{tabular}{|c|c|c|c|c|c|c|}
\hline $2 \& 3$ & 29920.10 & 30738.54 & $\mathrm{Y}-12$ & 868.33 & 871.39 & 67.00 \\
\hline $2 \& 3$ & 29900.61 & 30720.30 & $\mathrm{Y}-12$ & 863.27 & 865.20 & 82.00 \\
\hline $2 \& 3$ & 29900.61 & 30720.30 & $\mathrm{Y}-12$ & 863.27 & 865.40 & 82.00 \\
\hline $2 \& 3$ & 29900.61 & 30720.30 & $\mathrm{Y}-12$ & 863.27 & 865.55 & 82.00 \\
\hline $2 \& 3$ & 29889.73 & 30773.29 & Y-12 & 870.61 & 873.04 & 26.00 \\
\hline $2 \& 3$ & 29890.25 & 30737.11 & Y-12 & 868.67 & 871.28 & 42.50 \\
\hline $2 \& 3$ & 29890.25 & 30737.11 & $\mathrm{Y}-12$ & 868.67 & 871.93 & 42.50 \\
\hline $2 \& 3$ & 29890.25 & 30737.11 & $\mathrm{Y}-12$ & 868.67 & 871.84 & 42.50 \\
\hline $2 \& 3$ & 29910.66 & 30738.09 & $\mathrm{Y}-12$ & 868.61 & 871.13 & 39.00 \\
\hline $2 \& 3$ & 29910.66 & 30738.09 & $\mathrm{Y}-12$ & 868.61 & 870.50 & 39.00 \\
\hline $2 \& 3$ & 29910.66 & 30738.09 & Y-12 & 868.61 & 870.87 & 39.00 \\
\hline $2 \& 3$ & 29900.41 & 30747.28 & $\mathrm{Y}-12$ & 869.21 & 871.18 & 40.50 \\
\hline $2 \& 3$ & 29900.41 & 30747.28 & $\mathrm{Y}-12$ & 869.21 & 871.46 & 40.50 \\
\hline $2 \& 3$ & 29900.41 & 30747.28 & $\mathrm{Y}-12$ & 869.21 & 871.96 & 40.50 \\
\hline $2 \& 3$ & 29908.58 & 30773.45 & $\mathrm{Y}-12$ & 870.98 & 874.28 & 26.00 \\
\hline $2 \& 3$ & 29911.80 & 30751.92 & Y-12 & 869.33 & 871.55 & 26.00 \\
\hline $2 \& 3$ & 29887.86 & 30718.88 & Y-12 & 863.81 & 864.30 & 20.50 \\
\hline $2 \& 3$ & 29908.82 & 30713.65 & $\mathrm{Y}-12$ & 863.01 & 866.32 & 16.80 \\
\hline $2 \& 3$ & 29914.27 & 30708.68 & $\mathrm{Y}-12$ & 862.98 & 865.25 & 21.00 \\
\hline $2 \& 3$ & 29902.43 & 30711.37 & $\mathrm{Y}-12$ & 863.14 & 866.81 & 17.00 \\
\hline $2 \& 3$ & 29922.49 & 30706.85 & $\mathrm{Y}-12$ & 863.22 & 867.27 & 21.50 \\
\hline $2 \& 3$ & 29921.53 & 30692.75 & Y-12 & 861.55 & 863.83 & 19.00 \\
\hline $2 \& 3$ & 29915.19 & 30693.65 & Y-12 & 861.12 & 864.69 & 17.00 \\
\hline $2 \& 3$ & 29908.18 & 30694.68 & $\mathrm{Y}-12$ & 860.69 & 862.85 & 14.10 \\
\hline $2 \& 3$ & 29912.84 & 30694.15 & $\mathrm{Y}-12$ & 861.10 & 863.99 & 19.30 \\
\hline $2 \& 3$ & 29917.50 & 30693.18 & $\mathrm{Y}-12$ & 861.34 & 863.75 & 16.50 \\
\hline $2 \& 3$ & 29920.31 & 30639.44 & Y-12 & 852.24 & 855.08 & 7.70 \\
\hline
\end{tabular}




$\begin{array}{ll}\begin{array}{c}\text { CURRENT } \\ \text { NAME }\end{array} & \text { FUNCTIONAL AREA } \\ \text { GW-496 } & \text { Gum Branch Road } \\ \text { GW-497 } & \text { Gum Branch Road } \\ \text { GW-498 } & \text { Gum Branch Road } \\ \text { GW-499 } & \text { Gum Branch Road } \\ \text { GW-499A } & \text { Gum Branch Road } \\ \text { GW-499AA } & \text { Gum Branch Road } \\ \text { GW-499AB } & \text { Gum Branch Road } \\ \text { GW-499AC } & \text { Gum Branch Road } \\ \text { GW-499AD } & \text { Gum Branch Road } \\ \text { GW-499AE } & \text { Gum Branch Road } \\ \text { GW-499AF } & \text { Gum Branch Road } \\ \text { GW-499B } & \text { Gum Branch Road } \\ \text { GW-499C } & \text { Gum Branch Road } \\ \text { GW-499D } & \text { Gum Branch Road } \\ \text { GW-499E } & \text { Gum Branch Road } \\ \text { GW-499F } & \text { Gum Branch Road } \\ \text { GW-499G } & \text { Gum Branch Road } \\ \text { GW-499H } & \text { Gum Branch Road } \\ \text { GW-499I } & \text { Gum Branch Road } \\ \text { GW-499J } & \text { Gum Branch Road } \\ \text { GW-499K } & \text { Gum Branch Road } \\ \text { GW-499L } & \text { Gum Branch Road } \\ \text { GW-499M } & \text { Gum Branch Road } \\ \text { GW-499N } & \text { Gum Branch Road } \\ \text { GW-4990 } & \text { Gum Branch Road } \\ \text { GW-499P } & \text { Gum Branch Road } \\ \text { GW-499Q } & \text { Gum Branch Road }\end{array}$

MAP NORTHING E

\begin{tabular}{|c|c|c|c|c|}
\hline $2 \& 3$ & 29917.51 & 30639.11 & Y-12 & 852.03 \\
\hline $2 \& 3$ & 29914.47 & 30639.84 & Y-12 & 851.87 \\
\hline $2 \& 3$ & 29911.65 & 30640.30 & Y-12 & 852.03 \\
\hline $2 \& 3$ & 29908.62 & 30640.74 & $\mathrm{Y}-12$ & 852.15 \\
\hline $2 \& 3$ & 29923.23 & 30638.43 & $Y-12$ & 852.35 \\
\hline $2 \& 3$ & 29669.30 & 30947.52 & Y-12 & 851.09 \\
\hline $2 \& 3$ & 29932.81 & 30637.55 & Y-12 & 850.97 \\
\hline $2 \& 3$ & 29915.09 & 30583.29 & $Y-12$ & 843.54 \\
\hline $2 \& 3$ & 29911.03 & 30575.22 & $Y-12$ & 842.71 \\
\hline $2 \& 3$ & 29921.27 & 30581.64 & $Y-12$ & 843.02 \\
\hline $2 \& 3$ & 29927.39 & 30583.48 & $Y-12$ & 842.91 \\
\hline $2 \& 3$ & 29898.85 & 30643.12 & Y-12 & 852.85 \\
\hline $2 \& 3$ & 29890.16 & 30642.22 & Y-12 & 853.03 \\
\hline $2 \& 3$ & 29882.73 & 30639.27 & $Y-12$ & 852.56 \\
\hline $2 \& 3$ & 29875.26 & 30636.15 & Y-12 & 852.33 \\
\hline $2 \& 3$ & 29903.81 & 30641.31 & Y-12 & 852.15 \\
\hline $2 \& 3$ & 29916.11 & 30667.88 & Y-12 & 857.74 \\
\hline $2 \& 3$ & 29913.72 & 30666.85 & Y-12 & 857.57 \\
\hline $2 \& 3$ & 29910.37 & 30665.71 & Y-12 & 857.41 \\
\hline $2 \& 3$ & 29907.68 & 30664.70 & Y-12 & 857.15 \\
\hline $2 \& 3$ & 29919.73 & 30680.58 & $Y-12$ & 859.44 \\
\hline $2 \& 3$ & 29922.96 & 30681.85 & $Y-12$ & 859.62 \\
\hline $2 \& 3$ & 29916.41 & 30679.86 & $Y-12$ & 859.39 \\
\hline $2 \& 3$ & 29913.23 & 30679.66 & Y-12 & 859.32 \\
\hline $2 \& 3$ & 29910.03 & 30681.70 & $Y-12$ & 859.20 \\
\hline $2 \& 3$ & 29907.35 & 30683.35 & $Y-12$ & 859.37 \\
\hline $2 \& 3$ & 29891.17 & 30669.93 & $Y-12$ & 857.64 \\
\hline
\end{tabular}

T.O.C. T.O.W.W.

T.D.

$\begin{array}{lr}854.68 & 6.70 \\ 854.57 & 8.20 \\ 854.48 & 5.00 \\ 854.85 & 5.00 \\ 854.69 & 6.20 \\ 854.55 & 9.81 \\ 853.78 & 8.80 \\ 843.88 & 4.40 \\ 844.89 & 5.00 \\ 844.15 & 3.60 \\ 843.85 & 4.40 \\ 855.50 & 8.70 \\ 855.98 & 15.00 \\ 855.93 & 13.15 \\ 855.47 & 14.85 \\ 853.44 & 4.25 \\ 859.76 & 14.00 \\ 859.77 & 12.50 \\ 859.75 & 10.50 \\ 859.60 & 10.00 \\ 862.32 & 12.30 \\ 862.26 & 15.00 \\ 862.35 & 12.30 \\ 862.27 & 13.00 \\ 862.63 & 13.00 \\ 860.87 & 15.00 \\ 857.86 & 51.50\end{array}$




\section{CURRENT \\ NAME}

GW-499R

GW-499S

GW-499T

GW-499U

GW-499V

GW-499W

GW-499X

GW-499Y

GW-499Z

GW-502

GW-505

GW-506

GW-507

GW-508

GW-509

GW-511

GW-512

GW-513

GW-514

GW-520

GW-521

GW-522

GW-526

GW-527

GW-528

GW-531

GW-532

\section{FUNCTIONAL AREA}

Gum Branch Road

Gum Branch Road

Gum Branch Road

Gum Branch Road

Gum Branch Road

Gum Branch Road

Gum Branch Road

Gum Branch Road

Gum Branch Road

Bear Creek Burial Grounds WMA

Rust Garage Area

Rust Garage Area

Rust Garage Area

Rust Garage Area

Rust Garage Area

Chestnut Ridge Security Pits

Filled Coal Ash Pond

Filled Coal Ash Pond

Filled Coal Ash Pond

Industrial Landfill ।

Industrial Landfill IV

Industrial Landfill IV

S-3 Site

Bear Creek Burial Grounds WMA

Bear Creek Burial Grounds WMA

Lysimeter Demo

Lysimeter Demo
MAP NORTHING EASTING

SYSTEM

ELEVATION

T.O.C.

T.O.W.W

T.D.

\begin{tabular}{crrrr}
2 \& 3 & 29883.69 & 30668.56 & $\mathrm{Y}-12$ & 857.13 \\
2 \& 3 & 29825.00 & 30810.46 & $\mathrm{Y}-12$ & 871.02 \\
2 \& 3 & 29935.25 & 30852.60 & $\mathrm{Y}-12$ & 876.67 \\
2 \& 3 & 29934.75 & 30713.36 & $\mathrm{Y}-12$ & 864.84 \\
2 \& 3 & 29932.88 & 30754.24 & $\mathrm{Y}-12$ & 869.06 \\
2 \& 3 & 29915.18 & 30633.18 & $\mathrm{Y}-12$ & 851.27 \\
2 \& 3 & 29907.07 & 30783.63 & $\mathrm{Y}-12$ & 872.78 \\
2 \& 3 & 29835.49 & 30980.25 & $\mathrm{Y}-12$ & 860.14 \\
2 \& 3 & 29937.06 & 31006.01 & $\mathrm{Y}-12$ & 864.78 \\
4 & 30269.49 & 43112.87 & $\mathrm{Y}-12$ & 977.77 \\
5 & 30400.04 & 53037.45 & $\mathrm{Y}-12$ & 1011.60 \\
5 & 30310.10 & 53013.00 & $\mathrm{Y}-12$ & 1007.06 \\
5 & 30302.30 & 53081.90 & $\mathrm{Y}-12$ & 1008.30 \\
5 & 30281.45 & 53148.44 & $\mathrm{Y}-12$ & 1009.41 \\
5 & 30312.10 & 52969.00 & $\mathrm{Y}-12$ & 1006.52 \\
5 & 28055.64 & 57739.43 & $\mathrm{Y}-12$ & 1090.70 \\
5 & 27601.02 & 57343.05 & $\mathrm{Y}-12$ & 998.99 \\
5 & 27606.52 & 57331.91 & $\mathrm{Y}-12$ & 998.99 \\
5 & 27575.29 & 57341.06 & $\mathrm{Y}-12$ & 998.66 \\
4 & 28884.78 & 46724.85 & $\mathrm{Y}-12$ & 985.18 \\
5 & 28541.02 & 52039.50 & $\mathrm{Y}-12$ & 1179.46 \\
5 & 28377.44 & 52612.31 & $\mathrm{Y}-12$ & 1172.04 \\
4 \& 5 & 30032.85 & 50708.14 & $\mathrm{Y}-12$ & 995.34 \\
4 & 30467.00 & 43643.00 & $\mathrm{Y}-12$ & 940.20 \\
4 & 30425.00 & 43649.00 & $\mathrm{Y}-12$ & 934.80 \\
\hline 5 & 30452.72 & 49331.48 & $\mathrm{Y}-12$ & 1002.19 \\
\hline & 30387.95 & 49299.99 & $\mathrm{Y}-12$ & 994.15
\end{tabular}

859.55

17.50

873.73

879.62

868.26

871.14

853.62

873.86

863.55

868.24

978.41

1014.61

1009.99

1011.28

1010.74

1009.51

1093.21

1001.54

1001.41

1001.22

987.38

1182.68

1175.31

997.45

941.60

936.85

1004.61

994.77
25.00

32.00

25.00

26.00

31.00

260.00

$1015.19 \quad 13.50$

9.50

9.00

15.00

9.00

153.70

61.00

$1001.41 \quad 125.30$

$1001.22 \quad 195.00$

80.30

$1182.88 \quad 136.00$

$1175.48 \quad 195.50$

$998.25 \quad 123.00$

5.60

6.00

$1004.85 \quad 39.50$

29.70 


\begin{tabular}{|c|c|c|c|c|c|c|c|c|c|}
\hline \multicolumn{10}{|c|}{ CURRENT } \\
\hline NAME & FUNCTIONAL AREA & $\underline{\text { MAP }}$ & NORTHING & EASTING & SYSTEM & ELEVATION & T.O.C. & T.O.W.W. & T.D. \\
\hline GW-533 & Lysimeter Demo & $4 \& 5$ & 30474.88 & 49405.30 & $\mathrm{Y}-12$ & 1001.35 & 1003.66 & & 30.10 \\
\hline GW-534 & Lysimeter Demo & $4 \& 5$ & 30392.82 & 49491.97 & $\mathrm{Y}-12$ & 997.43 & 1000.85 & & 47.00 \\
\hline GW-535 & Lysimeter Demo & $4 \& 5$ & 30333.84 & 49468.98 & Y-12 & 987.29 & 990.94 & & 19.50 \\
\hline GW-536 & Lysimeter Demo & $4 \& 5$ & 30289.83 & 49442.58 & $\mathrm{Y}-12$ & 982.45 & 983.40 & & 19.70 \\
\hline GW-537 & Oil Landfarm WMA & $4 \& 5$ & 30057.01 & 49538.95 & $\mathrm{Y}-12$ & 974.49 & 976.48 & 976.65 & 24.50 \\
\hline GW-538 & Lysimeter Demo & $4 \& 5$ & 30329.08 & 49378.67 & Y-12 & 993.97 & 996.89 & & 42.50 \\
\hline GW-539 & Industrial Landfill II & 5 & 27192.53 & 52278.26 & $\mathrm{Y}-12$ & 1090.39 & 1093.00 & 1093.20 & 156.00 \\
\hline GW-540 & Industrial Landfill II & 5 & 27489.06 & 52371.31 & $Y-12$ & 1069.38 & 1072.12 & 1072.31 & 171.50 \\
\hline GW-541 & Construction/Demolition Landfill VI & 5 & 27653.53 & 51738.09 & $\mathrm{Y}-12$ & 1055.79 & 1058.40 & & 104.50 \\
\hline GW-542 & Construction/Demolition Landfill VI & 5 & 27466.22 & 51641.74 & Y-12 & 1049.03 & 1051.60 & 1051.81 & 77.50 \\
\hline GW-543 & Construction/Demolition Landfill VI & 5 & 27072.06 & 51458.48 & Y-12 & 1021.19 & 1023.80 & 1024.01 & 94.00 \\
\hline GW-544 & Construction/Demolition Landfill VI & 5 & 26963.22 & 51819.56 & $\mathrm{Y}-12$ & 1042.53 & 1044.99 & 1045.19 & 110.00 \\
\hline GW-545 & Construction/Demolition Landfill VI & 5 & 27640.84 & 51728.54 & $\mathrm{Y}-12$ & 1055.43 & 1058.02 & & 67.00 \\
\hline GW-546 & Construction/Demolition Landfill VI & 5 & 27473.75 & 52366.34 & $\mathrm{Y}-12$ & 1069.64 & 1072.21 & & 84.50 \\
\hline GW-551 & South Side Chestnut Ridge & $5 \& 6$ & 27298.80 & 60263.02 & Y-12 & 990.20 & 993.95 & & 350.20 \\
\hline GW-552 & South Side Chestnut Ridge & $5 \& 6$ & 26610.51 & 60094.00 & $\mathrm{Y}-12$ & 968.68 & 972.78 & & 804.80 \\
\hline GW-553 & South Side Chestnut Ridge & 6 & 26362.62 & 61046.95 & Y-12 & 1045.30 & 1049.10 & & 599.30 \\
\hline GW-554 & South Side Chestnut Ridge & 6 & 25355.73 & 61287.60 & $\mathrm{Y}-12$ & 906.80 & 911.87 & & 303.05 \\
\hline GW-555 & South Side Chestnut Ridge & $5 \& 6$ & 25867.53 & 59850.81 & Y-12 & 934.53 & 937.42 & & 314.35 \\
\hline GW-556 & South Side Chestnut Ridge & 5 & 27358.98 & 58158.65 & Y-12 & 981.91 & 986.51 & & 354.50 \\
\hline GW-557 & Industrial Landfill V & $5 \& 6$ & 26450.11 & 59519.59 & $\mathrm{Y}-12$ & 1078.63 & 1081.16 & 1081.36 & 139.00 \\
\hline GW-558 & South Side Chestnut Ridge & 5 & 26103.95 & 58948.72 & Y-12 & 980.97 & 981.42 & & 87.00 \\
\hline GW-559 & South Side Chestnut Ridge & 5 & 26753.05 & 58226.65 & Y-12 & 1100.20 & 1102.79 & & 201.00 \\
\hline GW-560 & Construction/Demolition Landfill VII & $5 \& 6$ & 25691.87 & 60743.15 & $\mathrm{Y}-12$ & 945.76 & 948.85 & 949.05 & 117.00 \\
\hline GW-561 & South Side Chestnut Ridge & $5 \& 6$ & 27810.66 & 59323.38 & $\mathrm{Y}-12$ & 1030.74 & 1033.35 & & 94.00 \\
\hline GW-562 & Construction/Demolition Landfill VII & 6 & 26276.29 & 61640.17 & $\mathrm{Y}-12$ & 931.86 & 934.49 & 934.69 & 133.00 \\
\hline GW-563 & South Side Chestnut Ridge & 6 & 25367.63 & 61292.63 & Y-12 & 907.50 & 909.93 & & 104.00 \\
\hline
\end{tabular}




\begin{tabular}{|c|c|c|c|c|c|c|c|c|c|}
\hline \multicolumn{10}{|l|}{ CURRENT } \\
\hline NAME & FUNCTIONAL AREA & $\underline{\text { MAP }}$ & NORTHING & EASTING & $\underline{\text { SYSTEM }}$ & ELEVATION & T.O.C. & T.O.W.W. & $\underline{\text { T.D. }}$ \\
\hline GW-564 & Construction/Demolition Landfill VII & $5 \& 6$ & 25872.94 & 59865.28 & $\mathrm{Y}-12$ & 935.12 & 937.77 & 938.07 & 88.00 \\
\hline GW-565 & South Side Chestnut Ridge & 5 & 27362.65 & 58174.46 & $\mathrm{Y}-12$ & 982.68 & 985.29 & & 61.00 \\
\hline GW-566 & South Side Chestnut Ridge & 6 & 26360.15 & 61060.32 & $\mathrm{Y}-12$ & 1045.32 & 1047.97 & & 188.00 \\
\hline GW-567 & South Side Chestnut Ridge & $5 \& 6$ & 26622.47 & 60095.73 & $\mathrm{Y}-12$ & 968.51 & 971.07 & & 86.00 \\
\hline GW-568 & South Side Chestnut Ridge & $5 \& 6$ & 27775.19 & 61015.00 & $\mathrm{Y}-12$ & 1063.30 & & & 101.30 \\
\hline GW-569 & South Side Chestnut Ridge & $5 \& 6$ & 27794.42 & 60857.60 & $\mathrm{Y}-12$ & 1042.50 & 1047.60 & 1048.17 & 127.40 \\
\hline GW-570 & South Side Chestnut Ridge & $5 \& 6$ & 27724.68 & 60818.33 & $\mathrm{Y}-12$ & 1050.30 & 1051.50 & & 137.80 \\
\hline GW-571 & South Side Chestnut Ridge & 5 & 27672.31 & 58935.65 & $\mathrm{Y}-12$ & 1062.20 & 1063.10 & & 80.80 \\
\hline GW-572 & South Side Chestnut Ridge & 5 & 27750.63 & 58964.26 & $\mathrm{Y}-12$ & 1072.10 & 1076.80 & & 74.00 \\
\hline GW-573D & South Side Chestnut Ridge & $5 \& 6$ & 27028.24 & 60852.25 & $\mathrm{Y}-12$ & 979.95 & 985.07 & & 89.80 \\
\hline GW-573S & South Side Chestnut Ridge & $5 \& 6$ & 27044.36 & 60847.48 & $\mathrm{Y}-12$ & 980.04 & 982.57 & & 15.00 \\
\hline GW-576 & South Side Chestnut Ridge & $5 \& 6$ & 25697.78 & 60747.87 & $\mathrm{Y}-12$ & 945.98 & 948.89 & & 59.20 \\
\hline GW-580 & South Side Chestnut Ridge & $5 \& 6$ & 27846.29 & 59309.35 & $\mathrm{Y}-12$ & 1032.90 & 1036.20 & & 80.00 \\
\hline GW-601 & Oil Landfarm WMA & 4 & 28902.55 & 47629.49 & $\mathrm{Y}-12$ & 999.09 & 1002.20 & 1002.80 & 356.00 \\
\hline GW-602 & Oil Landfarm WMA & 4 & 28640.11 & 47430.35 & $\mathrm{Y}-12$ & 1075.06 & 1077.77 & & 212.00 \\
\hline GW-603 & New Hope Pond & 6 & 28429.84 & 64803.26 & $\mathrm{Y}-12$ & 959.41 & 961.32 & & 75.20 \\
\hline GW-604 & New Hope Pond & 6 & 28436.97 & 64836.82 & $\mathrm{Y}-12$ & 959.53 & 960.86 & & 112.40 \\
\hline GW-605 & Exit Pathway - Traverse I & 6 & 28706.83 & 62001.50 & $\mathrm{Y}-12$ & 916.97 & 918.88 & 919.06 & 40.50 \\
\hline GW-606 & Exit Pathway - Traverse I & 6 & 28708.32 & 61951.42 & $\mathrm{Y}-12$ & 916.98 & 919.39 & 919.59 & 175.00 \\
\hline GW-607 & Chestnut Ridge Security Pits & 5 & 27865.53 & 58922.12 & $\mathrm{Y}-12$ & 1072.86 & 1075.98 & & 151.30 \\
\hline GW-608 & Chestnut Ridge Security Pits & $5 \& 6$ & 27889.43 & 59724.35 & $\mathrm{Y}-12$ & 1071.00 & 1073.95 & 1075.38 & 220.00 \\
\hline GW-608A & Chestnut Ridge Security Pits & $5 \& 6$ & 27885.70 & 59758.88 & $\mathrm{Y}-12$ & 1069.01 & 1069.39 & & 125.00 \\
\hline GW-609 & Chestnut Ridge Security Pits & $5 \& 6$ & 28109.43 & 60039.73 & $\mathrm{Y}-12$ & 1109.70 & 1112.11 & 1112.31 & 269.00 \\
\hline GW-610 & Chestnut Ridge Security Pits & $5 \& 6$ & 28549.31 & 59471.94 & $\mathrm{Y}-12$ & 1056.78 & 1059.44 & 1059.44 & 117.40 \\
\hline GW-611 & Chestnut Ridge Security Pits & 5 & 28855.52 & 58058.92 & $\mathrm{Y}-12$ & 1045.43 & 1048.38 & 1048.38 & 121.60 \\
\hline GW-612 & Chestnut Ridge Security Pits & 5 & 28370.61 & 58503.62 & $\mathrm{Y}-12$ & 1128.65 & 1131.03 & 1131.03 & 254.00 \\
\hline GW-613 & S-3 Site & $4 \& 5$ & 30406.77 & 50231.77 & $\mathrm{Y}-12$ & 1010.17 & 1013.37 & 1013.58 & 42.00 \\
\hline
\end{tabular}




\section{CURRENT}

NAME

GW-614

GW-615

GW-616

GW-617

GW-618

GW-619

GW-620

GW-621

GW-622

GW-623

GW-624

GW-625

GW-626

GW-627

GW-628

GW-629

GW-630

GW-631

GW-632

GW-633

GW-634

GW-635

GW-636

GW-637

GW-638

GW-639

GW-640

\section{FUNCTIONAL AREA}

S-3 Site

S-3 Site

S-3 Site

Exit Pathway - Traverse E

Exit Pathway - Traverse E

Fire Training Facility

Fire Training Facility

Exit Pathway - Traverse B

Bear Creek Burial Grounds WMA

Bear Creek Burial Grounds WMA

Bear Creek Burial Grounds WMA

Bear Creek Burial Grounds WMA

Bear Creek Burial Grounds WMA

Bear Creek Burial Grounds WMA

Bear Creek Burial Grounds WMA

Bear Creek Burial Grounds WMA

Lysimeter Demo

Rust Garage Area

Rust Garage Area

Rust Garage Area

Rust Garage Area

Rust Garage Site

Oil Landfarm WMA

Oil Landfarm WMA

Oil Landfarm WMA

Bear Creek Burial Grounds WMA

Bear Creek Burial Grounds WMA

\section{MAP NORTHING}

EASTING

SYSTEM

4

$4 \&$
5
5
5

30425.77
30009.32
29723.83
29806.44
29798.46
29562.80
29564.54
29023.30
29367.74
29388.47
29421.33
29398.45
29535.32
29505.05
29449.54
29521.85
30316.48
30202.77
30236.97
30144.56
30222.60
30147.85
29482.30
29492.81
29485.32
29626.12
29670.10
29

50223.05

52223.78

51906.92

54755.65

54738.12

52905.94

52894.57

45032.91

44115.90

44137.84

43007.38

43009.03

42772.01

42774.10

43499.91

43046.50

49450.48

53144.93

53077.90

53100.41

53206.77

53288.91

46412.64

46402.39

46388.51

45259.76

45252.92

$$
\begin{aligned}
& \text { Y-12 } \\
& \text { Y-12 } \\
& \text { Y-12 } \\
& \text { Y-12 } \\
& \text { Y-12 } \\
& \text { Y-12 } \\
& \text { Y-12 } \\
& \text { Y-12 } \\
& \text { Y-12 } \\
& \text { Y-12 } \\
& \text { Y-12 } \\
& \text { Y-12 } \\
& \text { Y-12 } \\
& \text { Y-12 } \\
& \text { Y-12 } \\
& \text { Y-12 } \\
& \text { Y-12 } \\
& \text { Y-12 } \\
& \text { Y-12 } \\
& \text { Y-12 } \\
& \text { Y-12 } \\
& \text { Y-12 } \\
& \text { Y-12 } \\
& \text { Y-12 } \\
& \text { Y-12 } \\
& \text { Y-12 } \\
& \text { Y-12 }
\end{aligned}
$$

ELEVATION

1009.39

1014.17

1009.81

982.61

982.64

1012.74

1012.84

923.07

921.54

922.01

919.52

917.67

939.95

940.39

920.55

924.42

984.53

1004.10

1006.00

996.66

1007.48

994.85

938.08

938.26

938.14

937.98

942.59
T.O.C.

T.O.W.W.

$\underline{\text { T.D. }}$

$1012.22 \quad 1012.42 \quad 90.20$

$1016.75 \quad 1017.55 \quad 245.00$

$1011.81 \quad 1011.81 \quad 269.00$

$\begin{array}{lll}985.11 & 985.28 & 18.00\end{array}$

$984.94 \quad 985.14 \quad 37.00$

$1015.24 \quad 1015.42 \quad 40.80$

$1015.34 \quad 1015.57 \quad 75.00$

$\begin{array}{lll}925.24 & 925.45 & 43.00\end{array}$

$924.16 \quad 20.50$

$\begin{array}{lll}925.21 & 925.87 & 349.00\end{array}$

$\begin{array}{lll}921.96 & 922.15 & 27.20\end{array}$

$919.50 \quad 283.00$

$\begin{array}{lll}942.61 & 942.87 & 78.00\end{array}$

$942.85 \quad 943.51 \quad 270.00$

$923.25 \quad 288.00$

$\begin{array}{lll}927.16 & 928.03 \quad 312.00\end{array}$

$986.65 \quad 28.60$

$1004.00 \quad 1004.00 \quad 16.00$

$1005.70 \quad 15.00$

$\begin{array}{lll}996.43 & 996.43 \quad 15.00\end{array}$

$1007.31 \quad 15.00$

$994.85 \quad 15.00$

$941.92 \quad 117.00$

$941.83 \quad 27.50$

$941.77 \quad 12.00$

$940.70 \quad 940.95 \quad 125.50$

$945.38 \quad 47.40$ 


\begin{tabular}{|c|c|c|c|c|c|c|c|c|c|}
\hline \multicolumn{10}{|l|}{ CURRENT } \\
\hline NAME & FUNCTIONAL AREA & $\underline{\text { MAP }}$ & $\underline{\text { NORTHING }}$ & EASTING & $\underline{\text { SYSTEM }}$ & ELEVATION & T.O.C. & T.O.W.W. & $\underline{\text { T.D. }}$ \\
\hline GW-641 & Bear Creek Burial Grounds WMA & 4 & 29685.73 & 45250.72 & Y-12 & 944.01 & 946.66 & & 24.30 \\
\hline GW-642 & Bear Creek Burial Grounds WMA & 4 & 31215.27 & 44654.05 & $\mathrm{Y}-12$ & 1011.90 & 1014.70 & 1014.95 & 36.90 \\
\hline GW-643 & Bear Creek Burial Grounds WMA & 4 & 30656.47 & 45253.26 & $\mathrm{Y}-12$ & 979.90 & 982.42 & & 29.30 \\
\hline GW-644 & Oil Landfarm WMA & 4 & 29945.31 & 46608.69 & Y-12 & 956.78 & 959.75 & & 23.40 \\
\hline GW-645 & Oil Landfarm WMA & 4 & 28837.01 & 46649.34 & $\mathrm{Y}-12$ & 1003.50 & 1006.40 & & 85.80 \\
\hline GW-646 & Oil Landfarm WMA & 4 & 28872.66 & 47579.86 & $\mathrm{Y}-12$ & 1002.00 & 1004.50 & & 75.90 \\
\hline GW-647 & Oil Landfarm WMA & 4 & 28940.89 & 48770.23 & $\mathrm{Y}-12$ & 1030.10 & 1033.30 & & 91.00 \\
\hline GW-647A & Oil Landfarm WMA & 4 & 29053.05 & 48708.36 & Admin & & & & 43.00 \\
\hline GW-648 & Rust Spoil Area & $4 \& 5$ & 29088.15 & 49888.45 & Y-12 & 1026.50 & 1029.20 & 1029.20 & 80.10 \\
\hline GW-649 & S-3 Site & $4 \& 5$ & 30364.58 & 50142.75 & $\mathrm{Y}-12$ & 981.01 & 983.66 & & 20.80 \\
\hline GW-651 & Bear Creek Burial Grounds WMA & 4 & 29042.93 & 42535.39 & $\mathrm{Y}-12$ & 900.05 & 903.12 & & 52.00 \\
\hline GW-652 & Bear Creek Burial Grounds WMA & 4 & 29029.46 & 42452.49 & $\mathrm{Y}-12$ & 897.98 & 900.83 & & 31.20 \\
\hline GW-653 & Bear Creek Burial Grounds WMA & 4 & 29660.39 & 42317.29 & $\mathrm{Y}-12$ & 928.85 & 931.60 & 931.84 & 39.00 \\
\hline GW-654 & Bear Creek Burial Grounds WMA & 4 & 30548.76 & 41907.28 & $\mathrm{Y}-12$ & 938.26 & 940.79 & & 15.50 \\
\hline GW-655 & Bear Creek Burial Grounds WMA & 4 & 31403.07 & 42907.49 & $\mathrm{Y}-12$ & 1108.20 & 1111.42 & & 65.00 \\
\hline GW-656 & Y-12 Plant Site & 5 & 29894.69 & 57439.12 & $\mathrm{Y}-12$ & 954.90 & 954.79 & 954.79 & 21.50 \\
\hline GW-657 & Y-12 Plant Site & $5 \& 6$ & 29287.13 & 59598.78 & $\mathrm{Y}-12$ & 930.80 & 930.53 & & 17.10 \\
\hline GW-658 & Y-12 Fuel Station & 6 & 29638.24 & 62146.20 & $\mathrm{Y}-12$ & 942.04 & 944.81 & 944.81 & 19.10 \\
\hline GW-659 & Y-12 Fuel Station & 6 & 29614.54 & 62100.46 & $\mathrm{Y}-12$ & 941.79 & 941.16 & & 16.70 \\
\hline GW-660 & East Fork Poplar Creek & 6 & 32818.80 & 63543.11 & $\mathrm{Y}-12$ & 875.30 & 879.23 & & 11.00 \\
\hline GW-661 & East Fork Poplar Creek & 6 & 32793.69 & 63483.20 & $\mathrm{Y}-12$ & 878.80 & 881.52 & & 7.80 \\
\hline GW-662 & East Fork Poplar Creek & 7 & 34093.89 & 62401.88 & $\mathrm{Y}-12$ & 861.60 & 865.75 & & 17.50 \\
\hline GW-663 & East Fork Poplar Creek & 7 & 34515.14 & 61993.74 & $\mathrm{Y}-12$ & 861.00 & 864.21 & & 4.00 \\
\hline GW-664 & East Fork Poplar Creek & 7 & 35085.79 & 61748.97 & $\mathrm{Y}-12$ & 859.00 & 862.83 & & 5.90 \\
\hline GW-665 & East Fork Poplar Creek & 7 & 35457.01 & 61708.44 & $Y-12$ & 859.50 & 862.52 & & 30.70 \\
\hline GW-666 & East Fork Poplar Creek & 7 & 39754.19 & 55755.36 & $\mathrm{Y}-12$ & 837.20 & 841.06 & & 7.90 \\
\hline GW-667 & East Fork Poplar Creek & 7 & 39666.59 & 55792.90 & $\mathrm{Y}-12$ & 836.90 & 840.31 & & 8.00 \\
\hline
\end{tabular}




$\begin{array}{ll}\begin{array}{c}\text { CURRENT } \\ \text { NAME }\end{array} & \text { FUNCTIONAL AREA } \\ \text { GW-668 } & \text { East Fork Poplar Creek } \\ \text { GW-669 } & \text { East Fork Poplar Creek } \\ \text { GW-670 } & \text { East Fork Poplar Creek } \\ \text { GW-671 } & \text { East Fork Poplar Creek } \\ \text { GW-672 } & \text { Filled Coal Ash Pond } \\ \text { GW-673 } & \text { Filled Coal Ash Pond } \\ \text { GW-674 } & \text { Filled Coal Ash Pond } \\ \text { GW-675 } & \text { Filled Coal Ash Pond } \\ \text { GW-676 } & \text { Filled Coal Ash Pond } \\ \text { GW-677 } & \text { Filled Coal Ash Pond } \\ \text { GW-678 } & \text { Filled Coal Ash Pond } \\ \text { GW-679 } & \text { Filled Coal Ash Pond } \\ \text { GW-680 } & \text { Filled Coal Ash Pond } \\ \text { GW-681 } & \text { Filled Coal Ash Pond } \\ \text { GW-682 } & \text { Filled Coal Ash Pond } \\ \text { GW-683 } & \text { Exit Pathway - Traverse A } \\ \text { GW-684 } & \text { Exit Pathway - Traverse A } \\ \text { GW-685 } & \text { Exit Pathway - Traverse A } \\ \text { GW-686 } & \text { Coal Pile Trench } \\ \text { GW-687 } & \text { Coal Pile Trench } \\ \text { GW-688 } & \text { Coal Pile Trench } \\ \text { GW-689 } & \text { Coal Pile Trench } \\ \text { GW-690 } & \text { Coal Pile Trench } \\ \text { GW-691 } & \text { Coal Pile Trench } \\ \text { GW-692 } & \text { Coal Pile Trench } \\ \text { GW-693 } & \text { Coal Pile Trench } \\ \text { GW-694 } & \text { Exit Pathway - Traverse B } \\ & \end{array}$

\begin{tabular}{|c|c|c|c|c|c|c|c|}
\hline MAP & NORTHING & EASTING & SYSTEM & ELEVATION & T.O.C. & T.O.W.W. & T.D. \\
\hline 7 & 40289.58 & 53381.51 & Y-12 & 835.50 & 839.14 & & 23.10 \\
\hline 7 & 40249.42 & 50619.24 & Y-12 & 829.90 & 833.66 & & 9.40 \\
\hline 7 & 40236.83 & 50616.34 & Y-12 & 830.30 & 833.67 & & 21.80 \\
\hline 7 & 40106.43 & 50605.00 & Y-12 & 830.10 & 833.74 & & 8.90 \\
\hline 5 & 26269.06 & 57042.11 & Y-12 & 926.73 & 928.99 & & 28.00 \\
\hline 5 & 25567.33 & 56903.86 & Y-12 & 880.20 & 882.01 & & 133.00 \\
\hline 5 & 25578.48 & 56910.85 & Y-12 & 880.23 & 883.79 & & 16.50 \\
\hline & & & & & & & 179.00 \\
\hline 5 & 24225.57 & 56562.98 & Y-12 & 843.02 & 846.50 & & 17.00 \\
\hline 5 & 27483.90 & 56259.76 & Y-12 & 1027.80 & 1030.40 & & 165.00 \\
\hline 5 & 27414.81 & 56461.96 & $Y-12$ & 998.10 & 1000.70 & & 132.00 \\
\hline 5 & 27266.55 & 56765.81 & Y-12 & 1024.20 & 1026.90 & 1026.90 & 132.00 \\
\hline 5 & 27223.75 & 57934.81 & $Y-12$ & 999.80 & 1001.50 & 1001.50 & 120.00 \\
\hline 5 & 26869.88 & 58051.58 & Y-12 & 1068.50 & 1070.90 & & 230.00 \\
\hline 5 & 27023.03 & 58209.27 & $Y-12$ & 1041.80 & 1044.30 & & 166.00 \\
\hline 4 & 28281.78 & 41552.33 & Y-12 & 969.45 & 972.06 & 972.23 & 197.50 \\
\hline 4 & 28524.52 & 41353.53 & Y-12 & 895.53 & 898.66 & 898.83 & 129.60 \\
\hline 4 & 28667.40 & 41447.78 & $Y-12$ & 889.28 & 891.51 & 892.29 & 138.30 \\
\hline 5 & 29540.20 & 55956.31 & Y-12 & 964.43 & 963.76 & 963.76 & 17.00 \\
\hline 5 & 29551.29 & 55650.67 & Y-12 & 964.59 & 964.89 & & 32.00 \\
\hline 5 & 29687.56 & 55604.28 & Y-12 & 967.46 & 968.21 & & 55.00 \\
\hline 5 & 29682.96 & 55596.57 & Y-12 & 967.34 & 967.00 & & 20.00 \\
\hline 5 & 29787.18 & 55989.75 & $Y-12$ & 967.71 & 967.36 & 967.36 & 53.00 \\
\hline 5 & 29794.10 & 55983.33 & $Y-12$ & 968.09 & 968.59 & 968.59 & 20.00 \\
\hline 5 & 29653.12 & 56001.20 & Y-12 & 964.55 & 964.38 & 964.38 & 53.00 \\
\hline 5 & 29657.27 & 56001.54 & Y-12 & 964.55 & 964.01 & & 24.00 \\
\hline 4 & 28844.77 & 44893.28 & Y-12 & 938.58 & 941.38 & 941.98 & 204.50 \\
\hline
\end{tabular}




\section{CURRENT \\ NAME}

GW-695

GW-696

GW-697

GW-698

GW-699

GW-700

GW-701

GW-702

GW-703

GW-704

GW-705

GW-705A

GW-705B

GW-706

GW-707

GW-708

GW-709

GW-710

GW-711

GW-712

GW-713

GW-714

GW-715

GW-721

GW-722

GW-723

GW-724

\section{FUNCTIONAL AREA}

Exit Pathway - Traverse B

Y-12 Plant Site

Y-12 Plant Site

Building 8110

Y-12 Plant Site

Building 8110

Y-12 Plant Site

Y-12 Plant Site

Exit Pathway - Traverse B

Exit Pathway - Traverse B

Exit Pathway - Traverse B

Bear Creek Burial Grounds WMA

Bear Creek Burial Grounds WMA

Exit Pathway - Traverse B

Y-12 Plant Site

Y-12 Plant Site

Industrial Landfill II

Exit Pathway - Traverse W

Exit Pathway - Traverse W

Exit Pathway - Traverse W

Exit Pathway - Traverse W

Exit Pathway - Traverse W

Exit Pathway - Traverse W

Pine Ridge

Exit Pathway - Traverse $\mathrm{J}$

Exit Pathway - Traverse C

Exit Pathway - Traverse C

\section{MAP NORTHING}

EASTING

SYSTEM

ELEVATION

T.o.C.

T.O.W.W

T.D.

28844.73
29276.64
29276.59
29277.15
29168.40
29452.50
29461.34
29429.50
28806.34
28844.67
28944.60

44868.30

$\mathrm{Y}-12$

937.22

939.37

939.54

62.60

$56810.35 \quad \mathrm{Y}-12 \quad 969.78$

$56806.01 \quad \mathrm{Y}-12 \quad 969.81$

56803.74

$\mathrm{Y}-12$

$56843.96 \quad Y-12$

56827.67

56712.13

56969.62

$\mathrm{Y}-12$

44930.51

44934.98

$\mathrm{Y}-12$

$\mathrm{Y}-12$

$\mathrm{Y}-12$

$4 \quad 28946.41$

44943.63

Y-12

59578.51

59529.84

$\mathrm{Y}-12$

$\mathrm{Y}-12$

52371.88

36470.67

36535.38

36506.87

36434.40

36435.09

36453.11

63157.62

64925.78

49088.55

48995.17
$\mathrm{Y}-12$

$\mathrm{Y}-12$

$\mathrm{Y}-12$

$\mathrm{Y}-12$

$\mathrm{Y}-12$

$\mathrm{Y}-12$

$\mathrm{Y}-12$

$\mathrm{Y}-12$

$\mathrm{Y}-12$

$\mathrm{Y}-12$

$\mathrm{Y}-12$
970.09

966.39

957.78

958.20

955.55

951.80

941.99

924.96

969.78

969.81

970.09

971.14

960.18

961.42

958.44

954.69

944.73

928.67

969.78

32.50

21.00

$970.09 \quad 75.00$

13.90

31.00

25.00

21.00

$955.29 \quad 182.00$

$945.33 \quad 256.00$

307.00

66.00

59.00

925.78

931.16

931.03

903.84

906.83

901.96

873.61

877.83

872.30

872.17

1140.29

951.04

1019.31

976.62

928.67

930.91

930.87

906.60

906.42

905.20

877.09

880.63

875.08

874.72

1141.42

953.71

1022.23

979.27
$929.47 \quad 182.50$

26.00

13.90

$906.81 \quad 80.60$

$906.42 \quad 744.50$

$905.20 \quad 666.20$

$877.89 \quad 457.50$

$881.43 \quad 315.20$

$875.88 \quad 145.00$

$874.92 \quad 44.60$

6.00

644.30

444.50

301.60 


\begin{tabular}{|c|c|}
\hline \multicolumn{2}{|c|}{ CURRENT } \\
\hline NAME & FUNCTIONAL AREA \\
\hline GW-725 & Exit Pathway - Traverse C \\
\hline GW-726 & Bear Creek Burial Grounds WMA \\
\hline GW-727 & Bear Creek Burial Grounds WMA \\
\hline GW-728 & Bear Creek Burial Grounds WMA \\
\hline GW-729 & Bear Creek Burial Grounds WMA \\
\hline GW-730 & Bear Creek Burial Grounds WMA \\
\hline GW-731 & $\begin{array}{l}\text { Chestnut Ridge Sediment } \\
\text { Disposal Basin }\end{array}$ \\
\hline GW-732 & $\begin{array}{l}\text { Chestnut Ridge Sediment } \\
\text { Disposal Basin }\end{array}$ \\
\hline GW-733 & Exit Pathway - Traverse $\mathrm{J}$ \\
\hline GW-734 & Exit Pathway - Traverse $\mathrm{J}$ \\
\hline GW-735 & Exit Pathway - Traverse $\mathrm{J}$ \\
\hline GW-736 & Exit Pathway - Traverse C \\
\hline GW-737 & Exit Pathway - Traverse C \\
\hline GW-738 & Exit Pathway - Traverse C \\
\hline GW-739 & Exit Pathway - Traverse C \\
\hline GW-740 & Exit Pathway - Traverse C \\
\hline GW-742 & Chestnut Ridge Security Pits \\
\hline GW-743 & Chestnut Ridge Security Pits \\
\hline GW-744 & Y-12 Grid Well K1 \\
\hline GW-745 & Y-12 Grid Well K1 \\
\hline GW-746 & Y-12 Grid Well K1 \\
\hline GW-747 & Y-12 Grid Well K2 \\
\hline GW-748 & Y-12 Grid Well K2 \\
\hline GW-749 & Y-12 Grid Well K2 \\
\hline GW-750 & Exit Pathway - Traverse $\mathrm{J}$ \\
\hline GW-751 & Y-12 Grid Well J3 \\
\hline
\end{tabular}

\section{MAP NORTHING EA}

\begin{tabular}{|c|c|c|c|c|c|c|c|}
\hline $4 \& 5$ & 29405.44 & 48989.13 & Y-12 & 958.26 & 961.05 & 961.63 & 142.50 \\
\hline 4 & 29200.66 & 42467.12 & Y-12 & 922.77 & 925.39 & & 600.00 \\
\hline 4 & 28734.12 & 42540.07 & $\mathrm{Y}-12$ & 897.96 & 900.63 & & 1000.10 \\
\hline 4 & 28773.93 & 43009.74 & Y-12 & 907.00 & 909.96 & & 305.30 \\
\hline 4 & 28500.91 & 43656.85 & $\mathrm{Y}-12$ & 1002.24 & 1004.48 & & 1361.00 \\
\hline 4 & 28920.84 & 44607.57 & Y-12 & 922.64 & 926.09 & & 1424.80 \\
\hline 6 & 27463.65 & 63863.14 & Y-12 & 1045.75 & 1049.18 & 1049.38 & 180.40 \\
\hline 6 & 27716.72 & 64267.74 & $\mathrm{Y}-12$ & 1060.65 & 1064.09 & 1064.29 & 190.60 \\
\hline 6 & 28447.10 & 65067.17 & $\mathrm{Y}-12$ & 955.69 & 959.04 & 959.84 & 256.50 \\
\hline 6 & 28681.81 & 64942.67 & Y-12 & 937.37 & 939.93 & & 103.40 \\
\hline 6 & 28866.50 & 64872.27 & $\mathrm{Y}-12$ & 921.34 & 924.28 & 924.46 & 83.00 \\
\hline 4 & 29381.05 & 48935.90 & Y-12 & 957.55 & 960.12 & 960.37 & 105.00 \\
\hline 4 & 29365.49 & 48890.01 & $\mathrm{Y}-12$ & 957.50 & 959.91 & 960.07 & 89.50 \\
\hline $4 \& 5$ & 29149.69 & 49025.75 & Y-12 & 980.36 & 983.08 & 983.31 & 90.10 \\
\hline $4 \& 5$ & 29010.00 & 49125.60 & Y-12 & 1020.66 & 1023.74 & 1023.74 & 320.00 \\
\hline $4 \& 5$ & 29027.17 & 49055.41 & Y-12 & 1016.95 & 1019.63 & 1020.25 & 190.00 \\
\hline 5 & 28037.99 & 58908.05 & $\mathrm{Y}-12$ & 1097.83 & 1100.97 & 1100.97 & 420.00 \\
\hline 5 & 28056.00 & 58907.98 & $\mathrm{Y}-12$ & 1098.72 & 1100.36 & 1100.36 & 161.10 \\
\hline 6 & 30282.34 & 64323.62 & $\mathrm{Y}-12$ & 905.05 & 907.43 & 907.43 & 69.50 \\
\hline 6 & 30278.42 & 64309.35 & $\mathrm{Y}-12$ & 904.28 & 907.06 & 907.06 & 33.00 \\
\hline 6 & 30290.91 & 64311.83 & $\mathrm{Y}-12$ & 904.26 & 906.88 & 906.88 & 15.20 \\
\hline 6 & 29730.17 & 64569.39 & $\mathrm{Y}-12$ & 911.68 & 911.06 & 920.96 & 73.25 \\
\hline 6 & 29741.23 & 64579.16 & Y-12 & 911.71 & 911.20 & 911.20 & 20.02 \\
\hline 6 & 29726.40 & 64585.74 & Y-12 & 918.69 & 921.19 & 921.19 & 16.40 \\
\hline 6 & 28974.53 & 64835.48 & Y-12 & 915.96 & 918.86 & 919.03 & 72.80 \\
\hline 6 & 29310.00 & 63248.80 & Y-12 & 909.38 & 912.05 & & 60.80 \\
\hline
\end{tabular}




\begin{tabular}{ll}
$\begin{array}{c}\text { CURRENT } \\
\text { NAME }\end{array}$ & FUNCTIONAL AREA \\
\cline { 2 - 2 } GW-752 & Y-12 Grid Well J3 \\
GW-753 & Y-12 Grid Well J2 \\
GW-754 & Y-12 Grid Well J2 \\
GW-755 & Y-12 Grid Well J1 \\
GW-756 & Y-12 Grid Well J1 \\
GW-757 & Industrial Landfill II \\
GW-758 & Y-12 Grid Well G1 \\
GW-759 & Y-12 Grid Well G1 \\
GW-760 & Y-12 Grid Well G2 \\
GW-761 & Y-12 Grid Well G2 \\
GW-762 & Y-12 Grid Well J-Primary \\
GW-763 & Y-12 Grid Well J-Primary \\
GW-764 & Y-12 Grid Well E1 \\
GW-765 & Y-12 Grid Well E1 \\
GW-766 & Y-12 Grid Well I2 \\
GW-767 & Y-12 Grid Well I2 \\
GW-768 & Y-12 Grid Well I1 \\
GW-769 & Y-12 Grid Well G3 \\
GW-770 & Y-12 Grid Well G3 \\
GW-771 & Y-12 Grid Well C1 \\
GW-772 & Y-12 Grid Well C1 \\
GW-773 & Y-12 Grid Well H2 \\
GW-774 & Y-12 Grid Well H2 \\
GW-775 & Y-12 Grid Well H3 \\
GW-776 & Y-12 Grid Well H3 \\
GW-777 & Y-12 Grid Well B2 \\
GW-778 & Y-12 Grid Well B2 \\
&
\end{tabular}

MAP NORTHING E

\begin{tabular}{|c|c|c|c|c|c|c|c|}
\hline 6 & 29312.53 & 63226.77 & $\mathrm{Y}-12$ & 909.76 & 912.78 & & 16.00 \\
\hline 6 & 29638.00 & 63208.59 & $\mathrm{Y}-12$ & 924.73 & 927.69 & 927.86 & 70.70 \\
\hline 6 & 29644.34 & 63190.57 & $\mathrm{Y}-12$ & 925.63 & 928.59 & 928.78 & 24.10 \\
\hline 6 & 30203.59 & 63266.73 & $\mathrm{Y}-12$ & 927.41 & 930.36 & 930.54 & 60.70 \\
\hline 6 & 30195.42 & 63288.31 & $\mathrm{Y}-12$ & 924.89 & 927.92 & 928.11 & 16.70 \\
\hline 5 & 25409.50 & 53302.52 & $\mathrm{Y}-12$ & 958.65 & 961.43 & 961.64 & 166.50 \\
\hline $5 \& 6$ & 30853.74 & 60331.97 & $\mathrm{Y}-12$ & 989.65 & 992.70 & & 50.20 \\
\hline $5 \& 6$ & 30863.89 & 60312.58 & $\mathrm{Y}-12$ & 991.04 & 994.01 & & 30.20 \\
\hline $5 \& 6$ & 30160.44 & 60207.30 & $Y-12$ & 966.51 & 970.02 & 970.02 & 60.10 \\
\hline $5 \& 6$ & 30133.05 & 60201.31 & $Y-12$ & 964.91 & 968.23 & 968.23 & 15.30 \\
\hline 6 & 29114.91 & 63192.53 & $\mathrm{Y}-12$ & 911.85 & 915.34 & 915.56 & 60.20 \\
\hline 6 & 29117.17 & 63219.76 & $\mathrm{Y}-12$ & 911.38 & 914.85 & 915.03 & 17.00 \\
\hline 5 & 31026.68 & 58461.86 & $\mathrm{Y}-12$ & 1006.72 & 1009.87 & 1009.87 & 65.10 \\
\hline 5 & 31025.53 & 58481.71 & $\mathrm{Y}-12$ & 1005.53 & 1008.54 & 1008.54 & 32.50 \\
\hline 6 & 29717.66 & 62312.08 & $\mathrm{Y}-12$ & 944.67 & 948.37 & & 100.30 \\
\hline 6 & 29725.79 & 62294.12 & $\mathrm{Y}-12$ & 945.17 & 948.54 & & 18.00 \\
\hline 6 & 30330.21 & 62445.04 & $\mathrm{Y}-12$ & 956.65 & 959.73 & & 65.00 \\
\hline $5 \& 6$ & 29510.42 & 60230.01 & $\mathrm{Y}-12$ & 941.53 & 944.26 & 944.43 & 61.40 \\
\hline $5 \& 6$ & 29504.56 & 60255.00 & $\mathrm{Y}-12$ & 941.67 & 944.55 & 944.72 & 20.00 \\
\hline 5 & 31331.05 & 56360.67 & $Y-12$ & 1008.27 & 1011.20 & 1011.38 & 54.40 \\
\hline 5 & 31310.43 & 56356.69 & $Y-12$ & 1009.60 & 1012.66 & 1012.82 & 19.00 \\
\hline 6 & 29878.95 & 61388.81 & $\mathrm{Y}-12$ & 960.72 & 963.76 & & 59.60 \\
\hline 6 & 29876.52 & 61408.76 & $\mathrm{Y}-12$ & 960.09 & 963.16 & & 26.60 \\
\hline 6 & 29272.38 & 61277.82 & $\mathrm{Y}-12$ & 931.48 & 931.35 & 931.35 & 60.50 \\
\hline 6 & 29271.39 & 61309.10 & $\mathrm{Y}-12$ & 931.44 & 931.25 & 931.25 & 24.00 \\
\hline 5 & 30942.73 & 55118.94 & $\mathrm{Y}-12$ & 998.79 & 1002.30 & & 59.10 \\
\hline 5 & 30923.01 & 55120.61 & $\mathrm{Y}-12$ & 998.31 & 1001.84 & & 22.00 \\
\hline
\end{tabular}




\begin{tabular}{|c|c|c|c|c|c|c|c|c|c|}
\hline \multicolumn{10}{|c|}{ CURRENT } \\
\hline NAME & FUNCTIONAL AREA & $\underline{\text { MAP }}$ & NORTHING & EASTING & SYSTEM & ELEVATION & T.O.C. & T.O.W.W. & T.D. \\
\hline GW-779 & Y-12 Grid Well F2 & $5 \& 6$ & 30225.96 & 59246.86 & $Y-12$ & 959.89 & 963.19 & & 63.10 \\
\hline GW-780 & Y-12 Grid Well F2 & $5 \& 6$ & 30222.02 & 59267.14 & Y-12 & 960.22 & 963.40 & & 20.00 \\
\hline GW-781 & Y-12 Grid Well E3 & 5 & 29710.78 & 58117.83 & Y-12 & 944.66 & 947.72 & 947.89 & 69.60 \\
\hline GW-782 & Y-12 Grid Well E3 & 5 & 29718.84 & 58099.21 & $\mathrm{Y}-12$ & 944.48 & 947.56 & 947.73 & 36.00 \\
\hline GW-783 & Y-12 Grid Well E3 & 5 & 29734.28 & 58112.53 & $\mathrm{Y}-12$ & 945.81 & 948.32 & 948.49 & 16.30 \\
\hline GW-784 & Y-12 Grid Well D1 & 5 & 31251.62 & 57036.50 & $\mathrm{Y}-12$ & 1005.81 & 1009.27 & 1009.44 & 64.30 \\
\hline GW-785 & Y-12 Grid Well D1 & 5 & 31246.88 & 57050.04 & Y-12 & 1005.87 & 1009.43 & 1009.58 & 25.50 \\
\hline GW-786 & Y-12 Grid Well E2 & 5 & 30456.28 & 58589.75 & $\mathrm{Y}-12$ & 985.23 & 987.80 & 987.80 & 64.90 \\
\hline GW-787 & Y-12 Grid Well E2 & 5 & 30437.24 & 58588.32 & $\mathrm{Y}-12$ & 984.81 & 987.85 & 987.85 & 18.10 \\
\hline GW-788 & Y-12 Grid Well F3 & $5 \& 6$ & 29617.94 & 59043.21 & $\mathrm{Y}-12$ & 933.84 & 937.07 & 937.24 & 67.90 \\
\hline GW-789 & Y-12 Grid Well F3 & $5 \& 6$ & 29644.68 & 59044.56 & $\mathrm{Y}-12$ & 934.37 & 937.30 & 937.49 & 23.80 \\
\hline GW-790 & Bear Creek Burial Grounds WMA & 4 & 28670.02 & 43038.45 & $\mathrm{Y}-12$ & 909.49 & 911.51 & & 1040.30 \\
\hline GW-791 & Y-12 Grid Well D2 & 5 & 30482.73 & 57423.24 & $\mathrm{Y}-12$ & 988.51 & 991.96 & 992.13 & 70.60 \\
\hline GW-792 & Y-12 Grid Well D2 & 5 & 30481.15 & 57442.11 & $\mathrm{Y}-12$ & 989.60 & 992.57 & 992.74 & 29.20 \\
\hline GW-793 & $\begin{array}{l}\text { Above Grade Low Level Waste } \\
\text { Storage Fac. }\end{array}$ & 4 & 30235.82 & 46831.52 & $\mathrm{Y}-12$ & 965.05 & 968.18 & 968.38 & 29.00 \\
\hline GW-794 & $\begin{array}{l}\text { Above Grade Low Level Waste } \\
\text { Storage Fac. }\end{array}$ & 4 & 29273.17 & 46392.92 & Y-12 & 930.91 & 934.17 & 934.37 & 39.30 \\
\hline GW-795 & $\begin{array}{l}\text { Above Grade Low Level Waste } \\
\text { Storage Fac. }\end{array}$ & 4 & 29286.69 & 45630.06 & Y-12 & 922.92 & 925.98 & 926.18 & 20.10 \\
\hline GW-796 & Industrial Landfill $\vee$ & 5 & 27923.90 & 58206.40 & $\mathrm{Y}-12$ & 1048.80 & 1052.42 & 1052.62 & 139.70 \\
\hline GW-797 & Industrial Landfill V & 5 & 27446.60 & 58550.40 & Y-12 & 1056.10 & 1059.80 & 1060.00 & 134.10 \\
\hline GW-798 & Construction/Demolition Landfill VII & $5 \& 6$ & 27264.85 & 60309.95 & $\mathrm{Y}-12$ & 1002.42 & 1005.80 & 1006.00 & 151.32 \\
\hline GW-799 & Industrial Landfill $\vee$ & $5 \& 6$ & 26745.50 & 59961.20 & Y-12 & 978.10 & 981.09 & 981.29 & 92.00 \\
\hline GW-800 & Oil Landfarm WMA & 4 & 28981.60 & 48260.30 & $Y-12$ & 961.40 & 964.36 & 964.56 & 32.00 \\
\hline GW-801 & Industrial Landfill V & 5 & 26807.80 & 58779.90 & $\mathrm{Y}-12$ & 1093.82 & 1096.96 & 1097.16 & 188.90 \\
\hline GW-802 & Y-12 Fuel Station & 6 & 29654.70 & 62216.80 & Y-12 & 942.30 & 941.83 & & 26.50 \\
\hline GW-803 & Y-12 Fuel Station & 6 & 29558.70 & 62217.30 & $\mathrm{Y}-12$ & 939.29 & 942.53 & & 25.00 \\
\hline
\end{tabular}




\begin{tabular}{|c|c|c|c|c|c|c|c|c|c|}
\hline \multicolumn{10}{|c|}{ CURRENT } \\
\hline NAME & FUNCTIONAL AREA & $\underline{\text { MAP }}$ & NORTHING & EASTING & SYSTEM & ELEVATION & T.O.C. & T.O.W.W. & T.D. \\
\hline GW-804 & Y-12 Fuel Station & 6 & 29558.10 & 62110.80 & $\mathrm{Y}-12$ & 939.22 & 942.55 & & 25.00 \\
\hline GW-805 & Y-12 Plant Site & 5 & 29884.80 & 57402.70 & $\mathrm{Y}-12$ & 955.25 & 955.02 & & 13.80 \\
\hline GW-806 & Y-12 Plant Site & 5 & 29899.70 & 57461.00 & Y-12 & 952.16 & 951.95 & & 12.90 \\
\hline GW-807 & Y-12 Plant Site & 5 & 29866.00 & 57458.90 & $\mathrm{Y}-12$ & 951.52 & 955.36 & & 11.30 \\
\hline GW-808 & Y-12 Plant Site & $5 \& 6$ & 29318.00 & 59529.90 & $Y-12$ & 931.24 & 930.75 & & 40.40 \\
\hline GW-809 & Y-12 Plant Site & $5 \& 6$ & 29316.60 & 59548.40 & Y-12 & 931.27 & 931.04 & & 40.00 \\
\hline GW-810 & Y-12 Plant Site & $5 \& 6$ & 29323.80 & 59606.50 & $\mathrm{Y}-12$ & 931.68 & 931.44 & & 26.80 \\
\hline GW-811 & Spoil Area I & 5 & 29443.24 & 52324.63 & Y-12 & 1044.56 & 1048.26 & & 64.50 \\
\hline GW-812 & Spoil Area I & 5 & 29466.38 & 52477.07 & $\mathrm{Y}-12$ & 1028.76 & 1032.13 & & 44.90 \\
\hline GW-813 & SY-200 Yard & 5 & 29590.07 & 51474.27 & $\mathrm{Y}-12$ & 1003.83 & 1007.16 & & 25.50 \\
\hline GW-814 & SY-200 Yard & 5 & 29475.15 & 51102.53 & $Y-12$ & 998.37 & 1001.48 & & 24.30 \\
\hline GW-815 & SY-200 Yard & 5 & 29592.36 & 51121.37 & $\mathrm{Y}-12$ & 998.30 & 1000.96 & & 21.10 \\
\hline GW-816 & $\begin{array}{l}\text { Exit Pathway Scarboro Road/Pine } \\
\text { Ridge }\end{array}$ & 6 & 31581.50 & 64031.36 & $\mathrm{Y}-12$ & 894.56 & 898.21 & 898.42 & 16.10 \\
\hline GW-817 & Y-12 Grid Well K3 & 6 & 29269.15 & 64643.00 & $Y-12$ & 914.61 & 918.12 & 918.30 & 23.00 \\
\hline GW-818 & Building 9201-2 & $5 \& 6$ & 29118.66 & 60140.25 & Y-12 & 928.18 & 927.93 & & 20.40 \\
\hline GW-819 & Building 9201-2 & $5 \& 6$ & 29144.68 & 60086.58 & $\mathrm{Y}-12$ & 928.82 & 928.75 & 928.75 & 15.50 \\
\hline GW-820 & Building 9201-2 & $5 \& 6$ & 29174.65 & 59772.96 & Y-12 & 929.67 & 929.57 & 929.57 & 17.30 \\
\hline GW-821 & Gum Branch Road & $2 \& 3$ & 29621.25 & 29250.08 & Y-12 & 842.40 & 842.80 & & 50.50 \\
\hline GW-822 & Gum Branch Road & $2 \& 3$ & 29587.33 & 29270.74 & $\mathrm{Y}-12$ & 842.71 & 843.06 & & 70.00 \\
\hline GW-823 & Gum Branch Road & $2 \& 3$ & 29591.59 & 29214.43 & $Y-12$ & 840.42 & 840.65 & & 72.00 \\
\hline GW-824 & Y-12 Plant Site & $5 \& 6$ & 29454.00 & 60022.00 & $\mathrm{Y}-12$ & 930.00 & & & 11.70 \\
\hline GW-825 & Industrial Landfill II & 5 & 26852.33 & 52347.91 & $\mathrm{Y}-12$ & 1088.55 & 1091.24 & & 36.00 \\
\hline GW-826 & Industrial Landfill II & 5 & 26271.84 & 52774.69 & $\mathrm{Y}-12$ & 1062.59 & 1063.27 & & 36.00 \\
\hline GW-827 & Construction/Demolition Landfill VI & 5 & 27721.42 & 51826.32 & $\mathrm{Y}-12$ & 1048.13 & 1051.39 & 1051.60 & 135.00 \\
\hline GW-828 & S-3 Site & $4 \& 5$ & 30059.52 & 49995.02 & $\mathrm{Y}-12$ & 979.08 & 982.92 & & 168.00 \\
\hline GW-829 & S-3 Site & $4 \& 5$ & 29952.81 & 50035.53 & $\mathrm{Y}-12$ & 981.92 & 985.78 & 985.95 & 115.00 \\
\hline
\end{tabular}




\begin{tabular}{|c|c|}
\hline \multicolumn{2}{|c|}{ CURRENT } \\
\hline NAME & FUNCTIONAL AREA \\
\hline GW-830 & TSD Facility, Blair Road \\
\hline GW-831 & Chestnut Ridge Security Pits \\
\hline GW-832 & New Hope Pond \\
\hline GW-833 & Y-12 Plant Site \\
\hline GW-834 & S-3 Ponds \\
\hline GW-835 & S-3 Ponds \\
\hline GW-836 & S-3 Ponds \\
\hline GW-837 & S-3 Ponds \\
\hline GW-838 & Pine Ridge \\
\hline GW-839 & Pine Ridge \\
\hline GW-840 & Pine Ridge \\
\hline GW-841 & $\begin{array}{l}\text { South Campus Facility, Bethel } \\
\text { Valley }\end{array}$ \\
\hline GW-842 & $\begin{array}{l}\text { South Campus Facility, Bethel } \\
\text { Valley }\end{array}$ \\
\hline GW-843 & $\begin{array}{l}\text { South Campus Facility, Bethel } \\
\text { Valley }\end{array}$ \\
\hline GW-844 & $\begin{array}{l}\text { South Campus Facility, Bethel } \\
\text { Valley }\end{array}$ \\
\hline GW-845 & Y-12 Plant Site \\
\hline GW-854 & S-3 Ponds \\
\hline GW-855 & S-3 Ponds \\
\hline GW-857 & S-3 Ponds \\
\hline GW-858 & S-3 Ponds \\
\hline GW-859 & S-3 Ponds \\
\hline GW-860 & S-3 Ponds \\
\hline GW-861 & S-3 Ponds \\
\hline GW-862 & S-3 Ponds \\
\hline
\end{tabular}

\section{MAP NORTHING}

EASTING

SYSTEM

ELEVATION

T.O.C.

T.O.W.W.

T.D.

\begin{tabular}{|c|c|c|c|c|c|c|c|}
\hline 1 & 33096.25 & 18306.25 & Y-12 & 842.34 & 842.54 & & \\
\hline 5 & 26653.53 & 56593.48 & Y-12 & 1088.04 & 1091.09 & 1091.29 & 200.00 \\
\hline 6 & 29141.95 & 64133.61 & Y-12 & 906.83 & 906.18 & & 11.90 \\
\hline $5 \& 6$ & 29123.57 & 60085.40 & $\mathrm{Y}-12$ & 927.86 & & & 10.00 \\
\hline $4 \& 5$ & 30020.30 & 50855.00 & Y-12 & 991.93 & 995.04 & 995.21 & 16.80 \\
\hline 5 & 29822.02 & 51358.36 & Y-12 & 998.04 & 1000.91 & & 19.20 \\
\hline 5 & 29774.56 & 51356.24 & Y-12 & 998.04 & 1001.00 & & 24.40 \\
\hline 5 & 29969.06 & 52281.57 & Y-12 & 1005.22 & 1007.96 & & 27.90 \\
\hline 4 & 30395.55 & 46084.20 & Y-12 & 964.42 & 967.73 & & 31.50 \\
\hline 4 & 30775.51 & 46797.22 & Y-12 & 1037.62 & 1040.62 & & 62.00 \\
\hline 4 & 31173.36 & 47090.25 & Y-12 & 1024.24 & 1027.63 & & 32.50 \\
\hline 6 & 23166.92 & 66202.65 & Y-12 & 828.09 & 830.64 & 830.64 & 10.30 \\
\hline 6 & 23004.36 & 66235.19 & Y-12 & 820.14 & 822.34 & 822.34 & 25.00 \\
\hline 6 & 22993.10 & 66234.47 & Y-12 & 819.70 & 821.52 & 821.52 & 67.00 \\
\hline 6 & 22998.47 & 66242.47 & Y-12 & 819.73 & 821.94 & 821.94 & 177.00 \\
\hline 6 & 28600.91 & 64391.98 & Y-12 & 924.71 & 926.47 & & 438.30 \\
\hline $4 \& 5$ & 30050.68 & 50862.26 & Y-12 & 990.50 & 994.12 & & 12.90 \\
\hline $4 \& 5$ & 30123.76 & 51002.32 & Y-12 & 991.89 & 995.00 & & 17.80 \\
\hline $4 \& 5$ & 30017.36 & 50846.69 & Y-12 & 990.47 & 992.35 & & 14.80 \\
\hline $4 \& 5$ & 29928.39 & 50872.25 & Y-12 & 991.35 & 994.33 & & 17.50 \\
\hline 5 & 29781.15 & 51410.93 & $\mathrm{Y}-12$ & 998.28 & 1000.28 & & 14.60 \\
\hline 5 & 29757.29 & 51229.85 & Y-12 & 996.74 & 999.48 & & 20.70 \\
\hline 5 & 29745.83 & 51144.29 & Y-12 & 988.97 & 991.64 & & 16.90 \\
\hline 5 & 29958.44 & 52281.32 & Y-12 & 1004.51 & 1006.78 & & 14.30 \\
\hline
\end{tabular}




$\begin{array}{ll}\begin{array}{c}\text { CURRENT } \\ \text { NAME }\end{array} & \text { FUNCTIONAL AREA } \\ \text { GW-863 } & \text { S-3 Ponds } \\ \text { GW-864 } & \text { S-3 Ponds } \\ \text { GW-865 } & \text { Boneyard/Burnyard } \\ \text { GW-866 } & \text { Boneyard/Burnyard } \\ \text { GW-867 } & \text { Boneyard/Burnyard } \\ \text { GW-868 } & \text { S-3 Ponds } \\ \text { GW-869 } & \text { S-3 Ponds } \\ \text { GW-871 } & \text { S-3 Ponds } \\ \text { GW-872 } & \text { S-3 Ponds } \\ \text { GW-873 } & \text { Boneyard/Burnyard } \\ \text { GW-874 } & \text { Boneyard/Burnyard } \\ \text { GW-875 } & \text { Boneyard/Burnyard } \\ \text { GW-876 } & \text { Boneyard/Burnyard } \\ \text { GW-877 } & \text { S-3 Ponds } \\ \text { GW-878 } & \text { S-3 Ponds } \\ \text { GW-879 } & \text { S-3 Ponds } \\ \text { GW-880 } & \text { S-3 Ponds } \\ \text { GW-881 } & \text { S-3 Ponds } \\ \text { GW-882 } & \text { S-3 Ponds } \\ \text { GW-883 } & \text { S-3 Ponds } \\ \text { GW-884 } & \text { S-3 Ponds } \\ \text { GW-885 } & \text { S-3 Ponds } \\ \text { GW-886 } & \text { S-3 Ponds } \\ \text { GW-887 } & \text { S-3 Ponds } \\ \text { GW-888 } & \text { S-3 Ponds } \\ \text { GW-889 } & \text { S-3 Ponds } \\ \text { GW-890 } & \text { S-3 Ponds } \\ & \end{array}$

MAP NORTHING EASTING

T.D.

$\begin{array}{crrrrrr}5 & 29911.55 & 52062.66 & \text { Y-12 } & 1005.04 & 1007.16 & 23.30 \\ 5 & 29858.65 & 51838.90 & \text { Y-12 } & 1003.08 & 1005.53 & 17.30 \\ 4 \& 5 & 30063.55 & 49773.83 & \text { Y-12 } & 975.78 & 978.82 & 12.90 \\ 4 \& 5 & 29970.97 & 49551.56 & \text { Y-12 } & 970.18 & 972.73 & 17.40 \\ 4 \& 5 & 29912.20 & 49478.25 & \text { Y-12 } & 971.84 & 973.79 & 9.70 \\ 5 & 29768.96 & 51349.84 & \text { Y-12 } & 998.06 & 1000.27 & 17.50 \\ 5 & 29828.61 & 51363.03 & \text { Y-12 } & 998.21 & 999.44 & 15.30 \\ 5 & 29812.18 & 51286.82 & \text { Y-12 } & 997.47 & 999.97 & 20.60 \\ 5 & 29948.10 & 52420.49 & \text { Y-12 } & 1001.36 & 1004.01 & 11.20 \\ 4 \& 5 & 30009.87 & 49702.40 & \text { Y-12 } & 975.83 & 978.33 & 12.30 \\ 4 \& 5 & 29940.63 & 49752.14 & \text { Y-12 } & 981.03 & 983.44 & 16.70 \\ 4 \& 5 & 29873.18 & 49791.72 & \text { Y-12 } & 980.19 & 982.51 & 19.20 \\ 4 \& 5 & 29914.63 & 49471.15 & \text { Y-12 } & 972.96 & 975.62 & 21.10 \\ 4 \& 5 & 29965.39 & 50827.71 & \text { Y-12 } & 991.66 & 994.34 & 16.00 \\ 5 & 30074.96 & 52029.77 & \text { Y-12 } & 1007.68 & 1009.03 & 15.20 \\ 5 & 30151.22 & 51520.13 & \text { Y-12 } & 1000.47 & 1003.21 & 17.10 \\ 5 & 30244.31 & 51237.66 & \text { Y-12 } & 998.85 & 1001.49 & 18.90 \\ 5 & 30228.20 & 52001.47 & \text { Y-12 } & 1010.69 & 1012.76 & 20.10 \\ 5 & 29876.85 & 51465.49 & \text { Y-12 } & 999.95 & 1003.11 & 20.10 \\ 5 & 30024.42 & 51526.39 & \text { Y-12 } & 1000.16 & 1003.72 & 18.60 \\ 5 & 30052.04 & 52234.25 & \text { Y-12 } & 1007.42 & 1009.60 & 16.10 \\ 5 & 30053.34 & 52236.31 & \text { Y-12 } & 1007.29 & 1009.35 & 17.00 \\ 5 & 29966.77 & 52272.13 & \text { Y-12 } & 1005.26 & 1007.52 & 20.50 \\ 5 & 29968.93 & 52201.22 & \text { Y-12 } & 1006.37 & 1007.67 & 21.50 \\ 5 & 29960.92 & 52124.35 & \text { Y-12 } & 1005.97 & 1006.69 & 11.50 \\ 5 & 29980.41 & 52372.05 & \text { Y-12 } & 1003.42 & 1004.76 & \\ 5 & 29994.59 & 52432.36 & \text { Y-12 } & 1002.14 & 1003.35 & \end{array}$




$\begin{array}{ll}\begin{array}{c}\text { CURRENT } \\ \text { NAME }\end{array} & \text { FUNCTIONAL AREA } \\ \text { GW-902 } & \text { Gum Branch Road } \\ \text { GW-904 } & \text { Oil Landfarm WMA } \\ \text { GW-905 } & \text { Oil Landfarm WMA } \\ \text { GW-906 } & \text { Oil Landfarm WMA } \\ \text { GW-907 } & \text { Oil Landfarm WMA } \\ \text { GW-908 } & \text { Oil Landfarm WMA } \\ \text { GW-909 } & \text { Oil Landfarm WMA } \\ \text { GW-910 } & \text { Oil Landfarm WMA } \\ \text { GW-911 } & \text { Oil Landfarm WMA } \\ \text { GW-912 } & \text { Oil Landfarm WMA } \\ \text { GW-913 } & \text { Oil Landfarm WMA } \\ \text { GW-914 } & \text { Oil Landfarm WMA } \\ \text { GW-915 } & \text { Gum Branch Road } \\ \text { GW-916 } & \text { EMWMF } \\ \text { GW-917 } & \text { EMWMF } \\ \text { GW-918 } & \text { EMWMF } \\ \text { GW-919 } & \text { EMWMF } \\ \text { GW-920 } & \text { EMWMF } \\ \text { GW-921 } & \text { EMWMF } \\ \text { GW-922 } & \text { EMWMF } \\ \text { GW-923 } & \text { EMWMF } \\ \text { GW-924 } & \text { EMWMF } \\ \text { GW-925 } & \text { EMWMF } \\ \text { GW-926 } & \text { EMWMF } \\ \text { GW-927 } & \text { EMWMF } \\ \text { GW-928 } & \text { Y-12 Grid Well C1 } \\ \text { GW-929 } & \text { Y-12 Grid Well C1 }\end{array}$

$\begin{array}{crrrrrrr}\text { MAP } & \text { NORTHING } & \text { EASTING } & \text { SYSTEM } & \text { ELEVATION } & \text { T.O.C. } & \text { T.O.W.W. } & \text { T.D. } \\ 2 \& 3 & 29911.50 & 30756.05 & \text { Y-12 } & 870.30 & 872.60 & & 31.80 \\ 4 & 29783.61 & 45889.53 & \text { Y-12 } & 949.86 & 952.88 & & 300.00 \\ 4 & 30426.78 & 46121.23 & \text { Y-12 } & 970.11 & 973.59 & & 71.00 \\ 4 & 29899.14 & 46196.37 & \text { Y-12 } & 948.48 & 951.16 & & 39.90 \\ 4 & 30440.14 & 46374.36 & \text { Y-12 } & 992.98 & 995.30 & & \\ 4 & 30573.70 & 46980.35 & \text { Y-12 } & 983.71 & 985.59 & & 48.20 \\ 4 & 30616.07 & 46458.08 & \text { Y-12 } & 991.21 & 993.40 & & 26.10 \\ 4 & 30783.27 & 47775.47 & \text { Y-12 } & & & & 35.50 \\ 4 & 30512.13 & 47799.85 & \text { Y-12 } & 1003.66 & 1006.23 & & 55.40 \\ 4 & 30277.36 & 48226.71 & \text { Y-12 } & 976.23 & 978.91 & & 42.20 \\ 4 & 29981.98 & 47922.83 & \text { Y-12 } & 973.42 & 973.15 & & 22.00 \\ 4 & 29782.69 & 47508.31 & \text { Y-12 } & 958.77 & 961.58 & & 45.20 \\ 2 \& 3 & 29914.65 & 30702.20 & \text { Y-12 } & 870.30 & 872.60 & & \\ 4 & 31186.00 & 48275.78 & \text { Y-12 } & & 1002.85 & 1002.85 & 36.00 \\ 4 & 30462.57 & 47914.08 & \text { Y-12 } & & 997.10 & 997.10 & 51.00 \\ 4 & 31672.26 & 47549.25 & \text { Y-12 } & & 1067.96 & 1067.96 & 75.00 \\ 4 & 30739.25 & 47325.88 & \text { Y-12 } & 987.50 & 993.56 & 993.56 & 32.00 \\ 4 & 30192.58 & 47375.12 & \text { Y-12 } & & 967.43 & 967.43 & 55.00 \\ 4 & 30350.07 & 47138.93 & \text { Y-12 } & & 971.29 & 971.29 & 50.00 \\ 4 & 30024.00 & 47146.86 & \text { Y-12 } & & 956.91 & 956.91 & 46.00 \\ 4 & 30821.98 & 48183.73 & \text { Y-12 } & & 1016.73 & 1016.73 & 102.00 \\ 4 & 30184.77 & 46299.54 & \text { Y-12 } & & 968.90 & 968.90 & 54.00 \\ 4 & 30348.97 & 47128.22 & \text { Y-12 } & & 971.14 & 971.14 & 170.00 \\ 4 & 30184.96 & 46290.39 & \text { Y-12 } & & 968.94 & 968.94 & 145.00 \\ 4 & 30462.61 & 47905.71 & \text { Y-12 } & & 997.19 & 997.19 & 172.00 \\ 5 & 31725.61 & 56220.60 & \text { Y-12 } & 1046.83 & 1049.20 & 1049.23 & 44.00 \\ 5 & 31725.76 & 56240.51 & \text { Y-12 } & 1047.82 & 1050.25 & 1050.28 & 28.60\end{array}$




$\begin{array}{ll}\begin{array}{c}\text { CURRENT } \\ \text { NAME }\end{array} & \text { FUNCTIONAL AREA } \\ \text { GW-930 } & \text { Y-12 Grid Well D1 } \\ \text { GW-931 } & \text { Y-12 Grid Well D1 } \\ \text { GW-932 } & \text { EMWMF } \\ \text { GW-933 } & \text { EMWMF } \\ \text { GW-934 } & \text { New Hope Pond } \\ \text { GW-935 } & \text { EMWMF } \\ \text { GW-936 } & \text { EMWMF } \\ \text { GW-937 } & \text { EMWMF } \\ \text { GW-938 } & \text { EMWMF } \\ \text { GW-939 } & \text { EMWMF } \\ \text { GW-940 } & \text { EMWMF } \\ \text { GW-941 } & \text { EMWMF } \\ \text { GW-942 } & \text { EMWMF } \\ \text { GW-943 } & \text { EMWMF } \\ \text { GW-944 } & \text { EMWMF } \\ \text { GW-945 } & \text { EMWMF } \\ \text { GW-946 } & \text { EMWMF } \\ \text { GW-947 } & \text { EMWMF } \\ \text { GW-948 } & \text { EMWMF } \\ \text { GW-949 } & \text { EMWMF } \\ \text { GW-950 } & \text { EMWMF } \\ \text { GW-951 } & \text { EMWMF } \\ \text { GW-952 } & \text { EMWMF } \\ \text { GW-953 } & \text { Unknown } \\ \text { GW-954 } & \text { Y-12 Plant Site } \\ \text { GW-955 } & \text { Y-12 Plant Site } \\ \text { GW-956 } & \text { Y-12 Plant Site } \\ & \end{array}$

\begin{tabular}{|c|c|c|c|c|c|c|c|}
\hline MAP & NORTHING & EASTING & SYSTEM & ELEVATION & T.O.C. & T.O.W.W. & T.D. \\
\hline 5 & 31424.19 & 57053.12 & Y-12 & 1002.47 & 1005.58 & 1005.62 & 41.50 \\
\hline 5 & 31420.92 & 57080.37 & Y-12 & 1003.62 & 1006.92 & 1006.96 & 19.80 \\
\hline 4 & 30900.00 & 47300.00 & Y-12 & & & & \\
\hline 4 & 30775.00 & 46797.00 & $Y-12$ & & & & \\
\hline 6 & 28661.97 & 62965.47 & Y-12 & 914.81 & 916.60 & & 400.40 \\
\hline 4 & 31374.04 & 47426.46 & Y-12 & 1049.64 & 1051.62 & & 32.00 \\
\hline 4 & 31227.30 & 47381.26 & Y-12 & 1018.46 & 1020.79 & & 23.00 \\
\hline 4 & 31227.66 & 46914.61 & Y-12 & 1019.97 & 1022.27 & & 28.00 \\
\hline 4 & 31075.59 & 46663.11 & Y-12 & 1002.96 & 1004.95 & & 30.00 \\
\hline 4 & 30957.90 & 47011.71 & Y-12 & 1010.01 & 1012.15 & & 22.50 \\
\hline 4 & 30726.71 & 46941.75 & Y-12 & 997.27 & 999.27 & & 23.00 \\
\hline 4 & 30458.62 & 46610.49 & Y-12 & 984.88 & 986.82 & & 18.40 \\
\hline 4 & 30509.21 & 46235.87 & Y-12 & 969.23 & 971.39 & & 18.00 \\
\hline 4 & 30719.13 & 46300.79 & Y-12 & 975.57 & 977.74 & & 20.00 \\
\hline 4 & 30618.81 & 48206.51 & Y-12 & 1006.21 & 1007.98 & & 30.00 \\
\hline 4 & 30919.65 & 47452.39 & Y-12 & 1016.16 & 1018.20 & & 35.00 \\
\hline 4 & 31374.07 & 47430.62 & Y-12 & 1050.11 & 1052.31 & & 60.00 \\
\hline 4 & 31406.49 & 47694.11 & Y-12 & 1054.39 & 1056.47 & & 45.00 \\
\hline 4 & 31430.81 & 47911.13 & Y-12 & 1058.89 & 1060.98 & & 50.00 \\
\hline \multirow[t]{2}{*}{4} & 31193.69 & 48089.16 & Y-12 & 1051.18 & 1053.36 & & 50.00 \\
\hline & 31395.20 & 47561.80 & & 1050.86 & 1053.97 & & 46.00 \\
\hline 4 & 31395.80 & 47560.90 & $Y-12$ & 1050.90 & 1053.97 & & 45.00 \\
\hline 4 & 30604.24 & 47775.40 & Y-12 & 1019.60 & 1022.30 & & 45.00 \\
\hline 4 & 30648.78 & 46612.75 & Y-12 & 992.32 & 994.74 & & 35.00 \\
\hline 5 & 30621.63 & 58218.34 & Y-12 & 988.73 & 988.27 & & 170.00 \\
\hline 5 & 30621.67 & 58208.19 & Y-12 & 988.67 & 988.23 & & \\
\hline 6 & 29475.00 & 61050.00 & Y-12 & 940.00 & & & 170.00 \\
\hline
\end{tabular}




\begin{tabular}{ll} 
CURRENT & \\
NAME & FUNCTIONAL AREA \\
GW-959 & Y-12 Plant Site \\
GW-960 & Y-12 Grid Well F2 \\
GW-961 & EMWMF \\
GW-964 & EMWMF \\
GW-965 & EMWMF \\
GW-966 & Y-12 Fuel Station \\
GW-967 & Building 8110 \\
J-001 & Y-12 Plant Site \\
J-002 & Y-12 Plant Site \\
J-003 & Y-12 Plant Site \\
J-004 & Y-12 Plant Site \\
J-005 & Y-12 Plant Site \\
J-006 & Y-12 Plant Site \\
J-007 & Y-12 Plant Site \\
J-008 & Y-12 Plant Site \\
J-009 & Y-12 Plant Site \\
J-010 & Y-12 Plant Site \\
J-011 & Y-12 Plant Site \\
J-012 & Y-12 Plant Site \\
J-015 & Y-12 Plant Site \\
J-017 & Y-12 Plant Site \\
J-018 & Y-12 Plant Site \\
J-019 & Y-12 Plant Site \\
J-020 & Y-12 Plant Site \\
J-021 & Y-12 Plant Site \\
J-022 & Y-12 Plant Site \\
K-020 & Y-12 Plant Site \\
& \\
\hline
\end{tabular}

\section{MAP NORTHING}

EASTING

5

5 \&

\begin{tabular}{l}
29115.05 \\
30212.28 \\
30347.22 \\
30280.62 \\
30282.91 \\
29979.16 \\
29325.00 \\
29265.96 \\
29365.13 \\
29464.29 \\
29563.45 \\
29662.62 \\
29860.95 \\
29240.14 \\
29438.47 \\
29636.80 \\
29835.13 \\
29214.32 \\
29412.65 \\
29168.00 \\
29565.00 \\
29763.00 \\
29291.78 \\
29490.11 \\
29688.44 \\
29886.77 \\
\hline 5600.00 \\
\hline 57
\end{tabular}

60189.27

60189.27

4609151

46596.54

46606.35

61978.04

56835.00

56994.51

57007.42

57020.33

57033.24

57046.15

57081.97

57192.84

57218.66

57244.48

57270.30

57391.17

57416.99

57585.00

57639.00

57664.00

56796.18

56822.00

56847.82

56873.64

62400.00
SYSTEM

Y-12

$\mathrm{Y}-12$

$\mathrm{Y}-12$

Y-12

$\mathrm{Y}-12$

$\mathrm{Y}-12$

$\mathrm{Y}-12$

$\mathrm{Y}-12$

$\mathrm{Y}-12$

$\mathrm{Y}-12$

$\mathrm{Y}-12$

Y-12

$\mathrm{Y}-12$

$\mathrm{Y}-12$

$\mathrm{Y}-12$

Y-12

$\mathrm{Y}-12$

$\mathrm{Y}-12$

$\mathrm{Y}-12$

$\mathrm{Y}-12$

$\mathrm{Y}-12$

$\mathrm{Y}-12$

$\mathrm{Y}-12$

$\mathrm{Y}-12$

$\mathrm{Y}-12$

$\mathrm{Y}-12$

$\mathrm{Y}-12$
ELEVATION

T.O.C.

T.O.W.W.

T.D.

$\begin{array}{ll}928.32 & 927.69 \\ 959.88 & 963.26 \\ & 963.57 \\ & 977.40 \\ & 977.63 \\ 956.64 & 956.30\end{array}$

927.69

9.00

963.47

25.40

27.00

32.00

72.00

20.00

942.58

945.08

944.67

947.83

952.25

960.58

941.08

945.42

956.96

961.75

938.67

942.08

939.47

944.17

957.47

944.25

947.75

950.75

950.25

919.72
38.50

41.00

28.00

34.00

41.00

51.00

45.60

50.00

46.00

35.00

35.10

31.70

35.00

40.00

35.00

30.20

32.00

32.00

31.00

35.00 


\section{CURRENT}

K-021

K-022

$\mathrm{K}-023$

K-024

K1-015

K2-015

LL/HAZ-01

LL/HAZ-02

LL/HAZ-03

LL/HAZ-04

LL/HAZ-05

LL/HAZ-06

LL/HAZ-07

LL/HAZ-08

LL/HAZ-09

LL/HAZ-10

LL/HAZ-11

LL/HAZ-12

LL/HAZ-13

LL/HAZ-14

LL/HAZ-15

LL/HAZ-16

LL/HAZ-17

LL/HAZ-18

LL/HAZ-19

M-04

M-05
Y-12 Plant Site

Y-12 Plant Site

$\mathrm{Y}-12$ Plant Site

Y-12 Plant Site

Y-12 Plant Site

Y-12 Plant Site

Gum Branch Road

Gum Branch Road

Gum Branch Road

Gum Branch Road

Gum Branch Road

Gum Branch Road

Gum Branch Road

Gum Branch Road

Gum Branch Road

Gum Branch Road

Gum Branch Road

Gum Branch Road

Gum Branch Road

Gum Branch Road

Gum Branch Road

Gum Branch Road

Gum Branch Road

Gum Branch Road

Gum Branch Road

Gum Branch Road

Gum Branch Road

$\begin{array}{lll}28600.00 & 62800.00 & \mathrm{Y}-12 \\ 28600.00 & 63200.00 & \mathrm{Y}-12 \\ 28600.00 & 63600.00 & \mathrm{Y}-12 \\ 28600.00 & 64000.00 & \mathrm{Y}-12 \\ 28850.00 & 64000.00 & \mathrm{Y}-12 \\ 28850.00 & 64005.00 & \mathrm{Y}-12 \\ 30878.00 & 33054.00 & \mathrm{Y}-12 \\ 30849.00 & 33207.00 & \mathrm{Y}-12 \\ 29806.44 & 32789.13 & \mathrm{Y}-12 \\ 29773.63 & 32580.08 & \mathrm{Y}-12 \\ 29589.83 & 31450.34 & \mathrm{Y}-12 \\ 29770.43 & 32123.31 & \mathrm{Y}-12 \\ 30714.87 & 32106.57 & \mathrm{Y}-12 \\ 30582.01 & 32124.08 & \mathrm{Y}-12 \\ 30354.29 & 32122.72 & \mathrm{Y}-12 \\ 30471.13 & 32123.38 & \mathrm{Y}-12 \\ 30223.26 & 32138.85 & \mathrm{Y}-12 \\ 30086.28 & 32124.05 & \mathrm{Y}-12 \\ 30640.63 & 32192.72 & \mathrm{Y}-12 \\ 30236.57 & 32157.15 & \mathrm{Y}-12 \\ 29779.90 & 32153.06 & \mathrm{Y}-12\end{array}$

924.12

925.98

927.95

931.40

912.38

912.50

925.47

914.11

866.92

874.20

868.30

879.80

912.56

923.47

947.70

936.84

931.30

908.80

916.34

932.47

878.41

927.47

917.11

868.91

875.48

869.10

882.03

914.07

924.65

948.66

938.59

932.57

909.77

918.61

934.95

879.09

37.00

45.00

55.00

57.00

59.00

62.50

45.00

25.00

15.00

25.00

25.00

30.00

40.00

32.00

59.00

40.00

33.00

35.00

400.00

350.00

375.00

24.00

40.00

22.00

23.50
890.31

891.94

864.63

864.63

3
$32146.20 \quad Y-12$ 


\begin{tabular}{|c|c|}
\hline \multicolumn{2}{|c|}{ CURRENT } \\
\hline NAME & FUNCTIONAL AREA \\
\hline M-06 & Gum Branch Road \\
\hline M-07 & Gum Branch Road \\
\hline M-08 & Gum Branch Road \\
\hline M-09 & Gum Branch Road \\
\hline M-10 & Gum Branch Road \\
\hline OR-02 & Gum Branch Road \\
\hline OR-03 & Gum Branch Road \\
\hline OR-04 & Gum Branch Road \\
\hline OR-05 & Gum Branch Road \\
\hline OR-06 & Gum Branch Road \\
\hline OR-07 & BCV @ Hagwood Road \\
\hline OR-08 & BCV@ Hagwood Road \\
\hline OR-09 & BCV@ Hagwood Road \\
\hline OR-21 & Gum Branch Road \\
\hline OR-22 & Gum Branch Road \\
\hline OR-23 & Gum Branch Road \\
\hline$P \& A-1$ & Gum Branch Road \\
\hline$P \& A-2$ & Gum Branch Road \\
\hline$P \& A-3$ & Gum Branch Road \\
\hline PP-01 & EMWMF \\
\hline PP-02 & EMWMF \\
\hline PP-03 & EMWMF \\
\hline PP-04 & EMWMF \\
\hline PP-05 & EMWMF \\
\hline PP-06 & EMWMF \\
\hline PP-07 & EMWMF \\
\hline PP-08 & EMWMF \\
\hline
\end{tabular}

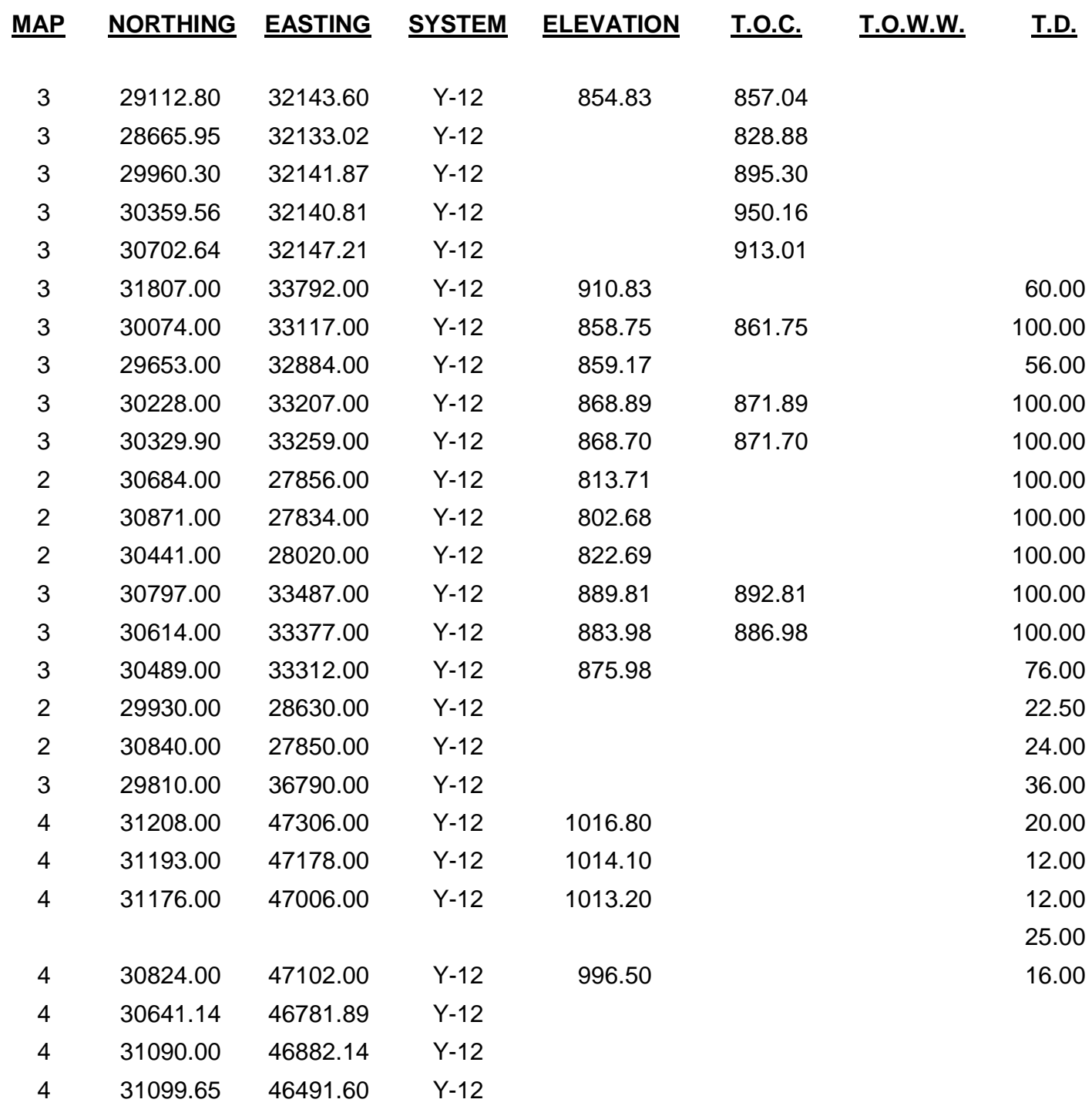




$\begin{array}{ll}\begin{array}{l}\text { CURRENT } \\ \text { NAME }\end{array} & \text { FUNCTIONAL ARE } \\ \text { PP-09 } & \text { EMWMF } \\ \text { RB-001 } & \text { Y-12 Plant Site } \\ \text { RB-005 } & \text { Y-12 Plant Site } \\ \text { RB-008 } & \text { Y-12 Plant Site } \\ \text { RB-009A } & \text { Y-12 Plant Site } \\ \text { RB-010A } & \text { Y-12 Plant Site } \\ \text { RB-011 } & \text { Y-12 Plant Site } \\ \text { RB-014 } & \text { Y-12 Plant Site } \\ \text { RB-015 } & \text { Y-12 Plant Site } \\ \text { RB-016 } & \text { Y-12 Plant Site } \\ \text { RB-017 } & \text { Y-12 Plant Site } \\ \text { RB-018 } & \text { Y-12 Plant Site } \\ \text { RB-019 } & \text { Y-12 Plant Site } \\ \text { RB-020 } & \text { Y-12 Plant Site } \\ \text { RB-031 } & \text { Y-12 Plant Site } \\ \text { RB-032 } & \text { Y-12 Plant Site } \\ \text { RB-033 } & \text { Y-12 Plant Site } \\ \text { RB-034 } & \text { Y-12 Plant Site } \\ \text { RB-035 } & \text { Y-12 Plant Site } \\ \text { RB-036 } & \text { Y-12 Plant Site } \\ \text { RB-037 } & \text { Y-12 Plant Site } \\ \text { RB-038 } & \text { Y-12 Plant Site } \\ \text { S3-1 } & \text { S-3 Ponds } \\ \text { S3-3 } & \text { S-3 Ponds } \\ \text { S3-5 } & \text { S-3 Ponds } \\ \text { S3-6 } & \text { S-3 Ponds } \\ \text { S3-7 } & \text { S-3 Ponds } \\ & \end{array}$

\section{MAP NORTHING}

EASTING

SYSTEM

ELEVATION

T.O.C.

T.O.W.W.

T.D.

\begin{tabular}{|c|c|c|c|c|}
\hline 4 & 30600.95 & 46421.30 & Y-12 & \\
\hline $5 \& 6$ & 29865.00 & 59355.00 & Y-12 & 949.50 \\
\hline $5 \& 6$ & 29555.00 & 59710.00 & Y-12 & 938.40 \\
\hline $5 \& 6$ & 29475.00 & 60100.00 & $\mathrm{Y}-12$ & 936.30 \\
\hline $5 \& 6$ & 29335.00 & 60265.00 & $\mathrm{Y}-12$ & 930.00 \\
\hline $5 \& 6$ & 29180.00 & 60420.00 & Y-12 & 926.10 \\
\hline $5 \& 6$ & 29410.00 & 60465.00 & $\mathrm{Y}-12$ & 936.50 \\
\hline $5 \& 6$ & 29500.00 & 59000.00 & Y-12 & 927.80 \\
\hline $5 \& 6$ & 29500.00 & 59500.00 & Y-12 & 931.30 \\
\hline $5 \& 6$ & 29200.00 & 59500.00 & $\mathrm{Y}-12$ & 930.80 \\
\hline $5 \& 6$ & 29200.00 & 59000.00 & $\mathrm{Y}-12$ & 927.80 \\
\hline $5 \& 6$ & 29350.00 & 59250.00 & Y-12 & 935.60 \\
\hline $5 \& 6$ & 29545.00 & 59085.00 & $\mathrm{Y}-12$ & 932.80 \\
\hline $5 \& 6$ & 29530.00 & 59355.00 & Y-12 & 931.20 \\
\hline $5 \& 6$ & 29480.00 & 59345.00 & Y-12 & 930.80 \\
\hline $5 \& 6$ & 29538.00 & 59215.00 & $\mathrm{Y}-12$ & 931.50 \\
\hline $5 \& 6$ & 29400.00 & 59425.00 & $Y-12$ & 932.20 \\
\hline $5 \& 6$ & 29480.00 & 59245.00 & Y-12 & 929.80 \\
\hline $5 \& 6$ & 29495.00 & 59075.00 & $\mathrm{Y}-12$ & 929.60 \\
\hline $5 \& 6$ & 29465.00 & 59245.00 & Y-12 & 929.20 \\
\hline $5 \& 6$ & 29480.00 & 59270.00 & Y-12 & 929.60 \\
\hline $5 \& 6$ & 29480.00 & 59220.00 & $\mathrm{Y}-12$ & 929.50 \\
\hline 5 & 30427.20 & 52537.40 & Y-12 & 1010.80 \\
\hline 5 & 30069.40 & 52118.40 & Y-12 & 1006.50 \\
\hline 5 & 30380.70 & 51801.90 & Y-12 & 1005.00 \\
\hline 5 & 30424.60 & 51936.50 & Y-12 & 1011.60 \\
\hline 5 & 30178.90 & 51991.30 & $\mathrm{Y}-12$ & 1008.10 \\
\hline
\end{tabular}

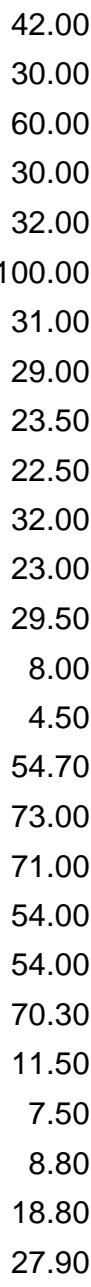

30.00

60.00

30.00

00.00

31.00

29.00

32.00

23.00

29.50

4.50

54.70

73.00

71.00

54.00

54.00

11.50

7.50

8.80

27.90 


\begin{tabular}{|c|c|c|c|c|c|c|c|c|c|}
\hline \multicolumn{10}{|l|}{ CURRENT } \\
\hline NAME & FUNCTIONAL AREA & MAP & $\underline{\text { NORTHING }}$ & EASTING & SYSTEM & ELEVATION & T.O.C. & T.O.W.W. & T.D. \\
\hline S3-8 & S-3 Ponds & 5 & 30173.30 & 52368.30 & $\mathrm{Y}-12$ & 1006.00 & & & 12.00 \\
\hline SAN-1 & Grassy Creek & & & & & & & & 98.00 \\
\hline SAN-10 & Grassy Creek & & & & & & & & 53.00 \\
\hline SAN-2 & Grassy Creek & & & & & & & & 43.00 \\
\hline SAN-3 & Grassy Creek & & & & & & & & 57.00 \\
\hline SAN-4 & Grassy Creek & & & & & & & & 35.00 \\
\hline SAN-5 & Grassy Creek & & & & & & & & 34.00 \\
\hline SAN-6 & Grassy Creek & & & & & & & & 42.00 \\
\hline SAN-7 & Grassy Creek & & & & & & & & 59.50 \\
\hline SAN-8 & Grassy Creek & & & & & & & & 39.00 \\
\hline SAN-9 & Grassy Creek & & & & & & & & 38.50 \\
\hline UNIDENT A & Filled Coal Ash Pond & & & & & & & & 36.20 \\
\hline UNIDENT B & Filled Coal Ash Pond & & & & & & & & 19.30 \\
\hline V-1 & Y-12 Plant Site & & & & & & & & 18.00 \\
\hline V-2 & Y-12 Plant Site & & & & & & & & 23.00 \\
\hline V-3 & Y-12 Plant Site & & & & & & & & 27.00 \\
\hline V-4 & Y-12 Plant Site & 5 & 29890.00 & 57781.25 & $\mathrm{Y}-12$ & & & & 4.10 \\
\hline V-5 & Y-12 Plant Site & 5 & 29880.50 & 57780.00 & $\mathrm{Y}-12$ & & & & 7.30 \\
\hline V-6 & Y-12 Plant Site & 5 & 29871.00 & 57780.75 & $\mathrm{Y}-12$ & & & & 6.30 \\
\hline V-7 & Y-12 Plant Site & & & & & & & & 27.50 \\
\hline
\end{tabular}


This Page Intentionally Left Blank 
APPENDIX B

Borehole and Well Nomenclature, Construction Type, and Status Summary 
This Page Intentionally Left Blank 


CURRE
NAME
001-L
$002-\mathrm{L}$
$003-\mathrm{L}$
$004-\mathrm{L}$
$005-\mathrm{L}$
$006-\mathrm{L}$
$007-\mathrm{L}$
$008-\mathrm{L}$
$009-\mathrm{L}$
$010-\mathrm{L}$
$011-\mathrm{L}$
$012-\mathrm{L}$
$013-\mathrm{L}$
$014-\mathrm{L}$
$015-\mathrm{L}$
$016-\mathrm{L}$
$017-\mathrm{L}$
$018-\mathrm{L}$
$019-\mathrm{L}$
$020-\mathrm{L}$
$021-\mathrm{L}$
$022-\mathrm{L}$
$023-\mathrm{L}$
$024-\mathrm{L}$
$025-\mathrm{L}$
$026-\mathrm{L}$

001-L

$002-L$

003-L

005-L

006-L

$09-L$

$12-\mathrm{L}$

13-L

16-L

7-L

$20-\mathrm{L}$

21-L

23-L

026-L
OTHER NAME

\section{WELL}

ORIGINATION

or P \& A ----- Water Levels ----.

SCREENED OPEN STATUS CONDITION

DATE

MIN MEAN

MAX

$\begin{array}{llll}\text { unknown } & \text { unknown } & \text { Inactive } & \text { Destroyed } \\ \text { unknown } & \text { unknown } & \text { Inactive } & \text { Destroyed } \\ \text { unknown } & \text { unknown } & \text { Inactive } & \text { Destroyed } \\ \text { unknown } & \text { unknown } & \text { Inactive } & \text { Destroyed } \\ \text { unknown } & \text { unknown } & \text { Inactive } & \text { Destroyed } \\ \text { unknown } & \text { unknown } & \text { Inactive } & \text { Destroyed } \\ \text { unknown } & \text { unknown } & \text { Inactive } & \text { Destroyed } \\ \text { unknown } & \text { unknown } & \text { Inactive } & \text { Destroyed } \\ \text { unknown } & \text { unknown } & \text { Inactive } & \text { Destroyed } \\ \text { unknown } & \text { unknown } & \text { Inactive } & \text { Destroyed } \\ \text { unknown } & \text { unknown } & \text { Inactive } & \text { Destroyed } \\ \text { unknown } & \text { unknown } & \text { Inactive } & \text { Destroyed } \\ \text { unknown } & \text { unknown } & \text { Inactive } & \text { Destroyed } \\ \text { unknown } & \text { unknown } & \text { Inactive } & \text { Destroyed } \\ \text { unknown } & \text { unknown } & \text { Inactive } & \text { Destroyed } \\ \text { unknown } & \text { unknown } & \text { Inactive } & \text { Destroyed } \\ \text { unknown } & \text { unknown } & \text { Inactive } & \text { Destroyed } \\ \text { unknown } & \text { unknown } & \text { Inactive } & \text { Destroyed } \\ \text { unknown } & \text { unknown } & \text { Inactive } & \text { Destroyed } \\ \text { unknown } & \text { unknown } & \text { Inactive } & \text { Destroyed } \\ \text { unknown } & \text { unknown } & \text { Inactive } & \text { Destroyed } \\ \text { unknown } & \text { unknown } & \text { Inactive } & \text { Destroyed } \\ \text { unknown } & \text { unknown } & \text { Inactive } & \text { Destroyed } \\ \text { unknown } & \text { unknown } & \text { Inactive } & \text { Destroyed } \\ \text { unknown } & \text { unknown } & \text { Inactive } & \text { Destroyed } \\ \text { unknown } & \text { unknown } & \text { Inactive } & \text { Destroyed } \\ & & & \\ \text { unown } & & \\ \text { unnown }\end{array}$




\begin{tabular}{|c|c|}
\hline \multicolumn{2}{|c|}{ CURRENT } \\
\hline NAME & OTHER NAME \\
\hline \multicolumn{2}{|l|}{ 027-L } \\
\hline \multicolumn{2}{|l|}{ 028-L } \\
\hline \multicolumn{2}{|l|}{ 029-L } \\
\hline \multicolumn{2}{|l|}{ 030-L } \\
\hline \multicolumn{2}{|l|}{ 031-L } \\
\hline \multicolumn{2}{|l|}{ 032-L } \\
\hline \multicolumn{2}{|l|}{ 033-L } \\
\hline \multicolumn{2}{|l|}{ 034-L } \\
\hline \multicolumn{2}{|l|}{ 035-L } \\
\hline 1001 & Y-03,YGMW-01A \\
\hline 1002 & AP-02,W-02,YAP-02,YGMW-01 \\
\hline 1003 & AP-03,YGMW-02,YMW-10 \\
\hline 1004 & AP-04,YGMW-03,YMW-09 \\
\hline 1005 & AP-05,YGMW-04,YMW-08 \\
\hline 1006 & AP-06,No. 03,W-01,YAP-01 \\
\hline 1007 & AP-07,No. 02,W-03,YAP-03 \\
\hline 1008 & AP-08,No. 04,W-06,YAP-06 \\
\hline 1009 & AP-09,No. 01,W-07,YAP-07 \\
\hline 1010 & AP-10,No. 06,W-04,YAP-04 \\
\hline 1011 & AP-11,No. 05,W-05,YAP-05 \\
\hline 1012 & AP-12,Y-02 \\
\hline 1013 & AP-13,Y-04 \\
\hline 1014 & AP-14,Y-05 \\
\hline 1015 & AP-15,Y-06 \\
\hline 1016 & AP-16,Y-07 \\
\hline 1017 & AP-17,Y-08 \\
\hline
\end{tabular}

\begin{tabular}{|c|c|c|c|}
\hline \multirow[b]{2}{*}{ SCREENED } & \multicolumn{3}{|c|}{ WELL } \\
\hline & OPEN & STATUS & CONDITIO \\
\hline unknown & unknown & Inactive & Destroyed \\
\hline unknown & unknown & Inactive & Destroyed \\
\hline unknown & unknown & Inactive & Destroyed \\
\hline unknown & unknown & Inactive & Destroyed \\
\hline unknown & unknown & Inactive & Destroyed \\
\hline unknown & unknown & Inactive & Destroyed \\
\hline unknown & unknown & Inactive & Destroyed \\
\hline unknown & unknown & Inactive & Destroyed \\
\hline unknown & unknown & Inactive & Destroyed \\
\hline no & yes & Inactive & $P \& A$ \\
\hline yes & no & Inactive & Destroyed \\
\hline yes & no & Inactive & $P \& A$ \\
\hline yes & no & Inactive & $P \& A$ \\
\hline yes & no & Inactive & $P \& A$ \\
\hline yes & no & Inactive & $P \& A$ \\
\hline yes & no & Inactive & $P \& A$ \\
\hline yes & no & Inactive & $P \& A$ \\
\hline yes & no & Inactive & $P \& A$ \\
\hline yes & no & Inactive & $P \& A$ \\
\hline yes & no & Inactive & $P \& A$ \\
\hline no & yes & Inactive & $P \& A$ \\
\hline no & yes & Inactive & $P \& A$ \\
\hline no & yes & Inactive & $P \& A$ \\
\hline no & yes & Inactive & $P \& A$ \\
\hline no & yes & Inactive & $P \& A$ \\
\hline no & yes & Inactive & $P \& A$ \\
\hline & B- 2 & & \\
\hline
\end{tabular}

ORIGINATION

or $P$ \& $A$

\section{DATE}

MIN

Water Levels ------

MEAN MAX

$\begin{array}{lrrr}05 / 26 / 92 & 1090.16 & 1096.82 & 1104.21 \\ 05 / 24 / 93 & 988.97 & 989.79 & 991.20 \\ 09 / 07 / 95 & 970.05 & 971.48 & 973.73 \\ 04 / 03 / 89 & 980.30 & 981.37 & 982.43 \\ 07 / 29 / 88 & 995.85 & 997.19 & 998.34 \\ 05 / 27 / 93 & 994.28 & 995.98 & 997.56 \\ 06 / 20 / 95 & 993.40 & 995.05 & 995.90 \\ 07 / 14 / 88 & 996.29 & 998.20 & 1002.26 \\ 05 / 26 / 93 & 1009.97 & 1010.85 & 1012.20 \\ 05 / 25 / 93 & 993.97 & 995.66 & 997.00 \\ 09 / 07 / 93 & 1042.79 & 1048.06 & 1053.09 \\ 08 / 10 / 93 & 1043.66 & 1050.21 & 1057.47 \\ 07 / 27 / 93 & 1074.13 & 1081.33 & 1090.04 \\ 05 / 22 / 92 & 1047.96 & 1053.07 & 1058.66 \\ 09 / 03 / 93 & 1030.52 & 1037.47 & 1045.45 \\ 08 / 10 / 93 & 1057.46 & 1067.30 & 1072.39\end{array}$




\begin{tabular}{|c|c|}
\hline \multicolumn{2}{|c|}{ CURRENT } \\
\hline NAME & OTHER NAME \\
\hline 1018 & AP-18,Y-09 \\
\hline 1019 & AP-19,Y-10 \\
\hline 1020 & AP-20,Y-11 \\
\hline 1021 & AP-21,Y-12 \\
\hline 1022 & AP-22,Y-13 \\
\hline 1023 & AP-23,Y-14 \\
\hline 1024 & AP-24,Y-15 \\
\hline 1025 & AP-25,Y-16 \\
\hline \multicolumn{2}{|l|}{ 1025A } \\
\hline 1026 & AP-26,Y-01 \\
\hline 1027 & BG-01,No. 07,YGMW-05 \\
\hline 1028 & BG-02,YGMW-06,YMW-11 \\
\hline 1029 & BG-03,No. 09,YGMW-07 \\
\hline 1030 & BG-04,YGMW-08,YMW-02 \\
\hline 1031 & BG-05,YGMW-09,YMW-04 \\
\hline 1032 & BG-06,YGMW-10,YMW-01 \\
\hline 1033 & BG-07,YGMW-11 \\
\hline 1034 & BG-08,YGMW-12,YMW-03 \\
\hline 1035 & BG-09,No. 03,YBG2-3,YGMW-13 \\
\hline 1036 & BG-10,No. 06,YBG2-6,YGMW-14 \\
\hline 1037 & BG-11,YGMW-15,YMW-07 \\
\hline 1038 & BG-12,No. 01 \\
\hline 1040 & BG-14,No. 04,YBG2-4 \\
\hline 1041 & BG-15,No. 05 \\
\hline 1043 & BG-17,USGS A,YBG2-7 \\
\hline 1044 & BG-18,USGS B \\
\hline
\end{tabular}

\begin{tabular}{|c|c|c|c|}
\hline \multirow[b]{2}{*}{ SCREENED } & \multicolumn{3}{|c|}{ WELL } \\
\hline & OPEN & STATUS & CONDI \\
\hline no & yes & Inactive & $P \& A$ \\
\hline no & yes & Inactive & $P \& A$ \\
\hline no & yes & Inactive & $P \& A$ \\
\hline no & yes & Inactive & $P \& A$ \\
\hline no & yes & Inactive & $P \& A$ \\
\hline no & yes & Inactive & $P \& A$ \\
\hline no & yes & Inactive & $P \& A$ \\
\hline no & yes & Inactive & $P \& A$ \\
\hline no & yes & Inactive & $P \& A$ \\
\hline no & yes & Inactive & $P \& A$ \\
\hline no & yes & Inactive & $P \& A$ \\
\hline yes & no & Inactive & $P \& A$ \\
\hline yes & no & Inactive & $P \& A$ \\
\hline yes & no & Inactive & $P \& A$ \\
\hline yes & no & Inactive & $P \& A$ \\
\hline yes & no & Inactive & $P \& A$ \\
\hline no & yes & Inactive & $P \& A$ \\
\hline yes & no & Inactive & $P \& A$ \\
\hline no & yes & Inactive & $P \& A$ \\
\hline no & yes & Inactive & $P \& A$ \\
\hline yes & no & Inactive & $P \& A$ \\
\hline yes & no & Inactive & $P \& A$ \\
\hline yes & no & Inactive & $P \& A$ \\
\hline yes & no & Inactive & $P \& A$ \\
\hline no & yes & Inactive & $P \& A$ \\
\hline no & yes & Inactive & $P \& A$ \\
\hline & B- 3 & & \\
\hline
\end{tabular}

ORIGINATION

\begin{tabular}{|c|c|c|c|}
\hline \multirow{2}{*}{$\begin{array}{c}\text { or P \& A } \\
\text { DATE }\end{array}$} & \multicolumn{3}{|c|}{----- Water Levels ----- } \\
\hline & $\underline{\mathrm{MIN}}$ & MEAN & $\underline{\operatorname{MAX}}$ \\
\hline 09/17/93 & 1044.29 & 1046.97 & 1050.68 \\
\hline 09/17/93 & 1044.81 & 1048.30 & 1052.52 \\
\hline 08/24/93 & 1025.84 & 1032.59 & 1038.31 \\
\hline 06/06/92 & 1022.82 & 1027.04 & 1029.84 \\
\hline 07/27/93 & 1028.62 & 1033.14 & 1039.81 \\
\hline 06/02/92 & 1027.10 & 1030.93 & 1035.27 \\
\hline 08/20/93 & 1059.76 & 1061.39 & 1063.08 \\
\hline 08/10/93 & 1034.59 & 1041.81 & 1049.39 \\
\hline \multicolumn{4}{|l|}{ 08/10/93 } \\
\hline 09/21/93 & 1026.03 & 1029.98 & 1039.35 \\
\hline \multicolumn{4}{|l|}{ 05/05/93 } \\
\hline \multicolumn{4}{|l|}{ 05/17/93 } \\
\hline \multicolumn{4}{|l|}{ 05/07/93 } \\
\hline \multicolumn{4}{|l|}{ 02/02/89 } \\
\hline \multicolumn{4}{|l|}{ 10/10/88 } \\
\hline \multicolumn{4}{|l|}{ 05/07/93 } \\
\hline 04/27/93 & 933.69 & 938.24 & 942.79 \\
\hline 05/16/95 & 924.25 & 926.35 & 928.65 \\
\hline \multicolumn{4}{|l|}{ 05/17/93 } \\
\hline \multicolumn{4}{|l|}{$05 / 17 / 93$} \\
\hline 05/07/93 & 905.99 & 909.70 & 915.53 \\
\hline \multicolumn{4}{|l|}{$05 / 14 / 93$} \\
\hline \multicolumn{4}{|l|}{ 05/17/93 } \\
\hline \multicolumn{4}{|l|}{ 05/17/93 } \\
\hline \multicolumn{4}{|l|}{ 02/12/93 } \\
\hline 09/30/88 & & & \\
\hline
\end{tabular}




\begin{tabular}{|c|c|}
\hline \multicolumn{2}{|c|}{ CURRENT } \\
\hline NAME & OTHER NAME \\
\hline 1045 & BG-19,USGS C \\
\hline 1046 & BG-20,USGS D \\
\hline 1047 & BG-21,CO-1,YGMW-16 \\
\hline $1047 \mathrm{~A}$ & CO-3 \\
\hline 1048 & No. 13, OD-01, YGMW-17 \\
\hline 1049 & OD-02,YGMW-18,YMW-05 \\
\hline 1050 & No. 10,OD-03,YGMW-19 \\
\hline 1051 & No. $12, \mathrm{OD}-04$ \\
\hline 1052 & No. $11, \mathrm{OD}-05$ \\
\hline 1055 & OB-06 \\
\hline 1056 & OB-02 \\
\hline 1057 & OB-07 \\
\hline 1058 & OB-03 \\
\hline 1059 & OB-01 \\
\hline 1060 & OB-08 \\
\hline 1061 & OB-05 \\
\hline 1062 & OB-04 \\
\hline 1063 & TW \\
\hline 1070 & \\
\hline 1071 & BG-13,No. 02 \\
\hline 1072 & BG-16,No. 08 \\
\hline 1073 & YGMW-20,YMW-06 \\
\hline 1080 & YGMW-23,YMW-15 \\
\hline 1081 & Well 1 \\
\hline 1082 & Well 2 \\
\hline 1083 & Well 3 \\
\hline
\end{tabular}

\begin{tabular}{l} 
SCREENED \\
\hline no \\
no \\
no \\
no \\
no \\
yes \\
no \\
yes \\
yes \\
yes \\
yes \\
yes \\
yes \\
yes \\
yes \\
yes \\
yes \\
yes \\
yes \\
yes \\
yes \\
yes \\
yes \\
yes \\
yes \\
yes
\end{tabular}

ORIGINATION$$
\begin{array}{lccc}
\text { WELL } & \text { or P \& A } & ----- & \text { Water Levels } \\
\text {------- }
\end{array}
$$

OPEN STATUS CONDITION $\underline{\text { DATE MIN MEAN MAX }}$

\begin{tabular}{|c|c|c|c|c|c|c|}
\hline yes & Inactive & $P \& A$ & $11 / 03 / 88$ & & & \\
\hline yes & Inactive & $P \& A$ & $11 / 03 / 88$ & & & \\
\hline yes & Inactive & $P \& A$ & $12 / 16 / 93$ & & & \\
\hline yes & Inactive & $P \& A$ & $12 / 22 / 93$ & & & \\
\hline yes & Inactive & $P \& A$ & $05 / 25 / 93$ & & & \\
\hline no & Inactive & $P \& A$ & $05 / 21 / 93$ & & & \\
\hline yes & Inactive & $P \& A$ & $05 / 25 / 93$ & & & \\
\hline no & Inactive & $P \& A$ & $05 / 21 / 93$ & & & \\
\hline no & Inactive & $P \& A$ & $05 / 25 / 93$ & & & \\
\hline no & Inactive & $P \& A$ & 09/13/88 & & & \\
\hline no & Inactive & $P \& A$ & 09/12/88 & & & \\
\hline no & Inactive & $P \& A$ & 09/16/88 & & & \\
\hline no & Inactive & $P \& A$ & $10 / 19 / 88$ & & & \\
\hline no & Inactive & $P \& A$ & $09 / 12 / 88$ & & & \\
\hline no & Inactive & $P \& A$ & $10 / 20 / 88$ & & & \\
\hline no & Inactive & $P \& A$ & $10 / 20 / 88$ & & & \\
\hline no & Inactive & $P \& A$ & $10 / 19 / 88$ & & & \\
\hline no & Inactive & $P \& A$ & $10 / 22 / 88$ & & & \\
\hline no & Inactive & $P \& A$ & $10 / 14 / 88$ & & & \\
\hline no & Inactive & $P \& A$ & $05 / 14 / 93$ & & & \\
\hline no & Inactive & $P \& A$ & 02/14/89 & & & \\
\hline no & Inactive & $P \& A$ & 02/01/89 & & & \\
\hline no & Inactive & $P \& A$ & $11 / 01 / 88$ & 1012.57 & 1022.71 & 1032.30 \\
\hline no & Inactive & $P \& A$ & $02 / 03 / 94$ & 815.80 & 817.10 & 821.12 \\
\hline no & Active & Open & & 801.91 & 812.48 & 819.01 \\
\hline no & Inactive & $P \& A$ & $02 / 03 / 94$ & 811.61 & 812.91 & 820.46 \\
\hline
\end{tabular}




$\begin{array}{ll}\begin{aligned} \text { CURRENT } \\ \text { NAME }\end{aligned} & \text { OTHER NAME } \\ 1084 & \text { Well 4 } \\ 1085 & \text { YMW-1,YMW-12 } \\ 1086 & \text { YMW-13,YMW-2 } \\ 1087 & \text { YMW-14,YMW-3 } \\ 1088 & \text { CO-6 } \\ 1090 & \text { YGMW-24,YUNC-16 } \\ 1091 & \text { YGMW-25,YUNC-17 } \\ 1092 & \text { YGMW-26,YUNC-18 } \\ 1095 & \text { SD-01,YGMW-21,YSD-11 } \\ 1096 & \text { SD-02,YGMW-22,YSD-12 } \\ 1097 & \text { Unknown,YC-1 } \\ 1100 & \text { BMW-1 } \\ 1101 & \text { I-1 } \\ 1102 & \text { BMW-3 } \\ 1103 & \text { MW-3 } \\ 1104 & \text { I-3 } \\ 1105 & \text { BMW-5 } \\ 1106 & \text { MW-5 } \\ 1107 & \text { BMW-6 } \\ 1108 & \text { MW-6 } \\ 1109 & \text { BMW-7 } \\ 1110 & \text { MW-9 } \\ 1111 & \text { MW-10 } \\ 1112 & \text { MW-11 } \\ 1130 & \\ 1131 & \\ \end{array}$

SCREENED
yes
yes
yes
yes
no
yes
yes
yes
yes
yes
no
yes
yes
yes
yes
yes
yes
yes
yes
yes
yes
yes
yes
yes
yes
yes

ORIGINATION

or $P$ \& $A$

WELL

DATE

MIN

Water Levels -------

OPEN STATUS CONDITION

Active Open

$\begin{array}{lll}887.02 & 903.20 \quad 908.18\end{array}$

Inactive $P \& A$

$04 / 23 / 92$

$935.65 \quad 938.30 \quad 943.45$

Inactive $P \& A$

$03 / 14 / 92$

988.90

$995.33 \quad 1009.75$

Inactive $P \& A$

Inactive $P \& A$

Active Open

Inactive $P \& A$

Inactive Destroyed

Inactive $P \& A$

Inactive $P \& A$

Inactive $P \& A$

Inactive $P \& A$

Inactive $P \& A$

Inactive $P \& A$

Inactive $P \& A$

Inactive $P \& A$

Inactive $P \& A$

Inactive $P \& A$

Inactive $P \& A$

Inactive $P \& A$

Inactive $P \& A$

Inactive $P \& A$

$02 / 08 / 91$

918.65

$921.08 \quad 923.28$

$06 / 15 / 93$

$\begin{array}{lll}1022.58 & 1048.09 & 1076.36\end{array}$

$11 / 27 / 91 \quad 1022.16 \quad 1027.02 \quad 1035.63$

03/05/96 $922.60 \quad 927.22 \quad 937.20$

03/04/96 $979.00 \quad 984.24 \quad 1035.10$

$09 / 14 / 93 \quad 1042.01 \quad 1046.57 \quad 1051.20$

04/07/95

$\begin{array}{llll}04 / 06 / 95 & 937.85 & 937.85 & 937.85\end{array}$

$\begin{array}{llll}04 / 05 / 95 & 901.65 & 901.92 & 902.35\end{array}$

$04 / 03 / 95$

$\begin{array}{llll}04 / 04 / 95 & 901.47 & 901.67 & 901.97\end{array}$

03/30/95 $897.64 \quad 901.69 \quad 909.34$

$\begin{array}{llll}04 / 03 / 95 & 898.27 & 905.44 & 910.17\end{array}$

$\begin{array}{llll}04 / 12 / 95 & 900.67 & 901.07 & 901.47\end{array}$

$\begin{array}{llll}04 / 12 / 95 & 900.42 & 900.57 & 900.72\end{array}$

$\begin{array}{llll}04 / 12 / 95 & 936.54 & 940.07 & 942.04\end{array}$

$\begin{array}{llll}04 / 12 / 95 & 948.46 \quad 948.46 & 948.46\end{array}$

Inactive Could not locate 06/17/86

Inactive Could not locate 06/17/86

Inactive P\&A 12/15/92

Inactive $P \& A \quad 12 / 15 / 92$ 


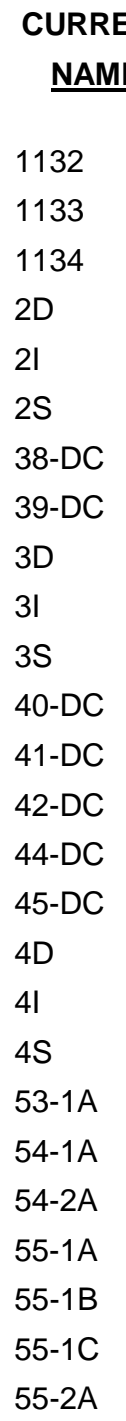

OTHER NAME

SCREENED

yes

yes

yes

yes

yes

yes

no

no

yes

yes

yes

no

no

no

no

no

yes

yes

yes

yes

yes

yes

yes

yes

yes

yes
ORIGINATION

or $P \& A$

WELL

\section{DATE}

MIN

Water Levels -------

\section{OPEN STATUS CONDITION}

Inactive $P \& A$

$12 / 15 / 92$

$03 / 18 / 93$

$06 / 16 / 93$

$03 / 16 / 92$

$03 / 16 / 92$

$03 / 16 / 92$

Inactive $P \& A$

$06 / 11 / 74$

Inactive P\&A

$\begin{array}{ll}\text { Could not locate } & 06 / 03 / 74 \\ \text { P\&A } & 04 / 14 / 92\end{array}$

Inactive

Could not locate

Inactive $P \& A$

04/14/92

Inactive $P \& A$

$04 / 14 / 92$

Inactive $P \& A$

$05 / 23 / 74$

Inactive

Could not locate

Inactive Could not locate

Inactive $P \& A$

$10 / 07 / 93$

Inactive Could not locate 06/14/74

Inactive Could not locate 06/13/74

Inactive $P \& A$

$03 / 03 / 92$

Inactive $P \& A$

$03 / 03 / 92$

$03 / 03 / 92$

03/04/13

$02 / 14 / 92$

971.67

988.95

$971.80 \quad 973.27$

968.81

970.72

$09 / 09 / 83$

$09 / 12 / 83$

975.84

975.63

975.01

$09 / 13 / 83$

970.93

967.72

968.24

990.67

975.24

972.98

976.57

976.16

Inactive Open

Open

08/08/83

975.83

968.94 


\begin{tabular}{|c|c|c|}
\hline \multicolumn{3}{|c|}{ CURRENT } \\
\hline NAME & OTHER NAME & SCREENED \\
\hline $55-2 B$ & & yes \\
\hline $55-2 C$ & & yes \\
\hline $55-3 A$ & & yes \\
\hline $55-3 B$ & & yes \\
\hline $55-3 C$ & & yes \\
\hline $55-4 B$ & & yes \\
\hline $55-4 C$ & & yes \\
\hline $55-5 A$ & & yes \\
\hline $55-6 \mathrm{~A}$ & & yes \\
\hline $55-7 A$ & No. 1 & yes \\
\hline $55-8 A$ & No. 3 & yes \\
\hline $55-8 B$ & No. 2 & yes \\
\hline $55-9 A$ & No. 4 & yes \\
\hline $56-1 A$ & & yes \\
\hline $56-1 C$ & & yes \\
\hline $56-2 A$ & & yes \\
\hline $56-2 B$ & & yes \\
\hline $56-2 C$ & & yes \\
\hline $56-3 A$ & & yes \\
\hline $56-3 B$ & & yes \\
\hline $56-3 C$ & & yes \\
\hline $56-4 A$ & & yes \\
\hline $56-4 B$ & & yes \\
\hline $56-4 C$ & & yes \\
\hline $56-5 A$ & & yes \\
\hline $56-5 B$ & & yes \\
\hline
\end{tabular}

ORIGINATION

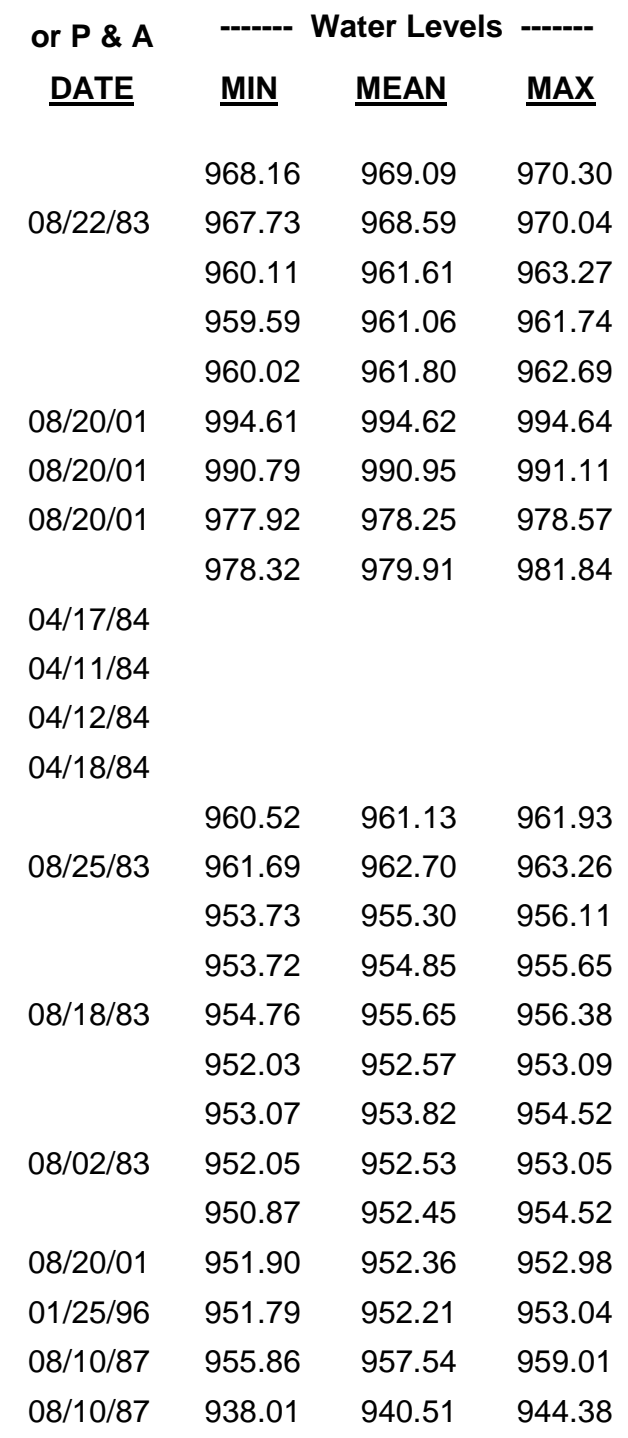




CURREN
NAME
$56-5 C$
$56-6 A$
$56-7 A$
$56-8 A$
$56-9 A$
$58-1 A$
$58-2 A$
$59-1 A$
$59-1 B$
$59-1 C$
$5 D$
51
5 S
$60-1 A$
$60-1 B$
$60-1 C$
$60-2 A$
AN:D-1
AN:D-10
AN:D-11
AN:D-2
AN:D-3
AN:D-4
AN:D-5
AN:D-6
AN:D-7

OTHER NAME

yes

yes

yes

yes

yes

yes

yes

yes

yes

yes

yes

yes

yes

yes

yes

unknown

yes

yes

yes

yes

yes

yes

yes

yes

yes

yes
WELL

OPEN STATUS CONDITION

Inactive $P \& A$

Active Open

Inactive Open

Active Open

Inactive $P \& A$

Inactive Unknown

Inactive Open

Inactive Open

Inactive Open

Inactive Open

Inactive $P \& A$

Inactive $P \& A$

Inactive $P \& A$

Active Open

Inactive Open

nknown Inactive $P \& A$

no Inactive $P \& A$

no Inactive $P \& A$

no Inactive $P \& A$

no Inactive $P \& A$

no Inactive $P \& A$

no Inactive $P \& A$

no Inactive $P \& A$

no Inactive $P \& A$

no Inactive $P \& A$

no Inactive $P \& A$

RIGINATION

\begin{tabular}{|c|c|c|c|}
\hline \multirow{2}{*}{$\begin{array}{c}\text { or P \& A } \\
\text { DATE }\end{array}$} & \multicolumn{2}{|c|}{------ Water Levels } & \multirow{2}{*}{ MAX } \\
\hline & $\underline{\text { MIN }}$ & MEAN & \\
\hline \multirow[t]{4}{*}{ 08/10/87 } & 932.39 & 937.52 & 942.76 \\
\hline & 947.75 & 948.47 & 950.28 \\
\hline & 937.78 & 941.33 & 942.83 \\
\hline & 941.74 & 943.54 & 945.53 \\
\hline \multirow{5}{*}{$\begin{array}{l}08 / 30 / 04 \\
11 / 29 / 83\end{array}$} & 950.54 & 952.85 & 954.90 \\
\hline & & & \\
\hline & 928.94 & 930.09 & 931.70 \\
\hline & 940.06 & 941.53 & 942.75 \\
\hline & 940.69 & 941.90 & 943.29 \\
\hline 09/14/83 & 941.07 & 942.26 & 943.77 \\
\hline \multicolumn{4}{|l|}{ 02/20/92 } \\
\hline \multicolumn{4}{|l|}{ 03/04/92 } \\
\hline \multicolumn{4}{|l|}{ 03/04/92 } \\
\hline \multirow[t]{2}{*}{ 08/12/83 } & 915.96 & 917.31 & 919.66 \\
\hline & 916.30 & 917.38 & 918.12 \\
\hline \multicolumn{4}{|l|}{ 09/09/83 } \\
\hline $04 / 07 / 04$ & 919.19 & 919.59 & 920.40 \\
\hline \multicolumn{4}{|l|}{$12 / 26 / 96$} \\
\hline \multicolumn{4}{|l|}{$12 / 13 / 96$} \\
\hline \multicolumn{4}{|l|}{$12 / 13 / 96$} \\
\hline \multicolumn{4}{|l|}{$12 / 24 / 96$} \\
\hline \multicolumn{4}{|l|}{$12 / 24 / 96$} \\
\hline \multicolumn{4}{|l|}{$12 / 24 / 96$} \\
\hline \multicolumn{4}{|l|}{$12 / 24 / 96$} \\
\hline \multicolumn{4}{|l|}{$12 / 26 / 96$} \\
\hline $12 / 16 / 96$ & & & \\
\hline
\end{tabular}




CURRENT
NAME
AN:D-8
AN:D-9
AN:D-UNKNOWN
B-1
B-3
BC-01
BC-03
BC-06
BC-07
BC-08
BC-09
BC-10
BC-11
BC-14
BC-15
BC-16
BC-17
BC-18
BC-19
BC-21
BC-22
BC-23
BC-25
BC-30
BC-31
BC-32

\begin{tabular}{|c|c|c|c|c|}
\hline & \multicolumn{3}{|c|}{ WELL } & \multirow{2}{*}{$\begin{array}{c}\text { or P \& A } \\
\text { DATE }\end{array}$} \\
\hline & OPEN & STATUS & CONDITION & \\
\hline yes & no & Inactive & $P \& A$ & $12 / 16 / 96$ \\
\hline yes & no & Inactive & $P \& A$ & $12 / 13 / 96$ \\
\hline yes & no & Inactive & $P \& A$ & $12 / 26 / 96$ \\
\hline yes & no & Inactive & $P \& A$ & 09/22/95 \\
\hline yes & no & Inactive & $P \& A$ & 09/22/95 \\
\hline unknown & unknown & Inactive & $P \& A$ & $01 / 11 / 94$ \\
\hline no & yes & Inactive & $P \& A$ & $11 / 30 / 94$ \\
\hline no & yes & Inactive & $P \& A$ & 03/07/94 \\
\hline no & yes & Inactive & $P \& A$ & 03/09/94 \\
\hline unknown & unknown & Inactive & Unknown & \\
\hline no & yes & Inactive & $P \& A$ & 03/16/94 \\
\hline no & yes & Inactive & $P \& A$ & $03 / 22 / 9$ \\
\hline unknown & unknown & Inactive & Unknown & \\
\hline no & yes & Inactive & $P \& A$ & $03 / 23 / 94$ \\
\hline yes & no & Inactive & $P \& A$ & $03 / 22 / 9$ \\
\hline unknown & unknown & Inactive & Could not locate & \\
\hline no & yes & Inactive & $P \& A$ & $04 / 20 / 9$ \\
\hline no & yes & Inactive & $P \& A$ & $04 / 22 / 94$ \\
\hline no & yes & Inactive & $P \& A$ & 03/31/94 \\
\hline unknown & unknown & Inactive & Open & \\
\hline no & yes & Inactive & $P \& A$ & 05/16/94 \\
\hline no & yes & Inactive & $P \& A$ & $03 / 31 / 94$ \\
\hline no & yes & Inactive & $P \& A$ & $12 / 02 / 94$ \\
\hline no & yes & Inactive & $P \& A$ & $11 / 23 / 94$ \\
\hline no & yes & Inactive & $P \& A$ & $11 / 11 / 9$ \\
\hline no & yes & Inactive & $P \& A$ & $11 / 15 / 94$ \\
\hline & & & & \\
\hline
\end{tabular}




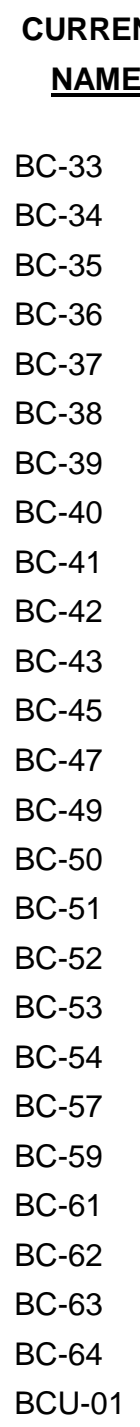

OTHER NAME

BC-33

BC-34

$\mathrm{BC}-35$

BC-36

BC-39

BC-40

C-41

$\mathrm{BC}-45$

BC-47

BC-49

BC-50

BC-51

C-52

$\mathrm{BC}-54$

$\mathrm{BC}-57$

C-59

-63

BCU-01

ORIGINATION

or P \& A ----- Water Levels ----.

\begin{tabular}{|c|c|c|c|c|c|c|}
\hline SCREENED & OPEN & STATUS & CONDITION & DATE & MIN & MEAN \\
\hline
\end{tabular}

\begin{tabular}{|c|c|c|c|c|}
\hline no & yes & Inactive & $P \& A$ & $11 / 15 / 94$ \\
\hline no & yes & Inactive & $P \& A$ & $11 / 15 / 94$ \\
\hline no & yes & Inactive & $P \& A$ & $04 / 14 / 94$ \\
\hline no & yes & Inactive & $P \& A$ & $11 / 09 / 94$ \\
\hline unknown & unknown & Inactive & Could not locate & \\
\hline unknown & unknown & Inactive & Could not locate & \\
\hline no & yes & Inactive & $P \& A$ & $11 / 22 / 94$ \\
\hline no & yes & Inactive & $P \& A$ & $11 / 15 / 94$ \\
\hline no & yes & Inactive & $P \& A$ & $11 / 21 / 94$ \\
\hline no & yes & Inactive & $P \& A$ & $11 / 08 / 94$ \\
\hline unknown & unknown & Inactive & Could not locate & \\
\hline unknown & unknown & Inactive & Open & \\
\hline yes & no & Inactive & $P \& A$ & 03/31/94 \\
\hline unknown & unknown & Inactive & Could not locate & \\
\hline no & yes & Inactive & $P \& A$ & $11 / 30 / 94$ \\
\hline unknown & unknown & Inactive & Unknown & \\
\hline unknown & unknown & Inactive & Unknown & \\
\hline unknown & unknown & Inactive & Unknown & \\
\hline unknown & unknown & Inactive & Unknown & \\
\hline unknown & unknown & Inactive & Unknown & \\
\hline unknown & unknown & Inactive & Unknown & \\
\hline unknown & unknown & Inactive & Could not locate & \\
\hline yes & no & Inactive & $P \& A$ & $11 / 15 / 94$ \\
\hline no & yes & Inactive & $P \& A$ & 05/05/94 \\
\hline unknown & unknown & Inactive & Destroyed & \\
\hline \multirow[t]{2}{*}{ no } & yes & Inactive & $P \& A$ & 05/10/94 \\
\hline & & & & \\
\hline
\end{tabular}




CURREN
NAME
BCU-02
BCU-03
BCU-04
BCU-05
BCU-06
BCU-07
BCU-08
BCU-09
BCU-10
BCU-11
BCU-12
BCU-13
BCU-14
BCU-15
BCU-16
BCU-17
BCU-18
BCU-19
BCU-20
BCU-21
BCU-22
BCU-23
BCU-24
BCU-25
BCU-26
BCU-27

OTHER NAME

BC-66
WELL

\section{SCREENED OPEN STATUS CONDITION}

Inactive $P \& A$

Inactive $P \& A$

Inactive $P \& A$

Inactive $P \& A$

Inactive $P \& A$

Inactive $P \& A$

Inactive $P \& A$

Inactive $P \& A$

Inactive $P \& A$

Inactive $P \& A$

Inactive $P \& A$

Inactive $P \& A$

Inactive $P \& A$

Inactive $P \& A$

Inactive $P \& A$

Inactive $P \& A$

Inactive $P \& A$

Inactive $P \& A$

Inactive $P \& A$

Inactive $P \& A$

Inactive $P \& A$

Inactive $P \& A$

Inactive $P \& A$

Inactive $P \& A$

Inactive $P \& A$

Inactive $P \& A$
ORIGINATION

or $P$ \& $A$

DATE

MIN

Water Levels -------

$04 / 18 / 94$

05/20/94

$06 / 23 / 94$

$05 / 27 / 94$

$05 / 25 / 94$

$05 / 23 / 94$

$05 / 20 / 94$

$05 / 17 / 94$

$05 / 11 / 94$

05/13/94

$09 / 15 / 94$

05/10/94

$11 / 09 / 94$

$11 / 08 / 94$

$04 / 18 / 94$

$11 / 14 / 94$

$11 / 14 / 94$

$11 / 11 / 94$

05/06/94

10/04/94

$11 / 15 / 94$

09/16/94

09/29/94

09/16/94

09/19/94

09/29/94 


$\begin{aligned} & \text { CURREN } \\ & \text { NAME }\end{aligned}$
BCU-28
BCU-29
BY-10
BY-11
BY-12
BY-13
BY-14
BY-15
BY-16
BY-17
BY-18
BY-19
BY-20
BY-21
BY-22
BY-23
BY-24
BY-25
BY-26
BY-27
BY-28
BY-29
BY-30
BY-31
BY-32
BY-33

OTHER NAME

CU-28

BCU-29

Y -12

BY-15

BY-16

BY-17

Y-18

BY-19

BY-20

BY -23

BY-24

BY-25

BY-26

BY-27

BY-28

YY-31

BY-33
ORIGINATION

or P \& A ----- Water Levels -----

\begin{tabular}{|c|c|c|c|c|c|c|}
\hline SCREENED & OPEN & STATUS & CONDITION & DATE & MIN & MEAN \\
\hline
\end{tabular}

yes

yes

yes

yes

yes

yes

yes

yes

yes

yes

yes

yes

yes

yes

yes

yes

yes

yes

yes

yes

yes

yes

yes

yes

yes

yes
Inactive P\&A 09/29/94

Inactive P\&A 10/03/94

Inactive Open

Inactive Open

Inactive Open

Inactive Open

Inactive Open

Inactive Open

Inactive Open

Inactive Open

Inactive Open

Inactive Open

Inactive Open

Inactive Open

Inactive Open

Inactive Open

Inactive Open

Inactive $P \& A$

Inactive $P \& A$

Inactive $P \& A$

Inactive $P \& A$

Inactive Open

Inactive $P \& A$

Inactive Open

Inactive Open

Inactive $P \& A$

B- 12 


CURREN
NAME
BY-34
BY-35
BY-36
BY-37
BY-38
BY-39
BY-40
BY-41
BY-43
BY-44
BY-45
BY-46
BY-47
BY-48
BY-49
BY-50
C-1
C-19
C-21
C-25
C-40
C-45
C-48
C-50
C-51
CH-001

OTHER NAME

BY-34

$B Y-35$

BY-36

Y-37

BY-39

BY-40

Y -41

Y-45

YY-46

-4 -49

Y -50

$-19$

C-21

$-25$

$-45$

-48

$\mathrm{CH}-001$
WELL

SCREENED OPEN STATUS CONDITION

Inactive $P \& A$

Inactive $P \& A$

Inactive Open

Inactive Open

Inactive Open

Inactive Open

Inactive Open

Inactive Open

Inactive Open

Inactive Open

Inactive Open

Inactive Open

Inactive Open

Inactive Open

Inactive Open

Inactive $P \& A$

Active Open

Inactive $P \& A$

Inactive $P \& A$

Inactive $P \& A$

Inactive $P \& A$

Active Open

Active Open

Active Open

Active Open

Destroyed

05/20/93

02/01/01

01/29/01

$01 / 26 / 01$

02/02/01

$05 / 20 / 93$

$05 / 20 / 93$

05/20/93

05/20/93

unknown

unknown Inactive Destroyed
Water Levels
MIN MEAN MAX 


\begin{tabular}{ll}
$\begin{array}{ll}\text { CURRENT } \\
\text { NAME }\end{array}$ & OTHER NAME \\
CH-002 & \\
CH-143 & \\
CH-157 & \\
CH-185 & \\
CH-189 & \\
CO-2 & \\
CO-4 & \\
DC WELL & DCWELL \\
ED/Y-12 No. 2 & \\
EMW-VWUNDRDR \\
FP-001 & Hole No. 1 \\
FP-002 & Hole No. 2 \\
FP-003 & Hole No. 3 \\
FP-004 & Hole No. 4 \\
FP-005 & Hole No. 5 \\
FP-006 & Hole No. 6 \\
FP-007 & Hole No. 7 \\
FP-008A & Hole No. 8A \\
FP-008B & Hole No. 8B \\
FP-009 & Hole No. 9 \\
FP-010 & Hole No. 10 \\
FP-011 & Hole No. 11 \\
FT-001 & \\
FT-002 & \\
FT-003 & \\
FW001 & \\
& \\
\hline
\end{tabular}

WELL SCREENED

unknown OPEN

STATUS

unknown Inactive

yes Inactive Open

yes Inactive Open

yes Inactive Open

yes Inactive Open

yes Inactive $P \& A$

yes Inactive P\&A

unknown Inactive Open

unknown Inactive Open

unknown N/A Unknown

unknown Inactive Destroyed

unknown Inactive Destroyed

unknown Inactive Destroyed

unknown Inactive Destroyed

unknown Inactive Destroyed

unknown Inactive Destroyed

unknown Inactive Destroyed

unknown Inactive Destroyed

unknown Inactive Destroyed

unknown Inactive Destroyed

unknown Inactive Destroyed

unknown Inactive Destroyed

unknown Inactive Destroyed

unknown Inactive Destroyed

unknown Inactive Destroyed

no Inactive Open

B- 14

unknown

yes
ORIGINATION

or $P \& A$ ----- Water Levels -------

DATE

$\underline{\text { MIN }}$

MEAN

MAX

$\begin{array}{llll}08 / 22 / 85 & 856.26 & 856.40 & 856.49 \\ 10 / 07 / 85 & 949.75 & 949.87 & 949.98 \\ 09 / 16 / 85 & 817.12 & 817.46 & 818.10 \\ 08 / 29 / 85 & 831.28 & 832.08 & 833.38\end{array}$

$03 / 20 / 95$

$03 / 17 / 95$

$01 / 11 / 00$

04/20/06

09/14/00 


\author{
CURRENT \\ NAME \\ FW002 \\ FW003 \\ FW004 \\ FW005 \\ GW-001 \\ GW-002 \\ GW-003 \\ GW-004 \\ GW-005 \\ GW-006 \\ GW-007 \\ GW-008 \\ GW-009 \\ GW-010 \\ GW-011 \\ GW-012 \\ GW-013 \\ GW-014 \\ GW-015 \\ GW-016 \\ GW-017 \\ GW-018 \\ GW-019 \\ GW-020 \\ GW-021 \\ GW-022
}

OTHER NAME
ORIGINATION

or $P$ \& $A$

WELL

SCREENED OPEN STATUS CONDITION

DATE

$\underline{\text { MIN }}$

Water Levels ------

OPEN STATUS CONDITION

$09 / 14 / 00$

yes
yes
yes
yes
yes
yes
yes

\section{no}

Inactive Open

Inactive Open

Inactive Open

Inactive Open

Active Open

Inactive $P \& A$

unknown

yes

yes

yes

yes

unknown

yes

yes

yes

yes

yes

yes

yes

yes

yes

unknown

yes

yes

yes
Inactive $P \& A$

no

unknown

no Inactive $P \& A$

no Active Open

unknown Inactive $P \& A$

no Active Open

no Inactive Open

no Active Open

no Inactive Open

no Active Open

no Inactive Open

no Active Open

no Inactive Open

no Inactive Open

unknown Inactive $P \& A$

no Inactive $P \& A$

no Inactive $P \& A$

no Inactive $P \& A$

B- 15 no Inactive $P \& A$

no Active Open

$\begin{array}{llll}\text { 09/18/00 } & & & \\ 09 / 26 / 00 & & & \\ 09 / 08 / 83 & 962.08 & 965.53 & 970.83 \\ 10 / 02 / 95 & 964.88 & 967.97 & 975.38 \\ 12 / 12 / 88 & 965.30 & 966.43 & 968.18 \\ 09 / 14 / 83 & & & \\ 07 / 11 / 02 & 957.31 & 962.16 & 963.99 \\ 09 / 17 / 83 & 953.34 & 956.92 & 959.47 \\ 09 / 21 / 95 & 949.40 & 956.23 & 959.40 \\ 09 / 21 / 83 & 946.59 & 950.52 & 952.91 \\ 09 / 16 / 83 & & & \\ 09 / 23 / 83 & 945.40 & 949.02 & 951.70 \\ 09 / 28 / 83 & 946.70 & 949.47 & 951.16 \\ 09 / 28 / 83 & 945.57 & 949.51 & 953.30 \\ 09 / 13 / 83 & 952.74 & 960.02 & 963.31 \\ 09 / 29 / 83 & 920.50 & 927.94 & 930.95 \\ 10 / 05 / 83 & 919.40 & 921.48 & 922.65 \\ 10 / 03 / 83 & 909.56 & 917.75 & 922.59 \\ 10 / 06 / 83 & 911.63 & 913.92 & 918.16 \\ 10 / 05 / 83 & 902.96 & 911.32 & 919.56 \\ 10 / 01 / 83 & & & \\ 04 / 19 / 89 & 915.83 & 916.56 & 918.10 \\ 01 / 24 / 89 & 915.70 & 919.39 & 922.65 \\ 02 / 01 / 89 & 920.77 & 924.00 & 925.66\end{array}$




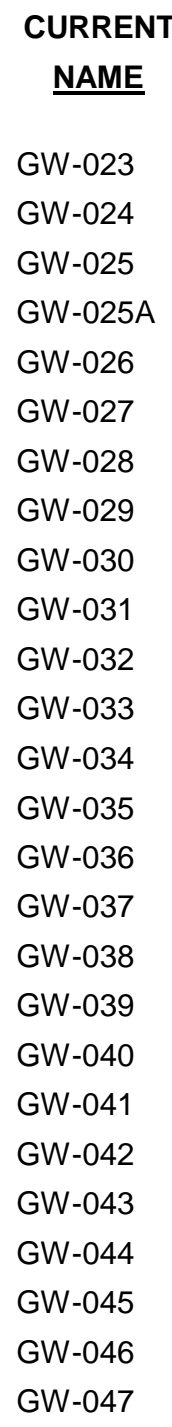

OTHER NAME

SCREENED

yes

yes

unknown

yes

yes

yes

yes

yes

yes

yes

yes

yes

yes

yes

yes

yes

yes

yes

yes

yes

yes

yes

yes

yes

yes

yes
WELL

OPEN STATUS CONDITION

no Inactive $P \& A$

no Inactive $P \& A$

unknown Inactive $P \& A$

no Inactive $P \& A$

no Inactive $P \& A$

no Inactive $P \& A$

no Inactive $P \& A$

no Inactive $P \& A$

no Inactive $P \& A$

no Inactive $P \& A$

no Inactive $P \& A$

no Inactive Destroyed

no Inactive Destroyed

no Inactive $P \& A$

no Inactive $P \& A$

no Inactive $P \& A$

no Inactive $P \& A$

no Inactive $P \& A$

no Inactive Open

no Active Open

no Inactive Open

no Inactive $P \& A$

no Inactive $P \& A$

no Inactive Open

no Active Open

no Active Open

B- 16
ORIGINATION

$\begin{array}{cccc}\text { or P \& A } & \text {----- } & \text { Water Levels } & \text {----- } \\ \text { DATE } & \text { MIN } & \text { MEAN } & \text { MAX } \\ \text { 03/16/89 } & 935.90 & 939.39 & 945.32 \\ \text { 02/13/89 } & 941.86 & 943.12 & 944.68 \\ \text { 09/26/83 } & & & \\ & & & \\ \text { 12/13/88 } & 934.83 & 943.17 & 948.32 \\ \text { 12/15/88 } & 944.60 & 947.05 & 948.99 \\ \text { 12/12/88 } & 962.80 & 964.74 & 972.68 \\ \text { 02/06/89 } & 956.09 & 959.62 & 962.00 \\ \text { 02/10/93 } & 938.07 & 943.04 & 956.90 \\ \text { 02/10/93 } & 938.43 & 942.57 & 953.23 \\ \text { 02/12/93 } & & & \\ \text { 10/17/83 } & 967.35 & 972.30 & 980.11 \\ \text { 10/31/83 } & 951.91 & 953.65 & 955.88 \\ \text { 02/16/93 } & 964.71 & 968.08 & 975.57 \\ \text { 02/11/93 } & 940.25 & 960.00 & 973.90 \\ \text { 02/11/93 } & 957.24 & 960.87 & 970.69 \\ \text { 02/11/93 } & 953.30 & 957.17 & 963.14 \\ \text { 02/06/89 } & 934.70 & 943.67 & 946.62 \\ \text { 09/30/83 } & 975.98 & 987.30 & 996.45 \\ \text { 09/27/83 } & 980.60 & 988.19 & 1002.48 \\ \text { 09/29/83 } & 974.80 & 982.61 & 986.88 \\ \text { 01/02/01 } & 981.24 & 989.14 & 1003.96 \\ \text { 02/02/01 } & 979.79 & 988.31 & 1005.00 \\ \text { 10/26/83 } & 892.59 & 898.77 & 906.09 \\ \text { 10/27/83 } & 909.05 & 914.47 & 919.80 \\ \text { 11/01/83 } & 908.60 & 920.22 & 924.50\end{array}$




\author{
CURRENT \\ NAME \\ GW-048 \\ GW-049 \\ GW-050 \\ GW-051 \\ GW-052 \\ GW-053 \\ GW-054 \\ GW-055 \\ GW-056 \\ GW-057 \\ GW-058 \\ GW-059 \\ GW-060 \\ GW-061 \\ GW-062 \\ GW-063 \\ GW-064 \\ GW-065 \\ GW-066 \\ GW-067 \\ GW-068 \\ GW-069 \\ GW-070 \\ GW-071 \\ GW-072 \\ GW-073
}

OTHER NAME
ORIGINATION

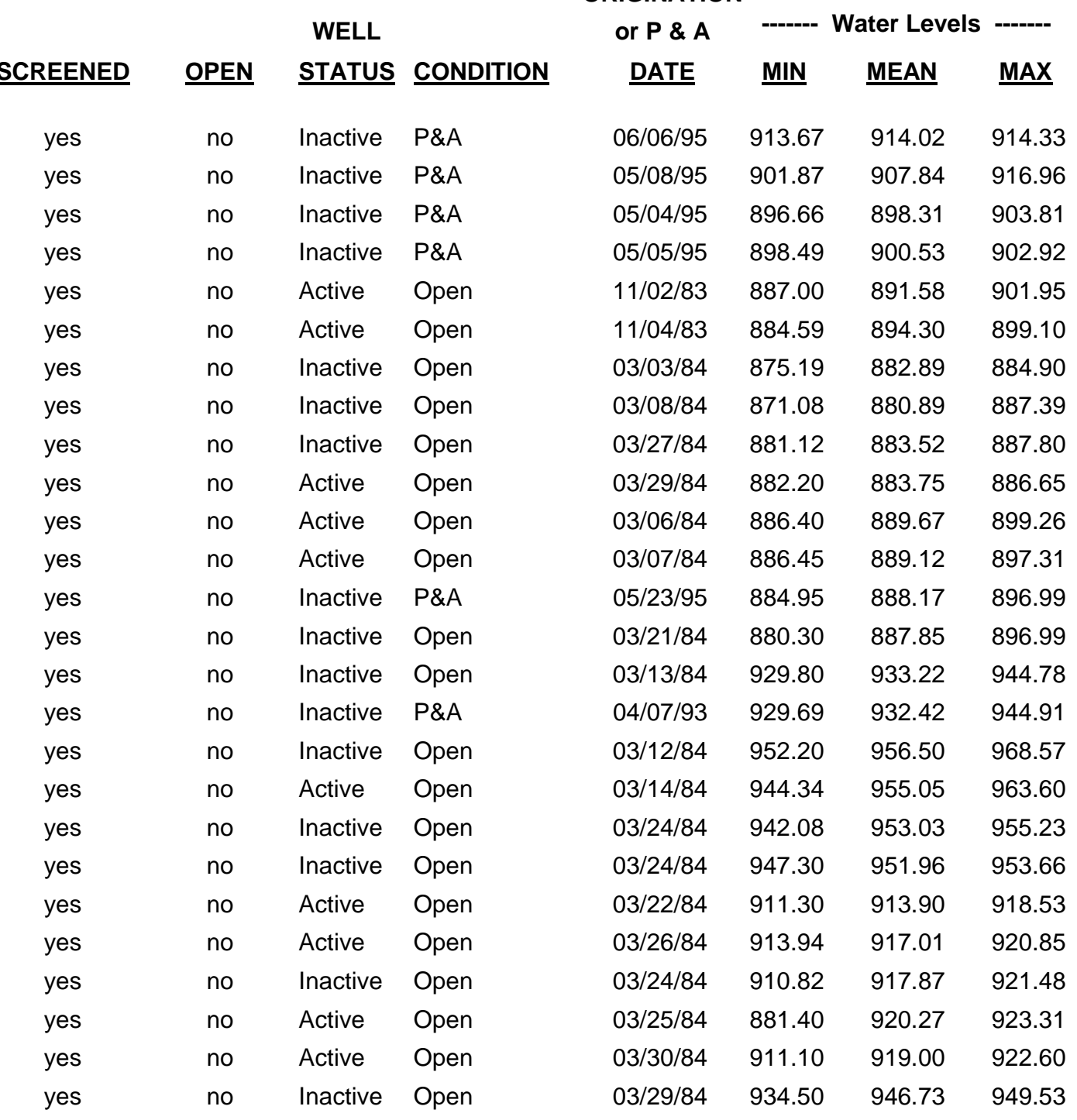




\begin{tabular}{|c|c|c|}
\hline \multicolumn{3}{|l|}{ CURRENT } \\
\hline NAME & OTHER NAME & SCREENED \\
\hline GW-074 & & yes \\
\hline GW-075 & & yes \\
\hline GW-076 & & yes \\
\hline GW-077 & & yes \\
\hline GW-078 & & yes \\
\hline GW-079 & & yes \\
\hline GW-080 & & yes \\
\hline GW-081 & & yes \\
\hline GW-082 & & yes \\
\hline GW-083 & & yes \\
\hline GW-084 & & yes \\
\hline GW-085 & & yes \\
\hline GW-086 & & yes \\
\hline GW-087 & & yes \\
\hline GW-088 & & yes \\
\hline GW-089 & & yes \\
\hline GW-090 & & yes \\
\hline GW-091 & & yes \\
\hline GW-092 & & yes \\
\hline GW-093 & P-02 & yes \\
\hline GW-093A & & yes \\
\hline GW-094 & P-03 & yes \\
\hline GW-095 & P-05 & yes \\
\hline GW-096 & $\mathrm{P}-07$ & yes \\
\hline GW-096A & & yes \\
\hline GW-097 & P-09 & yes \\
\hline
\end{tabular}

ORIGINATION

or $P \& A$

WELL

OPEN STATUS CONDITION

DATE

$\underline{\text { MIN }}$

Water Levels ------

$03 / 27 / 84$

03/28/84 $958.34 \quad 962.48 \quad 965.26$

07/11/02 958.26 $962.62 \quad 965.86$

03/29/84 906.27 $910.39 \quad 915.94$

03/30/84 $905.30 \quad 910.30 \quad 915.73$

03/23/84 $948.87 \quad 959.21 \quad 968.79$

$03 / 24 / 84 \quad 953.63 \quad 957.69 \quad 981.00$

03/16/84 $975.42 \quad 975.42 \quad 975.42$

03/17/84 $939.82 \quad 943.10 \quad 948.90$

03/16/84 $921.50 \quad 924.18 \quad 930.03$

03/17/84 $978.00 \quad 982.75 \quad 990.66$

$\begin{array}{llll}03 / 22 / 84 & 963.70 & 968.33 & 972.14\end{array}$

03/22/84 $963.97 \quad 967.86 \quad 971.97$

07/12/02 $948.20 \quad 952.20 \quad 954.34$

05/05/93 $944.54 \quad 946.25 \quad 952.74$

$944.16 \quad 958.02 \quad 963.06$

$952.28 \quad 954.68 \quad 957.98$

$936.28 \quad 942.34 \quad 948.13$

Active Open

Inactive Destroyed

Inactive $P \& A$

$10 / 14 / 88$

10/10/88 $905.74 \quad 911.24 \quad 912.99$

09/14/84 $899.20 \quad 904.02 \quad 907.90$

$09 / 19 / 84 \quad 900.50 \quad 905.48 \quad 909.40$

$09 / 11 / 84$

05/16/95 $\quad 872.43 \quad 906.96 \quad 913.31$

$\begin{array}{llll}09 / 11 / 84 & 932.80 & 934.88 & 936.98\end{array}$ 


$\begin{array}{ll}\begin{array}{c}\text { CURRENT } \\ \text { NAME }\end{array} & \\ \text { GW-097A } & \\ \text { GW-098 } & \\ \text { GW-099 } & \text { P-10 } \\ \text { GW-100 } & \text { P-13 } \\ \text { GW-101 } & \text { P-14 } \\ \text { GW-102 } & \text { P-15 } \\ \text { GW-103 } & \text { P-18 } \\ \text { GW-104 } & \text { P-19 } \\ \text { GW-105 } & \text { P-22 } \\ \text { GW-106 } & \text { P-23 } \\ \text { GW-107 } & \text { P-24 } \\ \text { GW-108 } & \text { P-25 } \\ \text { GW-109 } & \text { P-26 } \\ \text { GW-110 } & \\ \text { GW-111 } & \\ \text { GW-112 } & \\ \text { GW-113 } & \\ \text { GW-114 } & \\ \text { GW-115 } & \\ \text { GW-116 } & \text { P-01 } \\ \text { GW-117 } & \text { P-04 } \\ \text { GW-118 } & \text { P-06 } \\ \text { GW-119 } & \text { P-08 } \\ \text { GW-120 } & \text { P-11 } \\ \text { GW-121 } & \text { P-12 } \\ \text { GW-122 } & \text { P-16 }\end{array}$

ORIGINATION

\begin{tabular}{|c|c|c|c|}
\hline \multirow{2}{*}{$\begin{array}{c}\text { or P \& A } \\
\text { DATE }\end{array}$} & \multicolumn{3}{|c|}{----- Water Levels ------ } \\
\hline & $\underline{\mathrm{MIN}}$ & MEAN & $\underline{\text { MAX }}$ \\
\hline & 928.09 & 934.94 & 938.49 \\
\hline 09/21/84 & 923.80 & 932.06 & 938.40 \\
\hline 08/02/88 & 1091.85 & 1094.03 & 1096.47 \\
\hline 09/12/84 & 975.60 & 980.65 & 982.84 \\
\hline 09/12/84 & 995.50 & 997.84 & 1004.40 \\
\hline \multicolumn{4}{|l|}{ 06/27/88 } \\
\hline 06/16/88 & 995.03 & 996.73 & 1005.89 \\
\hline 08/02/88 & 994.70 & 997.90 & 1007.08 \\
\hline 09/18/84 & 1004.80 & 1008.81 & 1011.23 \\
\hline 09/26/84 & 996.75 & 1011.22 & 1012.94 \\
\hline 09/17/84 & 988.40 & 990.84 & 992.90 \\
\hline 09/26/84 & 974.36 & 990.37 & 992.60 \\
\hline 09/27/84 & 962.80 & 993.34 & 995.07 \\
\hline \multicolumn{4}{|l|}{$01 / 13 / 94$} \\
\hline \multicolumn{4}{|l|}{ 09/20/84 } \\
\hline 09/29/84 & 847.68 & 851.08 & 854.28 \\
\hline $10 / 05 / 84$ & 830.19 & 843.17 & 857.49 \\
\hline $01 / 24 / 94$ & 801.47 & 803.19 & 806.71 \\
\hline 11/01/84 & 1007.36 & 1041.99 & 1051.22 \\
\hline 11/21/88 & 923.09 & 924.54 & 928.34 \\
\hline 06/19/85 & 532.21 & 883.91 & 911.46 \\
\hline 08/03/85 & 429.91 & 833.65 & 909.49 \\
\hline 08/02/85 & 574.44 & 895.48 & 920.87 \\
\hline 08/25/85 & 937.49 & 941.32 & 943.87 \\
\hline 06/19/85 & 382.51 & 511.23 & 598.76 \\
\hline 07/25/85 & 984.73 & 989.41 & 998.21 \\
\hline
\end{tabular}




CURREN
NAME
GW-123
GW-124
GW-125
GW-126
GW-127
GW-128
GW-129
GW-130
GW-131
GW-132
GW-133
GW-134
GW-135
GW-136
GW-137
GW-138
GW-139
GW-140
GW-141
GW-142
GW-143
GW-144
GW-145
GW-146
GW-147
GW-148

ORIGINATION

\begin{tabular}{|c|c|c|c|}
\hline \multirow{2}{*}{$\begin{array}{c}\text { or P \& A } \\
\text { DATE }\end{array}$} & \multicolumn{3}{|c|}{------ Water Levels ------ } \\
\hline & $\underline{\text { MIN }}$ & MEAN & MAX \\
\hline $07 / 25 / 85$ & 633.89 & 972.49 & 1004.93 \\
\hline $07 / 23 / 85$ & 981.49 & 987.23 & 997.95 \\
\hline $07 / 22 / 85$ & 653.99 & 967.57 & 1003.98 \\
\hline \multirow[t]{2}{*}{$06 / 24 / 85$} & 904.92 & 910.14 & 918.25 \\
\hline & 987.20 & 991.27 & 997.57 \\
\hline $10 / 04 / 91$ & 898.99 & 899.71 & 900.50 \\
\hline 09/19/91 & 924.14 & 924.42 & 924.47 \\
\hline $06 / 13 / 91$ & 901.26 & 904.50 & 914.45 \\
\hline 03/08/90 & 910.87 & 918.71 & 988.14 \\
\hline 03/28/90 & 899.02 & 902.67 & 904.12 \\
\hline $03 / 25 / 90$ & 945.06 & 1012.58 & 1025.01 \\
\hline $03 / 12 / 90$ & 984.73 & 988.16 & 993.16 \\
\hline $03 / 14 / 90$ & 889.18 & 1002.54 & 1023.58 \\
\hline $12 / 16 / 87$ & 846.98 & 849.36 & 855.53 \\
\hline $10 / 09 / 91$ & 825.25 & 825.75 & 827.35 \\
\hline $11 / 01 / 91$ & 814.83 & 815.97 & 818.97 \\
\hline $10 / 14 / 91$ & 874.61 & 877.26 & 881.27 \\
\hline $11 / 15 / 91$ & 862.02 & 866.98 & 874.26 \\
\hline 09/04/87 & 1079.75 & 1091.57 & 1179.35 \\
\hline $10 / 03 / 85$ & 709.88 & 831.29 & 847.25 \\
\hline $10 / 24 / 85$ & 736.34 & 829.94 & 842.28 \\
\hline $10 / 24 / 85$ & 822.43 & 833.02 & 836.73 \\
\hline $10 / 14 / 85$ & 796.09 & 832.68 & 838.67 \\
\hline $10 / 09 / 85$ & 625.30 & 735.09 & 835.51 \\
\hline 09/25/85 & 788.20 & 833.55 & 839.31 \\
\hline 07/30/85 & 895.33 & 900.64 & 902.53 \\
\hline
\end{tabular}




\begin{tabular}{|c|c|}
\hline CURREN & \\
\hline GW-149 & \\
\hline GW-150 & \\
\hline GW-151 & \\
\hline GW-152 & \\
\hline GW-153 & \\
\hline GW-154 & \\
\hline GW-155 & \\
\hline GW-156 & \\
\hline GW-157 & \\
\hline GW-158 & \\
\hline GW-159 & \\
\hline GW-160 & \\
\hline GW-161 & \\
\hline GW-162 & USGS-1Na \\
\hline GW-163 & USGS-1Nb \\
\hline GW-164 & USGS-1Nc \\
\hline GW-165 & USGS-1Sa \\
\hline GW-166 & USGS-1Sb \\
\hline GW-167 & USGS-2Wa \\
\hline GW-168 & USGS-2Wb \\
\hline GW-169 & USGS-2Ea \\
\hline GW-170 & USGS-2Eb \\
\hline GW-171 & USGS-2Ed \\
\hline GW-172 & USGS-2Ee \\
\hline GW-173 & \\
\hline GW-174 & \\
\hline
\end{tabular}

WELL

SCREENED OPEN STATUS CONDITION

\begin{tabular}{|c|c|c|c|}
\hline yes & no & Active & Open \\
\hline yes & no & Inactive & Open \\
\hline yes & no & Active & Open \\
\hline yes & no & Active & Open \\
\hline yes & no & Active & Open \\
\hline yes & no & Active & Open \\
\hline yes & no & Inactive & $P \& A$ \\
\hline yes & no & Active & Open \\
\hline yes & no & Inactive & $P \& A$ \\
\hline no & yes & Inactive & Open \\
\hline yes & no & Active & Open \\
\hline no & yes & Active & Open \\
\hline no & yes & Active & Open \\
\hline no & yes & Inactive & Open \\
\hline no & yes & Inactive & Open \\
\hline no & yes & Inactive & Open \\
\hline no & yes & Inactive & Open \\
\hline no & yes & Inactive & Open \\
\hline yes & no & Active & Open \\
\hline no & yes & Active & Open \\
\hline yes & no & Active & Open \\
\hline no & yes & Active & Open \\
\hline yes & no & Active & Open \\
\hline no & yes & Active & Open \\
\hline yes & no & Active & Open \\
\hline yes & no & Active & Open \\
\hline
\end{tabular}

ORIGINATION

\begin{tabular}{|c|c|c|c|}
\hline \multirow{2}{*}{$\begin{array}{c}\text { or P \& A } \\
\text { DATE }\end{array}$} & \multicolumn{2}{|c|}{----- Water Levels } & ls ------- \\
\hline & $\underline{\mathrm{MIN}}$ & MEAN & MAX \\
\hline 08/14/85 & 891.86 & 899.41 & 902.76 \\
\hline 08/01/85 & 899.58 & 902.39 & 904.18 \\
\hline 08/14/85 & 898.82 & 901.59 & 903.96 \\
\hline 08/02/85 & 895.20 & 957.15 & 10920.18 \\
\hline $10 / 31 / 85$ & 895.95 & 902.14 & 911.54 \\
\hline 07/30/85 & 897.98 & 901.48 & 904.78 \\
\hline 09/04/91 & 903.52 & 907.41 & 921.89 \\
\hline $10 / 18 / 85$ & 900.18 & 906.08 & 927.35 \\
\hline 08/06/91 & 919.85 & 922.48 & 924.85 \\
\hline $10 / 25 / 85$ & 880.10 & 932.59 & 945.25 \\
\hline $10 / 18 / 85$ & 897.69 & 930.81 & 936.01 \\
\hline $07 / 18 / 87$ & 938.96 & 947.92 & 975.29 \\
\hline 07/07/87 & 928.91 & 933.34 & 940.44 \\
\hline $11 / 16 / 85$ & 978.64 & 1019.39 & 1028.36 \\
\hline $11 / 14 / 85$ & 980.19 & 990.82 & 1001.44 \\
\hline $11 / 08 / 85$ & 1037.74 & 1037.74 & 1037.74 \\
\hline $11 / 27 / 85$ & 980.94 & 1001.31 & 1019.98 \\
\hline $11 / 12 / 85$ & 980.63 & 1000.88 & 1020.64 \\
\hline 04/21/86 & 899.38 & 903.50 & 912.38 \\
\hline 04/21/86 & 892.50 & 902.52 & 909.54 \\
\hline 09/16/86 & 895.10 & 901.53 & 911.90 \\
\hline 04/01/86 & 892.01 & 899.38 & 904.65 \\
\hline 02/26/86 & 904.00 & 910.22 & 919.19 \\
\hline 05/05/86 & 903.67 & 909.42 & 918.58 \\
\hline 08/15/85 & 953.75 & 967.85 & 996.43 \\
\hline 08/15/85 & 968.90 & 996.78 & 1015.11 \\
\hline
\end{tabular}




CURREN
NAME
GW-175
GW-176
GW-177
GW-178
GW-179
GW-180
GW-181
GW-182
GW-183
GW-184
GW-185
GW-186
GW-187
GW-188
GW-189
GW-190
GW-191
GW-192
GW-193
GW-194
GW-195
GW-196
GW-197
GW-198
GW-199
GW-200

ORIGINATION

\begin{tabular}{|c|c|c|c|}
\hline or $P \& A$ & & Water Level & Is ------- \\
\hline DATE & $\underline{\text { MIN }}$ & MEAN & $\underline{\text { MAX }}$ \\
\hline 06/22/88 & 953.69 & 964.76 & 978.90 \\
\hline 08/27/85 & 1001.94 & 1008.01 & 1010.93 \\
\hline $10 / 24 / 85$ & 1023.85 & 1040.63 & 1139.88 \\
\hline 08/20/87 & 1039.06 & 1050.94 & 1061.27 \\
\hline 12/03/85 & 1009.10 & 1014.86 & 1020.85 \\
\hline 08/11/87 & 964.62 & 990.28 & 1058.43 \\
\hline 04/13/88 & 973.55 & 988.43 & 1033.03 \\
\hline 06/21/95 & 1018.85 & 1025.50 & 1032.05 \\
\hline 08/02/89 & 933.85 & 935.12 & 936.92 \\
\hline $11 / 15 / 85$ & 810.04 & 817.45 & 836.19 \\
\hline $12 / 10 / 85$ & 359.67 & 365.80 & 369.22 \\
\hline $12 / 20 / 85$ & 816.00 & 817.02 & 819.31 \\
\hline $12 / 04 / 85$ & 788.59 & 815.30 & 822.14 \\
\hline 10/03/85 & 806.73 & 817.06 & 819.22 \\
\hline $12 / 18 / 85$ & 785.53 & 816.84 & 818.37 \\
\hline 08/01/89 & 1019.82 & 1021.00 & 1022.52 \\
\hline 10/29/85 & 1001.22 & 1005.20 & 1008.34 \\
\hline 09/30/85 & 1000.17 & 1002.70 & 1005.65 \\
\hline 08/04/89 & 924.05 & 925.17 & 926.39 \\
\hline 09/30/85 & 978.77 & 988.36 & 989.74 \\
\hline 09/30/85 & 989.20 & 995.58 & 997.55 \\
\hline 09/30/85 & 1014.56 & 1017.90 & 1025.11 \\
\hline 09/30/85 & 998.62 & 999.60 & 1001.22 \\
\hline 09/11/85 & 941.69 & 943.28 & 947.03 \\
\hline 09/11/85 & 941.17 & 943.81 & 945.96 \\
\hline $10 / 30 / 85$ & 949.09 & 952.08 & 954.39 \\
\hline
\end{tabular}




$\begin{array}{ll}\begin{array}{c}\text { CURRENT } \\ \text { NAME }\end{array} & \\ \text { GW-201 } & \\ \text { OW-202 } & \\ \text { GW-203 } & \\ \text { GW-204 } & \\ \text { GW-205 } & \\ \text { GW-206 } & \\ \text { GW-207 } & \text { USGS-4Ea } \\ \text { GW-208 } & \text { USGS-4Eb } \\ \text { GW-209 } & \text { USGS-4EC } \\ \text { GW-210 } & \text { USGS-4W } \\ \text { GW-211 } & \text { USGS-4Wc } \\ \text { GW-212 } & \text { USGS-3Sa } \\ \text { GW-213 } & \text { USGS-3Sb } \\ \text { GW-214 } & \text { USGS-3Sc } \\ \text { GW-215 } & \text { USGS-3Na } \\ \text { GW-216 } & \text { USGS-3Nb } \\ \text { GW-217 } & \\ \text { GW-218 } & \\ \text { GW-219 } & \\ \text { GW-220 } & \\ \text { GW-221 } & \\ \text { GW-222 } & \\ \text { GW-223 } & \\ \text { GW-224 } & \\ \text { GW-225 } & \\ \text { GW-226 } & \end{array}$

ORIGINATION

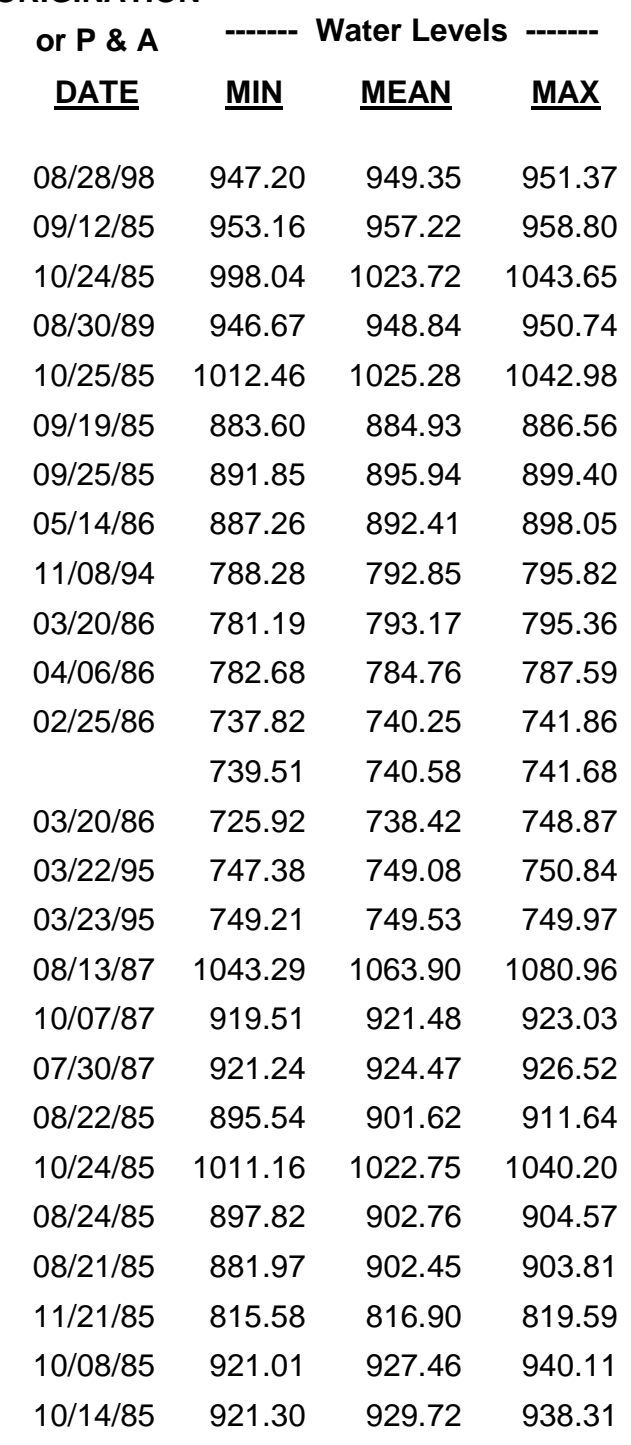




$\begin{array}{ll}\begin{array}{c}\text { CURRENT } \\ \text { NAME }\end{array} & \\ \text { GW-227 } & \text { OTHER NAM } \\ \text { GW-228 } & \\ \text { GW-229 } & \\ \text { GW-230 } & \text { USGS-2Ef } \\ \text { GW-231 } & \\ \text { GW-232 } & \text { USGS-2Ec } \\ \text { GW-233 } & \\ \text { GW-234 } & \\ \text { GW-235 } & \text { USGS-2Eg } \\ \text { GW-236 } & \\ \text { GW-237 } & \\ \text { GW-238 } & \text { USGS-3Sd } \\ \text { GW-239 } & \text { USGS-2Wc } \\ \text { GW-240 } & \\ \text { GW-241 } & \\ \text { GW-242 } & \\ \text { GW-243 } & \\ \text { GW-244 } & \\ \text { GW-245 } & \\ \text { GW-246 } & \\ \text { GW-247 } \\ \text { GW-248 } \\ \text { GW-249 } & \\ \text { GW-250 } & \\ \text { GW-251 } & \\ \text { GW-252 } & \end{array}$

\begin{tabular}{|c|c|c|c|}
\hline \multirow{2}{*}{ SCREENED } & \multicolumn{3}{|c|}{ WELL } \\
\hline & OPEN & STATUS & CONDIT \\
\hline no & yes & Active & Open \\
\hline no & yes & Inactive & Open \\
\hline no & yes & Active & Open \\
\hline no & yes & Active & Open \\
\hline yes & no & Active & Open \\
\hline no & yes & Inactive & Open \\
\hline yes & no & Inactive & $P \& A$ \\
\hline yes & no & Inactive & $P \& A$ \\
\hline unknown & unknown & Inactive & $P \& A$ \\
\hline yes & no & Active & Open \\
\hline yes & no & Inactive & Open \\
\hline no & yes & Inactive & Open \\
\hline no & yes & Inactive & Open \\
\hline yes & no & Active & Open \\
\hline yes & no & Active & Open \\
\hline yes & no & Active & Open \\
\hline yes & no & Active & Open \\
\hline yes & no & Active & Open \\
\hline yes & no & Active & Open \\
\hline yes & no & Active & Open \\
\hline yes & no & Active & Open \\
\hline yes & no & Inactive & Open \\
\hline yes & no & Active & Open \\
\hline yes & no & Inactive & Open \\
\hline yes & no & Active & Open \\
\hline yes & no & Inactive & Open \\
\hline & B- 2 & & \\
\hline
\end{tabular}

ORIGINATION

\begin{tabular}{crrr} 
or P \& A & ------ & Water Levels & ------ \\
DATE & MIN & MEAN & MAX \\
11/09/85 & 927.06 & 933.80 & 941.19 \\
10/23/85 & 924.47 & 932.21 & 940.51 \\
10/30/85 & 916.20 & 926.68 & 943.50 \\
05/12/86 & 903.40 & 907.80 & 912.99 \\
10/02/85 & 830.60 & 834.48 & 840.07 \\
03/27/86 & 894.92 & 899.50 & 904.71 \\
08/16/88 & 1072.44 & 1074.97 & 1077.21 \\
05/05/93 & & & \\
& & & \\
10/16/85 & 970.09 & 972.17 & 975.67 \\
11/01/85 & 904.92 & 908.32 & 912.02 \\
05/19/86 & 736.87 & 739.65 & 741.66 \\
04/21/86 & 900.55 & 903.78 & 908.25 \\
10/31/85 & 897.11 & 902.67 & 906.69 \\
01/30/86 & 923.40 & 932.31 & 959.54 \\
11/20/85 & 965.68 & 971.75 & 973.52 \\
03/10/86 & 992.98 & 996.88 & 1000.56 \\
03/10/86 & 993.03 & 996.51 & 999.44 \\
03/06/86 & 993.07 & 995.21 & 997.24 \\
03/11/86 & 993.67 & 995.31 & 997.65 \\
03/13/86 & 992.23 & 995.40 & 997.60 \\
03/02/87 & 921.13 & 929.35 & 936.45 \\
06/17/86 & 953.25 & 960.48 & 973.06 \\
06/17/86 & 951.65 & 959.35 & 970.05 \\
04/08/86 & 976.60 & 985.37 & 994.29 \\
04/10/86 & 973.52 & 999.00 & 1010.61 \\
& & & \\
\hline
\end{tabular}




CURREN
NAME
GW-253
GW-254
GW-255
GW-256
GW-257
GW-258
GW-259
GW-260
GW-261
GW-262
GW-263
GW-264
GW-265
GW-266
GW-267
GW-268
GW-269
GW-270
GW-271
GW-272
GW-273
GW-274
GW-275
GW-276
GW-277
GW-278

OTHER NAME

USGS-3Nd
WELL SCREENED

yes
yes
yes
yes
yes
yes
yes
unknown
yes
yes
yes
yes
yes
yes
yes
yes
yes
yes
yes
yes
yes
yes
yes
yes
yes
yes

ORIGINATION

$\begin{array}{crrr}\text { or P \& A } & \text {------ } & \text { Water Levels } & \text {------ } \\ \text { DATE } & \text { MIN } & \text { MEAN } & \text { MAX } \\ \text { 04/11/86 } & 974.10 & 994.46 & 1003.30 \\ \text { 09/15/87 } & 938.27 & 967.93 & 984.72 \\ \text { 04/07/86 } & 974.53 & 998.39 & 1010.63 \\ \text { 09/15/87 } & 978.33 & 984.90 & 988.28 \\ \text { 03/03/87 } & 926.38 & 930.17 & 936.76 \\ \text { 03/05/87 } & 926.30 & 928.94 & 936.39 \\ \text { 03/05/87 } & 925.43 & 928.42 & 939.53 \\ & 739.62 & 740.61 & 741.65 \\ \text { 05/22/86 } & 1027.57 & 1030.29 & 1033.54 \\ \text { 05/22/86 } & 1028.82 & 1030.80 & 1033.58 \\ \text { 05/16/86 } & 1023.90 & 1027.41 & 1034.78 \\ \text { 05/16/86 } & 1021.50 & 1026.85 & 1033.60 \\ \text { 06/16/86 } & 1010.44 & 1019.68 & 1023.68 \\ \text { 04/10/88 } & 1014.37 & 1015.25 & 1016.24 \\ \text { 04/10/88 } & 1013.34 & 1014.43 & 1015.41 \\ \text { 06/10/86 } & 1006.93 & 1008.86 & 1015.26 \\ \text { 06/16/86 } & 1004.28 & 1006.70 & 1012.90 \\ \text { 06/09/86 } & 1003.38 & 1005.09 & 1007.16 \\ \text { 06/05/86 } & 1002.99 & 1006.01 & 1008.41 \\ \text { 06/16/86 } & 995.18 & 1000.43 & 1003.91 \\ \text { 06/09/86 } & 995.97 & 997.27 & 998.37 \\ \text { 06/09/86 } & 986.94 & 989.93 & 992.08 \\ \text { 05/30/86 } & 928.82 & 990.28 & 993.16 \\ \text { 07/15/86 } & 991.82 & 994.68 & 997.65 \\ \text { 09/30/87 } & 985.06 & 988.46 & 991.85\end{array}$




\author{
CURRENT \\ NAME \\ GW-279 \\ GW-280 \\ GW-281 \\ GW-282 \\ GW-283 \\ GW-284 \\ GW-285 \\ GW-286 \\ GW-287 \\ GW-288 \\ GW-289 \\ GW-290 \\ GW-291 \\ GW-292 \\ GW-293 \\ GW-294 \\ GW-295 \\ GW-296 \\ GW-297 \\ GW-298 \\ GW-299 \\ GW-300 \\ GW-301 \\ GW-302 \\ GW-303 \\ GW-304
}

OTHER NAME
ORIGINATION

\begin{tabular}{|c|c|c|c|c|c|c|c|}
\hline \multirow[b]{2}{*}{ SCREENED } & \multicolumn{3}{|c|}{ WELL } & \multirow{2}{*}{$\begin{array}{c}\text { or P \& A } \\
\text { DATE }\end{array}$} & \multirow{2}{*}{ MIN } & \multirow{2}{*}{$\begin{array}{c}\text { Water Levels } \\
\qquad \text { MEAN }\end{array}$} & \multirow{2}{*}{ MAX } \\
\hline & OPEN & STATUS & CONDITION & & & & \\
\hline yes & no & Inactive & $P \& A$ & 09/30/87 & 971.31 & 987.98 & 992.57 \\
\hline yes & no & Inactive & $P \& A$ & 09/30/87 & 981.24 & 987.06 & 992.66 \\
\hline yes & no & Active & Open & 08/20/86 & 936.90 & 940.08 & 946.10 \\
\hline yes & no & Inactive & Open & 08/21/86 & 937.64 & 939.52 & 943.54 \\
\hline yes & no & Inactive & Open & 08/21/86 & 929.55 & 932.41 & 934.12 \\
\hline yes & no & Inactive & Open & 08/21/86 & 931.27 & 932.81 & 935.56 \\
\hline yes & no & Inactive & Open & $08 / 22 / 86$ & 931.56 & 932.87 & 935.63 \\
\hline yes & no & Inactive & Open & $11 / 20 / 86$ & 912.13 & 916.69 & 918.03 \\
\hline yes & no & Active & Open & $11 / 20 / 86$ & 910.37 & 917.33 & 918.89 \\
\hline yes & no & Inactive & Open & $11 / 13 / 86$ & 925.66 & 930.37 & 941.16 \\
\hline yes & no & Active & Open & $11 / 20 / 86$ & 925.44 & 930.15 & 942.41 \\
\hline yes & no & Inactive & Open & $11 / 13 / 86$ & 938.64 & 940.81 & 943.99 \\
\hline yes & no & Active & Open & $11 / 14 / 86$ & 934.96 & 938.75 & 944.10 \\
\hline yes & no & Active & Open & $05 / 22 / 87$ & 918.01 & 958.05 & 968.58 \\
\hline no & yes & Active & Open & $06 / 11 / 87$ & 941.39 & 947.27 & 970.37 \\
\hline yes & no & Active & Open & 05/01/87 & 979.70 & 985.21 & 993.77 \\
\hline yes & no & Inactive & $P \& A$ & 09/20/95 & 947.50 & 950.57 & 958.81 \\
\hline yes & no & Active & Open & $05 / 11 / 87$ & 967.63 & 972.05 & 975.29 \\
\hline yes & no & Inactive & $P \& A$ & 08/25/95 & 978.37 & 978.37 & 978.37 \\
\hline yes & no & Active & Open & $07 / 27 / 87$ & 937.10 & 941.19 & 959.76 \\
\hline yes & no & Active & Open & 08/03/87 & 945.23 & 955.32 & 966.19 \\
\hline yes & no & Active & Open & $07 / 17 / 87$ & 950.69 & 962.40 & 973.22 \\
\hline yes & no & Active & Open & $07 / 02 / 87$ & 948.73 & 954.30 & 966.38 \\
\hline yes & no & Active & Open & $11 / 10 / 89$ & 1032.82 & 1040.45 & 1053.42 \\
\hline no & yes & Active & Open & 09/25/87 & 912.84 & 920.09 & 936.21 \\
\hline yes & no & Active & Open & $04 / 25 / 88$ & 924.82 & 927.88 & 929.89 \\
\hline
\end{tabular}




\begin{tabular}{|c|c|c|}
\hline \multicolumn{3}{|c|}{ CURRENT } \\
\hline NAME & OTHER NAME & SCREENED \\
\hline GW-305 & & yes \\
\hline GW-306 & & yes \\
\hline GW-307 & & yes \\
\hline GW-308 & & yes \\
\hline GW-309 & & yes \\
\hline GW-310 & & yes \\
\hline GW-311 & & yes \\
\hline GW-312 & & yes \\
\hline GW-313 & & yes \\
\hline GW-314 & & yes \\
\hline GW-315 & & yes \\
\hline GW-316 & & yes \\
\hline GW-317 & & yes \\
\hline GW-318 & & yes \\
\hline GW-319 & & yes \\
\hline GW-320 & & yes \\
\hline GW-321 & & yes \\
\hline GW-322 & & no \\
\hline GW-323 & & yes \\
\hline GW-324 & & yes \\
\hline GW-325 & & yes \\
\hline GW-326 & ORP-2 & yes \\
\hline GW-327 & ORP-4 & yes \\
\hline GW-328 & ORP-6 & yes \\
\hline GW-329 & ORP-7 & yes \\
\hline GW-330 & ORP-8 & yes \\
\hline
\end{tabular}

ORIGINATION

\begin{tabular}{|c|c|c|c|}
\hline \multirow{2}{*}{$\begin{array}{c}\text { or } P \& A \\
\text { DATE }\end{array}$} & \multicolumn{2}{|c|}{--- Water Levels } & S ------ \\
\hline & $\underline{\mathrm{MIN}}$ & MEAN & $\underline{\text { MAX }}$ \\
\hline 08/25/87 & 1052.15 & 1061.09 & 1072.45 \\
\hline $09 / 25 / 87$ & 954.08 & 958.98 & 963.88 \\
\hline $07 / 15 / 87$ & 957.24 & 962.88 & 969.89 \\
\hline $07 / 21 / 87$ & 956.70 & 962.79 & 968.50 \\
\hline 09/24/87 & 963.19 & 966.87 & 970.00 \\
\hline $07 / 23 / 87$ & 972.20 & 974.30 & 977.73 \\
\hline $07 / 15 / 87$ & 956.65 & 963.32 & 978.77 \\
\hline $09 / 22 / 87$ & 956.54 & 961.76 & 972.13 \\
\hline 09/11/87 & 982.41 & 989.39 & 996.34 \\
\hline 09/09/87 & 983.91 & 989.01 & 996.61 \\
\hline $09 / 25 / 87$ & 982.78 & 989.84 & 1002.64 \\
\hline 09/18/87 & 979.46 & 989.75 & 997.67 \\
\hline $09 / 02 / 87$ & 982.18 & 989.13 & 999.78 \\
\hline 08/07/87 & 815.86 & 816.49 & 817.76 \\
\hline 08/24/87 & 815.96 & 816.14 & 816.26 \\
\hline 07/18/96 & 901.91 & 905.27 & 911.22 \\
\hline 07/18/96 & 899.08 & 906.19 & 917.33 \\
\hline $09 / 02 / 87$ & 965.13 & 981.10 & 1000.31 \\
\hline $10 / 05 / 87$ & 1032.01 & 1043.47 & 1044.90 \\
\hline $10 / 12 / 87$ & 984.44 & 991.03 & 999.44 \\
\hline $10 / 14 / 87$ & 983.53 & 989.94 & 999.33 \\
\hline 03/27/89 & & & \\
\hline 03/27/89 & & & \\
\hline 03/27/89 & & & \\
\hline 03/02/89 & & & \\
\hline 01/27/89 & & & \\
\hline
\end{tabular}




CURREN
NAME
GW-331
GW-332
GW-333
GW-334
GW-335
GW-336
GW-337
GW-338
GW-339
GW-340
GW-341
GW-342
GW-343
GW-344
GW-345
GW-346
GW-347
GW-348
GW-349
GW-350
GW-363
GW-364
GW-365
GW-366
GW-367
GW-368

ORIGINATION

$\begin{array}{crrr}\text { or P \& A } & \text {------ Water Levels } & \text {------ } \\ \text { DATE } & \text { MIN } & \text { MEAN } & \text { MAX } \\ \text { 08/05/87 } & 967.39 & 969.90 & 972.00 \\ \text { 08/11/87 } & 969.24 & 970.55 & 972.10 \\ \text { 08/11/87 } & 968.12 & 971.69 & 973.33 \\ \text { 08/07/87 } & 969.01 & 971.78 & 972.93 \\ \text { 08/06/87 } & 970.15 & 972.02 & 973.96 \\ \text { 08/06/87 } & 971.64 & 974.46 & 975.84 \\ \text { 08/12/87 } & 975.12 & 976.39 & 977.14 \\ \text { 08/12/87 } & 976.77 & 978.53 & 980.48 \\ \text { 12/04/89 } & 1043.29 & 1051.77 & 1069.84 \\ \text { 01/25/89 } & & & \\ \text { 12/07/88 } & & & \\ \text { 02/22/88 } & 1072.60 & 1076.37 & 1080.72 \\ \text { 02/16/88 } & 1058.93 & 1079.33 & 1086.33 \\ \text { 02/08/88 } & 1083.71 & 1083.71 & 1083.71 \\ \text { 06/14/88 } & 975.33 & 980.64 & 983.94 \\ \text { 06/07/88 } & 976.75 & 978.57 & 981.18 \\ \text { 05/25/87 } & 979.15 & 983.05 & 987.72 \\ \text { 05/18/88 } & 973.52 & 976.79 & 983.35 \\ \text { 05/31/88 } & 984.60 & 988.54 & 991.12 \\ \text { 05/27/88 } & 987.01 & 988.81 & 990.61 \\ \text { 03/16/88 } & 948.69 & 952.71 & 954.48 \\ \text { 05/04/88 } & 911.39 & 919.23 & 930.48 \\ \text { 05/02/88 } & 918.18 & 924.75 & 932.48 \\ \text { 05/16/88 } & 914.42 & 921.84 & 950.24 \\ \text { 05/12/88 } & 912.66 & 919.08 & 929.36 \\ \text { 06/13/88 } & 912.63 & 932.13 & 940.63\end{array}$




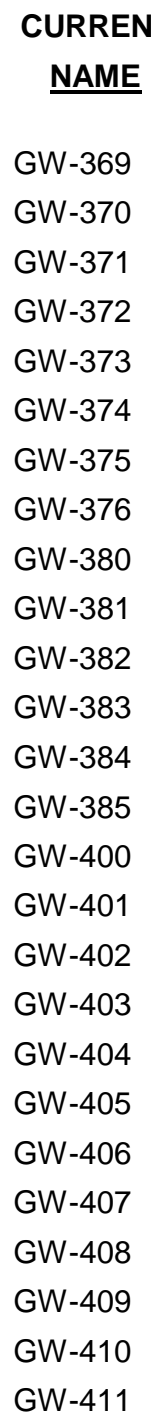

WELL

SCREENED OPEN STATUS CONDITION

ORIGINATION

\begin{tabular}{|c|c|c|c|c|c|c|c|}
\hline & & WELL & & or $P \& A$ & ------ & Water Levels & ------ \\
\hline REENED & OPEN & STATUS & CONDITION & DATE & MIN & MEAN & MAX \\
\hline no & yes & Active & Open & 05/31/88 & 913.72 & 923.22 & 936.32 \\
\hline yes & no & Active & Open & 03/11/88 & 941.14 & 945.93 & 953.64 \\
\hline no & yes & Inactive & Open & 03/09/88 & 939.83 & 943.14 & 945.02 \\
\hline yes & no & Active & Open & $03 / 04 / 88$ & 952.37 & 961.44 & 970.62 \\
\hline no & yes & Inactive & Open & 03/31/88 & 960.05 & 964.02 & 967.10 \\
\hline no & yes & Inactive & Open & 05/04/88 & 922.49 & 924.45 & 926.24 \\
\hline no & yes & Inactive & Open & $04 / 29 / 88$ & 908.53 & 913.13 & 917.23 \\
\hline no & yes & Inactive & Open & $01 / 14 / 88$ & 995.25 & 996.48 & 997.75 \\
\hline yes & no & Active & Open & 08/19/88 & 901.73 & 903.16 & 907.25 \\
\hline no & yes & Active & Open & $04 / 25 / 88$ & 899.27 & 902.61 & 904.64 \\
\hline no & yes & Active & Open & $04 / 11 / 88$ & 900.77 & 902.17 & 905.97 \\
\hline yes & no & Active & Open & $04 / 04 / 88$ & 897.65 & 899.18 & 902.20 \\
\hline no & yes & Active & Open & $03 / 22 / 88$ & 894.46 & 897.32 & 905.33 \\
\hline no & yes & Inactive & Open & 03/31/88 & 897.10 & 904.60 & 905.91 \\
\hline no & yes & Inactive & Open & $02 / 01 / 88$ & & & \\
\hline yes & no & Inactive & Open & $12 / 10 / 87$ & & & \\
\hline yes & no & Inactive & Open & $12 / 04 / 87$ & & & \\
\hline no & yes & Inactive & Open & 02/09/88 & & & \\
\hline no & yes & Inactive & Open & & & & \\
\hline yes & no & Inactive & Open & 03/18/87 & & & \\
\hline yes & no & Inactive & Open & 03/30/87 & & & \\
\hline yes & no & Inactive & Open & 03/17/87 & & & \\
\hline yes & no & Inactive & Open & $03 / 26 / 87$ & & & \\
\hline yes & no & Inactive & Open & 03/17/87 & & & \\
\hline yes & no & Inactive & Open & $03 / 30 / 87$ & & & \\
\hline yes & no & Inactive & Open & $03 / 31 / 87$ & & & \\
\hline
\end{tabular}




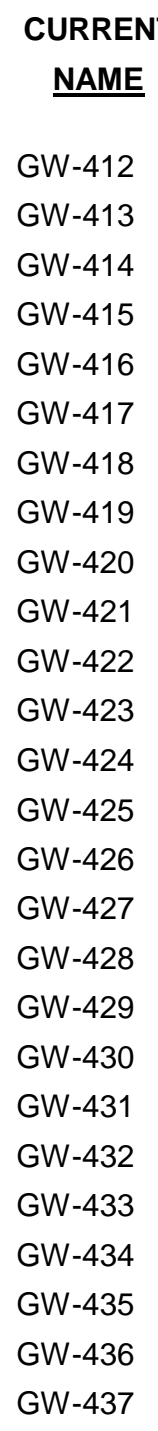

OTHER NAME

WELL

ORIGINATION

\begin{tabular}{|c|c|c|c|}
\hline or $P \& A$ & ------ & Water Levels & ------ \\
\hline DATE & MIN & MEAN & MAX \\
\hline
\end{tabular}

\begin{tabular}{|c|c|c|c|c|c|c|c|}
\hline SCREENED & OPEN & STATUS & CONDITION & DATE & MIN & MEAN & MAX \\
\hline
\end{tabular}

\begin{tabular}{|c|c|c|c|c|}
\hline yes & no & Inactive & Open & $03 / 27 / 87$ \\
\hline yes & no & Inactive & Open & $03 / 24 / 87$ \\
\hline yes & no & Inactive & Open & $03 / 28 / 87$ \\
\hline yes & no & Inactive & Open & $03 / 24 / 87$ \\
\hline yes & no & Inactive & Open & $03 / 23 / 87$ \\
\hline yes & no & Inactive & Open & $03 / 11 / 87$ \\
\hline yes & no & Inactive & Open & $03 / 23 / 87$ \\
\hline yes & no & Inactive & Open & 03/10/87 \\
\hline yes & no & Inactive & Open & $03 / 23 / 87$ \\
\hline yes & no & Inactive & Open & 05/01/87 \\
\hline yes & no & Inactive & Open & $05 / 20 / 87$ \\
\hline yes & no & Inactive & Open & $05 / 06 / 87$ \\
\hline yes & no & Inactive & Open & $05 / 21 / 87$ \\
\hline yes & no & Inactive & Open & $03 / 12 / 87$ \\
\hline yes & no & Inactive & Open & $03 / 23 / 87$ \\
\hline yes & no & Inactive & Open & $05 / 11 / 87$ \\
\hline yes & no & Inactive & Open & $05 / 20 / 87$ \\
\hline yes & no & Inactive & Open & $03 / 23 / 87$ \\
\hline yes & no & Inactive & Open & $03 / 13 / 87$ \\
\hline yes & no & Inactive & Open & 03/28/87 \\
\hline yes & no & Inactive & Open & $03 / 26 / 87$ \\
\hline unknown & unknown & Inactive & Open & \\
\hline yes & no & Inactive & Open & $03 / 27 / 87$ \\
\hline yes & no & Inactive & Open & $03 / 25 / 87$ \\
\hline yes & no & Inactive & Open & $03 / 20 / 87$ \\
\hline yes & no & Inactive & Open & 03/17/87 \\
\hline
\end{tabular}

B- 30 


\author{
CURRENT \\ NAME \\ GW-438 \\ GW-439 \\ GW-440 \\ GW-441 \\ GW-442 \\ GW-443 \\ GW-445 \\ GW-447 \\ GW-448 \\ GW-449 \\ GW-450 \\ GW-451 \\ GW-452 \\ GW-453 \\ GW-455 \\ GW-456 \\ GW-457 \\ GW-458 \\ GW-459 \\ GW-460 \\ GW-461 \\ GW-462 \\ GW-463 \\ GW-464 \\ GW-465 \\ GW-466
}

OTHER NAME
ORIGINATION

or $P \& A$

WELL

SCREENED OPEN STATUS CONDITION

DATE

MIN MEAN MAX

$03 / 23 / 87$

$03 / 16 / 87$

$03 / 23 / 87$

$03 / 23 / 87$

$03 / 24 / 87$

$05 / 22 / 87$

$04 / 07 / 87$

$820.59 \quad 823.07 \quad 825.39$

Inactive Open

Inactive Open

$04 / 18 / 95$

$08 / 30 / 95$

Inactive $P \& A$

Inactive Open

Inactive Open

Inactive Open

Inactive $P \& A$

Inactive Open

Inactive Open

Inactive Open

Inactive Open

Open

837.40

839.30

840.60

03/19/87

$07 / 23 / 87$

$08 / 29 / 95$

$01 / 13 / 88$

01/06/87

$11 / 13 / 87$

$11 / 13 / 87$

$11 / 14 / 87$

$11 / 14 / 87$

$11 / 17 / 87$

$11 / 17 / 87$

$11 / 17 / 87$

$12 / 04 / 87$

$12 / 01 / 87$

$12 / 04 / 87$

$12 / 02 / 87$ 


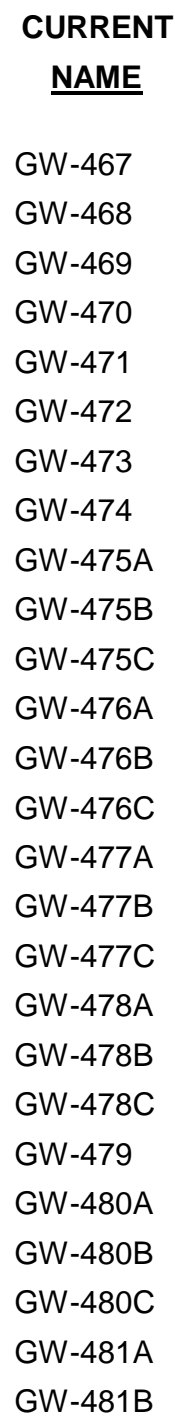

OTHER NAME

SCREENED

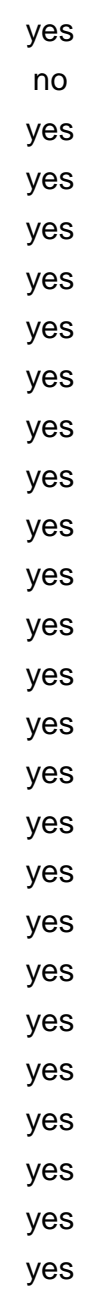

ORIGINATION

or $P$ \& $A$

WELL

OPEN STATUS CONDITION

DATE

MIN

Water Levels -------

$12 / 30 / 87$

$01 / 08 / 88$

$12 / 28 / 87$

$12 / 21 / 87$

$12 / 18 / 87$

$12 / 30 / 87$

$01 / 11 / 88$

$01 / 13 / 88$

$01 / 15 / 88$

$01 / 15 / 88$

$01 / 15 / 88$

$01 / 22 / 88$

$01 / 22 / 88$

$01 / 22 / 88$

$01 / 28 / 88$

$01 / 28 / 88$

$01 / 28 / 88$

$01 / 29 / 88$

$01 / 29 / 88$

$01 / 29 / 88$

$03 / 17 / 88$

$03 / 17 / 88$

03/17/88

03/17/88

$03 / 18 / 88$

03/18/88 


$\begin{gathered}\text { CURRENT } \\ \text { NAME }\end{gathered}$
GW-481C
GW-482A
GW-482B
GW-482C
GW-483
GW-484
GW-485
GW-486
GW-487
GW-488
GW-489
GW-490
GW-491
GW-492
GW-493
GW-494
GW-495
GW-496
GW-497
GW-498
GW-499
GW-499A
GW-499AA
GW-499AB
GW-499AC
GW-499AD

OTHER NAME

SREENED

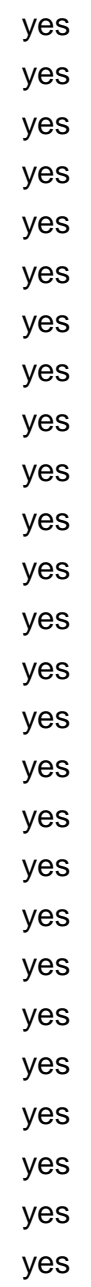

ORIGINATION

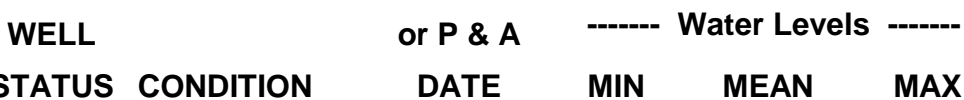

Inactive Open 03/18/88

$03 / 22 / 88$

$03 / 22 / 88$

$03 / 22 / 88$

$03 / 22 / 88$

$03 / 21 / 88$

$04 / 22 / 88$

$04 / 22 / 88$

$05 / 02 / 88$

$05 / 02 / 88$

$05 / 02 / 88$

05/04/88

$05 / 04 / 88$

05/04/88

05/16/88

05/17/88

$05 / 20 / 88$

$05 / 20 / 88$

05/20/88

05/20/88

$05 / 20 / 88$

$05 / 20 / 88$ 


$\begin{gathered}\text { CURRENT } \\ \text { NAME }\end{gathered}$
GW-499AE
GW-499AF
GW-499B
GW-499C
GW-499D
GW-499E
GW-499F
GW-499G
GW-499H
GW-499I
GW-499J
GW-499K
GW-499L
GW-499M
GW-499N
GW-499O
GW-499P
GW-499Q
GW-499R
GW-499S
GW-499T
GW-499U
GW-499V
GW-499W
GW-499X
GW-499Y

OTHER NAME

SREENED

yes

yes

yes

yes

yes

yes

yes

yes

yes

yes

yes

yes

yes

yes

yes

yes

yes

no

yes

yes

yes

yes

yes

yes

yes

yes
ORIGINATION

$\begin{array}{lclll}\text { WELL } & \text { or P \& A } & \text {----- Water Levels } & \text {------ } \\ \text { STATUS CONDITION } & \text { DATE } & \text { MIN } & \text { MEAN } & \text { MAX }\end{array}$

Inactive Could not locate

Inactive Could not locate

Inactive Open

Inactive Open

Inactive Open

Inactive Open

Inactive Could not locate

Inactive Open 06/28/88

Inactive Open 06/28/88

Inactive Open 06/28/88

Inactive Open 06/28/88

Inactive Open 06/30/88

Inactive Open 06/30/88

Inactive Open 06/30/88

Inactive Open 06/30/88

Inactive Open 06/30/88

Inactive Open 06/30/88

Inactive Open 03/07/89

Inactive Open 03/10/89

Inactive Open 03/10/89

Inactive Open 03/13/89

Inactive Open 03/14/89

Inactive Open 03/14/89

Inactive Could not locate 03/14/89

Inactive Open

Inactive Open 


$\begin{array}{ll}\begin{array}{l}\text { CURRENT } \\ \text { NAME }\end{array} & \\ \text { GW-4997 } & \\ \text { GW-502 } & \\ \text { GW-505 } & \\ \text { GW-506 } & \\ \text { GW-507 } & \\ \text { GW-508 } & \\ \text { GW-509 } & \\ \text { GW-511 } & \\ \text { GW-512 } & \\ \text { GW-513 } & \\ \text { GW-514 } & \\ \text { GW-520 } & \\ \text { GW-521 } & \\ \text { GW-522 } & \\ \text { GW-526 } & \\ \text { GW-527 } & \\ \text { GW-528 } & \text { ORP-13 } \\ \text { GW-531 } & \text { ORP-14 } \\ \text { GW-532 } & \text { W1 } \\ \text { GW-533 } & \\ \text { GW-534 } & \\ \text { GW-535 } & \text { W5 } \\ \text { GW-536 } & \text { W6 } \\ \text { GW-537 } & \text { W7 } \\ \text { GW-538 } & \\ \text { GW-539 } & \end{array}$

WELL

SCREENED OPEN STATUS CONDITION

yes
no
yes
yes
yes
yes
yes
yes
yes
yes
no
yes
yes
yes
no
yes
yes
yes
yes
yes
yes
yes
yes
yes
yes
yes

no Inactive Open

yes Inactive P\&A

no Active Open

no Inactive $P \& A$

no Inactive $P \& A$

no Active Open

no Inactive $P \& A$

no Active Open

no Active Open

no Inactive Open

yes Active Open

no Inactive Open

no Active Open

no Active Open

yes Active Open

no Inactive $P \& A$

no Inactive $P \& A$

no Active Open

no Inactive Open

no Inactive Open

no Inactive Open

no Inactive Open

no Inactive Destroyed

no Active Open

no Inactive Open

no Active Open
ORIGINATION

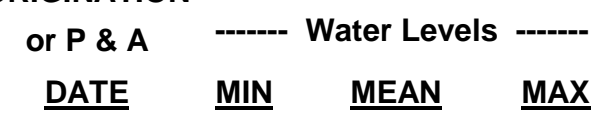

$\begin{array}{lrrr}02 / 11 / 93 & 944.47 & 949.00 & 952.27 \\ 04 / 06 / 88 & 1008.43 & 1009.73 & 1011.25 \\ 04 / 28 / 92 & & & \\ 04 / 28 / 92 & & & \\ 04 / 18 / 88 & 997.24 & 999.30 & 1000.84 \\ 04 / 28 / 92 & 1003.92 & 1003.92 & 1003.92 \\ 04 / 19 / 88 & 972.90 & 989.20 & 1030.11 \\ 04 / 04 / 88 & 969.29 & 978.14 & 995.44 \\ 03 / 30 / 88 & 965.59 & 978.38 & 995.66 \\ 03 / 24 / 88 & 964.45 & 978.00 & 996.37 \\ 05 / 23 / 88 & 917.58 & 924.24 & 933.27 \\ 09 / 14 / 88 & 1090.93 & 1098.43 & 1102.87 \\ 09 / 20 / 88 & 1061.76 & 1075.87 & 1089.61 \\ 06 / 13 / 88 & 982.84 & 984.81 & 986.09 \\ 03 / 27 / 89 & & & \\ 03 / 27 / 89 & & & \\ 09 / 14 / 88 & 980.58 & 987.75 & 997.07 \\ 09 / 15 / 88 & 978.57 & 980.09 & 984.35 \\ 05 / 02 / 90 & 985.06 & 987.79 & 991.36 \\ 04 / 03 / 89 & 980.15 & 981.76 & 983.05 \\ 09 / 15 / 88 & 971.43 & 976.51 & 980.09 \\ 09 / 15 / 88 & 970.04 & 971.58 & 973.12 \\ 09 / 14 / 88 & 967.58 & 969.77 & 972.65 \\ 04 / 03 / 89 & 977.14 & 978.66 & 979.89 \\ 05 / 11 / 89 & 973.50 & 988.50 & 1007.50\end{array}$




\author{
CURRENT \\ NAME \\ GW-540 \\ GW-541 \\ GW-542 \\ GW-543 \\ GW-544 \\ GW-545 \\ GW-546 \\ GW-551 \\ GW-552 \\ GW-553 \\ GW-554 \\ GW-555 \\ GW-556 \\ GW-557 \\ GW-558 \\ GW-559 \\ GW-560 \\ GW-561 \\ GW-562 \\ GW-563 \\ GW-564 \\ GW-565 \\ GW-566 \\ GW-567 \\ GW-568 \\ GW-569
}

OTHER NAME
WELL

SCREENED OPEN STATUS CONDITION

yes
yes
yes
yes
yes
yes
yes
yes
yes
yes
yes
yes
yes
yes
yes
yes
yes
yes
yes
yes
yes
yes
yes
yes
yes

ORIGINATION

or $P$ \& A

DATE

MIN

Water Levels -------

$\begin{array}{lllr}06 / 02 / 89 & 983.33 & 991.77 & 1017.12 \\ 05 / 11 / 89 & 992.30 & 994.47 & 1012.82 \\ 05 / 18 / 89 & 979.11 & 981.79 & 985.57 \\ 06 / 02 / 89 & 957.76 & 959.98 & 963.43 \\ 05 / 30 / 89 & 976.69 & 984.69 & 1003.33 \\ 02 / 07 / 95 & 991.57 & 995.37 & 1006.22 \\ 06 / 07 / 89 & 985.78 & 993.53 & 1010.84 \\ 01 / 06 / 93 & & & \end{array}$

$01 / 06 / 93$

$02 / 17 / 89$

$03 / 18 / 93$

$01 / 16 / 89$

$01 / 13 / 89$

$03 / 18 / 93$

$12 / 02 / 88$

$12 / 08 / 88$

$12 / 16 / 88$

$12 / 30 / 88$

$12 / 23 / 92$

$01 / 13 / 89$

$01 / 17 / 89$

$01 / 27 / 89$

$02 / 01 / 93$

$03 / 18 / 93$

$02 / 14 / 89$

04/17/89

04/20/89

$\begin{array}{lll}955.09 & 961.48 & 973.76 \\ 927.82 & 933.71 & 943.92 \\ 960.54 & 966.41 & 974.39 \\ 893.98 & 909.23 & 940.74 \\ & & \\ 915.69 & 926.81 & 934.69 \\ 870.77 & 870.77 & 870.77 \\ 924.62 & 927.46 & 935.67\end{array}$




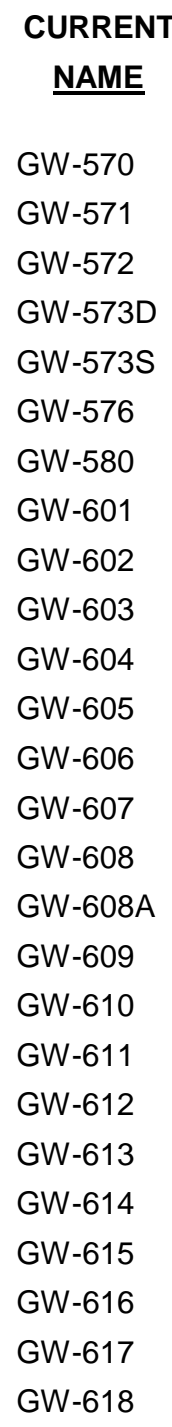

OTHER NAME

$\begin{array}{ll} & \text { WELL } \\ \text { STEENED OPEN STATUS }\end{array}$

yes

yes

yes

yes

yes

yes

yes

no

yes

yes

yes

yes

yes

yes

no

unknown

yes

yes

yes

no

yes

yes

no

no

yes

yes STATUS CONDITION

Inactive $P \& A$

Inactive $P \& A$

Inactive $P \& A$

Inactive $P \& A$

Inactive $P \& A$

Inactive Open

Inactive $P \& A$

Active Open

Inactive Open

Active Open

Inactive Open

Active Open

Active Open

Inactive P\&A

Active Open

unknown Inactive P\&A

no Active Open

no Active Open

no Active Open

yes Active Open

no Active Open

no Inactive Open

yes Active Open

yes Active Open

no Active Open

no Active Open

B- 37
ORIGINATION

or $P$ \& $A$

------ Water Levels ------

DATE MIN MEAN MAX

05/20/94

01/05/93

01/05/93

$03 / 18 / 93$

$03 / 18 / 93$

05/04/89

$12 / 18 / 92$

$08 / 31 / 89$

$10 / 12 / 89$

929.00

934.61

942.60

$09 / 15 / 89$

996.77

999.40

1002.06

09/25/89

902.94

909.41

$03 / 19 / 91$

$03 / 20 / 91$

04.67

$902.44 \quad 909.61$

$03 / 18 / 93$

864.91

907.79

908.85

10/05/89

972.13

904.72

908.47

933.85

10/18/90 $938.91 \quad 945.75 \quad 961.95$

01/02/90 $961.72 \quad 974.19 \quad 983.89$

01/19/90 $938.28 \quad 945.87 \quad 961.78$

$11 / 01 / 89 \quad 996.62 \quad 1007.54 \quad 1016.45$

$\begin{array}{llll}07 / 08 / 89 & 996.68 & 1000.34 & 1006.03\end{array}$

07/19/89 $1002.12 \quad 1006.67 \quad 1011.32$

02/13/90 $1002.49 \quad 1004.30 \quad 1006.75$

03/10/90 $996.90 \quad 1001.78 \quad 1003.81$

03/19/90 $968.41 \quad 971.30 \quad 972.79$

03/15/90 $968.54 \quad 971.37 \quad 972.64$ 


\author{
CURRENT \\ NAME \\ GW-619 \\ GW-620 \\ GW-621 \\ GW-622 \\ GW-623 \\ GW-624 \\ GW-625 \\ GW-626 \\ GW-627 \\ GW-628 \\ GW-629 \\ GW-630 \\ GW-631 \\ GW-632 \\ GW-633 \\ GW-634 \\ GW-635 \\ GW-636 \\ GW-637 \\ GW-638 \\ GW-639 \\ GW-640 \\ GW-641 \\ GW-642 \\ GW-643 \\ GW-644
}

OTHER NAME
ORIGINATION

$\begin{array}{cccc}\text { or P \& A } & \text {------ } & \text { Water Levels } & \text {----- } \\ \text { DATE } & \text { MIN } & \text { MEAN } & \text { MAX } \\ \text { 03/27/90 } & 984.04 & 992.00 & 1000.16 \\ \text { 03/27/90 } & 971.88 & 990.07 & 999.05 \\ \text { 09/19/89 } & 903.82 & 911.05 & 918.85 \\ \text { 12/01/89 } & 907.58 & 913.18 & 917.86 \\ \text { 08/02/90 } & 918.07 & 920.44 & 922.11 \\ \text { 01/05/90 } & 908.75 & 911.49 & 917.16 \\ \text { 01/05/90 } & & & \\ \text { 12/15/89 } & 912.05 & 916.70 & 922.07 \\ \text { 12/11/89 } & 916.71 & 918.93 & 921.51 \\ \text { 01/22/90 } & & & \\ \text { 02/02/90 } & 920.08 & 921.89 & 924.10 \\ \text { 05/03/90 } & 975.55 & 977.13 & 980.65 \\ \text { 05/02/90 } & 996.15 & 997.13 & 998.21 \\ \text { 05/02/90 } & 996.80 & 997.82 & 998.80 \\ \text { 05/03/90 } & 990.05 & 993.22 & 995.23 \\ \text { 05/03/90 } & 997.21 & 998.57 & 999.81 \\ \text { 02/07/91 } & & & \\ \text { 05/31/90 } & 931.32 & 935.90 & 940.27 \\ \text { 06/04/90 } & 926.33 & 931.81 & 936.13 \\ \text { 06/05/90 } & 927.97 & 934.01 & 936.20 \\ \text { 06/15/90 } & 927.00 & 929.49 & 931.65 \\ \text { 06/18/90 } & 923.48 & 927.36 & 931.78 \\ \text { 06/20/90 } & 923.66 & 927.43 & 931.96 \\ \text { 06/20/90 } & 986.10 & 993.88 & 997.52 \\ \text { 06/25/90 } & 958.62 & 963.48 & 968.34 \\ \text { 01/16/01 } & 946.35 & 949.78 & 953.25\end{array}$




$\begin{array}{ll}\begin{array}{l}\text { CURRENT } \\ \text { NAME }\end{array} & \\ \text { GW-645 } & \\ \text { GW-646 } & \\ \text { GW-647 } & \\ \text { GW-647A } & \\ \text { GW-648 } & \\ \text { GW-649 } & \\ \text { GW-651 } & \\ \text { GW-652 } & \\ \text { GW-653 } & \\ \text { GW-654 } & \\ \text { GW-655 } & \\ \text { GW-656 } & \\ \text { GW-657 } & \\ \text { GW-658 } & \\ \text { GW-659 } & \\ \text { GW-660 } & \text { AN:E-1 } \\ \text { GW-661 } & \text { AN:E-2 } \\ \text { GW-662 } & \text { AN:E-3 } \\ \text { GW-663 } & \text { AN:E-4 } \\ \text { GW-664 } & \text { AN:E-5 } \\ \text { GW-665 } & \text { AN:E-6 } \\ \text { GW-666 } & \text { AN:E-7 } \\ \text { GW-667 } & \text { AN:E-8 } \\ \text { GW-668 } & \text { AN:E-9 } \\ \text { GW-669 } & \text { AN:E-10 } \\ \text { GW-670 } & \text { AN:E-11 } \\ & \end{array}$

ORIGINATION

RIGINATION

WELL

SCREENED OPEN STATUS CONDITION

DATE

$\underline{\text { MIN }}$

Water Levels ------

Yes

$07 / 03 / 90$

925.84

MEAN

$\underline{\text { MAX }}$

yes

yes

no Active Open

Inactive Open

unknown

unknown Inactive P\&A

yes

yes

yes

yes

yes

yes

yes

yes

yes

yes

yes

yes

yes

yes

yes

yes

yes

yes

yes

yes

yes

yes
$08 / 24 / 90$

03/06/06

09/17/90

07/25/90

08/08/90

07/30/90

08/10/90

$08 / 13 / 90$

09/06/9

07/19/90

$10 / 11 / 90$

$08 / 31 / 90$

09/02/90

04/29/96

$12 / 26 / 96$

$12 / 26 / 96$

$12 / 23 / 96$

$12 / 23 / 96$

$12 / 23 / 96$

$12 / 20 / 96$

$12 / 16 / 96$

$12 / 19 / 96$

$04 / 30 / 96$

05/07/96
$944.80 \quad 963.16 \quad 964.80$

$957.70 \quad 962.36 \quad 972.23$

$972.86 \quad 975.12 \quad 977.26$

$891.52 \quad 895.48 \quad 899.72$

$887.90 \quad 890.33 \quad 893.20$

$890.17 \quad 908.70 \quad 912.80$

$931.29 \quad 933.30 \quad 934.69$

$1075.47 \quad 1082.61 \quad 1089.59$

$944.34 \quad 944.79 \quad 945.60$

$923.13 \quad 924.40 \quad 925.53$

$932.11 \quad 933.52 \quad 935.01$

$932.26 \quad 933.68 \quad 939.26$ 


$\begin{array}{ll}\begin{array}{l}\text { CURRENT } \\ \text { NAME }\end{array} & \text { OTHER NAME } \\ \text { GW-671 } & \text { AN:E-12 } \\ \text { GW-672 } & \\ \text { GW-673 } & \\ \text { GW-674 } & \\ \text { GW-675 } & \\ \text { GW-676 } & \text { WN-3 } \\ \text { GW-677 } & \text { WL-1 } \\ \text { GW-678 } & \text { WL-2 } \\ \text { GW-679 } & \text { WL-3 } \\ \text { GW-680 } & \text { WL-4 } \\ \text { GW-681 } & \text { WL-5 } \\ \text { GW-682 } & \text { WL-6 } \\ \text { GW-683 } & \\ \text { GW-684 } & \\ \text { GW-685 } & \text { WN5 Deep } \\ \text { GW-686 } & \text { MW2,WN3 } \\ \text { GW-687 } & \text { MW1 } \\ \text { GW-688 } & \text { WN3 Deep } \\ \text { GW-689 } & \text { WN3 Shallow } \\ \text { GW-690 } & \text { WN-2 Deep } \\ \text { GW-691 } & \\ \text { GW-692 } & \\ \text { GW-693 } & \\ \text { GW-694 } & \\ \text { GW-696 } & \\ \text { GW } & \\ \text { GW } & \\ \text { GW } & \\ \text { GW Shallow } \\ \text { GW }\end{array}$

\begin{tabular}{|c|c|c|c|}
\hline & & WELL & \\
\hline SCREENED & OPEN & STATUS & CONDIT \\
\hline yes & no & Inactive & $P \& A$ \\
\hline yes & no & Inactive & $P \& A$ \\
\hline no & yes & Inactive & Open \\
\hline yes & no & Active & Open \\
\hline unknown & unknown & Inactive & $P \& A$ \\
\hline yes & no & Active & Open \\
\hline yes & no & Active & Open \\
\hline yes & no & Active & Open \\
\hline yes & no & Active & Open \\
\hline yes & no & Active & Open \\
\hline yes & no & Inactive & Open \\
\hline yes & no & Inactive & Open \\
\hline yes & no & Active & Open \\
\hline yes & no & Active & Open \\
\hline no & yes & Active & Open \\
\hline yes & no & Active & Open \\
\hline yes & no & Inactive & $P \& A$ \\
\hline yes & no & Inactive & $P \& A$ \\
\hline yes & no & Inactive & $P \& A$ \\
\hline yes & no & Active & Open \\
\hline yes & no & Active & Open \\
\hline yes & no & Active & Open \\
\hline yes & no & Inactive & Open \\
\hline no & yes & Active & Open \\
\hline yes & no & Active & Open \\
\hline yes & no & Inactive & Open \\
\hline & B- 2 & & \\
\hline
\end{tabular}

ORIGINATION

$$
\begin{array}{cccc}
\text { or P \& A } & \text {---- Water Levels } & -\cdots \\
\text { DATE } & \underline{\text { MIN }} & \text { MEAN } & \text { MAX }
\end{array}
$$

05/07/96

07/18/96

$\begin{array}{llll}10 / 01 / 90 & 871.11 & 873.18 & 875.74\end{array}$

$\begin{array}{llll}09 / 03 / 90 & 873.21 & 875.95 & 877.95\end{array}$

$09 / 17 / 90$

$\begin{array}{llll}09 / 02 / 90 & 838.91 & 841.87 & 845.55\end{array}$

$\begin{array}{llll}10 / 24 / 90 & 993.09 & 1002.88 & 1011.28\end{array}$

$\begin{array}{llll}10 / 25 / 90 & 972.48 & 980.92 & 998.88\end{array}$

$\begin{array}{llll}10 / 27 / 90 & 967.78 & 976.35 & 989.41\end{array}$

$\begin{array}{llll}10 / 15 / 90 & 962.40 \quad 972.86 & 980.94\end{array}$

$10 / 24 / 90$

$10 / 10 / 90$

$\begin{array}{llll}12 / 03 / 90 & 881.03 & 883.66 & 892.26\end{array}$

$\begin{array}{llll}10 / 09 / 90 & 882.12 & 883.27 & 884.81\end{array}$

$11 / 12 / 90 \quad 882.01 \quad 883.73 \quad 885.01$

$10 / 18 / 90 \quad 950.47 \quad 951.10 \quad 952.21$

$\begin{array}{llll}05 / 29 / 03 & 953.19 & 953.99 & 954.78\end{array}$

$\begin{array}{llll}06 / 05 / 03 & & & \\ 05 / 29 / 03 & 958.24 & 958.24 & 958.24\end{array}$

$10 / 24 / 90 \quad 956.25 \quad 957.06 \quad 958.76$

$\begin{array}{llll}10 / 24 / 90 & 955.94 & 956.70 & 959.39\end{array}$

$\begin{array}{llll}10 / 25 / 90 & 953.73 \quad 955.00 & 956.20\end{array}$

$10 / 26 / 90$

$\begin{array}{llll}02 / 07 / 91 & 909.58 & 913.57 & 919.88\end{array}$

$\begin{array}{llll}02 / 21 / 91 & 907.42 & 911.60 & 917.56\end{array}$

$09 / 18 / 90 \quad 938.21 \quad 944.08 \quad 955.65$ 


$\begin{array}{ll}\begin{array}{cl}\text { CURRENT } \\ \text { NAME }\end{array} & \text { OTHER NAME } \\ \text { GW-697 } & \text { WN5 shallow } \\ \text { GW-698 } & \text { WN5 Bedrock } \\ \text { GW-699 } & \text { WN1 } \\ \text { GW-700 } & \text { WN3 } \\ \text { GW-701 } & \text { WN2 } \\ \text { GW-702 } & \text { WN4 } \\ \text { GW-703 } & \\ \text { GW-704 } & \\ \text { GW-705 } & \\ \text { GW-705A } & \\ \text { GW-705B } & \\ \text { GW-706 } & \\ \text { GW-707 } & \\ \text { GW-708 } & \\ \text { GW-709 } & \\ \text { GW-710 } & \\ \text { GW-711 } & \\ \text { GW-712 } & \\ \text { GW-713 } & \\ \text { GW-714 } & \\ \text { GW-715 } & \\ \text { GW-721 } & \\ \text { GW-722 } & \\ \text { GW-723 } & \\ \text { GW-724 } & \\ \text { GW-725 } & \end{array}$

WELL

SCREENED OPEN STATUS CONDITION

yes
yes
yes
yes
yes
yes
no
no
no
unknown
unknown

no

yes

yes

yes

no

no

no

no

no

yes

yes

no

no

no

no
ORIGINATION

or $P$ \& $A$

A ----- Water Levels ------

DATE MIN MEAN MAX

09/19/90

$\begin{array}{llll}11 / 02 / 90 & 927.15 & 937.23 & 953.11\end{array}$

$09 / 21 / 90 \quad 955.04 \quad 955.06 \quad 955.16$

$\begin{array}{llll}10 / 03 / 90 & 939.18 & 940.44 & 942.91\end{array}$

$10 / 09 / 90$

$10 / 10 / 90$

$\begin{array}{llll}12 / 07 / 90 & 906.09 & 912.64 & 928.09\end{array}$

$\begin{array}{llll}12 / 20 / 90 & 906.63 & 911.69 & 923.78\end{array}$

$01 / 18 / 91 \quad 646.42 \quad 855.70 \quad 913.57$

$04 / 02 / 91$

$04 / 02 / 91$

$\begin{array}{llll}01 / 27 / 91 & 909.67 & 913.41 & 919.77\end{array}$

$\begin{array}{llll}08 / 14 / 98 & 924.81 & 925.10 & 925.91\end{array}$

$\begin{array}{llll}08 / 14 / 98 & 925.32 & 925.97 & 926.57\end{array}$

$\begin{array}{llll}04 / 05 / 91 & 856.70 & 877.73 & 886.46\end{array}$

$\begin{array}{llll}07 / 02 / 91 & 824.02 & 840.78 & 848.28\end{array}$

$07 / 10 / 91 \quad 837.90 \quad 841.57 \quad 853.99$

$\begin{array}{llll}06 / 20 / 91 & 840.79 & 843.71 & 852.22\end{array}$

$\begin{array}{llll}01 / 13 / 92 & 839.03 & 843.82 & 849.83\end{array}$

$\begin{array}{llll}01 / 24 / 92 & 843.18 & 846.24 & 855.51\end{array}$

$\begin{array}{llll}01 / 29 / 92 & 844.59 & 847.42 & 856.42\end{array}$

09/19/95

08/09/91

$\begin{array}{llll}08 / 15 / 91 & 945.53 & 950.45 & 955.88\end{array}$

$\begin{array}{llll}08 / 12 / 91 & 943.87 & 949.06 & 955.14\end{array}$

$08 / 27 / 91 \quad 944.91 \quad 950.11 \quad 954.85$ 


$\begin{array}{ll}\begin{array}{ll}\text { CURRENT } \\ \text { NAME }\end{array} & \text { OTHER NAME } \\ \text { GW-726 } & \\ \text { GW-727 } & \\ \text { GW-728 } & \\ \text { GW-729 } & \\ \text { GW-730 } & \\ \text { GW-731 } & \\ \text { GW-732 } & \\ \text { GW-733 } & \\ \text { GW-734 } & \\ \text { GW-735 } & \\ \text { GW-736 } & \\ \text { GW-737 } & \\ \text { GW-738 } & \\ \text { GW-739 } & \\ \text { GW-740 } & \text { Grid Well: J-3 (shallow) } \\ \text { GW-742 } & \\ \text { GW-743 } & \\ \text { GW-744 } & \\ \text { GW-745 } & \\ \text { GW-746 } & \text { Grid Well: K-1 (deep) } \\ \text { GW-747 } & \\ \text { GW-748 } & \\ \text { GW-749-75-1 Well: K-1 (shallow) } \\ \end{array}$

\begin{tabular}{|c|c|c|c|}
\hline \multirow[b]{2}{*}{ SCREENED } & \multicolumn{3}{|c|}{ WELL } \\
\hline & OPEN & STATUS & CONDI \\
\hline no & yes & Active & Open \\
\hline no & yes & Active & Open \\
\hline no & yes & Inactive & Open \\
\hline no & yes & Active & Open \\
\hline no & yes & Active & Open \\
\hline yes & no & Active & Open \\
\hline yes & no & Active & Open \\
\hline no & yes & Active & Open \\
\hline no & yes & Active & Open \\
\hline yes & no & Active & Open \\
\hline yes & no & Active & Open \\
\hline yes & no & Inactive & Open \\
\hline yes & no & Active & Open \\
\hline no & yes & Active & Open \\
\hline no & yes & Active & Open \\
\hline no & yes & Inactive & Open \\
\hline yes & no & Active & Open \\
\hline yes & no & Active & Open \\
\hline yes & no & Inactive & Open \\
\hline yes & no & Active & Open \\
\hline yes & no & Active & Open \\
\hline yes & no & Active & Open \\
\hline yes & no & Inactive & $P \& A$ \\
\hline yes & no & Active & Open \\
\hline yes & no & Inactive & Open \\
\hline yes & no & Active & Open \\
\hline & B- 42 & & \\
\hline
\end{tabular}

ORIGINATION

$$
\begin{aligned}
& \text { or P \& A ------ Water Levels ------ } \\
& \text { DATE MIN MEAN MAX }
\end{aligned}
$$

$07 / 24 / 92$

$08 / 07 / 92$

$09 / 02 / 92$

$08 / 12 / 92$

$08 / 03 / 92$

$\begin{array}{llll}09 / 12 / 91 & 923.30 & 924.83 & 927.38\end{array}$

$\begin{array}{llll}09 / 11 / 91 & 903.99 & 907.34 & 930.49\end{array}$

$\begin{array}{llll}10 / 02 / 91 & 898.19 & 901.38 & 908.10\end{array}$

$\begin{array}{llll}10 / 05 / 91 & 899.13 & 901.39 & 906.63\end{array}$

$10 / 30 / 91 \quad 899.72 \quad 901.51 \quad 906.54$

$\begin{array}{llll}10 / 25 / 91 & 945.32 & 949.55 & 953.72\end{array}$

$\begin{array}{llll}11 / 07 / 91 & 945.21 & 949.30 & 954.47\end{array}$

$11 / 21 / 91 \quad 951.19 \quad 954.23 \quad 959.46$

$11 / 26 / 91 \quad 944.62 \quad 948.78 \quad 953.94$

$12 / 20 / 91 \quad 943.76 \quad 949.09 \quad 955.22$

$\begin{array}{lllll}12 / 05 / 91 & 962.09 & 972.87 & 1006.35\end{array}$

$\begin{array}{llll}12 / 13 / 91 & 961.03 & 977.13 & 1015.36\end{array}$

$\begin{array}{llll}01 / 08 / 92 & 898.73 & 900.76 & 902.38\end{array}$

$\begin{array}{llll}01 / 09 / 92 & 896.11 & 899.64 & 902.06\end{array}$

$01 / 10 / 92 \quad 897.64 \quad 901.19 \quad 903.51$

$\begin{array}{llll}01 / 28 / 92 & 901.26 & 909.65 & 917.53\end{array}$

$\begin{array}{llll}01 / 30 / 92 & 901.78 & 904.46 & 906.34\end{array}$

$\begin{array}{llll}03 / 14 / 06 & 910.19 & 914.69 & 917.59\end{array}$

$\begin{array}{llll}02 / 06 / 92 & 902.33 & 906.68 & 912.64\end{array}$

$\begin{array}{llll}02 / 18 / 92 & 903.55 & 906.78 & 908.35\end{array}$

$\begin{array}{llll}02 / 21 / 92 & 904.28 & 908.20 & 909.82\end{array}$ 


\section{CURRENT \\ NAME}

GW-753

GW-754

GW-755

GW-756

GW-757

GW-758

GW-759

GW-760

GW-761

GW-762

GW-763

GW-764

GW-765

GW-766

GW-767

GW-768

GW-769

GW-770

GW-771

GW-772

GW-773

GW-774

GW-775

GW-776

GW-777

GW-778

\section{OTHER NAME}

Grid Well: J-2 (deep)

Grid Well: J-2 (shallow)

Grid Well: J-1 (deep)

Grid Well: J-1 (shallow)

Grid Well: G-1 (deep)

Grid Well: G-1 (shallow)

Grid Well: G-2 (deep)

Grid Well: G-2 (shallow)

Grid Well: J-Primary (deep)

Grid Well: J-Primary (shallow)

Grid Well E-1 (deep)

Grid Well E-1 (shallow)

Grid Well I-2

Grid Well I-2

Grid Well I-1

Grid Well G-3

Grid Well G-3

Grid Well C-1

Grid Well C-1

Grid Well H-2

Grid Well H-2

Grid Well H-3

Grid Well H-3

Grid Well B-2

Grid Well B-2
WELL

SCREENED

yes

yes

yes

yes

yes

yes

yes

yes

yes

yes

yes

yes

yes

yes

yes

yes

yes

yes

yes

yes

yes

yes

yes

yes

yes

yes
ORIGINATION

or $P$ \& $A$

DATE MIN MEAN MAX

03/02/92 $915.99 \quad 920.12 \quad 923.69$

03/13/92 $914.59 \quad 918.47 \quad 924.79$

$03 / 25 / 92 \quad 920.56 \quad 922.31 \quad 924.01$

$\begin{array}{llll}03 / 27 / 92 & 920.12 & 921.76 & 923.45\end{array}$

04/24/92 $857.93 \quad 877.16 \quad 882.17$

04/28/92 $975.14 \quad 977.03 \quad 979.90$

04/30/92 $971.41 \quad 975.75 \quad 979.70$

05/01/92 $955.74 \quad 958.73 \quad 961.22$

05/04/92 $954.83 \quad 957.73 \quad 960.83$

05/15/92 $901.04 \quad 901.86 \quad 903.64$

05/13/92 $903.42 \quad 905.31 \quad 907.25$

05/08/92 $988.67 \quad 989.45 \quad 991.07$

05/13/92 $986.97 \quad 988.75 \quad 990.40$

05/18/92 $935.37 \quad 936.69 \quad 938.42$

05/20/92 $934.87 \quad 936.61 \quad 938.44$

05/28/92 $940.33 \quad 941.31 \quad 942.63$

06/04/92 $930.86 \quad 933.55 \quad 936.11$

06/04/92 $929.32 \quad 931.29 \quad 934.47$

06/23/03 $997.92 \quad 1001.04 \quad 1003.60$

06/11/03 $997.86 \quad 1001.10 \quad 1003.99$

06/24/92 $947.66 \quad 951.34 \quad 958.01$

06/25/92 $945.86 \quad 949.40 \quad 955.24$

07/16/92 $914.35 \quad 917.95 \quad 922.60$

07/21/92 $913.65 \quad 917.17 \quad 922.55$

07/30/92 $991.80 \quad 994.26 \quad 996.20$

07/31/92 $990.60 \quad 993.71 \quad 996.04$ 


CURREN
NAME
GW-779
GW-780
GW-781
GW-782
GW-783
GW-784
GW-785
GW-786
GW-787
GW-788
GW-789
GW-790
GW-791
GW-792
GW-793
GW-794
GW-795
GW-796
GW-797
GW-798
GW-799
GW-800
GW-801
GW-802
GW-803
GW-804

ORIGINATION

WELL

OTHER NAME

Grid Well F-2

Grid Well F-2

Grid Well E-3

Grid Well E-3

Grid Well E-3

Grid Well D-1

Grid Well D-1

Grid Well E-2

Grid Well E-2

Grid Well F-3

Grid Well F-3

Grid Well D-2

Grid Well D-2
SCREENED

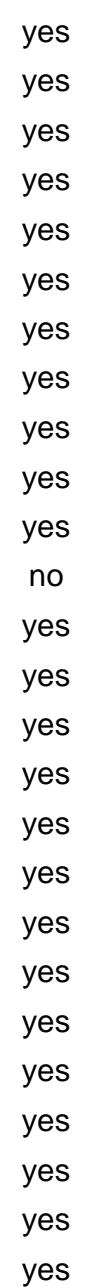

\section{or $P$ \& A} DATE

$08 / 02 / 92$

\section{6/01/06}

08/10/92

$08 / 12 / 92$

$08 / 13 / 92$

06/11/03

06/10/03

03/01/06

03/01/06

06/01/0

06/01/06

04/06/93

09/21/92

$09 / 24 / 92$

$01 / 25 / 01$

$10 / 08 / 92$

$10 / 13 / 92$

03/04/93

$03 / 16 / 93$

$03 / 18 / 93$

$03 / 25 / 93$

04/07/93

07/01/93

$06 / 25 / 93$

$07 / 13 / 93$

no Inactive Open 07/13/93

------ Water Levels -------

MIN MEAN MAX

$952.69 \quad 953.48 \quad 954.19$

$949.89 \quad 950.76 \quad 951.50$

$938.62 \quad 939.66 \quad 941.39$

$937.73 \quad 938.85 \quad 947.73$

$934.92 \quad 938.04 \quad 940.70$

$994.81 \quad 997.58 \quad 1000.60$

$995.11 \quad 998.62 \quad 1002.10$

$973.90 \quad 977.99 \quad 981.50$

$969.25 \quad 972.35 \quad 976.35$

$930.07 \quad 936.12 \quad 937.07$

$933.10 \quad 933.68 \quad 934.82$

$967.08 \quad 968.71 \quad 970.84$

$966.57 \quad 968.00 \quad 976.87$

$957.64 \quad 959.66 \quad 961.89$

$912.97 \quad 917.64 \quad 925.17$

$913.88 \quad 919.58 \quad 923.22$

$964.41 \quad 979.49 \quad 1040.25$

$\begin{array}{lll}924.10 & 987.80 & 1004.20\end{array}$

$916.58 \quad 928.79 \quad 962.56$

$954.33 \quad 965.49 \quad 979.09$

$942.43 \quad 945.81 \quad 957.20$

$916.16 \quad 987.77 \quad 1010.56$

$934.79 \quad 935.70 \quad 936.33$ 


$\begin{array}{ll}\begin{array}{c}\text { CURRENT } \\ \text { NAME }\end{array} & \\ \text { GW-805 } & \\ \text { OWHER NAME } \\ \text { GW-807 } \\ \text { GW-808 } \\ \text { GW-809 } \\ \text { GW-810 } \\ \text { GW-811 } \\ \text { GW-812 } \\ \text { GW-813 } \\ \text { GW-814 } \\ \text { GW-815 } \\ \text { GW-816 } \\ \text { GW-817 } \\ \text { GW-818 } \\ \text { GW-819 } \\ \text { GW-820 } \\ \text { GW-821 } \\ \text { GW-822 } \\ \text { GW-823 } \\ \text { GW-824 } \\ \text { GW-825 } \\ \text { GW-826 } \\ \text { GW-827 } \\ \text { GW-828 } \\ \text { GW-829 } \\ \text { GW-830 } \\ \end{array}$

\begin{tabular}{ccccc} 
SCREENED & OPEN & & STATUS & CONDI \\
\cline { 1 - 1 } yes & & no & Inactive & P\&A \\
yes & no & Inactive & P\&A \\
yes & no & Inactive & P\&A \\
yes & no & Inactive & P\&A \\
yes & no & Inactive & P\&A \\
yes & no & Inactive & P\&A \\
yes & no & Inactive & Open \\
yes & no & Inactive & Open \\
yes & no & Inactive & Open \\
yes & no & Inactive & Open \\
yes & no & Inactive & Open \\
yes & no & Active & Open \\
yes & no & Inactive & P\&A \\
yes & no & Inactive & P\&A \\
unknown & unknown & Inactive & Open \\
unknown & unknown & Active & Open \\
yes & no & Inactive & Open \\
yes & no & Inactive & Open \\
yes & no & Inactive & Open \\
unknown & unknown & Inactive & P\&A \\
yes & no & Inactive & Open \\
yes & no & Inactive & Open \\
yes & no & Active & Open \\
yes & no & Inactive & Open \\
yes & no & Active & Open \\
unknown & unknown & Inactive & Open
\end{tabular}

ORIGINATION

$$
\begin{aligned}
& \text { or } P \text { \& A ---- Water Levels --- } \\
& \text { DATE MIN MEAN } \underline{\text { MAX }}
\end{aligned}
$$

$08 / 14 / 98$

$08 / 14 / 98$

$08 / 14 / 98$

$08 / 14 / 98$

$08 / 14 / 98$

$08 / 14 / 98$

$10 / 27 / 93$

$11 / 12 / 93$

$12 / 30 / 93$

$12 / 30 / 93$

$12 / 30 / 93$

$\begin{array}{llll}06 / 02 / 94 & 883.28 & 885.69 & 887.42\end{array}$

$\begin{array}{llll}03 / 14 / 06 & 908.32 & 910.72 & 918.30\end{array}$

04/07/04 919.66 $919.66 \quad 919.66$

$919.17 \quad 919.70 \quad 920.54$

06/29/94

$06 / 29 / 94$

$06 / 30 / 94$

$07 / 22 / 94$

$12 / 07 / 94$

$12 / 08 / 94$

$\begin{array}{llll}01 / 24 / 95 & 1003.79 & 1010.16 & 1016.95\end{array}$

$\begin{array}{llll}03 / 06 / 95 & 968.12 & 977.07 & 982.52\end{array}$

$\begin{array}{llll}03 / 17 / 95 & 936.98 & 970.61 & 975.93\end{array}$

$795.04 \quad 795.04 \quad 795.04$ 


$\begin{array}{ll}\begin{array}{cl}\text { CURRENT } \\ \text { NAME }\end{array} & \text { OTHER NAME } \\ \text { GW-831 } & \\ \text { GW-832 } & \\ \text { GW-833 } & \text { Outfall 51 Spri } \\ \text { GW-834 } & \text { BPA-04 } \\ \text { GW-835 } & \text { BPA-02 } \\ \text { GW-836 } & \text { BPA-03 } \\ \text { GW-837 } & \text { BPA-01 } \\ \text { GW-838 } & \text { EBCVMW-838 } \\ \text { GW-839 } & \text { EBCVMW-839 } \\ \text { GW-840 } & \text { EBCVMW-840 } \\ \text { GW-841 } & \text { MW-19 } \\ \text { GW-842 } & \text { MW-43A } \\ \text { GW-843 } & \text { MW-43B } \\ \text { GW-844 } & \text { MW-43C } \\ \text { GW-845 } & 9422-22 \text { TF INF,EEVOC-INFLUENT } \\ \text { GW-854 } & \text { TPB-01 } \\ \text { GW-855 } & \text { TPB-02 } \\ \text { GW-857 } & \text { TPB-04 } \\ \text { GW-858 } & \text { TPB-05 } \\ \text { GW-859 } & \text { TPB-06 } \\ \text { GW-860 } & \text { TPB-07 } \\ \text { GW-861 } & \text { TPB-08 } \\ \text { GW-862 } & \text { TPB-09 } \\ \text { GW-863 } & \text { TPB-10 } \\ \text { GW-864 } & \text { TPB-11 } \\ \text { GW-865 } & \text { TPB-12 } \\ \end{array}$

\begin{tabular}{|c|c|c|c|c|c|c|c|}
\hline \multirow[b]{2}{*}{ SCREENED } & \multicolumn{3}{|c|}{ WELL } & \multirow{2}{*}{$\begin{array}{c}\text { or P \& A } \\
\text { DATE }\end{array}$} & \multirow{2}{*}{ 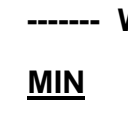 } & \multirow{2}{*}{$\begin{array}{c}\text { Water Levels } \\
\text { MEAN }\end{array}$} & \multirow{2}{*}{ MAX } \\
\hline & OPEN & STATUS & CONDITION & & & & \\
\hline yes & no & Active & Open & 07/30/96 & 956.68 & 963.00 & 969.61 \\
\hline yes & no & Active & Open & 05/09/96 & 897.58 & 899.05 & 899.51 \\
\hline no & yes & Inactive & $P \& A$ & $04 / 07 / 04$ & & & \\
\hline yes & no & Inactive & Open & 07/11/96 & & & \\
\hline yes & no & Active & Open & 07/11/96 & 985.21 & 985.93 & 987.08 \\
\hline yes & no & Inactive & Open & $07 / 16 / 96$ & & & \\
\hline yes & no & Inactive & Could not locate & $07 / 17 / 96$ & 995.77 & 995.77 & 995.77 \\
\hline yes & no & Inactive & $P \& A$ & 01/25/01 & & & \\
\hline yes & no & Inactive & $P \& A$ & 02/01/01 & & & \\
\hline yes & no & Inactive & $P \& A$ & 01/26/01 & & & \\
\hline yes & no & Active & Open & & 821.86 & 824.59 & 826.14 \\
\hline yes & no & Active & Open & $07 / 06 / 93$ & 805.64 & 816.58 & 819.62 \\
\hline yes & no & Inactive & Open & 07/21/93 & 815.49 & 818.38 & 821.02 \\
\hline yes & no & Inactive & Open & 07/28/93 & 661.44 & 661.44 & 661.44 \\
\hline no & yes & Active & Open & & & & \\
\hline yes & no & Inactive & Open & & & & \\
\hline yes & no & Inactive & Open & & & & \\
\hline yes & no & Inactive & $P \& A$ & $09 / 11 / 00$ & & & \\
\hline yes & no & Inactive & $P \& A$ & $09 / 14 / 00$ & & & \\
\hline yes & no & Inactive & Could not locate & & & & \\
\hline yes & no & Inactive & Could not locate & & & & \\
\hline yes & no & Inactive & Could not locate & & & & \\
\hline yes & no & Inactive & Could not locate & & & & \\
\hline yes & no & Inactive & Could not locate & & & & \\
\hline yes & no & Inactive & Could not locate & & & & \\
\hline yes & no & Inactive & Open & & & & \\
\hline & B- 46 & & & & & & \\
\hline
\end{tabular}




$\begin{array}{cl}\begin{array}{c}\text { CURRENT } \\ \text { NAME }\end{array} & \text { OTHER NAME } \\ \text { GW-866 } & \text { TPB-13 } \\ \text { GW-867 } & \text { TPB-14 } \\ \text { GW-868 } & \text { TPB-15 } \\ \text { GW-869 } & \text { TPB-16 } \\ \text { GW-871 } & \text { TPB-18 } \\ \text { GW-872 } & \text { TPB-19 } \\ \text { GW-873 } & \text { TPB-20 } \\ \text { GW-874 } & \text { TPB-21 } \\ \text { GW-875 } & \text { TPB-22 } \\ \text { GW-876 } & \text { TPB-23 } \\ \text { GW-877 } & \text { TPB-24 } \\ \text { GW-878 } & \text { TPB-25 } \\ \text { GW-879 } & \text { TPB-26 } \\ \text { GW-880 } & \text { TPB-27 } \\ \text { GW-881 } & \text { TPB-28 } \\ \text { GW-882 } & \text { TPB-29 } \\ \text { GW-883 } & \text { TPB-30 } \\ \text { GW-884 } & \text { TPB-31 } \\ \text { GW-885 } & \text { TPB-32 } \\ \text { GW-886 } & \text { B-2,B2 } \\ \text { GW-887 } & \text { B-3,B-3A,B3 } \\ \text { GW-888 } & \text { B-4,B4 } \\ \text { GW-889 } & \text { B-5,B5 } \\ \text { GW-890 } & \text { B-6,B6 } \\ \text { GW-902 } & \\ \text { GW-904 } & \\ & \end{array}$

\begin{tabular}{l} 
SCREENED \\
\hline yes \\
yes \\
yes \\
yes \\
yes \\
yes \\
yes \\
yes \\
yes \\
yes \\
yes \\
yes \\
yes \\
yes \\
yes \\
yes \\
yes \\
yes \\
yes \\
yes \\
yes \\
yes \\
yes \\
yes \\
yes \\
yes
\end{tabular}

ORIGINATION

$\begin{array}{lcccc}\text { WELL } & \text { or P \& A } & \text {--- } & \text { Water Levels } & \text {-..- } \\ \text { STATUS CONDITION } & \underline{\text { DATE }} & \underline{\text { MIN }} & \text { MEAN } & \text { MAX }\end{array}$

Inactive Open

Inactive Open

Inactive Could not locate

Inactive Could not locate

Inactive Could not locate

Inactive Could not locate

Inactive Open

Inactive Open

Inactive Open

Inactive Open

Inactive $P \& A$

Inactive Could not locate

Inactive Could not locate

Inactive Open

Inactive Could not locate

Inactive Could not locate

Inactive Open

Inactive Could not locate

Inactive Could not locate

Inactive Could not locate 04/24/97

Inactive Could not locate 04/25/97

Inactive Could not locate 04/26/97

Inactive Could not locate 04/26/97

Inactive Could not locate 04/26/97

Inactive Open 01/20/98

Inactive P\&A 01/18/01

B- 47 


CURREN
NAME
GW-905
GW-906
GW-907
GW-908
GW-909
GW-910
GW-911
GW-912
GW-913
GW-914
GW-915
GW-916
GW-917
GW-918
GW-919
GW-920
GW-921
GW-922
GW-923
GW-924
GW-925
GW-926
GW-927
GW-928
GW-929
GW-930

OTHER NAME

SCREENED

yes
yes
unknown
yes
yes
yes
yes
yes
yes
yes

unknown

yes

yes

yes

yes

yes

yes

yes

yes

yes

yes

yes

yes

yes

yes

yes
WELL

OPEN STATUS CONDITION

no Inactive $P \& A$

no Inactive $P \& A$

unknown Inactive $P \& A$

no Inactive $P \& A$

no Inactive $P \& A$

no Inactive $P \& A$

no Inactive $P \& A$

no Inactive Open

no Inactive Open

no Inactive Open

unknown Inactive Open

no Active Open

no Active Open

no Active Open

no Inactive P\&A

no Active Open

no Active Open

no Active Open

no Active Open

no Active Open

no Active Open

no Active Open

no Active Open

no Active Open

no Active Open

no Active Open

B- 48
ORIGINATION

or P \& A ----- Water Levels --.-.

DATE MIN MEAN MAX

01/25/01

$01 / 17 / 01$

02/01/01

$01 / 29 / 01$

$01 / 29 / 01$

$02 / 02 / 01$

$02 / 01 / 01$

$07 / 07 / 98$

$07 / 14 / 98$

$07 / 09 / 98$

$01 / 20 / 98$

01/29/01 $993.41 \quad 997.54 \quad 998.96$

01/22/01 971.27 $975.40 \quad 980.98$

02/02/01 $1058.96 \quad 1062.28 \quad 1063.76$

05/20/04 990.46 $991.20 \quad 991.76$

01/16/01 $957.32 \quad 959.74 \quad 961.73$

01/31/01 960.49 $964.42 \quad 966.54$

01/17/01 $951.21 \quad 951.77 \quad 952.19$

02/01/01 $982.15 \quad 987.51 \quad 1016.73$

01/29/01 953.45 $959.15 \quad 963.40$

02/05/01 954.78 966.36 971.04

02/01/01 $956.90 \quad 960.32 \quad 962.56$

02/01/01 $974.02 \quad 978.83 \quad 985.90$

$\begin{array}{llll}12 / 21 / 09 & 1037.23 & 1037.83 & 1038.23\end{array}$

$\begin{array}{llll}12 / 19 / 09 & 1035.28 & 1036.29 & 1038.28\end{array}$

$12 / 21 / 09 \quad 994.62 \quad 995.15 \quad 995.62$ 


CURREN
NAME
GW-931
GW-932
GW-933
GW-934
GW-935
GW-936
GW-937
GW-938
GW-939
GW-940
GW-941
GW-942
GW-943
GW-944
GW-945
GW-946
GW-947
GW-948
GW-949
GW-950
GW-951
GW-952
GW-953
GW-954
GW-955
GW-956

ORIGINATION

or $P \& A$

WELL

OTHER NAME

SCREENED OPEN STATUS CONDITION

DATE

MIN

12/20/09 $995.96 \quad 996.66 \quad 997.96$

$05 / 10 / 02$

$05 / 10 / 02$

$11 / 30 / 02$

$02 / 13 / 03$

10/31/03

05/19/04

02/12/03

05/21/04

02/11/03

$02 / 11 / 03$

$02 / 12 / 03$

$02 / 12 / 03$

05/24/04

05/19/04

$03 / 22 / 03$

$03 / 22 / 03$

$03 / 22 / 03$

$03 / 22 / 03$

$04 / 20 / 04$

04/20/04

04/20/04

05/19/04 


\begin{tabular}{|c|c|}
\hline $\begin{array}{c}\text { CURRENT } \\
\text { NAME }\end{array}$ & $\underline{\mathrm{OTH}}$ \\
\hline GW-959 & \\
\hline GW-960 & \\
\hline GW-961 & \\
\hline GW-964 & \\
\hline GW-965 & \\
\hline GW-966 & \\
\hline GW-967 & \\
\hline J-001 & $1-\mathrm{J}$ \\
\hline $\mathrm{J}-002$ & $2-\mathrm{J}$ \\
\hline $\mathrm{J}-003$ & $3-\mathrm{J}$ \\
\hline $\mathrm{J}-004$ & 4-J \\
\hline J-005 & 5-J \\
\hline J-006 & 6-J \\
\hline J-007 & 7-J \\
\hline $\mathrm{J}-008$ & 8-J \\
\hline J-009 & 9-J \\
\hline $\mathrm{J}-010$ & $10-\mathrm{J}$ \\
\hline J-011 & $11-\mathrm{J}$ \\
\hline $\mathrm{J}-012$ & $12-\mathrm{J}$ \\
\hline $\mathrm{J}-015$ & $15-\mathrm{J}$ \\
\hline $\mathrm{J}-017$ & $17-\mathrm{J}$ \\
\hline $\mathrm{J}-018$ & $18-\mathrm{J}$ \\
\hline J-019 & $19-\mathrm{J}$ \\
\hline $\mathrm{J}-020$ & $20-\mathrm{J}$ \\
\hline $\mathrm{J}-021$ & 21-J \\
\hline $\mathrm{J}-022$ & $22-\mathrm{J}$ \\
\hline
\end{tabular}

WELL

SCREENED

yes
yes
yes
yes
yes
yes
unknown
unknown
unknown
unknown
unknown
unknown
unknown
unknown
unknown
unknown
unknown
unknown
unknown
unknown
unknown
unknown
unknown
unknown
unknown
unknown

ORIGINATION

\begin{tabular}{|c|c|c|c|}
\hline$P \& A$ & ----- & ater Levels & ----- \\
\hline DATE & MIN & MEAN & MAX \\
\hline & 23.7 & 5 & 925.58 \\
\hline $07 / 28$ & 9.47 & 950.3 & 950.81 \\
\hline 09/09 & 49.77 & 950.67 & 951.75 \\
\hline $03 / 14$ & 960.72 & $962 . \varepsilon$ & 964. \\
\hline $3 / 14$ & 51.44 & 963.56 & 965.23 \\
\hline
\end{tabular}

$11 / 05 / 12$

$08 / 15 / 12$

06/07/43

06/07/43

$06 / 08 / 43$

$06 / 08 / 43$

$06 / 09 / 43$

$06 / 08 / 43$

$06 / 10 / 43$

$06 / 10 / 43$

$06 / 11 / 43$

$06 / 12 / 43$

$06 / 16 / 43$

$06 / 18 / 43$

$08 / 04 / 43$

$08 / 03 / 43$

06/14/43

$06 / 14 / 43$

$06 / 15 / 43$

$06 / 17 / 43$ 


\section{CURRENT \\ NAME}

$\begin{array}{ll}\mathrm{K}-020 & 20-\mathrm{K} \\ \mathrm{K}-021 & 21-\mathrm{K} \\ \mathrm{K}-022 & 22-\mathrm{K} \\ \mathrm{K}-023 & 23-\mathrm{K} \\ \mathrm{K}-024 & 24-\mathrm{K} \\ \mathrm{K} 1-015 & 15-\mathrm{K}-2 \\ \mathrm{~K} 2-015 & 15-\mathrm{K}-2\end{array}$

LL/HAZ-01

LL/HAZ-02

LL/HAZ-03

LL/HAZ-04

LL/HAZ-05

LL/HAZ-06

LL/HAZ-07

LL/HAZ-08

LL/HAZ-09

LL/HAZ-10

LL/HAZ-11

LL/HAZ-12

LL/HAZ-13

LL/HAZ-14

LL/HAZ-15

LL/HAZ-16

LL/HAZ-17

LL/HAZ-18

LL/HAZ-19
WELL

\section{SCREENED}

unknown

unknown

unknown

unknown

unknown

unknown

unknown

yes

yes

yes

yes

yes

yes

yes

yes

yes

yes

yes

yes

yes

yes

yes

yes

yes

yes

yes
ORIGINATION

or $P$ \& $A$

DATE

MIN

Water Levels -------

OPEN STATUS CONDITION

MEAN

$\underline{\text { MAX }}$

unknown Inactive Destroyed 07/13/43

unknown Inactive Destroyed 07/11/43

unknown Inactive Destroyed 07/11/43

unknown Inactive Destroyed 07/11/43

unknown Inactive Destroyed 07/15/43

unknown Inactive Destroyed 07/12/43

unknown Inactive Destroyed 07/14/43

no Inactive Open 09/09/80

$09 / 10 / 80$

$09 / 03 / 98$

$04 / 26 / 95$

$09 / 11 / 80$

$08 / 31 / 95$

$09 / 12 / 80$

903.27

912.50

899.41

904.51

920.67

Inactive $P \& A$

Inactive $P \& A$

Inactive Open

Inactive $P \& A$

Inactive Open

Inactive Open

Inactive Open

Inactive Open

Inactive $P \& A$

Inactive $P \& A$

Inactive Open

Inactive $P \& A$

Inactive $P \& A$

Inactive Open

Inactive Open

Inactive Open

0.51

864.38

863.13

$851.50 \quad 855.90$

$861.53 \quad 866.18$

866.18
894.97

900.75

$09 / 15 / 80$

921.21

09/16/80

913.26

908.69

912.22

03/04/96

907.97

914.07

09/05/95

885.87

892.57

$894.61 \quad 895.01$

$03 / 01 / 96$

889.75

891.85

908.91

866.51

866.88

860.30

869.93

897.07

902.45

929.86

915.69

920.87

902.97

$04 / 28 / 95$

$11 / 14 / 80$

$11 / 20 / 80$

$11 / 20 / 80$

$11 / 20 / 80$ 


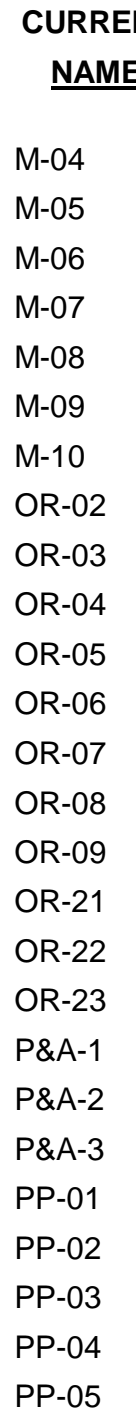

ORIGINATION

ORIGINATION

WELL

SCREENED OPEN STATUS CONDITION

DATE

MIN Water Levels -----
MEAN MAX

\begin{tabular}{|c|c|c|c|c|c|c|c|}
\hline unknown & unknown & Inactive & $P \& A$ & $10 / 21 / 93$ & & & \\
\hline unknown & unknown & Inactive & $P \& A$ & $11 / 09 / 93$ & & & \\
\hline unknown & unknown & Inactive & $P \& A$ & $11 / 02 / 93$ & & & \\
\hline unknown & unknown & Inactive & $P \& A$ & $10 / 28 / 93$ & & & \\
\hline unknown & unknown & Inactive & $P \& A$ & $11 / 02 / 93$ & 873.40 & 879.07 & 887.60 \\
\hline unknown & unknown & Inactive & $P \& A$ & $10 / 14 / 93$ & & & \\
\hline unknown & unknown & Inactive & $P \& A$ & $10 / 05 / 93$ & & & \\
\hline unknown & unknown & Inactive & Could not locate & & & & \\
\hline no & yes & Inactive & $P \& A$ & 03/27/95 & 850.55 & 852.80 & 854.15 \\
\hline unknown & unknown & Inactive & Could not locate & & & & \\
\hline unknown & unknown & Inactive & Could not locate & & 859.59 & 861.06 & 863.29 \\
\hline no & yes & Inactive & $P \& A$ & $11 / 05 / 93$ & 855.30 & 856.67 & 858.20 \\
\hline no & yes & Inactive & $P \& A$ & $12 / 06 / 93$ & & & \\
\hline no & yes & Inactive & $P \& A$ & $12 / 08 / 93$ & & & \\
\hline yes & no & Inactive & $P \& A$ & $11 / 18 / 93$ & & & \\
\hline no & yes & Inactive & $P \& A$ & $11 / 12 / 93$ & 876.11 & 876.11 & 876.11 \\
\hline no & yes & Inactive & $P \& A$ & $11 / 10 / 93$ & 873.98 & 873.98 & 873.98 \\
\hline unknown & unknown & Inactive & Could not locate & & & & \\
\hline yes & no & Inactive & $P \& A$ & $10 / 04 / 95$ & & & \\
\hline yes & no & Inactive & $P \& A$ & $10 / 09 / 95$ & & & \\
\hline yes & no & Inactive & $P \& A$ & $10 / 11 / 95$ & & & \\
\hline unknown & unknown & Active & Open & 04/19/04 & & & \\
\hline unknown & unknown & Active & Open & $04 / 19 / 04$ & & & \\
\hline unknown & unknown & Active & Open & 04/19/04 & & & \\
\hline unknown & unknown & Active & $P \& A$ & 04/19/04 & & & \\
\hline unknown & unknown & Active & Open & 04/19/04 & & & \\
\hline
\end{tabular}

B- 52 


$\begin{aligned} & \text { CURREN } \\ & \text { NAME }\end{aligned}$
PP-06
PP-07
PP-08
PP-09
RB-001
RB-005
RB-008
RB-009A
RB-010A
RB-011
RB-014
RB-015
RB-016
RB-017
RB-018
RB-019
RB-020
RB-031
RB-032
RB-033
RB-034
RB-035
RB-036
RB-037
RB-038
S3-1

WELL SCREENED

unknown
unknown
unknown
unknown
unknown
unknown
unknown
unknown
unknown
unknown
unknown
unknown
unknown
unknown
unknown
unknown
unknown
unknown
unknown
unknown
unknown
unknown
unknown
unknown
unknown
yes

ORIGINATION

$$
\begin{array}{ccc}
\text { or P \& A } & \text {---- } \text { Water Levels } & \text {---- } \\
\text { DATE } & \underline{\text { MIN }} & \text { MEAN }
\end{array}
$$

\begin{tabular}{|c|c|c|}
\hline unknown & Active & Open \\
\hline Inknown & Active & Open \\
\hline unknown & Active & Open \\
\hline nknown & Active & Open \\
\hline lown & Inactive & Destroye \\
\hline Inknown & Inactive & Destroyed \\
\hline Inknown & Inactive & Destroyed \\
\hline nown & Inactive & Destroyed \\
\hline inknown & Inactive & Destroyed \\
\hline unknown & Inactive & Destroyed \\
\hline known & Inactive & Destroyed \\
\hline own & Inactive & Destroyed \\
\hline unknown & Inactive & Destroyed \\
\hline unknown & Inactive & Destroyed \\
\hline Inknown & Inactive & Destroyed \\
\hline nown & Inactive & Destroyed \\
\hline unknown & Inactive & Destroyed \\
\hline lknown & Inactive & Destroyed \\
\hline unknown & Inactive & Destroyed \\
\hline unknown & Inactive & Destroyed \\
\hline unknown & Inactive & Destroyed \\
\hline unknown & Inactive & Destroyed \\
\hline unknown & Inactive & Destroyed \\
\hline nown & Inactive & Destroye \\
\hline nknov & Inactive & Destroye \\
\hline no & Inactive & $P \& A$ \\
\hline
\end{tabular}

no Inactive P\&A 06/19/95




CURRENT
NAME
S3-3
S3-5
S3-6
S3-7
S3-8
SAN-1
SAN-10
SAN-2
SAN-3
SAN-4
SAN-5
SAN-6
SAN-7
SAN-8
SAN-9
UNIDENT A
UNIDENT B
V-1
V-2
V-3
V-4
V-5
V-6
V-7

OTHER NAME

$\begin{array}{ll} & \text { WELL } \\ \text { OPEN STATUS }\end{array}$

yes

yes

yes

yes

yes

yes

yes

yes

yes

yes

yes

yes

yes

yes

yes

no

yes

unknown

unknown

unknown

unknown

unknown

unknown

unknown
ORIGINATION

or $P$ \& $A$ ----- Water Levels -------

\section{DATE}

MIN

MEAN

$\underline{\text { MAX }}$

06/19/95

06/20/95

06/19/95

06/19/95

06/19/95

$12 / 01 / 80$

$12 / 19 / 80$

$12 / 04 / 80$

$11 / 24 / 80$

$12 / 05 / 80$

$12 / 05 / 80$

$12 / 10 / 80$

$12 / 15 / 80$

$12 / 16 / 80$

$12 / 16 / 80$

$04 / 12 / 95$

04/12/95

10/17/91

10/17/91

10/16/91

10/15/91

10/15/91

10/15/91

10/16/91 
APPENDIX C

Borehole and Well Reference and Comments Summary 
This Page Intentionally Left Blank 


\section{CURRENT}

NAME

001-L

002-L

003-L

004-L

005-L

006-L

007-L

008-L

009-L

010-L

011-L

012-L

\section{REFERENCE}

Stone and Webster, Inc.(1943d)

Stone and Webster, Inc.(1943d)

Stone and Webster, Inc.(1943d)

Stone and Webster, Inc.(1943d)

Stone and Webster, Inc.(1943d)

Stone and Webster, Inc.(1943d)

Stone and Webster, Inc.(1943d)

Stone and Webster, Inc.(1943d)

Stone and Webster, Inc.(1943d)

Stone and Webster, Inc.(1943d)

Stone and Webster, Inc.(1943d)

Stone and Webster, Inc.(1943d)

\section{COMMENTS}

NX corehole drilled in 1943. Core hole converted to hole class of Unknown. Data base revised on 4/21/97 to reflect changes discovered during periodic review.

NX core hole drilled in 1943. Core hole converted to hole class of Unknown. Data base revised on 4/21/97 to reflect changes discovered during periodic review.

NX core hole drilled in 1943. Core hole converted to hole class of Unknown. Data base revised on 4/21/97 to reflect changes discovered during periodic review.

NX core hole drilled in 1943. Core hole converted to hole class of Unknown. Data base revised on 4/21/97 to reflect changes discovered during periodic review.

NX core hole drilled in 1943. Core hole converted to hole class of Unknown. Data base revised on 4/21/97 to reflect changes discovered during periodic review.

NX core hole drilled in 1943. Core hole converted to hole class of Unknown. Data base revised on 4/21/97 to reflect changes discovered during periodic review.

NX core hole drilled in 1943. Core hole converted to hole class of Unknown. Data base revised on 4/21/97 to reflect changes discovered during periodic review.

NX core hole drilled in 1943. Core hole converted to hole class of Unknown. Data base revised on 4/21/97 to reflect changes discovered during periodic review.

NX core hole drilled in 1943. Core hole converted to hole class of Unknown. Data base revised on 4/21/97 to reflect changes discovered during periodic review.

NX core hole drilled in 1943. Core hole converted to hole class of Unknown. Data base revised on 4/21/97 to reflect changes discovered during periodic review.

NX core hole drilled in 1943. Core hole converted to hole class of Unknown. Data base revised on 4/21/97 to reflect changes discovered during periodic review.

NX core hole drilled in 1943. Core hole converted to hole class of Unknown. Data base revised on 4/21/97 to reflect changes discovered during periodic review. 


\section{CURRENT}

NAME

013-L

014-L

015-L

016-L

017-L

018-L

019-L

020-L

021-L

022-L

\section{REFERENCE}

Stone and Webster, Inc.(1943d)

Stone and Webster, Inc.(1943d)

Stone and Webster, Inc.(1943d)

Stone and Webster, Inc.(1943d)

Stone and Webster, Inc.(1943d)

Stone and Webster, Inc.(1943d)

Stone and Webster, Inc.(1943d)

Stone and Webster, Inc.(1943d)

Stone and Webster, Inc.(1943d)

Stone and Webster, Inc.(1943d)

Stone and Webster, Inc.(1943d)

Stone and Webster, Inc.(1943d)

\section{COMMENTS}

NX core hole drilled in 1943. Core hole converted to hole class of Unknown. Data base revised on 4/21/97 to reflect changes discovered during periodic review.

NX core hole drilled in 1943. Core hole converted to hole class of Unknown. Data base revised on 4/21/97 to reflect changes discovered during periodic review.

NX core hole drilled in 1943. Core hole converted to hole class of Unknown. Data base revised on 4/21/97 to reflect changes discovered during periodic review.

NX core hole drilled in 1943. Core hole converted to hole class of Unknown. Data base revised on 4/21/97 to reflect changes discovered during periodic review.

NX core hole drilled in 1943. Core hole converted to hole class of Unknown. Data base revised on 4/21/97 to reflect changes discovered during periodic review.

NX core hole drilled in 1943. Core hole converted to hole class of Unknown. Data base revised on 4/21/97 to reflect changes discovered during periodic review.

NX core hole drilled in 1943. Core hole converted to hole class of Unknown. Data base revised on 4/21/97 to reflect changes discovered during periodic review.

NX core hole drilled in 1943. Core hole converted to hole class of Unknown. Data base revised on 4/21/97 to reflect changes discovered during periodic review.

NX core hole drilled in 1943. Core hole converted to hole class of Unknown. Data base revised on 4/21/97 to reflect changes discovered during periodic review.

NX core hole drilled in 1943. Core hole converted to hole class of Unknown. Data base revised on 4/21/97 to reflect changes discovered during periodic review.

NX core hole drilled in 1943. Core hole converted to hole class of Unknown. Data base revised on 4/21/97 to reflect changes discovered during periodic review.

NX core hole drilled in 1943. Core hole converted to hole class of Unknown. Data base revised on 4/21/97 to reflect changes discovered during periodic review. 


\section{CURRENT}

NAME

025-L

026-L

027-L

028-L

029-L

030-L

031-L

032-L

033-L

034-L

035-L

1002

\section{REFERENCE}

Stone and Webster, Inc.(1943d)

Stone and Webster, Inc.(1943d)

Stone and Webster, Inc.(1943d)

Stone and Webster, Inc.(1943d)

Stone and Webster, Inc.(1943d)

Stone and Webster, Inc.(1943d)

Stone and Webster, Inc.(1943d)

Stone and Webster, Inc.(1943d)

Stone and Webster, Inc.(1943d)

Stone and Webster, Inc.(1943d)

Stone and Webster, Inc.(1943d)

Brunton, G. A.(1978), SAIC(1992b)

Geotek Engineering, Inc.(1982a)

\section{COMMENTS}

NX core hole drilled in 1943. Core hole converted to hole class of Unknown. Data base revised on 4/21/97 to reflect changes discovered during periodic review.

NX core hole drilled in 1943. Core hole converted to hole class of Unknown. Data base revised on 4/21/97 to reflect changes discovered during periodic review.

NX core hole drilled in 1943. Core hole converted to hole class of Unknown. Data base revised on 4/21/97 to reflect changes discovered during periodic review.

NX core hole drilled in 1943. Core hole converted to hole class of Unknown. Data base revised on 4/21/97 to reflect changes discovered during periodic review.

NX core hole drilled in 1943. Core hole converted to hole class of Unknown. Data base revised on 4/21/97 to reflect changes discovered during periodic review.

NX core hole drilled in 1943. Core hole converted to hole class of Unknown. Data base revised on 4/21/97 to reflect changes discovered during periodic review.

NX core hole drilled in 1943. Core hole converted to hole class of Unknown. Data base revised on 4/21/97 to reflect changes discovered during periodic review.

NX core hole drilled in 1943. Core hole converted to hole class of Unknown. Data base revised on 4/21/97 to reflect changes discovered during periodic review.

NX core hole drilled in 1943. Core hole converted to hole class of Unknown. Data base revised on 4/21/97 to reflect changes discovered during periodic review.

NX core hole drilled in 1943. Core hole converted to hole class of Unknown. Data base revised on 4/21/97 to reflect changes discovered during periodic review.

NX core hole drilled in 1943. Core hole converted to hole class of Unknown. Data base revised on 4/21/97 to reflect changes discovered during periodic review.

OWI well. **TOC elevation missing so TOC estimated as GS+3ft, (best GS was for 01/01/1901 ) from meas_pt_elevation

Destroyed. Replaced by GW-115. 


\section{CURRENT}

\section{NAME}

1003

1004

1005

1006

1007

1008

1009

1010

1011

1012

1013

1014

1015

1016

1017

1018

1019

1020

1021

1022

1023

\section{REFERENCE}

Butz, T. R.(1981), SAIC(1993h)

Butz, T. R.(1981), SAIC(1997c)

Butz, T. R.(1981)

Geotek Engineering, Inc.(1982a)

Geotek Engineering, Inc.(1982a), SAIC(1993h)

Geotek Engineering, Inc.(1982a), SAIC(1995b)

Geotek Engineering, Inc.(1982a)

Geotek Engineering, Inc.(1982a), SAIC(1993h)

Geotek Engineering, Inc.(1982a), SAIC(1993h)

Brunton, G. A.(1978), SAIC(1994d)

Brunton, G. A.(1978), SAIC(1993h)

Brunton, G. A.(1978), SAIC(1993h)

Brunton, G. A.(1978), SAIC(1992b)

Brunton, G. A.(1978), SAIC(1994d)

Brunton, G. A.(1978), SAIC(1993h)

Brunton, G. A.(1978), SAIC(1994d)

Brunton, G. A.(1978), SAIC(1994d)

Brunton, G. A.(1978), SAIC(1994d)

Brunton, G. A.(1978), SAIC(1992b)

Brunton, G. A.(1978), SAIC(1993h)

Brunton, G. A.(1978), SAIC(1992b)

\section{COMMENTS}

Destroyed. Replaced by GW-236.

Drilled to $24 \mathrm{ft}$, hole collapsed and bottom $6 \mathrm{ft}$ filled in

OWI well. **TOC elevation missing so TOC estimated as GS+3ft, (best GS was for 01/01/1901 ) from meas_pt_elevation

OWI well. **TOC elevation missing so TOC estimated as GS+3ft, (best GS was for 01/01/1901) from meas_pt_elevation

OWI well. **TOC elevation missing so TOC estimated as GS+3ft, (best GS was for 01/01/1901) from meas_pt_elevation

OWI well. **TOC elevation missing so TOC estimated as GS+3ft, (best GS was for 01/01/1901) from meas_pt_elevation

OWI well. ${ }^{* *} \mathrm{TOC}$ elevation missing so TOC estimated as GS+3ft, (best GS was for 01/01/1901 ) from meas_pt_elevation

OWI well. **TOC elevation missing so TOC estimated as GS+3ft, (best GS was for 01/01/1901 ) from meas_pt_elevation

OWI well. **TOC elevation missing so TOC estimated as GS+3ft, (best GS was for 01/01/1901 ) from meas pt elevation

OWI well. **TOC elevation missing so TOC estimated as GS+3ft, (best GS was for 01/01/1901 ) from meas pt elevation

OWI well. **TOC elevation missing so TOC estimated as GS+3ft, (best GS was for 01/01/1901) from meas_pt_elevation

OWI well. **TOC elevation missing so TOC estimated as GS+3ft, (best GS was for 01/01/1901 ) from meas_pt_elevation

OWI well. **TOC elevation missing so TOC estimated as GS+3ft, (best GS was for 01/01/1901 ) from meas_pt_elevation

OWI well. **TOC elevation missing so TOC estimated as GS+3ft, (best GS was for 01/01/1901) from meas_pt_elevation 


\section{CURRENT}

\section{NAME}

1024

1025

$1025 \mathrm{~A}$

1026

1027

1028

1029

1030

1031

1032

1033

1034

1035

1036

1037

1038

1040

1041

1043

1044

\section{REFERENCE}

Brunton, G. A.(1978), SAIC(1993h)

Brunton, G. A.(1978), SAIC(1993h)

\section{SAIC(1993h)}

Brunton, G. A.(1978), SAIC(1994d)

Geotek Engineering, Inc.(1979), SAIC(1993h)

Butz, T. R.(1981), SAIC(1993h)

Geotek Engineering, Inc.(1979), SAIC(1993h)

Butz, T. R.(1981)

Butz, T. R.(1981)

Butz, T. R.(1981), SAIC(1993h)

SAIC(1993h)

Butz, T. R.(1981), SAIC(1995b)

Geotek Engineering, Inc.(1979), SAIC(1993h)

Geotek Engineering, Inc.(1979), SAIC(1993h)

Butz, T. R.(1981), SAIC(1993h)

Geotek Engineering, Inc.(1979), SAIC(1993h)

Geotek Engineering, Inc.(1979), SAIC(1993h)

Geotek Engineering, Inc.(1979), SAIC(1993h)

Sanders, M.(1985), SAIC(1993h)

Sanders, M.(1985)

\section{COMMENTS}

OWI well. Could not be located 24Jun86. **TOC elevation missing so TOC estimated as GS+3ft, (best GS was for 01/01/1901) from meas_pt_elevation

OWI well. **TOC elevation missing so TOC estimated as GS+3ft, (best GS was for 01/01/1901 ) from meas_pt_elevation

OWI well. **TOC elevation missing so TOC estimated as GS+3ft, (best GS was for 01/01/1901) from meas_pt_elevation

NXWL core hole to $50 \mathrm{ft}$. Borehole reamed and casing set. Hole deepened to TD. Hole converted to Open-interval well. Data base revised on $3 / 27 / 97$ to reflect changes discovered during periodic review.

Data base revised on $3 / 27 / 97$ to reflect changes discovered during periodic review.

NXWL core hole to $50 \mathrm{ft}$. Borehole reamed and casing set. Hole deepened to TD. Borehole converted to Open-interval well. Data base revised on $3 / 27 / 97$ to reflect changes discovered during periodic review.

Data base revised on $3 / 27 / 97$ to reflect changes discovered during periodic review.

Data base revised on $3 / 27 / 97$ to reflect changes discovered during periodic review.

Data base revised on $3 / 27 / 97$ to reflect changes discovered during periodic review.

Data base revised on $3 / 27 / 97$ to reflect changes discovered during periodic review.

Forms cluster with GW-093. 


\section{CURRENT}

\section{NAME}

1045

1046

1047

1047A

1048

1049

1050

1051

1052

1055

1056

1057

1058

1059

1060

1061

1062

1063

\section{REFERENCE}

Sanders, M.(1985)

Sanders, M.(1985)

Sanders, M.(1985), SAIC(1994d)

SAIC(1994d)

Geotek Engineering, Inc.(1979), SAIC(1993h)

Butz, T. R.(1981), SAIC(1993h)

Geotek Engineering, Inc.(1979), SAIC(1993h)

Geotek Engineering, Inc.(1979), SAIC(1993h)

Geotek Engineering, Inc.(1979), SAIC(1993h)

Law Engineering, Inc.(1983)

Law Engineering, Inc.(1983)

Law Engineering, Inc.(1983)

Law Engineering, Inc.(1983)

Law Engineering, Inc.(1983)

Law Engineering, Inc.(1983)

Law Engineering, Inc.(1983)

Law Engineering, Inc.(1983)

Law Engineering, Inc.(1983)

\section{COMMENTS}

Forms cluster with GW-094.

Data base revised on $4 / 28 / 97$ to reflect changes discovered during periodic review.

Location coordinates scaled from map. Data base revised on 4/28/97 to reflect changes discovered during periodic review.

NXWL core hole to $50 \mathrm{ft}$. Borehole reamed and casing set. Hole deepened to TD. Borehole converted to Open-interval well. Data base revised on 3/27/97 to reflect changes discovered during periodic review.

Data base revised on $3 / 27 / 97$ to reflect changes discovered during periodic review.

Data base revised on $3 / 27 / 97$ to reflect changes discovered during periodic review.

Data base revised on $3 / 27 / 97$ to reflect changes discovered during periodic review.

Data base revised on 4/18/97 to reflect changes discovered during periodic review.

Data base revised on $4 / 18 / 97$ to reflect changes discovered during periodic review.

Data base revised on $4 / 18 / 97$ to reflect changes discovered during periodic review.

Data base revised on $4 / 18 / 97$ to reflect changes discovered during periodic review.

Data base revised on $4 / 18 / 97$ to reflect changes discovered during periodic review.

Data base revised on $4 / 18 / 97$ to reflect changes discovered during periodic review.

Data base revised on 4/21/97 to reflect changes discovered during periodic review.

Data base revised on $4 / 21 / 97$ to reflect changes discovered during periodic review.

Data base revised on $4 / 21 / 97$ to reflect changes discovered during periodic review. 


\section{CURRENT}

\section{NAME}

1070

1071

1072

1073

1080

1081

1082

1083

1084

1085

1086

1087

1088

1090

1091

1092

1095

1096

1097

1100

110

1102

\section{REFERENCE}

Unknown

Geotek Engineering, Inc.(1979), SAIC(1993h)

Geotek Engineering, Inc.(1979)

Butz, T. R.(1981)

Butz, T. R.(1981)

Farmer, C. D.(1984), SAIC(1994d)

Farmer, C. D.(1984)

Farmer, C. D.(1984), SAIC(1994d)

Farmer, C. D.(1984)

Butz, T. R.(1981), Martin Marietta Energy

Systems(1985), SAIC(1992b)

Butz, T. R.(1981), Martin Marietta Energy

Systems(1985), SAIC(1992b)

Butz, T. R.(1981), Martin Marietta Energy

Systems(1985), SAIC(1991b)

Sanders, M.(1985), SAIC(1993h)

Geotek Engineering, Inc.(1982b)

Geotek Engineering, Inc.(1982b), SAIC(1992b)

Geotek Engineering, Inc.(1982b)

Geotek Engineering, Inc.(1982a), SAIC(1997c)

Geotek Engineering, Inc.(1982a), SAIC(1997c)

Brunton, G. A.(1978), SAIC(1994d)

Geotek Engineering, Inc.(1986), King, H.L., Haase, C.S., and LaRue, D.L.(1989), SAIC(1995b)

Geotek Engineering, Inc.(1986), King, H.L., Haase C.S., and LaRue, D.L.(1989), SAIC(1995b)

Geotek Engineering, Inc.(1986), King, H.L., Haase, C.S., and LaRue, D.L.(1989), SAIC(1995b)

\section{COMMENTS}

Data base revised on $3 / 27 / 97$ to reflect changes discovered during periodic review.

Data base revised on $3 / 27 / 97$ to reflect changes discovered during periodic review.

Forms cluster with GW-187 and GW-224.

NX core hole. Core hole converted to Open Hole.

OWI well. NX core hole. Core hole converted to Open Hole. ${ }^{* *}$ TOC elevation missing so TOC estimated as GS+3ft, (best GS was for 01/01/1901 ) from meas pt_elevation

Data base revised on $4 / 28 / 97$ to reflect changes discovered during periodic review.

Data base revised on $4 / 28 / 97$ to reflect changes discovered during periodic review.

Forms cluster with 1103 and 1104 . Data base revised on 4/28/97 to reflect changes discovered during periodic review. 


\section{CURRENT}

NAME

1103

1104

1105

1106

1107

1108

1109

1110

1111

1112

1130

1131

1132

1133

1134

2D

21

$2 S$

\section{REFERENCE}

Geotek Engineering, Inc.(1986), King, H.L., Haase, C.S., and LaRue, D.L.(1989), SAIC(1995b)

Geotek Engineering, Inc.(1986), King, H.L., Haase, C.S., and LaRue, D.L.(1989), SAIC(1995b)

Geotek Engineering, Inc.(1986), King, H.L., Haase, C.S., and LaRue, D.L.(1989), SAIC(1995b)

Geotek Engineering, Inc.(1986), King, H.L., Haase, C.S., and LaRue, D.L.(1989), SAIC(1995b)

Geotek Engineering, Inc.(1986), King, H.L., Haase, C.S., and LaRue, D.L.(1989), SAIC(1995b)

Geotek Engineering, Inc.(1986), King, H.L., Haase, C.S., and LaRue, D.L.(1989), SAIC(1995b)

Geotek Engineering, Inc.(1986), King, H.L., Haase, C.S., and LaRue, D.L.(1989), SAIC(1995b)

Geotek Engineering, Inc.(1986), King, H.L., Haase C.S., and LaRue, D.L.(1989), SAIC(1995b)

Geotek Engineering, Inc.(1986), King, H.L., Haase, C.S., and LaRue, D.L.(1989)

Geotek Engineering, Inc.(1986), King, H.L., Haase C.S., and LaRue, D.L.(1989)

Ketelle, R. H.(1992), SAIC(1993h)

Ketelle, R. H.(1992), SAIC(1993h)

Ketelle, R. H.(1992), SAIC(1993h)

Ketelle, R. H.(1992), SAIC(1993h)

Ketelle, R. H.(1992), SAIC(1993h)

SAIC(1992b)

SAIC $(1992 b)$

$\operatorname{SAIC}(1992 b)$

\section{COMMENTS}

Forms cluster with 1102 and 1104 . Data base revised on 4/28/97 to reflect changes discovered during periodic review.

Forms cluster with 1102 and 1103 . Data base revised on 4/28/97 to reflect changes discovered during periodic review.

Forms cluster with 1106 . Data base revised on 4/28/97 to reflect changes discovered during periodic review.

Forms cluster with 1105 . Data base revised on 4/28/97 to reflect changes discovered during periodic review.

Forms cluster with 1108 . Data base revised on 4/28/97 to reflect changes discovered during periodic review.

Forms cluster with 1107. Data base revised on 4/28/97 to reflect changes discovered during periodic review.

Data base revised on $4 / 28 / 97$ to rflect changes discovered during periodic review.

Data base revised on $4 / 28 / 97$ to reflect changes discovered during periodic review.

Several attempts have been made to locate well 1111, all unsuccessful. Data base revised on $4 / 28 / 97$ to reflect changes discovered during periodic review.

Several attempts have been made to locate well 1112, all unsuccessful.

Data base revised on 4/28/97 to reflect changes discovered during periodic review.

Data base revised on $3 / 26 / 97$ to reflect changes discovered during periodic review.

Data base revised on $3 / 26 / 97$ to reflect changes discovered during periodic review.

Data base revised on 3/26/97 to reflect changes disacovered during periodic review.

Data base revised on $3 / 26 / 97$ to reflect changes discovered during periodic review.

Data base revised on $3 / 26 / 97$ to reflect changes discovered during periodic review.

Piezometer is the deep component of Nest 2.

Piezometer is the intermediate depth component of Nest 2.

Piezometer is the shallow component of NEST 2. 


\section{CURRENT}

\section{NAME}

38-DC

39-DC

$3 \mathrm{D}$

31

35

40-DC

$41-\mathrm{DC}$

42-DC

44-DC

$45-\mathrm{DC}$

41

$4 \mathrm{~S}$

53-1A

54-1A

54-2A

55-1A

55-1B

\section{REFERENCE}

Union Carbide Corporation Nuclear Division(1980)

Union Carbide Corporation Nuclear Division(1980)

$\operatorname{SAIC}(1992 b)$

$\operatorname{SAIC}(1992 b)$

$\operatorname{SAIC}(1992 b)$

Union Carbide Corporation Nuclear Division(1980)

Union Carbide Corporation Nuclear Division(1980)

Union Carbide Corporation Nuclear Division(1980), $\operatorname{SAIC}(1994 d)$

Union Carbide Corporation Nuclear Division(1980)

Union Carbide Corporation Nuclear Division(1980)

$\operatorname{SAIC}(1992 b)$

$\operatorname{SAIC}(1992 b)$

SAIC(1992b)

Rothschild, E. R., R. R. Turner, S. H. Stow, M. A. Bogle, L. K. Hyder, O. M. Sealand, and H. J. Wyrick(1984)

Rothschild, E. R., R. R. Turner, S. H. Stow, M. A. Bogle, L. K. Hyder, O. M. Sealand, and H. J. Wyrick(1984), SAIC(1992b)

Rothschild, E. R., R. R. Turner, S. H. Stow, M. A. Bogle, L. K. Hyder, O. M. Sealand, and H. J. Wyrick(1984)

Rothschild, E. R., R. R. Turner, S. H. Stow, M. A. Bogle, L. K. Hyder, O. M. Sealand, and H. J. Wyrick(1984)

Rothschild, E. R., R. R. Turner, S. H. Stow, M. A. Bogle, L. K. Hyder, O. M. Sealand, and H. J. Wyrick(1984)

\section{COMMENTS}

Core hole converted to Open-interval well. Data base revised on 4/21/97 to reflect changes discovered during periodic review.

Core hole converted to Open-interval well. Data base revised on 4/21/97 to reflect changes discovered during periodic review.

Piezometer is the deep component of Nest 3.

Piezometer is the intermediate depth component of Nest 3.

Piezometer is the shallow component of Nest 3.

Core hole converted to Open-interval well. Data base revised on 4/21/97 to reflect changes discovered during periodic review.

Core hole converted to Open-interval well. Data base revised on 4/21/97 to reflect changes discovered during periodic review.

Core hole converted to Open-interval well. Data base revised on 4/21/97 to reflect changes discovered during periodic review.

Core hole converted to Open-interval well. Data base revised on 4/21/97 to reflect changes discovered during periodic review.

Core hole converted to Open-interval well. Data base revised on 4/21/97 to reflect changes discovered during periodic review.

Piezometer is the deep component of Nest 4.

Piezometer is the intermediate depth component of Nest 4.

Piezometer is the shallow component of Nest 4.

Data base revised on 4/9/97 to reflect changes discovered during periodic review.

Data base revised on $4 / 9 / 97$ to reflect changes discovered during periodic review.

Data base revised on 4/9/97 to reflect changes discovered during periodic review.

Data base revised on 4/9/97 to reflect changes discovered during periodic review. TOWW elevation changed 06/06/2005 to reflect proper WW configuration.

Well is located near Building 9201-5. Data base revised on 4/9/97 to reflect changes discovered during periodic review. TOWW elevation changed 06/06/2005 to reflect proper WW configuration. 


\section{NAME}

$55-1 \mathrm{C}$

$55-2 \mathrm{~A}$

$55-2 B$

$55-2 \mathrm{C}$

$55-3 \mathrm{~A}$

$55-3 B$

$55-3 C$

$55-4 B$

$55-4 \mathrm{C}$

$55-5 A$

$55-6 \mathrm{~A}$

55-7A

$55-8 \mathrm{~A}$

$55-8 B$

\section{REFERENCE}

Rothschild, E. R., R. R. Turner, S. H. Stow, M. A. Bogle, L. K. Hyder, O. M. Sealand, and H. J. Wyrick(1984)

Rothschild, E. R., R. R. Turner, S. H. Stow, M. A. Bogle, L. K. Hyder, O. M. Sealand, and H. J. Wyrick(1984)

Rothschild, E. R., R. R. Turner, S. H. Stow, M. A. Bogle, L. K. Hyder, O. M. Sealand, and H. J. Wyrick(1984)

Rothschild, E. R., R. R. Turner, S. H. Stow, M. A. Bogle, L. K. Hyder, O. M. Sealand, and H. J. Wyrick(1984)

Rothschild, E. R., R. R. Turner, S. H. Stow, M. A. Bogle, L. K. Hyder, O. M. Sealand, and H. J. Wyrick(1984)

Rothschild, E. R., R. R. Turner, S. H. Stow, M. A. Bogle, L. K. Hyder, O. M. Sealand, and H. J. Wyrick(1984)

Rothschild, E. R., R. R. Turner, S. H. Stow, M. A. Bogle, L. K. Hyder, O. M. Sealand, and H. J. Wyrick(1984)

Rothschild, E. R., R. R. Turner, S. H. Stow, M. A. Bogle, L. K. Hyder, O. M. Sealand, and H. J. Wyrick(1984)

Rothschild, E. R., R. R. Turner, S. H. Stow, M. A. Bogle,

L. K. Hyder, O. M. Sealand, and H. J. Wyrick(1984)

Rothschild, E. R., R. R. Turner, S. H. Stow, M. A. Bogle, L. K. Hyder, O. M. Sealand, and H. J. Wyrick(1984)

Rothschild, E. R., R. R. Turner, S. H. Stow, M. A. Bogle,

L. K. Hyder, O. M. Sealand, and H. J. Wyrick(1984)

Rothschild, E. R., G. A. Gillis, C. D. Farmer, and O. M. Sealand(1984)

Rothschild, E. R., G. A. Gillis, C. D. Farmer, and O. M. Sealand(1984)

Rothschild, E. R., G. A. Gillis, C. D. Farmer, and O. M. Sealand(1984)

\section{COMMENTS}

Data base revised on 4/9/97 to reflect changes discovered during periodic review.

Well is located near Building 9201-5. data base revised on 4/10/97 to reflect changes discovered during periodic review. Measurement point changed to TOWW with no change in elevation due to low clearance cap.

Data base revised on 4/10/97 to reflect changes discovered during periodic review. TOWW value estimated for this well.

NX core hole. Reamed and finished as monitoring well. Well is located near Building 9201-5. Data base revised on 4/10/97 to reflect changes discovered during periodic review. TOWW value estimated for this well.

Data base revised on 4/10/97 to reflect changes discovered during periodic review. Measurement point changed to TOWW with no change in elevation due to low clearance cap.

Data base revised on 4/10/97 to reflect changes discovered during periodic review. Measurement point changed to TOWW with no change in elevation due to low clearance cap.

Well is located near Building 9201-5. Data base revised on 4/10/97 to reflect changes discovered during periodic review. Measurement point changed to TOWW with no change in elevation due to low clearance cap.

Data base revised on 4/10/97 to reflect changes discovered during periodic review. Well is believed to have been romoved, no documentation.

NX core hole. Reamed and finished as monitoring well. Data base revised on $4 / 10 / 97$ to reflect changes discovered during periodic review. Well is believed to have been removed, no documentation.

Data base revised on 4/10/97 to reflect changes discovered during periodic review. Well is believed to have been removed, no documentation.

Data base revised on $4 / 10 / 97$ to reflect changes discovered during periodic review. TOWW elevation changed 06/06/2005 to reflect proper WW configuration

Location covered by coal pile.

Forms cluster with 55-8B. NX core hole reamed and finished as monitoring well. Location covered by coal pile.

Forms cluster with 55-8A. NX core hole reamed and finished as monitoring well. Location covered by coal pile. 


\section{REFERENCE}

Rothschild, E. R., G. A. Gillis, C. D. Farmer, and O. M. Sealand(1984)

$56-1 \mathrm{~A}$

$56-1 C$

$56-2 A$

$56-2 B$

$56-2 C$

$56-3 \mathrm{~B}$

$56-3 C$

$56-4 \mathrm{~A}$

$56-4 B$

$56-4 \mathrm{C}$
Rothschild, E. R., R. R. Turner, S. H. Stow, M. A. Bogle, L. K. Hyder, O. M. Sealand, and H. J. Wyrick(1984)

Rothschild, E. R., R. R. Turner, S. H. Stow, M. A. Bogle, L. K. Hyder, O. M. Sealand, and H. J. Wyrick(1984)

Rothschild, E. R., R. R. Turner, S. H. Stow, M. A. Bogle, L. K. Hyder, O. M. Sealand, and H. J. Wyrick(1984)

Rothschild, E. R., R. R. Turner, S. H. Stow, M. A. Bogle, L. K. Hyder, O. M. Sealand, and H. J. Wyrick(1984)

Rothschild, E. R., R. R. Turner, S. H. Stow, M. A. Bogle, L. K. Hyder, O. M. Sealand, and H. J. Wyrick(1984)

Rothschild, E. R., R. R. Turner, S. H. Stow, M. A. Bogle, L. K. Hyder, O. M. Sealand, and H. J. Wyrick(1984)

Rothschild, E. R., R. R. Turner, S. H. Stow, M. A. Bogle, L. K. Hyder, O. M. Sealand, and H. J. Wyrick(1984)

Rothschild, E. R., R. R. Turner, S. H. Stow, M. A. Bogle, L. K. Hyder, O. M. Sealand, and H. J. Wyrick(1984)

Rothschild, E. R., R. R. Turner, S. H. Stow, M. A. Bogle, L. K. Hyder, O. M. Sealand, and H. J. Wyrick(1984)

Rothschild, E. R., R. R. Turner, S. H. Stow, M. A. Bogle, L. K. Hyder, O. M. Sealand, and H. J. Wyrick(1984)

Rothschild, E. R., R. R. Turner, S. H. Stow, M. A. Bogle, L. K. Hyder, O. M. Sealand, and H. J. Wyrick(1984), SAIC $(1997 c)$

\section{COMMENTS}

Location covered by coal pile.

Data base revised on 4/10/97 to reflect changes discovered during periodic review. Measurement point changed to TOWW with no change in elevation due to low clearance cap.

NX core hole. Reamed and finished as monitoring well. Data base revised on 4/10/97 to reflect changes discovered during periodic review.

Data base revised on 4/10/97 to reflect changes discovered during periodic review. TOWW elevation changed 06/06/2005 to reflect proper WW configuration.

Data base revised on 4/10/97 to reflect changes discovered during periodic review. TOWW elevation changed 06/06/2005 to reflect proper WW configuration.

NX core hole. Reamed and finished as monitoring well. Well is located near Building 9201-4. Data base revised on 4/10/97 to reflect changes discovered during periodic review.

Data base revised on 4/10/97 to reflect changes discovered during periodic review. Measurement point changed to TOWW with no change in elevation due to low clearance cap.

Data base revised on 4/10/97 to reflect changes discovered during periodic review. Well re-survey 12/22/2004 due to well casing repair.

Measurement point changed to TOWW with no change in elevation due to low clearance cap.

Well is located near Building 9201-4. Data base revised on 4/10/97 to reflect changes discovered during periodic review. Measurement point changed to TOWW with no change in elevation due to low clearance cap.

Data base revised on 4/10/97 to reflect changes discovered during periodic review. Measurement point changed to TOWW with no change in elevation due to low clearance cap.

Data base revised on 4/10/97 to reflect changes discovered during periodic review. Well is beleived to have been removed, no documentation.

NX core hole. Reamed and finished as monitoring well. Well located near Building 9201-4. Data base revised on 4/10/97 to reflect changes discovered during periodic review. 


\section{REFERENCE}

Rothschild, E. R., R. R. Turner, S. H. Stow, M. A. Bogle L. K. Hyder, O. M. Sealand, and H. J. Wyrick(1984)

$56-5 B$

$56-5 \mathrm{C}$

$56-6 \mathrm{~A}$

$56-8 \mathrm{~A}$

$56-9 A$

$58-1 \mathrm{~A}$

$58-2 \mathrm{~A}$

$59-1 \mathrm{~A}$

$59-1 B$
Rothschild, E. R., R. R. Turner, S. H. Stow, M. A. Bogle, L. K. Hyder, O. M. Sealand, and H. J. Wyrick(1984)

Rothschild, E. R., R. R. Turner, S. H. Stow, M. A. Bogle, L. K. Hyder, O. M. Sealand, and H. J. Wyrick(1984)

Rothschild, E. R., R. R. Turner, S. H. Stow, M. A. Bogle, L. K. Hyder, O. M. Sealand, and H. J. Wyrick(1984)

Rothschild, E. R., R. R. Turner, S. H. Stow, M. A. Bogle, L. K. Hyder, O. M. Sealand, and H. J. Wyrick(1984)

Rothschild, E. R., R. R. Turner, S. H. Stow, M. A. Bogle, L. K. Hyder, O. M. Sealand, and H. J. Wyrick(1984)

Rothschild, E. R., R. R Turner, S. H. Stow, M. A. Bogle, L. K. Hyder, O. M. Sealand, and H. J. Wyrick(1984), E. R. Schultz(2004)

Rothschild, E. R., R. R. Turner, S. H. Stow, M. A. Bogle, L. K. Hyder, O. M. Sealand, and H. J. Wyrick(1984)

Rothschild, E. R., R. R. Turner, S. H. Stow, M. A. Bogle, L. K. Hyder, O. M. Sealand, and H. J. Wyrick(1984)

Rothschild, E. R., R. R. Turner, S. H. Stow, M. A. Bogle, L. K. Hyder, O. M. Sealand, and H. J. Wyrick(1984)

Rothschild, E. R., R. R. Turner, S. H. Stow, M. A. Bogle, L. K. Hyder, O. M. Sealand, and H. J. Wyrick(1984)

\section{COMMENTS}

Data base revised on 4/10/97 to reflect changes discovered during periodic review. An electric logging probe was probably lost in this corehole or a nearby borehole during geophysical logging activities in the 1984-1985 timeframe.

Data base revised on $4 / 10 / 97$ to reflect changes discovered during periodic review. An electric logging probe was probably lost in this corehole or a nearby borehole during geophysical logging activities in the 1984-1985 timeframe.

NX core hole. Reamed and finished as monitoring well. Well is located near Building 81-10. Data base revised on 4/10/97 to reflect changes discovered during periodic review. An electric logging probe was probably lost in this corehole or a nearby borehole during geophysical logging activities in the 1984-1985 timeframe.

Data base revised on 4/10/97 to reflect changes discovered during periodic review. Measurement point changed to TOWW with no change in elevation due to low clearance cap.

ata base revised on $4 / 10 / 97$ to reflect changes discovered during periodic review.

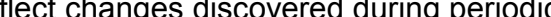
review. Measurement point changed to TOWW with no change in elevation due to low clearance cap.

Data base revised on 4/10/97 to reflect changes discovered during periodic review.

Well installed in boring 58-1/BN. Well is located near Building 9733-1.

Data base revised on $4 / 10 / 97$ to reflect changes discovered during periodic review.

Data base revised on 4/10/97 to reflect changes discovered during periodic review.

Data base revised on 4/10/97 to reflect changes discovered during periodic review. TOWW elevation of $.20 \mathrm{ft}$. added 6/16/2003.

base revised on 4/10/97 to reflect changes discovered during periodic review. TOWW elevation of $.25 \mathrm{ft}$. added 6/16/2003. 


\section{CURRENT}

NAME

59-1C

$5 \mathrm{D}$

51

$5 S$

60-1A

$60-1 \mathrm{~B}$

$60-1 C$

$60-2 A$

AN:D-1

AN:D-10

AN:D-11

AN:D-2

AN:D-3

$A N: D-4$

AN:D-5

AN:D-6

$A N: D-7$

AN:D-8

AN:D-9

AN:D-UNKNOWN

B-1

\section{REFERENCE}

Rothschild, E. R., R. R. Turner, S. H. Stow, M. A. Bogle, L. K. Hyder, O. M. Sealand, and H. J. Wyrick(1984)

SAIC $(1992 b)$

$\operatorname{SAIC}(1992 b)$

$\operatorname{SAIC}(1992 b)$

Rothschild, E. R., R. R. Turner, S. H. Stow, M. A. Bogle,

L. K. Hyder, O. M. Sealand, and H. J. Wyrick(1984)

Rothschild, E. R., R. R. Turner, S. H. Stow, M. A. Bogle, L. K. Hyder, O. M. Sealand, and H. J. Wyrick(1984)

Rothschild, E. R., R. R. Turner, S. H. Stow, M. A. Bogle,

L. K. Hyder, O. M. Sealand, and H. J. Wyrick(1984)

Rothschild, E. R., R. R. Turner, S. H. Stow, M. A. Bogle, L. K. Hyder, O. M. Sealand, and H. J. Wyrick(1984), William Burress and Dave Painter(2004)

$\operatorname{SAIC}(1994 a)$

SAIC(1994a)

$\operatorname{SAIC}(1994 a)$

SAIC(1994a)

SAIC(1994a)

$\operatorname{SAIC}(1994 a)$

$\operatorname{SAIC}(1994 a)$

SAIC(1994a)

$\operatorname{SAIC}(1994 a)$

SAIC(1994a)

SAIC(1994a)

Unknown

Environmental Consulting Engineers, Inc.(1993)

Ogden Environmental Services(1993), SAIC(1997c)

\section{COMMENTS}

NX core hole. Reamed and finished as a monitoring well. Well is located near Building 9202. Data base revised on 4/10/97 to reflect changes discovered during periodic review. TOWW value of $.25 \mathrm{ft}$. added 06/16/2003.

Piezometer is the deep component of Nest 5.

Piezometer is the intermediate depth component of Nest 5.

Piezometer is the shallow component of Nest 5.

Well is located near Building 9201-2. Data base revised on 4/10/97 to reflect changes discovered during periodic review. Measurement point changed to TOWW with no change in elevation due to low clearance cap.

Data base revised on 4/10/97 to reflect changes discovered during periodic review. TOWW value of $.20 \mathrm{ft}$. added 06/16/2003.

NX core hole. Borehole collapsed before casing installed. Data base revised on 4/10/97 to reflect changes discovered during periodic review. Status changed to P\&A 02/25/2003 review. Location re-surveyed 10/18/2002.

Location coordinates are estimated.

Location coordinates are estimated.

Location coordinates are estimated.

Location coordinates are estimated.

Location coordinates are estimated.

Location coordinates are estimated.

Location coordinates are estimated.

Location coordinates are estimated.

Location coordinates are estimated.

Location coordinates are estimated.

Location coordinates are estimated

Location coordinates are estimated. 


\section{CURRENT}

\section{NAME}

B-3

BC-01

$\mathrm{BC}-03$

BC-06

BC-07

BC-08

BC-09

BC-10

$\mathrm{BC}-11$

BC-14

$\mathrm{BC}-15$

BC-16

BC-17

\section{REFERENCE}

Environmental Consulting Engineers, Inc.(1993), Ogden Environmental Services(1993), SAIC(1997c)

Law Engineering, Inc.(1975), SAIC(1994d)

Law Engineering, Inc.(1975), SAIC(1995b)

Law Engineering, Inc.(1975), SAIC(1994d)

Law Engineering, Inc.(1975), SAIC(1994d)

Law Engineering, Inc.(1975)

Law Engineering, Inc.(1975), SAIC(1994d)

Law Engineering, Inc.(1975), SAIC(1994d)

Law Engineering, Inc.(1975)

Law Engineering, Inc.(1975), SAIC(1994d)

Law Engineering, Inc.(1975), SAIC(1994d)

Law Engineering, Inc.(1975)

Law Engineering, Inc.(1975), SAIC(1994d)

\section{COMMENTS}

NX core hole. Core @ Bldg 7042/ORNL. Core hole converted to hole class of Unknown. Data base revised on 4/22/97 to reflect changes discovered during periodic review. Loc "TORCed" to Y-12 grid 8/21/2000

NX core hole. Core @ BIdg 7042/ORNL. Re-survey 06/09/1994. Core hole converted to hole class of Unknown. Data base revised on 4/23/97 to reflect changes discovered during periodic review.

NX core hole. Core @ Bldg 7042/ORNL. Core hole converted to hole class of Unknown. Data base revised on 4/22/97 to reflect changes discovered during periodic review.

NX core hole. Core @ BIdg 7042/ORNL. Core hole converted to hole class of Unknown. Data base revised on 4/22/97 to reflect changes discovered during periodic review.

NX core hole. Core @ Bldg 7042/ORNL. No casing found for P\&A. Core hole converted to hole class of Unknown. Location Coord. TORC'ed $08 / 31 / 2000$

NX core hole. Core @ Bldg 7042/ORNL. Re-survey 06/09/1994. Core hole converted to hole class of Unknown. Data base revised on 4/22/97 to reflect changes discovered during periodic review.

NX core hole. Core @ Bldg 7042/ORNL. Core hole converted to hole class of Unknown. Data base revised on 4/22/97 to rflect changes discovered during periodic review.

NX core hole. Core @ BIdg 7042/ORNL. Core hole converted to hole class of Unknown.

NX core hole. Core @ Bldg 7042/ORNL. Core hole converted to hole class of Unknown. Data base revised on 4/22/97 to reflect changes discovered during periodic review.

NX core hole. Core @ Bldg 7042/ORNL. Core hole converted to hole class of Unknown. Data base revised on 4/22/97 to reflect changes discovered during periodic review.

NX core hole. Core @ Bldg 7042/ORNL. Core hole converted to hole class of Unknown. Location Coord. TORC'ed 08/31/2000.

NX core hole. Core @ Bldg 7042/ORNL. Core hole converted to hole class of Unknown. Data base revised on 4/22/97 to reflect changes discovered during periodic review. 


\section{CURRENT}

\section{NAME}

BC-18

BC-19

BC-21

BC-22

BC-25

BC-30

BC-31

BC-32

$\mathrm{BC}-33$

BC-34

BC-35

\section{REFERENCE}

Law Engineering, Inc.(1975), SAIC(1994d)

Law Engineering, Inc.(1975), SAIC(1994d)

Law Engineering, Inc.(1975)

Law Engineering, Inc.(1975), SAIC(1994d)

Law Engineering, Inc.(1975), SAIC(1994d)

Law Engineering, Inc.(1975), SAIC(1995b)

Law Engineering, Inc.(1975), SAIC(1995b)

Law Engineering, Inc.(1975), SAIC(1995b)

Law Engineering, Inc.(1975), SAIC(1995b)

Law Engineering, Inc.(1975), SAIC(1995b)

Law Engineering, Inc.(1975), SAIC(1995b)

Law Engineering, Inc.(1975), SAIC(1994d)

\section{COMMENTS}

NX core hole. Core @ Bldg 7042/ORNL. Core hole converted to hole class of Unknown. Data base revised on 4/22/97 to reflect changes discovered during periodic review.

NX core hole. Core @ Bldg 7042/ORNL. Core hole converted to hole class of Unknown. Data base revised on 4/22/97 to reflect changes discovered during periodic review.

NX core hole. Core @ Bldg 7042/ORNL. Core hole converted to hole class of Unknown. Data base revised on 4/22/97 to reflect changes discovered during periodic review. Location Coord. TORC'ed 08/31/2000. NX core hole. Core @ Bldg 7042/ORNL. Core hole converted to hole class of Unknown. Data base revised on 4/22/97 to reflect changes discovered during periodic review.

NX core hole. Core @ Bldg 7042/ORNL. Core hole converted to hole class of Unknown. Data base revised on 4/22/97 to reflect changes discovered during periodic review.

NX core hole. Core @ Bldg 7042/ORNL. Core hole converted to hole class of Unknown. Data base revised on 4/23/97 to reflect changes discovered during periodic review. Location Coord. TORC'ed 08/31/2000.

NX core hole. Core @ Bldg 7042/ORNL. Re-survey 06/09/1994. Core hole converted to hole class of Unknown. Data base revised on 4/23/97 to reflect changes discovered during periodic review.

NX core hole. Core @ Bldg 7042/ORNL. Core hole converted to hole class of Unknown. Data base revised on 4/23/97 to reflect changes discovered during periodic review.

NX core hole. Core @ Bldg 7042/ORNL. Core hole converted to hole class of Unknown. Data base revised on 4/23/97 to reflect changes discovered during periodic review.

NX core hole. Core @ Bldg 7042/ORNL. Core hole converted to hole class of Unknown. Data base revised on 4/23/97 to reflect changes discovered during periodic review.

NX core hole. Core @ Bldg 7042/ORNL. Core hole converted to hole class of Unknown. Data base revised on 4/23/97 to reflect changes discovered during periodic review.

NX core hole. Core @ Bldg 7042/ORNL. Core hole converted to hole class of Unknown. Data base revised on 4/22/97 to reflect changes discovered during periodic review. 


\section{CURRENT}

NAME

BC-36

BC-37

BC-38

BC-39

BC-40

BC-41

BC-42

BC-43

BC-45

BC-47

BC-49

BC-50

BC-51

BC-52

\section{REFERENCE}

Law Engineering, Inc.(1975), SAIC(1995b)

Law Engineering, Inc.(1975)

Law Engineering, Inc.(1975)

Law Engineering, Inc.(1975), SAIC(1995b)

Law Engineering, Inc.(1975), SAIC(1995b)

Law Engineering, Inc.(1975), SAIC(1995b)

Law Engineering, Inc.(1975), SAIC(1995b)

Law Engineering, Inc.(1975)

Law Engineering, Inc.(1975)

Law Engineering, Inc.(1975), SAIC(1994d)

Law Engineering, Inc.(1975)

Law Engineering, Inc.(1975), SAIC(1995b)

Law Engineering, Inc.(1975)

Law Engineering, Inc.(1975)

\section{COMMENTS}

NX core hole. Core @ Bldg 7042/ORNL. Core hole converted to hole class of Unknown. Data base revised on 4/23/97 to reflect changes discovered during periodic review.

NX core hole. Core @ Bldg 7042/ORNL. Core hole converted to hole class of Unknown. Location Coord. TORC'ed 08/31/2000.

NX core hole. Core @ Bldg 7042/ORNL. Core hole converted to hole class of Unknown. Location Coord. TORC'ed 08/31/2000.

NX core hole. Core @ Bldg 7042/ORNL. Core hole converted to hole class of Unknown. Data base revised on 4/23/97 to reflect changes discovered during periodic review.

NX core hole. Core @ Bldg 7042/ORNL. Core hole converted to hole class of Unknown. Data base revised on 4/23/97 to reflect changes discovered during periodic review.

NX core hole. Core @ Bldg 7042/ORNL. Core hole converted to hole class of Unknown. Data base revised on 4/23/97 to reflect changes discovered during periodic review.

NX core hole. Core @ Bldg 7042/ORNL. Core hole converted to hole class of Unknown. Data base revised on 4/23/97 to reflect changes discovered during periodic review.

NX core hole. Core @ Bldg 7042/ORNL. Core hole converted to hole class of Unknown. Location Coord. TORC'ed 08/31/2000.

NX core hole. Core @ BIdg 7042/ORNL. Core hole converted to hole class of Unknown. Location Coord. TORC'ed 08/31/2000.

NX core hole. Core @ Bldg 7042/ORNL. Core hole converted to hole class of Unknown. Data base revised on 4/22/97 to reflect changes discovered during periodic review.

NX core hole. Core @ BIdg 7042/ORNL. Core hole converted to hole class of Unknown. Location Coord. TORC'ed 08/31/2000.

NX core hole. Core @ Bldg 7042/ORNL. Core hole converted to hole class of Unknown. Data base revised on 4/23/97 to reflect changes discovered during periodic review. Location Coord. TORC'ed 08/31/2000.

NX core hole. Core @ Bldg 7042/ORNL. Core hole converted to hole class of Unknown. Location Coord. TORC'ed 08/31/2000.

NX core hole. Core @ Bldg 7042/ORNL. Core hole converted to hole class of Unknown. Location Coord. TORC'ed 08/31/2000. 


\begin{tabular}{|c|c|}
\hline CURREN & \\
\hline NAME & REFERENCE \\
\hline BC-53 & Law Engineering, Inc.(1975) \\
\hline BC-54 & Law Engineering, Inc.(1975) \\
\hline BC-57 & Law Engineering, Inc.(1975) \\
\hline BC-59 & Law Engineering, Inc.(1975) \\
\hline BC-61 & Law Engineering, Inc.(1975) \\
\hline BC-62 & Law Engineering, Inc.(1975), SAIC(1995b) \\
\hline BC-63 & Law Engineering, Inc.(1975), SAIC(1994d) \\
\hline BC-64 & Law Engineering, Inc.(1975) \\
\hline BCU-01 & SAIC(1994d) \\
\hline BCU-02 & SAIC(1994d) \\
\hline BCU-03 & SAIC(1994d) \\
\hline BCU-04 & SAIC(1994d) \\
\hline BCU-05 & SAIC(1994d) \\
\hline BCU-06 & SAIC(1994d) \\
\hline BCU-07 & SAIC(1994d) \\
\hline BCU-08 & SAIC(1994d) \\
\hline
\end{tabular}

\section{COMMENTS}

NX core hole. Core @ Bldg 7042/ORNL. Core hole converted to hole class of Unknown. Location Coord. TORC'ed 08/31/2000.

NX core hole. Core @ Bldg 7042/ORNL. Core hole converted to hole class of Unknown. Location Coord. TORC'ed 08/31/2000.

NX core hole. Core @ Bldg 7042/ORNL. Core hole converted to hole class of Unknown. Location Coord. TORC'ed 08/31/2000.

NX core hole. Core @ Bldg 7042/ORNL. Core hole converted to hole class of Unknown. Location Coord. TORC'ed 08/31/2000.

NX core hole. Core @ Bldg 7042/ORNL. Core hole converted to hole class of Unknown. Location Coord. TORC'ed 08/31/2000.

NX core hole. Core @ Bldg 7042/ORNL. Core hole converted to hole class of Unknown. Data base revised on 4/24/97 to reflect changes discovered during periodic review.

NX core hole. Core @ Bldg 7042/ORNL. Core hole converted to hole class of Unknown. Data base revised on 4/22/97 to reflect changes discovered during periodic review.

NX core hole. Core @ Bldg 7042/ORNL. Core hole converted to hole class of Unknown. Location Coord. TORC'ed 08/31/2000.

Data base revised on $4 / 24 / 97$ to reflect changes discovered during periodic review.

Data base revised on $4 / 24 / 97$ to reflect changes discovered during periodic review.

Data base revised on $4 / 24 / 97$ to reflect changes discovered during periodic review.

Data base revised on $4 / 24 / 97$ to reflect changes discovered during periodic review.

Data base revised on $4 / 24 / 97$ to reflect changes discovered during periodic review.

Data base revised on $4 / 24 / 97$ to reflect changes discovered during periodic review.

Data base revised on $4 / 24 / 97$ to reflect changes discovered during periodic review.

Data base revised on $4 / 25 / 97$ to reflect changes discovered during periodic review. 


\begin{tabular}{ll}
$\begin{array}{c}\text { CURRENT } \\
\text { NAME }\end{array}$ & REFERENCE \\
BCU-09 & SAIC(1994d) \\
BCU-10 & SAIC(1994d) \\
BCU-11 & SAIC(1994d) \\
BCU-12 & SAIC(1995b) \\
BCU-13 & SAIC(1994d) \\
BCU-14 & SAIC(1995b) \\
BCU-15 & SAIC(1995b) \\
BCU-16 & SAIC(1994d) \\
BCU-17 & SAIC(1995b) \\
BCU-18 & SAIC(1995b) \\
BCU-19 & SAIC(1995b) \\
BCU-20 & SAIC(1994d) \\
BCU-21 & SAIC(1995b) \\
BCU-22 & SAIC(1995b) \\
BCU-23 & SAIC(1995b) \\
BCU-24 & SAIC(1995b) \\
BCU-25 & SAIC(1994d), SAIC(1995b) \\
\hline
\end{tabular}

\section{COMMENTS}

Re-survey 06/09/1994. Data base revised on 4/25/97 to reflect changes discovered during periodic review.

Data base revised on $4 / 25 / 97$ to reflect changes discovered during periodic review.

Data base revised on $4 / 25 / 97$ to reflect changes discovered during periodic review.

Data base revised on $4 / 25 / 97$ to reflect changes discovered during periodic review.

Data base revised on $4 / 25 / 97$ to reflect changes discovered during periodic review.

Data base revised on $4 / 25 / 97$ to reflect changes discovered during periodic review.

Data base revised on $4 / 25 / 97$ to reflect changes discovered during periodic review.

Grout from 146.3 to $47.6 \mathrm{ft}$, hole collapsed from $47.6-45.0 \mathrm{ft}$, grout from 45.0-1.0 ft. Data base revised on 4/25/97 to reflect changes discovered during periodic review.

Data base revised on $4 / 25 / 97$ to reflect changes discovered during periodic review.

Data base revised on $4 / 25 / 97$ to reflect changes discovered during periodic review.

Data base revised on $4 / 25 / 97$ to reflect changes discovered during periodic review.

Data base revised on $4 / 25 / 97$ to reflect changes discovered during periodic review.

Data base revised on $4 / 25 / 97$ to reflect changes discovered during periodic review.

Data base revised on $4 / 25 / 97$ to reflect changes discovered during periodic review.

Data base revised on $4 / 25 / 97$ to reflect changes discovered during periodic review.

Data base revised on $4 / 25 / 97$ to reflect changes discovered during periodic review.

Data base revised on $4 / 25 / 97$ to reflect changes discovered during periodic review. 


$\begin{array}{ll}\begin{array}{c}\text { CURRENT } \\ \text { NAME }\end{array} & \\ \text { BCU-26 } & \text { REFERENCE } \\ \text { BCU-27 } & \text { SAIC(1995b) } \\ \text { BCU-28 } & \text { SAIC(1995b) } \\ \text { BCU-29 } & \text { SAIC(1995b) } \\ \text { BY-10 } & \text { SAIC(1995b) } \\ \text { BY-11 } & \\ \text { BY-12 } & \text { SAIC(1998b) } \\ \text { BY-13 } & \text { SAIC(1998b) } \\ \text { BY-14 } & \text { SAIC(1998b) } \\ \text { BY-15 } & \text { SAIC(1998b) } \\ \text { BY-16 } & \text { SAIC(1998b) } \\ \text { BY-17 } & \text { SAIC(1998b) } \\ \text { BY-18 } & \text { SAIC(1998b) } \\ \text { BY-19 } & \text { SAIC(1998b) } \\ \text { BY-20 } & \text { SAIC(1998b) } \\ \text { BY-21 } & \text { SAIC(1998b) } \\ \text { BY-22 } & \text { SAIC(1998b) } \\ \text { BY-23 } & \text { SAIC(1998b) } \\ \text { BY-24 } & \text { SAIC(1998b) } \\ \text { BY-25 } & \text { SAIC(1998b) } \\ \text { BY-26 } & \text { SAIC(1998b) } \\ \text { BY-27 } & \text { SAIC(1998b) } \\ \text { BY-28 } & \text { SAIC(1998b) } \\ \text { BY-29 } & \text { SAIC(1998b) } \\ \text { BY-30 } & \text { SAIC(1998b) } \\ \text { BY-31 } & \text { SAIC(1998b) } \\ \text { BY-32 } & \text { SAIC(1998b) } \\ & \text { SAIC(1998b) } \\ & \text { SAIC(1998b) }\end{array}$

\section{COMMENTS}

Re-survey 06/09/1994. Data base revised on 4/25/97 to reflect changes discovered during periodic review.

Data base revised on $4 / 25 / 97$ to reflect changes discovered during periodic review.

Data base revised on $4 / 25 / 97$ to reflect changes discovered during periodic review.

Data base revised on $4 / 25 / 97$ to reflect changes discovered during periodic review. 


\section{CURRENT}

NAME

BY-33

BY-34

BY-35

BY-36

BY-37

BY-38

BY-39

BY-40

BY-41

BY-43

BY-44

BY-45

BY-46

BY-47

BY-48

BY-49

BY-50

C-1

C-19

C-21

C-25

C- 40

C- 45

C-48

C-50

C-51

CH-001

CH-002

\section{REFERENCE}

SAIC(1998b)

SAIC(1998b)

SAIC(1998b)

SAIC(1998b)

SAIC(1998b)

SAIC(1998b)

SAIC(1998b)

SAIC(1998b)

SAIC(1998b)

SAIC(1998b)

SAIC(1998b)

SAIC(1998b)

SAIC(1998b)

SAIC(1998b)

SAIC(1998b)

SAIC(1998b)

SAIC(1998b)

Union Carbide Corporation Nuclear Division(1965)

Union Carbide Corporation Nuclear Division(1965)

\section{COMMENTS}

NX core hole. History of hole unknown. Core hole converted to hole class of Unknown.

NX core hole. History of hole unknown. Core hole converted to hole class of Unknown. 


\section{CURRENT}

\section{NAME}

$\mathrm{CH}-143$

$\mathrm{CH}-157$

$\mathrm{CH}-185$

$\mathrm{CH}-189$

DC WELL

ED/Y-12 No. 2

EMW-VWUNDRDR

FP-001

FP-002
Stone and Webster, Inc.(1945)

\section{REFERENCE}

Haase, C. S., G. A. Gillis, and H. L. King(1987)

Haase, C. S., G. A. Gillis, and H. L. King(1987)

Haase, C. S., G. A. Gillis, and H. L. King(1987)

Haase, C. S., G. A. Gillis, and H. L. King(1987)

SAIC(1995b)

SAIC(1995b)

\section{Unknown}

Unknown

Stone and Webster, Inc.(1945)

\section{COMMENTS}

NC core hole. Core @ Bldg 7042/ORNL. Core hole converted to Openinterval well. Data base revised on $4 / 8 / 97$ to reflect changes discovered during periodic review.

NC core hole. Core @ BIdg 7042/ORNL. Core hole converted to Openinterval well. Data base revised on 4/8/97 to reflect changes discovered during periodic review.

NC core hole. Core @ Bldg 7042/ORNL. Core hole converted to Openinterval well. Data base revised on 4/8/97 to reflect changes discovered during periodic review.

NC core hole. Core @ BIdg 7042/ORNL. Core hole converted to Openinterval well. Data base revised on 4/8/97 to reflect changes discovered during periodic review.

Corehole (?) installed by USGS; northern most hole in a N-S transect, age of hole is indeterminant. Data base revised on $5 / 5 / 97$ to reflect changes discovered during periodic review.

Corehole (?) installed by USGS; one of a N-S transect, age of hole is indeterminant. Data base revised on $5 / 5 / 97$ to reflect changes discovered during periodic review.

Location Geog. Info. updated to reflect better location information. Survey 06/19/2002.

NX core hole. SW corner of Bldg 9201-3. Core @ Bldg 7042/ORNL. Core hole converted to hole class of Unknown.

I had a conversation with Steve Kucera, BJC, regarding this location and he indicated it is essentially a horizontal well beneath the landfill used to relieve hydrostatic pressure (i.e. lower the water table away from the bottom of the landfill liners). He indicated that when they built the site they cleaned out NT-4 and backfilled it with native material (sand, gravel, silt, and I assume some so

NX core hole drilled in 1945. Core hole converted to hole class of Unknown. Data base revised on 4/21/97 to reflect changes discovered during periodic review.

NX core hole drilled in 1945. Core hole converted to hole class of Unknown. Data base revised on 4/21/97 to reflect changes discovered during periodic review. 


\section{CURRENT}

\section{NAME}

FP-003

FP-004

FP-005

FP-006

FP-007

FP-008A

FP-008B

FP-009

FP-010

FP-011

FT-001

FT-002

FT-003

FW001

FW002

\section{REFERENCE}

Stone and Webster, Inc.(1945)

Stone and Webster, Inc.(1945)

Stone and Webster, Inc.(1945)

Stone and Webster, Inc.(1945)

Stone and Webster, Inc.(1945)

Stone and Webster, Inc.(1945)

Stone and Webster, Inc.(1945)

Stone and Webster, Inc.(1945)

Stone and Webster, Inc.(1945)

Stone and Webster, Inc.(1945)

Union Carbide Corporation Nuclear Division(1971)

Union Carbide Corporation Nuclear Division(1971)

Union Carbide Corporation Nuclear Division(1971)

Fairlie, T.(2001)

Fairlie, T.(2001)

\section{COMMENTS}

NX core hole drilled in 1945. Core hole converted to hole class of Unknown. Data base revised on 4/21/97 to reflect changes discovered during periodic review.

NX core hole drilled in 1945. Core hole converted to hole class of Unknown. Data base revised on 4/21/97 to reflect changes discovered during periodic review.

NX core hole drilled in 1945. Core hole converted to hole class of Unknown. Data base revised on 4/21/97 to reflect changes discovered during periodic review.

NX core hole drilled in 1945. Core hole converted to hole class of Unknown. Data base revised on 4/21/97 to reflect changes discovered during periodic review.

NX core hole drilled in 1945. Core hole converted to hole class of Unknown. Data base revised on 4/21/97 to reflect changes discovered during periodic review.

NX core hole drilled in 1945. Core hole converted to hole class of Unknown. Data base revised on 4/21/97 to reflect changes discovered during periodic review.

NX core hole drilled in 1945. Core hole converted to hole class of Unknown. Data base revised on 4/21/97 to reflect changes discovered during periodic review.

NX core hole drilled in 1945. Core hole converted to hole class of Unknown. Data base revised on 4/21/97 to reflect changes discovered during periodic review.

NX core hole drilled in 1945. Core hole converted to hole class of Unknown. Data base revised on 4/21/97 to reflect changes discovered during periodic review.

NX core hole drilled in 1945. Core hole converted to hole class of Unknown. Data base revised on 4/21/97 to reflect changes discovered during periodic review.

NABIR FRC Phase 1 well, Drive Point, Area 2

NABIR FRC Phase 1 well, drive point, Area 2 


\section{CURRENT}

\section{NAME}

FW003

FW004

FW005

GW-001

GW-002

GW-003

GW-004

GW-005

GW-006

GW-007

GW-008

GW-009

GW-010

GW-011

GW-012

\section{REFERENCE}

Fairlie, T.(2001)

Fairlie, T.(2001)

Fairlie, T.(2001)

Bechtel National Corp.(1984b)

Bechtel National Corp.(1984b), SAIC(1997c)

Bechtel National Corp.(1984b)

Bechtel National Corp.(1984b)

Bechtel National Corp.(1984b)

Bechtel National Corp.(1984b)

Bechtel National Corp.(1984b), SAIC(1997c)

Bechtel National Corp.(1984b)

Bechtel National Corp.(1984b)

Bechtel National Corp.(1984b)

Bechtel National Corp.(1984b)

Bechtel National Corp.(1984b)

\section{COMMENTS}

NABIR FRC Phase 1 well, Drive Point, Area 2

NABIR FRC Phase 1 well, Drive Point, Area 2

NABIR FRC Phase 1 well, Drive Point, Area 2

Bottom of borehole caved. Sealed with bentonite to approximately $25 \mathrm{ft}$. Data base revised on $1 / 28 / 97$ to reflect changes discovered during periodic review.

Data base reviewed on $1 / 28 / 97$ to reflect changes discovered during periodic review.

Data base revised on $1 / 28 / 97$ to reflect changes discovered during periodic review.

NX core hole. Borehole drilled on an angle, 60 degrees from horizontal. Total depth of boring is $58.4 \mathrm{ft}$. Vertical depth from ground surface to TD is $50.6 \mathrm{ft}$. Borehole drilled from 0 to $8.0 \mathrm{ft} @ 4.75$-in. dia., then NQ wireline core to TD. Borehole backfilled with cement at the conclusion of drilling

NX core hole. Finished as monitoring well. Data base revised on 1/28/97 to reflect changes discovered during periodic review.

Data base revised on $1 / 28 / 97$ to reflect changes discovered during periodic review. TOWW elevation changed 06/06/2005 to reflect proper WW configuration.

Data base revised on $1 / 28 / 97$ to reflect changes discovered during periodic review.

Data base revised on $1 / 28 / 97$ to reflect changes discovered during periodic review. TOWW value estimated for this well.

Borehole was drilled on an angle, 60 degrees from horizontal. Total depth was $63.8 \mathrm{ft}$, and the vertical depth at TD was $55.3 \mathrm{ft}$. Borehole was drilled from 0 to $10.0 \mathrm{ft} @ 4.75$-in. dia. then NQ wireline core to TD. Borehole was backfilled immediately after drilling: no well was installed. Data base revised on $1 / 28 / 97$ to reflect changes discovered during periodic review.

Data base revised on $1 / 28 / 97$ to reflect changes discovered during periodic review.

Data base revised on $1 / 28 / 97$ to reflect changes discovered during periodic review. TOWW value estimated for this well.

Data base revised on $1 / 28 / 97$ to reflect changes discovered during periodic review. 
CURRENT

NAME

GW-013

GW-014

GW-015

GW-016

GW-017

GW-018

GW-019

GW-020

GW-021

GW-022

GW-023

GW-024

GW-025

GW-025A

\section{REFERENCE}

Bechtel National Corp.(1984b)

Bechtel National Corp.(1984a,d)

Bechtel National Corp.(1984a,d)

Bechtel National Corp.(1984a,d)

Bechtel National Corp.(1984a,d)

Bechtel National Corp.(1984a,d)

Bechtel National Corp.(1984a,d)

Bechtel National Corp.(1984a,d)

Bechtel National Corp.(1984a,d)

Bechtel National Corp.(1984a,d)

Bechtel National Corp.(1984a,d)

Bechtel National Corp.(1984a,d)

Bechtel National Corp.(1984a,d)

Bechtel National Corp.(1984a,d)

\section{COMMENTS}

Data base revised on $1 / 28 / 97$ to reflect changes discovered during periodic review. TOWW value estimated for this well.

Data base revised on $1 / 28 / 97$ to reflect changes discovered during periodic review.

Data base revised on $1 / 28 / 97$ to reflect changes discovered during periodic review.

Data base revised on $1 / 28 / 97$ to reflect changes discovered during periodic review. TOWW value estimated for this well.

Data base revised on $1 / 28 / 97$ to reflect changes discovered during periodic review.

Data base revised on $1 / 28 / 97$ to reflect changes discovered during periodic review.

Borehole was drilled on an angle, 60 degrees from horizontal. Total depth of the borehole was $62.2 \mathrm{ft}$. The vertical distance from to the ground surface at TD was $53.9 \mathrm{ft}$. Borehole was drilled @ 4.75-in. dia. from 0 to $11.0 \mathrm{ft}$, then NQ wireline core to TD. The borehole was grouted immediately after drilling. Data base revised on 1/28/97 to reflect changes discovered during periodic review.

Data base revised on $1 / 28 / 97$ to reflect changes discovered during periodic review.

Data base revised on $1 / 28 / 97$ to reflect changes discovered during periodic review.

Data base revised on $1 / 28 / 97$ to reflect changes discovered during periodic review.

Data base revised on $1 / 29 / 97$ to reflect changes discovered during periodic review.

Data base revised on $1 / 29 / 97$ to reflect changes discovered during periodic review.

Borehole was drilled on an angle, 60 degrees from horizontal. The total depth of the borehole was $61.7 \mathrm{ft}$. The vertical distance from the ground surface to TD was $54.3 \mathrm{ft}$. Borehole was drilled @ 4.75-in. dia. from 0 to $6.0 \mathrm{ft}$, then NQThe borehole was grouted after drilling: no well was installed in the borehole. Data base revised on 1/29/97 to reflect changes discovered during periodic review.

Borehole not plotted on borehole location map. Status changed to P\&A $02 / 25 / 2003$ 


\section{CURRENT}

\section{NAME}

GW-026

GW-027

GW-028

GW-029

GW-030

GW-031

GW-032

GW-033

GW-034

GW-035

GW-036

GW-037

GW-038

GW-039

GW-040

GW-041

GW-042

\section{REFERENCE}

Bechtel National Corp.(1984a,d)

Bechtel National Corp.(1984a,d)

Bechtel National Corp.(1984a,d)

Bechtel National Corp.(1984a,d)

Bechtel National Corp.(1984a,d), SAIC(1993h)

Bechtel National Corp.(1984a,d), SAIC(1993h)

Bechtel National Corp.(1984a,d), SAIC(1993h)

Bechtel National Corp.(1984a,d)

Bechtel National Corp.(1984a,d)

Bechtel National Corp.(1984a,d), SAIC(1993h)

Bechtel National Corp.(1984a,d), SAIC(1993h)

Bechtel National Corp.(1984a,d), SAIC(1993h)

Bechtel National Corp.(1984a,d), SAIC(1993h)

Bechtel National Corp.(1984a,d)

Bechtel National Corp.(1984a,d)

Bechtel National Corp.(1984a,d)

Bechtel National Corp.(1984a,d)

\section{COMMENTS}

Data base revised on $1 / 29 / 97$ to reflect changes discovered during periodic review.

Data base revised on $1 / 29 / 97$ to reflect changes discovered during periodic review.

Data base revised on $1 / 29 / 97$ to reflect changes discovered during periodic review.

Data base revised on $1 / 29 / 97$ to reflect changes discovered during periodic review.

Forms cluster with GW-031. Data base revised on 1/29/97 to reflect changes discovered during periodic review.

Forms cluster with GW-030. Data base revised on 1/29/97 to reflect changes discovered during periodic review.

Borehole was drilled on an angle, 60 degrees from horizontal. Data base revised on 1/29/97 to reflect changes discovered during periodic review.

Data base revised on $1 / 29 / 97$ to reflect changes discovered during periodic review.

Data base revised on $1 / 29 / 97$ to reflect changes discovered during periodic review.

Data base revised on $1 / 29 / 97$ to reflect changes discovered during periodic review.

Data base revised on 1/29/97 to reflect changes discovered during periodic review.

Data base revised on $1 / 29 / 97$ to reflect changes discovered during periodic review.

Data base revised on $1 / 29 / 97$ to reflect changes discovered during periodic review.

Data base revised on $1 / 29 / 97$ to reflect changes discovered during periodic review.

Data base revised on $1 / 30 / 97$ to reflect changes discovered during periodic review. TOWW value estimated for this well.

Data base revised on 1/30/97 to reflect changes discovered during periodic review. TOWW value estimated for this well.

Data base revised on 1/30/97 to reflect changes discovered during periodic review. TOWW value estimated for this well. 


\section{CURRENT}

\section{NAME}

GW-043

GW-044

GW-045

GW-046

GW-047

GW-048

GW-049

GW-050

GW-051

GW-052

GW-053

GW-054

GW-055

GW-056

GW-057

\section{REFERENCE}

Bechtel National Corp.(1984b)

Bechtel National Corp.(1984b)

Bechtel National Corp.(1984a,d)

Bechtel National Corp.(1984a,d)

Bechtel National Corp.(1984a,d)

Bechtel National Corp.(1984a,d), SAIC(1995b)

Bechtel National Corp.(1984a,d), SAIC(1995b)

Bechtel National Corp.(1984a,d), SAIC(1995b)

Bechtel National Corp.(1984a,d), SAIC(1995b)

Bechtel National Corp.(1984a,d)

Bechtel National Corp.(1984a,d)

Bechtel National Corp.(1984c)

Bechtel National Corp.(1984c)

Bechtel National Corp.(1984c)

Bechtel National Corp.(1984c)

\section{COMMENTS}

Data base revised on $1 / 30 / 97$ to reflect changes discovered during periodic review.

Data base revised on $1 / 30 / 97$ to reflect changes discovered during periodic review.

Forms cluster with GW-095. Data base revised on 1/30/97 to reflect changes discovered during periodic review.

Data base revised on 1/30/97 to reflect changes discovered during periodic review. TOWW value estimated for this well.

Forms cluster with GW-374. Data base revised on 1/30/97 to reflect changes discovered during periodic review. TOWW value estimated for this well.

Data base revised on $1 / 30 / 97$ to reflect changes discovered during periodic review.

Well re-discovered during FY 1994 well inspection, scheduled for $P \& A$, replaced by GW-237. Data base revised on $1 / 30 / 97$ to reflect changes discovered during periodic review.

Data base revised on 1/30/1997 to reflect changes discovered during periodic review.

Data base revised on $1 / 30 / 97$ to reflect changes discovered during periodic review.

Data base revised on $1 / 30 / 97$ to reflect changes discovered during periodic review. TOWW elevation changed 06/06/2005 to reflect proper WW low clearance cap configuration.

Data base revised on 1/30/97 to reflect changes discovered during periodic review.

Data base revised on 1/30/97 to reflect changes discovered during periodic review. TOWW elevation changed 06/06/2005 to reflect proper WW configuration.

Data base revised on 1/30/97 to reflect changes discovered during periodic review.

Data base revised on 1/30/97 to reflect changes discovered during periodic review. TOWW elevation changed 06/06/2005 to reflect proper WW configuration.

Data base revised on 1/30/97 to reflect changes discovered during periodic review. 


\section{CURRENT}

\section{NAME}

GW-058

GW-059

GW-060

GW-061

GW-062

GW-063

GW-064

GW-065

GW-066

GW-067

GW-068

GW-069

GW-070

\section{REFERENCE}

Bechtel National Corp.(1984c)

Bechtel National Corp.(1984c)

Bechtel National Corp.(1984c), SAIC(1995b)

Bechtel National Corp.(1984c)

Bechtel National Corp.(1984c)

Bechtel National Corp.(1984c), SAIC(1993h)

Bechtel National Corp.(1984c), Geraghty and Miller, Inc.(1988b)

Bechtel National Corp.(1984c)

Bechtel National Corp.(1984c)

Bechtel National Corp.(1984c)

Bechtel National Corp.(1984c)

Bechtel National Corp.(1984c)

Bechtel National Corp.(1984c)

\section{COMMENTS}

DData base revised on $1 / 30 / 97$ to reflect changes discovered during periodic review. Well Wizard pump and low clearance cap installed.

Data base revised on 1/30/97 to reflect changes discovered during periodic review.

Data base revised on 1/30/97 to reflect changes discovered during periodic review. TOWW value estimated for this well.

Data base revised on 1/30/97 to reflect changes discovered during periodic review. TOWW elevation changed 06/06/2005 to reflect proper WW low clearance cap configuration.

Borehole drilled to $56.7 \mathrm{ft} /$ Caved to $52.7 \mathrm{ft}$. Data base revised on 1/30/97 to reflect changes discovered during periodic review. TOWW value estimated for this well. TOWW elevation changed 06/06/2005 to reflect proper WW configuration.

Borehole drilled to $35.0 \mathrm{ft} /$ Caved to $32.7 \mathrm{ft}$. Data base revised on 1/30/97 to reflect changes discovered during periodic review.

Bolehole drilled to $57.0 \mathrm{ft} /$ Caved to $53.8 \mathrm{ft}$. Data base revised on 1/30/97 to reflect changes discovered during periodic review. TOWW value estimated for this well.

Borehole drilled to $35.0 \mathrm{ft} /$ Caved to $34.0 \mathrm{ft} .4 .75 \mathrm{in}$. dia. from $0-30.1 \mathrm{ft} \mathrm{bgs}$. and $4.0 \mathrm{in}$. dia. from 30.1-35.0 ft. bgs. Data base revised on 1/30/97 to reflect changes discovered during periodic review.

Data base revised on 1/30/97 to reflect changes discovered during periodic review. Well re-survey 06/20/2003. TOWW elevation changed 06/06/2005 to reflect proper WW low clearance cap configuration.

Data base revised on 1/30/97 to reflect changes discovered during periodic review. Well casing has been damaged beyond practical repair $(07 / 15 / 03)$. P/A request 05-001P/A has been submitted.

Data base revised on 1/30/97 to reflect changes discovered during periodic review. TOWW value estimated for this well.

Data base revised on 1/30/97 to reflect changes discovered during periodic review.

Borehole drilled to $148.5 \mathrm{ft} /$ Caved to $140.5 \mathrm{ft}$. Data base revised on $1 / 30 / 97$ to reflect changes discovered during periodic review. 


\section{CURRENT}

NAME

GW-071

GW-072

GW-073

GW-074

GW-075

GW-076

GW-077

GW-078

GW-079

GW-080

GW-081

GW-082

GW-083

GW-084

GW-085

\section{REFERENCE}

Bechtel National Corp.(1984c)

Bechtel National Corp.(1984c)

Bechtel National Corp.(1984c)

Bechtel National Corp.(1984c)

Bechtel National Corp.(1984c)

Bechtel National Corp.(1984c)

Bechtel National Corp.(1984c)

Bechtel National Corp.(1984c)

Bechtel National Corp.(1984c)

Bechtel National Corp.(1984c)

Bechtel National Corp.(1984c)

Bechtel National Corp.(1984c)

Bechtel National Corp.(1984c)

Bechtel National Corp.(1984c)

Bechtel National Corp.(1984c)

\section{COMMENTS}

06/27/05 - WW pump can not be pulled. Obstruction at or directly below ground surface. Obstruction also at approximately $135 \mathrm{ft}$. Original WW pump installed to $135 \mathrm{ft}$., and was not in the screened interval. Tubing was added, pump passed the obstruction at $135 \mathrm{ft}$. and was set $9.0 \mathrm{ft}$ off of bottom from TOWW. Data base revised on 1/30/97 to reflect changes discovered during periodic review.

Data base revised on 1/30/97 to reflect changes discovered during periodic review.

Data base revised on $1 / 30 / 97$ to reflect changes discoverd during periodic review. TOWW value estimated for this well.

Data base revised on 1/30/97 to reflect changes discovered during periodic review.

Data base revised on 1/30/97 to reflect changes discovered during periuodic review.

Data base revised on 1/30/97 to reflect changes discovered during periodic review.

Data base revised on 1/30/97 to reflect changes discovered during periodic review. TOWW value estimated for this well.

Data base revised on $1 / 30 / 97$ to reflect changes discovered during periodic review. TOWW value estimated for this well.

Data base revised on 1/30/97 to reflect changes discovered during periodic review. TOWW value estimated for this well.

Data base revised on $1 / 30 / 97$ to reflect changes discovered during periodic review.

Destroyed. Replaced by GW-242. Data base revised on 1/30/97 to reflect changes discovered during periodic review. TOWW elevation changed 06/06/2005 to reflect proper WW configuration.

Data base revised on $1 / 31 / 97$ to reflect changes discovered during periodic review.

Data base revised on $1 / 31 / 97$ to reflect changes discovered during periodic review.

Data base revised on 1/31/97 to reflect changes discovered during periodic review. TOWW value estimated for this well.

Borehole drilled to $62.0 \mathrm{ft} /$ Caved to $60.0 \mathrm{ft}$. Data base revised on 1/31/97

to reflect changes discovered during periodic review. TOWW value estimated for this well. 


\section{CURRENT}

NAME

GW-086

GW-087

GW-088

GW-089

GW-090

GW-091

GW-092

GW-093

GW-093A

GW-094

GW-095

GW-096

GW-096A

GW-097

GW-097A

GW-098

GW-099

GW-100

\section{REFERENCE}

Bechtel National Corp.(1984c)

Bechtel National Corp.(1984c)

Gillis, G. A.(1984), SAIC(1993h)

Gillis, G. A.(1984)

Gillis, G. A.(1984)

Gillis, G. A.(1984)

Gillis, G. A.(1984)

Bechtel National Corp.(1984e,f)

Gillis, G. A.(1984)

Bechtel National Corp.(1984e,f)

Bechtel National Corp.(1984e,f)

Bechtel National Corp.(1984e,f)

Gillis, G. A.(1984), SAIC(1995b)

Bechtel National Corp.(1984e,f)

Gillis, G. A.(1984)

Bechtel National Corp.(1984e,f)

Geraghty and Miller, Inc.(1989), King, H.L., Haase, C.S., and LaRue, D.L.(1989)

Bechtel National Corp.(1984e,f), Geraghty and Miller, Inc. (1988b)

\section{COMMENTS}

Data base revised on $1 / 31 / 97$ to reflect changes discovered during periodic review.

Data base revised on 1/31/97 to reflect changes discovered during periodic review. TOWW value estimated for this well.

Well re-survey 06/19/2002. TOWW elevation changed 06/06/2005 to reflect proper WW low clearance cap configuration.

Forms cluster with 1044. Data base revised on 1/31/97 to reflect changes discovered during periodic review.

Observation well immediately adjacent to GW-093. Borehole not plotted on map.

Forms cluster with 1045. Grout plug from $115.3 \mathrm{ft}$ to $131.0 \mathrm{ft}$. Data base revised on 1/31/97 to reflect changes discovered during periodic review.

TOWW value estimated for this well. TOWW elevation changed

06/06/2005 to reflect proper WW configuration.

Forms cluster with GW-045. Data base revised on 1/31/97 to reflect changes discovered during periodic review. TOWW value estimated for this well.

Forms cluster with GW-119, GW-237, and GW-375. Data base revised on $1 / 31 / 97$ to reflect changes discovered during periodic review.

Observation well immediately adjacent to GW-096.

Forms cluster with GW-98. Data base revised on 2/3/97 to reflect changes discovered during periodic review.

Observation well immediately adjacent to GW-097. Status Date: Sep84

Forms cluster with GW-097. Data base revised on 2/3/97 to reflect changes discovered during periodic review.

Destroyed/Plugged and abandoned. Replaced by GW-521. Data base revised on $2 / 3 / 97$ to reflect changes discovered during periodic review.

Data base revised on 2/3/97 to reflect changes discovered during periodic review. TOWW value estimated for this well. Screen style updated on 06/21/2007 to reflect proper screen style. 


\section{CURRENT}

NAME

GW-101

GW-102

GW-103

GW-104

GW-105

GW-106

GW-107

GW-108

GW-109

GW-110

GW-111

\section{REFERENCE}

Bechtel National Corp.(1984e,f)

Bechtel National Corp.(1984e,f)

Bechtel National Corp.(1984e,f)

Bechtel National Corp.(1984e,f)

Bechtel National Corp.(1984e,f), Geraghty and Miller Inc.(1988b)

Bechtel National Corp.(1984e,f), Geraghty and Miller, Inc.(1988b)

Bechtel National Corp.(1984e,f), Geraghty and Miller Inc.(1988b)

Bechtel National Corp.(1984e,f), Geraghty and Miller Inc.(1988b)

Bechtel National Corp.(1984e,f), Geraghty and Miller Inc.(1988b)

Bechtel National Corp.(1984e,f), SAIC(1994d)

Bechtel National Corp.(1984e,f)

\section{COMMENTS}

Data base revised on $2 / 3 / 97$ to reflect changes discovered during periodic review. TOWW value estimated for this well. TOWW elevation changed 06/06/2005 to reflect proper WW low clearance cap configuration. Screen style updated on 06/21/2007 to reflect proper screen style.

Destroyed/Plugged and abandoned. Replaced by GW-127. Data base revised on 2/3/97 to reflect changes discovered during periodic review.

Forms cluster with GW-104. Data base revised on 2/3/97 to reflect changes discovered during periodic review.

Forms cluster with GW-103. Data base revised on 2/3/97 to reflect changes discovered during periodic review.

Forms cluster with GW-106. Data base revised on 2/3/97 to reflect changes discovered during periodic review. TOWW value estimated for this well. Screen style updated on 06/21/2007 to reflect proper screen style.

Forms cluster with GW-105. Data base revised on 2/4/97 to reflect changes discovered during periodic review. TOWW value estimated for this well. TOWW elevation changed 06/06/2005 to reflect proper WW configuration. Screen style updated on $06 / 21 / 2007$ to reflect proper screen style.

Forms cluster with GW-108 and GW-109. Data base revised on 2/4/97 to reflect changes discovered during periodic review. TOWW value estimated for this well. Screen style updated on 06/21/2007 to reflect proper screen style.

Forms cluster with GW-107 and GW-109. Bottom of screen @ 55.70, bottom of sump @ 56.80. Data base revised on 2/4/97 to reflect changes discovered during periodic review. TOWW value estimated for this well.

Forms cluster with GW-107 and GW-108. Grout: 128.5 to $147.6 \mathrm{ft}$. Data base revised on 2/4/97 to reflect changes discovered during periodic review. TOWW value estimated for this well. TOWW elevation changed 06/06/2005 to reflect proper WW configuration.

Data base revised on $2 / 4 / 97$ to reflect changes discovered during periodic review.

NX core hole. Core @ Bldg 7042/ORNL. Data base revised on 2/4/97 to reflect changes discovered during periodic review. Borehole (core hole) drilled to a total depth of $300.0 \mathrm{ft}$ (5-in. bore: 0.0 to $30.3 \mathrm{ft}$, NX core: 30.3 to $300.0 \mathrm{ft}$ ). Borehole grouted to ground surface at conclusion of geophysical logging. Initial depth to water was $53.6 \mathrm{ft}$. The borehole was not surveyed. 
CURRENT

NAME

GW-112

GW-113

GW-114

GW-115

GW-116

GW-117

GW-118

GW-119

GW-120

GW-121

GW-122

GW-123

GW-124

GW-125

GW-126

\section{REFERENCE}

Bechtel National Corp.(1984e,f)

Bechtel National Corp.(1984e,f)

Bechtel National Corp.(1984e,f), SAIC(1994d)

Bechtel National Corp.(1984f), Ciravella, P. A.(1984), Geraghty and Miller, Inc.(1985), Geraghty and Miller, Inc.(1988b)

Geraghty and Miller, Inc.(1986b)

Geraghty and Miller, Inc.(1986b)

Geraghty and Miller, Inc.(1986b)

Geraghty and Miller, Inc.(1986b)

Geraghty and Miller, Inc.(1986b)

Geraghty and Miller, Inc.(1986b)

Geraghty and Miller, Inc.(1986b), Geraghty and Miller, Inc.(1988b)

Geraghty and Miller, Inc.(1986b), Geraghty and Miller, Inc.(1988b)

Geraghty and Miller, Inc.(1986b), Geraghty and Miller, Inc.(1988b)

Geraghty and Miller, Inc.(1986b), Geraghty and Miller, Inc.(1988b)

Geraghty and Miller, Inc.(1986b)

\section{COMMENTS}

Forms cluster with GW-113. Data base revised on 2/4/97 to reflect changes discovered during periodic review. Location Coord. TORC'ed 08/31/2000.

Forms cluster with GW-112. Data base revised on 2/4 97 to reflect changes discovered during periodic review. Location Coord. TORC'ed $08 / 31 / 2000$.

Data base revised on 2/4/97 to reflect changes discovered during periodic review.

Replaces 1002. Data base revised on 2/4/97 to reflect changes discovered during periodic review. TOWW value estimated for this well.

Data base revised on 2/4/97 to reflect changes discovered during periodic review.

T.O.C elevation may be in error? Data base revised on 2/4/97 to reflect changes discovered during periodic review.

Data base revised on 2/4/97 to reflect changes discovered during periodic review.

Forms cluster with GW-096, GW-237, and GW-375. Data base revised on 2/4/97 to reflect changes discovered during periodic review.

Data base revised on 2/4/97 to reflect changes discovered during periodic review.

Data base revised on 2/4/97 to reflect changes discovered during periodic review.

Data base revised on 2/4/97 to reflect changes discovered during periodic review. TOWW elevation changed 06/06/2005 to reflect proper WW low clearance cap configuration.

Data base revised on 2/4/97 to reflect changes discovered during periodic review. Well Wizard pump and low clearance cap installed.

Data base revised on 2/4/97 to reflect changes discovered during periodic review.

Data base revised on 2/4/97 to reflect changes discovered during periodic review.

Data base revised on 2/4/97 to reflect changes discovered during periodic review. Well Wizard pump and low clearance cap installed. 


\section{CURRENT}

NAME

GW-127

GW-128

GW-129

GW-130

GW-131

GW-132

GW-133

GW-134

GW-135

GW-136

GW-137

GW-138

\section{REFERENCE}

Gillis, G. A.(1984), Geraghty and Miller, Inc.(1988b)

King, H. L., and C. S. Haase(1987), SAIC(1991b)

King, H. L., and C. S. Haase(1987), SAIC(1991b)

King, H. L., and C. S. Haase(1987), SAIC(1991b)

King, H. L., and C. S. Haase(1987), Martin Marietta Energy Systems(1995)

King, H. L., and C. S. Haase(1987), Martin Marietta Energy Systems(1995)

King, H. L., and C. S. Haase(1987), Martin Marietta Energy Systems(1995)

King, H. L., and C. S. Haase(1987), Martin Marietta Energy Systems(1995)

King, H. L., and C. S. Haase(1987), Martin Marietta Energy Systems(1995)

King, H. L., and C. S. Haase(1987), Golder Associates(1988b), Martin Marietta Energy Systems(1988a)

King, H. L., and C. S. Haase(1987), Martin Marietta Energy Systems(1988a), SAIC(1991b)

King, H. L., and C. S. Haase(1987), Martin Marietta Energy Systems(1988a), SAIC(1992b)

\section{COMMENTS}

Replaces GW-102. Data base revised on 2/13/97 to reflect changes discovered during periodic review. TOWW elevation changed 06/06/2005 to reflect proper WW low clearance cap configuration.

NC core hole. Core @ Bldg 7042/ORNL. Core hole converted to Open Hole. Data base revised on 2/12/97 to reflect changes discovered during periodic review.

NC core hole. Core @ Bldg 7042/ORNL. Core hole converted to Open

Hole. Data base revised on 2/12/97 to reflect changes discovered during periodic review.

NC core hole. Core @ Bldg 7042/ORNL. Core hole converted to Open Hole.

NC core @ Bldg 7042/ORNL. Completed w/ Westbay Multi-port monitoring system. Data base revised on 2/12/97 to reflect changes discovered during periodic review.

NC core @ Bldg 7042/ORNL.Completed w/ Westbay Multi-port monitoring system. Data base revised on 2/12/97 to reflect changes discovered during periodic review.

NC core @ Bldg 7042/ORNL.Completed w/ Westbay Multi-port monitoring system. Data base revised on 2/12/97 to reflect changes discovered during periodic review.

NC core @ Bldg 7042/ORNL.Completed w/ Westbay Multi-port monitoring system. Data base revised on 2/12/97 to reflect changes discovered during periodic review.

NC core @ Bldg 7042/ORNL.Completed w/ Westbay Multi-port monitoring system. Data base revised on 2/12/97 to reflect changes discovered during periodic review.

NC core hole. Core @ Bldg 7042/ORNL. Hole grouted from 800.0 to 443.6 $\mathrm{ft}$, sealed with bentonite to 440.0, open to 419.9, packer 419.9-417.4,

installed 1 in OD PVC casing, hole grouted/bentonite to sfc. Core hole converted to screened well. Data base revised on 2/25/97 to reflect changes discovered during periodic review.

NC core hole. Core @ Bldg 7042/ORNL. Core hole converted to Open Hole.

NC core hole. Core @ Bldg 7042/ORNL. Core hole converted to Open Hole. 


\section{CURRENT}

\section{NAME}

GW-139

GW-140

GW-141

GW-142

GW-143

GW-144

GW-145

GW-146

GW-147

GW-148

GW-149

GW-150

\section{REFERENCE}

King, H. L., and C. S. Haase(1987), SAIC(1991b)

King, H. L., and C. S. Haase(1987), SAIC(1992b)

Geraghty and Miller, Inc.(1989), King, H.L., Haase, C.S., and LaRue, D.L.(1989)

Haase, C. S., G. A. Gillis, and H. L. King(1987)

Haase, C. S., G. A. Gillis, and H. L. King(1987)

Haase, C. S., G. A. Gillis, and H. L. King(1987)

Haase, C. S., G. A. Gillis, and H. L. King(1987)

Haase, C. S., G. A. Gillis, and H. L. King(1987)

Haase, C. S., G. A. Gillis, and H. L. King(1987)

Haase, C. S., G. A. Gillis, and H. L. King(1987)

Haase, C. S., G. A. Gillis, and H. L. King(1987)

Haase, C. S., G. A. Gillis, and H. L. King(1987)

\section{COMMENTS}

NC core hole. Core @ Bldg 7042/ORNL. Core hole converted to Open Hole. Location Coord. TORC'ed 08/31/2000.

NC core hole. Core @ Bldg 7042/ORNL. Core hole converted to Open Hole. Location Coord. TORC'ed 08/31/2000.

Data base revised on 2/4/97 to reflect changes discovered during periodic review. TOWW value estimated for this well.

NC core hole.Core @ Bldg 7042/ORNL. Data base revised on 2/4/97 to reflect changes discovered during periodic review. TOWW value estimated for this well.

Forms cluster with GW-144. Data base revised on 2/4/97 to reflect changes discovered during periodic review. TOWW value estimated for this well.

Forms cluster with GW-143. Data base revised on 2/4/97 to reflect changes discovered during periodic review. TOWW value estimated for this well. Screen style updated on 06/21/2007 to reflect proper screen

style.

Forms cluster with GW-146. Data base revised on 2/4/97 to reflect changes discovered during periodic review. TOWW value estimated for this well. Screen style updated on 06/21/2007 to reflect proper screen style.

Forms cluster with GW-145. Data base revised on 2/4/97 to reflect changes discovered during periodic review.

Forms cluster with GW-231. Data base revised on 2/4/97 to reflect changes discovered during periodic review. Screen style updated on 06/21/2007 to reflect proper screen style.

Forms cluster with GW-149. Data base revised on 2/4/97 to reflect changes discovered during periodic review. TOWW elevation changed $06 / 06 / 2005$ to reflect proper WW configuration. Screen style updated on $06 / 21 / 2007$ to reflect proper screen style.

Forms cluster with GW-148. Data base revised on 2/4/97 to reflect changes discovered during periodic review. Screen style updated on 06/21/2007 to reflect proper screen style.

Forms cluster with GW-151 and GW-220. Data base revised on 2/4/97 to reflect changes discovered during periodic review. Screen style updated on $06 / 21 / 2007$ to reflect proper screen style. 


\section{CURRENT}

\section{NAME}

GW-151

GW-152

GW-153

GW-154

GW-155

GW-156

GW-157

GW-158

GW-159

GW-160

GW-161

GW-162

\section{REFERENCE}

Haase, C. S., G. A. Gillis, and H. L. King(1987)

Haase, C. S., G. A. Gillis, and H. L. King(1987)

Haase, C. S., G. A. Gillis, and H. L. King(1987)

Haase, C. S., G. A. Gillis, and H. L. King(1987)

Haase, C. S., G. A. Gillis, and H. L. King(1987), $\operatorname{SAIC}(1991 b)$

Haase, C. S., G. A. Gillis, and H. L. King(1987)

Haase, C. S., G. A. Gillis, and H. L. King(1987), $\operatorname{SAIC}(1991 b)$

Haase, C. S., G. A. Gillis, and H. L. King(1987)

Haase, C. S., G. A. Gillis, and H. L. King(1987)

Geraghty and Miller, Inc.(1989), King, H.L., Haase, C.S., and LaRue, D.L.(1989)

Geraghty and Miller, Inc.(1989), King, H.L., Haase, C.S., and LaRue, D.L.(1989)

Haase, C. S., G. A. Gillis, and H. L. King(1987)

\section{COMMENTS}

Forms cluster with GW-150 and GW-220. Data base revised on 2/5/97 to reflect changes discovered during periodic review. TOWW value estimated for this well. Screen style updated on 06/21/2007 to reflect proper screen style.

Forms cluster with GW-153 and GW-240. Data base revised on 2/5/97 to reflect changes discovered during periodic review. Screen style updated on $06 / 21 / 2007$ to reflect proper screen style.

Forms cluster with GW-152 and GW-240. Data base revised on 2/5/97 to reflect changes discovered during periodic review. TOWW value estimated for this well. Screen style updated on 06/21/2007 to reflect proper screen style.

Forms cluster with GW-222 and GW-223. Data base revised on 2/5/97 to reflect changes discovered during periodic review. TOWW value estimated for this well. Screen style updated on 06/21/2007 to reflect proper screen style.

Data base revised on $2 / 5 / 97$ to reflect changes discovered during periodic review.

Data base revised on $2 / 5 / 97$ to reflect changes discovered during periodic review. TOWW value estimated for this well. Screen style updated on 06/21/2007 to reflect proper screen style.

Data base revised on $2 / 5 / 97$ to reflect changes discovered during periodic review.

Forms cluster with GW-241. Data base revised on 2/5/97 to reflect changes discovered during periodic review.

Measured depth of well from TOC was 155.8 after rehabilitation on 300 ct91. This differs from construction information which lists a depth of $159.4 \mathrm{ft}$ from TOC. Data base revised on 2/5/97 to reflect changes discovered during periodic review. TOWW value estimnated for this well. Screen style updated on 06/21/2007 to reflect proper screen style.

Forms cluster with GW-161 and GW-295. Data base revised on 2/5 97 to reflect changes discovered during periodic review.

Forms cluster with GW-160 and GW-295. Data base revised on 2/5/97 to reflect changes discovered during periodic review.

Forms cluster with GW-163 and GW-164. Data base revised on 2/5/97 to reflect changes discovered during periodic review. TOWW value estimated for this well. 


\section{CURRENT}

\section{NAME}

GW-163

GW-164

GW-165

GW-166

GW-167

GW-168

GW-169

GW-170

GW-171

GW-172

GW-173

GW-174

GW-175

\section{REFERENCE}

Haase, C. S., G. A. Gillis, and H. L. King(1987)

Haase, C. S., G. A. Gillis, and H. L. King(1987)

Haase, C. S., G. A. Gillis, and H. L. King(1987)

Haase, C. S., G. A. Gillis, and H. L. King(1987)

Webster, D. A.(1986), Bailey, Z. C. and D. B. Withington(1988), King, H.L., Haase, C.S., and LaRue, D.L.(1989)

Webster, D. A.(1986), Bailey, Z. C. and D. B.

Withington(1988), King, H.L., Haase, C.S., and LaRue, D.L.(1989)

Webster, D. A.(1986), Bailey, Z. C. and D. B.

Withington(1988), King, H.L., Haase, C.S., and LaRue,

D.L.(1989)

Webster, D. A.(1986), Bailey, Z. C. and D. B.

Withington(1988), King, H.L., Haase, C.S., and LaRue,

D.L.(1989)

Webster, D. A.(1986), Bailey, Z. C. and D. B.

Withington(1988), King, H.L., Haase, C.S., and LaRue,

D.L.(1989)

Webster, D. A.(1986), Bailey, Z. C. and D. B.

Withington(1988), King, H.L., Haase, C.S., and LaRue,

D.L.(1989)

Haase, C. S., G. A. Gillis, and H. L. King(1987)

Haase, C. S., G. A. Gillis, and H. L. King(1987)

EDGe, Inc.(1989), King, H.L., Haase, C.S., and LaRue, D.L.(1989)

\section{COMMENTS}

Forms cluster with GW-162 and GW-164. Data base revised on 2/5/97 to reflect changes discovered during periodic review.

Forms cluster with GW-162 and GW-163. Data base revised on 2/5/97 to reflect changes discovered during periodic review.

Forms cluster with GW-166. Data base revised on 2/5/97 to reflect changes discovered during periodic review.

Forms cluster with GW-165. Data base revised on 2/5/97 to reflect changes discovered during periodic review.

Forms cluster with GW-168 and GW-239. Data base revised on 2/5/97 to reflect changes discovered during periodic review. TOWW value estimated for this well.

Re-survey $\sim 04 / 27 / 1994$ due to well head modifications. Forms cluster with GW-167 and GW-239. Data base revised on 2/5/97 to reflect changes discovered during periodic review.

Forms cluster with GW-170 and GW-232. Original measuring point was (elevation) $931.87 \mathrm{ft}$. Data base revised on 2/5/97 to reflect changes discovered during periodic review. TOWW value estimated for this well.

Forms cluster with GW-169 and GW-232. Data base revised on 2/5/97 to reflect changes discovered during periodic review. TOWW value estimated for this well.

Forms cluster with GW-172 and GW-230. Data base revised on 2/5/97 to reflect changes discovered during periodic review. TOWW value estimated for this well.

Forms cluster with GW-171 and GW-230. Well resurveyed due to well head modifications. Data base revised on 2/5/97 to reflect changes discovered during periodic review. TOWW value estimated for this well.

Data base revised on 2/5/97 to reflect changes discovered during periodic review. TOWW elevation changed 06/06/2005 to reflect proper WW low clearance cap configuration.

Data base revised on $2 / 5 / 97$ to reflect changes discovered during periodic review.

Data base revised on $2 / 5 / 97$ to reflect changes discovered during periodic review. 


\section{CURRENT}

NAME

GW-176

GW-177

GW-178

GW-179

GW-180

GW-181

GW-182

GW-183

GW-184

GW-185

GW-186

GW-187

GW-188

GW-189

GW-190

\section{REFERENCE}

Haase, C. S., G. A. Gillis, and H. L. King(1987)

Haase, C. S., G. A. Gillis, and H. L. King(1987)

Geraghty and Miller, Inc.(1989), King, H.L., Haase, C.S., and LaRue, D.L.(1989)

Haase, C. S., G. A. Gillis, and H. L. King(1987)

Geraghty and Miller, Inc.(1989), King, H.L., Haase, C.S., and LaRue, D.L.(1989)

EDGe, Inc.(1989), King, H.L., Haase, C.S., and LaRue, D.L.(1989)

EDGe, Inc.(1988), EDGe, Inc.(1989), King, H.L., Haase, C.S., and LaRue, D.L.(1989), SAIC(1995b) ERCE(1991)

Haase, C. S., G. A. Gillis, and H. L. King(1987)

Haase, C. S., G. A. Gillis, and H. L. King(1987)

Haase, C. S., G. A. Gillis, and H. L. King(1987)

Haase, C. S., G. A. Gillis, and H. L. King(1987)

Haase, C. S., G. A. Gillis, and H. L. King(1987)

Haase, C. S., G. A. Gillis, and H. L. King(1987)

$\operatorname{ERCE}(1991)$

\section{COMMENTS}

Measured depth of well from TOC was 147.3 after rehabilitation on 13Mar92. This differs from construction information which lists a depth of $148.1 \mathrm{ft}$ from TOC. Data base revised on 2/5/97 to reflect changes

discovered during periodic review. TOWW elevation changed 06/06/2005 to reflect proper WW low clearance cap configuration.

Data base revised on 2/5/97 to reflect changes discovered during periodic review. TOWW value estimated for this well.

Data base revised on 2/5/97 to reflect changes discovered during periodic review. TOWW elevation changed 06/06/2005 to reflect proper WW low clearance cap configuration.

Data base revised on 2/5/97 to reflect changes discovered during periodic review. TOWW elevation changed 06/06/2005 to reflect proper WW low clearance cap configuration.

Data base revised on $2 / 5 / 97$ to reflect changes discovered during periodic review.

Data base revised on $2 / 5 / 97$ to reflect chages discovered during periodic review.

Core hole converted to Open Hole. Data base revised on 2/5/97 to reflect changes discovered during periodic review.

Data base revised on 2/5/97 to reflect changes discovered during periodic review.

Data base revised on 2/5/97 to reflect changes discovered during periodic review. Screen style updated on 06/21/2007 to reflect proper screen style. Forms cluster with $\mathrm{GW}-186$ and $\mathrm{GW}-189$. Data base revised on 2/5/97 to reflect changes discovered during periodic review.

Forms cluster with GW-185 and GW-189. Data base revised on 2/6/97 to reflect changes discovered during periodic review.

Forms cluster with 1081 and GW-224. Data base revised on 2/6/97 to reflect changes discovered during periodic review.

Data base revised on 2/6/97 to reflect changes discovered during periodic review.

Forms cluster with GW-185 and GW-186. Data base revised on 2/6/97 to reflect changes discovered during periodic review.

Data base revised on 2/6/97 to reflect changes discovered during periodic review. TOWW elevation changed 06/06/2005 to reflect proper WW low clearance cap configuration. 


\section{CURRENT}

\section{NAME}

GW-191

GW-192

GW-193

GW-194

GW-195

GW-196

GW-197

GW-198

GW-199

GW-200

GW-201

GW-202

GW-203

GW-204

\section{REFERENCE}

Haase, C. S., G. A. Gillis, and H. L. King(1987)

Haase, C. S., G. A. Gillis, and H. L. King(1987)

ERCE(1991)

Haase, C. S., G. A. Gillis, and H. L. King(1987)

Haase, C. S., G. A. Gillis, and H. L. King(1987)

Haase, C. S., G. A. Gillis, and H. L. King(1987)

Haase, C. S., G. A. Gillis, and H. L. King(1987)

Haase, C. S., G. A. Gillis, and H. L. King(1987)

Haase, C. S., G. A. Gillis, and H. L. King(1987)

Haase, C. S., G. A. Gillis, and H. L. King(1987)

Haase, C. S., G. A. Gillis, and H. L. King(1987), $\operatorname{SAIC}(1998 b)$

Haase, C. S., G. A. Gillis, and H. L. King(1987)

Haase, C. S., G. A. Gillis, and H. L. King(1987)

$\operatorname{ERCE}(1991)$

\section{COMMENTS}

Forms cluster with GW-192. Data base revised on 2/6/97 to reflect changes discovered during periodic review. TOWW value estimated for this well. Screen style updated on 06/21/2007 to reflect proper screen

style.

Forms cluster with GW-191. Data base revised on 2/6/97 to reflect changes discovered during periodic review. TOWW value estimated for this well. Screen style updated on 06/21/2007 to reflect proper screen style.

Data base revised on $2 / 6 / 97$ to reflect changes discovered during periodic review. TOWW value estimated for this well.

Data base revised on 2/6 97 to reflect changes discovered during periodic review.

Data base revised on $2 / 6 / 97$ to reflect changes discovered during periodic review.

Data base revised on 2/6/97 to reflect changes discovered during periodic review.

Data base revised on $2 / 6 / 97$ to reflect changes discovered during periodic review.

Data base revised on $2 / 6 / 97$ to reflect changes discovered during periodic review.

Data base revised on $2 / 6 / 97$ to reflect changes discovered during periodic review.

Forms cluster with GW-201. Data base revised on 2/6/97 to reflect changes discovered during periodic review.

Forms cluster with GW-200. Data base revised on 2/6/97 to reflect changes discovered during periodic review.

Data base revised on 2/6/97 to reflect changes discovered during periodic review.

Measured depth of well from TOC was $157.4 \mathrm{ft}$ after rehabilitation on 04Mar92. This differs from construction information which lists a depth of $159.8 \mathrm{ft}$ from TOC. Data base revised on $2 / 6 / 97$ to reflect changes discovered during periodic review. TOWW value estimated for this well.

Data base revised on $2 / 6 / 97$ to reflect changes discovered during periodic review. 


\section{CURRENT}

NAME

GW-205

GW-206

GW-207

GW-208

GW-209

GW-210

GW-211

GW-212

GW-213

GW-214

\section{REFERENCE}

Haase, C. S., G. A. Gillis, and H. L. King(1987)

Webster, D. A.(1986), Bailey, Z. C. and D. B.

Withington(1988), King, H.L., Haase, C.S., and LaRue, D.L.(1989)

Webster, D. A.(1986), Bailey, Z. C. and D. B.

Withington(1988), King, H.L., Haase, C.S., and LaRue D.L.(1989)

Webster, D. A.(1986), Bailey, Z. C. and D. B. Withington(1988), King, H.L., Haase, C.S., and LaRue, D.L.(1989)

Webster, D. A.(1986), Bailey, Z. C. and D. B. Withington(1988), King, H.L., Haase, C.S., and LaRue D.L.(1989), SAIC(1995b)

Webster, D. A.(1986), Bailey, Z. C. and D. B.

Withington(1988), King, H.L., Haase, C.S., and LaRue,

D.L.(1989)

Webster, D. A.(1986), Bailey, Z. C. and D. B. Withington(1988), King, H.L., Haase, C.S., and LaRue D.L.(1989)

Webster, D. A.(1986), Bailey, Z. C. and D. B. Withington(1988), King, H.L., Haase, C.S., and LaRue, D.L.(1989)

Webster, D. A.(1986), Bailey, Z. C. and D. B. Withington(1988), King, H.L., Haase, C.S., and LaRue, D.L.(1989)

Webster, D. A.(1986), Bailey, Z. C. and D. B.

Withington(1988), King, H.L., Haase, C.S., and LaRue, D.L.(1989)

\section{COMMENTS}

Measured depth of well from TOC was $155.1 \mathrm{ft}$ after rehabilitation on 03Mar92. This differs from construction information which lists a depth of $166.5 \mathrm{ft}$ from TOC. Data base revised on 2/6/97 to reflect changes

discovered during periodic review. TOWW value estimated for this well.

Forms cluster with GW-207 and GW-208. Data base revised on 2/6/97 to reflect changes discovered during periodic review.

Forms cluster with GW-206 and GW-208. Original measurement point was (elevation) $901.81 \mathrm{ft}$. Data base revised on 2/6/97 to reflect changes discovered during periodic review. Location re-surveyed 10/18/2002. Dedicated portable WW pump used in this well, TOC and TOWW are at the same elevation.

Forms cluster with GW-206 and GW-207. Data base revised on 2/6/97 to reflect changes discovered during periodic review. Location re-surveyed $10 / 18 / 2002$. Dedicated portable WW pump used in this well, TOC and TOWW are at the same elevation.

Forms cluster with GW-210 and GW-211. Data base revised on 2/6/97 to reflect changes discovered during periodic review.

Forms cluster with GW-209 and GW-211. Data base revised on 2/6/97 to reflect changes discovered during periodic review.

Forms cluster with GW-209 and GW-210. Data base revised on 2/6/97 to reflect changes discovered during periodic review.

Forms cluster with GW-214 and GW-238. Data base revised on 2/6/97 to reflect changes discovered during periodic review. Location Coord.

TORC'ed 08/31/2000.

Borehole abandoned during construction. Not plotted on borehole location map. Data base revised on 2/6/97 to reflect changes discovered during periodic review. Location Coord. TORC'ed 08/31/2000. Status changed to P\&A 02/25/2003

Forms cluster with GW-212 and GW-238. Data base revised on 2/6/97 to reflect changes discovered during periodic review. Location Coord. TORC'ed 08/31/2000. 


\section{CURRENT}

NAME

GW-215

GW-216

GW-217

GW-218

GW-219

GW-220

GW-221

GW-222

GW-223

GW-224

\section{REFERENCE}

Webster, D. A.(1986), Bailey, Z. C. and D. B. Withington(1988), King, H.L., Haase, C.S., and LaRue, D.L.(1989), SAIC(1995b)

Webster, D. A.(1986), King, H.L., Haase, C.S., and LaRue, D.L.(1989), SAIC(1995b)

Geraghty and Miller, Inc.(1989), King, H.L., Haase, C.S., and LaRue, D.L.(1989)

Geraghty and Miller, Inc.(1989), King, H.L., Haase, C.S., and LaRue, D.L.(1989)

Geraghty and Miller, Inc.(1989), King, H.L., Haase, C.S., and LaRue, D.L.(1989)

Haase, C. S., G. A. Gillis, and H. L. King(1987)

Haase, C. S., G. A. Gillis, and H. L. King(1987)

Haase, C. S., G. A. Gillis, and H. L. King(1987)

Haase, C. S., G. A. Gillis, and H. L. King(1987)

Haase, C. S., G. A. Gillis, and H. L. King(1987)

\section{COMMENTS}

Data base revised on 2/7/97 to reflect changes discovered during periodic review. Location Coord. TORC'ed 08/31/2000.

Borehole abandoned during construction. Not plotted on borehole location map. Data base revised on 2/7/97 to reflect changes discovered during periodic review. Location Coord. TORC'ed 08/31/2000.

Data base revised on 2/7/97 to reflect changes discovered during periodic review. TOWW value estimated for this well.

Data base revised on 2/7/97 to reflect changes discovered during periodic review.

Data base revised on $2 / 7 / 97$ to reflect changes discovered during periodic review.

Forms cluster with GW-150 and GW-151. Data base revised on 2/7/97 to reflect changes discovered during periodic review. TOWW value estimated for this well. TOWW elevation changed 06/06/2005 to reflect proper WW low clearance cap configuration. Screen style updated on $06 / 21 / 2007$ to reflect proper screen style.

Measured depth of well from TOC was $156.8 \mathrm{ft}$ after rehabilitation on 05Mar92. This differs from construction information which lists a depth of $160.6 \mathrm{ft}$ from TOC. Data base revised on 2/7/97 to reflect changes discovered during periodic review. TOWW value estimated for this well. Screen style updated on 06/21/2007 to reflect proper screen style.

Forms cluster with GW-154 and GW-223. Data base revised on 2/7/97 to reflect changes discovered during periodic review. TOWW elevation changed 06/06/2005 to reflect proper WW low clearance cap configuration. Screen style updated on $06 / 21 / 2007$ to reflect proper screen style.

Forms cluster with GW-154 and GW-222. Data base revised on 2/7/97 to reflect changes discovered during periodice review. TOWW elevation changed 06/06/2005 to reflect proper WW low clearance cap

configuration. Screen style updated on $06 / 21 / 2007$ to reflect proper screen style.

Forms cluster with 1081 and GW-187. Data base revised on 2/7/97 to reflect changes discovered during periodic review. Screen style updated on $06 / 21 / 2007$ to reflect proper screen style. 


\section{CURRENT}

NAME

GW-225

GW-226

GW-227

GW-228

GW-229

GW-230

GW-231

GW-232

GW-233

GW-234

GW-235

GW-236

\section{REFERENCE}

Geraghty and Miller, Inc.(1986b)

Geraghty and Miller, Inc.(1986b)

Geraghty and Miller, Inc.(1986b)

Geraghty and Miller, Inc.(1986b)

Geraghty and Miller, Inc.(1986b)

Webster, D. A.(1986), Bailey, Z. C. and D. B. Withington(1988), King, H.L., Haase, C.S., and LaRue, D.L.(1989)

Haase, C. S., G. A. Gillis, and H. L. King(1987)

Webster, D. A.(1986), Bailey, Z. C. and D. B. Withington(1988)

Geraghty and Miller, Inc.(1989), King, H.L., Haase, C.S., and LaRue, D.L.(1989)

Gillis, G. A.(1985), SAIC(1993h)

Webster, D. A.(1986), Bailey, Z. C. and D. B. Withington(1988), King, H.L., Haase, C.S., and LaRue, D.L.(1989)

Gillis, G. A.(1985), Geraghty and Miller, Inc.(1988b)

\section{COMMENTS}

Forms cluster with GW-226. Data base revised on 2/7/97 to reflect changes discovered during periodic review. TOWW elevation changed 06/06/2005 to reflect proper WW low clearance cap configuration.

Forms cluster with GW-225. Data base revised on 2/7/97 to reflect changes discovered during periodic review.

Forms cluster with GW-228. Data base revised on 2/7/97 to reflect changes discovered during periodic review. TOWW elevation changed 06/06/2005 to reflect proper WW low clearance cap configuration.

Forms cluster with GW-227. Data base revised on 2/7/97 to reflect changes discovered during periodice review. TOWW elevation changed 06/06/2005 to reflect proper WW low clearance cap configuration.

Borehole located in old channel of Bear Creek. Data base revised on $2 / 7 / 97$ to reflect changes discovered during periodic review. TOWW elevation changed 06/06/2005 to reflect proper WW low clearance cap configuration.

Forms cluster with GW-171 and GW-172. Well resurveyed due to well head modifications. Data base revised on 2/7 97 to reflect changes discovered during periodic review. TOWW value estimated for this well.

Forms cluster with GW-147. Data base revised on 2/7/97 to reflect changes discovered during periodic review. TOWW value estimated for this well. Screen style updated on 06/21/2007 to reflect proper screen style.

Measured depth of well from TOC was $405.2 \mathrm{ft}$ after rehabilitation on 17Dec91. This differs from construction information which lists a depth of 413.4 ft from TOC.Forms cluster with GW-169 and GW-170. Data base revised on $2 / 7 / 97$ to reflect changes discovered during periodic review. TOWW value estimated for this well.

Destroyed/Plugged and abandoned. Replaced by GW-522. Data base revised on 2/7/97 to reflect changes discovered during periodic review.

Destroyed

Borehole abandoned during construction. Not plotted on borehole location map. Data base revised on 2/7/97 to reflect changes discovered during periodic review. Status changed to P\&A 02/26/2003

Replaces 1004. TOWW elevation changed 06/06/2005 to reflect proper WW low clearance cap configuration. Screen style updated on 06/21/2007 to reflect proper screen style. 
CURRENT

NAME

GW-237

GW-238

GW-239

GW-240

GW-242

$\mathrm{GW}-243$

GW-244

GW-245

\section{REFERENCE}

Gillis, G. A.(1985)

Webster, D. A.(1986), Bailey, Z. C. and D. B. Withington(1988), King, H.L., Haase, C.S., and LaRue, D.L.(1989)

Webster, D. A.(1986), Bailey, Z. C. and D. B. Withington(1988), King, H.L., Haase, C.S., and LaRue, D.L.(1989)

Haase, C. S., G. A. Gillis, and H. L. King(1987)

Haase, C. S., G. A. Gillis, and H. L. King(1987)

Gillis, G. A.(1985)

Geraghty and Miller, Inc.(1986a), Geraghty and Miller, Inc.(1988b), King, H.L., Haase, C.S., and LaRue, D.L.(1989)

Geraghty and Miller, Inc.(1986a), Geraghty and Miller, Inc.(1988b), King, H.L., Haase, C.S., and LaRue, D.L.(1989)

Geraghty and Miller, Inc.(1986a), Geraghty and Miller, Inc.(1988b), King, H.L., Haase, C.S., and LaRue, D.L.(1989)

\section{COMMENTS}

Replaces GW-049. Forms cluster with GW-096, GW-119, and GW-375. Survey information taken from Geraghty and Miller, Inc. (1989) Location re-survey 06/19/2002. TOWW elevation changed 06/06/2005 to reflect proper WW low clearance cap configuration.

Forms cluster with GW-212 and GW-214. Data base revised on 2/7/97 to reflect changes discovered during periodic review. Location Coord. TORC'ed 08/31/2000

Forms cluster with GW-167 and GW-168. Data base revised on 2/7/97 to reflect changes discovered during periodic review.

Forms cluster with GW-152 and GW-153. Data base revised on 2/7/97 to reflect changes discovered during periodic review. TOWW elevation changed 06/09/2005 to reflect proper WW low clearance cap

configuration. Screen style updated on 06/21/2007 to reflect proper screen style.

Forms cluster with GW-158. Data base revised on 2/7/97 to reflect changes discovered during periodic review.

Replaces GW-081.

Constructed for S3 Ponds pumping test. Data base revised on 2/7/97 to reflect changes discovered during periodic review. TOWW value estimated for this well. TOWW elevation changed 06/06/2005 to reflect proper WW configuration. Screen style updated on $06 / 21 / 2007$ to reflect proper screen style.

Constructed for S3 Ponds pumping test. Data base revised on 2/7/97 to reflect changes discovered during periodic review. TOWW elevation changed 06/09/2005 to reflect proper WW low clearance cap configuration. Screen style updated on 06/21/2007 to reflect proper screen style.

Constructed for S3 Ponds pumping test. Data base revised on 2/10/97 to reflect changes discovered during periodic review. TOWW elevation changed 06/09/2005 to reflect proper WW low clearance cap configuration. Screen style updated on $06 / 21 / 2007$ to reflect proper screen style. 
CURRENT

NAME

GW-246

GW-247

GW-249

GW-250

GW-251

GW-252

GW-253

GW-254

GW-255

GW-256

GW-257

GW-258

\section{REFERENCE}

Geraghty and Miller, Inc.(1986a), Geraghty and Miller, Inc.(1988b), King, H.L., Haase, C.S., and LaRue,

D.L.(1989)

Geraghty and Miller, Inc.(1986a), Geraghty and Miller, Inc.(1988b), King, H.L., Haase, C.S., and LaRue, D.L.(1989)

Geraghty and Miller, Inc.(1987a), King, H.L., Haase, C.S., and LaRue, D.L.(1989)

Geraghty and Miller, Inc.(1987c), King, H.L., Haase, C.S., and LaRue, D.L.(1989)

Geraghty and Miller, Inc.(1987c), King, H.L., Haase, C.S., and LaRue, D.L.(1989)

Geraghty and Miller, Inc.(1987b,c), King, H.L., Haase, C.S., and LaRue, D.L.(1989)

Geraghty and Miller, Inc.(1987c), Geraghty and Miller, Inc.(1988b), King, H.L., Haase, C.S., and LaRue,

D.L.(1989)

Geraghty and Miller, Inc.(1987b,c), King, H.L., Haase, C.S., and LaRue, D.L.(1989)

Geraghty and Miller, Inc.(1987b,c), King, H.L., Haase, C.S., and LaRue, D.L.(1989)

Geraghty and Miller, Inc.(1987b,c), Geraghty and Miller, Inc.(1988b), King, H.L., Haase, C.S., and LaRue

D.L.(1989)

Geraghty and Miller, Inc.(1987b,c), King, H.L., Haase, C.S., and LaRue, D.L.(1989)

Geraghty and Miller, Inc.(1987a), King, H.L., Haase, C.S., and LaRue, D.L.(1989)

Geraghty and Miller, Inc.(1987a), King, H.L., Haase, C.S., and LaRue, D.L.(1989)

\section{COMMENTS}

Constructed for S3 Ponds pumping test. Data base revised on 2/10/97 to reflect changes discovered during periodic review. TOWW elevation changed 06/09/2005 to reflect proper WW low clearance cap configuration. Screen style updated on $06 / 21 / 2007$ to reflect proper screen style.

Constructed for $\$ 3$ Ponds pumping test. Data base revised on 2/10/97 to reflect changes discovered during periodic review. TOWW elevation changed 06/09/2005 to reflect proper WW low clearance cap

configuration. Screen style updated on 06/21/2007 to reflect proper screen style.

Forms cluster with GW-257. Data base revised on 2/10/97 to reflect changes discovered during periodic review.

Data base revised on 2/10/97 to reflect changes discovered during periodice review/

Data base revised on 2/10/97 to reflect changes discovered during periodic review.

Data base revised on 2/12/97 to reflect changes discovered during periodic review. TOWW value estimated for this well.

Forms cluster with GW-255. Data base revised on 2/12/97 to reflect changes discovered during periodic review.

Data base revised on 2/12/97 to reflect changes discovered during periodic review. TOWW value estimated for this well.

Forms cluster with GW-256. Data base revised on 2/12/97 to reflect changes discovered during periodic review.

Forms cluster with GW-252. Data base revised on 2/12/97 to reflect changes discovered during periodic review.

Forms cluster with GW-254. Data base revised on 2/12/97 to reflect changes discovered during periodic review. Measurement point changed to TOWW with no change in elevation due to low clearance cap.

Forms cluster with GW-248. Data base revised on 2/10/97 to reflect changes discovered during periodic review. TOWW elevation changed 06/09/2005 to reflect proper WW low clearance cap configuration.

Forms cluster with GW-259. Data base revised on 2/10/97 to reflect changes discovered during periodic review. 


\section{CURRENT}

NAME

GW-259

GW-260

GW-261

GW-262

GW-263

GW-264

GW-265

GW-266

GW-267

GW-268

GW-269

GW-270

GW-271

GW-272

\section{REFERENCE}

Geraghty and Miller, Inc.(1987a), King, H.L., Haase, C.S., and LaRue, D.L.(1989)

Webster, D. A.(1986), Bailey, Z. C. and D. B. Withington(1988), King, H.L., Haase, C.S., and LaRue D.L.(1989)

Geraghty and Miller, Inc.(1987c), King, H.L., Haase, C.S., and LaRue, D.L.(1989)

Geraghty and Miller, Inc.(1987c), King, H.L., Haase, C.S., and LaRue, D.L.(1989)

Geraghty and Miller, Inc.(1987c), King, H.L., Haase, C.S., and LaRue, D.L.(1989)

Geraghty and Miller, Inc.(1987c), King, H.L., Haase, C.S., and LaRue, D.L.(1989)

Geraghty and Miller, Inc.(1987c), King, H.L., Haase, C.S., and LaRue, D.L.(1989)

Geraghty and Miller, Inc.(1987c), King, H.L., Haase, C.S., and LaRue, D.L.(1989)

Geraghty and Miller, Inc.(1987c), King, H.L., Haase, C.S., and LaRue, D.L.(1989)

Geraghty and Miller, Inc.(1987c), King, H.L., Haase, C.S., and LaRue, D.L.(1989)

Geraghty and Miller, Inc.(1987c), King, H.L., Haase, C.S., and LaRue, D.L.(1989)

Geraghty and Miller, Inc.(1987c), Geraghty and Miller, Inc.(1988b), King, H.L., Haase, C.S., and LaRue,

D.L.(1989)

Geraghty and Miller, Inc.(1987c), Geraghty and Miller, Inc.(1988b), King, H.L., Haase, C.S., and LaRue, D.L.(1989)

Geraghty and Miller, Inc.(1987c), Geraghty and Miller, Inc.(1988b), King, H.L., Haase, C.S., and LaRue, D.L.(1989)

\section{COMMENTS}

Forms cluster with GW-258. Data base revised on 2/10/97 to reflect changes discovered during periodic review.

Borehole abandoned during construction. Not plotted on borehole location map. Data base revised on 2/10/97 to reflect changes discovered during periodic review. Location Coord. TORC'ed 08/31/2000. Status changed to P\&A 02/25/2003

Forms cluster with GW-262. Data base revised on 2/10/97 to reflect changes discovered during periodic review.

Forms cluster with GW-261. Data base revised on 2/10/97 to reflect changes discovered during periodic review.

Forms cluster with GW-264. Data base revised on 2/10/97 to reflect changes discovered during periodic review.

Forms cluster with GW-263. Data base revised on 2/10/97 to reflect changes discovered during periodic review.

Data base revised on 2/10/97 to reflect changes discovered during periodic review. Well Wizard pump and low clearance cap installed.

Data base revised on 2/10/97 to reflect changes discovered during periodic review.

Data base revised on 2/10/97 to reflect changes discovered during periodic review.

Data base revised on 2/10/97 to reflect changes discovered during periodic review.

Data base revised on 2/10/97 to reflect changes discovered during periodic review. TOWW elevation changed 06/09/2005 to reflect proper WW low clearance cap configuration.

Forms cluster with GW-271. Data base revised on 2/10/97 to reflect changes discovered during periodic review. TOWW elevation changed 06/09/2005 to reflect proper WW low clearance cap configuration.

Forms cluster with GW-270. Data base revised on 2/10/97 to reflect changes discovered during periodic review. TOWW elevation changed 06/09/2005 to reflect proper WW low clearance cap configuration.

Data base revised on 2/10/97 to reflect changes discovered during periodic review. TOWW elevation changed 06/09/2005 to reflect proper WW low clearance cap configuration. 
CURRENT

NAME

GW-273

GW-274

GW-275

GW-276

GW-277

GW-278

GW-279

GW-280

GW-281

GW-282

GW-283

GW-284

GW-285

\section{REFERENCE}

Geraghty and Miller, Inc.(1987c), King, H.L., Haase, C.S., and LaRue, D.L.(1989)

Geraghty and Miller, Inc.(1987c), Geraghty and Miller, Inc.(1988b), King, H.L., Haase, C.S., and LaRue,

D.L.(1989)

Geraghty and Miller, Inc.(1987c), Geraghty and Miller, Inc.(1988b), King, H.L., Haase, C.S., and LaRue,

D.L.(1989)

Geraghty and Miller, Inc.(1987c), Geraghty and Miller,

Inc.(1988b), King, H.L., Haase, C.S., and LaRue,

D.L.(1989)

Geraghty and Miller, Inc.(1987c), Geraghty and Miller, Inc.(1988b), King, H.L., Haase, C.S., and LaRue, D.L.(1989)

Geraghty and Miller, Inc.(1987c), Geraghty and Miller, Inc.(1988b), King, H.L., Haase, C.S., and LaRue, D.L.(1989)

Geraghty and Miller, Inc.(1987c), Geraghty and Miller, Inc.(1988b), King, H.L., Haase, C.S., and LaRue,

D.L.(1989)

Geraghty and Miller, Inc.(1987c), Geraghty and Miller, Inc.(1988b), King, H.L., Haase, C.S., and LaRue,

D.L.(1989)

IT Corp.(1986), King, H.L., Haase, C.S., and LaRue, D.L.(1989)

IT Corp.(1986), King, H.L., Haase, C.S., and LaRue, D.L.(1989)

IT Corp.(1986), King, H.L., Haase, C.S., and LaRue, D.L.(1989)

IT Corp.(1986), King, H.L., Haase, C.S., and LaRue, D.L.(1989)

IT Corp.(1986), King, H.L., Haase, C.S., and LaRue, D.L.(1989)

\section{COMMENTS}

Data base revised on 2/10/97 to reflect changes discovered during periodic review. TOWW elevation changed 06/09/2005 to reflect proper WW low clearance cap configuration.

Forms cluster with GW-275. Data base revised on 2/10/97 to reflect changes discovered during periodic review. TOWW value estimated for this well.

Forms cluster with GW-274. Data base revised on 2/11/97 to reflect changes discovered during periodic review.

Forms cluster with GW-277. Data base revised on 2/11/97 to reflect changes discovered during periodic review. TOWW value estimated for this well.

Forms cluster with GW-276. Data base revised on 2/11/97 to reflect changes discovered during periodic review. TOWW elevation changed 06/09/2005 to reflect proper WW low clearance cap configuration.

Forms cluster with GW-279 and GW-280. Data base revised on 2/11/97 to reflect changes discovered during periodic review.

Forms cluster with GW-278 and GW-280. Data base revised on 2/11/97 to reflect changes discovered during periodic review.

Forms cluster with GW-278 and GW-279. Data base revised on 2/11/97 to reflect changes discovered during periodic review.

Re-survey $\sim 04 / 27 / 1994$. Data base revised on 2/11/97 to reflect changes discovered during periodic review.

Data base revised on 2/11/97 to reflect changes discovered during periodic review.

Data base revised on $2 / 11 / 97$ to reflect changes discovered during periodic review.

Data base revised on 2/11/97 to reflect changes discovered during periodic review. No TOWW for this well, field verified 05/12/2005 no WW present.

Data base revised on 2/11/97 to reflect changes discovered during periodic review. 


\section{CURRENT}

NAME

GW-286

GW-287

GW-288

GW-289

GW-290

GW-291

GW-292

GW-293

GW-294

GW-295

GW-296

GW-297

GW-298

GW-299

GW-300

\section{REFERENCE}

Geraghty and Miller, Inc.(1987a), King, H.L., Haase, C.S., and LaRue, D.L.(1989)

Geraghty and Miller, Inc.(1987a), King, H.L., Haase, C.S., and LaRue, D.L.(1989)

Geraghty and Miller, Inc.(1987a), King, H.L., Haase, C.S., and LaRue, D.L.(1989)

Geraghty and Miller, Inc.(1987a), King, H.L., Haase, C.S., and LaRue, D.L.(1989)

Geraghty and Miller, Inc.(1987a), King, H.L., Haase, C.S., and LaRue, D.L.(1989)

Geraghty and Miller, Inc.(1987a), King, H.L., Haase, C.S., and LaRue, D.L.(1989)

Geraghty and Miller, Inc.(1989), King, H.L., Haase, C.S., and LaRue, D.L.(1989)

Geraghty and Miller, Inc.(1989), King, H.L., Haase, C.S., and LaRue, D.L.(1989)

Geraghty and Miller, Inc.(1989), King, H.L., Haase, C.S., and LaRue, D.L.(1989)

Geraghty and Miller, Inc.(1989), King, H.L., Haase, C.S., and LaRue, D.L.(1989), SAIC(1997c)

Geraghty and Miller, Inc.(1989), King, H.L., Haase, C.S., and LaRue, D.L.(1989)

Geraghty and Miller, Inc.(1989), King, H.L., Haase, C.S., and LaRue, D.L.(1989), SAIC(1997c)

Geraghty and Miller, Inc.(1989), King, H.L., Haase, C.S., and LaRue, D.L.(1989)

Geraghty and Miller, Inc.(1989), King, H.L., Haase, C.S., and LaRue, D.L.(1989)

Geraghty and Miller, Inc.(1989), King, H.L., Haase, C.S., and LaRue, D.L.(1989)

\section{COMMENTS}

Forms cluster with GW-287. Data base revised on 2/11/97 to reflect changes discovered during periodic review. TOWW elevation added $02 / 07 / 2006$ to reflect conditions in the field.

Forms cluster with GW-286. Data base revised on 2/11/97 to reflect changes discovered during periodic review. TOWW value estimated for this well.

Forms cluster with GW-289. Data base revised on 2/11/97 to reflect changes discovered during periodic review. TOWW elevation changed 06/09/2005 to reflect proper WW low clearance cap configuration.

Forms cluster with GW-288. Data base revised on 2/11/97 to reflect changes discovered during periodic review. TOWW value estimated for this well.

Forms cluster with GW-291. Data base revised on 2/11/97 to reflect changes discovered during periodic review.

Forms cluster with GW-290. Data base revised on 2/11/97 to reflect changes discovered during periodic review. TOWW value estimated for this well. Well re-survey 06/20/2003.

Data base revised on 2/11/97 to reflect changes discovered during periodic review.

Data base revised on $2 / 11 / 97$ to reflect changes discovered during periodic review.

Data base revised on 2/11/97 to reflect changes discovered during periodic review.

Forms cluster with GW-160 and GW-161. Data base revised on 2/11/97 to reflect changes discovered during periodic review.

Data base revised on $2 / 11 / 97$ to reflect changes discovered during periodic review.

Data base revised on $2 / 11 / 97$ to reflect changes discovered during periodic review.

Data base revised on 2/11/97 toreflect changes discovered during periodic review.

Data base revised on 2/11/97 to reflect changes discovered during periodic review.

Data base revised on 2/11/97 to reflect changes discovered during periodic review. TOWW elevation changed 06/09/2005 to reflect proper WW low clearance cap configuration. 


\section{CURRENT}

NAME

GW-301

GW-302

GW-303

GW-304

GW-305

GW-306

GW-307

GW-308

GW-309

GW-310

GW-311

GW-312

GW-313

GW-314

GW-315

\section{REFERENCE}

Geraghty and Miller, Inc.(1989), King, H.L., Haase, C.S., and LaRue, D.L.(1989)

$\operatorname{ERCE}(1991)$

Geraghty and Miller, Inc.(1989), King, H.L., Haase, C.S., and LaRue, D.L.(1989)

EDGe, Inc.(1989), King, H.L., Haase, C.S., and LaRue, D.L.(1989)

Geraghty and Miller, Inc.(1989), King, H.L., Haase, C.S., and LaRue, D.L.(1989)

Geraghty and Miller, Inc.(1989), King, H.L., Haase, C.S., and LaRue, D.L.(1989)

Geraghty and Miller, Inc.(1989), King, H.L., Haase, C.S., and LaRue, D.L.(1989)

Geraghty and Miller, Inc.(1989), King, H.L., Haase, C.S., and LaRue, D.L.(1989)

Geraghty and Miller, Inc.(1989), King, H.L., Haase, C.S., and LaRue, D.L.(1989)

Geraghty and Miller, Inc.(1989), King, H.L., Haase, C.S., and LaRue, D.L.(1989)

Geraghty and Miller, Inc.(1989), King, H.L., Haase, C.S., and LaRue, D.L.(1989)

Geraghty and Miller, Inc.(1989), King, H.L., Haase, C.S., and LaRue, D.L.(1989)

Geraghty and Miller, Inc.(1989), King, H.L., Haase, C.S., and LaRue, D.L.(1989)

Geraghty and Miller, Inc.(1989), King, H.L., Haase, C.S., and LaRue, D.L.(1989)

Geraghty and Miller, Inc.(1989), King, H.L., Haase, C.S., and LaRue, D.L.(1989)

\section{COMMENTS}

6-in borehole drilled from $163.5 \mathrm{ft}$ to $182.0 \mathrm{ft} /$ Backfilled with bentonite. Data base revised on $2 / 11 / 97$ to reflect changes discovered during periodic review. TOWW value estimated for this well.

Data base revised on 2/14/97 to reflect changes discovered during periodic review. TOWW value estimated for this well.

Data base revised on $2 / 14 / 97$ to reflect changes discovered during periodic review.

Data base revised on $2 / 14 / 97$ to reflect changes discovered during periodic review.

Data base revised on 2/14/97 to reflect changes dizcovered during periodic review. TOWW value estimated for this well.

Data base revised on 2/14/97 to reflect changes discovered during periodic review.

Data base revised on 2/14/97 to reflect changes discovered during periodic review. TOWW elevation changed 06/09/2005 to reflect proper WW low clearance cap configuration.

Data base revised on 2/14/97 to reflect changes discovered during periodic review.

Data base revised on 2/14/97 to reflect changes discovered during periodic review.

Data base revised on 2/14/97 to reflect changes discovered during periodic review. TOWW elevation added 02/07/2006 to reflect conditions in the field.

Data base revised on 2/14/97 to reflect changes discovered during periodic review. TOWW value estimated for this well.

Borehole caved from $24.8 \mathrm{ft}$ to $27.3 \mathrm{ft}$. Interval sealed with bentonite. Data base revised on 2/14/97 to reflect changes discovered during periodic review. TOWW value estimated for this well.

Data base revised on 2/14/97 to reflect changes discovered during periodic review. TOWW elevation changed 06/09/2005 to reflect proper WW low clearance cap configuration.

Data base revised on 2/14/97 to reflect changes discovered during periodic review.

Data base revised on 2/14/97 to reflect changes discovered during periodic review. TOWW value estimated for this well. 
CURRENT

NAME

GW-316

GW-317

GW-318

GW-319

GW-320

GW-321

GW-322

GW-323

GW-324

GW-325

GW-326

GW-327

GW-328

GW-329

GW-330

\section{REFERENCE}

Geraghty and Miller, Inc.(1989), King, H.L., Haase, C.S., and LaRue, D.L.(1989)

Geraghty and Miller, Inc.(1989), King, H.L., Haase, C.S., and LaRue, D.L.(1989)

Gillis, G. A.(1987), King, H.L., Haase, C.S., and LaRue D.L.(1989)

Gillis, G. A.(1987), King, H.L., Haase, C.S., and LaRue, Forms cluster with GW-318.

D.L.(1989)

Gillis, G. A.(1987), King, H.L., Haase, C.S., and LaRue,

D.L.(1989), SAIC(1997c)

Gillis, G. A.(1987), King, H.L., Haase, C.S., and LaRue,

D.L.(1989), SAIC(1997c)

Geraghty and Miller, Inc.(1989), King, H.L., Haase,

C.S., and LaRue, D.L.(1989)

Geraghty and Miller, Inc.(1989), King, H.L., Haase,

C.S., and LaRue, D.L.(1989)

Geraghty and Miller, Inc.(1988b), Geraghty and Miller,

Inc.(1989), King, H.L., Haase, C.S., and LaRue,

D.L.(1989)

Geraghty and Miller, Inc.(1988b), Geraghty and Miller, Inc.(1989), King, H.L., Haase, C.S., and LaRue,

D.L.(1989)

Geraghty and Miller, Inc.(1988a), King, H.L., Haase,

C.S., and LaRue, D.L.(1989)

Geraghty and Miller, Inc.(1988a), King, H.L., Haase,

C.S., and LaRue, D.L.(1989)

Geraghty and Miller, Inc.(1988a), King, H.L., Haase,

C.S., and LaRue, D.L.(1989)

Geraghty and Miller, Inc.(1988a), King, H.L., Haase,

C.S., and LaRue, D.L.(1989)

Geraghty and Miller, Inc.(1988a), King, H.L., Haase,

C.S., and LaRue, D.L.(1989)

\section{COMMENTS}

Data base revised on $2 / 14 / 97$ to reflect changes discovered during periodic review.

Data base revised on $2 / 14 / 97$ to reflect changes discovered during periodic review. TOWW value estimated for this well.

Forms cluster with GW-319.

Borehole drilled to $200 \mathrm{ft} /$ Grout plug $124 \mathrm{ft}$ to $200 \mathrm{ft}$. Bentonite $118 \mathrm{ft}$ to $124 \mathrm{ft}$.

10-in borehole drilled to $135 \mathrm{ft}$; 7 -in casing to $128 \mathrm{ft}$. Cuttings/grout from $128 \mathrm{ft}$ to $135 \mathrm{ft}$. Data base revised on 2/14/97 to reflect changes discovered during periodic review. TOWW elevation changed 06/06/2005 to reflect proper WW configuration.

Data base revised on 2/14/97 to reflect changes discovered during periodic review.

Forms cluster with GW-325. Data base revised on 2/14/97 to reflect changes discovered during periodic review.

Forms cluster with GW-324. Data base revised on 2/14/97 to reflect changes discovered during periodic review. 


\section{CURRENT}

NAME

GW-331

GW-332

GW-333

GW-334

GW-335

GW-336

GW-337

GW-338

GW-339

GW-340

GW-341

GW-342

GW-343

GW-344

GW-345

GW-346

\section{REFERENCE}

Geraghty and Miller, Inc.(1989)

Geraghty and Miller, Inc.(1989), King, H.L., Haase, C.S., and LaRue, D.L.(1989)

Geraghty and Miller, Inc.(1989), King, H.L., Haase, C.S., and LaRue, D.L.(1989)

Geraghty and Miller, Inc.(1989), King, H.L., Haase, C.S., and LaRue, D.L.(1989)

Geraghty and Miller, Inc.(1989), King, H.L., Haase, C.S., and LaRue, D.L.(1989)

Geraghty and Miller, Inc.(1989), King, H.L., Haase, C.S., and LaRue, D.L.(1989)

Geraghty and Miller, Inc.(1989), King, H.L., Haase, C.S., and LaRue, D.L.(1989)

Geraghty and Miller, Inc.(1989), King, H.L., Haase, C.S., and LaRue, D.L.(1989)

ERCE(1991)

Geraghty and Miller, Inc.(1988a), King, H.L., Haase, C.S., and LaRue, D.L.(1989)

Geraghty and Miller, Inc.(1988a), King, H.L., Haase, C.S., and LaRue, D.L.(1989)

EDGe, Inc.(1989), King, H.L., Haase, C.S., and LaRue, D.L.(1989)

EDGe, Inc.(1989), King, H.L., Haase, C.S., and LaRue, D.L.(1989)

EDGe, Inc.(1989), King, H.L., Haase, C.S., and LaRue, D.L.(1989)

EDGe, Inc.(1989), King, H.L., Haase, C.S., and LaRue, D.L.(1989)

EDGe, Inc.(1989), King, H.L., Haase, C.S., and LaRue,

D.L.(1989)

\section{COMMENTS}

Data base revised on 2/14/97 to reflect changes discovered during periodic review.

Data base revised on 2/14/97 to reflect changes discovered during periodic review. TOWW value estimated for this well.

Data base revised on 2/14/97 to reflect changes discovered during periodic review.

Data base revised on 2/14/97 to reflect changes discovered during periodic review.

Data base revised on 2/14/97 to reflect changes discovered during periodic review.

Data base revised on 2/14/97 to reflect changes discovered during periodic review. TOWW elevation changed 06/09/2005 to reflect proper WW low clearance cap configuration.

Data base revised on 2/14/97 to reflect changes discovered during periodic review. TOWW value estimated for this well.

Data base revised on 2/14/97 to reflect changes discovered during periodic review. TOWW value estimated for this well.

Data base revised on 2/14/97 to reflect changes discovered during periodic review. TOWW value estimated for this well.

Forms cluster with GW-343 and GW-344. Data base revised on 2/14/97 to reflect changes discovered during periodic review.

Forms cluster with GW-342 and GW-344. Data base revised on 2/14/97 to reflect changes discovered during periodic review.

Forms cluster with GW-342 and GW-343. Data base revised on 2/14/97 to reflect changes discovered during periodic review.

Forms cluster with GW-346 and GW-526. Data base revised on 2/14/97 to reflect changes discovered during periodic review.

Forms cluster with GW-345 and GW-526. Data base revised on 2/14 97 to reflect changes discovered during periodic review. 
CURRENT

NAME

GW-347

GW-348

GW-349

GW-350

GW-363

GW-364

GW-365

GW-366

GW-367

GW-368

GW-369

GW-370

GW-371

\section{REFERENCE}

EDGe, Inc.(1989), King, H.L., Haase, C.S., and LaRue D.L.(1989)

EDGe, Inc.(1989), King, H.L., Haase, C.S., and LaRue, D.L.(1989)

EDGe, Inc.(1989), King, H.L., Haase, C.S., and LaRue, D.L.(1989)

EDGe, Inc.(1989), King, H.L., Haase, C.S., and LaRue, D.L.(1989)

EDGe, Inc.(1989), King, H.L., Haase, C.S., and LaRue, D.L.(1989)

EDGe, Inc.(1989), King, H.L., Haase, C.S., and LaRue, D.L.(1989)

EDGe, Inc.(1989), King, H.L., Haase, C.S., and LaRue D.L.(1989)

EDGe, Inc.(1989), King, H.L., Haase, C.S., and LaRue D.L.(1989)

EDGe, Inc.(1989), King, H.L., Haase, C.S., and LaRue D.L.(1989)

EDGe, Inc.(1989), King, H.L., Haase, C.S., and LaRue D.L.(1989)

EDGe, Inc.(1989), King, H.L., Haase, C.S., and LaRue D.L.(1989)

EDGe, Inc.(1989), King, H.L., Haase, C.S., and LaRue, D.L.(1989)

EDGe, Inc.(1989), King, H.L., Haase, C.S., and LaRue, D.L.(1989)

\section{COMMENTS}

Forms cluster with GW-348. Data base revised on 2/14/97 to reflect changes discovered during periodic review. No WW found in this well. TOC elevation revised to reflect current data.

Forms cluster with GW-347. Data base revised on 2/14/97 to reflect changes discovered during periodic review. TOWW value estimated for this well.

Forms cluster with GW-350. Data base revised on 2/18/97 to reflect changes discovered during periodic review. TOWW elevation changed 06/09/2005 to reflect proper WW low clearance cap configuration.

Forms cluster with GW-349. Data base revised on 2/18/97 to reflect changes discovered during periodic review. TOWW elevation changed 06/09/2005 to reflect proper WW low clearance cap configuration.

Data base revised on 2/18/97 to reflect changes discovered during periodic review. No WW at this location, field verified 05/05/2005. TOWW elevation changed 06/09/2005 to reflect proper WW low clearance cap configuration.

Forms cluster with GW-365. Data base revised on 2/18/97 to reflect changes discovered during periodic review.

Forms cluster with GW-364. Data base revised on 2/18/97 to reflect changes discovered during periodic review. TOWW elevation changed 06/09/2005 to reflect proper WW low clearance cap configuration.

Forms cluster with GW-367 and GW-520. Data base revised on 2/18/97 to reflect changes discovered during periodic review.

Forms cluster with GW-366 and GW-520. Data base revised on 2/18/97 to reflect changes discovered during periodic review.

Forms cluster with GW-369. Data base revised on 2/18/97 to reflect changes discovered during periodic review. TOWW elevation changed 06/09/2005 to reflect proper WW low clearance cap configuration.

Forms cluster with GW-368. Data base revised on 2/18/97 to reflect changes discovered during periodic review.

Forms cluster with GW-371. Data base revised on 2/18/97 to reflect changes discovered during periodic review. TOWW value estimated for this well.

Forms cluster with GW-370. Data base revised on 2/18/97 to reflect changes discovered during periodic review. 
CURRENT

NAME

GW-372

GW-373

GW-374

GW-375

GW-376

GW-380

GW-381

GW-382

GW-383

GW-384

GW-385

GW-400

\section{REFERENCE}

EDGe, Inc.(1989), King, H.L., Haase, C.S., and LaRue D.L.(1989)

EDGe, Inc.(1989), King, H.L., Haase, C.S., and LaRue, D.L.(1989)

EDGe, Inc.(1989), King, H.L., Haase, C.S., and LaRue, D.L.(1989)

EDGe, Inc.(1989), King, H.L., Haase, C.S., and LaRue, D.L.(1989)

EDGe, Inc.(1989), King, H.L., Haase, C.S., and LaRue, D.L.(1989)

EDGe, Inc.(1989), King, H.L., Haase, C.S., and LaRue, D.L.(1989)

EDGe, Inc.(1989), King, H.L., Haase, C.S., and LaRue, D.L.(1989)

EDGe, Inc.(1989)

EDGe, Inc.(1989), King, H.L., Haase, C.S., and LaRue, D.L.(1989)

EDGe, Inc.(1989), King, H.L., Haase, C.S., and LaRue, D.L.(1989)

EDGe, Inc.(1989), King, H.L., Haase, C.S., and LaRue D.L.(1989)

Golder Associates(1988b), Martin Marietta Energy Systems(1988a)

\section{COMMENTS}

Forms cluster with GW-373 and GW-643. Data base revised on 2/18/97 to reflect changes discovered during periodic review. TOWW value estimated for this well.

Forms cluster with GW-372 and GW-643. Data base revised on 2/18/97 to reflect changes discovered during periodic review.

Forms cluster with GW-047. Data base revised on 2/18/97 to reflect changes discovered during periodic review.

Forms cluster with GW-096, GW-119, and GW-237. Data base revised on $2 / 18 / 97$ to reflect changes discovered during periodic review.

Data base revised on 2/18/97 to reflect changes discovered during periodic review. This well is configured as the water supply well for the Lysimeter Demonstration Site. It is fitted with hardware and piping and is inaccessible.

Forms cluster with GW-381 and GW-382. Data base revised on 2/18/97 to reflect changes discovered during periodic review. Dedicated portable WW pump used in this well, TOC and TOWW are at the same elevation. Forms cluster with GW-380 and GW-382. Data base revised on 2/18/97 to reflect changes discovered during periodic review. Dedicated portable WW pump used in this well, TOC and TOWW are at the same elevation. Forms cluster with GW-380 and GW-381. Data base revised on 2/18/97 to reflect changes discovered during periodic review. March 1997, TDECDOE-Oversite injected Fluorescein dye in this well

Forms cluster with GW-384 and GW-385. Data base revised on 2/18/97 to reflect changes discovered during periodic review. TOWW value estimated for this well.

Forms cluster with GW-383 and GW-385. Data base revised on 2/18/97 to reflect changes discovered during periodic review.

Forms cluster with GW-383 and GW-384 Data base revised on 2/18/97 to reflect changes discovered during periodic review. Note: no WW installed in this well.

TD-1251 ft. Backfilled w/grout, 1251.0-1134.0 ft, w/bentonite, 1134.0 to $650.0 \mathrm{ft}$, w/grout 650.0 to $577.0 \mathrm{ft}$. Core hole converted to Open Hole. Data base revised on $2 / 25 / 97$ to reflect changes discovered during periodic review. 


\section{CURRENT}

NAME

GW-401

GW-402

GW-403

GW-404

GW-405

GW-406

GW-407

GW-408

GW-409

GW-410

GW-411

GW-412

GW-413

GW-414

GW-415

\section{REFERENCE}

Golder Associates(1988b), Martin Marietta Energy Systems(1988a)

Golder Associates(1988b), Martin Marietta Energy Systems(1988a)

Golder Associates(1988b), Martin Marietta Energy Systems(1988a)

Martin Marietta Energy Systems(1988a), Ketelle, R. H.(1993)

Energy Division(1988), Martin Marietta Energy Systems(1988a)

Energy Division(1988), Martin Marietta Energy Systems(1988a)

Energy Division(1988), Martin Marietta Energy Systems(1988a)

Energy Division(1988), Martin Marietta Energy Systems(1988a)

Energy Division(1988), Martin Marietta Energy Systems(1988a)

Energy Division(1988), Martin Marietta Energy Systems(1988a)

Energy Division(1988), Martin Marietta Energy Systems(1988a)

Energy Division(1988), Martin Marietta Energy Systems(1988a)

Energy Division(1988), Martin Marietta Energy Systems(1988a)

Energy Division(1988), Martin Marietta Energy Systems(1988a)

Energy Division(1988), Martin Marietta Energy Systems(1988a)

\section{COMMENTS}

1 in. OD PVC/\#80 w/ perforated (3/8 in dia holes) galvanized steel from 852-812.1 Core hole converted to screened well. Data base revised on $2 / 25 / 97$ to reflect changes discovered during periodic review.

1 in OD PVC/\#80 w/perforated (3/8 in dia holes) black steel pipe from 373.4-399.3 Core hole converted to screened well. Data base revised on $2 / 25 / 97$ to reflect changes discovered during periodic review

Hole tremied w/bentonite 613-357, grout 357-300, 1 in OD PVC/\#80 sfc-

275. Core hole converted to screened well. Data base revised on $2 / 25 / 97$ to reflect changes discovered during periodic review.

Core hole converted to Open Hole. Functional Area: Gum Branch Road Area

Forms cluster with GW-406. Data base revised on 2/20/97 to reflect changes discovered during periodic review.

Forms cluster with GW-405. Hole drilled to 6.8, caved to 6.6. Data base revised on 2/20/97 to reflect changes discovered during periodic review.

Forms cluster with GW-408. Data base revised on 2/20/97 to reflect changes discovered during periodic review.

Forms cluster with GW-407. Hole drilled to to 6.5, caved to 5.9. Data base revised on 2/20/97 to reflect changes discovered during periodic review.

Forms cluster with GW-410. Data base revised on 2/20/97 to reflect changes discovered during periodic review.

Forms cluster with GW-409. Hole drilled to 16.8 , caved to 13.75 . Data base revised on $2 / 20 / 97$ to reflect changes discovered during periodic review.

Forms cluster with GW-412. Hole drilled to 8.7, caved to 8.35. Data base revised on $2 / 20 / 97$ to reflect changes discovered during periodic review.

Forms cluster with GW-411. Data base revised on 2/20/97 to reflect changes discovered during periodic review.

Forms cluster with GW-414. Hole drilled to 15.0, caved to 13.55. Data base revised on 2/21/97 to reflect changes discovered during periodic review.

Forms cluster with GW-413. Data base revised on 2/21/97 to reflect changes discovered during periodic review.

Forms cluster with GW-416. Data base revised on 2/21/97 to reflect changes discovered during periodic review. 


\section{CURRENT}

NAME

GW-416

GW-417

GW-418

GW-419

GW-420

GW-421

GW-422

GW-423

GW-424

GW-425

GW-426

GW-427

GW-428

GW-429

GW-430

GW-431

GW-432

\section{REFERENCE}

Energy Division(1988), Martin Marietta Energy Systems(1988a)

Energy Division(1988), Martin Marietta Energy Systems(1988a)

Energy Division(1988), Martin Marietta Energy Systems(1988a)

Energy Division(1988), Martin Marietta Energy Systems(1988a)

Energy Division(1988), Martin Marietta Energy Systems(1988a)

Energy Division(1988), Martin Marietta Energy Systems(1988a)

Energy Division(1988), Martin Marietta Energy Systems(1988a)

Energy Division(1988), Martin Marietta Energy Systems(1988a)

Energy Division(1988), Martin Marietta Energy Systems(1988a)

Energy Division(1988), Martin Marietta Energy Systems(1988a)

Energy Division(1988), Martin Marietta Energy Systems(1988a)

Energy Division(1988), Martin Marietta Energy Systems(1988a)

Energy Division(1988), Martin Marietta Energy Systems(1988a)

Energy Division(1988), Martin Marietta Energy Systems(1988a)

Energy Division(1988), Martin Marietta Energy Systems(1988a)

Energy Division(1988), Martin Marietta Energy Systems(1988a)

Energy Division(1988), Martin Marietta Energy Systems(1988a)

\section{COMMENTS}

Forms cluster with GW-415. Data base revised on 2/21/97 to reflect changes discovered during periodic review.

Forms cluster with GW-418. Data base revised on 2/21/97 to reflect changes discovered during periodic review.

Forms cluster with GW-417. Data base revised on 2/21/97 to reflect changes discovered during periodic review.

Forms cluster with GW-420. Data base revised on 2/21/97 to reflect changes discovered during periodic review.

Forms cluster with GW-419. Data base revised on 2/21/97 to reflect changes discovered during periodic review.

Forms cluster with GW-422. Data base revised on 2/21/97 to reflecr changes discovered during periodic review.

Forms cluster with GW-421. Data base revised on 2/21/97 to reflect changes discovered during periodic review.

Forms cluster with GW-424. Data base revised on 2/21/97 to reflect changes discovered during periodic review.

Forms cluster with GW-423. Data base revised on 2/21/97 to reflect changes discovered during periodic review.

Forms cluster with GW-426. Data base revised on 2/21/97 to reflect changes discovered during periodic review.

Forms cluster with GW-425. Data base revised on 2/21/97 to reflect changes discovered during periodic review.

Forms cluster with GW-428. Data base revised on 2/21/97 to reflect changes discovered during periodic review.

Forms cluster with GW-427. Data base revised on 2/21/97 to reflect changes discovered during periodic review.

Forms cluster with GW-430. Data base revised on 2/21/97 to reflect changes discovered during periodic review.

Forms cluster with GW-429. Data base revised on 2/21/97 to reflect changes discovered during periodic review.

Forms cluster with GW-432. Data base revised on 2/21/97 to reflect changes discovered during periodic review.

Forms cluster with GW-431. Data base revised on 2/21/97 to reflect changes discovered during periodic review. 


\section{CURRENT}

NAME

GW-433

GW-434

GW-435

GW-436

GW-437

GW-438

GW-439

GW-440

GW-441

GW-442

GW-443

GW-445

GW-447

GW-448

GW-449

\section{REFERENCE}

Energy Division(1988), Martin Marietta Energy Systems(1988a)

Energy Division(1988), Martin Marietta Energy Systems(1988a)

Energy Division(1988), Martin Marietta Energy Systems(1988a)

Energy Division(1988), Martin Marietta Energy Systems(1988a)

Energy Division(1988), Martin Marietta Energy Systems(1988a)

Energy Division(1988), Martin Marietta Energy Systems(1988a)

Energy Division(1988), Martin Marietta Energy Systems(1988a)

Energy Division(1988), Martin Marietta Energy Systems(1988a)

Energy Division(1988), Martin Marietta Energy Systems(1988a)

Energy Division(1988), Martin Marietta Energy Systems(1988b)

Energy Division(1988), Martin Marietta Energy Systems(1988a), King, H.L., Haase, C.S., and LaRue, D.L.(1989)

Energy Division(1988), Martin Marietta Energy Systems(1988a), King, H.L., Haase, C.S., and LaRue, D.L.(1989)

Energy Division(1988), Martin Marietta Energy Systems(1988a), SAIC(1995b)

Energy Division(1988), Martin Marietta Energy Systems(1988a), SAIC(1997c)

Energy Division(1988), Martin Marietta Energy Systems(1988a)

\section{COMMENTS}

Re-survey 04/24/95. Functional Area: Gum Branch Road Area

Forms cluster with GW-433. Data base revised on 2/21/97 to reflect changes discovered during periodic review.

Forms cluster with GW-436. Hole drilled to 9.0, caved to 8.4. Re-survey $04 / 24 / 95$. Data base revised on $2 / 21 / 97$ to reflect changes discovered during periodic review.

Forms cluster with GW-435. Data base revised on 2/21/97 to reflect changes discovered during periodic review.

Forms cluster with GW-438. Re-survey by Ogden 1995. Data base revised on 2/21/97 to reflect changes discovered during periodic review. Forms cluster with GW-437. Re-survey by Ogden 1995. Data base revised on 2/21/97 to reflect changes discovered during periodic review. Forms cluster with GW-440. Re-survey by Ogden 1995. Data base revised on 2/21/97 to reflect changes discovered during periodic review. Forms cluster with GW-439. Data base revised on 2/21/97 to reflect changes discovered during periodic review.

Forms cluster with GW-442. Data base revised on 2/21/97 to reflect changes discovered during periodic review.

Forms cluster with GW-441. Re-survey 04/24/95. Data base revised on $2 / 21 / 97$ to reflect changes discovered during periodic review.

Re-survey 04/24/95. Data base revised on $2 / 21 / 97$ to reflect changes discovered during periodic review.

Re-survey 04/24/95. Data base revised on 2/21/97 to reflect changes discovered during periodic review.

Re-survey by Ogden 1995. Data base revised on 2/21/97 to reflect changes discovered during periodic review.

Re-survey by Ogden 1995. Data base revised on 2/21/97 to reflect changes discovered during periodic review.

Forms cluster with GW-450. Hole drilled to 12.2, caved to 11.5. Status Date: ?Mar87. Data base revised on 2/21/97 to reflect changes discovered during periodic review. 


\section{CURRENT}

NAME

GW-450

GW-451

GW-452

GW-453

GW-455

GW-456

GW-457

GW-458

GW-459

GW-460

GW-461

GW-462

GW-463

GW-464

GW-465

GW-466

\section{REFERENCE}

Energy Division(1988), Martin Marietta Energy Systems(1988a)

Energy Division(1988), Martin Marietta Energy Systems(1988a)

Energy Division(1988), Martin Marietta Energy

Systems(1988a), SAIC(1997c)

Golder Associates(1988b), Martin Marietta Energy Systems(1988a)

Golder Associates(1988a), Martin Marietta Energy Systems(1988a)

Golder Associates(1988b), Martin Marietta Energy Systems(1988a)

Golder Associates(1988b), Martin Marietta Energy Systems(1988a)

Golder Associates(1988b), Martin Marietta Energy Systems(1988a)

Golder Associates(1988b), Martin Marietta Energy Systems(1988a)

Golder Associates(1988b), Martin Marietta Energy Systems(1988a)

Golder Associates(1988b), Martin Marietta Energy Systems(1988a)

Golder Associates(1988b), Martin Marietta Energy Systems(1988a)

Golder Associates(1988b), Martin Marietta Energy Systems(1988a)

Golder Associates(1988b), Martin Marietta Energy Systems(1988a)

Golder Associates(1988b), Martin Marietta Energy Systems(1988a)

Golder Associates(1988b), Martin Marietta Energy Systems(1988a)

\section{COMMENTS}

Forms cluster with GW-449. Data base revised on 2/21/97 to reflect changes discovered during periodic review.

Data base revised on 2/28/97 to reflect changes discovered during periodic review.

Auger refusal at $19.0 \mathrm{ft}$. Re-survey by Ogden 1995. Data base revised on $2 / 28 / 97$ to reflect changes discovered during periodic review.

Existing core hole modified to this status. Core hole converted to screened well. Data base revised on 2/25/97 to reflect changes discovered during periodic review.

Sfc casing depth listed as 10.2 \& 11.4, TD-195.5 grouted, 195.5-193.0 bentonite slurry. Data base revised on $2 / 26 / 97$ to reflect changes discovered during periodic review.

Installed in same hole as GW-457. Data base revised on 2/26/97 to reflect changes discovered during periodic review.

located in same hole as GW-456. Data base revised on 2/26/97 to reflect changes discovered during periodic review.

Located in same hole as GW-459. Data base revised on 2/26/97 to reflect changes discovered during periodic review.

Located in same hole as GW-458. Data base revised on 2/26/97 to reflect changes discovered during periodic review.

Located in same hole as GW-461. Data base revised on 2/26/97 to reflect changes discovered during periodic review.

Located in same hole as GW-460. Data base revised on 2/26/97 to reflect changes discovered during periodic review.

Data base revised on 2/26/97 to reflect changes discovered during peiodic review.

No hole diameter given in log, assume to be 8.75-in. diam. Data base revised on 2/26/97 to reflect changes discovered during periodic review.

Data base revised on 2/26/97 to reflect changes discovered during periodic review.

Hole caved to $41.09 \mathrm{ft}$. Data base revised on 2/26/97 to reflect changes discovered during periodic review.

Hole drilled to 48 , caved in to 42.2. Data base revised on 2/26/97 to reflect changes discovered during periodic review. 
CURRENT

NAME

GW-467

GW-468

GW-469

GW-470

GW-471

GW-472

GW-473

GW-474

GW-475A

GW-475B

GW-475C

GW-476A

GW-476B

GW-476C

\section{REFERENCE}

Golder Associates(1988b), Martin Marietta Energy Systems(1988a)

Golder Associates(1988b), Martin Marietta Energy Systems(1988a)

Golder Associates(1988b), Martin Marietta Energy Systems(1988a)

Golder Associates(1988b), Martin Marietta Energy Systems(1988a)

Golder Associates(1988a), Martin Marietta Energy Systems(1988a)

Golder Associates(1988b), Martin Marietta Energy Systems(1988a)

Golder Associates(1988a), Martin Marietta Energy Systems(1988a)

Golder Associates(1988a), Martin Marietta Energy Systems(1988a)

Golder Associates(1988a), Martin Marietta Energy Systems(1988a)

Golder Associates(1988a), Martin Marietta Energy Systems(1988a)

Golder Associates(1988a), Martin Marietta Energy Systems(1988a)

Golder Associates(1988a), Martin Marietta Energy Systems(1988a)

Golder Associates(1988a), Martin Marietta Energy Systems(1988a)

Golder Associates(1988a), Martin Marietta Energy Systems(1988a)

\section{COMMENTS}

Borehole collapsed to approximately $17.5 \mathrm{ft}$, filterpack sand bridged with top at $15.0 \mathrm{ft}$, bottom depth unknown. Section of 6.00-in. steel casing installed from 35-15 ft? Data base revised on 2/26/97 to reflect changes discovered during periodic review.

Core hole converted to Open Hole. Functional Area: Gum Branch Road Area

Data base revised on $2 / 26 / 97$ to reflect changes discovered during periodic review.

Data base revised on $2 / 26 / 97$ to reflect changes discovered during periodic review.

3.70 in corehole reamed with a 3.875 roller bit. Data base revised on $2 / 26 / 97$ to reflect changes discovered during periodic review.

Data base revised on $2 / 26 / 97$ to reflect changes discovered during periodic review.

Bentonite cap from 68-66, sand 66-65.5, grout to sfc. Data base revised on $2 / 26 / 97$ to reflect changes discovered during periodic review.

Data base revised on 2/27/97 to reflect changes discovered during periodic review.

Wells GW-475A, GW-475B, and GW-475C located in same drill hole. Data abase revised on $2 / 27 / 97$ to reflect changes discovered during periodic review.

Wells GW-475A, GW-475B, and GW-475C located in same drill hole.

Data base revised on $2 / 27 / 97$ to reflect changes discovered during periodic review.

Wells GW-475A, GW-475B, and GW-475C located in same drill hole.

Data base revised on $2 / 27 / 97$ to reflect changes discovered during periodic review.

Wells GW-476A, GW-476B, and GW-476C located in same drill hole. Data base revised on $2 / 27 / 97$ to reflect changes discovered during periodic review.

Wells GW-476A, GW-476B, and GW-476C located in same drill hole. Data base revised on $2 / 27 / 97$ to reflect changes discovered during periodic review.

Wells GW-476A, GW-476B, and GW-476C located in same drill hole.

Data base revised on $2 / 27 / 97$ to reflect changes discovered during periodic review. 
CURRENT

NAME

GW-477A

GW-477B

GW-477C

GW-478A

GW-478B

GW-478C

GW-479

GW-480A

GW-480B

GW-480C

GW-481A

GW-481B

\section{REFERENCE}

Golder Associates(1988a), Martin Marietta Energy Systems(1988a)

Golder Associates(1988a), Martin Marietta Energy Systems(1988a)

Golder Associates(1988a), Martin Marietta Energy Systems(1988a)

Golder Associates(1988a), Martin Marietta Energy Systems(1988a)

Golder Associates(1988a), Martin Marietta Energy Systems(1988a)

Golder Associates(1988a), Martin Marietta Energy Systems(1988a)

Golder Associates(1988a), Martin Marietta Energy Systems(1988a)

Golder Associates(1988a), Martin Marietta Energy Systems(1988a)

Golder Associates(1988a), Martin Marietta Energy Systems(1988a)

Golder Associates(1988a), Martin Marietta Energy Systems(1988a)

Golder Associates(1988a), Martin Marietta Energy Systems(1988a)

Golder Associates(1988a), Martin Marietta Energy Systems(1988a)

\section{COMMENTS}

Wells GW-477A, GW-477B, and GW-477C located in same drill hole. Data base revised on $2 / 27 / 97$ to reflect changes discovered during periodic review.

Wells GW-477A, GW-477B, and GW-477C located in same drill hole.

Data base revised on $2 / 27 / 97$ to reflect changes discovered during periodic review.

Wells GW-477A, GW-477B, and GW-477C located in same drill hole.

Data base revised on $2 / 27 / 97$ to reflect changes discovered during periodic review.

Wells GW-478A, GW-478B, and GW-478C located in same drill hole.

Data base revised on $2 / 27 / 97$ to reflect changes discovered during periodic review.

Wells GW-478A, GW-478B, and GW-478C located in same drill hole.

Data base revised on $2 / 27 / 97$ to reflect changes discovered during periodic review.

Wells GW-478A, GW-478B, and GW-478C located in same drill hole.

Data base revised on $2 / 27 / 97$ to reflect changes discovered during periodic review.

Data base revised on $2 / 27 / 97$ to reflect changes discovered during periodic review.

Wells GW-480A, GW-480B, and GW-480C located in same drill hole.

Data base revised on $2 / 27 / 97$ to reflec tchanges discovered during periodic review.

Wells GW-480A, GW-480B, and GW-480C located in same drill hole.

Data base revised on $2 / 27 / 97$ to reflect changes discovered during periodic review.

Wells GW-480A, GW-480B, and GW-480C located in same drill hole. Data base revised on $2 / 27 / 97$ to reflect changes dicovered during periodic review.

Wells GW-481A, GW-481B, and GW-481C located in same drill hole. Data base revised on $2 / 27 / 97$ to reflect changes discovered during periodic review.

Wells GW-481A, GW-481B, and GW-481C located in same drill hole.

Data base revised on $2 / 27 / 97$ to reflect changes discovered during periodic review. 


\section{CURRENT}

\section{NAME}

GW-481C

GW-482A

GW-482B

GW-482C

GW-483

GW-484

GW-485

GW-486

GW-487

GW-488

GW-489

GW-490

GW-491

GW-492

GW-493

\section{REFERENCE}

Golder Associates(1988a), Martin Marietta Energy Systems(1988a)

Golder Associates(1988a), Martin Marietta Energy Systems(1988a)

Golder Associates(1988a), Martin Marietta Energy Systems(1988a)

Golder Associates(1988a), Martin Marietta Energy Systems(1988a)

Golder Associates(1988a), Martin Marietta Energy Systems(1988a)

Golder Associates(1988a), Martin Marietta Energy Systems(1988a)

Golder Associates(1988a), Martin Marietta Energy Systems(1988a)

Golder Associates(1988a), Martin Marietta Energy Systems(1988a)

Golder Associates(1988a), Martin Marietta Energy Systems(1988a)

Golder Associates(1988a), Martin Marietta Energy Systems(1988a)

Golder Associates(1988a), Martin Marietta Energy Systems(1988a)

Golder Associates(1988a), Martin Marietta Energy Systems(1988a)

Golder Associates(1988a), Martin Marietta Energy Systems(1988a)

Golder Associates(1988a), Martin Marietta Energy Systems(1988a)

Golder Associates(1988a), Martin Marietta Energy Systems(1988a)

\section{COMMENTS}

Wells GW-481A, GW-481B, and GW-481C located in same drill hole. Data base revised on $2 / 27 / 97$ to reflect changes discovered during periodic review.

Wells GW-482A, GW-482B, and GW-482C located in same drill hole.

Data base revised on $2 / 27 / 97$ to reflect changes discovered during periodic review.

Wells GW-482A, GW-482B, and GW-482C located in same drill hole.

Data base revised on $2 / 27 / 97$ to reflect changes discovered during periodic review.

Wells GW-482A, GW-482B, and GW-482C located in same drill hole.

Data base revised on $2 / 27 / 97$ to reflect changes discovered during periodic review.

Data base revised on $2 / 27 / 97$ to reflect changes discovered during periodic review.

Data base revised on $2 / 27 / 97$ to reflect changes discovered during periodic review.

Auger refusal @ $20.5 \mathrm{ft}$. Data base revised on 2/27/97 to reflect changes discovered during periodic review.

Auger refusal @ $16.8 \mathrm{ft}$. Data base revised on 2/27/97 to reflect changes discovered during periodic review.

Auger refusal @ $21 \mathrm{ft}$. Data base revised on 2/27/97 to reflect changes discovered during periodic review.

Auger refusal @ $17 \mathrm{ft}$. Data base revised on 2/27/97 to reflect changes discovered during periodic review.

No hole diameter given in well log, assume it to be 6-in. diameter. Auger refusal @ $21.5 \mathrm{ft}$. Data base revised on 2/27/97 to reflect changes discovered during periodic review.

Auger refusal @ $19 \mathrm{ft}$. Data base revised on 2/27/97 to reflect changes discovered during periodic review.

Auger refusal @ $17 \mathrm{ft}$. Data base revised on 2/27/97 to reflect changes discovered during periodic review.

Auger refusal at $14.1 \mathrm{ft}$, assume refusal on weathered bedrock. Data base revised on 2/27/97 to reflect changes discovered during periodic review.

Auger refusal at $19.3 \mathrm{ft}$, assume refusal on weathered bedrock. Data base revised on $2 / 27 / 97$ to reflect changes discovered during periodic review. 
CURRENT

NAME

GW-494

GW-495

GW-496

GW-497

GW-498

GW-499

GW-499A

GW-499AA

GW-499AB

GW-499AC

GW-499AD

GW-499AE

GW-499AF

GW-499B

GW-499C

\section{REFERENCE}

Golder Associates(1988a), Martin Marietta Energy Systems(1988a)

Golder Associates(1988a), Martin Marietta Energy Systems(1988a)

Golder Associates(1988a), Martin Marietta Energy Systems(1988a)

Golder Associates(1988a), Martin Marietta Energy Systems(1988a)

Golder Associates(1988a), Martin Marietta Energy Systems(1988a)

Golder Associates(1988a), Martin Marietta Energy Systems(1988a)

Golder Associates(1988a), Martin Marietta Energy

Systems(1988a)

Martin Marietta Energy Systems(1988a), Ketelle, R. H.(1993)

Martin Marietta Energy Systems(1988a), Ketelle, R. H.(1993)

Martin Marietta Energy Systems(1988a), Ketelle, R. H.(1993)

Martin Marietta Energy Systems(1988b), Ketelle, R. H.(1993)

Martin Marietta Energy Systems(1988a), Ketelle, R. H.(1993)

Martin Marietta Energy Systems(1988a), Ketelle, R. H.(1993)

Martin Marietta Energy Systems(1988a), Ketelle, R. H.(1993)

Martin Marietta Energy Systems(1988a), Ketelle, R. H.(1993)

\section{COMMENTS}

Auger refusal at $16.5 \mathrm{ft}$, assume refusal on weathered bedrock. Hole caved to14.2 ft. Data base revised on 2/27/97 to reflect changes discovered during periodic review.

Auger refusal at $7.7 \mathrm{ft}$, assume refusal on weathered bedrock. Hole caved to 6.3. Data base revised on $2 / 27 / 97$ to reflect changes discovered during periodic review.

Hole diameter not listed, assume it to be 6 -in. Auger refusal at $6.7 \mathrm{ft}$, assume refusal on weathered bedrock. Hole caved to $6.0 \mathrm{ft}$. Data base revised on $2 / 27 / 97$ to reflect changes discovered during periodic review. Auger refusal at $8.2 \mathrm{ft}$, asume refusal on weathered bedrock.. Hole caved to $6.7 \mathrm{ft}$. Data base revised on 2/27/97 to reflect changes discovered during periodic review.

Auger refusal at $5.0 \mathrm{ft}$, assume refusal on weathered bedrock. Data base reviesc on $2 / 27 / 97$ to reflect changes discovered during periodic review.

Auger refusal at $5.0 \mathrm{ft}$, assume refusal on weathered bedrock. Data base revised on 2/27/97 to reflect changes discovered during periodic review.

Auger refusal at $6.2 \mathrm{ft}$, assume refusal on weathered bedrock. Bottom of screen taken from Materials Inventory no bottom of screen listed on construction diagram. Data base revised on 2/28/97 to reflect changes discovered during periodic review.

Data base revised on $4 / 18 / 97$ to reflect changes discovered during periodic review.

Data base revised on $4 / 18 / 97$ to reflect changes discovered during periodic review.

Data base revised on 4/18/97 to reflect changes discovered during periodic review. Could not locate well during 2001 well inspection.

Data base revised on 4/18/97 to reflect changes discovered during periodic review. Could not locate well during 2001 well inspection.

Data base revised on $4 / 18 / 97$ to reflect changes discovered during periodic review. Could not locate well during 2001 well inspection.

Data base revised on 4/18/97 to reflect changes discovered during periodic review. Could not locate well during 2001 well inspection.

Shallow hand augered piezometer. Data base revised on 4/18/97 to reflect changes discovered during periodic review.

Shallow hand augered piezometer. Data base revised on 4/18/97 to reflect changes discovered during periodic review. 


\section{CURRENT} NAME

GW-499D

GW-499E

GW-499F

GW-499G

GW-499H

GW-499I

GW-499J

GW-499K

GW-499L

GW-499M

GW-499N

GW-4990

GW-499P

\section{REFERENCE}

Martin Marietta Energy Systems(1988a), Ketelle, R. H.(1993)

Martin Marietta Energy Systems(1988a), Ketelle, R. H.(1993)

Martin Marietta Energy Systems(1988a), Ketelle, R. H.(1993)

Golder Associates(1988a), Martin Marietta Energy Systems(1988a)

Golder Associates(1988a), Martin Marietta Energy Systems(1988a)

Golder Associates(1988a), Martin Marietta Energy Systems(1988a)

Golder Associates(1988a), Martin Marietta Energy Systems(1988a)

Golder Associates(1988a), Martin Marietta Energy Systems(1988a)

Golder Associates(1988a), Martin Marietta Energy Systems(1988a)

Golder Associates(1988a), Martin Marietta Energy Systems(1988a)

Golder Associates(1988a), Martin Marietta Energy Systems(1988a)

Golder Associates(1988a), Martin Marietta Energy Systems(1988a)

Golder Associates(1988a), Martin Marietta Energy Systems(1988a)

\section{COMMENTS}

Shallow hand augered piezometer. Data base revised on 4/18/97 to reflect changes discovered during periodic review.

Shallow hand augered piezometer. Data base revised on 4/18/97 to reflect changes discovered during periodic review.

Shallow hand augered piezometer. Data base revised on 4/18/97 to reflect changes discovered during periodic review. Could not locate well during 2001 well inspection.

Auger refusal at $14 \mathrm{ft}$, assume refusal on weathered bedrock. Data base revised on $2 / 28 / 97$ to reflect changes discovered during periodic review.

Auger refusal at $12.5 \mathrm{ft}$, assume refusal on weathered bedrock. Hole caved to $11 \mathrm{ft}$. Data base revised on 2/28/97 to reflect changes discovered during periodic review.

Auger refusal at $10.5 \mathrm{ft}$, assume refusal on weathered bedrock. Hole caved to $9 \mathrm{ft}$. Data base revised on 2/28/97 to reflect changes discovered during periodic review.

Auger refusal at $10 \mathrm{ft}$, assume refusal on weathered bedrock. Hole caved to $8.6 \mathrm{ft}$. Data base revised on 2/28/97 to reflect changes discovered during periodic review.

Auger refusal at $12.3 \mathrm{ft}$, assume refusal on weathered bedrock. Hole caved to $10 \mathrm{ft}$. Data base revised on 2/28/97 to reflect changes discovered during periodic review.

Auger refusal at $15 \mathrm{ft}$, assume refusal on weathered bedrock. Hole caved

to $12 \mathrm{ft}$. Data base revised on 2/28/97 to reflect changes discovered during periodic review.

Auger refusal at $12.3 \mathrm{ft}$, assume refusal on weathered bedrock. Hole caved to $11.6 \mathrm{ft}$. Data base revised on 2/28/97 to reflect changes discovered during periodic review.

Auger refusal at $13 \mathrm{ft}$, assume refusal on weathered bedrock. Hole caved at $12 \mathrm{ft}$. Data base revised on 2/28/97 to reflect changes discovered during periodic review.

Auger refusal at $13 \mathrm{ft}$, assume refusal on weathered bedrock. Hole caved to $11.3 \mathrm{ft}$. No hole diameter given, assume it to be 6-in. Data base revised on $2 / 28 / 97$ to reflect changes discovered during periodic review.

Auger refusal at $15 \mathrm{ft}$, assume refusal on weathered bedrock. Hole caved to $13.2 \mathrm{ft}$. Data base revised on 2/28/97 to reflect changes discovered during periodic review. 
CURRENT NAME

GW-499Q

GW-499R

GW-499S

GW-499T

GW-499U

GW-499V

GW-499W

GW-499X

GW-499Y

GW-499Z

GW-502

GW-505

GW-506

GW-507

GW-508

\section{REFERENCE}

Martin Marietta Energy Systems(1988a), Ketelle, R. H.(1993)

Martin Marietta Energy Systems(1988a), Ketelle, R. H.(1993)

Martin Marietta Energy Systems(1988a), Ketelle, R. H.(1993)

Martin Marietta Energy Systems(1988a), Ketelle, R. H.(1993)

Martin Marietta Energy Systems(1988a), Ketelle, R. H.(1993)

Martin Marietta Energy Systems(1988a), Ketelle, R. H.(1993)

Martin Marietta Energy Systems(1988a), Ketelle, R. H.(1993)

Martin Marietta Energy Systems(1988a), Ketelle, R. H.(1993)

Martin Marietta Energy Systems(1988a), Ketelle, R. H.(1993)

Martin Marietta Energy Systems(1988a), Ketelle, R. H.(1993)

EDGe, Inc.(1989), King, H.L., Haase, C.S., and LaRue, D.L.(1989), SAIC(1993h)

EDGe, Inc.(1989), King, H.L., Haase, C.S., and LaRue, D.L.(1989)

EDGe, Inc.(1989), King, H.L., Haase, C.S., and LaRue, D.L.(1989), SAIC(1992b)

EDGe, Inc.(1989), King, H.L., Haase, C.S., and LaRue D.L.(1989), SAIC(1992b)

EDGe, Inc.(1989), King, H.L., Haase, C.S., and LaRue D.L.(1989)

\section{COMMENTS}

No hole diameter given

No hole diameter given

No hole diameter given. No Information on packed interval given.

No hole diameter given. No Information on packed interval given.

T.D. unknown.

T.D. unknown.

T.D. unknown.

Data base revised on $2 / 28 / 97$ to reflect changes discovered during periodic review.

Well resurveyed due to well head modifications. Data base revised on $2 / 28 / 97$ to reflect changes discovered during periodic review. TOWW value estimated for this well.

Well completely removed while excavating adjacent underground storage tank. Data base revised on 2/28/97 to reflect changes discovered during periodic review.

Well damaged; reconfigured as flush-mount design, but not resurveyed. Data base revised on $2 / 28 / 97$ to reflect changes discovered during periodic review.

Well resurveyed due to well head modifications. Data base revised on $3 / 3 / 97$ to reflect changes discovered during periodic review. Well casing is constricted close to ground surface. Probably due to freeze-thaw issues above the frost line. Pump cannot be removed (SBJ 9/15/2011) 
CURRENT

NAME

GW-509

GW-511

GW-512

GW-513

GW-514

GW-520

GW-521

GW-522

GW-526

GW-527

GW-528

GW-531

GW-532

GW-533

GW-534

\section{REFERENCE}

EDGe, Inc.(1989), King, H.L., Haase, C.S., and LaRue D.L.(1989), SAIC(1992b)

EDGe, Inc.(1989), King, H.L., Haase, C.S., and LaRue D.L.(1989)

EDGe, Inc.(1989), King, H.L., Haase, C.S., and LaRue,

D.L.(1989)

EDGe, Inc.(1989), King, H.L., Haase, C.S., and LaRue, D.L.(1989)

EDGe, Inc.(1989), King, H.L., Haase, C.S., and LaRue D.L.(1989)

EDGe, Inc.(1989), King, H.L., Haase, C.S., and LaRue D.L.(1989)

EDGe, Inc.(1989), King, H.L., Haase, C.S., and LaRue, D.L.(1989)

EDGe, Inc.(1989), King, H.L., Haase, C.S., and LaRue D.L.(1989)

EDGe, Inc.(1989), King, H.L., Haase, C.S., and LaRue D.L.(1989)

Geraghty and Miller, Inc.(1988a), King, H.L., Haase, C.S., and LaRue, D.L.(1989)

Geraghty and Miller, Inc.(1988a), King, H.L., Haase, C.S., and LaRue, D.L.(1989)

EDGe, Inc.(1989), King, H.L., Haase, C.S., and LaRue D.L.(1989)

EDGe, Inc.(1989), King, H.L., Haase, C.S., and LaRue, D.L.(1989)

$\operatorname{ERCE}(1991)$

$\operatorname{ERCE}(1991)$

\section{COMMENTS}

Well damaged; reconfigured as flush-mount design, but not resurveyed. Data base revised on $3 / 3 / 97$ to reflect changes discovered during periodic review.

Data base revised on $3 / 3 / 97$ to reflect changes discovered during periodic review.

Forms cluster with GW-513 and GW-514. Data base revised on 3/3/97 to reflect changes discovered during periodic review.

Forms cluster with GW-512 and GW-514. Data base revised on 3/3/97 to reflect changes discovered during periodic review. WW installed with a LCC.

Forms cluster with GW-512 and GW-513. Data base revised on 3/3/97 to reflect changes discovered during periodic review. Well Wizard pump and low clearance cap installed.

Forms cluster with GW-366 and GW-367. Data base revised on 3/3/97 to reflect changes discovered during periodic review.

Replaces GW-099. Data base revised on 3/3/97 to reflect changes discovered during periodic review. TOWW value estimated for this well.

Replaces GW-233. data base revised on 3/3/97 to reflect changes discovered during periodic review. TOWW value estimated for this well.

Forms cluster with GW-345 and GW-346. Data base revised on 3/3/97 to reflect changes discovered during periodic review. TOWW value estimated for this well.

Data base revised on $3 / 3 / 97$ to reflect changes discovered during periodic review.

Data base revised on $3 / 3 / 97$ to reflect changes discovered during periodic review.

Data base revised on 3/4/97 to reflect changes discovered during periodic review.

\section{Data bas}

Data base revised on $3 / 4 / 97$ to reflect changes discovered during periodic review. Re-survey 06/19/2002.

Data base revised on $3 / 4 / 97$ to reflect changes discovered during periodic review. 
CURRENT

NAME

GW-535

GW-536

GW-537

GW-538

GW-539

GW-540

GW-541

GW-542

GW-543

GW-544

GW-545

GW-546

GW-551

GW-552

GW-553

\section{REFERENCE}

EDGe, Inc.(1989), King, H.L., Haase, C.S., and LaRue D.L.(1989)

EDGe, Inc.(1989), King, H.L., Haase, C.S., and LaRue, D.L.(1989)

EDGe, Inc.(1989), King, H.L., Haase, C.S., and LaRue D.L.(1989)

ERCE(1991)

ERCE(1991)

$\operatorname{ERCE}(1991)$

ERCE(1991)

ERCE(1991)

$\operatorname{ERCE}(1991)$

$\operatorname{ERCE}(1991)$

ERCE(1991), SAIC(1995b)

ERCE(1991)

Golder Associates(1989), SAIC(1993h)

Golder Associates(1989)

Golder Associates(1989), SAIC(1993h)

\section{COMMENTS}

Data base revised on 3/4/97 to reflect changes discovered during periodic review.

Well destroyed during construction activities. Replaced by GW-630. Data base revised on $3 / 4 / 97$ to reflect changes discovered during periodic review.

Taped depth of $27.5 \mathrm{ft}$ which differs from constructed depth of $25.05 \mathrm{ft}$.

Data base revised on 3/4/97 to reflect changes discovered during periodic review. TOWW value estimated for this well. Well re-survey 06/20/2003.

Data base revised on $3 / 4 / 97$ to reflect changes discovered during periodic review.

Data base revised on $3 / 4 / 97$ to reflect changes discovered during periodic review. TOWW value estimated for this well.

Forms cluster with GW-546. Data base revised on 3/4/97 to reflect changes discovered during periodic review. TOWW value estimated for this well.

Forms cluster with GW-545. Data base revised on 3/4/97 to reflect changes discovered during periodic review. A WW was installed \& removed, no WW present at this location at this time.

Data base revised on $3 / 4 / 97$ to reflect changes discovered during periodic review. TOWW value estimated for this well.

Data base revised on $3 / 4 / 97$ to reflect changes discovered during periodic review. TOWW value estimated for this well.

Data base revised on $3 / 4 / 97$ to reflect changes discovered during periodic review. TOWW value estimated for this well.

Forms cluster with GW-541. Data base revised on 3/5/97 to reflect changes discovered during periodic review.

Forms cluster with GW-540. Data base revised on 3/5/97 to reflect changes discovered during periodic review.

Casing depth listed as both 90.0 and $90.5 \mathrm{ft}$. Core hole converted to screened well. Data base revised on 3/5/97 to reflect changes discovered during periodic review.

Casing depth listed as both 102.8 and $101.0 \mathrm{ft}$. Core hole converted to screened well. Data base revised on $3 / 5 / 97$ to reflect changes discovered during periodic review.

Core hole converted to screened well. Data base revised on 3/5/97 to reflect changes discovered during periodic review. 


\section{CURRENT}

\section{NAME}

GW-554

GW-555

GW-556

GW-557

GW-558

GW-559

GW-560

GW-561

GW-562

GW-563

GW-564

GW-565

\section{REFERENCE}

Golder Associates(1989)

Golder Associates(1989)

Golder Associates(1989), SAIC(1993h)

Golder Associates(1989)

Golder Associates(1989)

Golder Associates(1989)

Golder Associates(1989)

Golder Associates(1989), SAIC(1993h)

Golder Associates(1989)

Golder Associates(1989)

Golder Associates(1989)

Golder Associates(1989), SAIC(1993h)

\section{COMMENTS}

Core hole converted to screened well. Data base revised on $3 / 5 / 97$ to reflect changes discovered during periodic review.

TD listed as both 314.35 and $314.1 \mathrm{ft}$. Core hole converted to screened well. Data base revised on $3 / 5 / 97$ to reflect changes discovered during periodic review.

TD listed as both 354.5 and $356.5 \mathrm{ft}$. Core hole converted to screened well. Data base revised on 3/5/97 to reflect changes discovered during periodic review.

Data base revised on 3/5/97 to reflect changes discovered durinmg periodic review. TOWW value estimated for this well.

Bentonite seal: 63.0-61.8 ft, sand from $61.8 \mathrm{ft}-61.2 \mathrm{ft}$, Bentonite from 61.2 to $60.2 \mathrm{ft}$, grout from $60.2 \mathrm{ft}$ to surface. Data base revised on $3 / 5 / 97$ to reflect changes discovered during periodic review.

Data base revised on $3 / 5 / 97$ to reflect changes discovered during periodic review.

TD listed as both 117.00 and $114.00 \mathrm{ft}$. Data base revised on 3/5 97 to reflect changes discovered during periodic revision. Well casing extended approx.10 $\mathrm{ft}$ due to construction activity at the CD Landfill VII. TOWW value estimated for this well.

A 1-in OD PVC/sl/.01 piezometer placed adjacent to 4-in OD SS well. Screened interval from 27.05 to $15.05 \mathrm{ft}$, sand pack from $28.00-14.00 \mathrm{ft}$, bentonite cap with grout to surface. Data base revised on $3 / 5 / 97$ to reflect changes discovered during periodic review.

Hole filled with gravel from 133.00-67.00 ft, bentonite from 67.00-60.00 ft. Data base revised on $3 / 5 / 97$ to reflect changes discovered during periodic review. TOWW value estimated for this well.

Hole caved from 104-96.8, sandpack 96.8-72.5, sand/pea gravel from 72.5$63 \mathrm{ft}$. Data base revised on 3/5/97 to reflect changes discovered during periodic review.

Hole caved from 88-81 ft, 47.5-52 ft, and from 33-30 ft, bentonite from 30$25 \mathrm{ft}$. Data base revised on 3/5/97 to reflect chamges discovered during periodic review. TOWW value estimated for this well.

Data base revised on $3 / 5 / 97$ to reflect changes discovered during periodic review. 


\section{CURRENT \\ NAME}

GW-566

GW-567

GW-568

GW-569

GW-570

GW-571

GW-572

GW-573D

GW-573S

GW-576

GW-580

GW-601

GW-602

GW-603

GW-604

\section{REFERENCE}

Golder Associates(1989), SAIC(1993h)

Golder Associates(1989)

Golder Associates(1989)

Golder Associates(1989)

Golder Associates(1989), SAIC(1994d)

Golder Associates(1989), SAIC(1993h)

Golder Associates(1989), SAIC(1993h)

Golder Associates(1989), SAIC(1993h)

Golder Associates(1989), SAIC(1993h)

Golder Associates(1988b), Golder Associates(1989)

Golder Associates(1988b), Golder Associates(1989), SAIC(1993h)

ERCE(1991)

ERCE(1991)

$\operatorname{ERCE}(1991)$

ERCE(1991)

\section{COMMENTS}

Hole caved from 188-174 ft, screened interval contains sand, plus gravel and clay (from partial collapse of the borehole). Data base revised on $3 / 5 / 97$ to reflect changes discovered during periodic review.

Hole caved from TD - $80 \mathrm{ft}$, sand pack $80-71 \mathrm{ft}$, gravel pack $71-57 \mathrm{ft}$. Sand pack 57.00-55.00 ft. Grout 55.00-sfc, (no bentonite seal). Data base revised on $3 / 5 / 97$ to reflect changes discovered during periodic review.

Final disposition of this location is unknown. Documentation is limited but it appears that this location may have only been a borehole with no well components installed. The location probably was backfilled after drilling.

Data base revised on $3 / 5 / 97$ to reflect changes discovered during periodic review.

Data base revised on $3 / 5 / 97$ to reflect changes discovered during periodic review.

Data base revised on $3 / 6 / 97$ to reflect changes discovered during periodic review.

Data base revised on $3 / 6 / 97$ to reflect changes discovered during periodic review.

Data base revised on $3 / 6 / 97$ to reflect changes discovered during periodic review.

Data base revised on $3 / 6 / 97$ to reflect changes discovered during periodic review.

Data base revised on $3 / 6 / 97$ to reflect changes discovered during periodic review.

Data base revised on $3 / 6 / 97$ to reflect changes discovered during periodic review.

Measured depth of well from TOC was $211.4 \mathrm{ft}$ after rehabilitation on 16Mar92. This differs from construction information which lists a depth of $214.2 \mathrm{ft}$ from TOC. Data base revised on 3/6/97 to relfect changes discovered during periodic review.

Forms cluster with GW-604. Data base revised on 3/6/97 to reflect changes discovered during periodic review.

Forms cluster with GW-603. Data base revised on 3/6/97 to reflect changes discovered during periodic review. 


\section{CURRENT}

\section{NAME}

GW-605

GW-606

GW-607

GW-608

GW-608A

GW-609

GW-610

GW-611

GW-612

GW-613

GW-614

GW-615

GW-616

GW-617

\section{REFERENCE}

SAIC(1991a)

SAIC(1991a)

ERCE(1991), SAIC(1993h)

ERCE(1991)

ERCE(1991)

$\operatorname{ERCE}(1991)$

ERCE(1991)

ERCE(1991)

ERCE(1991)

ERCE(1991)

ERCE(1991)

ERCE(1991)

ERCE(1991)

ERCE(1991)

\section{COMMENTS}

Data base revised on $3 / 6 / 97$ to reflect changes discovered during periodic review. TOWW value estimated for this well.

Data base revised on $3 / 6 / 97$ to reflect changes discovered during periodic review. TOWW value estimated for this well.

Data base revised on $3 / 6 / 97$ to reflect changes discovered during periodic review.

Data base revised on 3/6/97 to reflect changes discovered during periodic review. TOWW value estimated for this well. TOWW elevation changed 06/06/2005 to reflect proper WW configuration.

Well abandoned. Hole grouted closed with bit cemented in bottom of hole. Functional Area: Chestnut Ridge Security Pits

Data base revised on $3 / 6 / 97$ to reflect changes discovered during periodic review. TOWW value estimated for this well.

Data base revised on $3 / 6 / 97$ to reflect changes discovered during periodic review. WW installed with a LCC, TOC and TOWW are the same elevation.

Data base revised on $3 / 6 / 97$ to reflect changes discovered during periodic review. A WW pump with a LCC is used in this well, TOC and TOWW are at the same elevation.

Data base revised on 3/6/97 to reflect changes discovered during periodic review. A WW pump with a LCC is used in this well, TOC and TOWW are at the same elevation.

Forms cluster with GW-614. Data base revised on 3/6/97 to reflect changes discovered during periodic review. TOWW value estimated for this well.

Forms cluster with GW-613. Data base revised on 3/6/97 to reflect changes discovered during periodic review. TOWW value estimated for this well.

Data base revised on $3 / 6 / 97$ to reflect changes discovered during periodic review. TOWW value estimated for this well.

Data base revised on $3 / 6 / 97$ to reflect changes discovered during periodic review. Well Wizard pump and low clearance cap installed.

Forms cluster with GW-618. Data base revised on 3/6/97 to reflect changes discovered during periodic review. TOWW value estimated for this well. 


\section{CURRENT \\ NAME}

GW-618

GW-619

GW-620

GW-621

GW-622

GW-623

GW-624

GW-625

GW-626

GW-627

GW-628

GW-629

GW-630
ERCE(1991)

ERCE(1991)

\section{REFERENCE}

ERCE(1991)

ERCE(1991)

ERCE(1991)

ERCE(1991)

ERCE(1991)

ERCE(1991)

ERCE(1991)

ERCE(1991)

ERCE(1991)

ERCE(1991)

ERCE(1991)

\section{COMMENTS}

Forms cluster with GW-617. Data base revised on 3/6/97 to reflect changes discovered during periodic review. TOWW value estimated for this well.

Forms cluster with GW-620. Functional Area: Fire Training Facility. Data base revised on 3/7/97 to reflect changes discovered during periodic review. TOWW value estimated for this well.

Forms cluster with GW-619. Functional Area: Fire Training Facility. Data base revised on 3/7/97 to reflect changes discovered during periodic review. TOWW value estimated for this well.

Data base revised on 3/7/97 to reflect changes discovered during periodic review. TOWW value estimated for this well.

Data base revised on $3 / 7 / 97$ to reflect changes discovered during periodic review.

Borehole drilled to $349 \mathrm{ft}$, backfilled to $276 \mathrm{ft}$ with grout and to $274.5 \mathrm{ft}$ with bentonite. Well was originally intended as an open-interval well, was

completed as a screened well on 8-2-90. Data base revised on 3/12/97 to reflect changes discovered during periodic review.

Forms cluster with GW-625. Data base revised on 3/7/97 to reflect changes discovered during periodic review. TOWW value estimated for this well.

Forms cluster with GW-624; completed with 2-in to 7-in swedge with locking valve. Data base revised on 3/7 97 to reflect changes discovered during periodic review.

Forms cluster with GW-627. Data base revised on 3/7/97 to reflect changes discovered during periodic review. TOWW value estimated for this well.

Forms cluster with GW-626. Data base revised on 3/7/97 to reflect changes discovered during periodic review. TOWW value estimated for this well.

Completed with 2-in to 7 -in swedge with locking valve. Data base revised on $3 / 7 / 97$ to reflect changes discovered during periodic review.

Data base revised on $3 / 7 / 97$ to reflect changes discovered during periodic review. TOWW value estimated for this well.

Replaces GW-536. Data base revised on 3/7/97 to reflect changes discovered during periodic review. 


\section{CURRENT}

NAME

GW-631

GW-632

GW-633

GW-634

GW-635

GW-636

GW-637

GW-638

GW-639

GW-640

GW-641

GW-642

GW-643

\section{REFERENCE}

Eaton, J. M., and F. R. Van Ryn(1991)

Eaton, J. M., and F. R. Van Ryn(1991)

Eaton, J. M., and F. R. Van Ryn(1991)

Eaton, J. M., and F. R. Van Ryn(1991)

Eaton, J. M., and F. R. Van Ryn(1991), SAIC(1991b)

ERCE(1991)

ERCE(1991)

$\operatorname{ERCE}(1991)$

ERCE(1991)

ERCE(1991)

$\operatorname{ERCE}(1991)$

$\operatorname{ERCE}(1991)$

$\operatorname{ERCE}(1991)$

\section{COMMENTS}

Well is located on the SE corner of Building 9983-29. Data base revised on $3 / 26 / 97$ to reflect changes discovered during periodic review.

Dedicated portable WW pump used in this well, TOC and TOWW are at the same elevation.

Well is located on the NW corner of Building 9983-29. Data base revised on $3 / 26 / 97$ to reflect changes discovered during periodic review. From several well inspections, and subsequent walkdown, it is believed the well was destroyed or covered with the construction of a concrete ramp to

building 9831 (per Beth Schultz).2/22/2012 - Well discovered during annual inspection sweep by T. J. Coffey. Well determined to be open and usable [S. B. Jones]

Data base revised on $3 / 26 / 97$ to reflect changes discovered during periodic review. Location re-survey $06 / 19 / 2002$. A dedicated portable WW pump is used in this well, TOC and TOWW are at the same elevation.

Data base revised on $3 / 26 / 97$ to reflect changes discovered during periodic review.

Data base revised on $3 / 26 / 97$ to reflect changes discovered during periodic review.

Forms cluster with GW-637 and GW-638. Data base revised on 3/7/97 to reflect changes discovered during periodic review.

Forms cluster with GW-636 and GW-638. Data base revised on 3/7/97 to reflect changes discovered during periodic review.

Forms cluster with GW-636 and GW-637. Data base revised on 3/7/97 to reflect changes discovered during periodic review.

Forms cluster with GW-640 and GW-641. Data base revised on 3/7/97 to reflect changes discovered during periodic review. TOWW elevation changed 06/06/2005 to reflect proper WW configuration.

Forms cluster with GW-639 and GW-641. Data base revised on 3/7/97 to reflect changes discovered during periodic review.

Forms cluster with GW-639 and GW-640. Data base revised on 3/7/97 to reflect changes discovered during periodic review.

Data base revised on 3/7/97 to reflect changes discovered during periodic review. TOWW value estimated for this well.

Forms cluster with GW-372 and GW-373. Data base revised on 3/7/97 to reflect changes discovered during periodic review. 


\begin{tabular}{|c|c|}
\hline CURRENT & \\
\hline$\underline{\text { NAME }}$ & REFERENCE \\
\hline GW-644 & ERCE(1991) \\
\hline GW-645 & ERCE(1991), Shevenell, L. A.(1993) \\
\hline GW-646 & ERCE(1991), Shevenell, L. A.(1993) \\
\hline GW-647 & $\operatorname{ERCE}(1991)$ \\
\hline GW-647A & $\operatorname{ERCE}(1991)$ \\
\hline GW-648 & $\operatorname{ERCE}(1991)$ \\
\hline GW-649 & ERCE(1991) \\
\hline GW-651 & ERCE(1991) \\
\hline GW-652 & ERCE(1991) \\
\hline GW-653 & ERCE(1991) \\
\hline GW-654 & ERCE(1991) \\
\hline GW-655 & ERCE(1991) \\
\hline GW-656 & ERCE(1991) \\
\hline GW-657 & ERCE(1991) \\
\hline
\end{tabular}

\section{COMMENTS}

Data base revised on $3 / 7 / 97$ to reflect changes discovered during periodic review.

*Shevenell suggests Rock Formations (Appendix G) as CON:Maynardville is suspect, tenatively recommends KNOX:Copper Ridge. Data base revised on 3/7/97 to reflect changes discovered during periodic review.

*Shevenell suggests Rock Formations (Appendix G) as CON:Maynardville is suspect, tenatively recommends KNOX:Copper Ridge. Data base revised on 3/7/97 to reflect changes discovered during periodic review.

Measured well depth from TOC was $91.9 \mathrm{ft}$ after rehabilitation on

11Dec91. This differs from construction information which lists a depth of

$94.1 \mathrm{ft}$ from TOC. Located $18 \mathrm{ft}$ west of first drilling attempt, GW-647A which was abandoned. Data base revised on 3/7/97 to reflect changes discovered during periodic review.

Borehole caved below $13 \mathrm{ft}$ depth; hole plugged and abandoned; replaced with GW-647. Well condition changed to P\&A 03/06/2006 to reflect actual condition.

Data base revised on $3 / 7 / 97$ to reflect changes discovered during periodic review.

Data base revised on $3 / 7 / 97$ to reflect changes discovered during periodic review.

Forms cluster with GW-652. Data base revised on 3/7/97 to reflect changes discovered during periodic review.

Forms cluster with GW-651. Data base revised on 3/7/97 to reflect changes discovered during periodic review.

Data base revised on 3/7/97 to reflect changes discovered during periodic review. TOWW value estimated for this well.

Data base revised on $3 / 7 / 97$ to reflect changes discovered during periodic review.

Data base revised on 3/7/97 to reflect changes discovered during periodic review.

Data base revised on $3 / 7 / 97$ to reflect changes discovered during periodic review. Dedicated portable WW pump used in this well, TOC and TOWW are at the same elevation.

Data base revised on 3/7/97 to reflect changes discovered during periodic review. 


\section{CURRENT \\ NAME}

GW-658

GW-659

GW-660

GW-661

GW-662

GW-663

GW-664

GW-665

GW-666

GW-667

GW-668

GW-669

GW-670

GW-671

GW-672

GW-673

GW-674

\section{REFERENCE}

ERCE(1991)

ERCE(1991)

SAIC(1994a), SAIC(1997c)

SAIC(1994a)

SAIC(1994a)

SAIC(1994a)

SAIC(1994a)

SAIC(1994a)

SAIC(1994a)

SAIC(1994a)

SAIC(1994a)

SAIC(1994a), SAIC(1997c)

SAIC(1994a), SAIC(1997c)

SAIC(1994a), SAIC(1997c)

CH2M Hill(1991), SAIC(1997c)

CH2M Hill(1991)

CH2M Hill(1991)

\section{COMMENTS}

Data base revised on $3 / 7 / 97$ to reflect changes discovered during periodic review. No WW at this location, field verified 05/12/2005. Dedicated portable WW pump used in this well, TOC and TOWW are at the same elevation.

Data base revised on 3/7/97 to reflect changes discovered during periodic review.

Data base revised on 3/12/97 to reflect changes discovered during periodic review.

Data base revised on $3 / 12 / 97$ to reflect changes discovered during periodic review.

Data base revised on $3 / 12 / 97$ to reflect changes discovered during periodic review.

Data base revised on $3 / 12 / 97$ to reflect changes discovered during periodic review.

Data base revised on $3 / 12 / 97$ to reflect changes discovered during periodic review.

TD listed as both 30.7 and $30.9 \mathrm{ft}$. Data base revised on 3/12/97 to reflect changes discovered during periodic review.

Data base revised on 3/12 97 to reflect changes discovered during periodic review.

Data base revised on 3/12/97 to reflect changes discovered during periodic review.

TD listed as both 23.1 and $23.0 \mathrm{ft}$. Data base revised on 3/12/97 to reflect changes discovered during periodic review.

Hole backfilled with bentonite from TD $(9.4 \mathrm{ft})$ to $6.8 \mathrm{ft}$. Data base revised on $3 / 12 / 97$ to reflect changes discovered during periodic review.

TD listed as both 21.84 and $21.0 \mathrm{ft}$. Data base revised on 3/12/97 to reflect changes discovered during periodic review.

TD listed as both 8.2 and $8.9 \mathrm{ft}$. Data base revised on 3/12/97 to reflect changes discovered during periodic review.

Additional 10 -in. diameter conductor casing installaed to $24.0 \mathrm{ft}$. Data base revised on 3/12 97 to reflect changes discovered during periodic review. 


\section{CURRENT}

NAME

GW-675

GW-676

GW-677

GW-678

GW-679

GW-680

GW-681

GW-682

GW-683

GW-684

GW-685

GW-686

GW-687

GW-688

GW-689

\section{REFERENCE}

CH2M Hill(1991)

CH2M Hill(1991)

CH2M Hill(1991)

CH2M Hill(1991)

CH2M Hill(1991)

CH2M Hill(1991)

CH2M Hill(1991)

CH2M Hill(1991)

ERCE(1991), Shevenell, L. A., R. B. Dreier, and W. K. Jago(1992)

ERCE(1991), Shevenell, L. A., R. B. Dreier, and W. K. Jago(1992)

ERCE(1991), Shevenell, L. A., R. B. Dreier, and W. K. Jago(1992)

The Earth Technology Corporation(1991)

The Earth Technology Corporation(1991), Vicki Brumbeck (SAIC)(2004)

The Earth Technology Corporation(1991), Vicki Brumbeck (SAIC)(2004)

The Earth Technology Corporation(1991), Vicki Brumbeck (SAIC)(2004)

\section{COMMENTS}

Well abandoned, borehole location adjacent to well GW-676. Data base revised on 3/12/97 to reflect changes discovered during periodic review.

Data base revised on $3 / 12 / 97$ to reflect changes discovered during periodic review.

Data base revised on $3 / 12 / 97$ to reflect changes discovered during periodic review.

Data base revised on 3/12/97 to reflect changes discovered during periodic review.

Data base revised on $3 / 12 / 97$ to reflect changes discovered during periodic review. A WW pump with a LCC is used in this well, TOC and TOWW are at the same elevation.

Data base revised on 3/12/97 to reflect changes discovered during periodic review. A WW pump with a LCC is used in this well, TOC and TOWW are at the same elevation.

Borehole collapsed to $178 \mathrm{ft}$, packed interval and Cav @ $175 \mathrm{ft}$ filled with pea gravel. Data base revised on 3/12/97 to reflect changes discovered during periodic review.

Data base revised on $3 / 12 / 97$ to reflect changes discovered during periodic review.

Data base revised on $3 / 7 / 97$ to reflect changes discovered during periodic review. TOWW value estimated for this well.

Data base revised on 3/7/97 to reflect changes discovered during periodic review. TOWW value estimated for this well.

Data base revised on 3/7/97 to reflect changes discovered during periodic review.

Data base revised on $4 / 24 / 97$ to reflect changes discovered during periodic review. Well Wizard pump and low clearance cap installed.

Data base revised on 4.24 .97 to reflect changes discovered during periodic review. Well P\&A'd 05/29/2003.

Data base revised on $4 / 24 / 97$ to reflect changes discovered during periodic review. Well P\&A'd 06/05/2003.

Data base revised on $4 / 24 / 97$ to reflect changes discovered during periodic review. Location covered by coal pile. Well P\&A'd 05/29/2003. 


\section{CURRENT}

\section{NAME}

GW-690

GW-691

GW-692

GW-693

GW-694

GW-695

GW-696

GW-697

GW-698

GW-699

GW-700

GW-701

GW-702

GW-703

\section{REFERENCE}

The Earth Technology Corporation(1991)

The Earth Technology Corporation(1991)

The Earth Technology Corporation(1991)

The Earth Technology Corporation(1990)

SAIC(1991a)

$\operatorname{SAIC}(1991 \mathrm{a})$

The Earth Technology Corporation(1991)

The Earth Technology Corporation(1991)

The Earth Technology Corporation(1991)

The Earth Technology Corporation(1991)

The Earth Technology Corporation(1991)

The Earth Technology Corporation(1991)

The Earth Technology Corporation(1991)

$\operatorname{ERCE}(1991)$

\section{COMMENTS}

Data base revised on $4 / 24 / 97$ to reflect changes discovered during periodic review. Dedicated portable WW pump used in this well, TOC and TOWW are at the same elevation.

Data base revised on $4 / 24 / 97$ to reflect changes discovered during periodic review. A WW pump with a LCC is used in this well, TOC and TOWW are at the same elevation.

Data base revised on 4/24/97 to reflect changes discovered during periodic review. A WW pump with a LCC is used in this well, TOC and TOWW are at the same elevation.

Data base revised on $4 / 17 / 97$ to reflect changes discovered during periodic review.

Data base revised on 3/7/97 to reflect changes discovered during periodic review. TOWW value estimated for this well.

Data base revised on 3/7/97 to reflect changes discovered during periodic review. TOWW value estimated for this well.

Well is located south of Building 81-10 site. Data base revised on 4/24/97 to reflect changes discovered during periodic review. Dedicated portable WW pump used in this well, TOC and TOWW are at the same elevation.

Well is located south of Building 81-10 site. Data base revised on 4/24/97 to reflect changes discovered during periodic review.

Well is located south of Building 81-10 site. Data base revised on 4/24/97 to reflect changes discovered during periodic review. TOWW value estimated for this well. TOWW elevation changed 06/06/2005 to reflect proper WW low clearance cap configuration.

Well is located south of Building 81-10 site. Data base revised on 4/24/97 to reflect changes discovered during periodic review. Well contains $<0.5 \mathrm{ft}$ of water in the well $(01 / 18 / 05)$

Well is located north of Building 81-10 site. Data base revised on 4/24/97 to reflect changes discovered during periodic review. WW installed with a LCC.

Well is located north of Building 81-10 site. Data base revised on 4/24/97 to reflect changes discovered during periodic review.

Well is located northeast of Building 81-10 site. Data base revised on $4 / 24 / 97$ to reflect changes discovered during periodic review.

Data base revised on $3 / 13 / 97$ to reflect changes discovered during periodic review. 
CURRENT

NAME

GW-704

GW-705

GW-705A

GW-705B

GW-706

GW-707

GW-708

GW-709

GW-710

GW-711

GW-712

GW-713

GW-714

GW-715

GW-721

GW-722

\section{REFERENCE}

ERCE(1991)

SAIC(1991a)

SAIC(1991a)

SAIC(1991a)

SAIC(1991a), Shevenell, L. A., R. B. Dreier, and W. K. Jago(1992)

ERCE(1991), SAIC(1998b)

ERCE(1991)

$\operatorname{SAIC}(1991 \mathrm{a})$

SAIC(1991a), Shevenell, L. A., R. B. Dreier, and W. K. Jago(1992)

SAIC(1991a), Shevenell, L. A., R. B. Dreier, and W. K. Jago(1992)

SAIC(1991a), Shevenell, L. A., R. B. Dreier, and W. K. Jago(1992)

SAIC(1992a)

$\operatorname{SAIC}(1992 a)$

SAIC(1992a)

SAIC(1997c)

SAIC(1991a), Shevenell, L. A., R. B. Dreier, and W. K. Jago(1992), Martin Marietta Energy Systems(1995)

\section{COMMENTS}

Data base revised on $3 / 13 / 97$ to reflect changes discovered during periodic review.

Data base revised on $3 / 13 / 97$ to reflect changes discovered during periodic review.

Soft drilling to $64 \mathrm{ft}$, hole caved, $P$ \& A due to poor/no circulation. No well installaed in borehole. Data base revised on $3 / 13 / 97$ to reflect changes discovered during periodic review.

Hole $P$ \& A due to ratty drilling, no/poor circulation. Data base revised on $3 / 13 / 97$ to reflect changes discovered during periodic review.

Data base revised on $3 / 13 / 97$ to reflect changes discovered during periodic review. TOWW value estimated for this well.

Borehole backfilled with sand 17.2-26.0 ft prior to completion. Data base revised on $3 / 13 / 97$ to reflect changes discovered during periodic review.

Strong odor of gasoline noted during drilling. Data base revised on 3/13/97 to reflect changes discovered during periodic review.

Replaces plugged and abandoned well 1087. Data base revised on $3 / 13 / 97$ to reflect changes discovered during periodic review. TOWW value estimated for this well.

Slow water accumulation in zone between 699.5-744.5 ft. Data base revised on $3 / 13 / 97$ to reflect changes discovered during periodic review. Re-survey 06/19/2002. Well Wizard pump and low clearance cap installed.

ata base revised on $3 / 13 / 97$ to reflect changes discovered durinm periodic review. Well Wizard pump and low clearance cap installed.

Data base revised on 3/13/97 to reflect changes discovered during periodic review. TOWW value estimated for this well.

Data base revised on 3/13/97 to reflect changes discovered during periodic review. TOWW value estimated for this well.

Data base revised on 3/13/97 to reflect changes discovered during periodic review. TOWW value estimated for this well.

85 sacks of $\mathrm{J}-20$ sand used for sand pack (due to cavities). Data base revised on 3/13/97 to reflect changes discovered during periodic review. TOWW value estimated for this well.

Pine Ridge East Repeater Station (Tank 2315-U) Product Recovery Well.

Completed w/ Westbay Multi-port monitoring system. Data base revised on $3 / 13 / 97$ to reflect changes discovered during periodic review. 
CURRENT

NAME

GW-723

GW-724

GW-725

GW-726

GW-727

GW-728

GW-729

GW-730

GW-731

GW-732

GW-733

GW-734

GW-735

\section{REFERENCE}

SAIC(1991a), Shevenell, L. A., R. B. Dreier, and W. K Jago(1992)

SAIC(1991a), Shevenell, L. A., R. B. Dreier, and W. K. Jago(1992)

$\operatorname{SAIC}(1991 \mathrm{a})$

SAIC(1993a), Dreier, R. B. and A. J. Caldanaro(1994)

SAIC(1993b), Dreier, R. B. and A. J. Caldanaro(1994)

$\operatorname{SAIC}(1993 d)$

SAIC(1993e), Dreier, R. B. and A. J. Caldanaro(1994)

SAIC(1993c), Dreier, R. B. and A. J. Caldanaro(1994)

$\operatorname{SAIC}(1991 \mathrm{a})$

$\operatorname{SAIC}(1991 \mathrm{a})$

$\operatorname{SAIC}(1992 a)$

SAIC(1992a)

$\operatorname{SAIC}(1992 a)$

\section{COMMENTS}

Data base revised on $3 / 13 / 97$ to reflect changes discovered during periodic review. WW installed with LCC, database updated 02/07/2006 to reflect conditions in the field.

Data base revised on $3 / 13 / 97$ to reflect changes discovered during periodic review.

Data base revised on $3 / 13 / 97$ to reflect changes discovered during periodic review.

Completed w/ Westbay Multi-port monitoring system. Data base revised on 3/19/97 to reflect changes discovered during periodic review.

Completed w/ Westbay Multi-port monitoring system. Data base revised on $3 / 19 / 97$ to reflect changes discovered during periodic review.

Due to drilling difficulities this well was turned into a piezometer and was replaced by GW-790. Data base revised on 3/20/97 to reflect changes discovered during periodic review.

Re-survey $\sim 04 / 27 / 1994$. Completed w/ Westbay Multi-port monitoring system. Data base revised on 3/20/97 to reflect changes discovered during periodic review.

Completed w/ Westbay Multi-port monitoring system. Data base revised on $3 / 20 / 97$ to reflect changes discovered during periodic review.

Replaces downgradient well GW-157. Data base revised on 3/13/97 to reflect changes discovered during periodic review. TOWW value estimated for this well. TOWW elevation changed 06/06/2005 to reflect proper WW configuration.

Replaces downgradient well GW-157. Data base revised on 3/13/97 to reflect changes discovered during periodic review. TOWW value estimated for this well.

Data base revised on $3 / 14 / 97$ to reflect changes discovered during periodic review. TOWW value estimated for this well.

Cav encountered at 59.4 pushed/drilled to $103.4 \mathrm{ft}$, bottom of Cav was not encountered. Set steel casing at top of cavity, hole is to be used as an open hole piezometer to monitor hydrologic and geochemical parameters. Data base revised on $3 / 14 / 97$ to reflect changes discovered during periodic review. March 1997, TDEC-DOE-Oversite injected Rhodamine dye

Data base revised on $3 / 14 / 97$ to reflect changes discovered during periodic review. TOWW value estimated for this well. 
CURRENT

NAME

GW-736

GW-737

GW-738

GW-739

GW-740

GW-742

GW-744

GW-745

GW-746

GW-747

\section{REFERENCE}

SAIC(1992a), Shevenell, L. A., R. B. Dreier, and W. K Jago(1992)

SAIC(1992a)

$\operatorname{SAIC}(1992 a)$

SAIC(1992a)

SAIC(1992a)

$\operatorname{SAIC}(1992 a)$

SAIC(1992a)

SAIC(1992a)

$\operatorname{SAIC}(1992 a)$

SAIC(1992a)

SAIC(1992a)

\section{COMMENTS}

Data base revised on $3 / 14 / 97$ to reflect changes discovered during periodic review. TOWW elevation changed 06/06/2005 to reflect proper WW configuration.

Data base revised on $3 / 14 / 97$ to reflect changes discovered during periodic review.

Soft drilling from 89.1-90.1 (mW). Data base revised on 3/14/97 to reflect changes discovered during periodic review.

Data base revised on $3 / 14 / 97$ to reflect changes discovered during periodic review. WW installed with LCC, database updated 02/07/2006 to reflect conditions in the field.

Data base revised on $3 / 14 / 97$ to reflect changes discovered during periodic review. TOWW elevation changed 06/06/2005 to reflect proper WW configuration.

WB @ 397.5-420 was diffuse with traces of naturally occurring oil. Data base revised on $3 / 14 / 97$ to reflect changes discovered during periodic

review. A WW pump with a LCC is used in this well, TOC and TOWW are at the same elevation.

Data base revised on $3 / 14 / 97$ to reflect changes discovered during periodic review. A WW pump with a LCC is used in this well, TOC and TOWW are at the same elevation.

Y-12 Grid Well (grid location K1 deep). Data base revised on 3/14/97 to reflect changes discovered during periodic review. TOWW value estimated for this well. TOWW elevation changed 06/06/2005 to reflect proper WW low clearance cap configuration.

Y-12 Grid Well (grid location K1 intermediate). Data base revised on $3 / 14 / 97$ to reflect changes discovered during periodic review. Dedicated portable WW pump used in this well, TOC and TOWW are at the same elevation.

Y-12 Grid Well (grid location K1 shallow). Data base revised on 3/14/97 to reflect changes discovered during periodic review. Dedicated portable WW pump used in this well, TOC and TOWW are at the same elevation.

Y-12 Grid Well (grid location K2 deep). Data base revised on 3/14/97 to reflect changes discovered during periodic review. TOWW value estimated for this well. TOWW elevation changed 06/06/2005 to reflect proper WW low clearance cap configuration. Well re-survey due to change to flush mount configuration. 


\section{CURRENT \\ NAME}

GW-748

GW-749

GW-750

GW-751

GW-752

GW-753

GW-754

GW-755

GW-756

GW-757

GW-758

GW-759

GW-760

\section{REFERENCE}

SAIC(1992a)

$\operatorname{SAIC}(1992 a)$

SAIC(1992a), Shevenell, L. A., R. B. Dreier, and W. K. Jago(1992)

$\operatorname{SAIC}(1992 a)$

$\operatorname{SAIC}(1992 a)$

$\operatorname{SAIC}(1992 a)$

$\operatorname{SAIC}(1992 a)$

$\operatorname{SAIC}(1992 a)$

$\operatorname{SAIC}(1992 a)$

$\operatorname{SAIC}(1992 a)$

$\operatorname{SAIC}(1992 a)$

$\operatorname{SAIC}(1992 a)$

SAIC(1992a)

\section{COMMENTS}

Y-12 Grid Well (grid location K2 intermediate). Data base revised on $3 / 14 / 97$ to reflect changes discovered druing periodic review. Dedicated portable WW pump used in this well, TOC and TOWW are at the same elevation. Well re-survey due to change to flush mount configuration.

Y-12 Grid Well (grid location K2 shallow). Data base revised on 3/14/97 to reflect changes discovered during periodic review. Dedicated portable WW pump used in this well, TOC and TOWW are at the same elevation. Please see P/A request \#06-001P/A

Y-12 Grid Well (grid location K3). Data base revised on 3/14/97 to reflect changes discovered during periodic review. TOWW value estimated for this well.

Y-12 Grid Well (grid location J3 deep). Data base revised on 3/14/97 to reflect changes discovered during periodic review.

Y-12 Grid Well (grid location J3 shallow). Data base revised on 3/14/97 to reflect changes discovered during periodic review.

Y-12 Grid Well (grid location J2 deep). Data base revised on 3/14/97 to reflect changes discovered during periodic review. TOWW value estimated for this well.

Y-12 Grid Well (grid location J2 shallow). Data base revised on 3/14/97 to reflect changes discovered during periodic review. TOWW value estimated for this well.

Y-12 Grid Well (grid location J1 deep). Data base revised on 3/14/97 to reflect changes discovered during periodic review. TOWW value estimated for this well.

Y-12 Grid Well (grid location J1 shallow). Data base revised on 3/14/97 to reflect changes discovered during periodic review. TOWW value estimated for this well.

Data base revised on 3/14/97 to reflect changes discovered during periodic review. TOWW value estimated for this well.

Y-12 Grid Well (grid location G-1 deep). Data base revised on 3/18/97 to reflect changes discovered during periodic review.

Y-12 Grid Well (grid location G-1 shallow). Data base revised on 3/18/97 to reflect changes discovered during periodic review.

Y-12 Grid Well (grid location G-2 deep). Data base revised on 3/18/97 to reflect changes discovered during periodic review. A WW pump with a LCC is used in this well, TOC and TOWW are at the same elevation. 


\section{CURRENT \\ NAME}

GW-761

GW-762

GW-763

GW-764

GW-765

GW-766

GW-767

GW-768

GW-769

GW-770

GW-771

GW-772

\section{REFERENCE}

SAIC(1992a)

SAIC(1992a)

$\operatorname{SAIC}(1992 a)$

SAIC(1992a)

$\operatorname{SAIC}(1992 a)$

$\operatorname{SAIC}(1992 a)$

$\operatorname{SAIC}(1992 a)$

SAIC(1992a)

$\operatorname{SAIC}(1992 a)$

$\operatorname{SAIC}(1992 a)$

SAIC(1992a), Vicki Brumbeck (SAIC)(2004)

SAIC(1992a), Vicki Brumbeck (SAIC)(2004)

\section{COMMENTS}

Y-12 Grid Well (grid location G-2 shallow). Data base revised on 3/18/97 to reflect changes discovered during periodic review. Well Wizard pump and low clearance cap installed.

Y-12 Grid Well (grid location J-primary deep). Data base revised on $3 / 18 / 97$ to reflect changes discovered during periodic review. TOWW elevation estimated for this well.

Y-12 Grid Well (grid location J-primary shallow). Data base revised on $3 / 18 / 97$ to reflect changes discovered during periodic review. TOWW value estimated for this well.

Y-12 Grid Well (grid location E-1 deep). Data base revised on 3/18/97 to reflect changes discovered during periodic review. Well Wizard pump and low clearance cap installed.

Y-12 Grid Well (grid location E-1 shallow). Data base revised on 3/18/97 to reflect changes discovered during periodic review. TOWW value estimated for this well. TOWW elevation changed 06/06/2005 to reflect proper WW low clearance cap configuration.

Hole drilled to 100.3, grout plug from 51.9-100.3, bentonite plug 47.6-51.9 ft. Y-12 Grid Well (grid location I-2 deep). Data base revised on 3/18/97 to reflect changes discovered during periodic review.

Y-12 Grid Well (grid location I-2 shallow). Data base revised on 3/18/97 to reflect changes discovered during periodic review.

Y-12 Grid Well (grid location I-1 deep). Data base revised on 3/18/97 to reflect changes discovered during periodic review.

Y-12 Grid Well (grid location G-3 deep). Data base revised on 3/18/97 to reflect changes discovered during periodic review. TOWW value estimated for this well.

Y-12 Grid Well (grid location G-3 shallow). Data base revised on 3/18/97 to reflect changes discovered during periodic review. TOWW value estimated for this well.

Y-12 Grid Well (grid location C-1 deep). Data base revised on 3/18/97 to reflect changes discovered during periodic review. Location P\&A'd 06/23/2003.

Y-12 Grid Well (grid location C-1 shallow). Data base revised on 3/18/97 to reflect changes discovered during periodic review. Well P\&A'd 06/11/2003. 


\section{CURRENT \\ NAME}

GW-773

GW-774

GW-775

GW-776

SAIC(1992a)

GW-777

GW-778

GW-779

GW-780

GW-781

GW-782

GW-783

GW-784

GW-785

\section{REFERENCE}

SAIC(1992a)

SAIC(1992a)

SAIC(1992a)

SAIC(1992a)

SAIC(1992a)

SAIC(1992a)

SAIC(1992a)

SAIC(1992a)

SAIC(1992a)

SAIC(1992a)
SAIC(1992a), Vicki Brumbeck (SAIC)(2004)

SAIC(1992a), Vicki Brumbeck (SAIC)(2004)

\section{COMMENTS}

Y-12 Grid Well (grid location H-2 deep). Data base revised on 3/18/97 to reflect changes discovered during periodic review.

Y-12 Grid Well (grid location H-2 shallow). Data base revised on 3/18/97 to reflect changes discovered during periodic review.

Flush mounted well. Y-12 Grid Well (grid location H-3 deep). Data base revised on $3 / 18 / 97$ to reflect changes discovered during periodic review. Dedicated portable WW pump used in this well, TOC and TOWW are at the same elevation.

Re-survey $\sim 04 / 27 / 1994$. Flush mounted well. Y-12 Grid Well (grid location $\mathrm{H}-3$ shallow). Data base revised on 3/18/97 to reflect changes discovered during periodic review. Dedicated portable WW pump used in this well, TOC and TOWW are at the same elevation.

Y-12 Grid Well Grid location B-2 deep). Data base revised on 3/18/97 to reflect changes discovered during periodic review.

Y-12 Grid Well (grid location B-2 shallow). Data base revised on 3/18/97 to reflect changes discovered during periodic review.

Y-12 Grid Well (grid location F-2 deep). Data base revised on 3/19/97 to reflect changes discovered during periodic review.

Y-12 Grid Well (grid location F-2 shallow). Data base revised on 3/19/97 to reflect changes discovered during periodic review. Well P\&A'd during May, 2006.

Y-12 Grid Well (grid location E-3 deep). Data base revised on 3/19/97 to reflect changes discovered during periodic review. TOWW value estimated for this well.

Y-12 Grid Well (grid location E-3 intermediate). Data base revised on $3 / 19 / 97$ to reflect changes discovered during periodic review. TOWW value estimated for this well.

Y-12 Grid Well (grid location E-3 shallow). Data base revised on 3/19/97 to reflect changes discovered during periodic review. TOWW value estimated for this well.

Estimated water table @18.1 ft Y-12 Grid Well (grid location D-1 deep) Data base revised on $3 / 19 / 97$ to reflect changes discovered during periodic review. Well P\&A'd 06/10/2003.

Depth to weathered and fresh bedrock estimated. Y-12 Grid Well (grid location D-1 shallow). Data base revised on $3 / 19 / 97$ to reflect changes discovered during periodic review. Well P\&A'd 06/10/2003. 


\section{CURRENT \\ NAME}

GW-786

GW-787

GW-788

GW-789

GW-790

GW-791

GW-792

GW-793

GW-794

GW-795

GW-796

GW-797

\section{REFERENCE}

SAIC(1992a)

SAIC(1992a)

SAIC(1992a), T. Coffey(2007)

SAIC(1992a)

SAIC(1993f), Dreier, R. B. and A. J. Caldanaro(1994)

$\operatorname{SAIC}(1992 a)$

$\operatorname{SAIC}(1992 a)$

SAIC(1993g)

$\operatorname{SAIC}(1993 g)$

$\operatorname{SAIC}(1993 g)$

$\operatorname{SAIC}(1993 g)$

$\operatorname{SAIC}(1993 g)$

\section{COMMENTS}

Y-12 Grid Well (grid location E-2 deep). Data base revised on 3/19/97 to reflect changes discovered during periodic review. Well Wizard pump and low clearance cap installed.

Y-12 Grid Well (grid location E-2 shallow). Data base revised on 3/19/97 to reflect changes discovered during periodic review. Well Wizard pump and low clearance cap installed.

Y-12 Grid Well Grid location F-3 deep). Data base revised on 3/19/97 to reflect changes discovered during periodic review. TOWW value estimated for this well. Well P\&A'd 06/01/2006

Top of weathered bedrock not identified. Y-12 Grid Well (grid location F-3 shallow). Data base revised on 3/19/97 to reflect changes discovered during periodic review. TOWW value estimated for this well. Well P\&A'd 06/01/2006

Re-survey 07/06/1994. Replaces GW-728. Completed w/ Westbay Multiport monitoring system. Data base revised on 3/19.97 to reflect changes discovered during periodic review.

Y-12 Grid Well (grid location D-2 deep). Data base revised on 3/19/97 to reflect changes discovered during periodic review. TOWW value estimated for this well.

Estimated water table at $19.0 \mathrm{ft}$. Re-survey due to well head modifications. Y-12 Grid Well (grid location D-2 shallow). Data base revised on 3/19/97 to reflect changes discovered during periodic review. TOWW value estimated for this well.

Functional Area: LLWAGSF. Data base revised on 3/20/97 to reflect changes discovered during periodic review. TOWW value estimated for this well.

Functional Area: LLWAGSF. Data base revised on 3/20/97 to reflect changes discovered during periodic review. TOWW value estimated for this well.

Functional Area: LLWAGSF. Data base revised on 3/20/97 to reflect changes discovered during periodic review. TOWW value estimated for this well.

Data base revised on $3 / 20 / 97$ to reflect changes discovered during periodic review. TOWW value estimated for this well.

Data base revised on $3 / 20 / 97$ to reflect changes discovered during periodic review. TOWW value estimated for this well. 


\section{CURRENT \\ NAME}

GW-798

GW-799

GW-800

GW-801

GW-802

GW-803

GW-804

GW-805

GW-806

GW-807

GW-808

GW-809

GW-810

\section{REFERENCE}

SAIC(1993g)

SAIC(1993g)

SAIC(1993g)

SAIC(1993g)

SAIC(1993g)

SAIC(1993g)

SAIC(1993g)

SAIC(1993g), SAIC(1998b)

SAIC(1993g), SAIC(1998b)

SAIC(1993g), SAIC(1998b)

SAIC(1993g), SAIC(1998b)

SAIC(1993g), SAIC(1998b)

SAIC(1993g), SAIC(1998b)

\section{COMMENTS}

15.2 feet of casing has been added to well GW-798 due to fill/grading of area around well. Database depths list construction information prior to stick-up addition and fill. Data base revised on 3/20/97 to reflect changes discovered during periodic review. TOWW value estimated for this well.

Data base revised on 3/20/97 to reflect changes discovered during periodic review. TOWW value estimated for this well.

Data base revised on $3 / 21 / 97$ to reflect changes discovered during periodic review. A WW was installed \& removed, no WW present at this location at this time.

Data base revised on $3 / 21 / 97$ to reflect changes discovered during periodic review.

Flush mount well for UST program. Data base revised on 3/21/97 to reflect changes discovered during periodic review.

Well installed for UST Program. Data base revised on 3/21/97 to reflect changes discovered during periodic review.

Well installed for UST Program. Data base revised on 3/21/97 to reflect changes discovered during periodic review.

Flush mount well for UST program. Well is located near Building 9204-2. Data base revised on $3 / 21 / 97$ to reflect changes discovered during periodic review.

Flush mount well for UST program. Well is located near Building 9204-2. Data base revised on 3/21/97 to reflect changes discovered during periodic review.

Well installed for UST Program. Well is located near Building 9204-2.

Data base revised on $3 / 21 / 97$ to reflect changes discovered during periodic review.

Flush mount well for UST program. Well is located near Building 9201-1.

Data base revised on $3 / 21 / 97$ to reflect changes discovered during periodic review.

Flush mount well for UST program. Well is located near Building 9201-1.

Data base revised on $3 / 21 / 97$ to reflect changes discovered during periodic review.

Flush mount well for UST program. Well is located near Building 9201-1.

Data base revised on $3 / 21 / 97$ to reflect changes discovered during periodic review. 


\section{CURRENT}

\section{NAME}

GW-811

GW-812

GW-813

GW-814

GW-815

GW-816

GW-817

GW-818

GW-819

GW-820

GW-821

GW-822

\section{REFERENCE}

SAIC(1994b)

$\operatorname{SAIC}(1994 b)$

$\operatorname{SAIC}(1994 b)$

$\operatorname{SAIC}(1994 b)$

SAIC(1994b)

SAIC(1994C)

SAIC(1994c)

Unknown

Unknown

Unknown

Moline, G. R. and M. E. Schreiber(1996)

Moline, G. R. and M. E. Schreiber(1996)

\section{COMMENTS}

BCV ER OU 2. Data base revised on 3/21/97 to reflect changes discovered during periodic review.

BCV ER OU 2. Data base revised on 3/24/97 to reflect changes discovered during periodic review.

BCV ER OU 2. Data base revised on 3/24/97 to reflect changes discovered during periodic review.

BCV ER OU 2. Data base revised on 3/24/97 to reflect changes discovered during periodic review.

BCV ER OU 2. Data base revised on 3/24/97 to reflect changes discovered during periodic review.

Well is located along Scarboro Road. Data base revised on 3/24/97 to reflect changes discoverd during periodic review. TOWW value estimated for this well.

End cap welded to bottom of screen. Y-12 Grid Well (grid location K-3). Data base revised on $3 / 24 / 97$ to reflect changes discovered during periodic review. TOWW value estimated for this well. See P/A request 06-002P/A.

Flush mount well discovered by H.L. King in 1994, survey performed by MKF. Ground elevation hand measured by S.B. Jones. No history on wells available. Well depth measured $20.65 \mathrm{ft}$ from TOC. Re-survey 06/30/1994. Well is located near Building 9201-2, on the south side.

Flush mount well discovered by H.L. King in 1994, survey performed by MKF. Ground elevation hand measured by S.B. Jones. No history on wells available. Well depth measured $15.57 \mathrm{ft}$ from TOC. Well is located near Building 9201-2, on the south side. Dedicated portable WW pump used in this well, TOC and TOWW are at the same elevation.

Flush mount well discovered by H.L. King in 1994, survey performed by MK

F. Ground elevation hand measured by S.B. Jones. No history on wells available. Well depth measures $17.4 \mathrm{ft}$ from TOC. Well is located near Building 9201-2, on the south side. Dedicated portable WW pump used in this well, TOC and TOWW are at the same elevation.

Solinst well installed in borehole drilled with Rotosonic drill rig. Data base revised on 3/24/97 to reflect changes discovered during periodic review.

Solinst well installed in borehole drilled with Rotosonic drill rig. Functional Area: Gum Branch Are Data base revised on 3/24/97 to reflect changes discovered during periodic review. 


\section{CURRENT \\ NAME}

GW-823

GW-824

GW-825

GW-826

GW-827

GW-828

GW-829

SAIC(1995a)

GW-830

GW-831

GW-832

GW-833

GW-834

GW-835

GW-836

\section{REFERENCE}

SAIC(1994c)

SAIC(1995a)

SAIC(1995a)

SAIC(1995a)

SAIC(1995a)

Unknown

SAIC(1997b)

$\operatorname{SAIC}(1997 b)$ Painter(2004)

SAIC(1997a)

SAIC(1997a)

SAIC(1997a)
Moline, G. R. and M. E. Schreiber(1996)

Law Engineering, Inc.(), William Burress and Dave

\section{COMMENTS}

Solinst well installed in a borehole drilled with Rotosonic drill rig. Functional Area: Gum Branch Are Data base revised on 3/24/97 to reflect changes discovered during periodic review.

Hole P\&A due to dry hole. Northing, Easting and Elevation scaled from S16A map. Borehole is located near Building 9201-2. Data base revised on $3 / 24 / 97$ to reflect changes discovered during periodic review.

Pea gravel filter pack. Methane gas monitoring probe. Data base revised on $3 / 24 / 97$ to reflect changes discovered during periodic review.

Pea gravel filter pack. Methane gas monitoring probe. Data base revised on $3 / 24 / 97$ to reflect changes discovered during periodic review.

Replacement well for GW-545. Re-survey 04/24/1995. Data base revised on $3 / 24 / 97$ to reflect changes discovered during periuodic review. TOWW value estimated for this well.

A temporary well (GW-828-100) was installed in the same borehole at 82.7 $\mathrm{ft}$. The temporary well was sampled and removed. The borehole was then extended to its present depth and sampled as a temporary well at $168 \mathrm{ft}$

(GW-828-200). At this depth is was completed as a permanent well. Data base revised on $12 / 12 / 2000$ to reflect changes discovered during periodic review [SBJ].

A temporary well (GW-829-29) was installed in the same borehole at 30.0 $\mathrm{ft}$. The temporary well was sampled and removed. The borehole was then extended to its present depth and sampled as a temporary well (GW-829$116)$ and then completed as a permanent well. Database revised on $12 / 12 / 2000$ to reflect changes discovered during periodic review. TOWW value estimated for this well [SBJ].

Abandoned water supply well possibly installed by Stone \& Webster, no longer in service.

TOWW value estimated for this well.

Well penetrates a French Drain beneath the East Fork Poplar Creek.

Well intercepts partially-filled spring box constructed in the 1940's to divert water from the spring to Upper East Fork Poplar Creek.

Survey coordinates changed 12/07/1999 to reflect incorrect values in database. The values given in the SAIC report Y/ER-286 were used and are considered valid. TOWW value estimated for this well. 


\begin{tabular}{|c|c|}
\hline \multicolumn{2}{|c|}{ CURRENT } \\
\hline NAME & REFERENCE \\
\hline GW-837 & $\operatorname{SAIC}(1997 a)$ \\
\hline GW-838 & Jacobs Engineering(1997) \\
\hline GW-839 & Jacobs Engineering(1997) \\
\hline GW-840 & Jacobs Engineering(1997) \\
\hline GW-841 & United States Department of Energy(1992) \\
\hline GW-842 & Jacobs Engineering() \\
\hline GW-843 & Jacobs Engineering() \\
\hline GW-844 & Jacobs Engineering() \\
\hline GW-845 & SAIC(1998b) \\
\hline GW-854 & SAIC(1997a) \\
\hline GW-855 & SAIC(1997a) \\
\hline GW-857 & SAIC(1997a), Collins, R.(2000) \\
\hline GW-858 & SAIC(1997a), Collins, R.(2000) \\
\hline GW-859 & SAIC(1997a) \\
\hline GW-860 & SAIC(1997a) \\
\hline GW-861 & SAIC(1997a) \\
\hline GW-862 & SAIC(1997a) \\
\hline GW-863 & SAIC(1997a) \\
\hline GW-864 & SAIC(1997a) \\
\hline GW-865 & SAIC(1997a) \\
\hline GW-866 & SAIC(1997a) \\
\hline GW-867 & SAIC(1997a) \\
\hline GW-868 & SAIC(1997a) \\
\hline GW-869 & SAIC(1997a) \\
\hline
\end{tabular}

\section{COMMENTS}

Survey coordinates updated 11/07/2002 to reflect changes discovered in the database. Source of correct information is SAIC report Y/ER-286.

Origional well used PVC materials on 10/22/1996, PVC removed and replaced with SS casing, completion date of 7/14/98 reflects this date . New well re-surveyed \& exact date of re-survey unknown.

PVC casing replaced with SS on $7 / 15 / 1998$. Origional construction completion date $10.21 / 1996$. Location re-survey completion date unknown. Dedicated portable WW pump used in this well, TOC and TOWW are at the same elevation.

Dedicated portable WW pump used in this well, TOC and TOWW are at the same elevation.

Dedicated portable WW pump used in this well, TOC and TOWW are at the same elevation.

Dedicated portable WW pump used in this well, TOC and TOWW are at the same elevation.

Survey date not actual survey date, actual date unknown

Hole P\&A'd 09/11/2000

Location P\&A'd 09/14/00 


$\begin{array}{cl}\begin{array}{c}\text { CURRENT } \\ \text { NAME }\end{array} & \text { REFERENCE } \\ \text { GW-871 } & \text { SAIC(1997a) } \\ \text { GW-872 } & \text { SAIC(1997a) } \\ \text { GW-873 } & \text { SAIC(1997a) } \\ \text { GW-874 } & \text { SAIC(1997a) } \\ \text { GW-875 } & \text { SAIC(1997a) } \\ \text { GW-876 } & \text { SAIC(1997a) } \\ \text { GW-877 } & \text { SAIC(1997a), King, H.(2000) } \\ \text { GW-878 } & \text { SAIC(1997a) } \\ \text { GW-879 } & \text { SAIC(1997a) } \\ \text { GW-880 } & \text { SAIC(1997a) } \\ \text { GW-881 } & \text { SAIC(1997a) } \\ \text { GW-882 } & \text { SAIC(1997a) } \\ \text { GW-883 } & \text { SAIC(1997a) } \\ \text { GW-884 } & \text { SAIC(1997a) } \\ \text { GW-885 } & \text { SAIC(1997a) } \\ \text { GW-886 } & \text { Watson, D.(1997), Mumby, M.(1998) } \\ \text { GW-887 } & \text { Watson, D.(1997), Mumby, M.(1998) } \\ \text { GW-888 } & \text { Watson, D.(1997), Mumby, M.(1998) } \\ \text { GW-889 } & \text { Watson, D.(1997), Mumby, M.(1998) } \\ \text { GW-890 } & \text { Watson, D.(1997), Mumby, M.(1998) } \\ \text { GW-902 } & \text { SAIC(1998b) } \\ \text { GW-904 } & \text { SAIC(1998a) } \\ \text { GW-905 } & \text { SAIC(1998a) } \\ \text { GW-906 } & \text { Kucera, S. P.(2001) } \\ \text { GW-907 } & \text { Kucera, S. P.(2001) } \\ \text { GW-908 } & \text { Kucera, S. P.(2001) } \\ \text { GW-909 } & \text { Kucera, S. P.(2001) } \\ \text { GW-910 } & \text { Kucera, S. P.(2001) } \\ \text { GW-911 } & \end{array}$

\section{COMMENTS}

Well P \& A 08/02/2000 by Ron Collins (BJC)

Core hole part of S-3 Site "trench" MSE Technology Application Core hole part of S-3 Site "trench" MSE Technology Application Core hole part of S-3 Site "trench" MSE Technology Application Core hole part of S-3 Site "trench" MSE Technology Application Core hole part of S-3 Site "trench" MSE Technology Application GS elevation revised 05/09/2005, previous measure appeared to be a typo (100 ft below realistic point).

Exact survey date unknown.

Exact Survey date unknown 


\begin{tabular}{|c|c|}
\hline CURREN & \\
\hline NAME & REFERENCE \\
\hline GW-912 & Kucera, S. P.(2001) \\
\hline GW-913 & Kucera, S. P.(2001) \\
\hline GW-914 & Kucera, S. P.(2001) \\
\hline GW-915 & Kucera, S. P.(2001) \\
\hline GW-916 & Kucera, S. P.(2001) \\
\hline GW-917 & Kucera, S. P.(2001) \\
\hline GW-918 & Kucera, S. P.(2001) \\
\hline GW-919 & Kucera, S. P.(2001) \\
\hline GW-920 & Kucera, S. P.(2001) \\
\hline GW-921 & Kucera, S. P.(2001) \\
\hline GW-922 & Kucera, S. P.(2001) \\
\hline GW-923 & Kucera, S. P.(2001) \\
\hline GW-924 & Kucera, S. P.(2001) \\
\hline GW-925 & Kucera, S. P.(2001) \\
\hline GW-926 & Kucera, S. P.(2001) \\
\hline GW-927 & Kucera, S. P.(2001) \\
\hline GW-928 & \\
\hline GW-929 & \\
\hline GW-930 & \\
\hline GW-931 & \\
\hline
\end{tabular}

\section{COMMENTS}

Piezometer installed as part of Waste Management Facility Phase III Well test pit

A WW pump with a LCC is used in this well, TOC and TOWW are at the same elevation.

A WW pump with a LCC is used in this well, TOC and TOWW are at the same elevation.

A WW pump with a LCC is used in this well, TOC and TOWW are at the same elevation.

TOC elevation updated 01/31/2003 with information provided by BJC (email from Steve Kucera). A WW pump with a LCC is used in this well, TOC and TOWW are at the same elevation. Well P\&A'd on 05/20/2004. A WW pump with a LCC is used in this well, TOC and TOWW are at the same elevation.

A WW pump with a LCC is used in this well, TOC and TOWW are at the same elevation.

A WW pump with a LCC is used in this well, TOC and TOWW are at the same elevation.

A WW pump with a LCC is used in this well, TOC and TOWW are at the same elevation.

A WW pump with a LCC is used in this well, TOC and TOWW are at the same elevation.

A WW pump with a LCC is used in this well, TOC and TOWW are at the same elevation.

A WW pump with a LCC is used in this well, TOC and TOWW are at the same elevation.

A WW pump with a LCC is used in this well, TOC and TOWW are at the same elevation. 


\section{CURRENT}

\section{NAME}

GW-932

GW-933

GW-934

GW-935

GW-936

GW-937

GW-938

GW-939

GW-940

GW-941

GW-942

GW-943

GW-944

GW-945

GW-946

GW-947

GW-948

GW-949

GW-950

GW-951

GW-952

GW-953

GW-954

GW-955

GW-956 surveyed by BJC. Survey info updated $07 / 08 / 2008$. Concrete pad elevation 914.81 feet, Top of Casing (TOC) elevation estimated using a tape measure from the top of the concrete pad/GS (8/4/2010, SBJ)

EMWMF monitoring well Well installed 02/13/2003 per Steve Jones
BARCAD instrumented corehole- 3 zone (GW-954-1, -2, -3) well installation.

BARCAD instrumented corehole, 4 zone well (GW-956-1, -2, -3, -4), location coordinates are estimated from a map 


\section{CURRENT}

NAME

GW-959

GW-960

GW-961

GW-964

GW-965

GW-966

GW-967

$\mathrm{J}-001$

$\mathrm{J}-002$

$\mathrm{J}-003$

J-004

J-005

J-006

$\mathrm{J}-007$

\section{REFERENCE}

Stone and Webster, Inc.(1943b)

Stone and Webster, Inc.(1943b)

Stone and Webster, Inc.(1943b)

Stone and Webster, Inc.(1943b)

Stone and Webster, Inc.(1943b)

Stone and Webster, Inc.(1943b)

Stone and Webster, Inc.(1943b)

Stone and Webster, Inc.(1943b)

\section{COMMENTS}

Well replaced sampling locations taken out for Big Spring Water Treatment Facility. Flush mount construction. Dedicated portable WW pump used in this well, TOC and TOWW are at the same elevation. Well installation date changed to $03 / 11 / 1005$ to reflect proper date.

Well installed to replace GW-780.

1 mile west of Bear Creek Road

EMWMF Cell 6

Installed by M\&W Drilling, Site Geologist Matthew Teglas, Quantum Environmental \& Engineering Services, LLC

NX corehole completed in 1943. Located west of Building 9204. Core hole converted to hole class of Unknown. Data base revised on 4/21/97 to reflect changes discovered during periodic review.

NX corehole completed in 1943. Located west of Building 9204. Core hole converted to hole class of Unknown. Data base revise on 4/21/97 to reflect changes discovered during periodic review.

NX corehole completed in 1943. Located west of Building 9204. Core hole converted to hole class of Unknown. Data base revised on 4/21/97 to reflect changes discovered during periodic review.

NX corehole completed in 1943. Located west of Building 9204. Core hole converted to hole class of Unknown. Data base revised on 4/21/97 to reflect changes discovered during periodic review.

NX corehole completed in 1943. Located west of Building 9204. Core hole converted to hole class of Unknown. Data base revised on 4/21/97 to reflect changes discovered during periodic review.

NX corehole completed in 1943. Located west of Building 9204. Core hole converted to hole class of Unknown. Data base revised on 4/21/97 to reflect changes discovered during periodic review.

NX corehole completed in 1943. Located west of Building 9204. Core hole converted to hole class of Unknown. Data Base revised on 4/21/97 to reflect changes discovered during periodic review.

NX corehole completed in 1943. Located west of Building 9204. Core hole converted to hole class of Unknown. Data base revised on 4/21/97 to reflect changes discovered during periodic review. 


\section{CURRENT} NAME

J-009

$\mathrm{J}-010$

J-011

\section{REFERENCE}

Stone and Webster, Inc.(1943b)

Stone and Webster, Inc.(1943c)

Stone and Webster, Inc.(1943c)

Stone and Webster, Inc.(1943c)

Stone and Webster, Inc.(1943c)

Stone and Webster, Inc.(1943c)

Stone and Webster, Inc.(1943c)

Stone and Webster, Inc.(1943c)

Stone and Webster, Inc.(1943c)

Stone and Webster, Inc.(1943c)

Stone and Webster, Inc.(1943c)

Stone and Webster, Inc.(1943e)

\section{COMMENTS}

NX corehole completed in 1943. Located west of Building 9204. Core hole converted to hole class of Unknown. Data base revised on 4/21/97 to reflect changes discovered during periodic review.

NX corehole completed in 1943. Located west of Building 9204. Core hole converted to hole class of Unknown. Data base revised on 4/21/97 to reflect chnages discovered during periodic review.

NX corehole completed in 1943. Located west of Building 9204. Core hole converted to hole class of Unknown. Data base revised on 4/21/97 to reflect changes discovered during periodic review.

NX corehole completed in 1943. Located west of Building 9204. Core hole converted to hole class of Unknown. Data base revised on 4/21/97 to reflect changes discovered during periodic review.

NX corehole completed in 1943. Located west of Building 9204. Core hole converted to hole class of Unknown. Data base revised on 4/21/97 to reflect changes discovered during periodic review.

NX corehole completed in 1943. Located west of Building 9204. Core hole converted to hole class of Unknown. Data base revised on 4/21/97 to reflect changes discovered during periodic review.

NX corehole completed in 1943. Located west of Building 9204. Core hole converted to hole class of Unknown. Data base revised on 4/21/97 to reflect changes discovered during periodic review.

NX corehole completed in 1943. Located west of Building 9204. Core hole converted to hole class of Unknown. Data base revised on 4/21/97 to reflect changes discovered during periodic review.

NX corehole completed in 1943. Located west of Building 9204. Core hole converted to hole class of Unknown. Data base revised on 4/21/97 to reflect changes discovered during periodic review.

NX corehole completed in 1943. Located west of Building 9204. Core hole converted to hole class of Unknown. Data base revised on 4/21/97 to reflect changes discovered during periodic review.

NX corehole completed in 1943. Located west of Building 9204. Core hole converted to hole class of Unknown. Data base revised on 4/21/97 to reflect changes discovered during periodic review.

NX corehole completed in 1943. Core hole converted to hole class of Unknown. Data base revised on 4/21/97 to reflect changes discovered during periodic review. 


\section{CURRENT}

\section{NAME}

K-021

$\mathrm{K}-022$

$\mathrm{K}-023$

$\mathrm{K}-024$

$\mathrm{K} 1-015$

K2-015

LL/HAZ-01

LL/HAZ-02

LL/HAZ-03

LL/HAZ-04

LL/HAZ-05

LL/HAZ-06

LL/HAZ-07

LL/HAZ-08

LL/HAZ-09

\section{REFERENCE}

Stone and Webster, Inc.(1943e)

Stone and Webster, Inc.(1943e)

Stone and Webster, Inc.(1943e)

Stone and Webster, Inc.(1943e)

Stone and Webster, Inc.(1943e)

Stone and Webster, Inc.(1943e)

Geotek Engineering, Inc.(1981)

Geotek Engineering, Inc.(1981)

Geotek Engineering, Inc.(1981), SAIC(1998b)

Geotek Engineering, Inc.(1981), SAIC(1995b)

Geotek Engineering, Inc.(1981)

Geotek Engineering, Inc.(1981), SAIC(1997c)

Geotek Engineering, Inc.(1981)

Geotek Engineering, Inc.(1981)

Geotek Engineering, Inc.(1981)

\section{COMMENTS}

NX corehole completed in 1943. Core hole converted to hole class of Unknown. Data base revised on 4/21/97 to reflect changes discovered during periodic review.

NX corehole completed in 1943. Core hole converted to hole class of Unknown. Data base revised on 4/21/97 to reflect changes discovered during periodic review.

NX corehole completed in 1943. Core hole converted to hole class of Unknown. Data base revised on 4/21/97 to reflect changes discovered during periodic review.

NX corehole completed in 1943. Core hole converted to hole class of Unknown. Data base revised on 4/21/97 to reflect changes discovered during periodic review.

NX corehole completed in 1943. Core hole converted to hole class of Unknown. Data base revised on 4/21/97 to reflect changes discovered during periodic review.

NX corehole completed in 1943. Core hole converted to hole class of Unknown. Data base revised on 4/21/97 to reflect changes discovered during periodic review.

PVC well, $2.0 \mathrm{ft}$ stickup. Data base revised on 4/8/97 to reflect changes discovered during periodic review.

PVC well, $3.0 \mathrm{ft}$ stickup. Data base revised on $4 / 8 / 97$ to reflect changes discovered during periodic review.

PVC well, $1.0 \mathrm{ft}$ stickup. Re-survey 04/24/1995. Data base revised on $4 / 8 / 97$ to reflect changes discovered during periodic review.

PVC well, $2.0 \mathrm{ft}$ stickup. Re-survey by Ogden 1995. Data base revised on 4/8/97 to reflect changes discovered during periodic review.

PVC well, $2.0 \mathrm{ft}$ stickup. Re-survey by Ogden 1995. Data base revised on 4/8/97/to reflect changes discovered during periodic review.

PVC well, $1.0 \mathrm{ft}$ stickup. Re-survey 04/24/1995. Data base revised on $4 / 8 / 97$ to reflect changes discovered during periodic review.

PVC well, $2.5 \mathrm{ft}$ stickup. Re-survey 04/24/1995. Data base revised on $4 / 8 / 97$ to reflect changes discovered during periodic review.

PVC well, $2.0 \mathrm{ft}$ stickup. Re-survey 04/24/1995. Data base revised on 4/8/97/to reflect changes discovered during periodic review.

PVC well, $1.0 \mathrm{ft}$ stickup. Re-survey by Ogden 1995. Data base revised on $4 / 8 / 97$ to reflect changes discovered during periodic review. 


\section{CURRENT}

\section{NAME}

LL/HAZ-10

LL/HAZ-11

LL/HAZ-12

LL/HAZ-13

LL/HAZ-14

LL/HAZ-15

LL/HAZ-16

LL/HAZ-17

LL/HAZ-18

LL/HAZ-19

M-04

M-05

M-06

M-07

$\mathrm{M}-08$

\section{REFERENCE}

Geotek Engineering, Inc.(1981)

Geotek Engineering, Inc.(1981), SAIC(1997c)

Geotek Engineering, Inc.(1981), SAIC(1997c)

Geotek Engineering, Inc.(1981), Energy Division(1988), Martin Marietta Energy Systems(1988a)

Geotek Engineering, Inc.(1981), Energy Division(1988) Martin Marietta Energy Systems(1988a), SAIC(1997c)

Geotek Engineering, Inc.(1981), Energy Division(1988) Martin Marietta Energy Systems(1988a), SAIC(1995b)

Geotek Engineering, Inc.(1981)

Geotek Engineering, Inc.(1981)

Geotek Engineering, Inc.(1981)

Geotek Engineering, Inc.(1981)

Union Carbide Corporation Nuclear Division(1980) $\operatorname{SAIC}(1994 d)$

Union Carbide Corporation Nuclear Division(1980), SAIC(1994d)

Union Carbide Corporation Nuclear Division(1980), SAIC(1994d)

Union Carbide Corporation Nuclear Division(1980), SAIC(1994d)

Union Carbide Corporation Nuclear Division(1980), $\operatorname{SAIC}(1994 d)$

\section{COMMENTS}

PVC well, $1.0 \mathrm{ft}$ stickup. Re-survey 04/24/1995. Data base revised on 4/8/97 to reflect changes discovered during periodic review.

PVC well, $1.0 \mathrm{ft}$ stickup. Re-survey by Ogden 1995. Data base revised on 4/8/97 to reflect changes discovered during periodic review.

PVC well, $1.0 \mathrm{ft}$ stickup. Re-survey by Ogden 1995. Data base revised on $4 / 8 / 97$ to reflect changes discovered during periodic review.

NX core hole.Core @ Bldg 7042/ORNL.Hole modified in 1987 to this status Status Date: ?87. Data base revised on 4/8/97 to reflect changes discovered during periodic review.

NX core hole. Core @ Bldg 7042/ORNL. PVC casing (2-in. diam.) installed in original borehole to 63.0ft. Casing removed, and hole modified in 1987 to this status. Status Date: ?87. Data base revised on 4/8/97 to reflect changes discovered during periodic review.

, NX core hole. Core @ Bldg 7042/ORNL. PVC casing (2-in. diam.) installed in oroginal borehole to $98.0 \mathrm{ft}$. Casing removed, and hole modified in 1987 to this status. Data base revised on $4 / 8 / 97$ to reflect changes discovered during periodic review.

PVC well, $3.0 \mathrm{ft}$ stickup. Data base revised on 4/8/97 to reflect changes discovered during periodic review.

PVC well, $3.0 \mathrm{ft}$ stickup. Data base revised on $4 / 8 / 97$ to reflect changes discovered during periodic review.

PVC well, $3.0 \mathrm{ft}$ stickup. Data base revised on 4/8/97 to reflect changes discovered during periodic review.

PVC well, $2.5 \mathrm{ft}$ stickup. Data base revised on 4/9/97 to reflect changes discovered during periodic review.

Data base revised on $4 / 21 / 97$ to reflect changes discovered during periodic review.

Data base revised on $4 / 21 / 97$ to reflect changes discovered during periodic review.

Data base revised on $4 / 21 / 97$ to reflect changes discovered during periodic review.

Data base revised on 4/21/97 to reflect changes discovered during periodic review.

Data base revised on $4 / 21 / 97$ to reflect changes discovered during periodic review. 


\section{CURRENT}

NAME

M-09

M-10

OR-02

OR-03

OR-04

OR-05

OR-06

OR-07

OR-08

OR-09

OR-21

OR-22

OR-23

$P \& A-1$

$P \& A-2$

$P \& A-3$

\section{REFERENCE}

Union Carbide Corporation Nuclear Division(1980) SAIC(1994d)

Union Carbide Corporation Nuclear Division(1980), SAIC(1994d)

Brunton, G. A.(1978)

Brunton, G. A.(1978), SAIC(1995b)

Brunton, G. A.(1978)

Brunton, G. A.(1978)

Brunton, G. A.(1978), SAIC(1994d)

Brunton, G. A.(1978), SAIC(1994d)

Brunton, G. A.(1978), SAIC(1994d)

Brunton, G. A.(1978), SAIC(1994d)

Brunton, G. A.(1978), SAIC(1994d)

Brunton, G. A.(1978), SAIC(1994d)

Brunton, G. A.(1978)

$\operatorname{SAIC}(1997 \mathrm{c})$

$\operatorname{SAIC}(1997 \mathrm{c})$

$\operatorname{SAIC}(1997 \mathrm{c})$

\section{COMMENTS}

Data base revised on $4 / 21 / 97$ to reflect changes discovered during periodic review.

Data base revised on $4 / 21 / 97$ to reflect changes discovered during periodic review.

Core hole converted to hole class of Unknown.

Core hole converted to hole class of Unknown. Data base revised on $4 / 29 / 97$ to reflect changes discovered during periodic review. ${ }^{* *}$ TOC elevation missing so TOC estimated as GS+3ft, (best GS was for 01/01/1901 ) from meas_pt_elevation

Core hole converted to hole class of Unknown.

Core hole converted to hole class of Unknown. ${ }^{* *} \mathrm{TOC}$ elevation missing so TOC estimated as GS+3ft, (best GS was for 01/01/1901) from meas_pt_elevation

Core hole converted to hole class of Unknown. Data base revised on $4 / 28 / 97$ to reflect changes discovered during periodic review. ${ }^{* *} \mathrm{TOC}$ elevation missing so TOC estimated as GS+3ft, (best GS was for 07/09/1997 ) from meas pt elevation

Core hole converted to hole class of Unknown. Data base revised on $4 / 28 / 97$ to reflect changes discovered during periodic review.

Core hole converted to hole class of Unknown. Data base revised on $4 / 28 / 97$ to reflect changes discovered during periodic review.

Core hole converted to hole class of Unknown. Data base revised on $4 / 28 / 97$ to reflect changes discovered during periodic review.

Core hole converted to hole class of Unknown. Data base revised on $4 / 28 / 97$ to reflect changes discovered during periodic review. ${ }^{* *} \mathrm{TOC}$ elevation missing so TOC estimated as GS+3ft, (best GS was for $01 / 01 / 1901$ ) from meas pt_elevation

Core hole converted to hole class of Unknown. Data base revised on $4 / 28 / 97$ to reflect changes discovered during periodic review. ${ }^{* \star}$ TOC elevation missing so TOC estimated as GS+3ft, (best GS was for 01/01/1901 ) from meas_pt_elevation

Core hole converted to hole class of Unknown. 


\section{CURRENT}

\section{NAME}

PP-01

PP-02

PP-03

PP-04

PP-05

PP-06

PP-07

PP-08

PP-09

RB-001

RB-005

RB-008

RB-009A

RB-010A

RB-011

RB-014

RB-015

\section{REFERENCE}

Stone and Webster, Inc.(1943a)

Stone and Webster, Inc.(1943a)

Stone and Webster, Inc.(1943a)

Stone and Webster, Inc.(1943a)

Stone and Webster, Inc.(1943a)

Stone and Webster, Inc.(1943a)

Stone and Webster, Inc.(1943a)

Stone and Webster, Inc.(1943a)

\section{COMMENTS}

Exploratory boring, finished in 1943. Core hole converted to hole class of Unknown. Data base revised on 4/21/97 to reflect changes discovered during periodic review.

Exploratory boring, finished in 1943. Core hole converted to hole class of Unknown. Data base revised on 4/21/97 to reflect changes discovered during periodic review.

Exploratory boring, finished in 1943. Core hole converted to hole class of Unknown. Data base revised on 4/21/97 to reflect changes discovered during periodic review.

Exploratory boring, finished in 1943. Core hole converted to hole class of Unknown. Data base revised on 4/21/97 to reflect changes discovered during periodic review.

Exploratory boring, finished in 1943. Core hole converted to hole class of Unknown. Data base revised on 4/21/97 to reflect changes discovered during periodic review.

Exploratory boring, finished in 1943. Core hole converted to hole class of Unknown. Data base revised on 4/21/97 to reflect changes discovered during periodic review.

Exploratory boring, finished in 1943. Core hole converted to hole class of Unknown. Data base revised on 4/21/97 to reflect changes discovered during periodic review.

Exploratory boring, finished in 1943. Core hole converted to hole class of Unknown. Data base revised on 4/21/97 to reflect changes discovered during periodic review. 


\section{CURRENT}

\section{NAME}

RB-016

RB-017

RB-018

RB-019

RB-020

RB-031

RB-032

RB-033

RB-034

RB-035

RB-036

RB-037

\section{REFERENCE}

Stone and Webster, Inc.(1943a)

Stone and Webster, Inc.(1943a)

Stone and Webster, Inc.(1943a)

Stone and Webster, Inc.(1943a)

Stone and Webster, Inc.(1943a)

Stone and Webster, Inc.(1943a)

Stone and Webster, Inc.(1943a)

Stone and Webster, Inc.(1943a)

Stone and Webster, Inc.(1943a)

Stone and Webster, Inc.(1943a)

Stone and Webster, Inc.(1943a)

Stone and Webster, Inc.(1943a)

\section{COMMENTS}

Exploratory boring, finished in 1943. Core hole converted to hole class of Unknown. Data base revised on 4/21/97 to reflect changes discovered during periodic review.

Exploratory boring, finished in 1943. Core hole converted to hole class of Unknown. Data base revised on 4/21/97 to reflect changes discovered during periodic review.

Exploratory boring, finished in 1943. Core hole converted to hole class of Unknown. Data base revised on 4/21/97 to reflect changes discovered during periodic review.

Exploratory boring, finished in 1943. Core hole converted to hole class of Unknown. Data base revised on 4/21/97 to reflect changes discovered during periodic review.

Exploratory boring, finished in 1943. Core hole converted to hole class of Unknown. Data base revised on 4/21/97 to reflect changes discovered during periodic review.

Exploratory boring, finished in 1943. Core hole converted to hole class of Unknown. Data base revised on 4/21/97 to reflect changes discovered during periodic review.

Exploratory boring, finished in 1943. Core hole converted to hole class of Unknown. Data base revised on 4/21/97 to reflect changes discovered during periodic review.

Exploratory boring, finished in 1943. Core hole converted to hole class of Unknown. Data base revised on 4/21/97 to reflect changes discovered during periodic review.

Exploratory boring, finished in 1943. Core hole converted to hole class of Unknown. Data base revised on 4/21/97 to reflect changes discovered during periodic review.

Exploratory boring, finished in 1943. Core hole converted to hole class of Unknown. Data base revised on 4/21/97 to reflect changes discovered during periodic review.

Exploratory boring, finished in 1943. Core hole converted to hole class of Unknown. Data base revised on 4/21/97 to reflect changes discovered during periodic review.

Exploratory boring, finished in 1943 Core hole converted to hole class of Unknown. Data base revised on 4/21/97 to reflect changes discovered during periodic review. 


\section{CURRENT NAME}

RB-038

S3-1

S3-3

S3-5

S3-6

S3-7

S3-8

SAN-1

SAN-10

SAN-2

SAN-3

SAN-4

SAN-5

SAN-6

SAN-7

SAN-8

SAN-9

UNIDENT A

UNIDENT B

$\mathrm{V}-1$

$\mathrm{V}-2$

$\mathrm{V}-3$

$V-4$

$V-5$

$\mathrm{V}-6$

$\mathrm{V}-7$

\section{REFERENCE}

Stone and Webster, Inc.(1943a)

SAIC(1995b)

SAIC(1995b)

SAIC(1995b)

SAIC(1995b)

SAIC(1995b)

SAIC(1995b)

Geotek Engineering, Inc.(1981), Payne, A.(1981)

Geotek Engineering, Inc.(1981), Payne, A.(1981)

Geotek Engineering, Inc.(1981), Payne, A.(1981)

Geotek Engineering, Inc.(1981), Payne, A.(1981)

Geotek Engineering, Inc.(1981), Payne, A.(1981)

Geotek Engineering, Inc.(1981), Payne, A.(1981)

Geotek Engineering, Inc.(1981), Payne, A.(1981)

Geotek Engineering, Inc.(1981), Payne, A.(1981)

Geotek Engineering, Inc.(1981), Payne, A.(1981)

Geotek Engineering, Inc.(1981), Payne, A.(1981)

SAIC(1995b)

SAIC(1995b)

SAIC(1992b)

SAIC(1992b)

SAIC(1992b)

SAIC(1992b)

SAIC(1992b)

SAIC(1992b)

SAIC(1992b)

\section{COMMENTS}

Exploratory boring, finished in 1943. Core hole converted to hole class of Unknown. Data base revised on 4/21/97 to reflect changes discovered during periodic review.

Well is located on west Chestnut Ridge, near the New Zion Cemetary. Data base revised on $4 / 17 / 97$ to reflect changes discovered during periodic review.

Well is located on west Chestnut Ridge, near the New Zion Cemetary. Well is located on west Chestnut Ridge, near the New Zion Cemetary. Well is located on west Chestnut Ridge, near the New Zion Cemetary. Well is located on west Chestnut Ridge, near the New Zion Cemetary. Well is located on west Chestnut Ridge, near the New Zion Cemetary. Well is located on west Chestnut Ridge, near the New Zion Cemetary. Well is located on west Chestnut Ridge, near the New Zion Cemetary. Well is located on west Chestnut Ridge, near the New Zion Cemetary. Well is located on west Chestnut Ridge, near the New Zion Cemetary.

Boring is located on the west side of Building 9206. Boring is located on the west side of Building 9206. Boring is located on the west side of Building 9206. Boring is located on the east side of Building 9206. Boring is located on the east side of Building 9206. Boring is located on the east side of Building 9206. Boring is located on the west side of Building 9206. 
This Page Intentionally Left Blank 
APPENDIX D

Screened Well Construction Summary 
This Page Intentionally Left Blank 


\begin{tabular}{|c|c|c|c|c|c|c|c|c|c|c|}
\hline $\begin{array}{c}\text { CURRENT } \\
\text { NAME }\end{array}$ & $\begin{array}{c}\text { CASING } \\
\text { TYPE }\end{array}$ & $\begin{array}{c}\text { CASING } \\
\text { O.D. }\end{array}$ & $\begin{array}{c}\text { CASING } \\
\text { I.D. }\end{array}$ & $\begin{array}{c}\text { HOLE } \\
\text { DIAMETER }\end{array}$ & $\begin{array}{c}\text { HOLE } \\
\text { DEPTH }\end{array}$ & SCREEN TYPE & $\begin{array}{l}\text { PAC } \\
\text { INTE }\end{array}$ & $\begin{array}{l}\text { CKED } \\
\text { RVAL }\end{array}$ & $\begin{array}{l}\text { SCF } \\
\text { INT }\end{array}$ & $\begin{array}{l}\text { EENED } \\
\text { ERVAL }\end{array}$ \\
\hline 1002 & PVC40 & 6.50 & & 8.00 & 48.00 & PVC/SL/ & & to & 28.00 & to 48.00 \\
\hline 1003 & PVC40 & 6.50 & & 8.00 & 48.00 & $\mathrm{PVC/SL/}$ & & to & 28.00 & to 48.00 \\
\hline 1004 & PVC40 & 6.50 & & 8.00 & 29.00 & PVC/SL/ & & to & 19.00 & to 29.00 \\
\hline 1005 & PVC40 & 6.50 & & 8.00 & 24.00 & PVC/SL/ & & to & 8.00 & to 18.00 \\
\hline 1006 & & & & & 20.50 & UNKN/UNKN/ & 8.00 & to 20.50 & 10.00 & to 20.00 \\
\hline 1007 & & & & & 18.00 & UNKN/UNKN/ & 7.00 & to 18.00 & 8.00 & to 18.00 \\
\hline 1008 & & & & & 20.00 & UNKN/UNKN/ & 7.30 & to 20.00 & 8.30 & to 18.30 \\
\hline 1009 & & & & & 22.50 & UNKN/UNKN/ & 11.50 & to 22.50 & 12.50 & to 22.50 \\
\hline 1010 & & & & & 20.00 & UNKN/UNKN/ & 9.00 & to 20.00 & 10.00 & to 20.00 \\
\hline 1011 & & & & & 22.50 & UNKN/UNKN/ & 11.00 & to 22.00 & 12.00 & to 22.00 \\
\hline 1028 & PVC40 & 6.50 & & 8.00 & 29.00 & PVC/SL/ & & to & 19.00 & to 29.00 \\
\hline 1029 & PVC40 & 6.50 & & 7.50 & 18.50 & PVC/PER/ & 2.00 & to 15.50 & 5.00 & to 18.50 \\
\hline 1030 & PVC40 & 6.50 & & 8.00 & 47.00 & PVC/SL/ & & to & 37.00 & to 47.00 \\
\hline 1031 & PVC40 & 6.50 & & 8.00 & 47.00 & PVC/SL/ & & to & 37.00 & to 47.00 \\
\hline 1032 & PVC40 & 6.50 & & 8.00 & 57.00 & PVC/SL/ & & to & 47.00 & to 57.00 \\
\hline 1034 & PVC40 & 6.50 & & 8.00 & 45.00 & PVC/SL/ & & to & 35.00 & to 45.00 \\
\hline 1037 & PVC40 & 6.50 & & 8.00 & 45.00 & PVC/SL/ & & to & 35.00 & to 45.00 \\
\hline 1038 & PVC40 & 6.50 & & 7.50 & 26.00 & PVC/PER/ & 2.00 & to 23.00 & 5.00 & to 26.00 \\
\hline 1040 & PVC40 & 6.50 & & 7.50 & 23.00 & PVC/PER/ & 2.00 & to 20.00 & 5.00 & to 23.00 \\
\hline 1041 & PVC40 & 6.50 & & 7.50 & 29.50 & PVC/PER/ & 2.00 & to 26.50 & 5.00 & to 29.50 \\
\hline 1049 & PVC40 & 6.50 & & 8.00 & 20.00 & PVC/SL/ & & to & 10.00 & to 20.00 \\
\hline 1051 & PVC40 & 6.50 & & 7.50 & 26.50 & PVC/PER/ & 2.00 & to 25.00 & 5.00 & to 28.00 \\
\hline 1052 & PVC40 & 6.50 & & 7.50 & 20.50 & PVC/PER/ & 2.00 & to 19.00 & 5.00 & to 22.00 \\
\hline 1055 & PVC40 & 4.50 & 4.00 & 8.00 & 70.00 & PVC/SL/ .02 & 10.00 & to 70.00 & 20.00 & to 70.00 \\
\hline 1056 & PVC40 & 4.50 & 4.00 & 8.00 & 70.00 & PVC/SL/ .02 & 10.00 & to 70.00 & 20.00 & to 70.00 \\
\hline 1057 & PVC40 & 4.50 & 4.00 & 8.00 & 70.00 & PVC/SL/ .02 & 10.00 & to 70.00 & 20.00 & to 70.00 \\
\hline 1058 & PVC40 & 4.50 & 4.00 & 8.00 & 70.00 & PVC/SL/ .02 & 10.00 & to 70.00 & 20.00 & to 70.00 \\
\hline
\end{tabular}




\begin{tabular}{|c|c|c|c|c|c|c|c|c|c|c|}
\hline $\begin{array}{c}\text { CURRENT } \\
\text { NAME }\end{array}$ & $\begin{array}{l}\text { CASING } \\
\text { TYPE }\end{array}$ & $\begin{array}{l}\text { CASING } \\
\underline{\text { O.D. }}\end{array}$ & $\begin{array}{c}\text { CASING } \\
\text { I.D. }\end{array}$ & $\begin{array}{c}\text { HOLE } \\
\text { DIAMETER }\end{array}$ & $\begin{array}{c}\text { HOLE } \\
\text { DEPTH }\end{array}$ & SCREEN TYPE & \multicolumn{2}{|c|}{$\begin{array}{r}\text { PACKED } \\
\text { INTERVAL }\end{array}$} & \multicolumn{2}{|c|}{$\begin{array}{l}\text { SCREENED } \\
\text { INTERVAL }\end{array}$} \\
\hline 1059 & PVC40 & 4.50 & 4.00 & 8.00 & 70.00 & PVC/SL/ .02 & 10.00 & to 70.00 & 20.00 & to 70.00 \\
\hline 1060 & PVC40 & 4.50 & 4.00 & 8.00 & 70.00 & PVC/SL/ .02 & 10.00 & to 70.00 & 20.00 & to 70.00 \\
\hline 1061 & PVC40 & 4.50 & 4.00 & 8.00 & 70.00 & $\mathrm{PVC} / \mathrm{SL} / .02$ & 10.00 & to 70.00 & 20.00 & to 70.00 \\
\hline 1062 & PVC40 & 4.50 & 4.00 & 8.00 & 70.00 & $\mathrm{PVC/SL/} .02$ & 10.00 & to 70.00 & 20.00 & to 70.00 \\
\hline 1063 & PVC40 & 6.50 & 6.00 & 11.75 & 70.00 & PVC/SL/ .01 & 10.00 & to 70.00 & 20.00 & to 70.00 \\
\hline 1070 & PVC40 & & & & 41.40 & UNKN/UNKN/ & & to & & to \\
\hline 1071 & PVC40 & 6.50 & & 7.50 & 20.00 & PVC/PER/ & 2.00 & to 17.00 & 5.00 & to 20.00 \\
\hline 1072 & PVC40 & 6.50 & & 7.50 & 20.00 & PVC/PER/ & 2.00 & to 17.00 & 5.00 & to 20.00 \\
\hline 1073 & PVC40 & 6.50 & & & 21.00 & UNKN/UNKN/ & & to & & to \\
\hline 1080 & PVC40 & 6.50 & & & 181.00 & PVC/UNKN/ & 160.00 & to 181.00 & 161.00 & to 181.00 \\
\hline 1081 & SS304 & 2.37 & & & 38.00 & SLS/SW/ .01 & 27.00 & to 38.00 & 28.00 & to 38.00 \\
\hline 1082 & SS304 & 2.37 & & & 50.00 & SLS/SW/ .01 & 39.00 & to 50.00 & 40.00 & to 50.00 \\
\hline 1083 & SS304 & 2.37 & & & 58.50 & SLS/SW/ .01 & 47.00 & to 58.50 & 48.50 & to 58.50 \\
\hline 1084 & SS304 & 2.37 & & & 144.50 & SLS/SW/ .01 & 133.00 & to 144.00 & 134.50 & to 144.50 \\
\hline 1085 & PVC40 & 6.50 & & & 122.00 & PVC/SL/ & 106.00 & to 122.00 & 107.00 & to 122.00 \\
\hline 1086 & PVC40 & 6.50 & & & 156.00 & PVC/SL/ & 140.00 & to 156.00 & 141.00 & to 156.00 \\
\hline 1087 & PVC40 & 6.50 & & & 145.00 & PVC/SL/ & 129.00 & to 145.00 & 130.00 & to 145.00 \\
\hline 1090 & PVC40 & 6.50 & & & 96.70 & PVC/SL/ & & to & & to \\
\hline 1091 & PVC40 & 6.50 & & & 102.00 & PVC/SL/ & & to & & to \\
\hline 1092 & PVC40 & 6.50 & & & & $\mathrm{PVC/SL/}$ & & to & & to \\
\hline 1095 & PVC40 & 6.50 & & 8.00 & 118.00 & $\mathrm{PVC/SL/}$ & 117.00 & to 118.00 & 108.00 & to 118.00 \\
\hline 1096 & PVC40 & 6.50 & & 8.00 & 68.00 & PVC/SL/ & 57.00 & to 68.00 & 58.00 & to 68.00 \\
\hline 1100 & PVC40 & 2.37 & 2.00 & 6.50 & 27.00 & PVC/SL/ .01 & 21.20 & to 27.00 & 22.00 & to 27.00 \\
\hline 1101 & PVC & 2.70 & & 6.50 & 28.40 & $\mathrm{PVC/SL/} \mathrm{.03}$ & 17.40 & to 28.90 & 18.40 & to 28.40 \\
\hline 1102 & PVC40 & 2.37 & 2.00 & 3.00 & 75.10 & PVC/SL/ .01 & 64.60 & to 75.10 & 65.10 & to 75.10 \\
\hline 1103 & PVC40 & 2.37 & 2.00 & 6.50 & 25.00 & PVC/SL/ .01 & 18.90 & to 25.00 & 20.00 & to 25.00 \\
\hline 1104 & PVC & 2.70 & & 6.50 & 53.40 & $\mathrm{PVC/SL/} \mathrm{.03}$ & 47.00 & to 54.30 & 48.50 & to 53.50 \\
\hline
\end{tabular}




\begin{tabular}{|c|c|c|c|c|c|c|c|c|c|c|}
\hline $\begin{array}{c}\text { CURRENT } \\
\text { NAME }\end{array}$ & $\begin{array}{l}\text { CASING } \\
\text { TYPE }\end{array}$ & $\begin{array}{c}\text { CASING } \\
\text { O.D. }\end{array}$ & $\begin{array}{c}\text { CASING } \\
\text { I.D. }\end{array}$ & $\begin{array}{c}\text { HOLE } \\
\text { DIAMETER }\end{array}$ & $\begin{array}{c}\text { HOLE } \\
\text { DEPTH }\end{array}$ & SCREEN TYPE & \multicolumn{2}{|c|}{$\begin{array}{r}\text { PACKED } \\
\text { INTERVAL }\end{array}$} & \multicolumn{2}{|c|}{$\begin{array}{l}\text { SCREENED } \\
\text { INTERVAL }\end{array}$} \\
\hline 1105 & PVC40 & 2.37 & 2.00 & 3.00 & 56.40 & PVC/SL/ .01 & 44.70 & to 56.40 & 46.40 & to 56.40 \\
\hline 1106 & PVC40 & 2.37 & 2.00 & 6.50 & 34.00 & PVC/SL/ .01 & 27.00 & to 34.50 & 29.00 & to 34.00 \\
\hline 1107 & PVC40 & 2.37 & 2.00 & 3.00 & 32.20 & $\mathrm{PVC} / \mathrm{SL} / .01$ & 20.50 & to 32.20 & 22.20 & to 32.20 \\
\hline 1108 & PVC40 & 2.37 & 2.00 & 6.50 & 9.00 & $\mathrm{PVC/SL/} \mathrm{.01}$ & 3.90 & to 9.00 & 4.00 & to 9.00 \\
\hline 1109 & PVC40 & 2.37 & 2.00 & 6.50 & 39.40 & PVC/SL/ .01 & 17.00 & to 41.80 & 29.40 & to 39.40 \\
\hline 1110 & PVC & 2.00 & 1.75 & & 8.00 & PVC/SL/ .01 & & to 8.00 & 1.00 & to 8.00 \\
\hline 1111 & PVC & 2.00 & 1.75 & & 8.00 & PVC/SL/ .01 & & to 8.00 & 1.00 & to 8.00 \\
\hline 1112 & PVC & 2.00 & 1.75 & & 8.00 & $\mathrm{PVC} / \mathrm{SL} / .01$ & & to 8.00 & 1.00 & to 8.00 \\
\hline 1130 & PVC40 & 4.00 & & 9.63 & 23.50 & $\mathrm{PVC} / \mathrm{SL} / .02$ & 11.00 & to 23.50 & 13.00 & to 23.00 \\
\hline 1131 & PVC40 & 4.00 & & 9.63 & 30.00 & $\mathrm{PVC/SL/} .02$ & 17.50 & to 30.00 & 19.50 & to 29.50 \\
\hline 1132 & PVC40 & 4.00 & & 9.63 & 27.50 & PVC/SL/ .02 & 13.00 & to 27.50 & 17.00 & to 27.00 \\
\hline 1133 & PVC40 & 4.00 & & 9.63 & 24.00 & PVC/SL/ .02 & 11.30 & to 24.00 & 13.50 & to 23.50 \\
\hline 1134 & PVC40 & 4.00 & & 8.00 & 189.00 & PVC/SL/ .02 & 174.40 & to 190.00 & 178.50 & to 188.50 \\
\hline $2 \mathrm{D}$ & STL & 1.75 & & & 43.80 & & 43.20 & to 44.40 & & to \\
\hline 21 & STL & 1.75 & & & 34.00 & & & to & & to \\
\hline $2 S$ & STL & 1.75 & & & 29.20 & & 28.00 & to 29.60 & & to \\
\hline $3 D$ & STL & 1.75 & & & 42.80 & & 41.00 & to 42.80 & & to \\
\hline 31 & STL & 1.75 & & & 32.00 & & & to & & to \\
\hline $3 S$ & STL & 1.75 & & & 27.10 & & 26.00 & to 27.20 & & to \\
\hline $4 \mathrm{D}$ & STL & 1.75 & & & 45.60 & & 44.50 & to 46.20 & & to \\
\hline $4 \mathrm{I}$ & STL & 1.75 & & & 36.40 & & & to & & to \\
\hline $4 S$ & STL & 1.75 & & & 30.80 & & 29.70 & to 31.20 & & to \\
\hline $53-1 \mathrm{~A}$ & SS304 & 2.37 & 2.00 & 6.00 & 22.10 & SLS/SW/ .01 & 14.00 & to 16.00 & 17.10 & to 22.10 \\
\hline $54-1 A$ & PVC40 & 4.50 & 4.00 & 6.00 & 23.00 & SLS/SW/ .01 & 15.00 & to 17.00 & 18.00 & to 23.00 \\
\hline $54-2 A$ & PVC40 & 4.50 & 4.00 & 6.00 & 26.30 & SLS/SW/ .01 & 18.30 & to 20.30 & 21.30 & to 26.30 \\
\hline $55-1 \mathrm{~A}$ & PVC40 & 4.50 & 4.00 & 6.00 & 19.30 & SLS/SW/ .01 & 13.30 & to 19.30 & 14.30 & to 19.30 \\
\hline $55-1 B$ & PVC40 & 4.50 & 4.00 & 6.00 & 38.80 & SLS/SW/ .01 & 30.80 & to 32.80 & 33.80 & to 38.80 \\
\hline
\end{tabular}




\begin{tabular}{|c|c|c|c|c|c|c|}
\hline $\begin{array}{c}\text { CURRENT } \\
\text { NAME }\end{array}$ & $\begin{array}{l}\text { CASING } \\
\text { TYPE }\end{array}$ & $\begin{array}{c}\text { CASING } \\
\text { O.D. }\end{array}$ & $\begin{array}{c}\text { CASING } \\
\text { I.D. }\end{array}$ & $\begin{array}{c}\text { HOLE } \\
\text { DIAMETER }\end{array}$ & $\begin{array}{c}\text { HOLE } \\
\text { DEPTH }\end{array}$ & SCREEN TYPE \\
\hline $55-1 C$ & PVC40 & 4.50 & 4.00 & 6.00 & 75.70 & SLS/SW/ .01 \\
\hline $55-2 A$ & PVC40 & 4.50 & 4.00 & 6.00 & 14.10 & SLS/SW/ .01 \\
\hline $55-2 B$ & PVC40 & 4.50 & 4.00 & 6.00 & 27.60 & SLS/SW/ .01 \\
\hline $55-2 C$ & PVC40 & 4.50 & 4.00 & 6.00 & 75.90 & SLS/SW/ .01 \\
\hline $55-3 A$ & PVC40 & 4.50 & 4.00 & 6.00 & 14.30 & SLS/SW/ .01 \\
\hline $55-3 B$ & PVC40 & 4.50 & 4.00 & 6.00 & 38.10 & SLS/SW/ .01 \\
\hline $55-3 C$ & PVC40 & 4.50 & 4.00 & 6.00 & 77.50 & SLS/SW/ .01 \\
\hline $55-4 B$ & PVC40 & 4.50 & 4.00 & 6.00 & 25.50 & SLS/SW/ .01 \\
\hline $55-4 C$ & PVC40 & 4.50 & 4.00 & 6.00 & 72.60 & SLS/SW/ .01 \\
\hline $55-5 A$ & PVC40 & 4.50 & 4.00 & 6.00 & 10.90 & SLS/SW/ .01 \\
\hline $55-6 \mathrm{~A}$ & PVC40 & 4.50 & 4.00 & 6.00 & 12.90 & SLS/SW/ .01 \\
\hline $55-7 \mathrm{~A}$ & SS304 & 2.37 & & 6.00 & 22.30 & SLS/SW/ .01 \\
\hline $55-8 \mathrm{~A}$ & SS304 & 2.37 & & 4.00 & 22.00 & SLS/SW/ .01 \\
\hline $55-8 B$ & SS304 & 2.37 & & 4.00 & 45.00 & SLS/SW/ .01 \\
\hline $55-9 A$ & SS304 & 2.37 & & 6.00 & 18.50 & SLS/SW/ .01 \\
\hline $56-1 \mathrm{~A}$ & PVC40 & 4.50 & 4.00 & 6.00 & 19.00 & SLS/SW/ .01 \\
\hline $56-1 C$ & PVC40 & 4.50 & 4.00 & 6.00 & 75.30 & SLS/SW/ .01 \\
\hline $56-2 A$ & PVC40 & 4.50 & 4.00 & 6.00 & 15.10 & SLS/SW/ .01 \\
\hline $56-2 B$ & PVC40 & 4.50 & 4.00 & 6.00 & 38.80 & SLS/SW/ .01 \\
\hline $56-2 C$ & PVC40 & 4.50 & 4.00 & 6.00 & 77.30 & SLS/SW/ .01 \\
\hline $56-3 A$ & PVC40 & 4.50 & 4.00 & 6.00 & 17.80 & SLS/SW/ .01 \\
\hline $56-3 B$ & PVC40 & 4.50 & 4.00 & 6.00 & 33.40 & SLS/SW/ .01 \\
\hline $56-3 C$ & PVC40 & 4.50 & 4.00 & 6.00 & 55.50 & SLS/SW/ .01 \\
\hline $56-4 A$ & PVC40 & 4.50 & 4.00 & 6.00 & 12.10 & SLS/SW/ .01 \\
\hline $56-4 B$ & PVC40 & 4.50 & 4.00 & 6.00 & 32.60 & SLS/SW/ .01 \\
\hline $56-4 C$ & PVC40 & 4.50 & 4.00 & 6.00 & 76.30 & SLS/SW/ .01 \\
\hline $56-5 A$ & PVC40 & 4.50 & 4.00 & 6.00 & 12.60 & SLS/SW/ .01 \\
\hline
\end{tabular}

\begin{tabular}{|c|c|c|c|}
\hline IN & $\begin{array}{l}\text { CKED } \\
\text { ERVAL }\end{array}$ & $\begin{array}{l}\text { SCF } \\
\text { INT }\end{array}$ & $\begin{array}{l}\text { EENED } \\
\text { ERVAL }\end{array}$ \\
\hline 9.70 & to 75.70 & 70.70 & to 75.70 \\
\hline 6.10 & to 8.10 & 9.10 & to 14.10 \\
\hline 19.60 & to 21.60 & 22.60 & to 27.60 \\
\hline 67.90 & to 69.90 & 70.90 & to 75.90 \\
\hline 6.30 & to 8.30 & 9.30 & to 14.30 \\
\hline 30.10 & to 32.10 & 33.10 & to 38.10 \\
\hline 69.50 & to 71.50 & 72.50 & to 77.50 \\
\hline 17.50 & to 19.50 & 20.50 & to 25.50 \\
\hline 64.60 & to 66.60 & 67.60 & to 72.60 \\
\hline 2.90 & to 4.90 & 5.90 & to 10.90 \\
\hline 4.90 & to 6.90 & 7.90 & to 12.9 \\
\hline 15.30 & to 22.30 & 17.30 & to 22.30 \\
\hline 15.00 & to 22.00 & 17.00 & to 22.00 \\
\hline 33.00 & to 45.00 & 35.00 & to 45.00 \\
\hline 11.50 & to 18.50 & 13.50 & to 18.50 \\
\hline 11.00 & to 13.00 & 14.00 & to 19.00 \\
\hline 67.30 & to 69.30 & 70.30 & to 75.30 \\
\hline 7.10 & to 9.10 & 10.10 & to 15.10 \\
\hline 30.80 & to 32.80 & 33.80 & to 38.80 \\
\hline 69.30 & to 71.30 & 72.30 & to 77.30 \\
\hline 9.80 & to 11.80 & 12.80 & to 17.80 \\
\hline 25.40 & to 27.40 & 28.40 & to 33.40 \\
\hline 47.50 & to 49.50 & 50.50 & to 55.50 \\
\hline 4.10 & to 6.10 & 7.10 & to 12.10 \\
\hline 24.60 & to 26.60 & 27.60 & to 32.60 \\
\hline 68.30 & to 70.30 & 71.30 & to 76.30 \\
\hline 6.60 & to & 7.60 & \\
\hline
\end{tabular}




\begin{tabular}{|c|c|c|c|c|c|c|c|c|c|c|}
\hline $\begin{array}{c}\text { CURRENT } \\
\text { NAME }\end{array}$ & $\begin{array}{c}\text { CASING } \\
\text { TYPE }\end{array}$ & $\begin{array}{l}\text { CASING } \\
\text { O.D. }\end{array}$ & $\begin{array}{c}\text { CASING } \\
\text { I.D. }\end{array}$ & $\begin{array}{c}\text { HOLE } \\
\text { DIAMETER }\end{array}$ & $\begin{array}{c}\text { HOLE } \\
\text { DEPTH }\end{array}$ & SCREEN TYPE & \multicolumn{2}{|c|}{$\begin{array}{r}\text { PACKED } \\
\text { INTERVAL } \\
\end{array}$} & \multicolumn{2}{|c|}{$\begin{array}{l}\text { SCREENED } \\
\text { INTERVAL }\end{array}$} \\
\hline $56-5 B$ & PVC40 & 4.50 & 4.00 & 6.00 & 35.10 & SLS/SW/ .01 & 27.10 & to 29.10 & 30.10 & to 35.10 \\
\hline $56-5 C$ & PVC40 & 4.50 & 4.00 & 6.00 & 71.60 & SLS/SW/ .01 & 63.60 & to 65.60 & 66.60 & to 71.60 \\
\hline $56-6 \mathrm{~A}$ & PVC40 & 4.50 & 4.00 & 6.00 & 21.00 & SLS/SW/ .01 & 13.00 & to 15.00 & 16.00 & to 21.00 \\
\hline $56-7 A$ & PVC40 & 4.50 & 4.00 & 6.00 & 21.10 & SLS/SW/ .01 & 15.10 & to 21.10 & 16.10 & to 21.10 \\
\hline $56-8 \mathrm{~A}$ & PVC40 & 4.50 & 4.00 & 6.00 & 25.60 & SLS/SW/ .01 & 19.60 & to 25.60 & 20.60 & to 25.60 \\
\hline $56-9 \mathrm{~A}$ & PVC40 & 4.50 & 4.00 & 6.00 & 17.30 & SLS/SW/ .01 & 9.30 & to 11.30 & 12.30 & to 17.30 \\
\hline $58-1 A$ & PVC40 & 4.50 & 4.00 & 6.00 & 11.20 & SLS/SW/ .01 & 3.20 & to 5.20 & 6.20 & to 11.20 \\
\hline $58-2 A$ & PVC40 & 4.50 & 4.00 & 6.00 & 9.90 & SLS/SW/ .01 & 3.90 & to 9.90 & 4.90 & to 9.90 \\
\hline $59-1 A$ & PVC40 & 4.50 & 4.00 & 6.00 & 13.20 & SLS/SW/ .01 & 7.20 & to 13.20 & 8.20 & to 13.20 \\
\hline $59-1 B$ & PVC40 & 4.50 & 4.00 & 6.00 & 36.90 & SLS/SW/ .01 & 30.90 & to 36.90 & 31.90 & to 36.90 \\
\hline $59-1 C$ & PVC40 & 4.50 & 4.00 & 6.00 & 73.90 & SLS/SW/ .01 & 65.90 & to 67.90 & 68.90 & to 73.90 \\
\hline $5 \mathrm{D}$ & STL & 1.75 & & & 41.00 & & 38.80 & to 41.80 & & to \\
\hline 51 & STL & 1.75 & & & 30.80 & & & to & & to \\
\hline $5 S$ & STL & 1.75 & & & 25.80 & & 23.00 & to 26.10 & & to \\
\hline $60-1 A$ & PVC40 & 4.50 & 4.00 & 6.00 & 23.20 & SLS/SW/ .01 & 15.20 & to 17.20 & 18.20 & to 23.20 \\
\hline $60-1 B$ & PVC40 & 4.50 & 4.00 & 6.00 & 29.20 & SLS/SW/ .01 & 21.20 & to 23.20 & 24.20 & to 29.20 \\
\hline $60-2 A$ & PVC40 & 4.50 & 4.00 & 6.00 & 13.50 & SLS/SW/ .01 & 5.50 & to 7.50 & 8.50 & to 13.50 \\
\hline AN:D-1 & SLS & 2.37 & 2.00 & 6.00 & 4.90 & SLS/SW/ .01 & 1.80 & to 4.90 & 1.90 & to 4.90 \\
\hline AN:D-10 & SLS & 2.37 & 2.00 & 6.00 & 9.30 & SLS/SW/ .01 & 3.50 & to 9.30 & 5.30 & to 9.30 \\
\hline AN:D-11 & SLS & 2.37 & 2.00 & 6.00 & 8.40 & SLS/SW/ .01 & 3.00 & to 8.40 & 5.40 & to 8.40 \\
\hline AN:D-2 & SLS & 2.37 & 2.00 & 6.00 & 8.20 & SLS/SW/ .01 & 2.50 & to 8.20 & 4.20 & to 8.20 \\
\hline AN:D-3 & SLS & 2.37 & 2.00 & 6.00 & 9.70 & SLS/SW/ .01 & 3.20 & to 9.70 & 4.70 & to 9.70 \\
\hline AN:D-4 & SLS & 2.37 & 2.00 & 6.00 & 4.90 & SLS/SW/ .01 & 1.80 & to 4.90 & 2.90 & to 4.90 \\
\hline AN:D-5 & SLS & 2.37 & 2.00 & 6.00 & 6.10 & SLS/SW/ .01 & 2.50 & to 6.10 & 3.10 & to 6.10 \\
\hline AN:D-6 & SLS & 2.37 & 2.00 & 6.00 & 3.80 & SLS/SW/ .01 & 1.20 & to 3.80 & 1.80 & to 3.80 \\
\hline AN:D-7 & SLS & 2.37 & 2.00 & 6.00 & 8.00 & SLS/SW/ .01 & 3.50 & to 8.00 & 5.00 & to 8.00 \\
\hline AN:D-8 & SLS & 2.37 & 2.00 & 6.00 & 8.40 & SLS/SW/ .01 & 3.60 & to 8.40 & 4.40 & to 8.40 \\
\hline
\end{tabular}




\begin{tabular}{|c|c|c|c|c|c|c|c|c|c|c|}
\hline $\begin{array}{c}\text { CURRENT } \\
\text { NAME }\end{array}$ & $\begin{array}{l}\text { CASING } \\
\text { TYPE }\end{array}$ & $\begin{array}{c}\text { CASING } \\
\text { O.D. }\end{array}$ & $\begin{array}{c}\text { CASING } \\
\text { I.D. }\end{array}$ & $\begin{array}{c}\text { HOLE } \\
\text { DIAMETER }\end{array}$ & $\begin{array}{c}\text { HOLE } \\
\text { DEPTH }\end{array}$ & SCREEN TYPE & $\begin{array}{r}\text { PA } \\
\text { INTI }\end{array}$ & $\begin{array}{l}\text { CKED } \\
\text { ERVAL }\end{array}$ & & $\begin{array}{l}\text { EENED } \\
\text { ERVAL }\end{array}$ \\
\hline AN:D-9 & SLS & 2.37 & 2.00 & 6.00 & 7.00 & SLS/SW/ & 3.00 & to 10.00 & 3.00 & to 7.00 \\
\hline AN:D-UNKNOWN & SLS & 2.37 & 2.00 & & 18.00 & SLS/SW/ .01 & & to 18.00 & 13.00 & to 18.00 \\
\hline B-1 & PVC & 2.37 & 2.00 & 8.00 & 21.00 & PVC/SL/ .01 & 4.80 & to 17.40 & 6.00 & to 16.00 \\
\hline B-3 & PVC & 2.37 & 2.00 & 8.00 & 36.00 & PVC/SL/ .01 & 7.00 & to 18.20 & 7.80 & to 17.80 \\
\hline BC-15 & PVC & & 1.00 & 3.00 & 100.20 & PVC/SL/ & & to & 40.50 & to 100.20 \\
\hline BC-47 & & & & 3.00 & 50.00 & PVC/SL/ & & to & & to 50.00 \\
\hline BC-62 & PVC & 1.25 & 1.00 & 3.00 & 56.30 & PVC/SL/ & & to & & to 56.30 \\
\hline BCU-03 & PVC & 1.25 & 1.00 & 5.00 & 57.60 & PVC/SL/ & 3.60 & to 57.60 & & to 57.60 \\
\hline BCU-04 & PVC & 4.50 & 4.00 & 8.00 & 60.20 & PVC/SL/ & 50.20 & to 60.20 & & to 60.20 \\
\hline BCU-05 & PVC & 1.25 & 1.00 & 5.00 & 61.00 & PVC/SL/ & 4.00 & to 61.00 & & to 61.00 \\
\hline BCU-06 & PVC & 4.50 & 4.00 & & 62.00 & PVC/SL/ & 5.00 & to 62.00 & & to 62.00 \\
\hline BCU-07 & PVC & 1.25 & 1.00 & 5.00 & 61.00 & PVC/SL/ & 3.60 & to 61.00 & & to 61.00 \\
\hline BCU-08 & PVC & 1.25 & 1.00 & 5.00 & 61.70 & PVC/SL/ & 2.00 & to 61.70 & & to 61.70 \\
\hline BCU-09 & PVC & 1.25 & 1.00 & 3.00 & 61.00 & PVC/SL/ & 4.00 & to 61.00 & & to 61.00 \\
\hline BCU-11 & PVC & 1.25 & 1.00 & 5.00 & & PVC/SL/ & 3.00 & to 45.00 & & to 45.00 \\
\hline BCU-17 & PVC & 1.25 & 1.00 & & 10.00 & PVC/UNKN/ & & to 10.00 & & to 10.00 \\
\hline BCU-18 & PVC & 1.25 & 1.00 & & 51.00 & UNKN/UNKN/ & & to 51.00 & & to 51.00 \\
\hline BCU-19 & PVC & 1.25 & 1.00 & & 34.00 & UNKN/UNKN/ & & to 34.00 & & to 34.00 \\
\hline BCU-22 & PVC & 4.50 & 4.00 & & 10.00 & PVC/SL/ & & to 10.00 & & to 10.00 \\
\hline BCU-23 & PVC & 1.25 & 1.00 & 5.00 & 58.30 & PVC/SL/ & & to 58.30 & 9.20 & to 58.30 \\
\hline BCU-24 & PVC & 1.25 & 1.00 & 5.00 & 59.80 & PVC/SL/ & & to 59.80 & & to 59.80 \\
\hline BCU-25 & PVC & 1.25 & 1.00 & 5.00 & 58.50 & PVC/SL/ & & to 58.50 & & to 58.50 \\
\hline BCU-26 & PVC & 1.25 & 1.00 & 5.00 & 59.10 & PVC/SL/ & & to & & to 59.10 \\
\hline BCU-27 & PVC & 1.25 & 1.00 & 5.00 & 58.30 & PVC/SL/ & 25.70 & to 58.30 & & to 58.30 \\
\hline BCU-28 & PVC & 1.25 & 1.00 & 5.00 & 58.30 & PVC/SL/ & & to 58.30 & & to 58.30 \\
\hline BCU-29 & PVC & 1.25 & 1.00 & 5.00 & 59.20 & PVC/SL/ & & to 59.20 & & to 59.20 \\
\hline BY-10 & PVC & & 1.00 & & 14.00 & PVC/SL/ & & to & 9.00 & to 14.00 \\
\hline
\end{tabular}




\begin{tabular}{|c|c|c|c|c|c|c|c|c|c|}
\hline $\begin{array}{c}\text { CURRENT } \\
\text { NAME }\end{array}$ & $\begin{array}{c}\text { CASING } \\
\text { TYPE }\end{array}$ & $\begin{array}{c}\text { CASING } \\
\text { O.D. }\end{array}$ & $\begin{array}{c}\text { CASING } \\
\text { I.D. }\end{array}$ & $\begin{array}{c}\text { HOLE } \\
\text { DIAMETER }\end{array}$ & $\begin{array}{c}\text { HOLE } \\
\text { DEPTH }\end{array}$ & SCREEN TYPE & $\begin{array}{c}\text { PACKED } \\
\text { INTERVAL }\end{array}$ & \multicolumn{2}{|c|}{$\begin{array}{l}\text { SCREENED } \\
\text { INTERVAL }\end{array}$} \\
\hline BY-11 & PVC & & 1.00 & & 16.00 & $\mathrm{PVC/SL/}$ & to & 11.00 & to 16.00 \\
\hline BY-12 & PVC & & 1.00 & & 18.00 & PVC/SL/ & to & 13.00 & to 18.00 \\
\hline BY-13 & PVC & & 1.00 & & 16.00 & PVC/SL/ & to & 11.00 & to 16.00 \\
\hline BY-14 & PVC & & 1.00 & & 15.00 & PVC/SL/ & to & 10.00 & to 15.00 \\
\hline BY-15 & PVC & & 1.00 & & 18.00 & PVC/SL/ & to & 13.00 & to 18.00 \\
\hline BY-16 & PVC & & 1.00 & & 17.00 & PVC/SL/ & to & 12.00 & to 17.00 \\
\hline BY-17 & PVC & & 1.00 & & 14.00 & PVC/SL/ & to & 9.00 & to 14.00 \\
\hline BY-18 & PVC & & 1.00 & & 16.00 & PVC/SL/ & to & 11.00 & to 16.00 \\
\hline BY-19 & PVC & & 1.00 & & 20.00 & PVC/SL/ & to & 15.00 & to 20.00 \\
\hline BY-20 & PVC & & 1.00 & & 17.00 & $\mathrm{PVC/SL/}$ & to & 12.00 & to 17.00 \\
\hline BY-21 & PVC & & 1.00 & & 12.00 & PVC/SL/ & to & 7.00 & to 12.00 \\
\hline BY-22 & PVC & & 1.00 & & 12.00 & PVC/SL/ & to & 7.00 & to 12.00 \\
\hline BY-23 & PVC & & 1.00 & & 11.00 & PVC/SL/ & to & 6.00 & to 11.00 \\
\hline BY-24 & PVC & & 1.00 & & 15.00 & PVC/SL/ & to & 10.00 & to 15.00 \\
\hline BY-25 & PVC & & 1.00 & & 15.00 & $\mathrm{PVC/SL/}$ & to & 10.00 & to 15.00 \\
\hline BY-26 & PVC & & 1.00 & & 15.50 & PVC/SL/ & to & 10.50 & to 15.50 \\
\hline BY-27 & PVC & & 1.00 & & 18.00 & PVC/SL/ & to & 13.00 & to 18.00 \\
\hline BY-28 & PVC & & 1.00 & & 18.00 & PVC/SL/ & to & 13.00 & to 18.00 \\
\hline BY-29 & PVC & & 1.00 & & 12.00 & PVC/SL/ & to & 7.00 & to 12.00 \\
\hline BY-30 & PVC & & 1.00 & & 16.00 & PVC/SL/ & to & 11.00 & to 16.00 \\
\hline BY-31 & PVC & & 1.00 & & 12.00 & PVC/SL/ & to & 7.00 & to 12.00 \\
\hline BY-32 & PVC & & 1.00 & & 12.00 & PVC/SL/ & to & 7.00 & to 12.00 \\
\hline BY-33 & PVC & & 1.00 & & 13.50 & PVC/SL/ & to & 8.50 & to 13.50 \\
\hline BY-34 & PVC & & 1.00 & & 16.00 & PVC/SL/ & to & 11.00 & to 16.00 \\
\hline BY-35 & PVC & & 1.00 & & 18.00 & PVC/SL/ & to & 13.00 & to 18.00 \\
\hline BY-36 & PVC & & 1.00 & & 16.00 & PVC/SL/ & to & 11.00 & to 16.00 \\
\hline BY-37 & PVC & & 1.00 & & 16.00 & PVC/SL/ & to & 11.00 & to 16.00 \\
\hline
\end{tabular}




\begin{tabular}{|c|c|c|c|c|c|c|}
\hline $\begin{array}{c}\text { CURRENT } \\
\text { NAME }\end{array}$ & $\begin{array}{c}\text { CASING } \\
\text { TYPE }\end{array}$ & $\begin{array}{c}\text { CASING } \\
\text { O.D. }\end{array}$ & $\begin{array}{c}\text { CASING } \\
\text { I.D. }\end{array}$ & $\begin{array}{c}\text { HOLE } \\
\text { DIAMETER }\end{array}$ & $\begin{array}{c}\text { HOLE } \\
\text { DEPTH }\end{array}$ & SCREEN TYPE \\
\hline BY-38 & PVC & & 1.00 & & 7.00 & PVC/SL/ \\
\hline BY-39 & PVC & & 1.00 & & 14.00 & $\mathrm{PVC/SL/}$ \\
\hline BY -40 & PVC & & 1.00 & & 12.00 & PVC/SL/ \\
\hline BY-41 & PVC & & 1.00 & & 8.00 & PVC/SL/ \\
\hline BY-43 & PVC & & 1.00 & & 15.00 & PVC/SL/ \\
\hline BY-44 & PVC & & 1.00 & & 14.00 & PVC/SL/ \\
\hline BY-45 & PVC & & 1.00 & & 12.00 & PVC/SL/ \\
\hline BY-46 & PVC & & 1.00 & & 16.00 & PVC/SL/ \\
\hline BY -47 & PVC & & 1.00 & & 16.00 & $\mathrm{PVC/SL/}$ \\
\hline BY-48 & PVC & & 1.00 & & 16.00 & $\mathrm{PVC/SL/}$ \\
\hline BY-49 & PVC & & 1.00 & & 17.00 & PVC/SL/ \\
\hline BY-50 & PVC & & 1.00 & & 25.50 & PVC/SL/ \\
\hline C-1 & PVC & 2.38 & 2.00 & 7.25 & 42.00 & PVC/SL/ \\
\hline C-19 & PVC & 2.38 & 2.00 & 7.25 & 25.20 & PVC/SL/ \\
\hline$C-21$ & PVC & 2.38 & 2.00 & 7.25 & 44.00 & PVC/SL/ \\
\hline$C-25$ & PVC & 2.38 & 2.00 & 3.00 & 75.00 & PVC/SL/ \\
\hline$C-40$ & PVC & 2.38 & 2.00 & 7.25 & 40.10 & PVC/SL/ \\
\hline C-45 & PVC & 2.38 & 2.00 & 3.00 & 93.00 & PVC/SL/ \\
\hline$C-48$ & PVC & 2.38 & 2.00 & & 115.00 & PVC/SL/ .01 \\
\hline$C-50$ & PVC & 2.38 & 2.00 & 7.25 & 36.00 & PVC/SL/ .01 \\
\hline$C-51$ & PVC & 2.38 & 2.00 & & 80.00 & $\mathrm{PVC/SL/} \mathrm{.01}$ \\
\hline FW001 & PVC40 & 1.05 & 0.80 & & 20.81 & \\
\hline FW002 & PVC40 & 1.05 & 0.80 & & 22.23 & \\
\hline FW003 & PVC40 & 1.05 & 0.80 & & 21.60 & \\
\hline FW004 & PVC40 & 1.05 & 0.80 & & 21.96 & \\
\hline FW005 & PVC40 & 1.05 & 0.80 & & 16.65 & \\
\hline GW-001 & SS304 & 2.37 & 2.00 & 3.00 & 23.10 & SLS/SW/ .01 \\
\hline
\end{tabular}

\begin{tabular}{|c|c|c|c|}
\hline \multicolumn{2}{|c|}{$\begin{array}{c}\text { PACKED } \\
\text { INTERVAL }\end{array}$} & \multicolumn{2}{|c|}{$\begin{array}{l}\text { SCREENED } \\
\text { INTERVAL }\end{array}$} \\
\hline & to & 2.00 & to 7.00 \\
\hline & to & 9.00 & to 14.00 \\
\hline & to & 7.00 & to 12.00 \\
\hline & to & 3.00 & to 8.00 \\
\hline & to & 10.00 & to 15.00 \\
\hline & to & 9.00 & to 14.00 \\
\hline & to & 7.00 & to 12.00 \\
\hline & to & 11.00 & to 16.00 \\
\hline & to & 11.00 & to 16.00 \\
\hline & to & 11.00 & to 16.00 \\
\hline & to & 12.00 & to 17.00 \\
\hline & to & 20.50 & to 25.50 \\
\hline 35.80 & to 42.00 & 36.80 & to 41.80 \\
\hline 19.00 & to 25.20 & 20.00 & to 25.00 \\
\hline 37.80 & to 44.00 & 38.80 & to 43.80 \\
\hline 69.00 & to 75.00 & 70.00 & to 75.00 \\
\hline 33.90 & to 40.10 & 34.90 & to 39.90 \\
\hline 86.80 & to 93.00 & 87.80 & to 92.80 \\
\hline 108.80 & to 115.00 & 109.80 & to 114.80 \\
\hline 24.80 & to 36.00 & 25.80 & to 35.80 \\
\hline 73.80 & to 80.00 & 74.80 & to 79.80 \\
\hline & to & & to \\
\hline & to & & to \\
\hline & to & & to \\
\hline & to & & to \\
\hline & to & & to \\
\hline 14.40 & to 25.70 & 18.10 & to 23.10 \\
\hline
\end{tabular}




\begin{tabular}{|c|c|c|c|c|c|c|c|c|c|c|}
\hline $\begin{array}{c}\text { CURRENT } \\
\text { NAME }\end{array}$ & $\begin{array}{c}\text { CASING } \\
\text { TYPE }\end{array}$ & $\begin{array}{l}\text { CASING } \\
\text { O.D. }\end{array}$ & $\begin{array}{c}\text { CASING } \\
\text { I.D. }\end{array}$ & $\begin{array}{c}\text { HOLE } \\
\text { DIAMETER }\end{array}$ & $\begin{array}{c}\text { HOLE } \\
\text { DEPTH }\end{array}$ & SCREEN TYPE & $\begin{array}{r}\text { PAC } \\
\text { INTE } \\
\end{array}$ & $\begin{array}{l}\text { CKED } \\
\text { ERVAL }\end{array}$ & $\begin{array}{l}\text { SCF } \\
\text { INT }\end{array}$ & $\begin{array}{l}\text { EENED } \\
\text { ERVAL }\end{array}$ \\
\hline GW-002 & SS304 & 2.37 & 2.00 & 3.00 & 57.70 & SLS/SW/ .01 & 40.30 & to 60.60 & 52.70 & to 57.70 \\
\hline GW-003 & SS304 & 2.37 & 2.00 & 4.50 & 26.80 & SLS/SW/ .01 & 18.00 & to 28.00 & 21.80 & to 26.80 \\
\hline GW-005 & SS304 & 2.37 & 2.00 & 6.00 & 10.30 & SLS/SW/ .01 & 3.00 & to 12.50 & 5.30 & to 10.30 \\
\hline GW-006 & SS304 & 2.37 & 2.00 & 4.50 & 42.30 & SLS/SW/ .01 & 15.30 & to 46.80 & 37.30 & to 42.30 \\
\hline GW-007 & SS304 & 2.37 & 2.00 & 6.00 & 14.30 & SLS/SW/ .01 & 8.70 & to 16.50 & 12.30 & to 14.30 \\
\hline GW-008 & SS304 & 2.37 & 2.00 & 4.50 & 20.70 & SLS/SW/ .01 & 13.00 & to 25.50 & 15.70 & to 20.70 \\
\hline GW-010 & SS304 & 2.37 & 2.00 & 6.00 & 12.70 & SLS/SW/ .01 & 5.30 & to 15.00 & 7.70 & to 12.70 \\
\hline GW-011 & SS304 & 2.37 & 2.00 & 4.00 & 45.30 & SLS/SW/ .01 & 28.00 & to 46.50 & 40.30 & to 45.30 \\
\hline GW-012 & SS304 & 2.37 & 2.00 & 6.00 & 13.50 & SLS/SW/ .01 & 8.70 & to 15.50 & 11.50 & to 13.50 \\
\hline GW-013 & SS304 & 2.37 & 2.00 & 6.00 & 10.40 & SLS/SW/ .01 & 6.00 & to 14.00 & 8.40 & to 10.40 \\
\hline GW-014 & SS304 & 2.37 & 2.00 & 6.00 & 13.20 & SLS/SW/ .01 & 5.00 & to 13.20 & 10.00 & to 12.00 \\
\hline GW-015 & SS304 & 2.37 & 2.00 & 6.00 & 6.70 & SLS/SW/ .01 & 1.00 & to 7.90 & 1.70 & to 6.70 \\
\hline GW-016 & SS304 & 2.37 & 2.00 & 6.00 & 15.90 & SLS/SW/ .01 & 6.50 & to 17.10 & 13.90 & to 15.90 \\
\hline GW-017 & SS304 & 2.37 & 2.00 & 4.00 & 59.20 & SLS/SW/ .01 & 35.60 & to 61.50 & 54.20 & to 59.20 \\
\hline GW-018 & SS304 & 2.37 & 2.00 & 6.00 & 17.70 & SLS/SW/ .01 & 12.00 & to 18.90 & 15.70 & to 17.70 \\
\hline GW-020 & SS304 & 2.37 & 2.00 & 4.00 & 57.90 & SLS/SW/ .01 & 37.00 & to 60.10 & 55.90 & to 57.90 \\
\hline GW-021 & SS304 & 2.37 & 2.00 & 4.25 & 13.00 & SLS/SW/ .01 & 5.50 & to 14.20 & 11.00 & to 13.00 \\
\hline GW-022 & SS304 & 2.37 & 2.00 & 4.00 & 51.40 & SLS/SW/ .01 & 20.00 & to 52.40 & 49.00 & to 51.40 \\
\hline GW-023 & SS304 & 2.37 & 2.00 & 4.00 & 52.40 & SLS/SW/ .01 & 31.00 & to 53.50 & 50.40 & to 52.40 \\
\hline GW-024 & SS304 & 2.37 & 2.00 & 4.00 & 74.60 & SLS/SW/ .01 & 45.00 & to 75.70 & 72.60 & to 74.60 \\
\hline GW-025A & SS304 & 2.37 & & 6.50 & 54.00 & SLS/SW/ .01 & 48.00 & to 54.00 & 49.00 & to 54.00 \\
\hline GW-026 & SS304 & 2.37 & 2.00 & 6.00 & 19.00 & SLS/SW/ .01 & 10.00 & to 20.20 & 17.00 & to 19.00 \\
\hline GW-027 & SS304 & 2.37 & 2.00 & 4.00 & 29.30 & SLS/SW/ .01 & 18.50 & to 30.50 & 27.30 & to 29.30 \\
\hline GW-028 & SS304 & 2.37 & 2.00 & 4.50 & 20.20 & SLS/SW/ .01 & 5.50 & to 21.40 & 15.20 & to 20.20 \\
\hline GW-029 & SS304 & 2.37 & 2.00 & 4.50 & 17.30 & SLS/SW/ .01 & 6.00 & to 21.10 & 15.30 & to 17.30 \\
\hline GW-030 & SS304 & 2.37 & 2.00 & 6.00 & 37.80 & SLS/SW/ .01 & 27.00 & to 39.00 & 27.80 & to 37.80 \\
\hline GW-031 & SS304 & 2.37 & 2.00 & 4.00 & 65.70 & SLS/SW/ .01 & 47.00 & to 67.00 & 63.70 & to 65.70 \\
\hline
\end{tabular}




\begin{tabular}{|c|c|c|c|c|c|c|c|c|c|c|}
\hline $\begin{array}{c}\text { CURRENT } \\
\text { NAME }\end{array}$ & $\begin{array}{c}\text { CASING } \\
\text { TYPE }\end{array}$ & $\begin{array}{l}\text { CASING } \\
\text { O.D. }\end{array}$ & $\begin{array}{c}\text { CASING } \\
\text { I.D. }\end{array}$ & $\begin{array}{c}\text { HOLE } \\
\text { DIAMETER }\end{array}$ & $\begin{array}{c}\text { HOLE } \\
\text { DEPTH }\end{array}$ & SCREEN TYPE & $\begin{array}{r}\text { PAC } \\
\text { INTE } \\
\end{array}$ & $\begin{array}{l}\text { CKED } \\
\text { ERVAL }\end{array}$ & $\begin{array}{l}\text { SCF } \\
\text { INT }\end{array}$ & $\begin{array}{l}\text { EENED } \\
\text { ERVAL }\end{array}$ \\
\hline GW-032 & SS304 & 2.37 & 2.00 & 3.00 & 57.10 & SLS/SW/ .01 & 26.00 & to 50.50 & 45.10 & to 49.50 \\
\hline GW-033 & SS304 & 2.37 & 2.00 & 6.00 & 36.70 & SLS/SW/ .01 & 30.00 & to 37.90 & 34.70 & to 36.70 \\
\hline GW-034 & SS304 & 2.37 & 2.00 & 4.50 & 44.00 & SLS/SW/ .01 & 27.00 & to 45.20 & 34.00 & to 44.00 \\
\hline GW-035 & SS304 & 2.37 & 2.00 & 4.00 & 59.10 & SLS/SW/ .01 & 37.50 & to 60.30 & 49.10 & to 59.10 \\
\hline GW-036 & SS304 & 2.37 & 2.00 & 6.00 & 36.90 & SLS/SW/ .01 & 8.50 & to 39.10 & 34.90 & to 36.90 \\
\hline GW-037 & SS304 & 2.37 & 2.00 & 4.00 & 66.50 & SLS/SW/ .01 & 42.00 & to 68.70 & 64.50 & to 66.50 \\
\hline GW-038 & SS304 & 2.37 & 2.00 & 6.00 & 51.20 & SLS/SW/ .01 & 38.90 & to 52.40 & 41.20 & to 51.20 \\
\hline GW-039 & SS304 & 2.37 & 2.00 & 6.00 & 19.90 & SLS/SW/ .01 & 6.00 & to 21.10 & 17.90 & to 19.90 \\
\hline GW-040 & SS304 & 2.37 & 2.00 & 6.00 & 27.70 & SLS/SW/ .01 & 21.00 & to 29.00 & 25.70 & to 27.70 \\
\hline GW-041 & SS304 & 2.37 & 2.00 & 4.50 & 38.60 & SLS/SW/ .01 & 6.00 & to 40.00 & 36.60 & to 38.60 \\
\hline GW-042 & SS304 & 2.37 & 2.00 & 6.00 & 26.90 & SLS/SW/ .01 & 13.40 & to 28.20 & 24.90 & to 26.90 \\
\hline GW-043 & SS304 & 2.37 & 2.00 & 6.00 & 32.80 & SLS/SW/ .01 & 10.00 & to 40.00 & 22.80 & to 32.80 \\
\hline GW-044 & SS304 & 2.37 & 2.00 & 4.00 & 58.00 & SLS/SW/ .01 & 42.50 & to 70.00 & 48.00 & to 58.00 \\
\hline GW-045 & SS304 & 2.37 & 2.00 & 6.00 & 13.00 & SLS/SW/ .01 & 3.00 & to 15.20 & 3.00 & to 13.00 \\
\hline GW-046 & SS304 & 2.37 & 2.00 & 6.00 & 18.10 & SLS/SW/ .01 & 5.00 & to 20.30 & 8.10 & to 18.10 \\
\hline GW-047 & SS304 & 2.37 & 2.00 & 6.00 & 23.50 & SLS/SW/ .01 & 12.50 & to 25.50 & 18.50 & to 23.50 \\
\hline GW-048 & SS304 & 2.37 & 2.00 & 6.00 & 7.30 & SLS/SW/ .01 & 2.00 & to 8.70 & 2.30 & to 7.30 \\
\hline GW-049 & SS304 & 2.37 & 2.00 & 4.50 & 18.60 & SLS/SW/ .01 & 6.30 & to 20.20 & 13.60 & to 18.60 \\
\hline GW-050 & SS304 & 2.37 & 2.00 & 6.00 & 13.00 & SLS/SW/ .01 & 6.00 & to 14.20 & 11.00 & to 13.00 \\
\hline GW-051 & SS304 & 2.37 & 2.00 & 6.00 & 9.60 & SLS/SW/ .01 & 2.00 & to 11.20 & 4.60 & to 9.60 \\
\hline GW-052 & SS304 & 2.37 & 2.00 & 6.00 & 18.30 & SLS/SW/ .01 & 4.00 & to 19.50 & 13.30 & to 18.30 \\
\hline GW-053 & SS304 & 2.37 & 2.00 & 4.00 & 31.60 & SLS/SW/ .01 & 11.40 & to 32.80 & 26.60 & to 31.60 \\
\hline GW-054 & SS304 & 2.37 & 2.00 & 4.00 & 37.20 & SLS/SW/ .01 & 31.10 & to 37.20 & 35.20 & to 37.20 \\
\hline GW-055 & SS304 & 2.37 & 2.00 & 4.00 & 20.00 & SLS/SW/ .01 & 15.50 & to 20.00 & 18.00 & to 20.00 \\
\hline GW-056 & SS304 & 2.37 & 2.00 & 4.00 & 55.20 & SLS/SW/ .01 & 50.40 & to 55.20 & 53.20 & to 55.20 \\
\hline GW-057 & SS304 & 2.37 & 2.00 & 4.00 & 22.80 & SLS/SW/ .01 & 17.50 & to 22.80 & 20.80 & to 22.80 \\
\hline GW-058 & SS304 & 2.37 & 2.00 & 3.00 & 44.20 & SLS/SW/ .01 & 38.80 & to 44.20 & 42.20 & to 44.20 \\
\hline
\end{tabular}




\begin{tabular}{|c|c|c|c|c|c|c|c|c|c|c|}
\hline $\begin{array}{c}\text { CURRENT } \\
\text { NAME }\end{array}$ & $\begin{array}{c}\text { CASING } \\
\text { TYPE }\end{array}$ & $\begin{array}{l}\text { CASING } \\
\text { O.D. }\end{array}$ & $\begin{array}{c}\text { CASING } \\
\text { I.D. }\end{array}$ & $\begin{array}{c}\text { HOLE } \\
\text { DIAMETER }\end{array}$ & $\begin{array}{c}\text { HOLE } \\
\text { DEPTH }\end{array}$ & SCREEN TYPE & $\begin{array}{l}\text { PAC } \\
\text { INTE } \\
\end{array}$ & $\begin{array}{l}\text { CKED } \\
\text { ERVAL }\end{array}$ & $\begin{array}{l}\text { SCF } \\
\text { INT }\end{array}$ & $\begin{array}{l}\text { EENED } \\
\text { ERVAL }\end{array}$ \\
\hline GW-059 & SS304 & 2.37 & 2.00 & 3.00 & 24.80 & SLS/SW/ .01 & 19.50 & to 24.70 & 22.80 & to 24.80 \\
\hline GW-060 & SS304 & 2.37 & 2.00 & 3.00 & 49.80 & SLS/SW/ .01 & 38.00 & to 49.80 & 47.80 & to 49.80 \\
\hline GW-061 & SS304 & 2.37 & 2.00 & 3.00 & 24.60 & SLS/SW/ .01 & 17.50 & to 24.60 & 19.60 & to 24.60 \\
\hline GW-062 & SS304 & 2.37 & 2.00 & 5.00 & 51.40 & SLS/SW/ .01 & 45.10 & to 51.40 & 49.40 & to 51.40 \\
\hline GW-063 & SS304 & 2.37 & 2.00 & 4.00 & 32.70 & SLS/SW/ .01 & 21.90 & to 32.70 & 27.70 & to 32.70 \\
\hline GW-064 & SS304 & 2.37 & 2.00 & 4.00 & 52.70 & SLS/SW/ .01 & 46.80 & to 52.70 & 50.70 & to 52.70 \\
\hline GW-065 & SS304 & 2.37 & 2.00 & 4.00 & 34.00 & SLS/SW/ .01 & 24.50 & to 34.00 & 29.00 & to 34.00 \\
\hline GW-066 & SS304 & 2.37 & 2.00 & 4.00 & 54.90 & SLS/SW/ .01 & 50.00 & to 54.90 & 52.90 & to 54.90 \\
\hline GW-067 & SS304 & 2.37 & 2.00 & 6.50 & 16.20 & SLS/SW/ .01 & 7.60 & to 16.20 & 11.20 & to 16.20 \\
\hline GW-068 & SS304 & 2.37 & 2.00 & 8.75 & 82.10 & SLS/SW/ .01 & 70.00 & to 83.60 & 71.90 & to 82.10 \\
\hline GW-069 & SS304 & 2.37 & 2.00 & 7.88 & 99.20 & SLS/SW/ .01 & 79.00 & to 99.20 & 89.00 & to 99.20 \\
\hline GW-070 & SS304 & 2.37 & 2.00 & 8.75 & 140.50 & SLS/SW/ .01 & 121.00 & to 140.50 & 124.90 & to 140.50 \\
\hline GW-071 & SS304 & 2.37 & 2.00 & 8.75 & 219.00 & SLS/SW/ .01 & 195.10 & to 219.00 & 198.40 & to 219.00 \\
\hline GW-072 & SS304 & 2.37 & 2.00 & 8.75 & 98.40 & SLS/SW/ .01 & 84.50 & to 98.40 & 87.80 & to 98.40 \\
\hline GW-073 & SS304 & 2.37 & 2.00 & 8.75 & 79.80 & SLS/SW/ .01 & 66.90 & to 79.80 & 69.80 & to 79.80 \\
\hline GW-074 & SS304 & 2.37 & 2.00 & 7.88 & 207.42 & SLS/SW/ .01 & 176.50 & to 200.60 & 180.00 & to 200.60 \\
\hline GW-075 & SS304 & 2.37 & 2.00 & 8.75 & 199.60 & SLS/SW/ .01 & 176.50 & to 199.60 & 179.60 & to 199.60 \\
\hline GW-076 & SS304 & 2.37 & 2.00 & 8.75 & 80.30 & SLS/SW/ .01 & 67.80 & to 80.30 & 69.70 & to 80.30 \\
\hline GW-077 & SS304 & 2.37 & 2.00 & 3.88 & 100.30 & SLS/SW/ .01 & 87.40 & to 100.30 & 90.30 & to 100.30 \\
\hline GW-078 & SS304 & 2.37 & 2.00 & 6.50 & 21.10 & SLS/SW/ .01 & 11.70 & to 21.10 & 16.10 & to 21.10 \\
\hline GW-079 & SS304 & 2.37 & 2.00 & 6.50 & 64.90 & SLS/SW/ .01 & 49.90 & to 64.90 & 59.90 & to 64.90 \\
\hline GW-080 & SS304 & 2.37 & 2.00 & 6.50 & 29.70 & SLS/SW/ .01 & 20.80 & to 29.70 & 24.70 & to 29.70 \\
\hline GW-081 & SS304 & 2.37 & 2.00 & 6.00 & 18.70 & SLS/SW/ .01 & 9.40 & to 18.70 & 13.70 & to 18.70 \\
\hline GW-082 & SS304 & 2.37 & 2.00 & 4.00 & 34.40 & SLS/SW/ .01 & 24.10 & to 34.40 & 29.40 & to 34.40 \\
\hline GW-083 & SS304 & 2.37 & 2.00 & 3.00 & 29.50 & SLS/SW/ .01 & 19.90 & to 29.50 & 24.50 & to 29.50 \\
\hline GW-084 & SS304 & 2.37 & 2.00 & 6.50 & 27.80 & SLS/SW/ .01 & 18.50 & to 27.80 & 22.80 & to 27.80 \\
\hline GW-085 & SS304 & 2.37 & 2.00 & 4.00 & 58.80 & SLS/SW/ .01 & 48.40 & to 58.80 & 53.80 & to 58.80 \\
\hline
\end{tabular}




\begin{tabular}{|c|c|c|c|c|c|c|c|c|c|c|}
\hline $\begin{array}{c}\text { CURRENT } \\
\text { NAME }\end{array}$ & $\begin{array}{c}\text { CASING } \\
\text { TYPE }\end{array}$ & $\begin{array}{l}\text { CASING } \\
\text { O.D. }\end{array}$ & $\begin{array}{c}\text { CASING } \\
\text { I.D. }\end{array}$ & $\begin{array}{c}\text { HOLE } \\
\text { DIAMETER }\end{array}$ & $\begin{array}{c}\text { HOLE } \\
\text { DEPTH }\end{array}$ & SCREEN TYPE & $\begin{array}{r}\text { PA } \\
\text { INTI }\end{array}$ & $\begin{array}{l}\text { CKED } \\
\text { ERVAL }\end{array}$ & $\begin{array}{l}\text { SCF } \\
\text { INT }\end{array}$ & $\begin{array}{l}\text { EENED } \\
\text { ERVAL }\end{array}$ \\
\hline GW-086 & SS304 & 2.37 & 2.00 & 4.00 & 29.60 & SLS/SW/ .01 & 21.00 & to 29.60 & 24.60 & to 29.60 \\
\hline GW-087 & SS304 & 2.37 & 2.00 & 6.50 & 19.00 & SLS/SW/ .01 & 7.50 & to 19.00 & 9.00 & to 19.00 \\
\hline GW-088 & SS304 & 2.37 & & 6.50 & 30.00 & SLS/SW/ .01 & 25.00 & to 30.00 & 28.00 & to 30.00 \\
\hline GW-089 & SS304 & 2.37 & & 6.50 & 25.00 & SLS/SW/ .01 & 20.00 & to 25.00 & 23.00 & to 25.00 \\
\hline GW-090 & SS304 & 2.37 & & 6.50 & 15.00 & SLS/SW/ .01 & 10.00 & to 15.00 & 13.00 & to 15.00 \\
\hline GW-091 & SS304 & 2.37 & & 6.50 & 25.00 & SLS/SW/ .01 & 20.00 & to 25.00 & 23.00 & to 25.00 \\
\hline GW-092 & SS304 & 2.37 & & 6.50 & 25.00 & SLS/SW/.01 & 20.00 & to 25.00 & 23.00 & to 25.00 \\
\hline GW-093 & SS304 & 2.37 & 2.00 & 6.50 & 48.20 & SLS/SW/ .01 & 39.50 & to 49.00 & 42.00 & to 47.00 \\
\hline GW-093A & SS304 & 2.37 & & 6.50 & 49.00 & SLS/SW/ .01 & 43.00 & to 49.00 & 44.00 & to 49.00 \\
\hline GW-094 & SS304 & 4.50 & 4.00 & 9.00 & 114.80 & SLS/SW/ .01 & 86.40 & to 115.30 & 93.80 & to 114.80 \\
\hline GW-095 & SS304 & 4.50 & 4.00 & 9.00 & 155.80 & SLS/SW/ .01 & 130.20 & to 156.00 & 134.80 & to 155.80 \\
\hline GW-096 & SS304 & 2.37 & & 9.00 & 60.60 & SLS/SW/ .01 & 42.20 & to 61.00 & 44.00 & to 59.40 \\
\hline GW-096A & SS304 & 2.37 & & 6.50 & 61.00 & SLS/SW/ .01 & 55.00 & to 61.00 & 56.00 & to 61.00 \\
\hline GW-097 & SS304 & 2.37 & 2.00 & 6.50 & 18.00 & SLS/SW/ .01 & 9.30 & to 19.20 & 11.80 & to 16.80 \\
\hline GW-097A & SS304 & 2.37 & & 6.50 & 19.00 & SLS/SW/ .01 & 13.00 & to 19.00 & 14.00 & to 19.00 \\
\hline GW-098 & SS304 & 4.50 & 4.00 & 9.00 & 103.40 & SLS/SW/ .01 & 76.60 & to 104.00 & 82.40 & to 103.40 \\
\hline GW-099 & SS304 & 4.50 & 4.00 & 10.00 & 126.50 & SLS/SW/ .01 & 112.50 & to 127.00 & 116.50 & to 126.50 \\
\hline GW-100 & PVC & 2.37 & 2.00 & 6.50 & 15.10 & PVC/SL/ .01 & 3.80 & to 20.70 & 10.20 & to 14.20 \\
\hline GW-101 & PVC & 2.37 & 2.00 & 6.50 & 17.20 & PVC/SL/ .01 & 10.10 & to 17.50 & 12.30 & to 16.30 \\
\hline GW-102 & PVC & 2.37 & 2.00 & 6.50 & 23.70 & PVC/SW/ .01 & 14.00 & to 24.00 & 18.80 & to 22.80 \\
\hline GW-103 & PVC & 2.37 & 2.00 & 6.50 & 25.00 & PVC/SW/ .01 & 17.80 & to 25.00 & 20.10 & to 24.10 \\
\hline GW-104 & PVC & 4.50 & 4.00 & 9.00 & 69.80 & PVC/SW/ .01 & 51.00 & to 74.00 & 59.80 & to 68.80 \\
\hline GW-105 & PVC & 2.37 & 2.00 & 6.50 & 17.00 & PVC/SL/ .01 & 9.50 & to 17.00 & 12.10 & to 16.10 \\
\hline GW-106 & PVC & 4.50 & 4.00 & 9.00 & 72.00 & PVC/SL/ .01 & 53.30 & to 75.00 & 61.90 & to 70.90 \\
\hline GW-107 & PVC & 2.37 & 2.00 & 6.50 & 13.40 & PVC/SL/ .01 & 6.40 & to 14.20 & 8.50 & to 12.50 \\
\hline GW-108 & PVC40 & 4.50 & 4.00 & 9.00 & 56.80 & PVC/SL/ .01 & 41.00 & to 58.60 & 46.70 & to 55.70 \\
\hline GW-109 & PVC40 & 4.50 & 4.00 & 9.00 & 123.00 & $\mathrm{PVC/SL/} \mathrm{.01}$ & 96.60 & to 128.50 & 102.90 & to 121.90 \\
\hline
\end{tabular}




\begin{tabular}{|c|c|c|c|c|c|c|c|c|c|c|}
\hline $\begin{array}{c}\text { CURRENT } \\
\text { NAME }\end{array}$ & $\begin{array}{c}\text { CASING } \\
\text { TYPE }\end{array}$ & $\begin{array}{l}\text { CASING } \\
\text { O.D. }\end{array}$ & $\begin{array}{c}\text { CASING } \\
\text { I.D. }\end{array}$ & $\begin{array}{c}\text { HOLE } \\
\text { DIAMETER }\end{array}$ & $\begin{array}{c}\text { HOLE } \\
\text { DEPTH }\end{array}$ & SCREEN TYPE & $\begin{array}{l}\text { PAC } \\
\text { INTE } \\
\end{array}$ & $\begin{array}{l}\text { CKED } \\
\text { ERVAL }\end{array}$ & $\begin{array}{l}\text { SCF } \\
\text { INT }\end{array}$ & $\begin{array}{l}\text { EENED } \\
\text { ERVAL }\end{array}$ \\
\hline GW-110 & SS304 & 2.37 & 2.00 & 3.00 & 39.80 & SLS/SW/ .01 & 27.00 & to 40.00 & 28.40 & to 38.60 \\
\hline GW-112 & SS304 & 2.37 & 2.00 & 6.00 & 244.60 & SLS/SW/ .01 & 218.90 & to 245.00 & 223.40 & to 243.40 \\
\hline GW-113 & SS304 & 2.37 & 2.00 & 6.00 & 159.80 & SLS/SW/ .01 & 132.10 & to 160.50 & 138.30 & to 158.70 \\
\hline GW-114 & SS304 & 2.37 & 2.00 & 6.00 & 120.00 & SLS/SW/ .01 & 88.60 & to 120.00 & 98.40 & to 118.80 \\
\hline GW-115 & SS304 & 2.37 & & & 52.00 & SLS/SW/ .01 & 37.60 & to 53.00 & 42.00 & to 52.00 \\
\hline GW-127 & SS304 & 2.37 & 2.00 & 7.00 & 22.80 & PVC/SL/ .01 & 14.00 & to 24.00 & 18.80 & to 22.80 \\
\hline GW-136 & SF25 & 1.00 & & 3.70 & 441.60 & STL/PER/ & & to & 419.90 & to 429.90 \\
\hline GW-141 & SS304 & 4.50 & 4.00 & 10.00 & 155.20 & SLS/SW/ .01 & 141.00 & to 156.00 & 144.50 & to 155.20 \\
\hline GW-144 & PVC40 & 4.50 & 4.00 & 11.00 & 190.00 & PVC/SL/ .01 & 148.00 & to 195.00 & 150.00 & to 190.00 \\
\hline GW-145 & PVC40 & 4.50 & 4.00 & 11.00 & 108.50 & PVC/SL/ .01 & 86.00 & to 110.00 & 88.50 & to 108.50 \\
\hline GW-147 & PVC40 & 4.50 & 4.00 & 10.00 & 69.00 & PVC/SL/ .01 & 52.00 & to 69.00 & 53.10 & to 68.10 \\
\hline GW-148 & PVC40 & 4.50 & 4.00 & 8.00 & 11.10 & PVC/SL/ .01 & 4.60 & to 11.10 & 5.60 & to 10.60 \\
\hline GW-149 & PVC40 & 4.50 & 4.00 & 11.00 & 47.50 & PVC/SL/ .01 & 36.00 & to 50.50 & 37.00 & to 47.00 \\
\hline GW-150 & PVC40 & 4.50 & 4.00 & 8.00 & 11.70 & PVC/SL/ .01 & 5.00 & to 11.70 & 6.20 & to 11.20 \\
\hline GW-151 & PVC40 & 4.50 & 4.00 & 11.00 & 96.50 & PVC/SL/ .01 & 85.00 & to 96.50 & 86.00 & to 96.00 \\
\hline GW-152 & PVC40 & 4.50 & 4.00 & 8.00 & 17.30 & PVC/SL/ .01 & 10.80 & to 17.30 & 11.80 & to 16.80 \\
\hline GW-153 & PVC40 & 4.50 & 4.00 & 10.00 & 59.50 & PVC/SL/ .01 & 45.00 & to 60.00 & 49.50 & to 59.50 \\
\hline GW-154 & PVC40 & 4.50 & 4.00 & 8.00 & 11.20 & PVC/SL/ .01 & 4.70 & to 11.20 & 5.70 & to 10.70 \\
\hline GW-155 & PVC40 & 4.50 & 4.00 & 8.50 & 177.00 & PVC/SW/ .01 & 165.00 & to 177.00 & 167.00 & to 177.00 \\
\hline GW-156 & PVC40 & 4.50 & 4.00 & 8.50 & 157.00 & PVC/SL/ .01 & 145.00 & to 157.60 & 147.00 & to 157.00 \\
\hline GW-157 & PVC40 & 4.50 & 4.00 & 8.00 & 145.00 & PVC/SWI .01 & 133.00 & to 145.00 & 135.00 & to 145.00 \\
\hline GW-159 & PVC40 & 4.50 & 4.00 & 8.50 & 157.00 & PVC/SL/ .01 & 145.00 & to 157.00 & 147.00 & to 157.00 \\
\hline GW-167 & PVC40 & 2.37 & 2.00 & 6.62 & 30.10 & PVC/SL/ .01 & 25.00 & to 30.10 & 26.00 & to 30.10 \\
\hline GW-169 & PVC40 & 2.37 & 2.00 & 8.00 & 34.80 & PVC/SL/ .01 & 28.70 & to 34.80 & 29.70 & to 34.70 \\
\hline GW-171 & PVC40 & 2.37 & 2.00 & 8.00 & 31.20 & PVC/SL/ .01 & 25.80 & to 31.20 & 26.80 & to 31.20 \\
\hline GW-173 & SS304 & 4.50 & 4.00 & 10.00 & 165.00 & SLS/SW/ .01 & 154.00 & to 165.00 & 155.00 & to 165.00 \\
\hline GW-174 & SS304 & 4.50 & 4.00 & 10.00 & 145.00 & SLS/SW/ .01 & 134.00 & to 145.00 & 135.00 & to 145.00 \\
\hline
\end{tabular}




\begin{tabular}{|c|c|c|c|c|c|c|c|c|c|c|}
\hline $\begin{array}{c}\text { CURRENT } \\
\text { NAME }\end{array}$ & $\begin{array}{c}\text { CASING } \\
\text { TYPE }\end{array}$ & $\begin{array}{c}\text { CASING } \\
\text { O.D. }\end{array}$ & $\begin{array}{c}\text { CASING } \\
\text { I.D. }\end{array}$ & $\begin{array}{c}\text { HOLE } \\
\text { DIAMETER }\end{array}$ & $\begin{array}{c}\text { HOLE } \\
\text { DEPTH }\end{array}$ & SCREEN TYPE & $\begin{array}{r}\text { PA } \\
\text { INTI } \\
\end{array}$ & $\begin{array}{l}\text { CKED } \\
\text { ERVAL }\end{array}$ & $\begin{array}{l}\mathrm{SCl} \\
\text { IN] }\end{array}$ & $\begin{array}{l}\text { REENED } \\
\text { ERVAL }\end{array}$ \\
\hline GW-175 & SS304 & 4.50 & 4.00 & 9.50 & 166.70 & SLS/SW/ .01 & 148.30 & to 166.70 & 150.60 & to 166.40 \\
\hline GW-176 & SS304 & 4.50 & 4.00 & 10.00 & 145.00 & SLS/SW/ .01 & 134.00 & to 145.00 & 135.00 & to 145.00 \\
\hline GW-177 & PVC40 & 4.50 & 4.00 & 8.00 & 143.00 & PVC/SL/ .01 & 130.00 & to 145.00 & 133.00 & to 143.00 \\
\hline GW-178 & SS304 & 4.50 & 4.00 & 10.00 & 132.00 & SLS/SW/ .01 & 117.50 & to 133.00 & 122.00 & to 132.00 \\
\hline GW-179 & SS304 & 4.50 & 4.00 & 8.00 & 117.00 & SLS/SW/ .01 & 106.00 & to 117.00 & 107.00 & to 117.00 \\
\hline GW-180 & SS304 & 4.50 & 4.00 & 10.00 & 143.00 & SLS/SW/ .01 & 126.00 & to 144.00 & 132.20 & to 143.00 \\
\hline GW-183 & SS304 & 4.50 & 4.00 & 8.00 & 29.80 & SLS/SW/ .01 & 5.00 & to 30.60 & 9.50 & to 29.50 \\
\hline GW-184 & PVC40 & 4.50 & 4.00 & 10.00 & 127.50 & PVC/SL/ .01 & 101.50 & to 130.00 & 107.50 & to 127.50 \\
\hline GW-186 & PVC40 & 4.50 & 4.00 & 10.00 & 170.00 & PVC/SL/ .01 & 142.00 & to 171.00 & 150.00 & to 170.00 \\
\hline GW-187 & PVC40 & 4.50 & 4.00 & 10.00 & 162.00 & PVC/SL/ .01 & 139.00 & to 162.00 & 147.00 & to 162.00 \\
\hline GW-188 & PVC40 & 4.50 & 4.00 & 11.00 & 67.50 & PVC/SL/ .01 & 49.00 & to 68.00 & 52.50 & to 67.50 \\
\hline GW-189 & PVC40 & 4.50 & 4.00 & 10.00 & 210.00 & PVC/SL/ .01 & 180.00 & to 210.00 & 190.00 & to 210.00 \\
\hline GW-190 & SS304 & 4.50 & 4.00 & 8.00 & 26.20 & SLS/SW/ .01 & 8.00 & to 26.20 & 9.80 & to 25.90 \\
\hline GW-191 & PVC40 & 4.50 & 4.00 & 11.00 & 60.00 & PVC/SL/ .01 & 45.00 & to 60.50 & 49.50 & to 59.50 \\
\hline GW-192 & PVC40 & 4.50 & 4.00 & 6.00 & 17.50 & PVC/SL/ .01 & 6.50 & to 17.50 & 7.50 & to 17.50 \\
\hline GW-193 & SS304 & 4.50 & 4.00 & 8.00 & 18.45 & SLS/SW/ .01 & 5.50 & to 18.45 & 8.15 & to 18.45 \\
\hline GW-194 & PVC40 & 4.50 & 4.00 & 8.00 & 12.50 & PVC/SL/ .01 & 6.50 & to 12.50 & 7.50 & to 12.50 \\
\hline GW-195 & PVC40 & 4.50 & 4.00 & 6.00 & 23.00 & PVC/SL/ .01 & 15.00 & to 23.00 & 17.00 & to 23.00 \\
\hline GW-196 & PVC40 & 4.50 & 4.00 & 8.00 & 27.00 & PVC/SL/ .01 & 21.00 & to 27.00 & 22.00 & to 27.00 \\
\hline GW-197 & PVC40 & 4.50 & 4.00 & 6.00 & 17.00 & PVC/SL/ .01 & 11.00 & to 17.00 & 12.00 & to 17.00 \\
\hline GW-198 & PVC40 & 4.50 & 4.00 & 8.00 & 26.50 & PVC/SL/ .01 & 15.50 & to 26.50 & 16.50 & to 26.50 \\
\hline GW-199 & PVC40 & 4.50 & 4.00 & 8.00 & 22.50 & PVC/SL/ .01 & 16.50 & to 22.50 & 17.50 & to 22.50 \\
\hline GW-200 & PVC40 & 4.50 & 4.00 & 11.00 & 57.50 & PVC/SL/ .01 & 44.00 & to 57.50 & 47.00 & to 57.00 \\
\hline GW-201 & PVC40 & 4.50 & 4.00 & 8.00 & 21.00 & PVC/SL/ .01 & 15.00 & to 21.00 & 16.00 & to 21.00 \\
\hline GW-202 & PVC40 & 4.50 & 4.00 & 8.00 & 20.10 & PVC/SL/ .01 & 14.10 & to 20.10 & 15.10 & to 20.10 \\
\hline GW-203 & PVC40 & 4.50 & 4.00 & 8.50 & 156.00 & PVC/SL/ .01 & 144.00 & to 156.00 & 146.00 & to 156.00 \\
\hline GW-204 & SS304 & 4.50 & 4.00 & 6.00 & 17.30 & SLS/SW/ .01 & 6.50 & to 17.30 & 7.30 & to 17.30 \\
\hline
\end{tabular}




\begin{tabular}{|c|c|c|c|c|c|c|c|c|c|c|c|}
\hline $\begin{array}{c}\text { CURRENT } \\
\text { NAME }\end{array}$ & $\begin{array}{c}\text { CASING } \\
\text { TYPE }\end{array}$ & $\begin{array}{l}\text { CASING } \\
\text { O.D. }\end{array}$ & $\begin{array}{c}\text { CASING } \\
\text { I.D. }\end{array}$ & $\begin{array}{c}\text { HOLE } \\
\text { DIAMETER }\end{array}$ & $\begin{array}{c}\text { HOLE } \\
\text { DEPTH }\end{array}$ & SCREEN TYPE & $\begin{array}{r}\text { PAC } \\
\text { INTE } \\
\end{array}$ & CKI & & $\begin{array}{l}\text { SCF } \\
\text { INT }\end{array}$ & $\begin{array}{l}\text { EENED } \\
\text { ERVAL }\end{array}$ \\
\hline GW-205 & PVC40 & 4.50 & 4.00 & 10.00 & 164.00 & $\mathrm{PVC/SL/} \mathrm{.01}$ & 152.00 & to & 164.00 & 154.00 & to 164.00 \\
\hline GW-206 & PVC40 & 2.37 & 2.00 & 8.00 & 16.90 & $\mathrm{PVC/SL/} \mathrm{.01}$ & 10.80 & to & 16.90 & 11.80 & to 16.90 \\
\hline GW-209 & PVC40 & 2.37 & 2.00 & 8.00 & 48.10 & PVC/SL/ .01 & 42.00 & to & 48.10 & 43.00 & to 48.10 \\
\hline GW-212 & PVC40 & 2.37 & 2.00 & 8.00 & 13.80 & PVC/SL/ & 8.40 & to & 13.80 & 9.40 & to 13.80 \\
\hline GW-215 & PVC40 & 2.37 & 2.00 & 8.00 & 15.20 & PVC/SW/ .01 & 9.60 & to & 15.20 & 10.60 & to 15.20 \\
\hline GW-217 & SS304 & 4.50 & 4.00 & 10.00 & 177.40 & SLS/SW/ .01 & 165.20 & to & 180.00 & 166.80 & to 177.40 \\
\hline GW-218 & SS304 & 4.50 & 4.00 & 8.00 & 27.10 & SLS/SW/ .01 & 14.40 & to & 27.50 & 17.10 & to 27.10 \\
\hline GW-219 & SS304 & 4.50 & 4.00 & 10.00 & 11.30 & SLS/SW/ .01 & 4.30 & to & 11.30 & 5.70 & to 11.30 \\
\hline GW-220 & PVC40 & 4.50 & 4.00 & 11.00 & 45.20 & PVC/SL/ .01 & 31.00 & to & 45.20 & 34.70 & to 44.70 \\
\hline GW-221 & PVC40 & 4.50 & 4.00 & 6.00 & 158.00 & PVC/SL/ .01 & 146.00 & to & 158.00 & 148.00 & to 158.00 \\
\hline GW-222 & PVC40 & 4.50 & 4.00 & 11.00 & 25.00 & PVC/SL/ .01 & 18.00 & to & 25.00 & 19.50 & to 24.50 \\
\hline GW-223 & PVC40 & 4.50 & 4.00 & 11.00 & 90.50 & PVC/SL/ .01 & 79.00 & to & 90.50 & 80.00 & to 90.00 \\
\hline GW-224 & PVC40 & 4.50 & 4.00 & 10.00 & 126.00 & PVC/SL/ .01 & 103.00 & to & 126.00 & 106.00 & to 126.00 \\
\hline GW-231 & PVC40 & 4.50 & 4.00 & 11.00 & 35.00 & PVC/SL/ .01 & 22.80 & to & 35.00 & 24.50 & to 34.50 \\
\hline GW-233 & SS304 & 4.50 & 4.00 & 10.00 & 167.50 & SLS/SW/ .01 & 152.00 & to & 170.00 & 157.00 & to 167.50 \\
\hline GW-234 & SS304 & 2.37 & & 8.00 & 16.50 & SLS/SW/ .01 & 8.00 & to & 16.50 & 10.20 & to 16.50 \\
\hline GW-236 & PVC40 & 4.50 & & 8.00 & 18.50 & PVC/SL/ .01 & 10.00 & to & 18.50 & 13.00 & to 18.00 \\
\hline GW-237 & SS304 & 2.37 & & 8.00 & 13.70 & SLS/SW/ .01 & 6.50 & to & 13.70 & 7.50 & to 12.50 \\
\hline GW-240 & PVC40 & 4.50 & 4.00 & & 29.00 & PVC/SL/ .01 & 21.00 & to & 29.50 & 24.00 & to 29.00 \\
\hline GW-241 & SS304 & 4.50 & 4.00 & 8.50 & 100.00 & SLS/SW/ .01 & 78.00 & to & 103.00 & 79.00 & to 100.00 \\
\hline GW-242 & SS304 & 2.37 & & 8.00 & 17.00 & SLS/SW/ .01 & 9.00 & to & 17.00 & 10.60 & to 17.00 \\
\hline GW-243 & PVC40 & 6.50 & 6.00 & 12.00 & 72.90 & PVC/SL/ .03 & 43.20 & to & 77.00 & 45.10 & to 72.90 \\
\hline GW-244 & PVC40 & 6.50 & 6.00 & 12.00 & 75.40 & PVC/SL/ .03 & 43.00 & to & 77.00 & 47.30 & to 75.40 \\
\hline GW-245 & PVC40 & 6.50 & 6.00 & 12.00 & 71.60 & PVC/SL/ .03 & 25.30 & to & 76.00 & 43.60 & to 71.60 \\
\hline GW-246 & PVC40 & 6.50 & 6.00 & 12.00 & 74.60 & PVC/SL/ .03 & 34.20 & to & 76.00 & 46.50 & to 74.60 \\
\hline GW-247 & PVC40 & 6.50 & 6.00 & 12.00 & 74.90 & PVC/SL/ .01 & 31.50 & to & 78.00 & 46.90 & to 74.90 \\
\hline GW-248 & SS304 & 4.50 & 4.00 & 9.00 & 61.70 & SLS/SW/ .01 & 49.10 & to & 62.00 & 50.70 & to 61.70 \\
\hline
\end{tabular}




\begin{tabular}{|c|c|c|c|c|c|c|c|c|c|c|}
\hline $\begin{array}{c}\text { CURRENT } \\
\text { NAME }\end{array}$ & $\begin{array}{c}\text { CASING } \\
\text { TYPE }\end{array}$ & $\begin{array}{l}\text { CASING } \\
\text { O.D. }\end{array}$ & $\begin{array}{c}\text { CASING } \\
\text { I.D. }\end{array}$ & $\begin{array}{c}\text { HOLE } \\
\text { DIAMETER }\end{array}$ & $\begin{array}{c}\text { HOLE } \\
\text { DEPTH }\end{array}$ & SCREEN TYPE & $\begin{array}{r}\text { PAC } \\
\text { INTE } \\
\end{array}$ & $\begin{array}{l}\text { CKED } \\
\text { ERVAL } \\
\end{array}$ & $\begin{array}{l}\text { SCF } \\
\text { INT }\end{array}$ & $\begin{array}{l}\text { EENED } \\
\text { ERVAL }\end{array}$ \\
\hline GW-249 & SS304 & 4.50 & 4.00 & 8.00 & 35.10 & SLS/SW/ .01 & 28.50 & to 35.10 & 29.90 & to 35.10 \\
\hline GW-250 & SS304 & 4.50 & 4.00 & 8.00 & 61.70 & SLS/SW/ .01 & 49.50 & to 61.70 & 51.30 & to 61.70 \\
\hline GW-251 & PVC40 & 4.50 & 4.00 & 8.25 & 47.10 & PVC/SL/ .01 & 35.00 & to 51.00 & 37.50 & to 47.10 \\
\hline GW-252 & SLS & 4.50 & 4.00 & 8.25 & 48.80 & SLS/SW/ .01 & 40.20 & to 49.00 & 43.80 & to 48.80 \\
\hline GW-253 & PVC40 & 4.50 & 4.00 & 8.25 & 46.70 & PVC/SL/ .01 & 36.20 & to 50.00 & 37.00 & to 46.70 \\
\hline GW-254 & SS304 & 4.50 & 4.00 & 9.62 & 56.60 & SLS/SW/ .01 & 43.80 & to 58.00 & 46.30 & to 56.60 \\
\hline GW-255 & SS304 & 4.50 & 4.00 & 10.00 & 81.30 & SLS/SW/ .01 & 66.30 & to 81.50 & 71.30 & to 81.30 \\
\hline GW-256 & SS304 & 4.50 & 4.00 & 8.25 & 17.70 & SLS/SW/ .01 & 10.30 & to 18.50 & 12.70 & to 17.70 \\
\hline GW-257 & SS304 & 4.50 & 4.00 & 9.00 & 33.70 & SLS/SW/ .01 & 19.00 & to 33.70 & 23.00 & to 33.70 \\
\hline GW-258 & SS304 & 4.50 & 4.00 & 9.00 & 49.30 & SLS/SW/ .01 & 37.00 & to 50.00 & 38.60 & to 49.30 \\
\hline GW-259 & SS304 & 4.50 & 4.00 & 9.00 & 33.00 & SLS/SW/ .01 & 21.10 & to 33.50 & 22.30 & to 33.00 \\
\hline GW-261 & SS304 & 4.50 & 4.00 & 10.00 & 23.70 & SLS/SW/ .01 & 16.70 & to 23.70 & 18.30 & to 23.70 \\
\hline GW-262 & SS304 & 4.50 & 4.00 & 10.00 & 70.60 & SLS/SW/ .01 & 57.70 & to 70.60 & 60.20 & to 70.60 \\
\hline GW-263 & SS304 & 4.50 & 4.00 & 10.00 & 30.80 & SLS/SW/ .01 & 23.00 & to 30.80 & 25.50 & to 30.80 \\
\hline GW-264 & SS304 & 4.50 & 4.00 & 10.00 & 70.70 & SLS/SW/ .01 & 58.50 & to 71.00 & 60.50 & to 70.70 \\
\hline GW-265 & PVC40 & 4.50 & 4.00 & 10.00 & 23.10 & PVC/SL/ .02 & 15.50 & to 23.10 & 17.50 & to 23.10 \\
\hline GW-266 & PVC40 & 4.50 & 4.00 & 8.00 & 24.90 & PVC/SL/ .02 & 17.50 & to 25.00 & 19.40 & to 24.90 \\
\hline GW-267 & PVC40 & 4.50 & 4.00 & 8.00 & 26.50 & PVC/SL/ .02 & 19.70 & to 27.00 & 21.00 & to 26.50 \\
\hline GW-268 & PVC40 & 4.50 & 4.00 & 8.00 & 33.70 & PVC/SL/ .02 & 26.50 & to 35.00 & 28.10 & to 33.70 \\
\hline GW-269 & PVC40 & 4.50 & 4.00 & 10.00 & 29.40 & PVC/SL/ .02 & 21.90 & to 30.00 & 23.70 & to 29.40 \\
\hline GW-270 & SS304 & 4.50 & 4.00 & 10.00 & 18.40 & SLS/SW/ .01 & 11.00 & to 18.50 & 13.00 & to 18.40 \\
\hline GW-271 & SS304 & 4.50 & 4.00 & 10.00 & 56.30 & SLS/SW/ .01 & 43.90 & to 56.30 & 45.90 & to 56.30 \\
\hline GW-272 & SS304 & 4.50 & 4.00 & 10.00 & 16.20 & SLS/SW/ .01 & 8.80 & to 16.20 & 10.90 & to 16.20 \\
\hline GW-273 & SS304 & 4.50 & 4.00 & 10.00 & 33.10 & SLS/SW/ .01 & 22.90 & to 33.10 & 27.80 & to 33.10 \\
\hline GW-274 & SS304 & 4.50 & 4.00 & 8.00 & 33.90 & SLS/SW/ .01 & 25.80 & to 35.00 & 28.50 & to 33.90 \\
\hline GW-275 & SS304 & 4.50 & 4.00 & 10.00 & 65.20 & SLS/SW/ .01 & 53.30 & to 65.50 & 54.80 & to 65.20 \\
\hline GW-276 & SS304 & 4.50 & 4.00 & 8.00 & 18.30 & SLS/SW/ .01 & 11.30 & to 18.50 & 13.00 & to 18.30 \\
\hline
\end{tabular}




\begin{tabular}{|c|c|c|c|c|c|c|c|c|c|c|}
\hline $\begin{array}{c}\text { CURRENT } \\
\text { NAME }\end{array}$ & $\begin{array}{c}\text { CASING } \\
\text { TYPE }\end{array}$ & $\begin{array}{l}\text { CASING } \\
\text { O.D. }\end{array}$ & $\begin{array}{c}\text { CASING } \\
\text { I.D. }\end{array}$ & $\begin{array}{c}\text { HOLE } \\
\text { DIAMETER }\end{array}$ & $\begin{array}{c}\text { HOLE } \\
\text { DEPTH }\end{array}$ & SCREEN TYPE & $\begin{array}{r}\text { PA } \\
\text { INTI }\end{array}$ & $\begin{array}{l}\text { CKED } \\
\text { ERVAL }\end{array}$ & $\begin{array}{l}\text { SCF } \\
\text { INT }\end{array}$ & $\begin{array}{l}\text { EENED } \\
\text { ERVAL }\end{array}$ \\
\hline GW-277 & SS304 & 4.50 & 4.00 & 8.00 & 77.40 & SLS/SW/ .01 & 62.20 & to 77.40 & 67.00 & to 77.40 \\
\hline GW-278 & SS304 & 4.50 & 4.00 & 8.00 & 21.10 & SLS/SW/ .01 & 14.00 & to 21.10 & 15.80 & to 21.10 \\
\hline GW-279 & SS304 & 4.50 & 4.00 & 8.00 & 76.40 & SLS/SW/ .01 & 61.50 & to 76.40 & 66.00 & to 76.40 \\
\hline GW-280 & SS304 & 4.50 & 4.00 & 8.00 & 152.00 & SLS/SW/ .01 & 126.60 & to 154.00 & 131.40 & to 152.00 \\
\hline GW-281 & SS304 & 4.50 & 4.00 & 6.00 & 17.50 & SLS/SL/ .01 & 4.00 & to 15.00 & 5.00 & to 15.00 \\
\hline GW-282 & SS304 & 4.50 & 4.00 & 6.00 & 14.00 & SLS/SL/ .01 & 3.00 & to 14.00 & 4.00 & to 14.00 \\
\hline GW-283 & SS304 & 4.50 & 4.00 & 6.00 & 20.00 & SLS/SL/ .01 & 4.00 & to 20.00 & 5.00 & to 20.00 \\
\hline GW-284 & SS304 & 4.50 & 4.00 & 6.00 & 18.00 & SLS/SL/ .01 & & to & 3.00 & to 18.00 \\
\hline GW-285 & SS304 & 4.50 & 4.00 & 6.00 & 21.00 & SLS/SL/ .01 & 3.50 & to 21.00 & 6.00 & to 21.00 \\
\hline GW-286 & SS304 & 4.50 & 4.00 & 9.00 & 32.00 & SLS/SW/ .01 & 19.50 & to 32.30 & 22.00 & to 32.00 \\
\hline GW-287 & SS304 & 4.50 & 4.00 & 9.00 & 12.30 & SLS/SW/ .01 & 5.60 & to 12.50 & 7.30 & to 12.30 \\
\hline GW-288 & SS304 & 4.50 & 4.00 & 9.00 & 59.10 & SLS/SW/ .01 & 46.00 & to 60.00 & 49.10 & to 59.10 \\
\hline GW-289 & SS304 & 4.50 & 4.00 & 9.00 & 40.60 & SLS/SW/ .01 & 28.90 & to 40.80 & 30.60 & to 40.60 \\
\hline GW-290 & SS304 & 4.50 & 4.00 & 9.00 & 32.10 & SLS/SW/ .01 & 19.30 & to 32.50 & 22.10 & to 32.10 \\
\hline GW-291 & SS304 & 4.50 & 4.00 & 9.00 & 13.70 & SLS/SW/ .01 & 6.70 & to 14.20 & 8.70 & to 13.70 \\
\hline GW-292 & SS304 & 4.50 & 4.00 & 10.00 & 184.80 & SLS/SW/ .01 & 172.10 & to 186.00 & 174.10 & to 184.80 \\
\hline GW-294 & SS304 & 4.50 & 4.00 & 10.00 & 128.00 & SLS/SW/ .01 & 113.00 & to 128.00 & 117.60 & to 128.00 \\
\hline GW-295 & SS304 & 4.50 & 4.00 & 10.00 & 146.00 & SLS/SW/ .01 & 130.40 & to 146.00 & 135.90 & to 146.00 \\
\hline GW-296 & SS304 & 4.50 & 4.00 & 10.00 & 146.80 & SLS/SW/ .01 & 134.40 & to 147.00 & 136.30 & to 146.80 \\
\hline GW-297 & SS304 & 4.50 & 4.00 & 10.00 & 120.00 & SLS/SW/ .01 & 107.00 & to 120.00 & 109.80 & to 120.00 \\
\hline GW-298 & SS304 & 4.50 & 4.00 & 10.00 & 186.00 & SLS/SW/ .01 & 171.10 & to 190.00 & 176.00 & to 186.00 \\
\hline GW-299 & SS304 & 4.50 & 4.00 & 10.00 & 166.60 & SLS/SW/ .01 & 153.00 & to 168.00 & 155.80 & to 166.60 \\
\hline GW-300 & SS304 & 4.50 & 4.00 & 10.00 & 145.40 & SLS/SW/ .01 & 132.00 & to 147.00 & 134.80 & to $145.4 \mathrm{C}$ \\
\hline GW-301 & SS304 & 4.50 & 4.00 & 10.00 & 161.00 & SLS/SW/ .01 & 148.50 & to 163.50 & 151.00 & to 161.00 \\
\hline GW-302 & SS304 & 4.50 & 4.00 & 9.50 & 134.80 & SLS/SW/ .01 & 121.50 & to 134.80 & 124.50 & to 134.80 \\
\hline GW-304 & SS304 & 4.50 & 4.00 & 9.75 & 167.00 & SLS/SW/ .01 & 148.50 & to 167.00 & 156.50 & to 166.50 \\
\hline GW-305 & SS304 & 4.50 & 4.00 & 10.00 & 179.60 & SLS/SW/ .01 & 165.30 & to 179.60 & 168.90 & to 179.60 \\
\hline
\end{tabular}




\begin{tabular}{|c|c|c|c|c|c|c|c|c|c|c|}
\hline $\begin{array}{c}\text { CURRENT } \\
\text { NAME }\end{array}$ & $\begin{array}{c}\text { CASING } \\
\text { TYPE }\end{array}$ & $\begin{array}{l}\text { CASING } \\
\text { O.D. }\end{array}$ & $\begin{array}{c}\text { CASING } \\
\text { I.D. }\end{array}$ & $\begin{array}{c}\text { HOLE } \\
\text { DIAMETER }\end{array}$ & $\begin{array}{c}\text { HOLE } \\
\text { DEPTH }\end{array}$ & SCREEN TYPE & $\begin{array}{r}\text { PAC } \\
\text { INTE } \\
\end{array}$ & $\begin{array}{l}\text { CKED } \\
\text { ERVAL } \\
\end{array}$ & $\begin{array}{l}\text { SCF } \\
\text { INT }\end{array}$ & $\begin{array}{l}\text { EENED } \\
\text { ERVAL }\end{array}$ \\
\hline GW-306 & SS304 & 4.50 & 4.00 & 8.00 & 58.10 & SLS/SW/ .01 & 46.20 & to 58.50 & 48.10 & to 58.10 \\
\hline GW-307 & SS304 & 4.50 & 4.00 & 10.00 & 41.60 & SLS/SW/ .01 & 28.70 & to 41.60 & 30.90 & to 41.60 \\
\hline GW-308 & SS304 & 4.50 & 4.00 & 10.00 & 37.70 & SLS/SW/ .01 & 22.00 & to 37.70 & 27.10 & to 37.70 \\
\hline GW-309 & SS304 & 4.50 & 4.00 & 8.00 & 37.00 & SLS/SW/ .01 & 25.00 & to 38.00 & 27.00 & to 37.00 \\
\hline GW-310 & SS304 & 4.50 & 4.00 & 10.00 & 27.10 & SLS/SW/ .01 & 19.50 & to 27.10 & 21.80 & to 27.10 \\
\hline GW-311 & SS304 & 4.50 & 4.00 & 10.00 & 40.30 & SLS/SW/ .01 & 25.60 & to 40.30 & 29.70 & to 40.30 \\
\hline GW-312 & SS304 & 4.50 & 4.00 & 12.00 & 40.50 & SLS/SW/ .01 & 29.60 & to 41.00 & 30.50 & to 40.50 \\
\hline GW-313 & SS304 & 4.50 & 4.00 & 10.00 & 112.10 & SLS/SW/ .01 & 98.20 & to 113.00 & 101.40 & to 112.10 \\
\hline GW-314 & SS304 & 4.50 & 4.00 & 10.00 & 113.00 & SLS/SW/ .01 & 101.00 & to 115.00 & 103.00 & to 113.00 \\
\hline GW-315 & SS304 & 4.50 & 4.00 & 10.00 & 103.30 & SLS/SW/ .01 & 90.00 & to 104.00 & 93.30 & to 103.30 \\
\hline GW-316 & SS304 & 4.50 & 4.00 & 10.00 & 79.40 & SLS/SW/ .01 & 67.50 & to 80.00 & 68.70 & to 79.40 \\
\hline GW-317 & SS304 & 4.50 & 4.00 & 10.00 & 130.50 & SLS/SW/ .01 & 117.00 & to 132.00 & 119.50 & to 130.50 \\
\hline GW-318 & SS304 & 4.50 & & 8.00 & 80.00 & SLS/UNKN/ & 67.60 & to 79.60 & 69.10 & to 79.60 \\
\hline GW-319 & SS304 & 4.50 & & 8.00 & 23.20 & SLS/UNKN/ & 10.20 & to 23.20 & 12.20 & to 23.20 \\
\hline GW-320 & SS304 & 4.50 & & 8.00 & 200.00 & SLS/SW/ .01 & 97.50 & to 115.00 & 100.00 & to 110.00 \\
\hline GW-321 & SS304 & 4.50 & & 8.00 & 98.60 & SLS/SW/ .01 & 84.00 & to 98.60 & 87.30 & to 98.00 \\
\hline GW-323 & SS304 & 4.50 & 4.00 & 10.00 & 107.00 & SLS/SW/ .01 & 94.00 & to 108.00 & 96.50 & to 107.00 \\
\hline GW-324 & SS304 & 4.50 & 4.00 & 10.00 & 79.00 & SLS/SW/ .01 & 66.50 & to 80.00 & 68.50 & to 79.00 \\
\hline GW-325 & SS304 & 4.50 & 4.00 & 10.00 & 17.30 & SLS/SW/ .01 & 5.10 & to 17.90 & 7.30 & to 17.30 \\
\hline GW-326 & PVC40 & 4.50 & & 8.00 & 7.90 & PVC/SW/ .01 & 1.50 & to 7.90 & 2.70 & to 7.70 \\
\hline GW-327 & PVC40 & 4.50 & & 8.00 & 7.90 & PVC/SW/ .01 & 1.50 & to 7.90 & 2.70 & to 7.70 \\
\hline GW-328 & PVC40 & 4.50 & & 8.00 & 16.80 & PVC/SW/ .01 & 1.00 & to 16.80 & 1.50 & to 16.50 \\
\hline GW-329 & PVC40 & 4.50 & & 8.00 & 26.80 & PVC/SW/ .01 & 1.50 & to 26.80 & 2.00 & to 26.50 \\
\hline GW-330 & PVC40 & 4.50 & & 8.00 & 27.00 & $\mathrm{PVC/SW/.01}$ & 1.00 & to 27.00 & 1.80 & to 26.80 \\
\hline GW-331 & SS304 & 4.50 & 4.00 & 10.00 & 30.10 & SLS/SW/ .01 & 23.00 & to 30.10 & 24.50 & to 30.10 \\
\hline GW-332 & SS304 & 4.50 & 4.00 & 10.00 & 24.10 & SLS/SW/ .01 & 16.80 & to 24.10 & 18.70 & to 24.10 \\
\hline GW-333 & SS304 & 4.50 & 4.00 & 10.00 & 25.10 & SLS/SW/ .01 & 13.70 & to 25.10 & 14.40 & to 25.10 \\
\hline
\end{tabular}




\begin{tabular}{|c|c|c|c|c|c|c|c|c|c|c|}
\hline $\begin{array}{c}\text { CURRENT } \\
\text { NAME }\end{array}$ & $\begin{array}{c}\text { CASING } \\
\text { TYPE }\end{array}$ & $\begin{array}{l}\text { CASING } \\
\text { O.D. }\end{array}$ & $\begin{array}{c}\text { CASING } \\
\text { I.D. }\end{array}$ & $\begin{array}{c}\text { HOLE } \\
\text { DIAMETER }\end{array}$ & $\begin{array}{c}\text { HOLE } \\
\text { DEPTH }\end{array}$ & SCREEN TYPE & $\begin{array}{r}\text { PAC } \\
\text { INTE } \\
\end{array}$ & $\begin{array}{l}\text { CKED } \\
\text { ERVAL } \\
\end{array}$ & $\begin{array}{l}\text { SCF } \\
\text { INT }\end{array}$ & $\begin{array}{l}\text { EENED } \\
\text { ERVAL }\end{array}$ \\
\hline GW-334 & SS304 & 4.50 & 4.00 & 10.00 & 27.20 & SLS/SW/ .01 & 19.80 & to 27.20 & 21.80 & to 27.20 \\
\hline GW-335 & SS304 & 4.50 & 4.00 & 10.00 & 15.70 & SLS/SW/ .01 & 7.60 & to 15.70 & 10.00 & to 15.70 \\
\hline GW-336 & SS304 & 4.50 & 4.00 & 10.00 & 21.40 & SLS/SW/ .01 & 13.20 & to 21.40 & 15.80 & to 21.40 \\
\hline GW-337 & SS304 & 4.50 & 4.00 & 10.00 & 22.10 & SLS/SW/ .01 & 15.00 & to 22.10 & 16.70 & to 22.10 \\
\hline GW-338 & SS304 & 4.50 & 4.00 & 10.00 & 17.60 & SLS/SW/ .01 & 11.20 & to 17.60 & 12.10 & to 17.60 \\
\hline GW-339 & SS304 & 4.50 & 4.00 & 9.50 & 114.00 & SLS/SW/ .01 & 101.00 & to 114.00 & 103.70 & to 114.00 \\
\hline GW-340 & PVC40 & 4.50 & & 8.00 & 12.80 & $\mathrm{PVC/SW/.01}$ & 1.00 & to 12.80 & 2.50 & to 12.50 \\
\hline GW-341 & PVC40 & 4.50 & & 8.00 & 18.00 & $\mathrm{PVC/SW/.01}$ & 1.30 & to 18.00 & 2.70 & to 17.70 \\
\hline GW-342 & SS304 & 4.50 & 4.00 & 9.50 & 75.00 & SLS/SW/ .01 & 61.00 & to 75.00 & 65.00 & to 75.00 \\
\hline GW-345 & SS304 & 4.50 & 4.00 & 14.50 & 26.30 & SLS/SW/ .01 & 15.20 & to 26.30 & 16.00 & to 26.00 \\
\hline GW-346 & SS304 & 4.50 & 4.00 & 9.50 & 64.90 & SLS/SW/ .01 & 51.50 & to 64.90 & 54.60 & to 64.60 \\
\hline GW-347 & SS304 & 4.50 & 4.00 & 9.50 & 27.80 & SLS/SW/ .01 & 9.50 & to 27.80 & 11.70 & to 27.50 \\
\hline GW-348 & SS304 & 4.50 & 4.00 & 9.50 & 80.90 & SLS/SW/ .01 & 68.10 & to 80.90 & 70.20 & to 80.60 \\
\hline GW-349 & SS304 & 4.50 & 4.00 & 8.00 & 24.30 & SLS/SW/ .01 & 5.00 & to 24.30 & 9.00 & to 24.00 \\
\hline GW-350 & SS304 & 4.50 & 4.00 & 9.50 & 43.70 & SLS/SW/ .01 & 28.00 & to 43.70 & 33.40 & to 43.40 \\
\hline GW-364 & SS304 & 4.50 & 4.00 & 10.60 & 60.30 & SLS/SW/ .01 & 47.00 & to 60.30 & 49.80 & to 59.80 \\
\hline GW-366 & SS304 & 4.50 & 4.00 & 9.50 & 101.00 & SLS/SW/ .01 & 88.00 & to 101.00 & 90.50 & to 100.50 \\
\hline GW-370 & SS304 & 4.50 & 4.00 & 9.50 & 33.10 & SLS/SW/ .01 & 21.10 & to 33.10 & 22.50 & to 32.50 \\
\hline GW-372 & SS304 & 4.50 & 4.00 & 9.50 & 51.60 & SLS/SW/ .01 & 39.80 & to 51.60 & 41.00 & to 51.00 \\
\hline GW-380 & SS304 & 4.50 & & 10.00 & 15.50 & SLS/SW/ .01 & 2.80 & to 15.50 & 4.80 & to 15.20 \\
\hline GW-383 & SS304 & 4.50 & 4.00 & 8.75 & 23.60 & SLS/SW/ .01 & 16.60 & to 23.60 & 18.10 & to 23.10 \\
\hline GW-401 & PVC80 & 1.00 & & 3.70 & 862.60 & STL/PER/ & & to & 812.10 & to 852.10 \\
\hline GW-402 & PVC40 & 1.00 & & 3.70 & 398.30 & STL/PER/ & & to & 373.40 & to 388.00 \\
\hline GW-405 & PVC40 & 2.37 & 2.00 & 6.13 & 34.50 & PVC/SL/ .01 & 23.80 & to 35.00 & 24.60 & to 34.50 \\
\hline GW-406 & PVC40 & 2.37 & 2.00 & 6.63 & 6.60 & PVC/SL/ .01 & 2.70 & to 6.60 & 2.90 & to 6.60 \\
\hline GW-407 & PVC40 & 2.37 & 2.00 & 6.13 & 40.50 & PVC/SL/ .01 & 31.40 & to 40.60 & 30.60 & to 40.50 \\
\hline GW-408 & PVC40 & 2.37 & 2.00 & 6.63 & 5.90 & PVC/SL/ .01 & 1.90 & to 5.90 & 2.90 & to 5.90 \\
\hline
\end{tabular}




\begin{tabular}{|c|c|c|c|c|c|c|c|c|c|c|}
\hline $\begin{array}{c}\text { CURRENT } \\
\text { NAME }\end{array}$ & $\begin{array}{c}\text { CASING } \\
\text { TYPE }\end{array}$ & $\begin{array}{l}\text { CASING } \\
\text { O.D. }\end{array}$ & $\begin{array}{c}\text { CASING } \\
\text { I.D. }\end{array}$ & $\begin{array}{c}\text { HOLE } \\
\text { DIAMETER }\end{array}$ & $\begin{array}{c}\text { HOLE } \\
\text { DEPTH }\end{array}$ & SCREEN TYPE & $\begin{array}{r}\text { PAC } \\
\text { INTE } \\
\end{array}$ & $\begin{array}{l}\text { CKED } \\
\text { ERVAL }\end{array}$ & $\begin{array}{l}\text { SCF } \\
\text { INT }\end{array}$ & $\begin{array}{l}\text { EENED } \\
\text { ERVAL }\end{array}$ \\
\hline GW-409 & PVC40 & 2.37 & 2.00 & 6.13 & 57.65 & PVC/SL/ .01 & 46.00 & to 58.20 & 47.75 & to 57.65 \\
\hline GW-410 & PVC40 & 2.37 & 2.00 & 6.63 & 13.75 & PVC/SL/ .01 & 2.80 & to 13.75 & 3.85 & to 13.75 \\
\hline GW-411 & PVC40 & 2.37 & 2.00 & 6.63 & 8.40 & PVC/SL/ .01 & 2.90 & to 8.35 & 3.35 & to 8.35 \\
\hline GW-412 & PVC40 & 2.37 & 2.00 & 6.13 & 40.00 & PVC/SL/ .01 & 27.20 & to 40.10 & 30.10 & to 40.00 \\
\hline GW-413 & PVC40 & 2.37 & 2.00 & 6.63 & 13.55 & PVC/SL/ .01 & 2.60 & to 13.55 & 3.65 & to 13.55 \\
\hline GW-414 & PVC40 & 2.37 & 2.00 & 6.13 & 55.70 & PVC/SL/ .01 & 43.70 & to 55.80 & 45.80 & to 55.70 \\
\hline GW-415 & PVC40 & 2.37 & 2.00 & 6.63 & 27.80 & PVC/SL/ .01 & 16.80 & to 27.80 & 17.90 & to 27.80 \\
\hline GW-416 & PVC40 & 2.37 & 2.00 & 6.13 & 61.00 & PVC/SL/ .01 & 47.90 & to 61.20 & 51.10 & to 61.00 \\
\hline GW-417 & PVC40 & 2.37 & 2.00 & 6.13 & 48.30 & PVC/SL/ .01 & 34.70 & to 49.10 & 33.30 & to 48.30 \\
\hline GW-418 & PVC40 & 2.37 & 2.00 & 6.63 & 21.10 & PVC/SL/ .01 & 10.10 & to 21.20 & 11.20 & to 21.10 \\
\hline GW-419 & PVC40 & 2.37 & 2.00 & 6.13 & 49.00 & PVC/SL/ .01 & 38.00 & to 50.00 & 39.00 & to 49.00 \\
\hline GW-420 & PVC40 & 2.37 & 2.00 & 6.63 & 13.75 & PVC/SL/ .01 & 2.80 & to 13.75 & 3.85 & to 13.75 \\
\hline GW-421 & PVC40 & 2.37 & 2.00 & 6.13 & 38.90 & PVC/SL/ .01 & 27.50 & to 38.90 & 29.00 & to 38.90 \\
\hline GW-422 & PVC40 & 2.37 & 2.00 & 6.63 & 7.60 & PVC/SL/ .01 & 2.10 & to 7.60 & 3.90 & to 7.60 \\
\hline GW-423 & PVC40 & 2.37 & 2.00 & 6.13 & 38.90 & PVC/SL/ .01 & 27.00 & to 39.00 & 29.00 & to 38.90 \\
\hline GW-424 & PVC40 & 2.37 & 2.00 & 6.63 & 4.40 & PVC/SL/ .01 & 3.00 & to 4.40 & 3.40 & to 4.40 \\
\hline GW-425 & PVC40 & 2.37 & 2.00 & 6.13 & 59.85 & PVC/SL/ .01 & 48.00 & to 60.00 & 49.95 & to 59.85 \\
\hline GW-426 & PVC40 & 2.37 & 2.00 & 6.63 & 31.00 & PVC/SL/ .01 & 20.00 & to 31.00 & 21.10 & to 31.00 \\
\hline GW-427 & PVC40 & 2.37 & 2.00 & 6.13 & 47.90 & PVC/SL/ .01 & 37.00 & to 48.00 & 38.00 & to 47.90 \\
\hline GW-428 & PVC40 & 2.37 & 2.00 & 6.63 & 13.90 & PVC/SL/ .01 & 2.90 & to 13.90 & 4.00 & to 13.90 \\
\hline GW-429 & PVC40 & 2.37 & 2.00 & 6.63 & 5.60 & PVC/SL/ .01 & 1.50 & to 5.60 & 1.60 & to 5.60 \\
\hline GW-430 & PVC40 & 2.37 & 2.00 & 6.13 & 37.00 & PVC/SL/ .01 & 25.40 & to 38.20 & 27.10 & to 37.00 \\
\hline GW-431 & PVC40 & 2.37 & 2.00 & 6.63 & 7.75 & PVC/SL/ .01 & 0.75 & to 7.75 & 0.75 & to 7.75 \\
\hline GW-432 & PVC40 & 2.37 & 2.00 & 6.13 & 43.50 & PVC/SL/ .01 & 32.00 & to 43.80 & 33.60 & to 43.50 \\
\hline GW-434 & PVC40 & 2.37 & 2.00 & 6.13 & 40.10 & PVC/SL/ .01 & 28.30 & to 40.30 & 30.00 & to 40.10 \\
\hline GW-435 & PVC40 & 2.37 & 2.00 & 6.63 & 8.40 & PVC/SL/ .01 & 1.60 & to 8.40 & 3.40 & to 8.40 \\
\hline GW-436 & PVC40 & 2.37 & 2.00 & 6.13 & 45.40 & PVC/SL/ .01 & 33.10 & to 45.50 & 35.50 & to 45.40 \\
\hline
\end{tabular}




\begin{tabular}{|c|c|c|c|c|c|c|c|c|c|c|}
\hline $\begin{array}{c}\text { CURRENT } \\
\text { NAME }\end{array}$ & $\begin{array}{l}\text { CASING } \\
\text { TYPE }\end{array}$ & $\begin{array}{c}\text { CASING } \\
\text { O.D. }\end{array}$ & $\begin{array}{c}\text { CASING } \\
\text { I.D. }\end{array}$ & $\begin{array}{c}\text { HOLE } \\
\text { DIAMETER }\end{array}$ & $\begin{array}{c}\text { HOLE } \\
\text { DEPTH }\end{array}$ & SCREEN TYPE & \multicolumn{2}{|c|}{$\begin{array}{r}\text { PACKED } \\
\text { INTERVAL } \\
\end{array}$} & \multicolumn{2}{|c|}{$\begin{array}{l}\text { SCREENED } \\
\text { INTERVAL }\end{array}$} \\
\hline GW-437 & PVC40 & 2.37 & 2.00 & 6.13 & 63.10 & PVC/SL/ .01 & 51.60 & to 63.20 & 53.20 & to 63.10 \\
\hline GW-438 & PVC40 & 2.37 & 2.00 & 6.63 & 22.95 & PVC/SL/ .01 & 12.00 & to 22.95 & 13.05 & to 22.95 \\
\hline GW-439 & PVC40 & 2.37 & 2.00 & 6.13 & 59.65 & PVC/SL/ .01 & 45.60 & to 60.00 & 49.75 & to 59.65 \\
\hline GW-440 & PVC40 & 2.37 & 2.00 & 6.63 & 26.65 & PVC/SL/ .01 & 15.70 & to 26.70 & 16.75 & to 26.65 \\
\hline GW-441 & PVC40 & 2.37 & 2.00 & 6.13 & 54.85 & PVC/SL/ .01 & 43.00 & to 54.85 & 44.95 & to 54.85 \\
\hline GW-442 & PVC40 & 2.37 & 2.00 & 6.63 & 14.80 & PVC/SL/ .01 & 3.80 & to 14.80 & 4.90 & to 14.80 \\
\hline GW-443 & SS304 & 4.50 & 4.00 & 10.00 & 49.50 & SLS/SW/ .01 & 37.50 & to 50.00 & 39.00 & to 49.50 \\
\hline GW-445 & SS304 & 4.50 & 4.00 & 10.00 & 45.70 & SLS/SW/ .01 & 33.00 & to 47.00 & 35.00 & to 45.70 \\
\hline GW-447 & PVC40 & 2.37 & 2.00 & 6.63 & 8.55 & PVC/SL/ .01 & 3.10 & to 8.55 & 3.55 & to 8.55 \\
\hline GW-448 & PVC40 & 2.37 & 2.00 & 6.13 & 44.30 & PVC/SL/ .01 & 31.00 & to 44.30 & 34.20 & to 44.30 \\
\hline GW-449 & PVC40 & 2.37 & 2.00 & 6.63 & 11.50 & PVC/SL/ .01 & 6.00 & to 11.50 & 6.50 & to 11.50 \\
\hline GW-450 & PVC40 & 2.37 & 2.00 & 6.13 & 55.90 & PVC/SL/ .01 & 44.20 & to 55.90 & 46.00 & to 55.90 \\
\hline GW-452 & PVC40 & 2.37 & 2.00 & 6.63 & 18.60 & PVC/SL/ .01 & 7.60 & to 19.00 & 8.70 & to 18.60 \\
\hline GW-453 & PVC40 & 1.00 & & 3.70 & 757.30 & STL/PER/ & & to & 737.30 & to 747.30 \\
\hline GW-455 & PVC40 & 2.37 & 2.00 & 3.70 & 182.50 & PVC/SL/ .01 & 155.40 & to 193.00 & 162.50 & to 182.50 \\
\hline GW-456 & PVC40 & 2.37 & 2.00 & 7.88 & 69.50 & PVC/SL/ .01 & 57.50 & to 70.00 & 59.20 & to 69.50 \\
\hline GW-457 & PVC40 & 2.37 & 2.00 & 7.88 & 25.00 & PVC/SL/ .01 & 13.50 & to 25.50 & 14.71 & to 25.00 \\
\hline GW-458 & PVC40 & 2.37 & 2.00 & 7.87 & 69.50 & PVC/SL/ .01 & 57.50 & to 70.00 & 59.20 & to 69.50 \\
\hline GW-459 & PVC40 & 2.37 & 2.00 & 7.87 & 26.00 & PVC/SL/ .01 & 12.50 & to 26.50 & 15.71 & to 26.00 \\
\hline GW-460 & PVC40 & 2.37 & 2.00 & 7.87 & 69.50 & PVC/SL/ .01 & 57.00 & to 70.00 & 59.20 & to 69.50 \\
\hline GW-461 & PVC40 & 2.37 & 2.00 & 7.87 & 25.00 & PVC/SL/ .01 & 13.00 & to 25.50 & 14.21 & to 25.00 \\
\hline GW-462 & PVC40 & 4.50 & 4.00 & 8.75 & 69.50 & PVC/SL/ .01 & 16.70 & to 70.00 & 19.45 & to 69.50 \\
\hline GW-463 & PVC40 & 2.37 & 2.00 & 8.75 & 55.80 & PVC/SL/ .01 & 43.70 & to 56.50 & 45.80 & to 55.80 \\
\hline GW-464 & PVC40 & 2.37 & 2.00 & 8.75 & 22.00 & PVC/SL/ .01 & 10.30 & to 22.50 & 12.00 & to 22.00 \\
\hline GW-465 & PVC40 & 2.37 & 2.00 & 8.75 & 41.09 & PVC/SL/ .01 & 28.30 & to 41.90 & 30.80 & to 41.90 \\
\hline GW-466 & PVC40 & 2.37 & 2.00 & 8.75 & 42.20 & PVC/SL/ .01 & 29.90 & to 39.50 & 32.20 & to 42.20 \\
\hline GW-467 & PVC40 & 4.50 & 4.00 & 7.88 & 58.50 & PVC/SL/ .02 & & to & 38.50 & to 58.50 \\
\hline
\end{tabular}




\begin{tabular}{|c|c|c|c|c|c|c|c|c|c|c|}
\hline $\begin{array}{c}\text { CURRENT } \\
\text { NAME }\end{array}$ & $\begin{array}{l}\text { CASING } \\
\text { TYPE }\end{array}$ & $\begin{array}{c}\text { CASING } \\
\text { O.D. }\end{array}$ & $\begin{array}{c}\text { CASING } \\
\text { I.D. }\end{array}$ & $\begin{array}{c}\text { HOLE } \\
\text { DIAMETER }\end{array}$ & $\begin{array}{c}\text { HOLE } \\
\text { DEPTH }\end{array}$ & SCREEN TYPE & $\begin{array}{r}\text { PA } \\
\text { INTI }\end{array}$ & $\begin{array}{l}\text { CKED } \\
\text { ERVAL }\end{array}$ & $\begin{array}{l}\text { SCF } \\
\text { INT }\end{array}$ & $\begin{array}{l}\text { EENED } \\
\text { ERVAL }\end{array}$ \\
\hline GW-469 & PVC40 & 4.50 & 4.00 & 8.75 & 469.00 & PVC/SL/ & 443.50 & to 469.00 & 448.80 & to 469.00 \\
\hline GW-470 & PVC40 & 4.50 & 4.00 & 8.75 & 262.50 & PVC/SL/ .01 & 238.40 & to 266.10 & 242.50 & to 262.50 \\
\hline GW-471 & PVC40 & 1.00 & & 3.70 & 101.50 & $\mathrm{PVC} / \mathrm{SL} / .01$ & 88.80 & to 103.80 & 91.50 & to 101.50 \\
\hline GW-472 & PVC80 & 4.50 & 4.00 & 8.75 & 310.00 & $\mathrm{PVC/SL/} \mathrm{.01}$ & 285.00 & to 313.00 & 290.00 & to 310.00 \\
\hline GW-473 & PVC80 & 4.50 & 4.00 & 8.75 & 90.00 & PVC/SL/ .02 & 68.00 & to 94.00 & 70.05 & to 90.00 \\
\hline GW-474 & PVC80 & 4.50 & 4.00 & 8.75 & 43.80 & PVC/SL/ .01 & 26.25 & to 44.50 & 28.75 & to 43.80 \\
\hline GW-475A & PVC40 & 2.37 & 2.00 & 9.50 & 97.00 & PVC/SL/ .01 & 85.00 & to 99.40 & 87.00 & to 97.00 \\
\hline GW-475B & PVC40 & 2.37 & 2.00 & 9.50 & 60.80 & PVC/SL/ .01 & 48.00 & to 61.00 & 50.40 & to 60.40 \\
\hline GW-475C & PVC40 & 2.37 & 2.00 & 9.50 & 18.00 & $\mathrm{PVC} / \mathrm{SL} / .01$ & 6.00 & to 18.50 & 8.00 & to 18.50 \\
\hline GW-476A & PVC40 & 2.37 & 2.00 & 9.50 & 80.20 & $\mathrm{PVC/SL/} \mathrm{.01}$ & 67.90 & to 81.00 & 70.00 & to 80.00 \\
\hline GW-476B & PVC40 & 2.37 & 2.00 & 9.50 & 46.40 & PVC/SL/ .01 & 34.00 & to 46.50 & 36.00 & to 46.00 \\
\hline GW-476C & PVC40 & 2.37 & 2.00 & 9.50 & 16.60 & PVC/SL/ .01 & 5.00 & to 16.70 & 6.20 & to 16.20 \\
\hline GW-477A & PVC40 & 2.37 & 2.00 & 9.50 & 65.40 & PVC/SL/ .01 & 53.00 & to 67.00 & 55.00 & to 65.40 \\
\hline GW-477B & PVC40 & 2.37 & 2.00 & 9.50 & 32.10 & PVC/SL/ .01 & 20.00 & to 32.60 & 21.50 & to 32.10 \\
\hline GW-477C & PVC40 & 2.37 & 2.00 & 9.50 & 16.40 & $\mathrm{PVC} / \mathrm{SL} / .01$ & 4.50 & to 16.40 & 6.00 & to 16.40 \\
\hline GW-478A & PVC40 & 2.37 & 2.00 & 9.50 & 78.40 & $\mathrm{PVC/SL/} \mathrm{.01}$ & 65.00 & to 80.40 & 68.00 & to 78.40 \\
\hline GW-478B & & 2.37 & 2.00 & 9.50 & 44.90 & PVC/SL/ .01 & 33.00 & to 45.00 & 34.50 & to 44.90 \\
\hline GW-478C & PVC40 & 2.37 & 2.00 & 9.50 & 16.20 & PVC/SL/ .01 & 4.50 & to 16.50 & 6.00 & to 16.20 \\
\hline GW-479 & PVC40 & 2.37 & 2.00 & 8.75 & 23.00 & PVC/SL/ .01 & 11.00 & to 23.50 & 13.00 & to 23.00 \\
\hline GW-480A & PVC40 & 1.00 & & 6.12 & 34.50 & $\mathrm{PVC} / \mathrm{SL} / .01$ & 31.00 & to 35.00 & 32.00 & to 34.50 \\
\hline GW-480B & PVC40 & 1.00 & & 6.12 & 29.00 & $\mathrm{PVC/SL/} \mathrm{.01}$ & 25.50 & to 29.50 & 26.50 & to 29.00 \\
\hline GW-480C & PVC40 & 1.00 & & 6.12 & 15.50 & PVC/SL/ .01 & 12.00 & to 16.00 & 13.00 & to 15.50 \\
\hline GW-481A & PVC40 & 1.00 & & 6.12 & 32.00 & PVC/SL/ .01 & 28.90 & to 32.50 & 29.50 & to 32.00 \\
\hline GW-481B & PVC40 & 1.00 & & 6.12 & 26.00 & PVC/SL/ .01 & 22.50 & to 26.50 & 23.52 & to 26.00 \\
\hline GW-481C & PVC40 & 1.00 & & 6.12 & 15.00 & PVC/SL/ .01 & 11.50 & to 15.40 & 12.50 & to 15.00 \\
\hline GW-482A & PVC40 & 1.00 & & 6.12 & 34.25 & $\mathrm{PVC} / \mathrm{SL} / .01$ & 30.75 & to 34.75 & 31.75 & to 34.25 \\
\hline GW-482B & PVC40 & 1.00 & & 6.12 & 27.25 & $\mathrm{PVC/SL/} \mathrm{.01}$ & 23.75 & to 27.75 & 24.75 & to 27.25 \\
\hline
\end{tabular}




\begin{tabular}{|c|c|c|c|c|c|c|c|c|c|c|}
\hline $\begin{array}{c}\text { CURRENT } \\
\text { NAME }\end{array}$ & $\begin{array}{c}\text { CASING } \\
\text { TYPE }\end{array}$ & $\begin{array}{c}\text { CASING } \\
\text { O.D. }\end{array}$ & $\begin{array}{l}\text { CASING } \\
\text { I.D. }\end{array}$ & $\begin{array}{c}\text { HOLE } \\
\text { DIAMETER }\end{array}$ & $\begin{array}{c}\text { HOLE } \\
\text { DEPTH }\end{array}$ & SCREEN TYPE & \multicolumn{2}{|c|}{$\begin{array}{r}\text { PACKED } \\
\text { INTERVAL }\end{array}$} & \multicolumn{2}{|c|}{$\begin{array}{l}\text { SCREENED } \\
\text { INTERVAL }\end{array}$} \\
\hline GW-482C & PVC40 & 1.00 & & 6.12 & 15.25 & PVC/SL/ .01 & 11.75 & to 15.75 & 12.75 & to 15.25 \\
\hline GW-483 & PVC40 & 2.37 & 2.00 & 6.12 & 23.70 & PVC/SL/ .01 & 11.40 & to 25.20 & 13.70 & to 23.70 \\
\hline GW-484 & PVC40 & 2.37 & 2.00 & 6.12 & 21.10 & PVC/SL/ .01 & 10.40 & to 23.75 & 12.10 & to 21.10 \\
\hline GW-485 & PVC40 & 2.37 & 2.00 & 6.00 & 19.50 & PVC/SL/ .01 & 8.30 & to 20.50 & 9.50 & to 19.50 \\
\hline GW-486 & PVC40 & 2.37 & 2.00 & 6.00 & 16.80 & PVC/SL/ .01 & 5.00 & to 16.80 & 6.80 & to 16.80 \\
\hline GW-487 & PVC40 & 2.37 & 2.00 & 6.00 & 18.00 & PVC/SL/ .01 & 6.00 & to 18.00 & 8.00 & to 18.00 \\
\hline GW-488 & PVC40 & 2.37 & 2.00 & 6.00 & 16.30 & PVC/SL/ .01 & 5.00 & to 16.30 & 6.30 & to 16.30 \\
\hline GW-489 & PVC40 & 2.37 & 2.00 & 6.00 & 20.50 & PVC/SL/ .01 & 8.00 & to 20.50 & 10.50 & to 20.50 \\
\hline GW-490 & PVC & 2.37 & 2.00 & 6.00 & 18.00 & PVC/SL/ .01 & 6.00 & to 18.00 & 8.00 & to 18.00 \\
\hline GW-491 & PVC40 & 2.37 & 2.00 & 6.00 & 16.50 & PVC/SL/ .01 & 4.50 & to 17.00 & 6.50 & to 16.50 \\
\hline GW-492 & PVC40 & 2.37 & 2.00 & 6.00 & 14.10 & PVC/SL/ .01 & 3.10 & to 14.10 & 4.10 & to 14.10 \\
\hline GW-493 & PVC40 & 2.37 & 2.00 & 6.00 & 16.80 & PVC/SL/ .01 & 4.00 & to 18.50 & 6.80 & to 16.80 \\
\hline GW-494 & PVC40 & 2.37 & 2.00 & 6.00 & 14.20 & PVC/SL/ .01 & 3.00 & to 14.20 & 4.20 & to 14.20 \\
\hline GW-495 & PVC40 & 2.37 & 2.00 & 6.00 & 6.30 & PVC/SL/ .01 & 2.30 & to 6.30 & 3.30 & to 6.30 \\
\hline GW-496 & PVC40 & 2.37 & 2.00 & 6.00 & 6.00 & PVC/SL/ .01 & 2.00 & to 6.00 & 3.00 & to 6.00 \\
\hline GW-497 & PVC40 & 2.37 & 2.00 & 6.00 & 6.70 & PVC/SL/ .01 & 2.70 & to 6.70 & 3.70 & to 6.70 \\
\hline GW-498 & PVC40 & 2.37 & 2.00 & 6.00 & 4.60 & PVC/SL/ .01 & 1.10 & to 5.00 & 1.60 & to 4.60 \\
\hline GW-499 & PVC40 & 2.37 & 2.00 & 6.00 & 4.00 & PVC/SL/ .01 & 1.00 & to 5.00 & 1.00 & to 4.00 \\
\hline GW-499A & PVC & 2.37 & 2.00 & 6.00 & 5.60 & PVC/SL/ .01 & 2.00 & to 6.20 & 2.60 & to 5.60 \\
\hline GW-499AA & PVC40 & 1.00 & & & & PVC/UNKN/ & & to & & to \\
\hline GW-499AB & PVC40 & 2.00 & & & 8.80 & ?/UNKN/ & & to & & to \\
\hline GW-499AC & PVC40 & 2.00 & & & 4.40 & PVC/UNKN/ & & to & & to \\
\hline GW-499AD & PVC40 & 2.00 & & & 5.00 & PVC/UNKN/ & & to & & to \\
\hline GW-499AE & PVC40 & 2.00 & & & 3.60 & PVC/UNKN/ & & to & & to \\
\hline GW-499AF & PVC40 & 2.00 & & & 4.40 & PVC/UNKN/ & & to & & to \\
\hline GW-499B & & & & & 8.70 & PVC/UNKN/ & & to & & to \\
\hline GW-499C & & & & & 15.00 & PVC/UNKN/ & & to & & to \\
\hline
\end{tabular}




\begin{tabular}{|c|c|c|c|c|c|c|c|c|c|c|}
\hline $\begin{array}{c}\text { CURRENT } \\
\text { NAME }\end{array}$ & $\begin{array}{c}\text { CASING } \\
\text { TYPE }\end{array}$ & $\begin{array}{l}\text { CASING } \\
\text { O.D. }\end{array}$ & $\begin{array}{c}\text { CASING } \\
\text { I.D. }\end{array}$ & $\begin{array}{c}\text { HOLE } \\
\text { DIAMETER }\end{array}$ & $\begin{array}{c}\text { HOLE } \\
\text { DEPTH }\end{array}$ & SCREEN TYPE & $\begin{array}{r}\text { PAC } \\
\text { INTE } \\
\end{array}$ & $\begin{array}{l}\text { EKED } \\
\text { RVAL }\end{array}$ & $\begin{array}{l}\text { SCR } \\
\text { INTI }\end{array}$ & $\begin{array}{l}\text { ENED } \\
\text { RVAL }\end{array}$ \\
\hline GW-499D & & & & & 13.15 & PVC/UNKN/ & & to & & to \\
\hline GW-499E & & & & & 14.85 & PVC/UNKN/ & & to & & to \\
\hline GW-499F & & & & & 4.26 & PVC/UNKN/ & & to & & to \\
\hline GW-499G & PVC40 & 2.37 & 2.00 & 6.00 & 12.80 & PVC/SL/ .01 & 5.30 & to 14.00 & 7.80 & to 12.80 \\
\hline GW-499H & PVC40 & 2.37 & 2.00 & 6.00 & 9.70 & PVC/SL/ .01 & 2.70 & to 11.00 & 4.70 & to 9.70 \\
\hline GW-499I & PVC40 & 2.37 & 2.00 & 6.00 & 9.00 & PVC/SL/ .01 & 2.70 & to 9.00 & 4.00 & to 9.00 \\
\hline GW-499J & PVC40 & 2.37 & 2.00 & 6.00 & 8.00 & PVC/SL/ .01 & 3.00 & to 8.00 & 3.60 & to 8.60 \\
\hline GW-499K & PVC40 & 2.37 & 2.00 & 6.00 & 10.00 & PVC/SL/ .01 & 4.00 & to 10.00 & 5.00 & to 10.00 \\
\hline GW-499L & PVC40 & 2.37 & 2.00 & 6.00 & 12.00 & PVC/SL/ .01 & 3.80 & to 12.00 & 7.00 & to 12.00 \\
\hline GW-499M & PVC40 & 2.37 & 2.00 & 6.00 & 11.60 & PVC/SL/ .01 & 4.30 & to 11.60 & 6.60 & to 11.60 \\
\hline GW-499N & PVC40 & 2.37 & 2.00 & 6.00 & 12.00 & PVC/SL/ .01 & 4.00 & to 12.00 & 7.00 & to 12.00 \\
\hline GW-4990 & PVC40 & 2.37 & 2.00 & & 11.30 & PVC/SL/ .01 & 4.00 & to 11.30 & 6.30 & to 11.30 \\
\hline GW-499P & PVC40 & 2.37 & 2.00 & 6.00 & 13.20 & PVC/SL/ .01 & 4.00 & to 13.20 & 8.20 & to 13.20 \\
\hline GW-499R & PVC40 & 2.00 & & 4.00 & 17.50 & PVC/SL/ & 10.00 & to 17.00 & 12.00 & to 17.00 \\
\hline GW-499S & PVC40 & 2.00 & & 4.00 & 25.00 & PVC/SL/ & 13.00 & to 22.50 & 17.50 & to 22.50 \\
\hline GW-499T & PVC40 & 2.00 & & & 32.00 & PVC/SL/ & 21.00 & to 30.00 & 20.00 & to 30.00 \\
\hline GW-499U & PVC40 & 2.00 & & & 25.00 & PVC/SL/ & 16.00 & to 22.50 & 17.50 & to 22.50 \\
\hline GW-499V & PVC40 & 2.00 & & & 26.00 & PVC/SL/ & & to & 20.10 & to 25.10 \\
\hline GW-499W & PVC40 & & & & 31.00 & PVC/SL/ & & to & 4.50 & to 9.50 \\
\hline GW-499X & & 4.00 & & & & UNKN/UNKN/ & & to & & to \\
\hline GW-499Y & PVC40 & 1.00 & & & & UNKN/UNKN/ & & to & & to \\
\hline GW-499Z & PVC40 & 1.00 & & & & UNKN/UNKN/ & & to & & to \\
\hline GW-505 & SS304 & 2.37 & 2.00 & 7.00 & 13.30 & SLS/SW/ .01 & 1.50 & to 13.50 & 1.90 & to 12.30 \\
\hline GW-506 & SS304 & 2.37 & 2.00 & 7.00 & 8.40 & SLS/SW/ .01 & 1.50 & to 9.50 & 2.10 & to 7.30 \\
\hline GW-507 & SS304 & 2.37 & 2.00 & 7.00 & 8.40 & SLS/SW/ .01 & 1.50 & to 9.00 & 2.10 & to 7.30 \\
\hline GW-508 & SS304 & 2.37 & 2.00 & 7.00 & 13.70 & SLS/SW/ .01 & 1.30 & to 15.00 & 2.20 & to 12.50 \\
\hline GW-509 & SS304 & 2.37 & 2.00 & 7.00 & 8.40 & SLS/SW/ .01 & 1.50 & to 9.00 & 2.10 & to 7.30 \\
\hline
\end{tabular}




\begin{tabular}{|c|c|c|c|c|c|c|c|c|c|c|}
\hline $\begin{array}{c}\text { CURRENT } \\
\text { NAME }\end{array}$ & $\begin{array}{l}\text { CASING } \\
\text { TYPE }\end{array}$ & $\begin{array}{c}\text { CASING } \\
\text { O.D. }\end{array}$ & $\begin{array}{c}\text { CASING } \\
\text { I.D. }\end{array}$ & $\begin{array}{c}\text { HOLE } \\
\text { DIAMETER }\end{array}$ & $\begin{array}{c}\text { HOLE } \\
\text { DEPTH }\end{array}$ & SCREEN TYPE & $\begin{array}{r}\text { PA } \\
\text { INTI }\end{array}$ & $\begin{array}{l}\text { CKED } \\
\text { ERVAL }\end{array}$ & $\begin{array}{l}\text { SCF } \\
\text { INT }\end{array}$ & $\begin{array}{l}\text { EENED } \\
\text { ERVAL }\end{array}$ \\
\hline GW-511 & SS304 & 4.50 & 4.00 & 9.60 & 153.70 & SLS/SW/ .01 & 140.00 & to 153.70 & 143.30 & to 153.30 \\
\hline GW-512 & SS304 & 4.50 & 4.00 & 9.50 & 61.00 & SLS/SW/ .01 & 48.00 & to 61.00 & 50.50 & to 60.50 \\
\hline GW-513 & SS304 & 4.50 & 4.00 & 9.50 & 125.30 & SLS/SW/ .01 & 111.00 & to 125.30 & 114.80 & to 124.80 \\
\hline GW-520 & SS304 & 4.50 & 4.00 & 9.50 & 80.30 & SLS/SW/ .01 & 68.00 & to 80.30 & 69.80 & to 80.10 \\
\hline GW-521 & SS304 & 4.50 & 4.00 & 9.50 & 135.60 & SLS/SW/ .01 & 123.20 & to 136.00 & 124.90 & to 135.20 \\
\hline GW-522 & SS304 & 4.50 & 4.00 & 9.50 & 195.30 & SLS/SW/ .01 & 183.00 & to 195.30 & 184.60 & to 195.00 \\
\hline GW-527 & PVC40 & 2.37 & 2.00 & 3.00 & 5.60 & PVC/SL/ & 2.00 & to 5.60 & 2.60 & to 5.60 \\
\hline GW-528 & PVC40 & 2.37 & 2.00 & 3.00 & 6.00 & PVC/SL/ & 1.00 & to 6.00 & 2.00 & to 6.00 \\
\hline GW-531 & SS304 & 4.50 & 4.00 & 8.75 & 38.50 & SLS/SW/ .01 & 22.00 & to 38.50 & 22.50 & to 38.20 \\
\hline GW-532 & SS304 & 4.50 & 4.00 & 8.75 & 28.70 & SLS/SW/ .01 & 11.00 & to 28.70 & 12.70 & to 28.40 \\
\hline GW-533 & SS304 & 4.50 & 4.00 & 9.50 & 30.10 & SLS/SW/ .01 & 18.00 & to 30.10 & 19.70 & to 30.10 \\
\hline GW-534 & SS304 & 4.50 & 4.00 & 9.25 & 46.30 & SLS/SW/ .01 & 29.40 & to 46.30 & 31.10 & to 46.15 \\
\hline GW-535 & SS304 & 4.50 & 4.00 & 8.75 & 19.50 & SLS/SW/ .01 & 3.00 & to 19.50 & 3.50 & to 19.30 \\
\hline GW-536 & SS304 & 4.50 & 4.00 & 8.75 & 19.70 & SLS/SW/ .01 & 3.20 & to 19.70 & 3.70 & to 19.40 \\
\hline GW-537 & SS304 & 4.50 & 4.00 & 8.75 & 23.30 & SLS/SW/ .01 & 4.80 & to 23.30 & 8.00 & to 23.00 \\
\hline GW-538 & SS304 & 4.50 & 4.00 & 9.25 & 42.20 & SLS/SL/ .01 & 25.50 & to 42.20 & 26.90 & to 41.90 \\
\hline GW-539 & SS304 & 4.50 & 4.00 & 9.25 & 156.00 & SLS/SL/ .01 & 136.40 & to 156.00 & 139.80 & to 155.70 \\
\hline GW-540 & SS304 & 4.50 & 4.00 & 9.25 & 171.50 & SLS/SL/ .01 & 158.50 & to 171.50 & 161.20 & to 171.50 \\
\hline GW-541 & SS304 & 4.50 & 4.00 & 9.25 & 104.50 & SLS/SL/ .01 & 86.70 & to 104.50 & 88.80 & to 104.50 \\
\hline GW-542 & SS304 & 4.50 & 4.00 & 9.25 & 76.50 & SLS/SL/ .01 & 59.00 & to 76.50 & 60.80 & to 76.50 \\
\hline GW-543 & SS304 & 4.50 & 4.00 & 9.25 & 93.60 & SLS/SL/ .01 & 76.20 & to 93.60 & 77.95 & to 93.60 \\
\hline GW-544 & SS304 & 4.50 & 4.00 & 9.25 & 109.30 & SLS/SL/ .01 & 91.00 & to 109.30 & 93.40 & to 109.30 \\
\hline GW-545 & SS304 & 4.50 & 4.00 & 9.25 & 67.00 & SLS/SL/ .01 & 55.20 & to 67.00 & 56.70 & to 67.00 \\
\hline GW-546 & SS304 & 4.50 & 4.00 & 9.25 & 84.35 & SLS/SL/ .01 & 66.20 & to 84.40 & 68.65 & to 84.35 \\
\hline GW-551 & STL & 1.00 & & 3.70 & 145.00 & STL/PER/ & & to & 111.00 & to 145.00 \\
\hline GW-552 & STL & 1.00 & & 3.70 & 227.50 & STL/PER/ & & to & 201.00 & to 227.50 \\
\hline GW-553 & STL & 1.00 & & 3.70 & 361.00 & STL/PER/ & & to & 340.00 & to 361.00 \\
\hline
\end{tabular}




\begin{tabular}{|c|c|c|c|c|c|c|c|c|c|c|}
\hline $\begin{array}{c}\text { CURRENT } \\
\text { NAME }\end{array}$ & $\begin{array}{l}\text { CASING } \\
\text { TYPE }\end{array}$ & $\begin{array}{c}\text { CASING } \\
\text { O.D. }\end{array}$ & $\begin{array}{c}\text { CASING } \\
\text { I.D. }\end{array}$ & $\begin{array}{c}\text { HOLE } \\
\text { DIAMETER }\end{array}$ & $\begin{array}{c}\text { HOLE } \\
\text { DEPTH }\end{array}$ & SCREEN TYPE & $\begin{array}{r}\text { PA } \\
\text { INTI }\end{array}$ & $\begin{array}{l}\text { EKED } \\
\text { RVAL }\end{array}$ & $\begin{array}{l}\text { SCF } \\
\text { INT }\end{array}$ & $\begin{array}{l}\text { EENED } \\
\text { ERVAL }\end{array}$ \\
\hline GW-554 & STL & 1.00 & & 3.70 & 303.05 & STL/PER/ & & to & 270.00 & to 303.05 \\
\hline GW-555 & STL & 1.00 & & 3.70 & 314.35 & STL/PER/ & & to & 275.00 & to 314.35 \\
\hline GW-556 & STL & 1.00 & & 3.70 & 272.60 & STL/PER/ & & to & 251.80 & to 272.60 \\
\hline GW-557 & SS304 & 4.50 & 4.00 & 9.50 & 135.75 & SLS/SW/ .01 & 112.90 & to 138.00 & 115.75 & to 135.75 \\
\hline GW-558 & SS304 & 4.50 & 4.00 & 9.50 & 84.55 & SLS/SW/ .01 & 63.00 & to 84.55 & 69.55 & to 84.55 \\
\hline GW-559 & SS304 & 4.50 & 4.00 & 9.50 & 188.11 & SLS/PPCK/.01 & 145.00 & to 201.00 & 167.41 & to 188.11 \\
\hline GW-560 & SS304 & 4.50 & 4.00 & 9.50 & 69.00 & SLS/SW/ .01 & 45.20 & to 69.00 & 49.00 & to 69.00 \\
\hline GW-561 & SS304 & 4.50 & 4.00 & 9.50 & 89.90 & SLS/SW/ .01 & 67.10 & to 91.00 & 69.90 & to 89.90 \\
\hline GW-562 & SS304 & 4.50 & 4.00 & 9.50 & 58.00 & SLS/SW/ .01 & 36.00 & to 60.00 & 38.00 & to 58.00 \\
\hline GW-563 & SLS & 4.50 & 4.00 & 9.50 & 95.20 & SLS/SW/ .01 & 72.50 & to 96.80 & 65.20 & to 95.20 \\
\hline GW-564 & SS304 & 4.50 & 4.00 & 9.50 & 75.30 & SLS/SW/ .01 & 52.00 & to 81.00 & 55.30 & to 75.30 \\
\hline GW-565 & SS304 & 4.50 & 4.00 & 9.50 & 57.30 & SLS/SW/ .01 & 34.30 & to 61.00 & 37.30 & to 57.30 \\
\hline GW-566 & SS304 & 4.50 & 4.00 & 9.50 & 173.00 & SLS/SW/ .01 & 151.50 & to 174.00 & 153.00 & to 173.00 \\
\hline GW-567 & SS304 & 4.50 & 4.00 & 9.50 & 77.50 & SLS/SW/ .01 & 71.00 & to 80.00 & 57.50 & to 77.50 \\
\hline GW-569 & SS304 & 2.37 & 2.00 & 6.00 & 127.40 & SLS/SW/ .01 & & to & 90.00 & to 112.80 \\
\hline GW-570 & SS304 & 2.37 & 2.00 & 6.00 & 137.80 & SLS/SW/ .01 & 90.00 & to 112.80 & 92.80 & to 112.80 \\
\hline GW-571 & SS304 & 2.37 & 2.00 & 6.00 & 73.30 & SLS/SW/ .01 & 60.00 & to 73.30 & 63.30 & to 73.30 \\
\hline GW-572 & SS304 & 2.37 & 2.00 & 6.00 & 67.40 & SLS/SW/ .01 & 50.00 & to 67.40 & 52.40 & to 67.40 \\
\hline GW-573D & SS304 & 2.37 & 2.00 & 6.00 & 89.80 & SLS/SW/ .01 & 69.80 & to 89.80 & 74.80 & to 89.80 \\
\hline GW-573S & SS304 & 2.00 & & 6.00 & 15.00 & SLS/SW/ .01 & 5.00 & to 15.00 & 10.00 & to 15.00 \\
\hline GW-576 & SS304 & 2.37 & 2.00 & 6.00 & 58.23 & SLS/SW/ .01 & 45.00 & to 58.23 & 48.23 & to 58.23 \\
\hline GW-580 & SS304 & 2.37 & 2.00 & 6.00 & 76.70 & SLS/SW/ .01 & 60.00 & to 76.70 & 61.70 & to 76.70 \\
\hline GW-602 & SS304 & 2.37 & 2.00 & 6.25 & 211.50 & SLS/SW/ .01 & 197.10 & to 211.50 & 199.70 & to 211.50 \\
\hline GW-603 & SS304 & 4.50 & 4.00 & 9.50 & 75.20 & SLS/SW/ .01 & 63.40 & to 75.20 & 64.90 & to 75.20 \\
\hline GW-604 & SS304 & 4.50 & 4.25 & 9.50 & 112.40 & SLS/SW/ .01 & 100.20 & to 112.40 & 102.50 & to 112.40 \\
\hline GW-605 & SS304 & 4.25 & 4.00 & 9.50 & 39.90 & SLS/SW/ .01 & 28.20 & to 39.90 & 29.70 & to 39.70 \\
\hline GW-606 & SS304 & 4.25 & 4.00 & 6.75 & 171.70 & SLS/?/ .01 & 155.00 & to 171.00 & 161.00 & to 171.00 \\
\hline
\end{tabular}




\begin{tabular}{|c|c|c|c|c|c|c|c|c|c|c|}
\hline $\begin{array}{c}\text { CURRENT } \\
\text { NAME }\end{array}$ & $\begin{array}{c}\text { CASING } \\
\text { TYPE }\end{array}$ & $\begin{array}{c}\text { CASING } \\
\text { O.D. }\end{array}$ & $\begin{array}{c}\text { CASING } \\
\text { I.D. }\end{array}$ & $\begin{array}{c}\text { HOLE } \\
\text { DIAMETER }\end{array}$ & $\begin{array}{c}\text { HOLE } \\
\text { DEPTH }\end{array}$ & SCREEN TYPE & $\begin{array}{r}\text { PA } \\
\text { INTI } \\
\end{array}$ & $\begin{array}{l}\text { CKED } \\
\text { ERVAL }\end{array}$ & $\begin{array}{l}\text { SCF } \\
\text { INT }\end{array}$ & $\begin{array}{l}\text { REENED } \\
\text { ERVAL }\end{array}$ \\
\hline GW-607 & SS304 & 4.50 & 4.25 & 9.50 & 151.30 & SLS/SW/ .01 & 138.00 & to 151.30 & 141.00 & to 151.30 \\
\hline GW-609 & SS304 & 4.50 & 4.25 & 9.50 & 269.00 & SLS/SW/ .01 & 256.40 & to 269.00 & 258.70 & to 269.00 \\
\hline GW-610 & SS304 & 4.50 & 4.25 & 9.50 & 117.40 & SLS/SW/ .01 & 105.10 & to 117.40 & 107.10 & to 117.40 \\
\hline GW-611 & SS304 & 4.50 & 4.25 & 9.50 & 117.30 & SLS/SW/ .01 & 101.50 & to 121.60 & 107.00 & to 117.00 \\
\hline GW-613 & SS304 & 4.50 & 4.25 & 9.50 & 42.00 & SLS/SW/ .01 & 30.00 & to 42.00 & 31.66 & to 42.00 \\
\hline GW-614 & SS304 & 4.50 & 4.25 & 9.50 & 90.20 & SLS/SW/ .01 & 71.60 & to 90.20 & 75.20 & to 90.20 \\
\hline GW-617 & SS304 & 4.50 & 4.25 & 9.50 & 18.00 & SLS/SW/ .01 & 6.80 & to 18.00 & 7.30 & to 17.60 \\
\hline GW-618 & SS304 & 4.50 & 4.25 & 9.50 & 37.00 & SLS/SW/ .01 & 26.00 & to 37.00 & 26.70 & to 37.00 \\
\hline GW-619 & SS304 & 4.50 & 4.25 & 9.50 & 40.80 & SLS/SW/ .01 & 26.80 & to 40.80 & 30.00 & to 40.80 \\
\hline GW-620 & SS304 & 4.50 & 4.25 & 9.50 & 75.00 & SLS/SW/ .01 & 61.70 & to 75.00 & 64.20 & to 75.00 \\
\hline GW-621 & SS304 & 4.50 & 4.25 & 7.88 & 40.50 & SLS/SW/ .01 & 23.30 & to 40.50 & 24.80 & to 40.50 \\
\hline GW-622 & SS304 & 4.50 & 4.25 & 10.63 & 18.90 & SLS/SW/ .01 & 6.80 & to 18.90 & 8.60 & to 18.70 \\
\hline GW-623 & SS304 & 2.37 & 2.00 & 6.00 & 274.20 & SLS/SW/ .01 & 238.10 & to 274.20 & 244.00 & to 274.20 \\
\hline GW-624 & SS304 & 4.50 & 4.25 & 9.50 & 27.20 & SLS/SW/ .01 & 14.40 & to 27.20 & 16.90 & to 27.20 \\
\hline GW-626 & SS304 & 4.50 & 4.25 & 9.50 & 78.00 & SLS/SW/ .01 & 63.00 & to 78.00 & 67.70 & to 77.70 \\
\hline GW-630 & SS304 & 4.50 & 4.25 & 9.50 & 28.60 & SLS/SW/ .01 & 14.80 & to 28.60 & 17.60 & to 28.30 \\
\hline GW-631 & PVC & 4.50 & 4.00 & 10.50 & 15.50 & PVC/SL/ .01 & 4.00 & to 16.00 & 5.00 & to 15.00 \\
\hline GW-632 & PVC & 4.50 & 4.00 & 7.00 & 14.50 & PVC/SL/ .01 & 3.50 & to 15.00 & 4.50 & to 14.50 \\
\hline GW-633 & PVC & 4.50 & 4.00 & 10.50 & 15.00 & PVC/SL/ .01 & 3.50 & to 15.00 & 5.00 & to 15.00 \\
\hline GW-634 & PVC & 4.50 & 4.00 & 10.50 & 14.80 & PVC/SL/ .01 & 3.50 & to 15.00 & 4.80 & to 14.80 \\
\hline GW-635 & PVC & 4.50 & 4.00 & 10.50 & 15.00 & PVC/SL/ .01 & 3.50 & to 15.00 & 5.00 & to 15.00 \\
\hline GW-637 & SS304 & 4.50 & 4.25 & 10.00 & 27.50 & SLS/SW/ .01 & 14.50 & to 27.50 & 17.20 & to 27.20 \\
\hline GW-638 & SS304 & 4.50 & 4.25 & 10.00 & 11.50 & SLS/SW/ .01 & 2.50 & to 11.50 & 6.20 & to 11.20 \\
\hline GW-640 & SS304 & 4.50 & 4.25 & 9.50 & 47.40 & SLS/SW/ .01 & 35.00 & to 47.40 & 36.00 & to 47.10 \\
\hline GW-641 & SS304 & 4.50 & 4.25 & 9.50 & 24.30 & SLS/SW/ .01 & 11.00 & to 24.30 & 13.60 & to 24.00 \\
\hline GW-642 & SS304 & 4.50 & 4.25 & 9.50 & 36.90 & SLS/SW/ .01 & 18.50 & to 36.90 & 20.30 & to 36.50 \\
\hline GW-643 & SS304 & 4.50 & 4.25 & 14.00 & 28.50 & SLS/SW/ .01 & 16.50 & to 28.50 & 17.50 & to 28.10 \\
\hline
\end{tabular}




\begin{tabular}{|c|c|c|c|c|c|c|c|c|c|c|}
\hline $\begin{array}{c}\text { CURRENT } \\
\text { NAME }\end{array}$ & $\begin{array}{c}\text { CASING } \\
\text { TYPE }\end{array}$ & $\begin{array}{l}\text { CASING } \\
\text { O.D. }\end{array}$ & $\begin{array}{c}\text { CASING } \\
\text { I.D. }\end{array}$ & $\begin{array}{c}\text { HOLE } \\
\text { DIAMETER }\end{array}$ & $\begin{array}{c}\text { HOLE } \\
\text { DEPTH }\end{array}$ & SCREEN TYPE & $\begin{array}{r}\text { PAC } \\
\text { INTE } \\
\end{array}$ & $\begin{array}{l}\text { CKED } \\
\text { ERVAL } \\
\end{array}$ & $\begin{array}{l}\text { SCF } \\
\text { INT }\end{array}$ & $\begin{array}{l}\text { EENED } \\
\text { ERVAL }\end{array}$ \\
\hline GW-644 & SS304 & 4.50 & 4.25 & 9.50 & 23.40 & SLS/SW/ .01 & 10.30 & to 23.40 & 12.70 & to 23.00 \\
\hline GW-645 & SLS & 4.50 & 4.25 & 10.00 & 80.20 & SLS/SW/ .01 & 57.60 & to 85.80 & 58.50 & to 79.80 \\
\hline GW-646 & SLS & 4.50 & 4.25 & 9.50 & 75.90 & SLS/SW/ .01 & 63.30 & to 75.90 & 64.80 & to 75.90 \\
\hline GW-647 & SS304 & 4.50 & 4.25 & 9.50 & 91.00 & SLS/SW/ .01 & 78.00 & to 91.00 & 81.00 & to 91.00 \\
\hline GW-648 & SS304 & 4.50 & 4.25 & 9.50 & 80.10 & SLS/SW/ .01 & 65.00 & to 80.10 & 70.10 & to 80.10 \\
\hline GW-649 & SS304 & 4.50 & 4.25 & 9.50 & 20.70 & SLS/SW/ .01 & 8.40 & to 20.70 & 9.90 & to 20.30 \\
\hline GW-651 & SS304 & 4.50 & 4.25 & 9.50 & 52.00 & SLS/SW/ .01 & 39.80 & to 52.00 & 42.00 & to 52.00 \\
\hline GW-652 & SS304 & 4.50 & 4.25 & 9.50 & 31.20 & SLS/SW/ .01 & 13.40 & to 31.20 & 14.60 & to 30.80 \\
\hline GW-653 & SS304 & 4.50 & 4.25 & 9.50 & 39.00 & SLS/SW/ .01 & 26.30 & to 39.00 & 29.00 & to 39.00 \\
\hline GW-654 & SS304 & 4.50 & 4.25 & 9.50 & 15.30 & SLS/SW/ .01 & 4.20 & to 15.30 & 5.30 & to 15.30 \\
\hline GW-655 & SS304 & 4.50 & 4.25 & 9.50 & 65.00 & SLS/SW/ .01 & 47.70 & to 65.00 & 49.10 & to 65.00 \\
\hline GW-656 & SS304 & 4.50 & 4.25 & 9.50 & 20.90 & SLS/SW/ .01 & 8.30 & to 21.50 & 10.70 & to 20.70 \\
\hline GW-657 & SS304 & 4.50 & 4.25 & 9.50 & 15.60 & SLS/SW/ .01 & 4.00 & to 16.00 & 5.30 & to 15.30 \\
\hline GW-658 & SS304 & 4.50 & 4.25 & 9.50 & 19.10 & SLS/SW/ .01 & 6.90 & to 19.10 & 8.80 & to 18.80 \\
\hline GW-659 & SS304 & 4.50 & 4.25 & 9.50 & 16.70 & SLS/SW/ .01 & 4.60 & to 16.70 & 5.70 & to 16.70 \\
\hline GW-660 & SS304 & 2.37 & 2.00 & 8.75 & 10.40 & SLS/SW/ .01 & 7.30 & to 10.40 & 8.40 & to 10.40 \\
\hline GW-661 & SS304 & 2.37 & 2.00 & 8.75 & 7.30 & SLS/SW/ .01 & 3.80 & to 7.30 & 4.80 & to 7.30 \\
\hline GW-662 & SS304 & 2.37 & 2.00 & 6.25 & 15.00 & SLS/SW/ .01 & 8.00 & to 15.00 & 10.00 & to 15.00 \\
\hline GW-663 & SS304 & 2.37 & 2.00 & 8.75 & 4.00 & SLS/SW/ .01 & 2.00 & to 4.00 & 3.00 & to 4.00 \\
\hline GW-664 & SS304 & 2.37 & 2.00 & 8.75 & 5.90 & SLS/SW/ .01 & 3.40 & to 5.90 & 4.90 & to 5.90 \\
\hline GW-665 & SS304 & 2.37 & 2.00 & 6.25 & 29.70 & SLS/SW/ .01 & 17.70 & to 29.70 & 19.70 & to 29.70 \\
\hline GW-666 & SS304 & 2.37 & 2.00 & 8.75 & 7.40 & SLS/SW/ .01 & 3.90 & to 7.40 & 4.90 & to 7.40 \\
\hline GW-667 & SS304 & 2.37 & 2.00 & 8.75 & 7.80 & SLS/SW/ .01 & 4.80 & to 7.80 & 5.80 & to 7.80 \\
\hline GW-668 & SS304 & 4.50 & 4.25 & 10.63 & 23.10 & SLS/SW/ .01 & 11.10 & to 23.10 & 13.10 & to 23.10 \\
\hline GW-669 & SS304 & 2.37 & 2.00 & 8.75 & 6.70 & SLS/SW/ .01 & 4.80 & to 6.80 & 5.70 & to 6.70 \\
\hline GW-670 & SS304 & 4.50 & 4.25 & 8.75 & 21.80 & SLS/SW/ .01 & 9.50 & to 20.00 & 10.00 & to 20.00 \\
\hline GW-671 & SS304 & 2.37 & 2.00 & 8.75 & 7.60 & SLS/SW/ .01 & 3.50 & to 7.60 & 5.60 & to 7.60 \\
\hline
\end{tabular}




\begin{tabular}{|c|c|c|c|c|c|c|c|c|c|c|c|}
\hline $\begin{array}{c}\text { CURRENT } \\
\text { NAME }\end{array}$ & $\begin{array}{c}\text { CASING } \\
\text { TYPE }\end{array}$ & $\begin{array}{c}\text { CASING } \\
\text { O.D. }\end{array}$ & $\begin{array}{c}\text { CASING } \\
\text { I.D. }\end{array}$ & $\begin{array}{c}\text { HOLE } \\
\text { DIAMETER }\end{array}$ & $\begin{array}{c}\text { HOLE } \\
\text { DEPTH }\end{array}$ & SCREEN TYPE & $\begin{array}{l}\text { PAC } \\
\text { INTE } \\
\end{array}$ & CKI & & & $\begin{array}{l}\text { EENED } \\
\text { ERVAL }\end{array}$ \\
\hline GW-672 & SS304 & 4.50 & 4.25 & 8.00 & 28.00 & SLS/SW/ .01 & 16.00 & to & 28.00 & 18.00 & to 28.00 \\
\hline GW-674 & SS304 & 4.50 & 4.25 & 8.00 & 16.50 & SLS/SW/ .01 & 7.00 & to & 16.50 & 9.00 & to 14.00 \\
\hline GW-676 & SS304 & 4.50 & 4.25 & 6.25 & 17.00 & SLS/SW/ .01 & 5.00 & to & 17.00 & 7.00 & to 17.00 \\
\hline GW-677 & PVC40 & 2.37 & 2.00 & 7.88 & 157.00 & PVC/SL/ .01 & 144.00 & to & 165.00 & 147.00 & to 157.00 \\
\hline GW-678 & PVC40 & 2.37 & 2.00 & 7.88 & 131.00 & $\mathrm{PVC} / \mathrm{SL} / .01$ & 117.00 & to & 132.00 & 121.00 & to 131.00 \\
\hline GW-679 & PVC40 & 2.37 & 2.00 & 7.88 & 132.00 & PVC/SL/ .01 & 117.00 & to & 132.00 & 122.00 & to 132.00 \\
\hline GW-680 & PVC40 & 2.37 & 2.00 & 7.88 & 120.00 & PVC/SL/ .01 & 107.00 & to & 120.00 & 110.00 & to 120.00 \\
\hline GW-681 & PVC40 & 2.37 & 2.00 & 7.88 & 180.00 & PVC/SL/ .01 & 150.00 & to & 178.00 & 170.00 & to 180.00 \\
\hline GW-682 & PVC40 & 2.37 & & 7.88 & 160.00 & PVC/?/ .01 & 148.00 & to & 166.00 & 150.00 & to 160.00 \\
\hline GW-683 & SS304 & 4.50 & 4.25 & 10.63 & 197.10 & SLS/PPCK/ .01 & 133.90 & to & 196.80 & 146.00 & to 196.80 \\
\hline GW-684 & SS304 & 4.50 & 4.25 & 10.50 & 128.80 & SLS/PPCK/ .01 & 106.40 & to & 129.60 & 113.80 & to 128.40 \\
\hline GW-686 & SS304 & 4.50 & 4.25 & 12.00 & 16.00 & SLS/SW/ .01 & 4.00 & to & 16.00 & 6.00 & to 16.00 \\
\hline GW-687 & SS304 & 4.50 & 4.25 & 12.00 & 32.00 & SLS/SW/ .01 & 20.00 & to & 32.00 & 22.00 & to 32.00 \\
\hline GW-688 & SS304 & 4.50 & 4.25 & 8.50 & 55.00 & SLS/SW/ .01 & 43.00 & to & 55.00 & 45.00 & to 55.00 \\
\hline GW-689 & SS304 & 4.50 & 4.25 & 12.00 & 20.00 & SLS/SW/ .01 & 8.00 & to & 20.00 & 10.00 & to 20.00 \\
\hline GW-690 & SS304 & 4.50 & 4.25 & 8.50 & 52.80 & SLS/SW/ .01 & 40.80 & to & 52.80 & 42.80 & to 52.80 \\
\hline GW-691 & SS304 & 4.50 & 4.25 & 12.00 & 20.00 & SLS/SW/ .01 & 8.00 & to & 20.00 & 10.00 & to 20.00 \\
\hline GW-692 & SS304 & 4.50 & 4.25 & 8.50 & 53.00 & SLS/SW/ .01 & 41.00 & to & 53.00 & 43.00 & to 53.00 \\
\hline GW-693 & SS304 & 4.50 & 4.25 & 12.00 & 24.00 & SLS/SW/ .01 & 12.00 & to & 24.00 & 14.00 & to 24.00 \\
\hline GW-695 & SS304 & 4.50 & 4.25 & 9.88 & 62.60 & SLS/SW/ .01 & 50.60 & to & 62.60 & 52.40 & to 62.40 \\
\hline GW-696 & SS304 & 4.50 & 4.25 & 7.50 & 32.50 & SLS/SW/ .01 & 18.50 & to & 32.50 & 22.50 & to 32.50 \\
\hline GW-697 & SS304 & 4.50 & 4.25 & 12.00 & 21.00 & SLS/SW/ .01 & 9.00 & to & 21.00 & 11.00 & to 21.00 \\
\hline GW-698 & SS304 & 4.50 & 4.25 & 8.50 & 75.00 & SLS/SW/ .01 & 63.00 & to & 75.00 & 65.00 & to 75.00 \\
\hline GW-699 & SS304 & 4.50 & 4.25 & 12.00 & 13.90 & SLS/SW/ .01 & 2.90 & to & 13.90 & 3.90 & to 13.90 \\
\hline GW-700 & SS304 & 4.50 & 4.25 & 11.00 & 31.00 & SLS/SW/ .01 & 19.00 & to & 31.00 & 21.00 & to 31.00 \\
\hline GW-701 & SS304 & 4.50 & 4.25 & 11.00 & 25.00 & SLS/SW/ .01 & 13.00 & to & 25.00 & 15.00 & to 25.00 \\
\hline GW-702 & SS304 & 4.50 & 4.25 & 11.00 & 21.00 & SLS/SW/ .01 & 9.00 & to & 21.00 & 11.00 & to 21.00 \\
\hline
\end{tabular}




\begin{tabular}{|c|c|c|c|c|c|c|c|c|c|c|}
\hline $\begin{array}{c}\text { CURRENT } \\
\text { NAME }\end{array}$ & $\begin{array}{l}\text { CASING } \\
\text { TYPE }\end{array}$ & $\begin{array}{c}\text { CASING } \\
\text { O.D. }\end{array}$ & $\begin{array}{c}\text { CASING } \\
\text { I.D. }\end{array}$ & $\begin{array}{c}\text { HOLE } \\
\text { DIAMETER }\end{array}$ & $\begin{array}{c}\text { HOLE } \\
\text { DEPTH }\end{array}$ & SCREEN TYPE & $\begin{array}{r}\text { PA } \\
\text { INTI }\end{array}$ & $\begin{array}{l}\text { CKED } \\
\text { ERVAL }\end{array}$ & $\begin{array}{l}\text { SCF } \\
\text { INT }\end{array}$ & $\begin{array}{l}\text { EENED } \\
\text { ERVAL }\end{array}$ \\
\hline GW-707 & SS304 & 4.50 & 4.25 & 9.50 & 17.20 & SLS/SW/ .01 & 5.50 & to 26.00 & 6.90 & to 16.90 \\
\hline GW-708 & SS304 & 4.50 & 4.25 & 9.50 & 13.70 & SLS/SW/ .01 & 2.70 & to 13.70 & 3.40 & to 13.40 \\
\hline GW-709 & SS304 & 4.25 & 4.00 & 10.60 & 80.60 & SLS/SW/ .01 & 68.70 & to 80.60 & 70.40 & to 80.40 \\
\hline GW-715 & SS304 & 4.25 & 4.00 & 10.60 & 43.10 & SLS/SW/ .01 & 32.00 & to 44.00 & 33.10 & to 43.10 \\
\hline GW-721 & PVC40 & 12.00 & & 22.00 & 6.00 & PVC/SL/ .02 & 3.00 & to 6.00 & 4.00 & to 6.00 \\
\hline GW-731 & SS304 & 4.50 & 4.25 & 10.60 & 175.40 & SLS/SW/ .01 & 164.00 & to 178.70 & 165.20 & to 175.20 \\
\hline GW-732 & SS304 & 4.50 & 4.25 & 10.60 & 189.50 & SLS/SW/ .01 & 178.30 & to 190.00 & 179.30 & to 189.30 \\
\hline GW-735 & SS304 & 4.50 & 4.25 & 10.60 & 78.10 & SLS/SW/ .01 & 67.50 & to 79.20 & 67.90 & to 77.90 \\
\hline GW-736 & SS304 & 4.50 & 4.25 & 10.60 & 102.50 & SLS/SW/ .01 & 92.00 & to 105.00 & 92.40 & to 102.40 \\
\hline GW-737 & SS304 & 4.50 & 4.25 & 10.60 & 89.50 & SLS/SW/ .01 & 79.00 & to 89.50 & 79.40 & to 89.40 \\
\hline GW-738 & SS304 & 4.50 & 4.25 & 10.60 & 87.50 & SLS/SW/ .01 & 63.50 & to 88.00 & 67.30 & to 87.30 \\
\hline GW-743 & SS304 & 4.50 & 4.25 & 10.60 & 160.30 & SLS/SW/ .01 & 150.10 & to 161.10 & 150.20 & to 160.20 \\
\hline GW-744 & SS304 & 4.50 & 4.25 & 10.60 & 67.00 & SLS/SW/ .01 & 55.00 & to 69.50 & 57.00 & to 66.90 \\
\hline GW-745 & SS304 & 4.50 & 4.25 & 9.88 & 32.50 & SLS/SW/ .01 & 21.20 & to 32.80 & 22.50 & to 32.40 \\
\hline GW-746 & SS304 & 4.50 & 4.25 & 9.88 & 14.75 & SLS/SW/ .01 & 3.20 & to 15.20 & 4.50 & to 14.50 \\
\hline GW-747 & SS304 & 4.50 & 4.25 & 9.88 & 72.55 & SLS/SW/ .01 & 60.75 & to 72.05 & 62.55 & to 72.45 \\
\hline GW-748 & SS304 & 4.50 & 4.25 & 9.88 & 19.82 & SLS/SW/ .01 & 7.62 & to 20.02 & 9.82 & to 19.72 \\
\hline GW-749 & SS304 & 4.50 & 4.25 & 9.88 & 16.00 & SLS/SW/ .01 & 4.80 & to 16.40 & 6.00 & to 15.90 \\
\hline GW-750 & SS304 & 4.50 & 4.25 & 10.60 & 72.40 & SLS/SW/ .01 & 61.20 & to 72.70 & 62.40 & to 72.30 \\
\hline GW-751 & SS304 & 4.50 & 4.25 & 9.88 & 60.10 & SLS/SW/ .01 & 49.00 & to 60.60 & 50.10 & to 60.00 \\
\hline GW-752 & SS304 & 4.50 & 4.25 & 9.88 & 15.50 & SLS/SW/ .01 & 4.80 & to 15.90 & 5.50 & to 15.40 \\
\hline GW-753 & SS304 & 4.50 & 4.25 & 9.88 & 70.40 & SLS/SW/ .01 & 59.60 & to 70.70 & 60.40 & to 70.30 \\
\hline GW-754 & SS304 & 4.50 & 4.25 & 9.88 & 24.00 & SLS/SW/ .01 & 13.00 & to 24.10 & 14.00 & to 23.90 \\
\hline GW-755 & SS304 & 4.50 & 4.25 & 9.88 & 60.00 & SLS/SW/ .01 & 49.20 & to 60.50 & 50.00 & to 59.90 \\
\hline GW-756 & SS304 & 4.50 & 4.25 & 9.88 & 16.00 & SLS/SW/ .01 & 5.30 & to 16.60 & 6.00 & to 15.90 \\
\hline GW-757 & SS304 & 4.50 & 4.25 & 9.63 & 165.70 & SLS/SW/ .01 & 134.00 & to 166.50 & 135.50 & to 165.50 \\
\hline GW-758 & SS304 & 4.50 & 4.25 & 9.88 & 50.20 & SLS/SW/ .01 & 38.40 & to 50.20 & 40.20 & to 50.10 \\
\hline
\end{tabular}




\begin{tabular}{|c|c|c|c|c|c|c|c|c|c|c|}
\hline $\begin{array}{c}\text { CURRENT } \\
\text { NAME }\end{array}$ & $\begin{array}{c}\text { CASING } \\
\text { TYPE }\end{array}$ & $\begin{array}{l}\text { CASING } \\
\text { O.D. }\end{array}$ & $\begin{array}{c}\text { CASING } \\
\text { I.D. }\end{array}$ & $\begin{array}{c}\text { HOLE } \\
\text { DIAMETER }\end{array}$ & $\begin{array}{c}\text { HOLE } \\
\text { DEPTH }\end{array}$ & SCREEN TYPE & $\begin{array}{r}\text { PAC } \\
\text { INTE } \\
\end{array}$ & $\begin{array}{l}\text { CKED } \\
\text { ERVAL }\end{array}$ & $\begin{array}{l}\text { SCF } \\
\text { INT }\end{array}$ & $\begin{array}{l}\text { EENED } \\
\text { ERVAL }\end{array}$ \\
\hline GW-759 & SS304 & 4.50 & 4.25 & 9.88 & 29.60 & SLS/SW/ .01 & 18.00 & to 30.20 & 18.90 & to 29.00 \\
\hline GW-760 & SS304 & 4.50 & 4.25 & 9.88 & 59.70 & SLS/SW/ .01 & 48.30 & to 60.10 & 49.70 & to 59.60 \\
\hline GW-761 & SS304 & 4.50 & 4.25 & 9.88 & 15.00 & SLS/SW/ .01 & 3.60 & to 15.30 & 5.30 & to 14.90 \\
\hline GW-762 & SS304 & 4.50 & 4.25 & 9.88 & 58.20 & SLS/SW/ .01 & 46.40 & to 58.70 & 48.20 & to 58.10 \\
\hline GW-763 & SS304 & 4.50 & 4.25 & 8.00 & 15.40 & SLS/SW/ .01 & 4.00 & to 16.00 & 5.15 & to 15.15 \\
\hline GW-764 & SS304 & 4.50 & 4.25 & 9.88 & 64.90 & SLS/SW/ .01 & 53.90 & to 65.00 & 54.90 & to 64.80 \\
\hline GW-765 & SS304 & 4.50 & 4.25 & 9.88 & 32.20 & SLS/SW/ .01 & 19.90 & to 32.40 & 21.20 & to 31.50 \\
\hline GW-766 & SS304 & 4.50 & 4.25 & 9.88 & 44.90 & SLS/SW/ .01 & 34.00 & to 47.60 & 34.90 & to 44.80 \\
\hline GW-767 & SS304 & 4.50 & 4.25 & 9.88 & 17.80 & SLS/SW/ .01 & 6.70 & to 18.00 & 7.80 & to 17.70 \\
\hline GW-768 & SS304 & 4.50 & 4.25 & 9.88 & 64.60 & SLS/SW/ .01 & 53.30 & to 65.00 & 54.60 & to 64.50 \\
\hline GW-769 & SS304 & 4.50 & 4.25 & 10.63 & 59.40 & SLS/SW/ .01 & 48.20 & to 60.30 & 49.40 & to 59.30 \\
\hline GW-770 & SS304 & 4.50 & 4.25 & 10.63 & 18.50 & SLS/SW/ .01 & 7.50 & to 19.00 & 8.50 & to 18.40 \\
\hline GW-771 & SS304 & 4.50 & 4.25 & 9.88 & 53.80 & SLS/SW/ .01 & 42.20 & to 54.40 & 44.00 & to 53.70 \\
\hline GW-772 & SS304 & 4.50 & 4.25 & 8.00 & 15.20 & SLS/SW/ .01 & 3.80 & to 17.00 & 5.10 & to 15.00 \\
\hline GW-773 & SS304 & 4.50 & 4.25 & 9.88 & 58.70 & SLS/SW/ .01 & 46.10 & to 59.60 & 48.60 & to 58.60 \\
\hline GW-774 & SS304 & 4.50 & 4.25 & 9.88 & 25.80 & SLS/SW/ .01 & 12.50 & to 26.60 & 16.00 & to 25.70 \\
\hline GW-775 & SS304 & 4.50 & 4.25 & 10.63 & 56.30 & SLS/SW/ .01 & 45.00 & to 56.40 & 46.30 & to 56.20 \\
\hline GW-776 & SS304 & 4.50 & 4.25 & 9.88 & 22.30 & SLS/SW/ .01 & 10.60 & to 23.00 & 12.30 & to 22.20 \\
\hline GW-777 & SS304 & 4.50 & 4.25 & 9.88 & 58.10 & SLS/SW/ .01 & 46.10 & to 59.00 & 48.10 & to 58.00 \\
\hline GW-778 & SS304 & 4.50 & 4.25 & 9.88 & 21.20 & SLS/SW/ .01 & 7.80 & to 21.90 & 10.80 & to 21.00 \\
\hline GW-779 & SS304 & 4.50 & 4.25 & 9.88 & 62.10 & SLS/SW/ .01 & 50.70 & to 62.90 & 52.10 & to 62.00 \\
\hline GW-780 & SS304 & 4.50 & 4.25 & 8.00 & 17.00 & SLS/SW/ .01 & 4.60 & to 18.60 & 7.00 & to 16.90 \\
\hline GW-781 & SS304 & 4.50 & 4.25 & 9.88 & 68.10 & SLS/SW/ .01 & 56.00 & to 69.30 & 57.80 & to 68.00 \\
\hline GW-782 & SS304 & 4.50 & 4.25 & 9.88 & 35.00 & SLS/SW/ .01 & 23.80 & to 35.90 & 25.00 & to 34.90 \\
\hline GW-783 & SS304 & 4.50 & 4.25 & 9.88 & 14.00 & SLS/SW/ .01 & 3.60 & to 16.30 & 4.20 & to 13.90 \\
\hline GW-784 & SS304 & 4.50 & 4.25 & 9.88 & 63.20 & SLS/SW/ .01 & 52.00 & to 64.30 & 53.20 & to 63.10 \\
\hline GW-785 & SS304 & 4.50 & 4.25 & 8.00 & 23.30 & SLS/SW/ .01 & 11.50 & to 25.40 & 13.30 & to 23.20 \\
\hline
\end{tabular}




\begin{tabular}{|c|c|c|c|c|c|c|c|c|c|c|}
\hline $\begin{array}{c}\text { CURRENT } \\
\text { NAME }\end{array}$ & $\begin{array}{c}\text { CASING } \\
\text { TYPE }\end{array}$ & $\begin{array}{l}\text { CASING } \\
\text { O.D. }\end{array}$ & $\begin{array}{c}\text { CASING } \\
\text { I.D. }\end{array}$ & $\begin{array}{c}\text { HOLE } \\
\text { DIAMETER }\end{array}$ & $\begin{array}{c}\text { HOLE } \\
\text { DEPTH }\end{array}$ & SCREEN TYPE & $\begin{array}{r}\text { PA } \\
\text { INTI }\end{array}$ & $\begin{array}{l}\text { CKED } \\
\text { ERVAL }\end{array}$ & $\begin{array}{l}\text { SCF } \\
\text { INT }\end{array}$ & $\begin{array}{l}\text { EENED } \\
\text { ERVAL }\end{array}$ \\
\hline GW-786 & SS304 & 4.50 & 4.25 & 9.88 & 64.20 & SLS/SW/ .01 & 52.90 & to 64.90 & 54.20 & to 64.10 \\
\hline GW-787 & SS304 & 4.50 & 4.25 & 9.88 & 16.90 & SLS/SW/ .01 & 5.30 & to 18.10 & 6.90 & to 16.60 \\
\hline GW-788 & SS304 & 4.50 & 4.25 & 9.88 & 66.80 & SLS/SW/ .01 & 55.90 & to 67.80 & 57.10 & to 66.70 \\
\hline GW-789 & SS304 & 4.50 & 4.25 & 9.88 & 22.70 & SLS/SW/ .01 & 10.70 & to 23.80 & 12.20 & to 22.20 \\
\hline GW-791 & SS304 & 4.50 & 4.25 & 9.88 & 69.00 & SLS/SW/ .01 & 57.50 & to 70.60 & 59.00 & to 68.90 \\
\hline GW-792 & SS304 & 4.50 & 4.25 & 9.88 & 28.30 & SLS/SW/ .01 & 17.00 & to 29.00 & 18.50 & to 28.20 \\
\hline GW-793 & SS304 & 4.50 & 4.25 & 9.88 & 28.40 & SLS/SW/ .01 & 16.90 & to 29.00 & 18.40 & to 28.30 \\
\hline GW-794 & SS304 & 4.50 & 4.25 & 9.88 & 38.80 & SLS/SW/ .01 & 25.80 & to 39.30 & 28.70 & to 38.70 \\
\hline GW-795 & SS304 & 4.50 & 4.25 & 9.88 & 19.80 & SLS/SW/ .01 & 7.50 & to 20.10 & 9.80 & to 19.70 \\
\hline GW-796 & SS304 & 4.50 & 4.25 & 9.50 & 136.50 & SLS/SW/ .01 & 122.90 & to 136.50 & 126.50 & to 136.40 \\
\hline GW-797 & SS304 & 4.50 & 4.25 & 9.50 & 133.50 & SLS/SW/ .01 & 118.00 & to 134.10 & 123.50 & to 133.40 \\
\hline GW-798 & SS304 & 4.50 & 4.25 & 9.50 & 150.32 & SLS/SW/ .01 & 137.82 & to 151.22 & 140.32 & to 150.22 \\
\hline GW-799 & SS304 & 4.50 & 4.25 & 9.50 & 91.00 & SLS/SW/ .01 & 78.70 & to 92.00 & 81.00 & to 90.90 \\
\hline GW-800 & SS304 & 4.50 & 4.25 & 10.63 & 29.90 & SLS/SW/ .01 & 19.30 & to 31.50 & 19.90 & to 29.40 \\
\hline GW-801 & SS304 & 4.50 & 4.25 & 9.88 & 188.10 & SLS/SW/ .01 & 175.80 & to 188.90 & 178.10 & to 188.00 \\
\hline GW-802 & PVC40 & 4.50 & 4.00 & 10.63 & 26.00 & PVC/SL/ .01 & 13.30 & to 26.50 & 15.50 & to 25.50 \\
\hline GW-803 & PVC40 & 4.50 & 4.00 & 10.63 & 23.70 & PVC/SL/ .01 & 11.30 & to 24.70 & 13.20 & to 23.20 \\
\hline GW-804 & PVC40 & 4.50 & 4.00 & 10.63 & 24.00 & PVC/SL/ .01 & 11.40 & to 25.00 & 13.50 & to 23.50 \\
\hline GW-805 & PVC40 & 4.50 & 4.00 & 10.63 & 12.70 & PVC/SL/ .01 & 5.20 & to 13.40 & 7.20 & to 12.20 \\
\hline GW-806 & PVC40 & 4.50 & 4.00 & 10.63 & 11.70 & PVC/SL/ .01 & 4.20 & to 12.70 & 6.20 & to 11.20 \\
\hline GW-807 & PVC40 & 4.50 & 4.00 & 10.63 & 10.00 & $\mathrm{PVC/SL/} \mathrm{.01}$ & 4.00 & to 10.50 & 4.50 & to 9.60 \\
\hline GW-808 & PVC40 & 4.50 & 4.00 & 10.63 & 36.90 & PVC/SL/ .01 & 24.00 & to 40.40 & 26.40 & to 36.40 \\
\hline GW-809 & PVC40 & 4.50 & 4.00 & 10.63 & 36.50 & PVC/SL/ .01 & 23.90 & to 37.00 & 26.00 & to 36.00 \\
\hline GW-810 & PVC40 & 4.50 & 4.00 & 10.63 & 26.30 & PVC/SL/ .01 & 13.90 & to 26.80 & 15.80 & to 25.80 \\
\hline GW-811 & SS304 & 4.50 & 4.00 & 11.00 & 64.00 & SLS/SW/ .01 & 52.00 & to 64.50 & 53.67 & to 63.70 \\
\hline GW-812 & SS304 & 4.50 & 4.00 & 11.00 & 44.25 & SLS/SW/ .01 & 31.50 & to 44.70 & 34.00 & to 43.90 \\
\hline GW-813 & SS304 & 4.50 & 4.00 & 10.25 & 24.50 & SLS/SW/ .01 & 11.40 & to 25.50 & 12.50 & to 22.50 \\
\hline
\end{tabular}




\begin{tabular}{|c|c|c|c|c|c|c|c|c|c|c|}
\hline $\begin{array}{c}\text { CURRENT } \\
\text { NAME }\end{array}$ & $\begin{array}{c}\text { CASING } \\
\text { TYPE }\end{array}$ & $\begin{array}{c}\text { CASING } \\
\text { O.D. }\end{array}$ & $\begin{array}{c}\text { CASING } \\
\text { I.D. }\end{array}$ & $\begin{array}{c}\text { HOLE } \\
\text { DIAMETER }\end{array}$ & $\begin{array}{c}\text { HOLE } \\
\text { DEPTH }\end{array}$ & SCREEN TYPE & $\begin{array}{r}\text { PAC } \\
\text { INTE } \\
\end{array}$ & $\begin{array}{l}\text { CKED } \\
\text { ERVAL }\end{array}$ & $\begin{array}{l}\text { SCF } \\
\text { INT }\end{array}$ & $\begin{array}{l}\text { EENED } \\
\text { ERVAL }\end{array}$ \\
\hline GW-814 & SS304 & 4.50 & 4.00 & 10.25 & 23.30 & SLS/SW/ .01 & 8.90 & to 24.30 & 11.20 & to 21.30 \\
\hline GW-815 & SS304 & 4.50 & 4.00 & 10.25 & 20.60 & SLS/SW/ .01 & 6.40 & to 21.10 & 8.50 & to 18.50 \\
\hline GW-816 & SS304 & 4.50 & 4.25 & 10.00 & 14.20 & SLS/SW/ .01 & 2.90 & to 15.80 & 4.20 & to 13.60 \\
\hline GW-817 & SS304 & 4.50 & 4.25 & 10.75 & 22.30 & SLS/SW/ .01 & 0.60 & to 23.00 & 1.00 & to 21.60 \\
\hline GW-818 & PVC & 4.50 & 4.20 & & 20.40 & & & to & & to \\
\hline GW-821 & & & & & 47.50 & & & to & & to \\
\hline GW-822 & & & & & 67.50 & & & to & & to \\
\hline GW-823 & & & & & 72.00 & & & to & & to \\
\hline GW-825 & PVC40 & 2.37 & 2.00 & 6.25 & 34.90 & PVC/SL/ .01 & 2.00 & to 35.40 & 4.60 & to 34.60 \\
\hline GW-826 & PVC40 & 2.37 & 2.00 & 6.25 & 35.50 & $\mathrm{PVC/SL/} \mathrm{.01}$ & 2.00 & to 35.90 & 5.20 & to 35.20 \\
\hline GW-827 & SS304 & 4.50 & 4.25 & 9.88 & 134.20 & SLS/SW/ .01 & 122.10 & to 134.80 & 124.10 & to 134.10 \\
\hline GW-828 & SS304 & 4.50 & 4.25 & & 165.90 & SLS/SW/ .01 & 153.00 & to 168.00 & 155.50 & to 165.40 \\
\hline GW-829 & SS304 & 4.50 & 4.25 & 9.88 & 114.60 & SLS/SW/ .01 & 102.90 & to 114.60 & 104.70 & to 114.50 \\
\hline GW-831 & SLS & 4.50 & 4.25 & 9.88 & 194.20 & SLS/SW/ .01 & 182.00 & to 199.60 & 183.20 & to 193.60 \\
\hline GW-832 & PVC & 6.63 & 6.00 & 12.00 & 11.10 & $\mathrm{PVC/SL/} \mathrm{.02}$ & 4.00 & to 11.80 & 5.90 & to 10.90 \\
\hline GW-834 & PVC & 4.50 & 4.00 & 6.25 & 16.80 & PVC/SL/ & 13.80 & to 16.80 & 14.80 & to 16.80 \\
\hline GW-835 & PVC & 4.50 & 4.00 & 6.25 & 19.20 & PVC/SL/ & 16.20 & to 19.20 & 17.20 & to 19.20 \\
\hline GW-836 & PVC & 4.50 & 4.00 & 6.25 & 24.40 & PVC/SL/ & 21.40 & to 24.40 & 22.40 & to 24.40 \\
\hline GW-837 & PVC & 4.50 & 4.00 & 6.25 & 27.90 & PVC/SL/ & 24.90 & to 27.90 & 25.90 & to 27.90 \\
\hline GW-838 & PVC & 2.37 & 2.00 & & 29.50 & PVC/SL/ .01 & 17.50 & to 31.50 & 19.50 & to 29.50 \\
\hline GW-839 & PVC & 2.37 & 2.00 & & 62.00 & PVC/SL/ .01 & 17.50 & to 62.00 & 22.00 & to 62.00 \\
\hline GW-840 & PVC & 2.37 & 2.00 & & 31.50 & PVC/SL/ .01 & 11.50 & to 32.50 & 11.50 & to 31.50 \\
\hline GW-841 & & & & & & & & to & & to \\
\hline GW-842 & SLS & 2.37 & 2.00 & 8.00 & 25.00 & SLS/SW/ .01 & 12.00 & to 25.00 & 13.00 & to 23.00 \\
\hline GW-843 & SLS & 2.37 & 2.00 & 8.00 & 67.00 & SLS/SW/ & 51.00 & to 67.00 & 56.00 & to 66.00 \\
\hline GW-844 & SLS & 2.37 & 2.00 & 8.00 & 175.00 & SLS/SW/ .01 & 159.00 & to 177.00 & 165.00 & to 175.00 \\
\hline GW-854 & PVC & 0.75 & 0.50 & 3.00 & 12.90 & PVC/SL/ & 9.90 & to 12.90 & 10.90 & to 12.90 \\
\hline
\end{tabular}




\begin{tabular}{|c|c|c|c|c|c|c|}
\hline $\begin{array}{c}\text { CURRENT } \\
\text { NAME }\end{array}$ & $\begin{array}{l}\text { CASING } \\
\text { TYPE }\end{array}$ & $\begin{array}{l}\text { CASING } \\
\text { O.D. }\end{array}$ & $\begin{array}{c}\text { CASING } \\
\text { I.D. }\end{array}$ & $\begin{array}{c}\text { HOLE } \\
\text { DIAMETER }\end{array}$ & $\begin{array}{c}\text { HOLE } \\
\text { DEPTH }\end{array}$ & SCREEN TYPE \\
\hline GW-855 & PVC & 0.75 & 0.50 & 3.00 & 17.80 & PVC/SL/ .01 \\
\hline GW-857 & PVC & 0.75 & 0.50 & 3.00 & 14.80 & PVC/SL/ .01 \\
\hline GW-858 & PVC & 0.75 & 0.50 & 3.00 & 17.50 & PVC/SL/ .01 \\
\hline GW-859 & PVC & 0.75 & 0.50 & 3.00 & 14.60 & PVC/SL/ .01 \\
\hline GW-860 & PVC & 0.75 & 0.50 & 3.00 & 20.70 & PVC/SL/ .01 \\
\hline GW-861 & PVC & 0.75 & 0.50 & 3.00 & 16.90 & PVC/SL/ .01 \\
\hline GW-862 & PVC & 0.75 & 0.50 & 3.00 & 14.30 & PVC/SL/ .01 \\
\hline GW-863 & PVC & 0.75 & 0.50 & 3.00 & 23.30 & $\mathrm{PVC} / \mathrm{SL} / .01$ \\
\hline GW-864 & PVC & 0.75 & 0.50 & 3.00 & 17.30 & PVC/SL/ .01 \\
\hline GW-865 & PVC & 0.75 & 0.50 & 3.00 & 12.90 & PVC/SL/ .01 \\
\hline GW-866 & PVC & 0.75 & 0.50 & 3.00 & 17.40 & PVC/SL/ .01 \\
\hline GW-867 & PVC & 0.75 & 0.50 & 3.00 & 9.70 & PVC/SL/ .01 \\
\hline GW-868 & PVC & 0.75 & 0.50 & 3.00 & 17.50 & PVC/SL/ .01 \\
\hline GW-869 & PVC & 0.75 & 0.50 & 3.00 & 15.30 & PVC/SL/ .01 \\
\hline GW-871 & PVC & 0.75 & 0.50 & 3.00 & 20.60 & PVC/SL/ .01 \\
\hline GW-872 & PVC & 0.75 & 0.50 & 3.00 & 11.20 & PVC/SL/ \\
\hline GW-873 & PVC & 0.75 & 0.50 & 3.00 & 12.30 & PVC/SL/ .01 \\
\hline GW-874 & PVC & 0.75 & 0.50 & 3.00 & 16.70 & PVC/SL/ .01 \\
\hline GW-875 & PVC & 0.75 & 0.50 & 3.00 & 19.20 & PVC/SL/ .01 \\
\hline GW-876 & PVC & 0.75 & 0.50 & 3.00 & 21.10 & PVC/SL/ .01 \\
\hline GW-877 & PVC & 0.75 & 0.50 & 3.00 & 16.00 & PVC/SL/ .01 \\
\hline GW-878 & PVC & 0.75 & 0.50 & 3.00 & 15.20 & PVC/SL/ .01 \\
\hline GW-879 & PVC & 0.75 & 0.50 & 3.00 & 17.10 & PVC/SL/ .01 \\
\hline GW-880 & PVC & 0.75 & 0.50 & 3.00 & 18.90 & PVC/SL/ .01 \\
\hline GW-881 & PVC & 1.00 & 0.75 & 2.00 & 19.90 & PVC/SL/ .01 \\
\hline GW-882 & PVC & 1.00 & 0.75 & 2.00 & 19.90 & PVC/SL/ .01 \\
\hline GW-883 & PVC & 1.00 & 0.75 & 2.00 & 18.40 & PVC/SL/ .01 \\
\hline
\end{tabular}

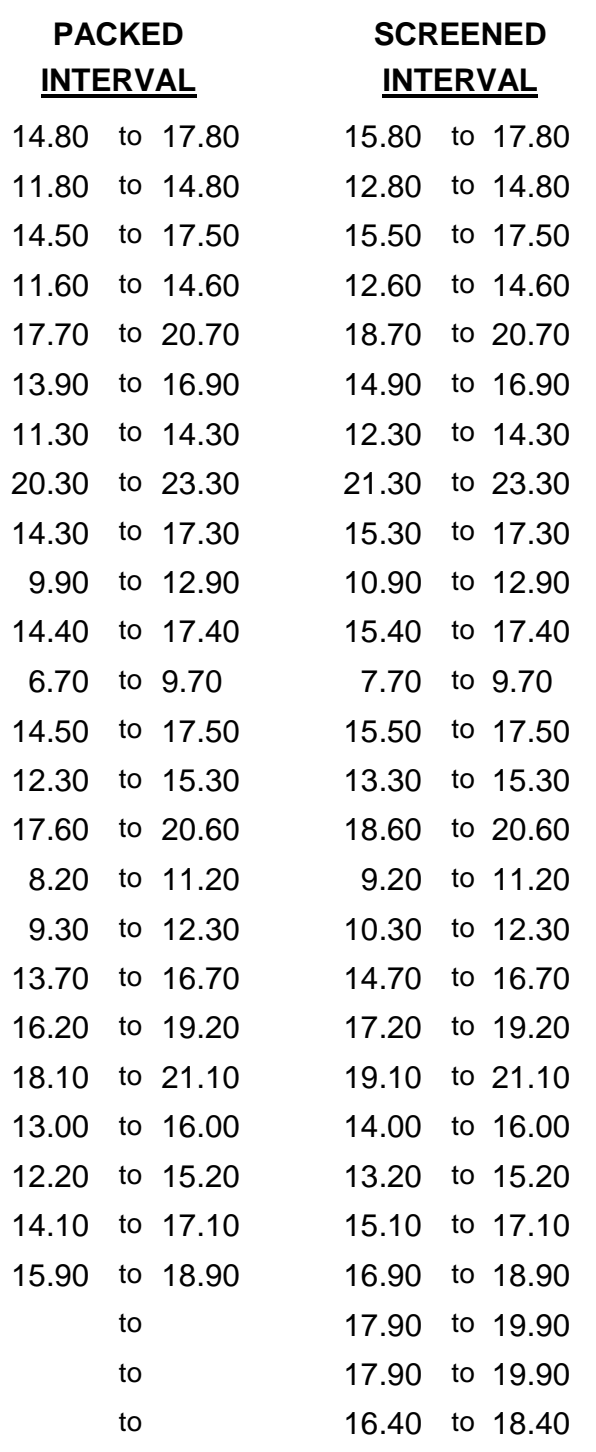




\begin{tabular}{|c|c|c|c|c|c|c|c|c|c|c|}
\hline $\begin{array}{c}\text { CURRENT } \\
\text { NAME }\end{array}$ & $\begin{array}{c}\text { CASING } \\
\text { TYPE }\end{array}$ & $\begin{array}{l}\text { CASING } \\
\text { O.D. }\end{array}$ & $\begin{array}{c}\text { CASING } \\
\text { I.D. }\end{array}$ & $\begin{array}{c}\text { HOLE } \\
\text { DIAMETER }\end{array}$ & $\begin{array}{c}\text { HOLE } \\
\text { DEPTH }\end{array}$ & SCREEN TYPE & $\begin{array}{r}\text { PAC } \\
\text { INTE } \\
\end{array}$ & $\begin{array}{l}\text { KED } \\
\text { RVAL }\end{array}$ & & $\begin{array}{l}\text { EENED } \\
\text { ERVAL }\end{array}$ \\
\hline GW-884 & PVC & 1.00 & 0.75 & 2.00 & 9.70 & PVC/SL/ .01 & & to & 7.70 & to 9.70 \\
\hline GW-885 & PVC & 1.00 & 0.75 & 2.00 & 15.90 & PVC/SL/ .01 & & to & 13.90 & to 15.90 \\
\hline GW-886 & PVC & & 0.75 & 2.00 & 17.00 & PVC/UNKN/ & & to & 12.00 & to 17.00 \\
\hline GW-887 & PVC & & 0.75 & 2.00 & 20.50 & PVC/UNKN/ & & to & 15.50 & to 20.50 \\
\hline GW-888 & PVC & & 0.75 & 2.00 & 21.50 & PVC/UNKN/.01 & & to & 16.50 & to 21.50 \\
\hline GW-889 & PVC & & 0.75 & 2.00 & 17.50 & PVC/UNKN/ & & to & 13.00 & to 18.00 \\
\hline GW-890 & PVC & 1.00 & 0.75 & 2.00 & 11.50 & PVC/UNKN/ & & to & 6.50 & to 11.50 \\
\hline GW-902 & PVC & 2.38 & 2.00 & 6.50 & 31.60 & PVC/SL/ .01 & & to & 23.60 & to 31.60 \\
\hline GW-904 & & & & & 293.50 & & & to & & to \\
\hline GW-905 & SLS & 4.50 & 4.00 & & 51.00 & SLS/SW/ & 39.00 & to 71.00 & 41.00 & to 51.00 \\
\hline GW-906 & PVC & 2.38 & 2.00 & & & PVC/SL/ & 14.50 & to 28.00 & 17.50 & to 27.50 \\
\hline GW-908 & PVC & 2.38 & 2.00 & & & PVC/SL/ & 20.00 & to 48.20 & 25.00 & to 35.00 \\
\hline GW-909 & PVC & 2.38 & 2.00 & 8.00 & 26.10 & PVC/SLI & 10.50 & to 26.10 & 16.10 & to 26.10 \\
\hline GW-910 & PVC & 2.38 & 2.00 & 8.00 & 35.30 & PVC/SL/ & 21.50 & to 35.50 & 25.50 & to 35.30 \\
\hline GW-911 & PVC & 2.38 & 2.00 & 7.00 & & PVC/SL/ & 11.00 & to 31.00 & 16.00 & to 31.00 \\
\hline GW-912 & & & & & & & & to & & to \\
\hline GW-913 & & & & & & & & to & & to \\
\hline GW-914 & & & & & & & & to & & to \\
\hline GW-916 & SS304 & 2.37 & 2.07 & 6.00 & 35.10 & SLS/SW/ .01 & 13.00 & to 36.00 & 15.00 & to 35.00 \\
\hline GW-917 & SS304 & 2.37 & 2.07 & 6.00 & 50.10 & SLS/SW/ .01 & 18.00 & to 51.00 & 20.00 & to 50.00 \\
\hline GW-918 & SS304 & & & 6.00 & 30.10 & SLS/SW/ .01 & 18.00 & to 33.00 & 20.00 & to 30.00 \\
\hline GW-919 & SS304 & 2.38 & 2.07 & & 31.50 & SLS/SW/ .01 & 11.60 & to 32.00 & 16.50 & to 31.50 \\
\hline GW-920 & SS304 & 2.37 & 2.07 & 7.63 & 54.10 & SLS/SW/ .01 & 22.00 & to 55.00 & 24.00 & to 54.00 \\
\hline GW-921 & SS304 & 2.37 & 2.07 & 6.50 & 48.10 & SLS/SW/ .01 & 16.00 & to 50.00 & 18.00 & to 48.00 \\
\hline GW-922 & SS304 & 2.37 & 2.07 & 6.00 & 45.10 & SLS/SW/ .01 & 23.00 & to 46.00 & 25.00 & to 45.00 \\
\hline GW-923 & SS304 & 2.37 & 2.07 & 6.00 & 70.10 & SLS/SW/ .01 & 36.00 & to 75.00 & 40.00 & to 70.00 \\
\hline GW-924 & SS304 & 2.37 & 2.07 & 6.00 & 53.10 & SLS/SW/ .01 & 21.00 & to 54.00 & 23.00 & to 53.00 \\
\hline
\end{tabular}




\begin{tabular}{|c|c|c|c|c|c|c|}
\hline $\begin{array}{c}\text { CURRENT } \\
\text { NAME }\end{array}$ & $\begin{array}{c}\text { CASING } \\
\text { TYPE }\end{array}$ & $\begin{array}{c}\text { CASING } \\
\text { O.D. }\end{array}$ & $\begin{array}{c}\text { CASING } \\
\text { I.D. }\end{array}$ & $\begin{array}{c}\text { HOLE } \\
\text { DIAMETER }\end{array}$ & $\begin{array}{c}\text { HOLE } \\
\text { DEPTH }\end{array}$ & SCREEN TYPE \\
\hline GW-925 & SS304 & 2.37 & 2.07 & 6.00 & 147.10 & SLS/SW/ .01 \\
\hline GW-926 & SS304 & 2.37 & 2.07 & 6.00 & 143.10 & SLS/SW/ .01 \\
\hline GW-927 & SS304 & 2.37 & 2.07 & 6.00 & 90.10 & SLS/SW/ .01 \\
\hline GW-928 & SS304 & 4.37 & 4.00 & 8.62 & 43.30 & SLS/SW/ .01 \\
\hline GW-929 & SS304 & 4.37 & 4.00 & 10.75 & 28.10 & SLS/SW/ .01 \\
\hline GW-930 & SS304 & 4.37 & 4.00 & 8.62 & 41.00 & SLS/SW/ .01 \\
\hline GW-931 & SS304 & 4.37 & 4.00 & 10.75 & 19.30 & SLS/SW/ .01 \\
\hline GW-935 & PVC & & 2.00 & 6.00 & 32.00 & PVC/SL/ .01 \\
\hline GW-936 & PVC & & 2.00 & 6.00 & 23.00 & PVC/SL/ .01 \\
\hline GW-937 & PVC & & 2.00 & 6.00 & 28.00 & PVC/SL/ .01 \\
\hline GW-938 & PVC & & 2.00 & 6.00 & 30.00 & PVC/SL/ .01 \\
\hline GW-939 & PVC & & 2.00 & 6.00 & 22.50 & PVC/SL/ .01 \\
\hline GW-940 & PVC & & 2.00 & 6.00 & 23.00 & PVC/SL/ .01 \\
\hline GW-941 & PVC & & 2.00 & 6.00 & 18.00 & PVC/SL/ .01 \\
\hline GW-942 & PVC & & 2.00 & 6.00 & 18.00 & PVC/SL/ .01 \\
\hline GW-943 & PVC & & 2.00 & 6.00 & 20.00 & PVC/SL/ .01 \\
\hline GW-944 & PVC & & 2.00 & 6.00 & 30.00 & PVC/SL/ .01 \\
\hline GW-945 & PVC & & 2.00 & 6.00 & 35.00 & PVC/SL/ .01 \\
\hline GW-946 & PVC & & 2.00 & 6.00 & 60.00 & PVC/SL/ .01 \\
\hline GW-947 & PVC & & 2.00 & 6.00 & 45.00 & PVC/SL/ .01 \\
\hline GW-948 & PVC & & 2.00 & 6.00 & 50.00 & PVC/SL/ .01 \\
\hline GW-949 & PVC & & 2.00 & 6.00 & 50.00 & PVC/SL/ .01 \\
\hline GW-950 & PVC & & 2.00 & 6.00 & 46.00 & PVC/SL/ \\
\hline GW-951 & PVC & & 2.00 & 6.00 & 45.00 & PVC/SL/ .01 \\
\hline GW-952 & PVC & & 2.00 & 6.00 & 45.00 & PVC/SL/ .01 \\
\hline GW-953 & SLS & & 2.00 & 6.00 & 35.00 & SLS/SW/ .01 \\
\hline
\end{tabular}

\begin{tabular}{|c|c|c|c|}
\hline \multicolumn{2}{|c|}{$\begin{array}{l}\text { PACKED } \\
\text { INTERVAL }\end{array}$} & \multicolumn{2}{|c|}{$\begin{array}{l}\text { SCREENED } \\
\text { INTERVAL }\end{array}$} \\
\hline 92.00 & to 148.00 & 97.00 & to 147.00 \\
\hline 103.00 & to 145.00 & 113.00 & to 143.00 \\
\hline 57.00 & to 92.00 & 60.00 & to 90.00 \\
\hline 31.20 & to 44.00 & 33.20 & to 43.20 \\
\hline 15.00 & to 28.60 & 18.00 & to 28.00 \\
\hline 28.00 & to 41.50 & 30.90 & to 40.90 \\
\hline 6.00 & to 19.80 & 9.20 & to 19.20 \\
\hline 9.00 & to 31.50 & 11.50 & to 31.50 \\
\hline 6.00 & to 23.00 & 8.00 & to 23.00 \\
\hline 11.00 & to 28.00 & 13.00 & to 28.00 \\
\hline 13.00 & to 30.00 & 15.00 & to 30.00 \\
\hline 5.00 & to 22.50 & 7.50 & to 22.50 \\
\hline 6.00 & to 23.00 & 8.00 & to 23.00 \\
\hline 6.00 & to 18.40 & 8.00 & to 18.00 \\
\hline 6.00 & to 18.00 & 8.00 & to 18.00 \\
\hline 8.00 & to 20.00 & 10.00 & to 20.00 \\
\hline 13.00 & to 30.00 & 15.00 & to 30.00 \\
\hline 23.00 & to 35.00 & 25.00 & to 35.00 \\
\hline 48.50 & to 60.00 & 50.00 & to 60.00 \\
\hline 28.00 & to 45.00 & 30.00 & to 45.00 \\
\hline 33.00 & to 50.00 & 35.00 & to 50.00 \\
\hline 33.00 & to 50.00 & 35.00 & to 50.00 \\
\hline 29.00 & to 46.00 & 31.00 & to 46.00 \\
\hline 28.00 & to 45.00 & 30.00 & to 45.00 \\
\hline 28.00 & to 45.00 & 30.00 & to 45.00 \\
\hline 23.00 & to 35.00 & 25.00 & to 35.00 \\
\hline
\end{tabular}

to 


\begin{tabular}{|c|c|c|c|c|c|c|c|c|c|c|}
\hline $\begin{array}{c}\text { CURRENT } \\
\text { NAME }\end{array}$ & $\begin{array}{c}\text { CASING } \\
\text { TYPE }\end{array}$ & $\begin{array}{c}\text { CASING } \\
\text { O.D. }\end{array}$ & $\begin{array}{c}\text { CASING } \\
\text { I.D. }\end{array}$ & $\begin{array}{c}\text { HOLE } \\
\text { DIAMETER }\end{array}$ & $\begin{array}{c}\text { HOLE } \\
\text { DEPTH }\end{array}$ & SCREEN TYPE & $\begin{array}{r}\text { PA } \\
\text { INTI } \\
\end{array}$ & $\begin{array}{l}\text { CKED } \\
\text { ERVAL } \\
\end{array}$ & $\begin{array}{l}\mathrm{SCl} \\
\text { IN] }\end{array}$ & $\begin{array}{l}\text { REENED } \\
\text { ERVAL }\end{array}$ \\
\hline GW-956 & & & & & & & & to & & to \\
\hline GW-959 & SLS & 4.50 & 4.00 & 7.50 & 8.50 & SLS/SW/ .01 & 2.40 & to 9.00 & 3.40 & to 8.40 \\
\hline GW-960 & PVC40 & 4.50 & 4.00 & & 24.80 & PVC/SL/ .01 & & to & 14.60 & to 24.60 \\
\hline GW-961 & SLS & 2.38 & 2.16 & 0.50 & 25.30 & SLS/SW/ .01 & 3.40 & to 25.50 & 5.30 & to 25.30 \\
\hline GW-964 & SLS & 2.40 & 2.00 & 6.50 & 32.00 & SLS/SW/ .01 & 7.90 & to 31.90 & 10.90 & to 30.90 \\
\hline GW-965 & SLS & 2.40 & 2.00 & 6.00 & 72.00 & SLS/SW/ .01 & 47.50 & to 72.00 & 51.80 & to 71.80 \\
\hline GW-966 & PVC & 2.38 & 2.00 & 0.50 & 19.70 & PVC/SL/ .01 & 2.50 & to 19.70 & 4.00 & to 19.50 \\
\hline LL/HAZ-01 & PVC40 & 6.50 & 6.00 & 8.50 & 45.00 & PVC/PER/ & 1.00 & to 45.00 & 30.00 & to 45.00 \\
\hline LL/HAZ-02 & PVC40 & 6.50 & 6.00 & 8.50 & 25.00 & PVC/PER/ & 1.00 & to 25.00 & 5.00 & to 25.00 \\
\hline LL/HAZ-03 & PVC40 & 6.50 & 6.00 & 8.50 & 15.00 & PVC/PER/ & 1.00 & to 15.00 & 5.00 & to 15.00 \\
\hline LL/HAZ-04 & PVC40 & 6.50 & 6.00 & 8.50 & 25.00 & PVC/PER/ & 1.00 & to 25.00 & 5.00 & to 25.00 \\
\hline LL/HAZ-05 & PVC40 & 6.50 & 6.00 & 8.50 & 25.00 & PVC/PER/ & 1.00 & to 25.00 & 10.00 & to 25.00 \\
\hline LL/HAZ-06 & PVC40 & 6.50 & 6.00 & 8.50 & 30.00 & PVC/PER/ & 1.00 & to 30.00 & 10.00 & to 30.00 \\
\hline LL/HAZ-07 & PVC40 & 6.50 & 6.00 & 8.50 & 33.50 & PVC/PER/ & 1.00 & to 33.50 & 13.50 & to 33.50 \\
\hline LL/HAZ-08 & PVC40 & 6.50 & 6.00 & 8.50 & 31.00 & PVC/PER/ & 1.00 & to 31.00 & 11.00 & to 31.00 \\
\hline LL/HAZ-09 & PVC40 & 6.50 & 6.00 & 8.50 & 52.00 & PVC/PER/ & 1.00 & to 52.00 & 27.00 & to 52.00 \\
\hline LL/HAZ-10 & PVC40 & 6.50 & 6.00 & 8.50 & 39.00 & PVC/PER/ & 1.00 & to 39.00 & 19.00 & to 39.00 \\
\hline LL/HAZ-11 & PVC40 & 6.50 & 6.00 & 8.50 & 33.00 & PVC/PER/ & 1.00 & to 33.00 & 13.00 & to 33.00 \\
\hline LL/HAZ-12 & PVC40 & 6.50 & 6.00 & 8.50 & 35.00 & PVC/PER/ & 1.00 & to 35.00 & 15.00 & to 35.00 \\
\hline LL/HAZ-13 & PVC40 & 2.37 & 2.00 & 6.13 & 399.00 & PVC/SL/ .01 & 387.00 & to 400.00 & 389.00 & to 399.00 \\
\hline LL/HAZ-14 & PVC40 & 2.37 & 2.00 & 6.13 & 349.00 & PVC/SL/ .01 & 338.00 & to 350.00 & 339.00 & to 349.00 \\
\hline LL/HAZ-15 & PVC40 & 2.37 & 2.00 & 6.13 & 372.00 & PVC/SL/ .01 & 360.00 & to 375.00 & 362.00 & to 372.00 \\
\hline LL/HAZ-16 & PVC40 & 6.50 & 6.00 & 8.50 & 24.00 & PVC/PER/ & & to 24.00 & 9.00 & to 24.00 \\
\hline LL/HAZ-17 & PVC40 & 6.50 & 6.00 & 8.50 & 40.00 & PVC/PER/ & & to 40.00 & 25.00 & to 40.00 \\
\hline LL/HAZ-18 & PVC40 & 6.50 & 6.00 & 8.50 & 22.00 & PVC/PER/ & & to 22.00 & 17.00 & to 22.00 \\
\hline LL/HAZ-19 & PVC40 & 6.50 & 6.00 & 8.50 & 23.50 & PVC/PER/ & & to 23.50 & 18.50 & to 23.50 \\
\hline OR-09 & PVC & 2.00 & 1.75 & 3.00 & 100.00 & $\mathrm{PVC/SL/}$ & & to 100.00 & & to 100.00 \\
\hline
\end{tabular}




\begin{tabular}{|c|c|c|c|c|c|c|}
\hline $\begin{array}{c}\text { CURRENT } \\
\text { NAME }\end{array}$ & $\begin{array}{l}\text { CASING } \\
\text { TYPE }\end{array}$ & $\begin{array}{c}\text { CASING } \\
\text { O.D. }\end{array}$ & $\begin{array}{c}\text { CASING } \\
\text { I.D. }\end{array}$ & $\begin{array}{c}\text { HOLE } \\
\text { DIAMETER }\end{array}$ & $\begin{array}{c}\text { HOLE } \\
\text { DEPTH }\end{array}$ & SCREEN TYPE \\
\hline$P \& A-1$ & PVC & 6.63 & 6.00 & & 22.00 & PVC/SL/ .01 \\
\hline$P \& A-2$ & PVC & 6.63 & 6.00 & & 23.90 & PVC/SL/ .01 \\
\hline$P \& A-3$ & PVC & 6.63 & 6.00 & & 35.80 & PVC/SL/ .01 \\
\hline S3-1 & PVC & 2.37 & 2.00 & 8.00 & 11.50 & PVC/SL/ .01 \\
\hline S3-3 & PVC & 2.37 & 2.00 & 8.00 & 7.50 & PVC/SL/ .01 \\
\hline S3-5 & PVC & 2.37 & 2.00 & 8.00 & 8.80 & PVC/SL/ .01 \\
\hline S3-6 & PVC & 2.37 & 2.00 & 8.00 & 18.80 & PVC/SL/ .01 \\
\hline S3-7 & PVC & 2.37 & 2.00 & 8.00 & 27.90 & PVC/SL/ .01 \\
\hline S3-8 & PVC & 2.37 & 2.00 & 8.00 & 12.00 & PVC/SL/ .01 \\
\hline SAN-1 & PVC & & & 8.00 & 98.00 & PVC/PER/ \\
\hline SAN-10 & PVC & 6.50 & 6.00 & 8.00 & 53.00 & PVC/PER/ \\
\hline SAN-2 & PVC & 6.50 & 6.00 & 8.00 & 43.00 & PVC/PER/ \\
\hline SAN-3 & PVC & 6.50 & 6.00 & 7.50 & 57.00 & PVC/PER/ \\
\hline SAN-4 & PVC & 6.50 & 6.00 & 8.00 & 35.00 & PVC/PER/ \\
\hline SAN-5 & PVC & 6.50 & 6.00 & 8.00 & 34.00 & PVC/PER/ \\
\hline SAN-6 & PVC & 6.50 & 6.00 & 8.00 & 42.00 & PVC/PER/ \\
\hline SAN-7 & PVC & 6.50 & 6.00 & 8.00 & 59.50 & PVC/PER/ \\
\hline SAN-8 & PVC & 6.50 & 6.00 & 8.00 & 39.00 & PVC/PER/ \\
\hline SAN-9 & PVC & 6.50 & 6.00 & 8.00 & 38.50 & PVC/PER/ \\
\hline UNIDENT B & PVC & 4.50 & 4.00 & & 19.30 & PVC/SL/ \\
\hline
\end{tabular}

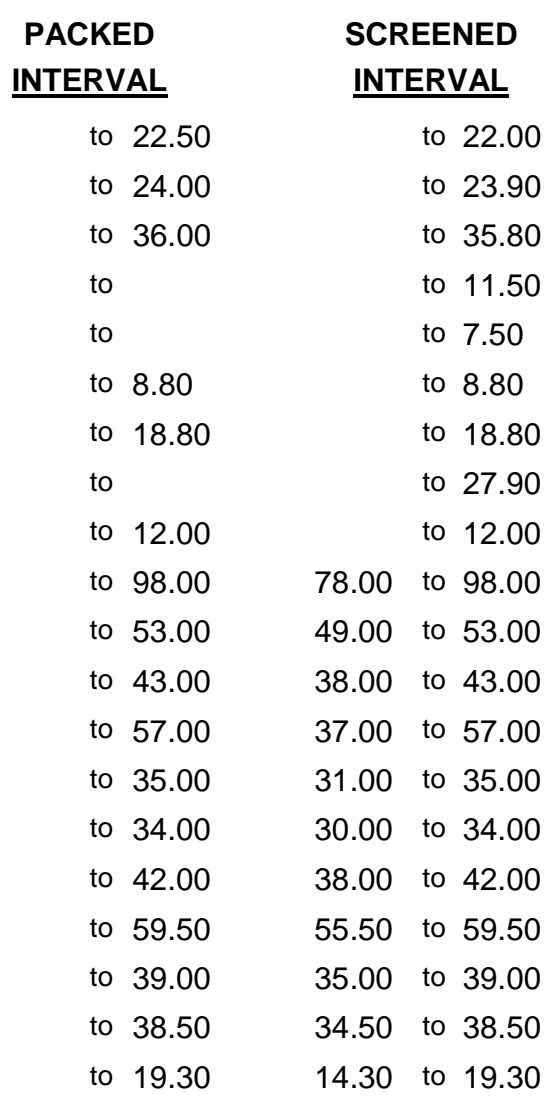


APPENDIX E

Open-interval Well Construction Summary 
This Page Intentionally Left Blank 


\begin{tabular}{|c|c|c|c|c|c|c|c|c|c|}
\hline \multirow{2}{*}{$\begin{array}{l}\text { CURRENT } \\
\text { NAME }\end{array}$} & \multirow{2}{*}{$\begin{array}{l}\text { CASING } \\
\text { TYPE }\end{array}$} & \multirow{2}{*}{$\begin{array}{l}\text { CASING } \\
\text { O.D. }\end{array}$} & \multirow{2}{*}{$\begin{array}{c}\text { CASING } \\
\text { I.D. }\end{array}$} & \multirow{2}{*}{$\begin{array}{l}\text { CASING } \\
\text { DEPTH }\end{array}$} & \multirow{2}{*}{$\begin{array}{c}\text { HOLE } \\
\text { DIAMETER }\end{array}$} & \multirow{2}{*}{$\begin{array}{l}\text { HOLE } \\
\text { DEPTH }\end{array}$} & \multirow{2}{*}{\multicolumn{2}{|c|}{ OPEN INTERVAL }} & \multirow{2}{*}{$\begin{array}{c}\text { OPEN INTERVAL } \\
\text { DIAMETER }\end{array}$} \\
\hline & & & & & & & & & \\
\hline 1001 & STL & 6.50 & & 30.00 & 8.00 & 30.00 & 30.00 & to 56.00 & \\
\hline 1012 & STL & 6.50 & & 40.00 & 8.00 & & & to 70.00 & \\
\hline 1013 & STL & 6.50 & & 28.00 & 8.00 & & & to 42.00 & \\
\hline 1014 & STL & 6.50 & & 36.00 & 8.00 & & & to 46.40 & \\
\hline 1015 & STL & 6.50 & & 29.50 & 8.00 & & & to 37.00 & \\
\hline 1016 & STL & 6.50 & & 32.00 & 8.00 & & & to 63.00 & \\
\hline 1017 & STL & 6.50 & & 29.00 & 8.00 & & & to 44.40 & \\
\hline 1018 & STL & 6.50 & & 36.00 & 8.00 & & & to 47.20 & \\
\hline 1019 & STL & 6.50 & & 40.00 & 8.00 & & & to 43.50 & \\
\hline 1020 & STL & 6.50 & & 21.60 & 8.00 & & & to 40.00 & \\
\hline 1021 & STL & 6.50 & & 23.00 & 8.00 & & & to 27.50 & \\
\hline 1022 & STL & 6.50 & & 30.00 & 8.00 & & & to 48.00 & \\
\hline 1023 & STL & 6.50 & & 21.00 & 8.00 & & & to 36.00 & \\
\hline 1024 & STL & 6.50 & & 20.00 & 8.00 & & & to 40.00 & \\
\hline 1025 & STL & 6.50 & & 30.00 & 8.00 & & & to 50.00 & \\
\hline $1025 \mathrm{~A}$ & STL & 6.50 & 6.00 & 27.60 & 8.00 & 27.60 & 27.60 & to 41.60 & 8.00 \\
\hline 1026 & STL & 6.50 & & 46.00 & 8.00 & & & to 70.00 & \\
\hline 1027 & PVC40 & 6.50 & & 28.00 & 7.50 & 28.00 & 28.00 & to 100.00 & 6.00 \\
\hline 1033 & S/GAL & 6.50 & & 110.00 & & 110.00 & 110.00 & to 160.00 & \\
\hline 1035 & PVC40 & 6.50 & & 28.00 & 7.50 & 28.00 & 28.00 & to 100.00 & 6.00 \\
\hline 1036 & PVC40 & 6.50 & & 33.30 & 7.50 & 33.30 & 33.30 & to 100.00 & 6.00 \\
\hline 1043 & STL & 6.62 & & & & & & to 150.00 & \\
\hline 1044 & STL & 6.62 & & & & & & to 160.00 & \\
\hline 1045 & STL & 6.62 & & & & & & to 155.00 & \\
\hline 1046 & STL & 6.62 & & & & & & to 75.00 & \\
\hline 1047 & STL & 4.00 & 3.50 & 4.90 & 3.00 & 4.90 & 4.90 & to 23.20 & 3.00 \\
\hline $1047 A$ & STL & 4.00 & 3.50 & 4.70 & 3.00 & 4.70 & 4.70 & to 170.00 & 3.00 \\
\hline 1048 & PVC40 & 6.50 & & 30.00 & 7.50 & 30.00 & 30.00 & to 100.00 & 6.00 \\
\hline
\end{tabular}




\begin{tabular}{|c|c|c|c|c|c|c|c|c|c|}
\hline CURRENT & CASING & CASING & CASING & CASING & HOLE & HOLE & & & OPEN INTERVAL \\
\hline NAME & TYPE & $\underline{\text { O.D. }}$. & I.D. & DEPTH & DIAMETER & DEPTH & OPEI & INTERVAL & DIAMETER \\
\hline 1050 & PVC40 & 6.50 & & 22.00 & 7.50 & 22.00 & 22.00 & to 100.00 & 6.00 \\
\hline 1088 & STL & 6.50 & & & & & & to 117.70 & \\
\hline 1097 & PVC40 & 4.50 & & 20.00 & 6.00 & 20.00 & 20.00 & to 40.00 & 3.00 \\
\hline 38-DC & STL & 6.62 & & 15.50 & & & & to 44.10 & \\
\hline 39-DC & STL & 6.62 & & 14.00 & & & & to 41.10 & \\
\hline 40-DC & STL & 6.62 & & 6.50 & & & & to 36.60 & \\
\hline 41-DC & STL & 6.62 & & 35.00 & & & & to 102.00 & \\
\hline 42-DC & STL & 6.62 & & 28.50 & & & & to 98.30 & \\
\hline 44-DC & STL & 6.62 & & 27.00 & & & & to 42.40 & \\
\hline $45-D C$ & STL & 6.62 & & 22.50 & & & & to 30.00 & \\
\hline BC-03 & PVC & 1.50 & 1.25 & 37.80 & 3.00 & 37.80 & 37.80 & to 349.10 & 3.00 \\
\hline BC-06 & PVC & 1.50 & 1.25 & 60.00 & 3.00 & 60.00 & 60.00 & to 352.50 & 3.00 \\
\hline BC-07 & PVC & 1.50 & 1.25 & 58.00 & 3.00 & 58.00 & 58.00 & to 351.90 & 3.00 \\
\hline BC-09 & $P V C$ & 1.50 & 1.25 & & & & & to 343.00 & 3.00 \\
\hline$B C-10$ & PVC & 1.50 & 1.25 & 58.90 & 3.00 & 58.90 & 58.90 & to 349.00 & 3.00 \\
\hline BC-14 & PVC & 4.50 & 4.00 & 17.80 & 3.00 & 17.80 & 17.80 & to 102.00 & 3.00 \\
\hline BC-17 & PVC & 1.50 & 1.25 & 19.10 & 3.00 & 19.10 & 19.10 & to 100.40 & 3.00 \\
\hline BC-18 & PVC & 1.50 & 1.25 & 58.80 & 3.00 & 58.80 & 58.80 & to 100.20 & 3.00 \\
\hline BC-19 & PVC & 1.50 & 1.25 & 38.90 & 3.00 & 38.90 & 38.90 & to 151.80 & 3.00 \\
\hline$B C-22$ & PVC & 1.25 & 1.00 & 39.60 & 3.00 & 39.60 & 39.60 & to 100.20 & 3.00 \\
\hline BC-23 & PVC & 4.50 & 4.00 & 17.90 & 3.00 & 17.90 & 17.90 & to 56.00 & 3.00 \\
\hline$B C-25$ & PVC & 4.50 & 4.00 & & & & & to 104.00 & 3.00 \\
\hline BC-30 & PVC & 1.75 & 1.50 & 18.80 & 3.00 & 18.80 & 18.80 & to 104.00 & 3.00 \\
\hline BC-31 & PVC & 4.50 & 4.00 & 12.50 & 3.00 & 12.50 & 12.50 & to 101.00 & 3.00 \\
\hline BC-32 & PVC & 1.25 & 1.00 & 62.40 & 3.00 & 62.40 & 62.40 & to 100.20 & 3.00 \\
\hline$B C-33$ & PVC & 1.25 & 1.00 & & & & & to 145.30 & 3.00 \\
\hline BC-34 & STL & 1.25 & 1.00 & & & & & to 126.30 & 3.00 \\
\hline BC-35 & PVC & 1.25 & 1.00 & 91.00 & 3.00 & 91.00 & 91.00 & to 325.00 & 3.00 \\
\hline
\end{tabular}




\begin{tabular}{|c|c|c|c|c|c|c|c|c|c|}
\hline CURRENT & CASING & CASING & CASING & CASING & HOLE & HOLE & OPRA & IITCDYA & OPEN INTERVAL \\
\hline BC-36 & PVC & 4.50 & 4.00 & 15.50 & 3.00 & 15.50 & 15.50 & to 350.00 & 3.00 \\
\hline BC-39 & STL & 1.25 & 1.00 & & & & & to 157.80 & 3.00 \\
\hline BC- -40 & PVC & 4.50 & 4.00 & & & & & to 135.50 & 3.00 \\
\hline BC-41 & STL & 1.25 & 1.00 & & & & & to 276.00 & 3.00 \\
\hline$B C-42$ & PVC & 1.25 & 1.00 & & & 66.40 & 66.40 & to 203.00 & 3.00 \\
\hline$B C-50$ & & & & & & 13.00 & 13.00 & to 100.00 & 3.00 \\
\hline BC-63 & PVC & 1.25 & 1.00 & 55.50 & 3.00 & 55.50 & 55.50 & to 283.70 & 3.00 \\
\hline BCU-01 & PVC & 1.25 & 1.00 & 37.90 & 3.00 & 37.90 & 37.90 & to 135.50 & 3.00 \\
\hline BCU-02 & PVC & 1.25 & 1.00 & 37.20 & 3.00 & 37.20 & 37.20 & to 178.00 & 3.00 \\
\hline BCU-10 & PVC & 1.25 & 1.00 & & & & & to 55.80 & 5.00 \\
\hline BCU-12 & PVC & 1.50 & 1.25 & 37.70 & 3.00 & 37.70 & 37.70 & to 201.00 & 3.00 \\
\hline BCU-13 & PVC & 4.50 & 4.00 & 8.90 & 5.00 & 8.90 & 8.90 & to 25.10 & 5.00 \\
\hline BCU-14 & PVC & 4.50 & 4.00 & 10.10 & 3.00 & 10.10 & 10.10 & to 15.00 & 3.00 \\
\hline BCU-15 & PVC & 4.50 & 4.00 & 10.00 & 3.00 & 10.00 & 10.00 & to 17.00 & 3.00 \\
\hline BCU-16 & PVC & 1.25 & 1.00 & 37.50 & 3.00 & 37.50 & 37.50 & to 146.30 & 3.00 \\
\hline BCU-20 & PVC & 1.25 & 1.00 & 43.30 & 3.00 & 43.30 & 43.30 & to 76.60 & 3.00 \\
\hline BCU-21 & PVC & 4.50 & 4.00 & 20.00 & 3.00 & 20.00 & 20.00 & to 242.00 & 3.00 \\
\hline $\mathrm{CH}-143$ & STL & 4.50 & 4.00 & 54.00 & 7.00 & 54.00 & 54.00 & to 600.00 & 3.70 \\
\hline $\mathrm{CH}-157$ & STL & 4.50 & 4.00 & 81.00 & 7.00 & 81.00 & 81.00 & to 540.00 & 3.70 \\
\hline CH-185 & STL & 4.50 & 4.00 & 30.30 & 7.00 & 30.30 & 30.30 & to 841.70 & 3.70 \\
\hline CH-189 & STL & 4.50 & 4.00 & 23.00 & 7.00 & 23.00 & 23.00 & to 763.00 & 3.70 \\
\hline $\mathrm{CO}-2$ & STL & 4.00 & & 10.00 & & 10.00 & 10.00 & to 107.20 & \\
\hline CO-4 & STL & 4.00 & & 37.00 & & 37.00 & 37.00 & to 57.00 & \\
\hline DC WELL & & & & & & & & to & \\
\hline GW-116 & SF25 & 4.50 & 4.00 & 235.00 & 10.00 & 235.00 & 235.00 & to 285.00 & 4.00 \\
\hline GW-117 & SF25 & 4.50 & 4.00 & 480.00 & 10.00 & 480.00 & 480.00 & to 530.00 & 4.00 \\
\hline GW-118 & SF25 & 4.50 & 4.00 & 525.00 & 10.00 & 525.00 & 525.00 & to 575.00 & 4.00 \\
\hline GW-119 & SF25 & 4.50 & 4.00 & 460.00 & 10.00 & 460.00 & 460.00 & to 510.00 & 4.00 \\
\hline
\end{tabular}




\begin{tabular}{|c|c|c|c|c|c|c|c|c|c|}
\hline CURRENT & CASING & CASING & CASING & CASING & HOLE & HOLE & OPRA & IOTCDYA & OPEN INTERVAL \\
\hline GW-120 & SF25 & 4.50 & 4.00 & 130.00 & 10.00 & 130.00 & 130.00 & to 180.00 & 4.00 \\
\hline GW-121 & SF25 & 4.50 & 4.00 & 550.00 & 10.00 & 550.00 & 550.00 & to 600.00 & 4.00 \\
\hline GW-122 & SF25 & 4.50 & 4.00 & 92.00 & 10.00 & 92.00 & 92.00 & to 142.00 & 4.00 \\
\hline GW-123 & SF25 & 4.50 & 4.00 & 522.00 & 10.00 & 522.00 & 522.00 & to 572.00 & 4.00 \\
\hline GW-124 & SF25 & 4.50 & 4.00 & 100.00 & 10.00 & 100.00 & 100.00 & to 150.00 & 4.00 \\
\hline GW-125 & SF25 & 4.50 & 4.00 & 502.00 & 10.00 & 502.00 & 502.00 & to 552.00 & 4.00 \\
\hline GW-126 & SF25 & 4.50 & 4.00 & 105.00 & 10.00 & 105.00 & 105.00 & to 155.00 & 4.00 \\
\hline GW-128 & SF25 & 4.50 & & 95.00 & 9.87 & 95.00 & 95.00 & to 914.00 & 3.70 \\
\hline GW-129 & SF25 & 4.50 & & 57.00 & 9.87 & 57.00 & 57.00 & to 953.50 & 3.70 \\
\hline GW-130 & SF25 & 4.50 & & 42.00 & 9.87 & 42.00 & 42.00 & to 1088.50 & 3.70 \\
\hline GW-131 & SF25 & 4.50 & & 43.00 & 9.87 & 1085.00 & & to & 3.70 \\
\hline GW-132 & SF25 & 4.50 & & 97.00 & 9.87 & 753.00 & & to & 3.70 \\
\hline GW-133 & SF25 & 4.50 & & 55.00 & 9.87 & 567.00 & & to & 3.70 \\
\hline GW-134 & SF25 & 4.50 & & 35.00 & 9.87 & 820.00 & & to & 3.70 \\
\hline GW-135 & SF25 & 4.50 & & 80.00 & 9.87 & 1241.00 & & to & 3.70 \\
\hline GW-137 & SF25 & 4.50 & & 40.00 & 9.87 & 40.00 & 40.00 & to 1000.00 & 3.70 \\
\hline GW-138 & SF25 & 4.50 & & 35.00 & 9.87 & 35.00 & 35.00 & to 1100.00 & 3.70 \\
\hline GW-139 & SF25 & 4.50 & & 70.00 & 9.87 & 70.00 & 70.00 & to 981.00 & 3.70 \\
\hline GW-140 & SF25 & 4.50 & & 101.00 & 9.87 & 101.00 & 101.00 & to 1200.00 & 3.70 \\
\hline GW-142 & SF25 & 6.62 & 6.12 & 248.50 & 11.00 & 248.50 & 248.50 & to 295.00 & 6.00 \\
\hline GW-143 & SF25 & 6.62 & 6.12 & 205.00 & 10.00 & 205.00 & 205.00 & to 253.00 & 6.00 \\
\hline GW-146 & SF25 & 6.62 & 6.12 & 190.00 & 11.00 & 190.00 & 190.00 & to 220.00 & 6.00 \\
\hline GW-158 & SF25 & 6.62 & 6.00 & 356.00 & 10.00 & 356.00 & 356.00 & to 441.00 & 6.00 \\
\hline GW-160 & SF25 & 6.62 & & 205.00 & 11.00 & 205.00 & 205.00 & to 235.00 & 6.00 \\
\hline GW-161 & SF25 & 6.62 & & 350.00 & 11.00 & 350.00 & 350.00 & to 400.00 & 11.00 \\
\hline GW-162 & SF25 & 6.62 & 6.12 & 92.00 & 10.00 & 92.00 & 92.00 & to 125.00 & 6.00 \\
\hline GW-163 & SF25 & 6.62 & 6.12 & 208.00 & 10.00 & 208.00 & 208.00 & to 225.00 & 6.00 \\
\hline GW-164 & SF25 & 6.62 & 6.12 & 370.00 & 8.50 & 370.00 & 370.00 & to 405.00 & 6.00 \\
\hline
\end{tabular}




\begin{tabular}{|c|c|c|c|c|c|c|c|c|c|}
\hline CURRENT & CASING & CASING & CASING & CASING & HOLE & HOLE & OPRA & IITCDYA & OPEN INTERVAL \\
\hline GW-165 & SF25 & 6.62 & 6.12 & 276.00 & 10.00 & 276.00 & 276.00 & to 325.00 & 6.00 \\
\hline GW-166 & SF25 & 6.62 & 6.00 & 380.00 & 10.00 & 380.00 & 380.00 & to 385.00 & 6.00 \\
\hline GW-168 & STL & 4.38 & 4.00 & 104.00 & 6.62 & 104.00 & 104.00 & to 135.40 & 3.88 \\
\hline GW-170 & STL & 4.38 & 4.00 & 104.00 & 6.62 & 104.00 & 104.00 & to 156.90 & 3.88 \\
\hline GW-172 & STL & 4.38 & 4.00 & 105.00 & 6.62 & 105.00 & 105.00 & to 133.80 & 3.63 \\
\hline GW-181 & SF25 & 6.62 & & 153.50 & 9.50 & 155.00 & 155.00 & to 168.00 & 6.60 \\
\hline GW-182 & SF25 & 4.50 & & 126.60 & 8.50 & 126.60 & 126.60 & to 402.10 & 3.70 \\
\hline GW-185 & SF25 & 6.62 & 6.00 & 405.00 & 10.00 & 405.00 & 405.00 & to 471.50 & 6.00 \\
\hline GW-207 & PVC40 & 4.38 & 4.00 & 100.00 & 7.87 & 100.00 & 100.00 & to 109.60 & 3.88 \\
\hline GW-208 & STL & 4.38 & 4.00 & 404.00 & 6.62 & 404.00 & 404.00 & to 412.80 & 3.63 \\
\hline GW-210 & STL & 4.38 & 4.00 & 104.00 & 6.62 & 104.00 & 104.00 & to 124.00 & 3.88 \\
\hline GW-211 & STL & 4.38 & 4.00 & 404.00 & 6.62 & 404.00 & 404.00 & to 410.00 & 3.88 \\
\hline GW-214 & STL & 4.38 & 4.00 & 414.60 & 6.62 & 414.60 & 414.60 & to 430.00 & 3.88 \\
\hline GW-225 & STL & 4.50 & 4.00 & 150.00 & 10.00 & 150.00 & 150.00 & to 200.00 & 4.00 \\
\hline GW-226 & STL & 4.50 & 4.00 & 45.00 & 10.00 & 45.00 & 45.00 & to 55.00 & 4.00 \\
\hline GW-227 & STL & 4.50 & 4.00 & 30.00 & 10.00 & 30.00 & 30.00 & to 40.00 & 4.00 \\
\hline GW-228 & STL & 4.50 & 4.00 & 80.00 & 10.00 & 80.00 & 80.00 & to 100.00 & 10.00 \\
\hline GW-229 & STL & 4.50 & 4.00 & 40.00 & 10.00 & 40.00 & 40.00 & to 55.00 & 4.00 \\
\hline GW-230 & STL & 4.38 & 4.00 & 341.00 & 5.50 & 341.00 & 341.00 & to 406.40 & 3.63 \\
\hline GW-232 & STL & 4.38 & 4.00 & 401.00 & 6.62 & 401.00 & 401.00 & to 411.70 & 3.88 \\
\hline GW-238 & STL & 4.38 & 4.00 & 100.00 & 7.50 & 100.00 & 100.00 & to 129.00 & 3.63 \\
\hline GW-239 & STL & 4.38 & 4.00 & 404.00 & 6.62 & 404.00 & 404.00 & to 433.30 & 3.88 \\
\hline GW-293 & SF25 & 6.62 & & 197.00 & 10.00 & 197.00 & 197.00 & to 214.00 & 6.00 \\
\hline GW-303 & STL & 7.00 & & 300.00 & 10.00 & 300.00 & 300.00 & to 321.00 & 6.00 \\
\hline GW-322 & STL & 7.00 & & 128.00 & 10.00 & 128.00 & 128.00 & to 193.00 & 6.00 \\
\hline GW-343 & SF25 & 6.62 & & 170.00 & 10.63 & 170.00 & 170.00 & to 185.00 & 6.00 \\
\hline GW-344 & SF25 & 6.62 & & 301.00 & 10.62 & 301.00 & 301.00 & to 316.00 & 6.00 \\
\hline GW-363 & SF25 & 6.62 & & 48.30 & 9.50 & 50.00 & 50.00 & to 75.00 & 6.00 \\
\hline
\end{tabular}




\begin{tabular}{|c|c|c|c|c|c|c|c|c|c|}
\hline CURRENT & CASING & CASING & CASING & CASING & HOLE & HOLE & OPRA & IITCDYA & OPEN INTERVAL \\
\hline GW-365 & SF25 & 6.62 & & 124.20 & 10.75 & 126.70 & 126.70 & to 150.00 & 6.00 \\
\hline GW-367 & SF25 & 6.62 & & 124.00 & 10.75 & 125.00 & 125.00 & to 151.40 & 6.00 \\
\hline GW-368 & SF25 & 6.62 & & 223.70 & 10.60 & 225.00 & 225.00 & to 245.00 & 6.10 \\
\hline GW-369 & SF25 & 6.62 & & 115.80 & 10.60 & 115.80 & 115.80 & to 150.20 & 6.10 \\
\hline GW-371 & SF25 & 6.37 & & 108.30 & 9.50 & 110.00 & 110.00 & to 125.00 & 6.00 \\
\hline GW-373 & SF25 & 6.62 & & 121.30 & 9.50 & 123.00 & 123.00 & to 158.00 & 6.00 \\
\hline GW-374 & SF25 & 6.62 & & 122.90 & 9.50 & 124.60 & 124.60 & to 150.10 & 6.10 \\
\hline GW-375 & SF25 & 6.62 & & 123.30 & 9.50 & 125.00 & 125.00 & to 161.50 & 6.10 \\
\hline GW-376 & SF25 & 6.62 & & 63.00 & 8.75 & 63.00 & 63.00 & to 218.00 & 6.00 \\
\hline GW-381 & SF25 & 6.62 & & 47.80 & 9.50 & 49.30 & 49.30 & to 60.40 & 6.10 \\
\hline GW-382 & SF25 & 6.62 & & 123.20 & 9.50 & 125.00 & 125.00 & to 173.00 & 6.12 \\
\hline GW-384 & SF25 & 6.62 & & 33.80 & 9.50 & 35.50 & 35.50 & to 55.70 & 6.00 \\
\hline GW-385 & SF25 & 6.62 & & 122.00 & 9.50 & 123.70 & 123.70 & to 178.70 & 6.00 \\
\hline GW-400 & STL & 2.00 & & 547.00 & 3.70 & 547.00 & 547.00 & to 577.00 & 3.70 \\
\hline GW-403 & PVC40 & 1.00 & & 275.00 & 3.70 & 275.00 & 275.00 & to 300.00 & 3.70 \\
\hline GW-404 & STL & 4.00 & & 20.00 & 3.75 & & & to 200.00 & 3.70 \\
\hline GW-451 & STL & 8.63 & 8.00 & 267.00 & 11.25 & 267.00 & 267.00 & to 300.00 & 6.00 \\
\hline GW-468 & STL & 4.50 & 4.00 & 27.00 & & 500.50 & 500.50 & to 500.50 & \\
\hline GW-499Q & & & & & & 51.50 & 51.50 & to 51.50 & \\
\hline GW-502 & SF25 & 6.62 & & 221.90 & 9.50 & 223.70 & 223.70 & to 260.00 & 6.10 \\
\hline GW-514 & SF25 & 6.62 & & 172.30 & 9.50 & 174.00 & 174.00 & to 195.00 & 6.00 \\
\hline GW-526 & SF25 & 6.62 & & 99.70 & 9.50 & 101.00 & 101.00 & to 123.00 & 6.10 \\
\hline GW-601 & STL & 7.00 & & 317.20 & 10.63 & 318.50 & 318.50 & to 356.00 & 6.25 \\
\hline GW-608 & & & & & & 148.00 & 148.00 & to 220.00 & 9.50 \\
\hline GW-612 & SF25 & 7.00 & 6.54 & 230.60 & 10.63 & 230.60 & 230.60 & to 254.00 & 6.25 \\
\hline GW-615 & SF25 & 7.00 & 6.54 & 221.20 & 10.63 & 222.50 & 222.50 & to 245.00 & 6.25 \\
\hline GW-616 & SF25 & 7.00 & 6.54 & 217.80 & 10.63 & 219.10 & 219.10 & to 269.00 & 6.25 \\
\hline GW-625 & SF25 & 7.00 & 6.54 & 230.20 & 10.63 & 231.50 & 231.50 & to 283.00 & 6.50 \\
\hline
\end{tabular}




\begin{tabular}{|c|c|c|c|c|c|c|c|c|c|}
\hline CURRENT & CASING & CASING & CASING & CASING & HOLE & HOLE & & & OPEN INTERVAL \\
\hline NAME & TYPE & $\underline{\text { O.D. }}$ & I.D. & DEPTH & DIAMETER & DEPTH & OPEN & INTERVAL & DIAMETER \\
\hline GW-627 & SF25 & 7.00 & 6.54 & 252.70 & 10.63 & 254.00 & 254.00 & to 270.00 & 6.25 \\
\hline GW-628 & SF25 & 7.00 & 6.54 & 234.50 & 10.63 & 235.80 & 235.80 & to 288.00 & 6.25 \\
\hline GW-629 & SF25 & 7.00 & 6.54 & 266.30 & 6.50 & 262.30 & 262.30 & to 312.00 & 6.50 \\
\hline GW-636 & SF25 & 7.00 & 6.54 & 97.50 & 10.75 & 98.50 & 98.50 & to 117.00 & 6.25 \\
\hline GW-639 & SF25 & 7.00 & 6.54 & 94.50 & 10.00 & 95.50 & 95.50 & to 125.50 & 6.25 \\
\hline GW-673 & SS304 & 4.00 & & 112.00 & 7.88 & 112.00 & 112.00 & to 133.00 & 4.00 \\
\hline GW-685 & SF25 & 7.00 & 6.54 & 87.30 & 10.63 & 88.30 & 88.30 & to 138.30 & 6.63 \\
\hline GW-694 & SF25 & 7.00 & 6.45 & 152.00 & 10.60 & 153.00 & 153.00 & to 204.50 & 6.25 \\
\hline GW-703 & SF25 & 7.00 & 6.54 & 132.80 & 10.63 & 133.80 & 133.80 & to 182.00 & 6.25 \\
\hline GW-704 & SF25 & 7.00 & 6.54 & 243.50 & 10.63 & 244.50 & 244.50 & to 256.00 & 6.25 \\
\hline GW-705 & SF25 & 7.00 & 6.54 & 236.00 & 10.60 & 237.00 & 237.00 & to 307.00 & 6.25 \\
\hline GW-706 & SF25 & 7.00 & 6.54 & 155.10 & 10.60 & 156.10 & 156.10 & to 182.50 & 6.25 \\
\hline GW-710 & SF25 & 7.00 & 6.54 & 538.40 & 10.60 & 539.70 & 539.70 & to 744.50 & 6.25 \\
\hline GW-711 & SF25 & 7.00 & 6.54 & 614.70 & 10.60 & 616.00 & 616.00 & to 666.20 & 6.25 \\
\hline GW-712 & SF25 & 7.00 & 6.54 & 440.20 & 10.60 & 441.50 & 441.50 & to 457.50 & 6.25 \\
\hline GW-713 & SF25 & 7.00 & 6.54 & 303.70 & 10.60 & 305.00 & 305.00 & to 315.20 & 6.25 \\
\hline GW-714 & SF25 & 7.00 & 6.54 & 113.80 & 10.60 & 115.10 & 115.10 & to 145.00 & 6.25 \\
\hline GW-722 & SJ55 & 4.50 & 4.00 & 74.50 & 5.00 & 640.00 & & to & 3.50 \\
\hline GW-723 & SF25 & 7.00 & 6.54 & 339.30 & 10.60 & 340.60 & 340.60 & to 444.50 & 6.25 \\
\hline GW-724 & SF25 & 7.00 & 6.54 & 288.30 & 10.60 & 289.60 & 289.60 & to 301.60 & 6.25 \\
\hline GW-725 & SF25 & 7.00 & 6.54 & 131.20 & 10.60 & 132.50 & 132.50 & to 142.50 & 6.25 \\
\hline GW-726 & SF25 & 7.00 & 6.54 & 39.90 & 10.63 & 596.00 & & to & 3.64 \\
\hline GW-727 & SF25 & 7.00 & 6.54 & 237.50 & 10.63 & 997.00 & & to & 3.64 \\
\hline GW-728 & STL/? & 4.50 & 4.00 & 295.00 & 6.25 & 105.80 & 105.80 & to 305.30 & 3.64 \\
\hline GW-729 & STL/? & 4.50 & 4.00 & 281.50 & 6.25 & 1361.00 & & to & 3.64 \\
\hline GW-730 & STL & 4.50 & 4.00 & 420.30 & 6.25 & 1384.00 & & to & 3.64 \\
\hline GW-733 & SF25 & 7.00 & 6.54 & 238.80 & 10.60 & 240.10 & 240.10 & to 256.50 & 6.25 \\
\hline GW-734 & SF25 & 7.00 & 6.54 & 57.00 & 10.60 & 57.00 & 57.00 & to 59.40 & 10.60 \\
\hline
\end{tabular}




\begin{tabular}{|c|c|c|c|c|c|c|c|c|c|}
\hline CURRENT & CASING & CASING & CASING & CASING & HOLE & HOLE & \multirow{2}{*}{\multicolumn{2}{|c|}{ OPEN INTERVAL }} & \multirow{2}{*}{$\begin{array}{l}\text { OPEN INTERVAL } \\
\text { DIAMETER }\end{array}$} \\
\hline NAME & TYPE & $\underline{\text { O.D. }}$ & I.D. & DEPTH & DIAMETER & DEPTH & & & \\
\hline GW-739 & SF25 & 7.00 & 6.54 & 287.90 & 10.60 & 289.20 & 289.20 & to 320.00 & 6.25 \\
\hline GW-740 & SF25 & 7.00 & 6.54 & 164.30 & 10.60 & 165.60 & 165.60 & to 190.00 & 6.25 \\
\hline GW-742 & SF25 & 7.00 & 6.54 & 348.70 & 10.60 & 350.00 & 350.00 & to 420.00 & 6.25 \\
\hline GW-790 & STL & 4.50 & 4.00 & 212.50 & 3.64 & 1040.30 & & to & 3.64 \\
\hline GW-833 & PVC & & 4.00 & 6.10 & 6.00 & 6.10 & 6.10 & to 10.00 & 6.00 \\
\hline GW-845 & & & & & & 156.90 & 156.90 & to 438.30 & 7.88 \\
\hline GW-934 & & & & 65.00 & 4.00 & 400.40 & & to & 4.00 \\
\hline OR-03 & PVC & 2.00 & 1.75 & & & & & to 100.00 & \\
\hline OR-06 & NONE & & & & & & & to 100.00 & 3.00 \\
\hline OR-07 & PVC & 2.00 & 1.75 & 36.00 & 3.00 & 36.00 & 36.00 & to 100.00 & 3.00 \\
\hline OR-08 & PVC & 2.00 & 1.75 & 42.00 & 3.00 & 42.00 & 42.00 & to 100.00 & 3.00 \\
\hline OR-21 & PVC & 2.00 & 1.75 & 37.50 & 3.00 & 37.50 & 37.50 & to 100.00 & 3.00 \\
\hline OR-22 & PVC & 2.00 & 1.75 & 28.20 & 3.00 & 28.20 & 28.20 & to 100.00 & 3.00 \\
\hline UNIDENT A & PVC & 4.50 & 4.00 & 19.30 & & 19.30 & 19.30 & to 36.20 & \\
\hline
\end{tabular}


APPENDIX F

Borehole and Well Hydrogeologic Data Summary 
This Page Intentionally Left Blank 


\begin{tabular}{|c|c|c|c|c|c|}
\hline \multirow{2}{*}{$\begin{array}{c}\text { CURRENT } \\
\text { NAME }\end{array}$} & \multicolumn{5}{|c|}{ FRACTURES, } \\
\hline & T.O.W.R & T.O.F.R & AQUIFER & CAVITIES, and WATER BREAKS & WELL SAMPLE HISTORY \\
\hline 001-L & 5.00 & & & & \\
\hline $002-L$ & 20.00 & & & & \\
\hline 003-L & 10.00 & & & & \\
\hline 004-L & 7.50 & & & & \\
\hline $005-\mathrm{L}$ & 12.30 & 29.00 & & CAV @ 14.2-18.7(m), 29(m) & \\
\hline 006-L & 6.00 & & & & \\
\hline 007-L & 7.00 & 31.00 & & & \\
\hline 008-L & 10.30 & 42.30 & & CAV@ 13.7-19.7, 24.7-31.7, 32-42.3 & \\
\hline $009-\mathrm{L}$ & 6.00 & & & & \\
\hline 010-L & 10.00 & 52.00 & & & \\
\hline 011-L & & 11.20 & & CAV@ 37.8-38.2 & \\
\hline 012-L & 5.00 & & & & \\
\hline 013-L & 3.00 & & & & \\
\hline 014-L & & 20.80 & & & \\
\hline 015-L & 10.00 & & & & \\
\hline 016-L & 10.00 & & & & \\
\hline 017-L & 10.50 & 12.50 & & CAV @ 12.2-12.5(m), 23.8-24.3(o) & \\
\hline 018-L & 15.00 & & & & \\
\hline 019-L & 5.00 & 17.00 & & & \\
\hline $020-\mathrm{L}$ & & 17.30 & & & \\
\hline 021-L & 5.00 & & & & \\
\hline 022-L & 9.00 & & & & \\
\hline 023-L & 4.10 & & & & \\
\hline 024-L & 17.00 & 30.00 & & CAV @ 21-21.1(m), 27.7-29.4(m) & \\
\hline 025-L & 5.00 & 29.00 & & & \\
\hline
\end{tabular}




\begin{tabular}{|c|c|c|c|c|c|}
\hline \multirow{2}{*}{$\begin{array}{c}\text { CURRENT } \\
\text { NAME }\end{array}$} & \multicolumn{5}{|c|}{ FRACTURES, } \\
\hline & $\underline{\text { T.O.W.R }}$ & T.O.F.R & $\underline{\text { AQUIFER }}$ & CAVITIES, and WATER BREAKS & WELL SAMPLE HISTORY \\
\hline 026-L & 15.00 & 30.70 & & & \\
\hline 027-L & 8.00 & 24.00 & & & \\
\hline 028-L & 9.00 & 13.00 & & & \\
\hline 029-L & 7.00 & 31.00 & & CAV@ 27.7-31 & \\
\hline 030-L & & 18.60 & & & \\
\hline 031-L & 5.50 & 26.00 & & CAV @ 20.2-21(m), 25-26(m) & \\
\hline 032-L & 3.50 & 17.70 & & CAV@ 10-10.6(m), 17.2-17.7 & \\
\hline 033-L & 16.00 & 51.00 & & & \\
\hline 034-L & & 11.00 & & & \\
\hline 035-L & 10.30 & 16.40 & & CAV@ 16-16.4 & \\
\hline 1001 & 30.00 & & $u$ & & \\
\hline 1002 & & & wB & & \\
\hline 1003 & 15.00 & 26.00 & wB & & \\
\hline 1004 & 12.00 & 27.00 & $w B$ & & \\
\hline 1005 & 24.00 & & $u$ & & \\
\hline 1006 & & & $u$ & & \\
\hline 1007 & & & $u$ & & \\
\hline 1008 & & & $u$ & & \\
\hline 1009 & & & U & & \\
\hline 1010 & & & U & & \\
\hline 1011 & & & U & & \\
\hline 1012 & 40.00 & & U & & \\
\hline 1013 & 28.00 & & U & & \\
\hline 1014 & 36.00 & & U & & \\
\hline 1015 & 29.50 & & U & & \\
\hline
\end{tabular}




\begin{tabular}{|c|c|c|c|c|c|}
\hline \multirow{2}{*}{$\begin{array}{c}\text { CURRENT } \\
\text { NAME }\end{array}$} & \multicolumn{5}{|c|}{ FRACTURES, } \\
\hline & T.O.W.R & T.O.F.R & AQUIFER & CAVITIES, and WATER BREAKS & WELL SAMPLE HISTORY \\
\hline 1016 & 32.00 & & $u$ & & \\
\hline 1017 & 29.00 & & $u$ & & \\
\hline 1018 & 36.00 & & $u$ & & \\
\hline 1019 & 40.00 & & $u$ & & \\
\hline 1020 & 21.60 & & $u$ & & \\
\hline 1021 & 23.00 & & $u$ & & \\
\hline 1022 & 30.00 & & $u$ & & \\
\hline 1023 & 21.00 & & $u$ & & \\
\hline 1024 & 20.00 & & $u$ & & \\
\hline 1025 & 30.00 & & $u$ & & \\
\hline $1025 A$ & & & $w B / B$ & & \\
\hline 1026 & 46.00 & & $u$ & & \\
\hline 1027 & 15.00 & 42.00 & $w B / B$ & & \\
\hline 1028 & & & $u$ & & \\
\hline 1029 & 6.00 & 18.50 & $\mathrm{U} / \mathrm{wB}$ & & \\
\hline 1030 & 16.00 & 40.00 & $w B$ & & \\
\hline 1031 & 10.00 & & wB & & \\
\hline 1032 & 3.00 & 38.00 & $w B$ & & \\
\hline 1033 & & & B & & \\
\hline 1034 & 15.00 & 30.00 & wB & & \\
\hline 1035 & 15.00 & 23.00 & B & & \\
\hline 1036 & 10.00 & 33.00 & B & & \\
\hline 1037 & & 15.00 & wB & & \\
\hline 1038 & & 25.00 & wB & & \\
\hline 1040 & 6.00 & 23.00 & $\mathrm{U} / \mathrm{wB}$ & WB @ 23 & \\
\hline
\end{tabular}




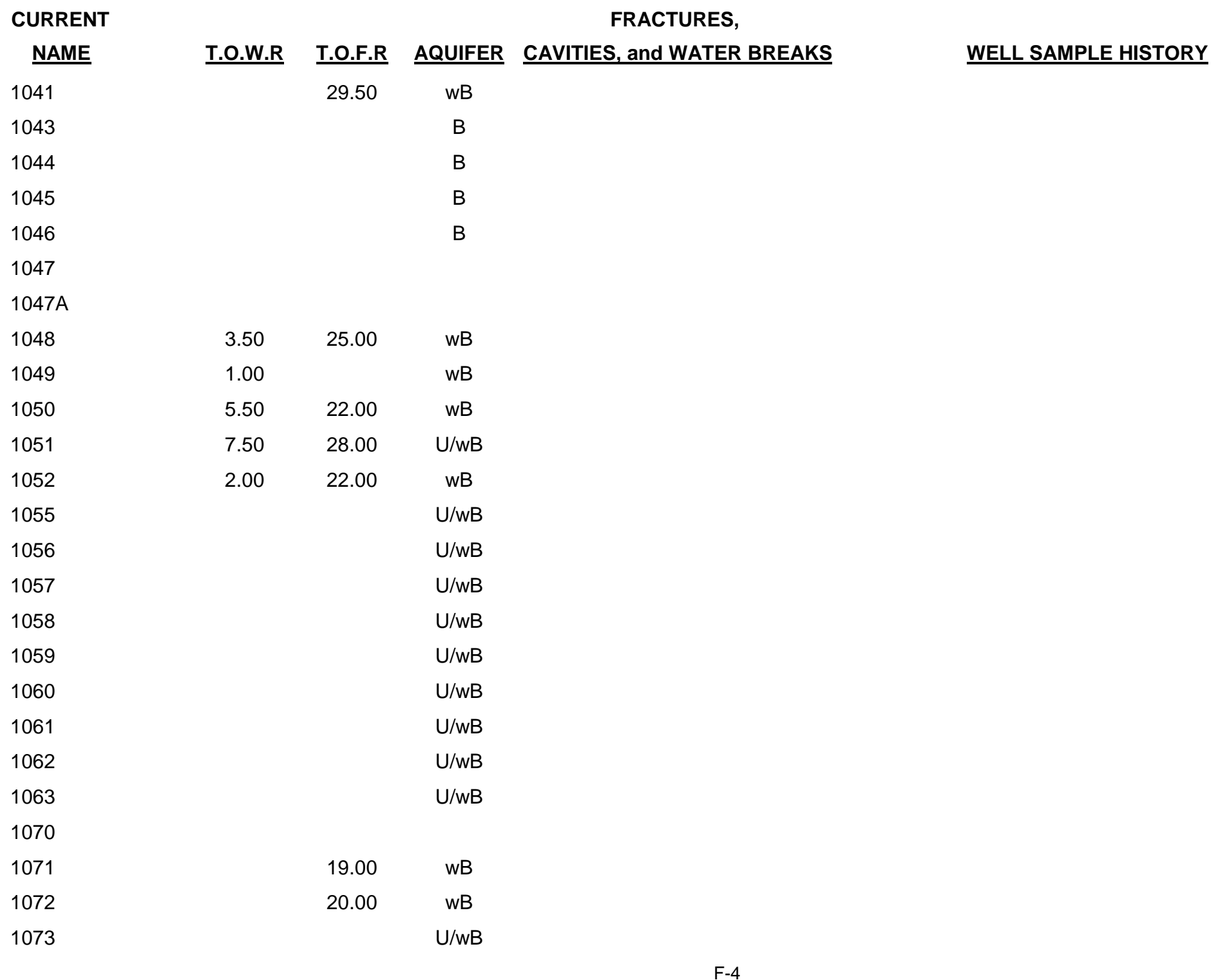




\begin{tabular}{|c|c|c|c|c|c|}
\hline CURRENT & & & & FRACTURES, & \\
\hline 1081 & & & wB & WB@ @ 33 & \\
\hline 1083 & & & wB & WB@ @ 54 & \\
\hline 1084 & & & B & WB @ 120-123 & \\
\hline 1087 & & 57.00 & B & CAV@ 72.5-77 / FRAC @ 130-131(w) & Q1/90 \\
\hline 1088 & & & $w B$ & & \\
\hline 1090 & & & wB & & $\begin{array}{l}\text { Q1/86-Q1/87, Q3/87, Q1/88-Q4/94, Q2/95, Q4/95, Q2/96, Q4/96, } \\
\text { Q2/97, Q4/97, Q3/98, Q1/99, Q3/99, Q1/00, Q3/00, Q1/01, } \\
\text { Q3/01, Q1/02, Q3/02, Q1/03, Q3/03, Q1/04, Q3/04, Q1/05, } \\
\text { Q3/05, Q1/06, Q3/06, Q1/07, }\end{array}$ \\
\hline 1096 & 60.00 & & $u$ & & Q1/86-Q2/86, Q1/87, Q3/87-Q4/88 \\
\hline 1097 & 20.00 & & $w B$ & & \\
\hline 1100 & & & $u$ & & \\
\hline 1101 & & & $u$ & & \\
\hline 1102 & & & $w B$ & & \\
\hline 1103 & & & $u$ & & \\
\hline 1104 & & & $w B$ & & \\
\hline 1105 & & & wB & & \\
\hline 1106 & & & $u$ & & \\
\hline
\end{tabular}




\begin{tabular}{|c|c|c|c|c|c|}
\hline \multirow{2}{*}{$\begin{array}{c}\text { CURRENT } \\
\text { NAME }\end{array}$} & \multicolumn{5}{|c|}{ FRACTURES, } \\
\hline & T.O.W.R & T.O.F.R & AQUIFER & CAVITIES, and WATER BREAKS & WELL SAMPLE HISTORY \\
\hline 1107 & & & wB & & \\
\hline 1108 & & & $\mathrm{U}$ & & \\
\hline 1109 & & & $u$ & & \\
\hline 1110 & & & $\mathrm{U}$ & & \\
\hline 1111 & & & $u$ & & \\
\hline 1112 & & & $u$ & & \\
\hline 1130 & 23.00 & & $\mathrm{U} / \mathrm{wB}$ & & \\
\hline 1131 & & & $U$ & & \\
\hline 1132 & & & $u$ & & \\
\hline 1133 & & & $u$ & & \\
\hline 1134 & 76.00 & 106.50 & $\mathrm{U} / \mathrm{wB}$ & & \\
\hline $2 \mathrm{D}$ & & & $u$ & & \\
\hline 21 & & & $u$ & & \\
\hline $2 S$ & & & $u$ & & \\
\hline 38-DC & 6.00 & 15.50 & $w B$ & & \\
\hline 39-DC & 4.50 & 14.00 & $w B$ & & \\
\hline 3D & & & $U$ & & \\
\hline 31 & & & $u$ & & \\
\hline $3 S$ & & & $U$ & & \\
\hline 40-DC & 6.50 & 18.00 & wB & & \\
\hline 41-DC & 15.00 & 35.50 & wB & & \\
\hline 42-DC & 1.00 & 28.50 & wB & & \\
\hline 44-DC & 7.50 & 35.40 & wB & & \\
\hline 45-DC & 10.50 & 22.50 & wB & & \\
\hline $4 \mathrm{D}$ & & & $u$ & & \\
\hline
\end{tabular}




\section{CURRENT NAME}

41

$4 S$

53-1A

54-1A

54-2A

55-1A

55-1B

55-1C

$55-2 A$

$55-2 B$

$55-2 C$

55-3A

55-3B

55-3C

55-4B

55-4C

55-5A

55-6A

55-7A

55-8A

55-8B

55-9A
FRACTURES,

T.O.W.R T.O.F.R AQUIFER CAVITIES, and WATER BREAKS

$U$

U

U

U

U

WB

$W B$

$W B / B$

$U / w B$

WB

47.20

B

U/wB

wB

B

wB

4.00

6.00

34.20

U

U

22.30

18.30

19.00

18.50

ER

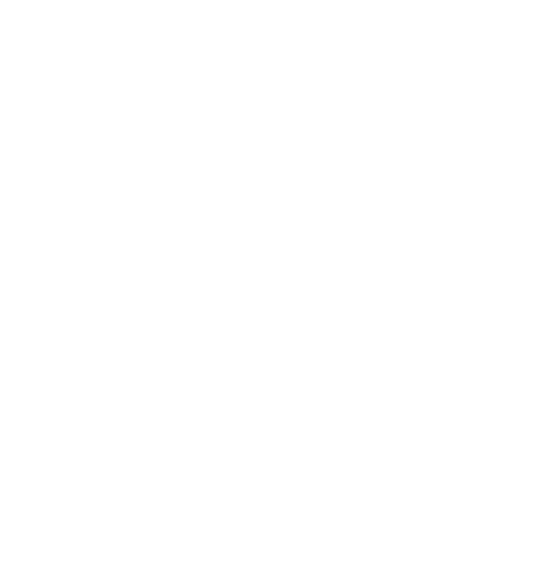

B




\begin{tabular}{|c|c|c|c|c|}
\hline CURRENT & & & & FRACTURES, \\
\hline$\underline{\text { NAME }}$ & $\underline{\text { T.O.W.R }}$ & T.O.F.R & AQUIFER & CAVITIES, and WATER BREAKS \\
\hline $56-1 A$ & 2.00 & & wB & \\
\hline $56-1 C$ & 6.00 & 10.00 & B & \\
\hline $56-2 A$ & & & $u$ & \\
\hline $56-2 B$ & & & $u$ & \\
\hline $56-2 C$ & 11.50 & 17.00 & B & \\
\hline $56-3 A$ & 3.50 & & $u$ & \\
\hline $56-3 B$ & & & $u$ & \\
\hline $56-3 C$ & 8.00 & & $w B$ & \\
\hline $56-4 \mathrm{~A}$ & 8.50 & & $\mathrm{U} / \mathrm{wB}$ & \\
\hline $56-4 B$ & 9.50 & & $w B$ & \\
\hline $56-4 \mathrm{C}$ & 8.30 & 28.50 & B & \\
\hline $56-5 A$ & & & $u$ & \\
\hline $56-5 B$ & & & $u$ & CAV@ 35.1 \\
\hline $56-5 C$ & 17.00 & 30.20 & B & CAV@ @ 34-37, 42-43.5 \\
\hline $56-6 A$ & & & $u$ & \\
\hline $56-7 A$ & & & $U$ & \\
\hline $56-8 \mathrm{~A}$ & & & $u$ & \\
\hline $56-9 \mathrm{~A}$ & & & $u$ & \\
\hline $58-1 \mathrm{~A}$ & 3.00 & & $\mathrm{U} / \mathrm{wB}$ & \\
\hline $58-2 A$ & & & U & \\
\hline
\end{tabular}

\section{WELL SAMPLE HISTORY}

Q2/06, Q4/06, Q2/07, Q4/07, Q2/08, Q3/09, Q1/10, Q2/11, $\mathrm{Q} 4 / 12, \mathrm{Q} 2 / 13$

Q2/08, Q3/09, Q1/10, Q2/11, Q4/12, Q2/13

$\mathrm{Q} 1 / 98, \mathrm{Q} 2 / 04, \mathrm{Q} 4 / 04, \mathrm{Q} 4 / 05-\mathrm{Q} 1 / 06, \mathrm{Q} 3 / 06, \mathrm{Q} 1 / 07-\mathrm{Q} 2 / 07, \mathrm{Q} 2 / 08$, Q3/09, Q1/10, Q2/11, Q4/12, Q2/13

Q1/98, Q2/04, Q4/04, Q4/05-Q1/06, Q3/06, Q1/07-Q2/07, Q2/08, Q3/09, Q1/10, Q2/11, Q4/12, Q2/13

Q1/98, Q2/00, Q4/00, Q2/03, Q4/03, Q1/06, Q3/06, Q1/07, Q2/08, Q3/09, Q1/10, Q2/11, Q4/12, Q2/13

Q1/06, Q3/06, Q1/07, Q3/07, Q2/08, Q3/09, Q1/10, Q2/11, $\mathrm{Q} 4 / 12, \mathrm{Q} 2 / 13$

Q1/06, Q3/06, Q1/07, Q3/07, Q2/08, Q3/09, Q1/10, Q2/11, Q4/12, Q2/13

Q1/06, Q3/06, Q1/07, Q3/07, Q2/08, Q3/09, Q1/10, Q2/11, Q4/12, Q2/13

Q1/06, Q3/06, Q1/07, Q3/07, Q2/08, Q2/09, Q1/10, Q2/11, Q4/12, Q2/13

Q1/06, Q3/06, Q1/07, Q3/07, Q2/08, Q4/10, Q2/12

Q2/06, Q4/06 


\begin{tabular}{|c|c|c|c|c|}
\hline \multirow{2}{*}{$\begin{array}{l}\text { CURRENT } \\
\text { NAME }\end{array}$} & \multirow[b]{2}{*}{$\underline{\text { T.O.W.R }}$} & \multirow[b]{2}{*}{$\underline{\text { T.O.F.R }}$} & \multicolumn{2}{|r|}{ FRACTURES, } \\
\hline & & & $\underline{\text { AQUIFER }}$ & CAVITIES, and WATER BREAKS \\
\hline $59-1 \mathrm{~A}$ & 5.00 & & $w B$ & \\
\hline $59-1 B$ & & & $u$ & \\
\hline $59-1 C$ & 22.00 & 60.00 & B & \\
\hline $5 \mathrm{D}$ & & & $u$ & \\
\hline 51 & & & $u$ & \\
\hline $5 S$ & & & $u$ & \\
\hline $60-1 \mathrm{~A}$ & & & $u$ & \\
\hline $60-1 B$ & & & $u$ & \\
\hline $60-1 C$ & & & & $\begin{array}{l}\text { FRAC @ 21.2, 30, 39.5-40 / CAV @ 31- } \\
33.5,42.5-43.2,44.5-46.5\end{array}$ \\
\hline $60-2 A$ & & & $u$ & \\
\hline AN:D-1 & 4.90 & & $u$ & \\
\hline AN:D-10 & & & $u$ & \\
\hline AN:D-11 & & & 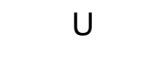 & \\
\hline AN:D-2 & 8.20 & & $u$ & \\
\hline AN:D-3 & 9.70 & & $u$ & \\
\hline AN:D-4 & 4.90 & & $u$ & \\
\hline AN:D-5 & 6.10 & & $u$ & \\
\hline AN:D-6 & 3.80 & & $u$ & \\
\hline AN:D-7 & 8.00 & & $u$ & \\
\hline AN:D-8 & 8.40 & & $u$ & \\
\hline AN:D-9 & & & $u$ & \\
\hline AN:D-UNKN & & & & \\
\hline B-1 & & & $u$ & \\
\hline B-3 & & & $u$ & \\
\hline
\end{tabular}

\section{WELL SAMPLE HISTORY}

Q1/98, Q2/03, Q4/03

Q1/98, Q2/03, Q4/03, Q2/04

Q1/98, Q2/03, Q4/03

Q2/06, Q4/06, Q2/07, Q4/07, Q4/08, Q2/10, Q2/12 Q2/03, Q4/03

Q2/03, Q4/03 


\begin{tabular}{|c|c|c|c|c|c|}
\hline \multirow{2}{*}{$\begin{array}{c}\text { CURRENT } \\
\text { NAME }\end{array}$} & \multicolumn{5}{|c|}{ FRACTURES, } \\
\hline & $\underline{\text { T.O.W.R }}$ & $\underline{\text { T.O.F.R }}$ & $\underline{\text { AQUIFER }}$ & CAVITIES, and WATER BREAKS & WELL SAMPLE HISTORY \\
\hline BC-01 & 12.00 & 20.40 & $w B / B$ & & \\
\hline BC-03 & 12.00 & 14.00 & $w B / B$ & & \\
\hline BC-06 & 7.00 & 44.50 & $w B / B$ & & \\
\hline BC-07 & 9.00 & 33.00 & $w B / B$ & & \\
\hline BC-08 & & 21.00 & $w B / B$ & & \\
\hline BC-09 & 12.00 & 24.00 & $w B / B$ & & \\
\hline BC-10 & 13.00 & 45.00 & $w B / B$ & & \\
\hline BC-11 & 23.00 & 48.50 & $w B / B$ & & \\
\hline BC-14 & 8.00 & 11.50 & wB & & \\
\hline BC-15 & 7.00 & 15.00 & wB & & \\
\hline BC-16 & 12.00 & 15.00 & wB & & \\
\hline BC-17 & 12.00 & 13.90 & wB & & \\
\hline BC-18 & 8.00 & 14.00 & wB & & \\
\hline BC-19 & 7.00 & 10.40 & $w B / B$ & & \\
\hline BC-21 & 7.00 & 10.00 & wB & & \\
\hline BC-22 & 3.00 & 10.20 & wB & & \\
\hline BC-23 & 7.00 & 14.50 & $w B$ & & \\
\hline BC-25 & 8.00 & 22.00 & wB & & \\
\hline BC-30 & & 16.00 & $w B$ & & \\
\hline BC-31 & 8.80 & 10.00 & $w B$ & & \\
\hline BC-32 & 16.50 & 18.80 & $w B$ & & \\
\hline BC-33 & 8.00 & 13.70 & $w B / B$ & & \\
\hline BC-34 & 13.00 & 21.00 & $w B / B$ & & \\
\hline BC-35 & 18.00 & 27.00 & $w B / B$ & & \\
\hline BC-36 & 15.00 & 16.00 & $w B / B$ & & \\
\hline & & & & F-10 & \\
\hline
\end{tabular}




\begin{tabular}{|c|c|c|c|c|c|}
\hline \multirow{2}{*}{$\begin{array}{c}\text { CURRENT } \\
\text { NAME }\end{array}$} & \multicolumn{5}{|c|}{ FRACTURES, } \\
\hline & T.O.W.R & T.O.F.R & $\underline{\text { AQUIFER }}$ & CAVITIES, and WATER BREAKS & WELL SAMPLE HISTORY \\
\hline BC-37 & 8.00 & 22.00 & $w B / B$ & & \\
\hline BC-38 & 3.00 & 11.80 & $w B / B$ & & \\
\hline BC-39 & 4.00 & 6.00 & $w B / B$ & & \\
\hline BC-40 & & 68.00 & $w B / B$ & & \\
\hline BC-41 & 3.00 & 21.80 & $w B / B$ & & \\
\hline BC-42 & 13.00 & 36.20 & $w B / B$ & & \\
\hline BC-43 & 8.00 & 45.00 & $w B / B$ & & \\
\hline BC-45 & 2.50 & 30.40 & wB/B & & \\
\hline BC-47 & 3.00 & 12.00 & $w B / B$ & & \\
\hline BC-49 & & 50.00 & wB & CAV@ @ 53.4-54.3 & \\
\hline BC-50 & 13.00 & 13.00 & $w B / B$ & CAV @ 41.4-41.9 & \\
\hline BC-51 & 19.00 & 21.20 & $w B / B$ & & \\
\hline$B C-52$ & 23.00 & 25.50 & $w B / B$ & & \\
\hline BC-53 & 29.80 & 30.00 & $w B / B$ & & \\
\hline BC-54 & 11.00 & 15.50 & $w B / B$ & & \\
\hline BC-57 & & 12.40 & $w B / B$ & CAV @ 47.3-47.4, 49.8-51.6 & \\
\hline BC-59 & & 11.00 & wB/B & & \\
\hline BC-61 & & 11.00 & $w B$ & & \\
\hline BC-62 & & 24.00 & wB & & \\
\hline BC-63 & & 39.30 & $w B / B$ & & \\
\hline BC-64 & & 2.00 & wB/B & & \\
\hline \multicolumn{6}{|l|}{ BCU-01 } \\
\hline \multicolumn{6}{|l|}{$\mathrm{BCU}-02$} \\
\hline \multicolumn{6}{|l|}{ BCU-03 } \\
\hline BCU-04 & & & & & \\
\hline & & & & F-11 & \\
\hline
\end{tabular}




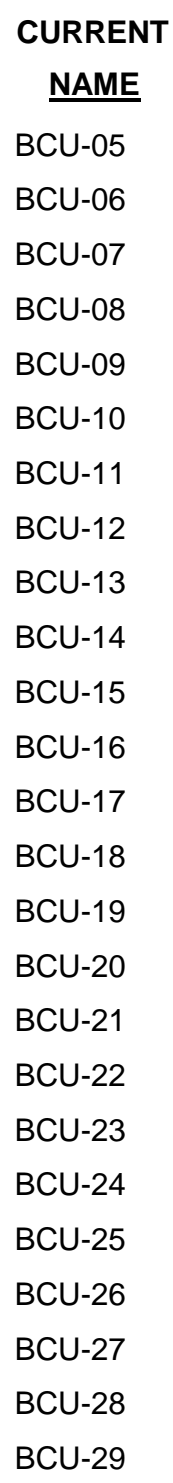




$$
\begin{aligned}
& \text { BY-10 } \\
& \text { BY-11 } \\
& \text { BY-12 } \\
& \text { BY-13 } \\
& \text { BY-14 } \\
& \text { BY-15 } \\
& \text { BY-16 } \\
& \text { BY-17 } \\
& \text { BY-18 } \\
& \text { BY-19 } \\
& \text { BY-20 } \\
& \text { BY-21 } \\
& \text { BY-22 } \\
& \text { BY-23 } \\
& \text { BY-24 } \\
& \text { BY-25 } \\
& \text { BY-26 } \\
& \text { BY-27 } \\
& \text { BY-28 } \\
& \text { BY-29 } \\
& \text { BY-30 } \\
& \text { BY-31 } \\
& \text { BY-32 } \\
& \text { BY-33 } \\
& \text { BY-34 }
\end{aligned}
$$




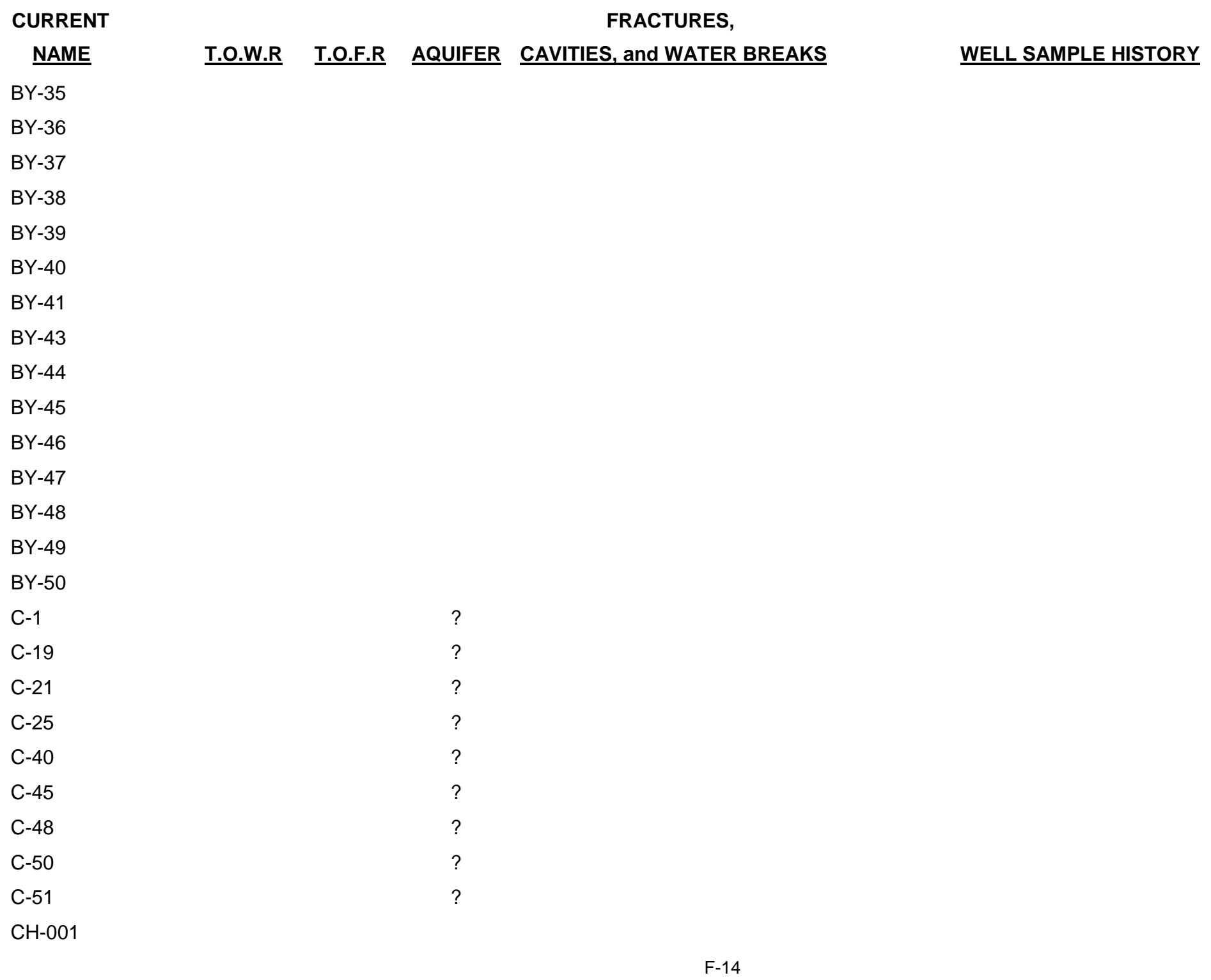




$\begin{array}{lc}\begin{array}{l}\text { CURRENT } \\ \text { NAME }\end{array} & \text { T.O.W.R } \\ \text { CH-002 } & \\ \text { CH-143 } & 9.00 \\ \text { CH-157 } & 63.00 \\ \text { CH-185 } & \\ \text { CH-189 } & \\ \text { CO-2 } & \\ \text { CO-4 } & \\ \text { DC WELL } & \\ \text { ED/Y-12 No. 2 } & \\ \text { EMW-VWUNDRDR } & \\ \text { FP-001 } & \\ \text { FP-002 } & 12.50 \\ \text { FP-003 } & 11.00 \\ \text { FP-004 } & 15.30 \\ \text { FP-005 } & 13.30 \\ \text { FP-006 } & 13.50 \\ \text { FP-007 } & 8.00 \\ \text { FP-008A } & 10.00 \\ \text { FP-008B } & 8.00 \\ \text { FP-009 } & 11.00 \\ \text { FP-010 } & 18.00 \\ \text { FP-011 } & 10.60 \\ \text { FT-001 } & 10.00 \\ \text { FT-002 } & 54.50 \\ \text { FT-003 } & \\ & \\ & \\ & \\ & \\ & \end{array}$

FRACTURES,

WELL SAMPLE HISTORY

Q1/99

$\mathrm{Q} 1 / 06-\mathrm{Q} 4 / 12$ 


$\begin{array}{lccc}\begin{array}{c}\text { CURRENT } \\ \text { NAME }\end{array} & \text { T.O.W.R } & \text { T.O.F.R } & \text { AQUIFER } \\ \text { FW001 } & & & \\ \text { FW002 } & & & \\ \text { FW003 } & & & \\ \text { FW004 } & & & \\ \text { FW005 } & & & \\ \text { GW-001 } & 2.50 & 36.00 & \text { WB } \\ \text { GW-002 } & 2.50 & 36.40 & \text { WB } \\ \text { GW-003 } & 8.90 & 18.10 & \text { WB/B } \\ \text { GW-004 } & 18.50 & 35.40 & \\ \text { GW-005 } & 9.70 & & \text { U/WB } \\ \text { GW-006 } & 6.50 & 16.50 & \text { B } \\ \text { GW-007 } & 6.50 & 16.50 & \text { WB } \\ \text { GW-008 } & 0.60 & & \text { WB } \\ & & & \\ \text { GW-009 } & & & \\ \text { GW-010 } & 9.25 & 17.00 & \\ \text { GW-011 } & 6.60 & & \text { U/WB } \\ \text { GW-012 } & 6.50 & 34.50 & \text { B } \\ \text { GW-013 } & 5.30 & 10.50 & \text { WB } \\ \text { GW-014 } & 4.00 & & \text { WB } \\ \text { GW-015 } & & & \\ \text { GW-016 } & 5.10 & & \text { U/wB } \\ \text { GW-017 } & 7.70 & & \text { WB } \\ & 4.00 & 29.00 & \text { B }\end{array}$

FRACTURES,

$B / B$

$/ \mathrm{wB}$

WB

wB

wB

B

\section{WELL SAMPLE HISTORY}

Q2/88-Q3/88

Q2/90-Q2/91

Q4/98-Q3/00, Q1/08, Q3/08, Q1/10, Q3/12 Q1/87-Q4/87, Q2/88-Q3/88, Q3/89-Q1/90

Q1/98, Q3/98, Q1/99, Q3/99, Q1/00, Q3/00, Q1/01, Q3/01 Q1/02, Q3/02, Q1/03, Q3/03, Q1/04, Q3/04, Q1/05, Q3/05, Q1/06, Q3/06, Q1/07, Q3/07, Q1/08, Q3/08, Q1/09, Q3/09, $\mathrm{Q} 1 / 10, \mathrm{Q} 3 / 10, \mathrm{Q} 1 / 11, \mathrm{Q} 3 / 11, \mathrm{Q} 1 / 1$

Q1/87-Q4/87, Q2/88-Q3/88, Q3/89-Q1/90

\section{Q2/90-Q4/93}

Q1/87-Q4/87, Q2/88-Q3/88, Q1/90, Q3/95, Q1/02, Q3/02, Q2/05, Q4/05-Q1/06, Q3/06, Q1/07, Q3/07, Q3/08, Q1/09, Q3/10, Q1/11, $\mathrm{Q} 3 / 12, \mathrm{Q} 1 / 13$ 


$\begin{array}{lcccc}\begin{array}{c}\text { CURRENT } \\ \text { NAME }\end{array} & \text { T.O.W.R } & \text { T.O.F.R } & \text { AQUIFER } & \begin{array}{c}\text { FRACTURES, } \\ \text { GAVITIES, and WATER BREAKS }\end{array} \\ \text { GW-018 } & & & \text { WB } \\ \text { GW-019 } & 7.00 & 29.00 & \\ \text { GW-020 } & 7.00 & 35.50 & \text { B } \\ \text { GW-021 } & 2.00 & & \text { WB } \\ \text { GW-022 } & 6.00 & 7.50 & \text { B } \\ \text { GW-023 } & 6.50 & 25.00 & \text { B } \\ \text { GW-024 } & 1.00 & 28.00 & \text { B } \\ \text { GW-025 } & & 31.80 & \\ \text { GW-025A } & & & \text { WB } \\ \text { GW-026 } & 3.10 & & \text { WB } \\ \text { GW-027 } & 4.50 & & \text { WB } \\ \text { GW-028 } & 3.70 & & \text { WB } \\ \text { GW-029 } & 3.00 & & \text { WB } \\ \text { GW-030 } & 9.60 & 38.50 & \text { WB } \\ \text { GW-031 } & 9.60 & 38.50 & \text { B } \\ \text { GW-032 } & & 28.60 & \text { B } \\ \text { GW-033 } & 1.00 & 31.00 & \text { B } \\ \text { GW-034 } & 1.40 & & \text { WB } \\ \text { GW-035 } & & 17.00 & \text { B } \\ \text { GW-036 } & 1.50 & & \text { WB } \\ \text { GW-037 } & 1.50 & 39.00 & \text { B } \\ \text { GW-038 } & 3.70 & & \text { WB } \\ \text { GW-039 } & 2.00 & & \text { WB } \\ \text { GW-040 } & & 22.00 & \text { B }\end{array}$

\section{WELL SAMPLE HISTORY}

Q1/91-Q2/91

Q1/87, Q3/87-Q4/87

Q1/87-Q4/87, Q2/88

Q2/90-Q2/91

Q1/87-Q4/87, Q2/88-Q4/88

Q1/87, Q3/87-Q4/87, Q2/88-Q1/89, Q3/89-Q1/90, Q4/91-Q1/96, Q3/96, Q1/97, Q3/97 


\section{CURRENT \\ NAME}

GW-041

GW-042

GW-043

GW-044

GW-045

GW-046

GW-047

GW-048

GW-049

GW-050

GW-051

GW-052

GW-053

GW-054

GW-055

GW-056

GW-057

GW-058

GW-059
FRACTURES,

T.O.W.R T.O.F.R AQUIFER CAVITIES, and WATER BREAKS

25.00

\section{$B$}

WB

$38.00 \quad w B$

$38.00 \quad B$

$U / w B$

$U / w B$

7.70

4.00

3.50

8.70

15.00

11.20

4.00

7.40

6.40

6.40

3.40

20.80

16.00
WB

$U / w B$

$U / w B$

$U$

U

$U$

WB

$40 \quad B$

B

B

\section{WELL SAMPLE HISTORY}

Q1/87-Q4/87, Q2/88-Q1/89, Q3/89-Q3/92, Q4/93-Q1/96, Q3/96, Q1/97, Q3/97

Q1/87-Q3/87, Q2/88, Q1/90-Q1/96, Q3/96, Q1/97, Q3/97, Q4/98$\mathrm{Q} 3 / 00$

Q2/90-Q1/96, Q3/96, Q1/97, Q3/97, Q4/98-Q3/00

Q1/87, Q2/88, Q1/89, Q3/89-Q1/90

Q1/87-Q4/87, Q2/88-Q3/88, Q3/89-Q1/90, Q3/95, Q1/98, Q3/98,

Q1/99, Q3/99, Q1/00, Q3/00, Q1/01, Q3/01, Q1/02, Q3/02,

Q1/03, Q3/03, Q1/04, Q3/04, Q1/05, Q3/05, Q1/06, Q3/06,

$\mathrm{Q} 1 / 07, \mathrm{Q} 3 / 07, \mathrm{Q} 1 / 08, \mathrm{Q} 3 / 08$,

Q1/87-Q4/87, Q2/88-Q1/89, Q3/89-Q1/90, Q1/94-Q3/95

Q2/90, Q1/91, Q4/92-Q2/93, Q1/04, Q3/04, Q1/07

Q2/90-Q3/92, Q1/94-Q1/96, Q3/96, Q1/97, Q3/97, Q1/98, Q3/98, Q1/99, Q3/99, Q1/00, Q3/00, Q1/01, Q3/01, Q2/05, Q4/05, $\mathrm{Q} 1 / 07, \mathrm{Q} 1 / 09, \mathrm{Q} 1 / 11, \mathrm{Q} 3 / 13$

Q2/90-Q3/92

Q2/90-Q1/94, Q3/94, Q1/95, Q3/95, Q1/96, Q3/96, Q1/97, Q3/97, Q1/98, Q3/98, Q1/99, Q3/99, Q1/00, Q3/00, Q1/01, Q2/04

Q2/90-Q2/91, Q1/93-Q1/94, Q3/94, Q1/95, Q3/95

Q4/87, Q2/88-Q1/89, Q3/89-Q3/92, Q2/06, Q1/08, Q1/09, Q3/10$\mathrm{Q} 1 / 11, \mathrm{Q} 3 / 13$ 


\begin{tabular}{|c|c|c|c|c|}
\hline CURRENT & & & & FRACTURES, \\
\hline NAME & T.O.W.R & T.O.F.R & AQUIFER & CAVITIES, and WATER BREAKS \\
\hline GW-060 & & 7.00 & B & \\
\hline GW-061 & & 15.70 & B & \\
\hline GW-062 & & 20.10 & B & \\
\hline GW-063 & & 16.70 & B & \\
\hline GW-064 & & 34.70 & B & \\
\hline GW-065 & & 30.10 & U/wB/B & \\
\hline GW-066 & & 16.20 & B & \\
\hline GW-067 & & 16.50 & U & \\
\hline GW-068 & & 25.00 & B & \\
\hline GW-069 & 3.00 & 24.00 & B & \\
\hline GW-070 & 2.00 & 22.00 & B & \\
\hline GW-071 & 5.50 & 16.00 & B & \\
\hline GW-072 & 5.00 & 34.00 & B & \\
\hline GW-073 & 6.00 & 36.00 & B & \\
\hline GW-074 & 1.00 & 46.00 & B & \\
\hline GW-075 & 6.00 & 14.00 & B & \\
\hline GW-076 & 12.00 & 48.00 & B & \\
\hline GW-077 & 7.00 & 13.00 & B & \\
\hline GW-078 & 6.50 & 8.50 & B & \\
\hline
\end{tabular}

\section{WELL SAMPLE HISTORY}

Q3/89-Q3/92, Q1/94-Q3/95, Q1/02, Q3/02, Q2/05, Q4/05

Q1/87-Q4/87, Q2/88-Q1/89, Q3/89-Q1/93

Q4/87, Q2/88-Q3/95, Q1/98, Q3/98, Q1/05, Q3/05

Q1/08, Q3/08, Q1/09, Q3/09, Q1/10, Q3/10, Q1/12

Q3/95, Q1/02, Q3/02, Q1/05, Q3/05

Q1/87-Q4/87, Q2/88-Q1/89, Q4/89-Q1/90

Q1/87-Q4/87, Q2/88, Q3/89, Q1/90, Q2/05, Q4/05, Q2/06, Q3/08,

Q1/09, Q3/10, Q1/11, Q3/12, Q1/13

Q2/90-Q1/96, Q3/96, Q1/97, Q3/97, Q1/02, Q3/02

Q1/87-Q4/87, O2/88, O3/89, O1/90-O2/91, Q1/02, O3/02, O1/04, $\mathrm{Q} 3 / 04, \mathrm{Q} 2 / 05, \mathrm{Q} 4 / 05-\mathrm{Q} 1 / 06, \mathrm{Q} 3 / 06, \mathrm{Q} 1 / 07, \mathrm{Q} 3 / 07, \mathrm{Q} 1 / 08, \mathrm{Q} 3 / 08$, Q1/09, Q3/09, Q1/10, Q3/10, Q1/11, Q3/11, Q3/13

Q1/87-Q4/87, Q2/88, Q3/89, Q1/90-Q2/91, Q1/02, Q3/02, Q2/05, Q4/05, Q1/07

Q1/87-Q4/87, Q2/88-Q1/89, Q3/89-Q1/90

$\mathrm{Q} 2 / 90-\mathrm{Q} 3 / 92$

Q2/90-Q3/92, Q1/06

$\mathrm{Q} 2 / 90-\mathrm{Q} 2 / 91, \mathrm{Q} 1 / 02$

Q2/90-Q2/91, Q2/97, Q1/98, Q3/98, Q1/99, Q3/99, Q1/00, Q3/00,

Q1/01, Q3/01, Q1/02, Q3/02, Q1/03, Q3/03, Q1/04, Q3/04

$\mathrm{Q} 1 / 05, \mathrm{Q} 3 / 05, \mathrm{Q} 1 / 06, \mathrm{Q} 3 / 06, \mathrm{Q} 1 / 07, \mathrm{Q} 3 / 07, \mathrm{Q} 1 / 08, \mathrm{Q} 3 / 08$,

$\mathrm{Q} 1 / 09, \mathrm{Q} 3 / 09, \mathrm{Q} 1 / 10, \mathrm{Q} 3 / 10$

Q2/90-Q2/91, Q2/97, Q1/98, Q3/98, Q1/99, Q3/99, Q1/00, Q3/00,

Q1/01, Q3/01, Q1/02, Q3/02, Q1/03, Q3/03, Q1/04, Q3/04,

$\mathrm{Q} 1 / 05, \mathrm{Q} 3 / 05, \mathrm{Q} 1 / 06, \mathrm{Q} 3 / 06, \mathrm{Q} 1 / 07, \mathrm{O} 3 / 07, \mathrm{Q} 1 / 08, \mathrm{O} 3 / 08$,

$\mathrm{Q} 1 / 09, \mathrm{Q} 3 / 09, \mathrm{Q} 1 / 10, \mathrm{Q} 3 / 10$ 


\section{CURRENT \\ NAME}

GW-079

GW-080

GW-081

GW-082

GW-083

GW-084

GW-085

GW-086

GW-087

GW-088

GW-089

GW-090

GW-091

GW-092

GW-093

GW-093A

GW-094
FRACTURES,

T.O.W.R T.O.F.R AQUIFER CAVITIES, and WATER BREAKS

4.00

26.50

B

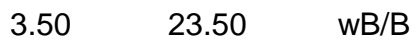

6.50

7.00

23.00

wB

B

4.00

13.70

B

4.00

2.00

40.00

WB

8.00

19.00

WB

U

U

U

$U$

U

U

29.50

2.00

17.00

\section{WELL SAMPLE HISTORY}

Q2/90-Q2/91, Q4/93-Q1/96, Q3/96, Q1/97, Q3/97, Q1/98, Q3/98, Q1/99, Q3/99, Q1/00, Q3/00, Q1/01, Q3/01, Q1/02, O3/02, $\mathrm{Q} 1 / 03, \mathrm{Q} 3 / 03, \mathrm{Q} 1 / 04, \mathrm{Q} 3 / 04, \mathrm{Q} 1 / 05, \mathrm{Q} 3 / 05, \mathrm{Q} 1 / 06, \mathrm{Q} 3 / 06$, $\mathrm{Q} 1 / 07, \mathrm{Q} 3 / 07, \mathrm{Q} 1 / 08, \mathrm{Q} 3 / 08$,

Q2/90-Q3/92, Q4/93-Q1/96, Q3/96, Q1/97, Q3/97, Q1/98, Q3/98, Q1/99, Q3/99, Q1/00, Q3/00, Q1/01, Q3/01, Q1/02, Q3/02, $\mathrm{Q} 1 / 03, \mathrm{Q} 3 / 03, \mathrm{Q} 1 / 04, \mathrm{Q} 3 / 04, \mathrm{Q} 1 / 05, \mathrm{Q} 3 / 05, \mathrm{Q} 1 / 06, \mathrm{Q} 3 / 06$, $\mathrm{Q} 1 / 07, \mathrm{Q} 3 / 07, \mathrm{Q} 1 / 08, \mathrm{Q} 3 / 08$,

Q1/87-Q4/87, Q2/88-Q1/89, Q3/89-Q4/93, Q2/97-Q3/97, Q2/98Q3/98, Q1/99, Q1/01, Q3/01, Q1/02, Q3/02, Q1/03, Q3/03, $\mathrm{Q} 1 / 04, \mathrm{Q} 3 / 04, \mathrm{Q} 2 / 05, \mathrm{Q} 4 / 05, \mathrm{Q} 2 / 06, \mathrm{Q} 1 / 07, \mathrm{Q} 3 / 08, \mathrm{Q} 3 / 09$, $\mathrm{Q} 1 / 10, \mathrm{Q} 3 / 11, \mathrm{Q} 1 / 12, \mathrm{Q} 3 / 13$

$\mathrm{Q} 2 / 90-\mathrm{Q} 3 / 92$

Q1/87-Q4/87, Q2/88-Q1/89, Q3/89-Q1/96, Q3/96, Q1/97, Q3/97

Q4/87, Q2/88-Q3/88, Q3/89-Q1/96, Q3/96, Q1/97, Q3/97, Q1/98, Q3/98, Q1/99, Q3/99, Q1/00, Q3/00, Q1/01, Q3/01, Q1/02, $\mathrm{Q} 3 / 02, \mathrm{Q} 1 / 03, \mathrm{Q} 3 / 03, \mathrm{Q} 1 / 04, \mathrm{Q} 3 / 04, \mathrm{Q} 1 / 05, \mathrm{Q} 3 / 05, \mathrm{Q} 1 / 06$, $\mathrm{Q} 3 / 06, \mathrm{Q} 1 / 07, \mathrm{Q} 3 / 07, \mathrm{Q} 1 / 08$,

Q2/90-Q2/91, Q1/93-Q4/93

Q1/87-Q4/87, Q2/88-Q1/89, Q4/89-Q1/90, Q3/95, Q2/97-Q3/97, Q2/98-Q3/98, Q1/99, Q1/02

Q2/90-Q3/92, Q1/07, Q3/08, Q3/13

Q1/02, Q3/02

Q1/87, Q3/87-Q4/87, Q2/88-Q1/89, Q3/89-Q1/90 


\section{CURRENT \\ NAME}

GW-095

GW-096

GW-096A

GW-097

GW-097A

GW-098

GW-099

GW-100

GW-101

GW-102

GW-103

GW-104

GW-105

GW-106

GW-107

GW-108

GW-109

GW-110

\section{T.O.W.R}

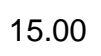

7.00

8.50

1.00

7.50

71.00

14.00

14.00

17.50

U/WB

$U$

wB

WB

U/wB

wB

WB

4.00

5.00

16.00

B

20.00

B

$W B$

$w B$

wB

B

\section{B}

J/wB

1.00

12.50

5.00

4.00

$$
w B
$$

29.30
FRACTURES,

CAVITIES, and WATER BREAKS

FRAC @ 86, 112

B

U

$w B / B$

\section{WELL SAMPLE HISTORY}

Q1/87-Q4/87, Q2/88-Q1/89, Q3/89-Q3/92, Q1/94-Q1/96, Q3/96

Q1/87-Q4/87, Q2/88-Q1/89, Q3/89-Q1/90, Q2/98-Q3/98, Q1/05, Q3/05

Q1/87-Q4/87, Q2/88-Q1/89, Q3/89-Q1/90, Q1/01, Q3/01, Q1/04, Q3/04, Q1/05, Q3/05, Q1/06, Q2/07, Q1/08, Q1/09, Q3/10,

Q1/11, Q3/12, Q1/13

Q1/88-Q2/88

Q1/86-Q2/86, Q1/87-Q2/87, Q4/87, Q3/88-Q1/90, Q3/95, Q1/04, Q3/04, Q2/07, Q1/09, Q3/10, Q1/11, Q3/12, Q1/13

Q1/86-Q2/86, Q2/90-Q2/91, Q1/04, Q3/04, Q2/07, Q1/09, Q3/10, $\mathrm{Q} 1 / 11, \mathrm{Q} 3 / 12, \mathrm{Q} 1 / 13$

\section{Q1/86-Q2/86}

Q1/86-Q2/86

Q1/86-Q2/86, Q1/87-Q2/87, Q4/87, Q2/88-Q1/90, Q2/03, Q4/03, $\mathrm{Q} 2 / 06, \mathrm{Q} 2 / 07, \mathrm{Q} 2 / 08$

Q1/86-Q2/86, Q1/87-Q1/90, Q2/03, Q4/03, Q2/06, Q2/07, Q2/08, Q4/10, Q4/12

Q2/86, Q1/87-Q1/90

Q1/86-Q2/86, Q1/87-Q1/90, Q2/95, Q1/96, Q4/96-Q1/97, Q3/97, Q1/98, Q3/98, Q1/99, Q3/99, Q1/00, Q3/00, Q1/01, Q3/01, $\mathrm{Q} 1 / 02, \mathrm{Q} 3 / 02, \mathrm{Q} 1 / 03, \mathrm{Q} 3 / 03, \mathrm{Q} 1 / 04, \mathrm{Q} 3 / 04, \mathrm{Q} 1 / 05, \mathrm{Q} 3 / 05$ Q1/06, Q3/06, Q1/07, Q3/07,

Q1/86-Q2/86, Q1/87-Q1/90, Q2/95, Q3/99, Q2/00, Q4/00, Q2/03, $\mathrm{Q} 4 / 03, \mathrm{Q} 2 / 06, \mathrm{Q} 2 / 07, \mathrm{Q} 2 / 08, \mathrm{Q} 4 / 08, \mathrm{Q} 4 / 10, \mathrm{Q} 4 / 12$ 


\section{CURRENT \\ NAME}

GW-111

GW-112

GW-113

GW-114

GW-115

GW-116

GW-117

GW-118

GW-119

GW-120

GW-121

GW-122

GW-123

GW-124

GW-125

GW-126

GW-127

GW-128

GW-129

GW-130

GW-131

GW-132
FRACTURES,

T.O.W.R T.O.F.R AQUIFER CAVITIES, and WATER BREAKS

30.30

20.00

19.20

18.00

20.00

$\begin{array}{ccl}40.50 & \text { B } & \\ 18.00 & \text { B } & \text { WB @ 30-55 } \\ 15.00 & \text { B } & \text { WB @ 45-80 } \\ 20.00 & \text { B } & \text { WB @ 30-35 } \\ 17.00 & \text { B } & \\ 20.00 & \text { B } & \text { WB @ 25-50 } \\ 37.00 & \text { B } & \text { WB @ 45-100 } \\ 38.00 & \text { B } & \text { WB @ 47-80 / FRAC @ 47-50 } \\ 30.00 & \text { B } & \text { WB @ 30-75 / FRAC @ 34-36 } \\ 25.00 & \text { B } & \text { CAV @ 40.5-42.5 / WB @ 50-100 } \\ 27.00 & \text { B } \\ & \text { U } \\ & \text { WB/B } \\ \text { WB/B } & \text { WB/B } \\ \text { U/WB/B } \\ \text { U/WB/B }\end{array}$

\section{WELL SAMPLE HISTORY}

Q1/87-Q2/87, Q4/87, Q2/88-Q1/94, Q3/94, Q1/95, Q3/95, Q1/96, Q3/96-Q1/97, Q3/97, Q1/98, Q3/98, Q1/99, Q3/99, Q1/00, Q3/00, Q1/01, Q3/01, Q1/02, Q3/02, Q1/03, Q3/03, Q1/04

Q2/87-Q4/87, Q2/88-Q4/88, Q2/89-Q3/92

Q2/87-Q4/87, Q2/88-Q4/88, Q2/89-Q4/93

Q1/87, Q3/87-Q4/87, Q2/88-Q4/88, Q2/89-Q3/92

Q1/87-Q4/87, Q2/88-Q1/89, Q3/89-Q1/90

Q4/86-Q2/87, Q4/87-Q2/91, Q1/05, Q3/05, Q1/06, Q2/07, Q1/08, Q1/09, Q3/11, Q1/13

Q4/86-Q4/87, Q2/88-Q2/89, Q4/89-Q3/92, Q1/03, Q3/03

Q4/86-Q2/87, Q4/87-Q1/90, Q3/95, Q1/01, Q3/01, Q1/05, Q3/05, Q1/08

Q4/86-Q2/89, Q4/89-Q3/92

Q1/87, Q3/87-Q4/87, Q2/88-Q1/89, Q3/89-Q1/90, Q1/99, Q2/06

Q4/86, Q2/88-Q1/90, Q2/05, Q4/05, Q2/07, Q1/09, Q3/11, Q1/13 


\section{CURRENT \\ NAME}

GW-133

GW-134

GW-135

GW-136

GW-137

GW-138

GW-139

GW-140

GW-141

GW-142

GW-143

18.00

GW-144

GW-145

12.00

GW-146

GW-147

40.00

11.00

6.00

(2)
FRACTURES,
T.O.W.R T.O.F.R AQUIFER CAVITIES, and WATER

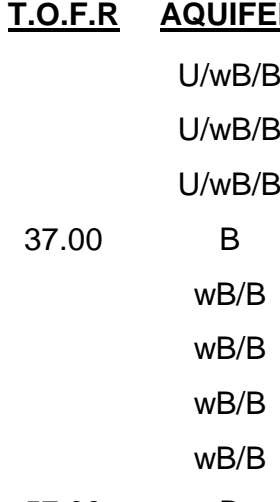

$57.00 \quad$ B

B

B

B

WB@ 170

B 146

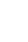

$B$
$B$
CAVITIES, and WATER BREAKS

FRAC @ 120, 145 / CAV @ 145.5 / WB @

FRAC @ 10-12.5(w), 19(w), 20-21(w), 24-

\section{WELL SAMPLE HISTORY}

Q1/88-Q1/95, Q3/95, Q1/96, Q3/96, Q1/97, Q3/97, Q1/98, Q3/98,

Q1/99, Q3/99, Q1/00, Q3/00, Q1/01, Q3/01, Q1/02, Q3/02, Q1/03, Q3/03, Q1/04, Q3/04, Q1/05, Q3/05, Q1/06, Q3/06, Q1/07, Q3/07, Q1/08, Q3/08

Q1/86-Q4/86, Q2/87-Q3/87, Q1/88-Q2/96, Q4/96, Q2/97-Q4/97, $\mathrm{Q} 2 / 98, \mathrm{Q} 4 / 98, \mathrm{Q} 2 / 99, \mathrm{Q} 4 / 99, \mathrm{Q} 2 / 00, \mathrm{Q} 4 / 00, \mathrm{Q} 2 / 01, \mathrm{Q} 4 / 01$

$\mathrm{Q} 2 / 02, \mathrm{Q} 4 / 02, \mathrm{Q} 2 / 03, \mathrm{Q} 4 / 03$

Q1/86-Q4/86, Q2/87-Q3/87, Q1/88-Q2/96, Q4/96, Q2/97-Q4/97,

$\mathrm{Q} 2 / 98, \mathrm{Q} 4 / 98, \mathrm{Q} 2 / 99, \mathrm{Q} 4 / 99, \mathrm{Q} 2 / 00, \mathrm{Q} 4 / 00, \mathrm{Q} 2 / 01, \mathrm{Q} 4 / 01$,

$\mathrm{Q} 2 / 02, \mathrm{Q} 4 / 02, \mathrm{Q} 2 / 03, \mathrm{Q} 4 / 03, \mathrm{Q} 2 / 04, \mathrm{Q} 4 / 04, \mathrm{Q} 2 / 05, \mathrm{Q} 4 / 05$,

Q2/06, Q4/06-Q1/07, Q3/08, Q1

Q1/86-Q4/86, Q2/87-Q3/87, Q1/88-Q2/96, Q4/96, Q2/97-Q4/97, $\mathrm{Q} 2 / 98, \mathrm{Q} 4 / 98, \mathrm{Q} 2 / 99, \mathrm{Q} 4 / 99, \mathrm{Q} 2 / 00, \mathrm{Q} 4 / 00, \mathrm{Q} 2 / 01, \mathrm{Q} 4 / 01$, $\mathrm{Q} 2 / 02, \mathrm{Q} 4 / 02, \mathrm{Q} 2 / 03, \mathrm{Q} 4 / 03, \mathrm{Q} 2 / 04, \mathrm{Q} 4 / 04, \mathrm{Q} 2 / 05, \mathrm{Q} 4 / 05$,

Q2/06, Q4/06-Q1/07, Q3/08, Q1

Q1/86-Q4/86, Q2/87-Q3/87, Q1/88-Q2/90, Q4/90-Q2/96, Q4/96, Q2/97-Q4/97, Q2/98, Q4/98, Q2/99, Q4/99, Q2/00, Q4/00, Q2/01, $\mathrm{Q} 4 / 01, \mathrm{Q} 2 / 02, \mathrm{Q} 4 / 02, \mathrm{Q} 2 / 03, \mathrm{Q} 4 / 03, \mathrm{Q} 2 / 04, \mathrm{Q} 4 / 04, \mathrm{Q} 2 / 05$, Q4/05, Q2/06, Q4/06-Q1/

Q1/86-Q4/86, Q2/87-Q3/87, Q1/88-Q2/90, Q4/90-Q2/96 $25,32.5-33(\mathrm{~m}), 46.5-47.5(\mathrm{~W}), 59.5-60.5$
Q1/86-Q4/86, Q2/87-Q3/87, Q1/88-Q2/90, Q4/90-Q1/91, Q3/91Q2/96 


\section{CURRENT \\ NAME}

GW-148

GW-149

GW-150

GW-151

GW-152

GW-153

GW-154

11.20

GW-155

GW-156

GW-157

GW-158

GW-159

GW-160

11.10

9.00

11.70

17.30

84.00

18.00

60.00
T.O.W.R

14.00

U

B

FRAC @ 29-30

U WB@ $@ 5$

89.00

93.00

87.00

94.00

93.00

100.00

U

WB @ 4, 25-28

FRAC @ 26(w) @ 150
FRACTURES,

AQUIFER CAVITIES, and WATER BREAKS

FRAC @ 130-136, 165-177(mw)

CAV @ 92-93, 101-103, 106-112 / FRAC

$\begin{array}{ll}\text { B } & \text { WB @ 74 / FRAC @ 114-116.5, 140(w) } \\ \text { B } & \text { FRAC @ 98(foam), 180(W), 220-230, 250- } \\ & 260(M W) \\ \text { B } & \text { CAV @ 112-117 / WB @ } 130\end{array}$

wB CAV@ 111, 115-118, 137-140, 169-171, 221-224 / FRAC @ 112, 118, 160, 171

\section{WELL SAMPLE HISTORY}

Q1/86-Q1/90, Q4/94-Q4/96, Q2/97, Q4/97, Q2/98, Q4/98, Q2/99, Q4/99, Q1/06, Q3/08, Q1/10, Q4/12

Q1/86-Q2/91, Q4/94-Q4/96

Q1/86-Q1/88

Q1/86-Q4/96, Q2/97, Q1/98, Q3/98, Q1/99, Q3/99, Q2/00-Q3/00, Q1/01, Q3/01, Q1/02, Q3/02, Q1/03, Q3/03, Q1/04, Q3/04, Q1/05, Q3/05, Q1/06, Q3/06, Q1/07, Q3/07, Q1/08, Q3/08, Q1/09, Q3/09, Q1/10, Q3/10,

Q1/86-Q1/88, Q3/88, Q1/89-Q3/89, Q1/90

Q1/86-Q3/89, Q1/90-Q2/91, Q4/94-Q4/96, Q2/97, Q4/97, Q2/98, Q4/98, Q2/99, Q4/99, Q2/00, Q4/00, Q2/01, Q4/01, Q2/02,

$\mathrm{Q} 4 / 02, \mathrm{Q} 2 / 03, \mathrm{Q} 4 / 03, \mathrm{Q} 2 / 04, \mathrm{Q} 4 / 04, \mathrm{Q} 2 / 05, \mathrm{Q} 4 / 05-\mathrm{Q} 1 / 06, \mathrm{Q} 1 / 07$, $\mathrm{Q} 3 / 08, \mathrm{Q} 4 / 09, \mathrm{Q} 2 / 10, \mathrm{Q}$

Q1/86-Q2/91, Q4/94-Q1/97, Q3/97, Q1/98, Q3/98, Q1/99, Q3/99, $\mathrm{Q} 2 / 00-\mathrm{Q} 3 / 00, \mathrm{Q} 1 / 01, \mathrm{Q} 3 / 01, \mathrm{Q} 1 / 02, \mathrm{Q} 3 / 02, \mathrm{Q} 1 / 03, \mathrm{Q} 3 / 03, \mathrm{Q} 1 / 04$, Q3/04, Q1/05, Q3/05, Q1/06, Q3/06, Q1/07, Q3/07, Q1/08,

Q3/08, Q1/09, Q3/09,

Q1/86-Q1/87, Q3/87-Q2/91

Q1/86-Q1/87, Q3/87-Q1/89, Q1/90-Q4/95, Q2/96, Q4/96, Q2/97,

Q4/97, Q2/98, Q4/98, Q2/99, Q4/99, Q2/00, Q4/00, Q2/01,

$\mathrm{Q} 4 / 01, \mathrm{Q} 2 / 02, \mathrm{Q} 4 / 02, \mathrm{Q} 2 / 03, \mathrm{Q} 4 / 03, \mathrm{Q} 2 / 04, \mathrm{Q} 4 / 04, \mathrm{Q} 2 / 05$, Q4/05, Q2/06, Q4/06-Q1/07, Q

Q1/86-Q1/87, Q3/87-Q1/89, Q3/89-Q1/91

Q1/86-Q2/86, Q1/87, Q3/87-Q3/89, Q1/90-Q3/95

Q1/86-Q1/87, Q3/87-Q4/95, Q2/96, Q4/96, Q2/97, Q4/97, Q2/98, $\mathrm{Q} 4 / 98, \mathrm{Q} 2 / 99, \mathrm{Q} 4 / 99, \mathrm{Q} 2 / 00, \mathrm{Q} 4 / 00, \mathrm{Q} 2 / 01, \mathrm{Q} 4 / 01, \mathrm{Q} 2 / 02$, Q4/02, Q2/03, Q4/03, Q2/04, Q4/04, Q2/05, Q4/05, Q2/06, Q4/06$\mathrm{Q} 1 / 07, \mathrm{Q} 3 / 08, \mathrm{Q} 1 / 09$

Q1/88-Q1/94, Q3/94, Q2/95, Q4/95, Q2/96 


\section{CURRENT \\ NAME}

GW-161

GW-162

GW-163

GW-164

GW-165

GW-166

GW-167

GW-168

GW-169

GW-170

GW-171

GW-172

GW-173

GW-174

GW-175

GW-176

\section{FRACTURES,}

\section{T.O.W.R T.O.F.R AQUIFER CAVITIES, and WATER BREAKS}

65.00

95.50

12.00

$B$

FRAC @ 98, 140, 170, 218, 236, 314, 362 I WB @ 370-374

12.00

10.00

70.00

116.00

50.00

67.00

29.00

30.00

15.00

19.00

73.00

105.00

51.00

80.00

46.00

98.50

84.00
B

U

WB@ 118

FRAC @ 150(w), 197(w), 280, 392(w)

FRAC @ 92-96, 100-102, 312 / CAV @ 103-113, 314-317(mW)

FRAC @ 287-290, 320-323, 358-363 / WB @ 360

$$
\text { U }
$$$$
\text { B }
$$$$
\text { U }
$$

B

CAV@ 16-19 / FRAC @ 117(w), 130(w)

FRAC @ 103.6 / CAV @ 108.9, 140-141 CAV@ 63-79.6, 91, 140 / FRAC @ 143

CAV@85 / WB @ 125-135 / FRAC @ 160, 161

\section{WELL SAMPLE HISTORY}

Q1/88-Q1/94, Q3/94, Q2/95, Q4/95, Q2/96, Q1/07, Q3/07, Q1/08, Q3/08, Q1/09, Q3/09, Q1/10, Q3/10, Q1/11, Q3/11, Q1/12, Q3/12 Q1/87, Q3/87-Q4/87, Q2/88-Q1/89, Q3/89-Q2/91, Q4/93-Q1/96, Q3/96, Q1/97, Q3/97

Q1/87-Q3/87, Q2/90-Q2/91

$\mathrm{Q} 2 / 87, \mathrm{Q} 2 / 90-\mathrm{Q} 2 / 91$

Q2/90-Q2/91

\section{Q2/90-Q2/91}

Q2/90-Q3/91, Q1/96

Q2/90-Q4/93, Q3/99

Q1/91-Q1/94, Q3/94-Q1/96, Q3/97, Q1/98, Q1/99, Q3/99, Q1/00Q3/00, Q2/01-Q3/01, Q1/02-Q3/05, Q1/06-Q3/06, Q1/07, Q3/07, Q1/08, Q1/09, Q3/09, Q1/10, Q3/10, Q1/11, Q1/12, Q3/12

Q2/90, Q1/91-Q1/94, Q3/94-Q2/96, Q3/97, Q2/98-Q3/98, Q1/99, Q3/99, Q1/00-Q3/06, Q1/07, Q3/07, Q1/08, Q3/08, Q1/09, Q3/09, $\mathrm{Q} 1 / 10, \mathrm{Q} 3 / 10, \mathrm{Q} 1 / 11, \mathrm{Q} 3 / 11, \mathrm{Q} 1 / 12, \mathrm{Q} 3 / 12$

Q3/94-Q2/96, Q2/97-Q3/97, Q1/98, Q3/98, Q1/99, Q3/99, Q1/00O3/00, Q1/01, Q3/01, Q1/02, Q3/02, Q3/03, Q1/04, Q3/04, Q2/05Q3/05, Q1/06, Q1/07, Q1/08, Q1/09, Q3/10, Q1/11, Q2/12

Q2/90, Q3/94-Q1/96, Q2/97-Q3/97, Q1/98, Q3/98, Q1/99, Q3/99, Q1/00-O3/00, Q1/01, O3/01, Q1/02, O3/02, O3/03, Q1/04, O3/04, Q1/05, Q3/05, Q1/06, Q1/07, Q1/08, Q1/09, Q3/10, Q1/11, Q1/12 Q1/86-Q3/92, Q2/04, Q4/04, Q2/08, Q4/08

Q1/86, Q1/87-Q3/92, Q3/96, Q1/01, Q3/01, Q2/07, Q4/09, Q3/11, $\mathrm{Q} 2 / 13$

Q3/88-Q1/96, Q1/01, Q3/01, Q2/04, Q4/04, Q2/06, Q4/08, Q4/09, $\mathrm{Q} 3 / 11, \mathrm{Q} 2 / 13$

Q1/86-Q3/92, Q2/04, Q4/04, Q2/07, Q4/09, Q3/11, Q2/13 


\section{CURRENT \\ NAME}

GW-177

GW-178

GW-179

GW-180

GW-181

GW-182

GW-183

GW-184

GW-185

GW-186

GW-187

GW-188

GW-189

GW-190

GW-191

GW-192

\section{T.O.W.R}

62.00

37.00

58.00

43.00

20.00

5.00

12.00

7.00

13.00

17.00

8.00

17.50
FRACTURES,

\section{AQUIFER CAVITIES, and WATER BREAKS}

98.00

\section{B}

CAV@ 68-73, 96-97.5 / FRAC @ 130-133

95.00

90.00

78.00

B

U/wB

8.00

17.00

14.50

15.00

14.00

19.00
B

B

B

B

CAV @ 43, 45.5, 52, 58, 64, 82 / FRAC @ 74, 80, 81, 84, 90, 100, 106, 121, 125 CAV@ @3-73 / FRAC @ 112

CAV @ 58, 68, 71, 78, 81, 83, 85 / FRAC @ 97-107, 132

CAV@ 147-149

FRAC @ 115(w), 126-130(w)

FRAC @ 0(W), 73(W), 170(w), 366

FRAC @ 25(w), 164-169(w)

FRAC @ 92(w), 115-121(w), 134-135, 159160(W)

B CAV @ 27-29(mw) / FRAC @ 47-48, 5558.5, 60-68

FRAC @ 44(w), 143-145(W), 164-165(W), 170(W), 200(W)

WB

WB

U

\section{WELL SAMPLE HISTORY}

Q1/86-Q1/96, Q1/01, Q3/01, Q1/02, Q3/02, Q1/03, Q3/03, Q1/04, Q3/04, Q1/05, Q3/05, Q1/06, Q3/06, Q1/07, Q3/07, Q1/08,

Q3/08, Q1/09, Q3/09, Q1/10, Q3/10, Q1/11, Q3/11, Q1/12, Q3/12 Q1/88-Q1/92, Q2/04, Q4/04, Q2/06, Q4/08, Q2/13

Q1/86-Q3/88, Q2/89, Q1/90-Q3/92, Q2/04, Q4/04, Q2/07, Q2/12 Q1/88-Q3/92, Q1/01, Q3/01, Q2/07, Q4/09, Q3/11, Q2/13

\section{Q3/88-Q4/95}

Q2/90-Q4/93

Q1/86-Q1/87, Q3/87, Q1/88, Q3/88, Q1/89, Q1/90, Q3/90, Q3/91Q1/94, Q2/95, Q4/95, Q2/96

Q1/86-Q1/87, Q3/87, Q1/88, Q3/88, Q1/89, Q1/90, Q3/90, Q3/91Q1/94, Q2/95, Q4/95, Q2/96

Q1/86-Q1/87, Q3/87, Q1/88, Q3/88, Q1/89, Q1/90, Q3/90, Q3/91Q1/94, Q2/95, Q4/95, Q2/96

Q1/86-Q1/87, Q3/87, Q1/88, Q3/88, Q1/89, Q1/90, Q3/90, Q3/91Q1/94, Q2/95, Q4/95, Q2/96

Q1/86-Q1/87, Q3/87, Q1/88, Q3/88

Q2/90-Q4/93, Q2/03, Q4/03, Q2/08, Q3/13

Q1/86-Q4/86, Q2/87, Q4/87, Q2/88, Q4/88, Q2/89, Q4/89-Q2/96, Q4/96

Q1/86-Q4/86, Q2/87, Q4/87, Q2/88, Q4/88, Q2/89, Q4/89-Q2/96, Q4/96, Q2/97, Q4/97, Q2/98, Q4/98, Q2/99, Q4/99, Q2/00, $\mathrm{Q} 4 / 00, \mathrm{Q} 2 / 01, \mathrm{Q} 4 / 01, \mathrm{Q} 2 / 06, \mathrm{Q} 2 / 07, \mathrm{Q} 2 / 08, \mathrm{Q} 3 / 13$ 


\section{CURRENT \\ NAME}

GW-193

GW-194

GW-195

GW-196

GW-197

GW-198

GW-199

GW-200

GW-201

GW-202

GW-203

GW-204

GW-205

GW-206

GW-207
FRACTURES,

T.O.W.R T.O.F.R AQUIFER CAVITIES, and WATER BREAKS

WB

CAV@ 9-12

$U$

$U$

U

U

17.00

0.60

26.50

22.50

19.00

21.00

20.10

93.00

86.00

10.00

$\mathrm{U} / \mathrm{wB}$

WB@ 5.6

$100.00 \quad 146.00$

B

U

B

\section{WELL SAMPLE HISTORY}

Q2/90-Q3/90, Q1/91-Q4/93, Q1/96, Q4/96-Q1/97, Q3/97, Q1/98, Q3/98, Q1/99, Q3/99, Q1/00, Q3/00, Q1/01, Q3/01, Q1/02, $\mathrm{O} 3 / 02, \mathrm{O} 1 / 03, \mathrm{O} 3 / 03, \mathrm{O} 1 / 04, \mathrm{O} 3 / 04, \mathrm{Q} 1 / 05, \mathrm{O} 3 / 05, \mathrm{Q} 1 / 06$, $\mathrm{Q} 3 / 06, \mathrm{Q} 1 / 07, \mathrm{Q} 3 / 07, \mathrm{Q} 1 / 08$,

Q1/86-Q4/86, Q2/87, Q4/87, Q2/88, Q4/88, Q2/89, Q4/89-Q1/90, Q3/91-Q2/96, Q4/96

Q1/86-Q4/86, Q2/87, Q4/87, Q2/88, Q4/88, Q2/89, Q4/89-Q1/90, Q3/91-Q2/96, Q4/96

Q1/86, Q3/86-Q4/86, Q2/87, Q4/87, Q2/88, Q4/88, Q2/89, Q4/89Q1/90

Q1/86-Q4/86, Q2/87, Q4/87, Q2/88, Q4/88, Q2/89, Q4/89-Q1/90 Q1/86-Q4/86, Q3/87-Q4/87, Q2/88, Q4/88

Q1/86-Q4/86, Q3/87-Q4/87, Q2/88, Q4/88, Q1/93-Q4/95

Q1/86-Q4/86, Q3/87-Q4/87, Q2/88, Q4/88

Q1/86-Q4/86, Q3/87-Q4/87, Q2/88, Q4/88

Q1/86-Q4/86, Q3/87-Q4/87, Q2/88, Q4/88

Q1/86-Q1/87, Q3/87, Q1/88-Q4/94, Q2/95, Q4/95, Q2/96, Q4/96, $\mathrm{Q} 2 / 97, \mathrm{Q} 4 / 97, \mathrm{Q} 3 / 98, \mathrm{Q} 1 / 99, \mathrm{Q} 3 / 99, \mathrm{Q} 1 / 00, \mathrm{Q} 3 / 00, \mathrm{Q} 1 / 01$, $\mathrm{Q} 3 / 01, \mathrm{Q} 1 / 02, \mathrm{Q} 3 / 02, \mathrm{Q} 1 / 03, \mathrm{Q} 3 / 03, \mathrm{Q} 1 / 04, \mathrm{Q} 3 / 04, \mathrm{Q} 1 / 05$, Q3/05, Q1/06, Q3/06, Q1/07,

Q2/90-Q4/93, Q1/96, Q3/99, Q2/00, Q4/00, Q2/01-Q4/01, Q2/02, $\mathrm{Q} 4 / 02, \mathrm{Q} 2 / 03, \mathrm{Q} 4 / 03, \mathrm{Q} 2 / 04, \mathrm{Q} 4 / 04, \mathrm{Q} 2 / 05, \mathrm{Q} 4 / 05, \mathrm{Q} 4 / 06$, Q4/07, Q4/08, Q2/09, Q4/10-Q1/11, Q3/12, Q1/13

Q1/86-Q1/87, Q3/87, Q1/88-Q4/94, Q2/95, Q4/95, Q2/96, Q4/96, Q2/97, Q4/97, Q3/98, Q1/99, Q3/99, Q1/00, Q3/00, Q1/01, Q3/01, Q1/02, Q3/02, Q1/03, Q3/03, Q1/04, Q3/04, Q1/05, $\mathrm{Q} 3 / 05, \mathrm{Q} 1 / 06, \mathrm{Q} 3 / 06, \mathrm{Q} 1 / 07$,

$\mathrm{Q} 2 / 90-\mathrm{Q} 4 / 95$

Q2/90-Q4/96, Q2/97, Q4/97, Q2/98, Q4/98, Q2/99, Q4/99, Q2/00, $\mathrm{Q} 4 / 00, \mathrm{Q} 2 / 01, \mathrm{Q} 4 / 01, \mathrm{Q} 2 / 02, \mathrm{Q} 4 / 02, \mathrm{Q} 2 / 03, \mathrm{Q} 4 / 03, \mathrm{Q} 2 / 04$, Q4/04, Q2/05, Q4/05 


\section{CURRENT \\ NAME}

GW-208

GW-209

GW-210

GW-211

GW-212

GW-213

GW-214

GW-215

GW-216

GW-217

GW-218

GW-219

GW-220

GW-221

36.00

90.00

10.00

37.00

18.00

55.00

75.00

6.00

11.00

GW-222
FRACTURES,

T.O.W.R T.O.F.R AQUIFER CAVITIES, and WATER BREAKS

$12.00 \quad B$

$75.00 \quad$ B

CAV@ $070-75$

CAV@ 83-88

WB

wB

CAV @ 30-33, 104-108, 111-115

FRAC@ 112, 138-154, 156, 286 / CAV @ 232

$U$

B

FRAC @125 / WB @ 165-180

WB

CAV@ 9, 22.5 / WB @ 22.5

U

WB

WB@ 11
FRAC @ 19

\section{WELL SAMPLE HISTORY}

Q2/90-Q4/96, Q2/97, Q4/97, Q2/98, Q4/98, Q2/99, Q4/99, Q2/00, $\mathrm{Q} 4 / 00, \mathrm{Q} 2 / 01, \mathrm{Q} 4 / 01, \mathrm{Q} 2 / 02, \mathrm{Q} 4 / 02, \mathrm{Q} 2 / 03, \mathrm{Q} 4 / 03, \mathrm{Q} 2 / 04$,

Q4/04, Q2/05, Q4/05

$\mathrm{Q} 2 / 90-\mathrm{Q} 2 / 91$

$\mathrm{Q} 2 / 90-\mathrm{Q} 2 / 91$

Q2/90-Q2/91

Q1/88-Q3/88, Q1/89, Q3/89-Q1/95, Q3/95, Q1/96, Q3/96, Q1/97, Q3/97, Q1/98, Q3/98, Q1/99, Q3/99, Q1/00, Q3/00, Q1/01, Q3/01, Q1/02, Q3/02, Q1/03, Q3/03, Q1/04, Q3/04, Q1/05, $\mathrm{Q} 3 / 05, \mathrm{Q} 1 / 06, \mathrm{Q} 3 / 06, \mathrm{Q} 1 / 07$,

Q2/96, Q4/99, Q2/00, Q4/00

$\mathrm{Q} 3 / 98, \mathrm{Q} 4 / 99, \mathrm{Q} 2 / 00, \mathrm{Q} 4 / 00, \mathrm{Q} 2 / 01, \mathrm{Q} 4 / 01, \mathrm{Q} 2 / 02, \mathrm{Q} 4 / 02$, $\mathrm{Q} 2 / 03, \mathrm{Q} 4 / 03, \mathrm{Q} 2 / 04, \mathrm{Q} 4 / 04, \mathrm{Q} 2 / 05, \mathrm{Q} 4 / 05, \mathrm{Q} 4 / 07, \mathrm{Q} 2 / 09$, $\mathrm{Q} 1 / 11, \mathrm{Q} 4 / 12-\mathrm{Q} 1 / 13$

Q1/86-Q4/96, Q2/97, Q4/97-Q4/99, Q2/00-Q4/00, Q2/01, Q4/01, $\mathrm{Q} 2 / 02, \mathrm{Q} 4 / 02, \mathrm{Q} 2 / 03, \mathrm{Q} 4 / 03, \mathrm{Q} 2 / 04, \mathrm{Q} 4 / 04, \mathrm{Q} 2 / 05, \mathrm{Q} 4 / 05$, Q2/06, Q4/06, Q2/07, Q4/07-Q4/08, Q2/09, Q4/09, Q3/10, Q1/11, $\mathrm{Q} 3 / 11, \mathrm{Q} 1 / 12, \mathrm{Q} 3 / 12, \mathrm{Q}$

Q1/86-Q1/87, Q3/87, Q1/88-Q4/94, Q2/95, Q4/95, Q2/96, Q4/96, Q2/97, Q4/97, Q3/98, Q1/99, Q3/99, Q1/00, Q3/00, Q1/01, $\mathrm{Q} 3 / 01, \mathrm{Q} 1 / 02, \mathrm{O} 3 / 02, \mathrm{Q} 1 / 03, \mathrm{Q} 3 / 03, \mathrm{Q} 1 / 04, \mathrm{Q} 3 / 04, \mathrm{Q} 1 / 05$, $\mathrm{Q} 3 / 05, \mathrm{Q} 1 / 06, \mathrm{Q} 3 / 06, \mathrm{Q} 1 / 07$,

Q1/86-Q2/89, Q4/89-Q2/91, Q4/94-Q4/96, Q2/00, Q4/00, Q2/04, $\mathrm{Q} 4 / 04, \mathrm{Q} 2 / 09$ 


\section{CURRENT \\ NAME}

GW-223

GW-224

GW-225

GW-226

GW-227

GW-228

GW-229

GW-230

GW-231

GW-232

GW-233

GW-234

GW-235
87.00

137.00

7.00

T.O.W.R

7.00

18.00

25.00

26.00

19.00

24.00

30.00

38.00

10.50

wB

B

B

wB

U

B

B
FRACTURES,

\section{AQUIFER CAVITIES, and WATER BREAKS}

FRAC @ 17-18, 27.5-29, 34-39, 66-67. 68.5-69.5 / CAV@ @7-59(m)

FRAC @ 0(W), 115-120

WB@30-110

WB @ 30-55

CAV@ 25.5-26(m), 34.5-35, 37-37.5

WB @ 35-100 / CAV @ 36-36.5(m), 40-41, 90-100

B CAV @ 30-38(MW) / FRAC @ 146147(m), 403-404(m)

FRAC @ 15-16.5, 17-17.5(w), 19-20.5(w) 23-24, 25-27, 28-29

FRAC @ 200(W)

FRAC @ 127, 134, 154.5, 159, 167 / CAV @ 130

CAV @ 7-14, 24-27, 34-42, 50-52

\section{WELL SAMPLE HISTORY}

Q1/86-Q2/89, Q4/89-Q2/91, Q4/94-Q4/96, Q3/99, Q2/00, Q4/00Q1/01, Q3/01, Q1/02, Q3/02, Q1/03, Q3/03, Q1/04, Q3/04, Q1/05, Q3/05, Q1/06, Q3/06, Q1/07, Q3/07, Q1/08, Q3/08, Q1/09, Q3/09, Q1/10, Q3/10, Q Q1/86-Q1/87, Q3/87, Q1/88, Q3/88

Q1/86-Q4/87, Q2/88-Q1/89, Q3/89-Q3/92, Q1/01, Q3/01, Q1/02, Q3/02, Q1/03, Q3/03, Q1/04, Q3/04, Q1/05, Q3/05, Q1/06, $\mathrm{Q} 3 / 06, \mathrm{Q} 1 / 07, \mathrm{Q} 3 / 07, \mathrm{Q} 1 / 08, \mathrm{Q} 3 / 08, \mathrm{Q} 1 / 09, \mathrm{Q} 3 / 10, \mathrm{Q} 1 / 11$

$\mathrm{Q} 3 / 12, \mathrm{Q} 1 / 13$

Q1/86-Q4/87, Q2/88-Q1/89, Q3/89-Q2/91, Q1/98, Q3/98, Q1/99, Q3/99, Q1/00, Q3/00, Q1/01, Q3/01, Q1/02, Q3/02, Q1/03,

Q3/03, Q1/04, Q3/04, Q1/05, Q3/05, Q1/06, Q3/06, Q1/07, Q3/07, Q1/08, Q3/08

Q1/86-Q4/87, Q2/88-Q1/89, Q3/89-Q3/92, Q1/05, Q3/05, Q1/08

Q1/86-Q4/87, Q2/88-Q1/89, Q3/89-Q1/90, Q3/95, Q3/99, Q1/05, Q3/05

Q1/86-Q4/87, Q2/88-Q1/89, Q3/89-Q1/90, Q3/95, Q1/02, Q3/02, Q1/03, Q3/03, Q1/04, Q3/04, Q1/05, Q3/05, Q1/06, Q2/07, Q1/08$\mathrm{Q} 2 / 08, \mathrm{Q} 4 / 08-\mathrm{Q} 1 / 09, \mathrm{Q} 3 / 10, \mathrm{Q} 1 / 11, \mathrm{Q} 3 / 12, \mathrm{Q} 1 / 13$

Q2/90, Q3/94-Q2/96, Q2/97, Q1/98, Q3/98, Q1/99, Q3/99, Q1/00$\mathrm{Q} 3 / 00, \mathrm{Q} 1 / 01, \mathrm{Q} 3 / 01, \mathrm{Q} 1 / 02, \mathrm{Q} 3 / 02, \mathrm{Q} 3 / 03, \mathrm{Q} 1 / 04, \mathrm{Q} 3 / 04, \mathrm{Q} 2 / 05-$ Q3/05, Q1/06, Q1/07, Q1/08, Q1/09, Q3/10, Q1/11, Q2/12

Q1/86-Q4/86, Q2/87-Q3/87, Q1/88-Q2/90, Q4/90-Q1/91, Q3/91Q2/96, Q4/96, Q2/97-Q4/97, Q2/98, Q4/98, Q2/99, Q4/99, Q2/00, $\mathrm{Q} 4 / 00, \mathrm{Q} 2 / 01, \mathrm{Q} 4 / 01, \mathrm{Q} 2 / 02, \mathrm{Q} 4 / 02, \mathrm{Q} 2 / 03, \mathrm{Q} 4 / 03, \mathrm{Q} 2 / 04$,

Q4/04, Q2/05, Q4/05, Q2/

Q2/90, Q1/91-Q1/94, Q3/94-Q1/96, Q2/97, Q1/98, Q3/98, Q1/99, Q3/99, Q1/00-Q3/06

Q1/88-Q2/88 


\section{CURRENT \\ NAME}

GW-236

GW-237

GW-238

GW-239

GW-240

GW-241

GW-242

GW-243

GW-244

GW-245

GW-246

GW-247

GW-248

GW-249

GW-250

GW-251

GW-252

GW-253
FRACTURES,

T.O.W.R T.O.F.R AQUIFER CAVITIES, and WATER BREAKS

9.00

18.50

wB

$U / w B$

15.20

27.00

B

CAV @ 30-33, 35-37 / FRAC @ 124, 127128(mw)

14.00

B

B

FRAC @ 29-30

67.00

94.60

20.00

37.00

22.50

26.00

26.00

36.00

9.00

34.00

9.00

33.00

32.50 $w B / B$

wB

\section{CAV @ 78-89 / FRAC @ 89-94.6}

B

B

B

WB

WB

B

WB@ 54

$w B / B$

wB

WB@ 71

\section{WELL SAMPLE HISTORY}

Q2/88-Q2/89, Q1/90, Q3/95, Q3/99, Q1/04, Q3/04, Q2/07, Q1/09, Q3/11, Q1/13

Q2/88, Q1/04, Q3/04

\section{Q2/90-Q4/93}

Q1/86-Q3/89, Q1/90-Q4/96, Q2/00, Q4/00, Q2/01, Q4/01, Q1/06, Q1/07, Q3/08, Q4/09, Q2/10, Q3/11, Q1/12, Q3/13

Q1/86-Q2/86, Q1/87, Q3/87-Q3/89, Q1/90-Q3/95, Q1/01, Q3/01 Q4/87, Q2/88-Q1/89, Q3/89-Q1/90, Q2/98-Q3/98, Q1/99, Q2/05, Q4/05, Q1/09, Q3/11, Q1/13

Q4/86-Q1/90, Q4/90-Q3/92, Q1/94, Q1/02, Q3/02

Q1/87-Q1/90, Q2/05, Q4/05

Q1/87-Q2/89, Q1/90, Q2/05, Q4/05

Q1/87-Q2/89, Q1/90, Q1/04, Q3/04, Q1/06, Q3/06, Q1/07, Q3/07, Q1/08-Q4/08, Q3/09, Q1/10-Q2/10, Q3/11, Q1/12, Q3/13

Q1/87-Q2/89, Q1/90, Q2/05, Q4/05

Q1/88-Q4/88, Q2/89-Q1/90, Q3/95

Q1/89, Q3/89-Q1/90

Q4/87, Q2/88-Q1/89, Q3/89-Q1/90

Q2/86-Q1/87, Q3/87-Q2/88, Q4/88-Q2/96, Q4/96, Q2/97, Q4/97-

$\mathrm{Q} 4 / 98, \mathrm{Q} 2 / 99, \mathrm{Q} 4 / 99, \mathrm{Q} 2 / 00, \mathrm{Q} 4 / 00, \mathrm{Q} 2 / 01, \mathrm{Q} 4 / 01, \mathrm{Q} 2 / 02$,

$\mathrm{Q} 4 / 02, \mathrm{Q} 2 / 03, \mathrm{Q} 4 / 03, \mathrm{Q} 2 / 04, \mathrm{Q} 4 / 04, \mathrm{Q} 2 / 05, \mathrm{Q} 4 / 05, \mathrm{Q} 2 / 06$,

$\mathrm{Q} 2 / 07, \mathrm{Q} 2 / 08, \mathrm{Q} 2 / 09, \mathrm{Q} 4 / 10-\mathrm{Q} 1$

Q2/86-Q1/87, Q3/87, Q1/88-Q4/93, Q2/94-Q2/95

Q2/86, Q4/86-Q1/87, Q3/87-Q2/88, Q4/88-Q1/89, Q2/90-Q2/91, Q1/96-Q2/96, Q2/98-Q3/98, Q1/99, Q3/99-Q4/99, Q2/00, Q4/00, $\mathrm{Q} 2 / 01, \mathrm{Q} 4 / 01, \mathrm{Q} 2 / 02, \mathrm{Q} 4 / 02, \mathrm{Q} 2 / 03, \mathrm{Q} 4 / 03, \mathrm{Q} 1 / 05, \mathrm{Q} 1 / 06$,

Q1/07, Q1/08, Q1/09, Q1/1 


\section{CURRENT \\ NAME}

GW-254

GW-255

GW-256

GW-257

GW-258

GW-259

GW-260

GW-261

GW-262

GW-263

GW-264

GW-265

GW-266

GW-267

GW-268

GW-269

GW-270

GW-271

GW-272

GW-273

GW-274

GW-275
T.O.W.R

10.00

5.00

5.00

2.50

10.00

5.00

5.00

5.00

5.00

3.00

5.00
FRACTURES,

\section{AQUIFER CAVITIES, and WATER BREAKS}

20.50

43.00

B

$U$

33.70

30.00

33.50

23.30

23.00

20.00

30.00

33.00

22.00

27.00

35.00

35.00

11.00

11.00

15.00

35.00

35.00
WB@ 30

WB@ @ 42.5

WB@ 19

CAV@ 100-112

WB@ 23

WB@ 55

B

$w B / B$

$B$

WB@ 60

$w B / B$

WB

$W B$

$W B$

$W B$

B

WB@ 12

WB@14, 41

$w B / B$

$W B$

WB

WB@ 17

WB@ 15

B WB @ 20, 40-50

\section{WELL SAMPLE HISTORY}

Q2/86-Q1/87, Q3/87

Q2/86-Q1/87, Q3/87-Q4/93, Q2/94-Q2/95

$\mathrm{Q} 2 / 86-\mathrm{Q} 1 / 87, \mathrm{Q} 3 / 87$

Q2/88, Q2/89-Q1/90, Q3/95, Q1/04, Q3/04, Q2/06, Q1/07, Q3/08

Q1/88-Q4/88, Q2/89-Q1/90

Q1/88-Q4/88, Q2/89-Q1/90, Q1/08, Q3/08, Q3/13

Q4/86-Q2/88, Q1/89, Q3/89-Q4/93, Q2/94-Q2/95

Q4/86-Q2/88, Q1/89-Q2/90, Q4/90-Q4/93, Q2/94-Q2/95

Q1/87-Q2/87, Q1/89-Q3/90, Q1/91-Q4/93, Q2/94-Q2/95

Q4/86-Q2/88, Q2/89-Q4/93, Q2/94-Q2/95

Q4/86-Q2/88, Q1/89, Q3/89-Q1/90, Q2/06, Q4/06, Q2/07, Q4/07,

Q2/08, Q2/11, Q4/12, Q2/13

$\mathrm{Q} 4 / 86-\mathrm{Q} 1 / 87$

Q4/86-Q1/87

Q4/86-Q2/88, Q1/89, Q3/89-Q1/90, Q2/11, Q4/12, Q2/13

Q4/86-Q2/88, Q1/89, Q3/89-Q1/90, Q2/03, Q4/03, Q2/06, Q4/06, $\mathrm{Q} 2 / 07, \mathrm{Q} 4 / 07, \mathrm{Q} 2 / 08, \mathrm{Q} 2 / 09, \mathrm{Q} 4 / 10, \mathrm{Q} 2 / 11, \mathrm{Q} 4 / 12, \mathrm{Q} 2 / 13$ Q4/86-Q1/90, Q2/03, Q4/03, Q2/07, Q2/09, Q2/11, Q2/13

$\mathrm{Q} 4 / 86-\mathrm{Q} 1 / 90, \mathrm{Q} 2 / 03, \mathrm{Q} 4 / 03$

Q4/86-Q1/90, Q2/03, Q4/03, Q1/07, Q2/09, Q2/11, Q2/13

Q4/86-Q1/88, Q4/88, Q2/03, Q4/03, Q2/07

Q4/86-Q1/90, Q4/95-Q1/96, Q2/00, Q4/00, Q2/03, Q4/03, Q1/06, $\mathrm{Q} 1 / 07, \mathrm{Q} 2 / 08, \mathrm{Q} 2 / 09, \mathrm{Q} 4 / 10, \mathrm{Q} 2 / 11, \mathrm{Q} 4 / 12, \mathrm{Q} 2 / 13$

Q4/86-Q1/90, Q4/95, Q2/00, Q4/00, Q2/03, Q4/03, Q1/06, Q1/07, Q2/08, Q2/09, Q4/10, Q2/11, Q4/12, Q2/13 


\section{CURRENT \\ NAME}

GW-276

$\begin{array}{lcccl}\text { GW-277 } & 17.50 & 65.00 & \text { WB/B } & \text { WB @ 50, 70 } \\ \text { GW-278 } & 21.00 & & U & \text { WB @ 16 } \\ \text { GW-279 } & & 27.00 & \text { B } & \text { WB @ 27, 71 } \\ \text { GW-280 } & 25.00 & 115.00 & \text { B } & \text { CAV @ 35-36, 38-39, 136-137 } \\ \text { GW-281 } & 17.50 & & U & \\ \text { GW-282 } & 16.00 & & U & \\ \text { GW-283 } & 20.00 & & U & \\ \text { GW-284 } & 18.00 & & U & \\ \text { GW-285 } & 21.00 & & U & \\ \text { GW-286 } & 5.00 & 10.00 & B & \\ \text { GW-287 } & 4.50 & 11.00 & \text { WB/B } & \\ \text { GW-288 } & 25.00 & 42.00 & B & \\ \text { GW-289 } & 24.00 & 38.00 & \text { WB/B } & \\ & & & & \\ \text { GW-290 } & & 17.00 & B & \\ \text { GW-291 } & 8.00 & & \text { U/wB } & \\ \text { GW-292 } & 32.00 & 59.50 & B & \text { CAV @ 56.5-58, 93-95.5 / FRAC @ 58, 66, } \\ \text { GW-293 } & 57.00 & 110.00 & B & \text { FRAC @ 59, 206 / CAV @ 107-110 / WB } \\ & & & & \text { @ 206 }\end{array}$

FRACTURES,

T.O.W.R T.O.F.R AQUIFER CAVITIES, and WATER BREAKS

U

@ 206

\section{WELL SAMPLE HISTORY}

Q4/86-Q1/90, Q1/94, Q3/94, Q1/95, Q3/95, Q1/96, Q3/96, Q1/97, Q3/97, Q1/98, Q3/98, Q1/99, Q3/99, Q1/00, Q3/00, Q1/01,

$\mathrm{Q} 3 / 01, \mathrm{Q} 1 / 02, \mathrm{Q} 3 / 02, \mathrm{Q} 1 / 03, \mathrm{Q} 3 / 03, \mathrm{Q} 1 / 04, \mathrm{Q} 3 / 04, \mathrm{Q} 1 / 05$,

Q3/05, Q1/06, Q3/06, Q1/07

Q4/86-Q1/90, Q1/05, Q3/05, Q2/07

$\mathrm{Q} 4 / 86-\mathrm{Q} 3 / 87$

$\mathrm{Q} 4 / 86-\mathrm{Q} 3 / 87$

$\mathrm{Q} 4 / 86-\mathrm{Q} 3 / 87$

Q2/89-Q4/93, Q2/03, Q2/04, Q2/05, Q2/06, Q2/07, Q2/08, Q3/09, $\mathrm{Q} 2 / 10, \mathrm{Q} 2 / 11, \mathrm{Q} 2 / 12$

$\mathrm{Q} 2 / 89-\mathrm{Q} 3 / 92$

$\mathrm{Q} 2 / 89-\mathrm{Q} 4 / 93$

Q2/89-Q4/93, Q1/96

$\mathrm{Q} 2 / 89-\mathrm{Q} 4 / 93$

Q1/87-Q1/89, Q3/89-Q1/90, Q3/91-Q4/93, Q2/05, Q4/05

Q1/87-Q1/89, Q3/89-Q1/90, Q3/91-Q1/96, Q3/96, Q1/97, Q3/97, Q1/98, Q3/98, Q1/99, Q3/99, Q1/00, Q3/00, Q2/05, Q4/05

Q1/88-Q1/89, Q3/89-Q1/90, Q3/95, Q1/02, Q3/02, Q2/05, Q4/05

Q1/88-Q1/89, Q3/89-Q1/90, Q3/95, Q2/98-Q3/98, Q1/02, Q3/02, Q2/05, Q4/05, Q2/06, Q1/07, Q3/08, Q3/09, Q1/10, Q3/11, $\mathrm{Q} 1 / 12, \mathrm{Q} 3 / 13$

Q1/88-Q4/88, Q3/89-Q1/90, Q1/91-Q2/91, Q3/95

Q1/88-Q4/88, Q3/89-Q1/90, Q1/91-Q2/91, Q3/95, Q2/98-Q3/98, Q1/02, Q3/02, Q2/05, Q4/05, Q3/10

Q4/87-Q4/88, Q2/89, Q4/89, Q2/90-Q2/94, Q4/94, Q2/95, Q4/95, Q2/96, Q1/07, Q3/07

Q4/87-Q4/88, Q2/89, Q4/89, Q2/90-Q2/94, Q4/94, Q2/95, Q4/95, Q2/96, Q1/07, Q3/07 


\section{CURRENT \\ NAME}

GW-294

GW-295

GW-296

GW-297

GW-298

GW-299

GW-300

GW-301

GW-302

GW-303

GW-304

GW-305

GW-306

GW-307

GW-308

GW-309

\section{T.O.W.R}

62.00

87.00

AQUIFER

\section{B}

$62.00 \quad 94.00$

67.00

81.50

B

B

77.00

65.00

94.00

80.00

66.00

102.00

94.00

70.00

106.00

136.00

63.00

102.00

37.00

111.00

35.00

53.00

92.00

84.00

44.00

41.60

37.70

20.00

U

U

wB
FRACTURES,

CAVITIES, and WATER BREAKS

FRAC @ 110, 115, 120, 135 / CAV @ 137139

CAV@ @1, 93

CAV@ @ 90 / FRAC @ 179, 182

FRAC @ 132, 156-168

FRAC @ 0, 141-144 / WB @ 141

CAV@ 0, 93.5, 121-136 / FRAC @ 109, 118 /WB@ 154

CAV@ 90-73, 86-88 / WB @ 99.8 / FRAC @ 127,131

CAV @ 39, 109 / FRAC @ 258 / WB @ 302, 310

WB@ 169

FRAC @ 48 / WB @ 48
WB @ 27 / FRAC @ 27, 30, 32.5, 35.7

\section{WELL SAMPLE HISTORY}

Q4/87-Q4/88, Q2/89, Q4/89, Q2/90-Q2/94, Q4/94, Q2/95, Q4/95, Q2/96, Q1/07, Q3/07, Q1/08, Q3/08, Q1/09, Q3/09, Q1/10,

Q3/10, Q1/11, Q3/11, Q1/12, Q3/12

Q1/89-Q1/94, Q3/94, Q2/95

Q4/87-Q4/88, Q2/89, Q4/89, Q2/90-Q2/94, Q4/94, Q2/95, Q4/95, Q2/96, Q1/07, Q3/07, Q1/08, Q3/08, Q1/09, Q3/09, Q1/10,

$\mathrm{Q} 3 / 10, \mathrm{Q} 1 / 11, \mathrm{Q} 3 / 11, \mathrm{Q} 1 / 12, \mathrm{Q} 3 / 12$

Q1/88-Q1/94, Q3/94, Q2/95, Q4/95, Q2/96, Q1/07, Q3/07, Q1/08, Q3/08, Q1/09, Q3/09, Q1/10, Q3/10, Q1/11, Q3/11, Q1/12, Q3/12 Q1/88-Q1/94, Q3/94, Q2/95, Q4/95, Q2/96

Q1/88-Q1/94, Q3/94, Q2/95, Q4/95, Q2/96, Q2/04, Q4/04

Q1/88-Q1/94, Q3/94, Q2/95, Q4/95, Q2/96, Q4/96-Q1/97, Q3/97,

$\mathrm{Q} 1 / 98, \mathrm{Q} 3 / 98, \mathrm{Q} 1 / 99, \mathrm{O} 3 / 99, \mathrm{O} 1 / 00, \mathrm{O} 3 / 00, \mathrm{Q} 1 / 01, \mathrm{Q} 3 / 01$

$\mathrm{Q} 1 / 02, \mathrm{Q} 3 / 02, \mathrm{Q} 1 / 03, \mathrm{Q} 3 / 03, \mathrm{Q} 1 / 04, \mathrm{Q} 3 / 04, \mathrm{Q} 1 / 05, \mathrm{Q} 3 / 05$

Q1/06, Q3/06, Q1/07, Q3/07,

Q2/90-Q4/94, Q2/95, Q4/95, Q2/96, Q4/96, Q2/97, Q4/97, Q3/98, $\mathrm{Q} 1 / 99, \mathrm{Q} 3 / 99, \mathrm{Q} 1 / 00, \mathrm{Q} 3 / 00, \mathrm{Q} 1 / 01, \mathrm{Q} 3 / 01, \mathrm{Q} 1 / 02, \mathrm{Q} 3 / 02$,

$\mathrm{Q} 1 / 03, \mathrm{Q} 3 / 03$

Q4/88-Q3/95

Q4/88-Q3/95

Q1/88-Q3/88, Q1/89, Q3/89-Q1/95, Q3/95, Q1/96, Q3/96, Q1/97, Q3/97, Q1/98, Q3/98, Q1/99, Q3/99-Q1/00, Q3/00-Q1/08, Q3/08, Q1/09, Q3/09-Q4/12

Q1/88-Q4/88, Q2/89, Q4/89-Q2/91, Q1/08, Q3/08, Q1/13

Q1/88-Q4/88, Q4/89-Q2/91, Q1/05, Q3/05, Q2/07, Q1/09, Q3/10, Q1/11, Q3/12, Q1/13

Q1/88-Q2/88, Q2/89-Q2/91

Q1/88-Q2/88, Q2/89-Q3/92, Q1/08, Q3/08, Q1/13 


\section{CURRENT \\ NAME}

GW-310

GW-311

GW-312

GW-313

GW-314

GW-315

GW-316

GW-317

GW-318

GW-319

GW-320

GW-321

GW-322

GW-323

GW-324

GW-325

GW-326

GW-327
FRACTURES,

T.O.W.R T.O.F.R AQUIFER CAVITIES, and WATER BREAKS

U

U

U

$30.00 \quad 34.50$

$47.00 \quad 49.00$

54.00

71.00

55.00

48.00

WB

CAV@ 39.5 / FRAC @ 69, 71, 76

FRAC @ 110, 122, 128

49.00

120.00

75.00

19.00

6.00

$$
\text { B }
$$

190

CAV@ $103-106$

CAV@ 30, 48 / FRAC @ 108 / WB @ 108.5

FRAC @ 61.5, 86.5, 94.5 / CAV@ 62, 68.5 / WB @ 94.5

FRAC @ 97, 100.5, 106.5 / WB @ 100.5 WB@ 18

$W B$

$U / w B$

U

\section{WELL SAMPLE HISTORY}

Q1/88-Q4/88, Q2/89-Q2/91, Q1/05, Q3/05, Q3/06, Q1/08, Q3/10, $\mathrm{Q} 1 / 12$

Q1/88-Q2/88, Q4/88, Q2/89-Q2/91, Q1/93-Q2/95, Q4/95-Q1/96, Q3/96, Q1/97, Q3/97, Q1/98, Q3/98, Q1/99, Q3/99, Q1/00, $\mathrm{Q} 3 / 00, \mathrm{O} 1 / 01, \mathrm{O} 3 / 01, \mathrm{O} 1 / 02, \mathrm{O} 3 / 02, \mathrm{Q} 1 / 03, \mathrm{O} 3 / 03, \mathrm{Q} 1 / 04$ $\mathrm{Q} 3 / 04, \mathrm{Q} 1 / 05, \mathrm{Q} 3 / 05, \mathrm{Q} 3 / 06$

Q1/88-Q2/88, Q4/88, Q2/89-Q2/91, Q1/93-Q3/95, Q1/05, Q3/05, $\mathrm{Q} 1 / 08, \mathrm{Q} 3 / 10, \mathrm{Q} 1 / 12$

Q1/88-Q3/88, Q1/90-Q3/92, Q1/05, Q3/05, Q2/07

Q1/88-Q4/88, Q1/90-Q3/92, Q1/08, Q3/08

Q1/88-Q4/88, Q1/90-Q1/96, Q3/96, Q1/97, Q3/97, Q1/98, Q3/98,

Q1/99, Q3/99, Q1/00, Q3/00, Q1/01, Q3/01, Q1/02, Q3/02,

$\mathrm{Q} 1 / 03, \mathrm{Q} 3 / 03, \mathrm{Q} 1 / 04, \mathrm{Q} 3 / 04, \mathrm{Q} 1 / 05, \mathrm{Q} 3 / 05, \mathrm{Q} 1 / 06, \mathrm{Q} 2 / 07$,

Q1/08, Q1/09, Q3/10, Q1/11,

Q1/88-Q4/88, Q1/90-Q4/93

$\mathrm{Q} 1 / 88-\mathrm{Q} 4 / 88, \mathrm{Q} 2 / 89, \mathrm{Q} 1 / 90-\mathrm{Q} 3 / 95$

Q1/88-Q4/88

Q1/88-Q4/88

$\mathrm{Q} 1 / 88-\mathrm{Q} 4 / 88$

Q1/89-Q2/94, Q4/94-Q2/95, Q4/95, Q2/96

Q1/88-Q3/92, Q1/98, Q2/04, Q4/04, Q2/06, Q4/08, Q4/09, Q2/10, $\mathrm{Q} 3 / 11, \mathrm{Q} 2 / 12, \mathrm{Q} 2 / 13$

Q1/88-Q4/88, Q1/90-Q4/93

Q4/87-Q2/91, Q1/93-Q1/94

Q4/87-Q2/91, Q1/93-Q1/94 


\section{CURRENT \\ NAME}

GW-328

GW-329

GW-330

GW-331

GW-332

GW-333

GW-334

GW-335

GW-336

GW-337

GW-338

GW-339

GW-340

GW-341

GW-342

GW-343

GW-344

GW-345

GW-346

GW-347

GW-348

GW-349
3.50

11.00

6.00

14.00

6.00

4.00

6.00

45.00

91.00

CAV@ @4, 67, 82

WB @ 61, 179 / CAV @ 100-104 / FRAC @ 154, 168

WB @ 109, 173 / FRAC @ 167, 306

$9.00 \quad 26.30$

$11.00 \quad 22.80$

26.50

35.80

25.50

\section{AQUIFER CAVITIES, and WATER BREAKS}

WB

U

WB

WB

$\mathrm{U} / \mathrm{wB}$

U WB@ 24

WB

WB

U

WB

$u$

WB

27.00

28.00

$W B$

B

WB@ 57

$U / w B$

36.60

$\mathrm{B}$
$\mathrm{U}$

WB @ 76.4

WB@ 13

\section{WELL SAMPLE HISTORY}

Q2/89-Q2/91

Q2/89-Q2/91, Q1/98-Q3/98, Q1/06, Q1/07, Q2/08, Q2/09, Q4/10$\mathrm{Q} 1 / 11, \mathrm{Q} 3 / 12, \mathrm{Q} 1 / 13$

$\mathrm{Q} 2 / 89-\mathrm{Q} 2 / 91$

$\mathrm{Q} 2 / 89-\mathrm{Q} 2 / 91$

$\mathrm{Q} 2 / 89-\mathrm{Q} 2 / 91$

Q2/89-Q2/91, Q2/03, Q4/03, Q1/06, Q1/07, Q2/08

Q2/89-Q2/95, Q3/98, Q3/99, Q2/00, Q4/00, Q2/03, Q4/03, Q1/06, $\mathrm{Q} 1 / 07, \mathrm{Q} 2 / 08, \mathrm{Q} 2 / 09, \mathrm{Q} 4 / 10-\mathrm{Q} 1 / 11, \mathrm{Q} 3 / 12, \mathrm{Q} 1 / 13$

$\mathrm{Q} 2 / 89-\mathrm{Q} 2 / 96, \mathrm{Q} 4 / 96, \mathrm{Q} 3 / 98$

Q2/90-Q4/94, Q2/95, Q4/95, Q2/96, Q4/96, Q2/97, Q4/97, Q3/98, $\mathrm{Q} 1 / 99, \mathrm{Q} 3 / 99, \mathrm{Q} 1 / 00, \mathrm{Q} 3 / 00, \mathrm{Q} 1 / 01, \mathrm{Q} 3 / 01, \mathrm{Q} 1 / 02, \mathrm{Q} 3 / 02$, $\mathrm{Q} 1 / 03, \mathrm{Q} 3 / 03$

Q2/90-Q3/92, Q4/93

Q3/88, Q4/89-Q3/92

Q2/90-Q3/92

Q3/88-Q2/91, Q1/93-Q1/94, Q1/99

Q3/88-Q1/90, Q3/95, Q1/99, Q2/05, Q4/05

Q3/88-Q4/88, Q2/89, Q2/90-Q4/90, Q2/91-Q3/95

Q3/88-Q4/88, Q2/89, Q2/90-Q4/90, Q2/91-Q3/95

Q3/88-Q1/90, Q2/02, Q4/02 


\section{CURRENT \\ NAME}

GW-350

GW-363

GW-364

GW-365

GW-366

GW-367

GW-368

GW-369

GW-370

GW-371

GW-372

GW-373

GW-374

GW-375

GW-376

GW-380

GW-381

GW-382
T.O.W.R

18.00

9.00

22.00

11.00

46.00

39.00

18.00

10.80

11.00

7.50

14.50

21.00

14.00

10.00

9.00

15.50

13.50

26.00

$w B / B$

CAV @ 16.3, 17, 19.6, 24.5

12.70

wB @ 27, 35

CAV@ 62

WB@ 225-235

WB@ 117

$W B / B$

$w B / B$

B

B $29,129-135,160.7$

WB@ $@ 42,65,160$

U

17.00
B
CAV@ 16 / WB @ 163-173
FRACTURES,

\section{AQUIFER CAVITIES, and WATER BREAKS}

CAV@ 22-24, 35 / FRAC @ 27, 40 / WB

FRAC @ 78-81, 142-143 / WB @ 142

FRAC @ 64, 68, 71 /WB @ 112, 127

CAV@ 15.4, 17.5, 19.8, 30.5, 36 /WB @

\section{WELL SAMPLE HISTORY}

Q3/88-Q1/90, Q2/02, Q4/02

Q3/88-Q1/89, Q3/89-Q1/90, Q1/94-Q1/96, Q3/96, Q1/97, Q3/97, $\mathrm{Q} 2 / 01-\mathrm{Q} 1 / 02, \mathrm{Q} 1 / 03-\mathrm{Q} 4 / 12$

Q3/88-Q1/89, Q3/89-Q4/93, Q1/01, Q3/01, Q1/05, Q3/05

Q3/88-Q1/89, Q3/89-Q4/93, Q1/01, Q3/01, Q1/05, Q3/05, Q1/08, Q1/09, Q3/10, Q1/11, Q3/12, Q1/13

$\mathrm{Q} 3 / 88-\mathrm{Q} 1 / 89, \mathrm{Q} 3 / 89-\mathrm{Q} 3 / 92$

Q3/88-Q1/89, Q3/89-Q2/91, Q1/08, Q3/08, Q1/13 Q3/88-Q1/89, Q3/89-Q1/90, Q1/05, Q3/05, Q2/07

Q3/88-Q1/89, Q3/89-Q2/91, Q2/07, Q1/08

Q3/88-Q1/89, Q3/89-Q3/95

Q3/88-Q1/89, Q3/89-Q1/90, Q3/91-Q4/93

Q3/88-Q1/89, Q3/89-Q1/96, Q3/96, Q1/97, Q3/97

Q3/88-Q1/89, Q3/89-Q1/96, Q3/96, Q1/97, Q3/97

Q3/88-Q1/89, Q3/89-Q1/90

Q3/88-Q1/89, Q3/89-Q1/90

Q4/88-Q1/89, Q4/89-Q1/90

Q4/88-Q4/96, Q2/97, Q4/97, Q2/98, Q4/98, Q2/99, Q4/99, Q2/00, Q1/02, Q3/02, Q1/03, Q3/03, Q1/04-Q3/04, Q1/05, Q3/05-Q4/05, $\mathrm{Q} 2 / 06, \mathrm{Q} 1 / 07, \mathrm{Q} 3 / 07, \mathrm{Q} 1 / 08, \mathrm{Q} 3 / 08, \mathrm{Q} 1 / 09, \mathrm{Q} 3 / 09, \mathrm{Q} 1 / 10$, Q3/10, Q1/11, Q3/11,

Q4/88-Q2/95, Q2/00, Q4/00, Q2/01, Q4/01, Q2/02, Q4/02, Q2/03, Q4/03, Q2/04, Q4/04, Q2/05, Q4/05, Q3/06, Q4/07, Q3/08, Q4/09, Q2/10, Q3/11, Q1/12, Q3/13

Q4/88-Q2/95, Q3/99, Q2/00-Q3/00, Q1/01, Q3/01, Q1/02, Q3/02, Q1/03, Q3/03, Q1/04, Q3/04, Q1/05, Q3/05, Q1/06, Q3/06, Q1/07, Q3/07, Q1/08, Q3/08, Q1/09, Q3/09, Q1/10, Q3/10, Q1/11, Q3/11, Q1/12, Q3/12 


\section{CURRENT}

NAME

GW-383

GW-384
GW-385
GW-400
GW-401
GW-402
GW-403
GW-404
GW-405
GW-406
GW-407
GW-408
GW-409
GW-410
GW-411
GW-412
GW-413
GW-414
GW-415
GW-416
GW-417
GW-418
GW-419

FRACTURES,

T.O.W.R T.O.F.R AQUIFER CAVITIES, and WATER BREAKS

11.50

12.00

11.00

4.00

10.00

27.00

8.00

8.00

20.00

30.00

10.00

10.00
WB

WB

13.00

23.00

B

B

B

B

B

B

WB

$U / w B$

B

WB

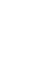

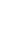

$w B$

$U$

wB

$U$

$U / w B$

$w B$

$U$

WB

$U$

$50.00 \quad \mathrm{wB} / \mathrm{B}$

$18.00 \quad \mathrm{wB} / \mathrm{B}$

25.00
$18.00 \quad B$

\section{WELL SAMPLE HISTORY}

Q3/88-Q4/96, Q2/97, Q4/97, Q2/98, Q4/98, Q2/99, Q4/99, Q2/00$\mathrm{Q} 4 / 00, \mathrm{Q} 2 / 01, \mathrm{Q} 4 / 01, \mathrm{Q} 2 / 02, \mathrm{O} 4 / 02, \mathrm{O} 2 / 03, \mathrm{Q} 4 / 03, \mathrm{Q} 2 / 04$ $\mathrm{Q} 4 / 04, \mathrm{Q} 2 / 05, \mathrm{Q} 4 / 05, \mathrm{Q} 2 / 06, \mathrm{Q} 4 / 06, \mathrm{Q} 2 / 07, \mathrm{Q} 4 / 07, \mathrm{Q} 3 / 08$, $\mathrm{Q} 4 / 09, \mathrm{Q} 2 / 10, \mathrm{Q} 3 / 11-\mathrm{Q} 1 / 12$

$\mathrm{Q} 3 / 88-\mathrm{Q} 4 / 96$

Q3/88-Q4/96 


\begin{tabular}{|c|c|c|c|c|c|}
\hline \multirow{2}{*}{$\begin{array}{l}\text { CURRENT } \\
\text { NAME }\end{array}$} & \multicolumn{5}{|c|}{ FRACTURES, } \\
\hline & T.O.W.R & T.O.F.R & AQUIFER & CAVITIES, and WATER BREAKS & $\underline{\text { WELL SAMPLE HISTORY }}$ \\
\hline GW-420 & & & $u$ & & \\
\hline GW-421 & 9.00 & & wB & & \\
\hline GW-422 & & & U & & \\
\hline GW-423 & 9.00 & 24.00 & B & & \\
\hline GW-424 & & & U & & \\
\hline GW-425 & 30.00 & 50.00 & $w B / B$ & & \\
\hline GW-426 & 30.00 & & $\mathrm{U} / \mathrm{wB}$ & & \\
\hline GW-427 & 8.00 & 16.00 & B & & \\
\hline GW-428 & 8.00 & & $\mathrm{U} / \mathrm{wB}$ & & \\
\hline GW-429 & & & U & & \\
\hline GW-430 & 8.00 & 26.00 & $w B / B$ & & \\
\hline GW-431 & & & U & & \\
\hline GW-432 & 10.00 & 15.00 & B & & \\
\hline \multicolumn{6}{|l|}{ GW-433 } \\
\hline GW-434 & 13.00 & 25.00 & B & & \\
\hline GW-435 & & & U & & \\
\hline GW-436 & 13.00 & 20.00 & B & & \\
\hline GW-437 & 20.00 & 33.00 & B & & \\
\hline GW-438 & 20.00 & & U/wB & & \\
\hline GW-439 & 25.00 & 28.00 & B & & \\
\hline GW-440 & 25.00 & & $\mathrm{U} / \mathrm{wB}$ & & \\
\hline GW-441 & & 22.00 & B & & \\
\hline GW-442 & & & U & & \\
\hline GW-443 & 4.00 & 43.00 & $w B / B$ & WB @ 42 / CAV @ 42-43 & \\
\hline GW-445 & 8.00 & 30.00 & B & WB @ 22, 43 & \\
\hline & & & & F-38 & \\
\hline
\end{tabular}




\begin{tabular}{|c|c|c|c|c|c|}
\hline \multirow{2}{*}{$\begin{array}{c}\text { CURRENT } \\
\text { NAME }\end{array}$} & \multicolumn{5}{|c|}{ FRACTURES, } \\
\hline & T.O.W.R & T.O.F.R & AQUIFER & CAVITIES, and WATER BREAKS & WELL SAMPLE HISTORY \\
\hline GW-447 & & & $u$ & & \\
\hline GW-448 & 12.00 & 15.00 & B & & \\
\hline GW-449 & & & $u$ & & \\
\hline GW-450 & & 25.00 & B & & \\
\hline GW-451 & 30.00 & 50.00 & B & & \\
\hline GW-452 & & & $u$ & & \\
\hline GW-453 & & 12.00 & B & & \\
\hline GW-455 & & 10.20 & B & & \\
\hline GW-456 & & 19.40 & B & & \\
\hline GW-457 & & 19.40 & $w B / B$ & & \\
\hline GW-458 & & 18.50 & B & & \\
\hline GW-459 & & 18.50 & $w B / B$ & & \\
\hline GW-460 & & 25.00 & B & & \\
\hline GW-461 & & 25.00 & $w B / B$ & & \\
\hline GW-462 & & 22.50 & $w B / B$ & & \\
\hline GW-463 & & 14.00 & B & CAV @ 45-50(m) & \\
\hline GW-464 & & 14.00 & $w B / B$ & & \\
\hline GW-465 & 10.00 & 47.00 & $w B / B$ & CAV @ 20-47(m) & \\
\hline GW-466 & & 8.00 & B & CAV @ 21-48(m) & \\
\hline GW-467 & 19.00 & 28.00 & $w B / B$ & & \\
\hline GW-468 & 16.20 & & B & & \\
\hline GW-469 & & 30.00 & B & & \\
\hline GW-470 & & 7.50 & B & & \\
\hline GW-471 & & 18.70 & B & & \\
\hline GW-472 & & 29.00 & B & & \\
\hline & & & & F-39 & \\
\hline
\end{tabular}




\begin{tabular}{|c|c|c|c|c|c|}
\hline \multirow{2}{*}{$\begin{array}{c}\text { CURRENT } \\
\text { NAME }\end{array}$} & \multicolumn{5}{|c|}{ FRACTURES, } \\
\hline & T.O.W.R & $\underline{\text { T.O.F.R }}$ & AQUIFER & CAVITIES, and WATER BREAKS & WELL SAMPLE HISTORY \\
\hline GW-473 & & 25.00 & B & & \\
\hline GW-474 & & 25.00 & $w B / B$ & & \\
\hline GW-475A & & 25.00 & B & & \\
\hline GW-475B & & 25.00 & B & & \\
\hline GW-475C & & 25.00 & $\mathrm{U} / \mathrm{wB}$ & & \\
\hline GW-476A & & 25.00 & B & & \\
\hline GW-476B & & 25.00 & B & & \\
\hline GW-476C & & 25.00 & $\mathrm{U} / \mathrm{wB}$ & & \\
\hline GW-477A & & 25.00 & B & & \\
\hline GW-477B & & 25.00 & $w B / B$ & & \\
\hline GW-477C & & 25.00 & $\mathrm{U} / \mathrm{wB}$ & & \\
\hline GW-478A & & 20.00 & B & & \\
\hline GW-478B & & 20.00 & B & & \\
\hline GW-478C & & 20.00 & $\mathrm{U} / \mathrm{wB}$ & & \\
\hline GW-479 & & 23.00 & $w B / B$ & & \\
\hline GW-480A & & 28.00 & B & & \\
\hline GW-480B & & 28.00 & $w B / B$ & & \\
\hline GW-480C & & 28.00 & $\mathrm{U} / \mathrm{wB}$ & & \\
\hline GW-481A & & 25.00 & B & & \\
\hline GW-481B & & 25.00 & $w B / B$ & & \\
\hline GW-481C & & 25.00 & $\mathrm{U} / \mathrm{wB}$ & & \\
\hline GW-482A & & 26.00 & B & & \\
\hline GW-482B & & 26.00 & $w B / B$ & & \\
\hline GW-482C & & 26.00 & $\mathrm{U} / \mathrm{wB}$ & & \\
\hline GW-483 & & 24.00 & $w B / B$ & & \\
\hline & & & & $\mathrm{F}-40$ & \\
\hline
\end{tabular}




CURRENT
NAME
GW-484
GW-485
GW-486
GW-487
GW-488
GW-489
GW-490
GW-491
GW-492
GW-493
GW-494
GW-495
GW-496
GW-497
GW-498
GW-499
GW-499A
GW-499AA
GW-499AB
GW-499AC
GW-499AD
GW-499AE
GW-499AF
GW-499B
GW-499C

FRACTURES,

T.O.W.R T.O.F.R AQUIFER CAVITIES, and WATER BREAKS

WELL SAMPLE HISTORY 


CURRENT
NAME
GW-499D
GW-499E
GW-499F
GW-499G
GW-499H
GW-499I
GW-499J
GW-499K
GW-499L
GW-499M
GW-499N
GW-4990
GW-499P
GW-499Q
GW-499R
GW-499S
GW-499T
GW-499U
GW-499V
GW-499W
GW-499X
GW-499Y
GW-499Z
GW-502

$\begin{array}{ccc}14.00 & & U \\ 12.50 & & U \\ 10.50 & & U \\ 10.00 & & U \\ 12.30 & & U \\ 15.00 & & U \\ 12.30 & & U \\ 13.00 & & U \\ 13.00 & & U \\ 15.00 & & U \\ & & B \\ & 17.50 & \mathrm{wB} / \mathrm{B} \\ & 24.50 & \mathrm{wB} / \mathrm{B} \\ & 30.00 & \mathrm{wB} / \mathrm{B} \\ & & \mathrm{wB} / \mathrm{B} \\ & & \mathrm{wB} / \mathrm{B} \\ & & \mathrm{wB}\end{array}$




\section{CURRENT \\ NAME}

GW-505

GW-506

GW-507

GW-508

GW-509

GW-511

GW-512

GW-513

GW-514

GW-520

GW-521

GW-522

GW-526

GW-527

GW-528

GW-531

GW-532

GW-533
3.50

40.50

55.00

67.00

44.00

40.00

85.00

130.00

B

CAV @ 102-104, 116

FRACTURES,

AQUIFER CAVITIES, and WATER BREAKS

$\mathrm{U}$

U

U

U

U

100.00

B

CAV@ 85-87, 152-153

97.00

B

CAV@ @5-82, 92-97

WB @ 44.3(m), 157 / CAV @ 44.3-48, 78 90, 157-158

63.00

54.00

B

CAV@ @4-63

CAV@ 127-129(sulfur)

$23.60 \quad B$

U

U

25.00

22.40

23.00

\section{WELL SAMPLE HISTORY}

Q4/88, Q2/90-Q1/94, Q1/96, Q2/03, Q4/03, Q2/07, Q2/09, Q4/11, Q2/13

Q4/90, Q4/91-Q1/92, Q3/92-Q1/94, Q2/08, Q4/08, Q2/09, Q4/11, $\mathrm{Q} 2 / 13$

Q4/88

Q3/88-Q4/95

Q4/88-Q2/94, Q4/94, Q2/95, Q4/95, Q2/96

Q4/88-Q2/94, Q4/94, Q2/95, Q4/95, Q2/96, Q2/04, Q4/04

Q4/88-Q2/94, Q4/94, Q2/95, Q4/95, Q2/96, Q1/01, Q3/01, Q2/06, Q4/08, Q2/10, Q2/12

Q3/88-Q1/89, Q2/90-Q3/92

Q3/89, Q1/90-Q1/95, Q3/95, Q1/96-Q1/97, Q3/97, Q1/98, Q3/98,

Q1/99, Q3/99, Q1/00, Q3/00, Q1/01, Q3/01, Q1/02, Q3/02,

Q1/03, Q3/03, Q1/04, Q3/04, Q1/05, Q3/05, Q1/06, Q3/06,

Q1/07, Q3/07, Q1/08, Q3/08,

Q3/89-Q1/95, Q3/95, Q1/96, Q3/96, Q1/97, Q3/97, Q1/98, Q3/98, Q1/99, Q3/99, Q1/00, Q3/00, Q1/01, Q3/01, Q1/02, Q3/02,

Q1/03, Q3/03, Q1/04, Q3/04, Q1/05, Q3/05, Q1/06, Q3/06,

Q1/07, Q3/07, Q1/08, Q3/08

Q3/88-Q4/93, Q2/97-Q3/97, Q1/98, Q3/98, Q1/99, Q1/00, Q3/00

Q1/01, Q3/01, Q1/02, Q3/02, Q1/03, Q3/03, Q1/04, Q3/04

$\mathrm{Q} 1 / 05, \mathrm{Q} 3 / 05, \mathrm{Q} 1 / 06, \mathrm{Q} 3 / 06, \mathrm{Q} 1 / 09, \mathrm{Q} 3 / 10, \mathrm{Q} 1 / 11, \mathrm{Q} 3 / 12, \mathrm{Q} 1 / 13$

Q4/88-Q1/89, Q4/89-Q2/91, Q3/08, Q1/13

Q1/89, Q4/89-Q2/91

Q4/90-Q2/91 


\section{CURRENT \\ NAME}

GW-534

GW-535

GW-536

GW-537

GW-538

GW-539

GW-540

GW-541

GW-542

GW-543

GW-544

GW-545

GW-546

GW-551

GW-552
FRACTURES,

T.O.W.R T.O.F.R AQUIFER CAVITIES, and WATER BREAKS

16.00

27.50

$B$

CAV@ 21, 22.5-23

10.60

10.90

14.90

18.50

37.00

74.00

110.00

150.00

wB/B

CAV@ @ 31, 37.5

WB@ 155

B

CAV@ 125-132, 139-143

56.00

69.50

B

CAV@ 69-69.5

U

WB@ 73

16.00

37.00

B

WB@ 86

47.00

52.50

B

WB@ 104, 107

54.50

74.50

90.50

U/WB

WB @ 55, 59

U/wB WB @ 82.5

102.80
B

CAV @ 122, 126.6-145, 213.2-214.4

\section{WELL SAMPLE HISTORY}

\section{Q4/90-Q2/91}

$\mathrm{Q} 4 / 89-\mathrm{Q} 2 / 91$

$\mathrm{Q} 4 / 89-\mathrm{Q} 1 / 90$

Q4/89-Q1/96, Q3/96, Q1/97-Q3/97, Q1/98, Q3/98, Q1/99, Q3/99, Q1/00, Q3/00, Q1/01, Q3/01, Q1/02, Q3/02, Q1/03, Q3/03,

Q1/04, Q3/04, Q1/05, Q3/05, Q1/06, Q2/07, Q3/08, Q3/09,

$\mathrm{Q} 1 / 10, \mathrm{Q} 3 / 11, \mathrm{Q} 1 / 12, \mathrm{Q} 3 / 13$

Q4/90-Q2/91

Q1/91-Q1/95, Q3/95-Q4/95, Q2/96, Q4/96, Q2/97, Q4/97, Q1/00, $\mathrm{Q} 3 / 00, \mathrm{Q} 1 / 01, \mathrm{Q} 3 / 01, \mathrm{Q} 1 / 02, \mathrm{Q} 3 / 02$

Q1/91-Q2/94, Q4/94, Q2/95, Q4/95, Q2/96, Q1/98-Q2/98, Q4/98, Q2/99, Q4/99-Q1/00, Q3/00, Q1/01, Q3/01, Q1/02, Q3/02, Q1/03, $\mathrm{Q} 3 / 03, \mathrm{Q} 1 / 04, \mathrm{Q} 3 / 04, \mathrm{Q} 1 / 05, \mathrm{Q} 3 / 05, \mathrm{Q} 1 / 06, \mathrm{Q} 3 / 06, \mathrm{Q} 1 / 07$, $\mathrm{Q} 3 / 07, \mathrm{Q} 1 / 08, \mathrm{Q} 3 / 08$,

Q1/91-Q2/94, Q4/94, Q2/95, Q4/95, Q2/96

Q1/91-Q2/94, Q4/94, Q2/95, Q4/95, Q2/96, Q4/96, Q2/97, Q4/97, $\mathrm{Q} 2 / 98, \mathrm{Q} 4 / 98, \mathrm{Q} 2 / 99, \mathrm{Q} 4 / 99-\mathrm{Q} 1 / 00, \mathrm{Q} 3 / 00, \mathrm{Q} 1 / 01, \mathrm{Q} 3 / 01, \mathrm{Q} 1 / 02$, Q3/02, Q1/03, Q3/03, Q1/04, Q3/04, Q1/05, Q3/05, Q1/06,

Q3/06, Q1/07, Q3/07

Q1/91-Q2/94, Q4/94, Q2/95, Q4/95, Q2/96, Q4/96, Q2/97, Q4/97, Q2/98, Q4/98, Q2/99, Q4/99-Q1/00, Q3/00, Q1/01, Q3/01, Q1/02, $\mathrm{Q} 3 / 02, \mathrm{Q} 1 / 03, \mathrm{Q} 3 / 03, \mathrm{Q} 1 / 04, \mathrm{Q} 3 / 04, \mathrm{Q} 1 / 05, \mathrm{Q} 3 / 05, \mathrm{Q} 1 / 06$, $\mathrm{Q} 3 / 06, \mathrm{Q} 1 / 07, \mathrm{Q} 3 / 07$

Q1/91-Q2/94, Q4/94, Q2/95, Q4/95, Q2/96, Q4/96, Q2/97, Q4/97, Q2/98, Q4/98, Q2/99, Q4/99-Q1/00, Q3/00, Q1/01, Q3/01, Q1/02, Q3/02, Q1/03, Q3/03, Q1/04, Q3/04, Q1/05, Q3/05, Q1/06, $\mathrm{Q} 3 / 06, \mathrm{Q} 1 / 07, \mathrm{Q} 3 / 07$

Q1/91-Q2/94, Q4/94

Q1/91-Q2/94, Q4/94, Q2/95, Q4/95, Q2/96 


\section{CURRENT \\ NAME}

GW-553

GW-554

GW-555

GW-556

GW-557

GW-558

GW-559

GW-560

GW-561

GW-562

GW-563

GW-564

GW-565

GW-566

GW-567

GW-568

GW-569
FRACTURES,

T.O.W.R T.O.F.R AQUIFER CAVITIES, and WATER BREAKS

99.70

47.50

B

94.10

70.30

113.80

134.00

74.00

151.00

U/wB/B CAV@ 50

B

92.00

U

70.00

$\mathrm{U} / \mathrm{wB} / \mathrm{B}$

52.00

$\mathrm{U} / \mathrm{wB} / \mathrm{B}$

70.00

$\mathrm{U} / \mathrm{wB} / \mathrm{B}$

72.00

$\mathrm{U} / \mathrm{wB} / \mathrm{B}$

46.50

$\mathrm{U} / \mathrm{wB} / \mathrm{B}$

67.00

53.00
CAV@ 102.6-103.5

CAV@75-80

西

Q2/93-Q4/94, Q2/95, Q4/95, Q2/96, Q4/96-Q1/97, Q3/97, Q1/98, $\mathrm{Q} 3 / 98, \mathrm{Q} 1 / 99, \mathrm{Q} 3 / 99, \mathrm{Q} 1 / 00, \mathrm{Q} 3 / 00, \mathrm{Q} 1 / 01, \mathrm{Q} 3 / 01, \mathrm{Q} 1 / 02$, Q3/02, Q1/03, Q3/03, Q1/04, Q3/04, Q1/05, Q3/05, Q1/06, Q3/06, Q1/07, Q3/07, Q1/08,

Q2/93-Q4/94, Q2/95, Q4/95, Q2/96, Q4/96-Q1/97, Q3/97, Q3/00Q4/00, Q2/01-Q1/02, Q3/02, Q1/03, Q3/03, Q1/04, Q3/04, Q1/05, Q3/05, Q1/06, Q3/06, Q1/07, Q3/07, Q1/08, Q3/08, Q1/09, Q3/09, Q1/10, Q3/10, Q

Q2/93-Q4/94, Q2/95, Q4/95, Q2/96, Q4/96-Q1/97, Q3/97, Q3/00Q4/00, Q2/01-Q1/02, Q3/02, Q1/03, Q3/03, Q1/04, Q3/04, Q1/05, Q3/05, Q1/06, Q3/06, Q1/07, Q3/07, Q1/08, Q3/08, Q1/09, Q3/09, Q1/10, Q3/10, Q

Q1/12

Q2/93-Q4/94, Q2/95, Q4/95, Q2/96, Q4/96-Q1/97, Q3/97, Q3/00Q4/00, Q2/01-Q1/02, Q3/02, Q1/03, Q3/03, Q1/04, Q3/04, Q1/05, Q3/05, Q1/06, Q3/06, Q1/07, Q3/07, Q1/08, Q3/08, Q1/09, Q3/09, Q1/10, Q3/10, Q 


\section{CURRENT \\ NAME}

GW-570

GW-571

GW-572

GW-573D

GW-573S

GW-576

GW-580

GW-601

GW-602

GW-603

GW-604

GW-605

GW-606

GW-607

GW-608

GW-608A

GW-609

GW-610

GW-611
T.O.W.R T.O.F.R

\section{$\underline{A Q U}$}

$U$
$U / w B$
$U / w B$
$U / w B$
$U$
$U$
$U / w B$

75.00

8.00

54.00

55.00

24.00

31.00

100.00

60.00

55.00

9.50

10.80

\author{
B
}

WB @ 8, 24 / CAV @ 58-61, 67-68

107.00

140.00

B

WB@ 143

113.00

107.00

39.00

30.00

FRACTURES,

\section{CAVITIES, and WATER BREAKS}

CAV@ 23.5

CAV@ 65-67

CAV@ 39, 45-47, 55-58 / WB @ 67

CAV@ 35-39, 43-47, 52.5-54.5 / WB @ 86, 104.5

WB@ 33

50.00

63.80

B
WB/B
B
B
B

CAV@ 114-135, 216-218(mw)

WB/B

WB @ 97 / CAV @ 125-129.5

CAV@ $@ 5-63$

CAV@ 55-60 / FRAC @ 85-90

\section{WELL SAMPLE HISTORY}

Q1/90-Q1/94, Q1/99, Q1/05, Q3/05, Q2/07, Q1/09, Q3/10, Q1/11, Q3/12, Q1/13

Q1/90-Q3/92

Q1/90-Q4/96

Q1/90-Q4/96

Q3/91-Q1/97, Q3/97, Q1/98-Q3/98, Q1/99, Q3/99, Q1/00, Q3/00

$\mathrm{Q} 1 / 01, \mathrm{Q} 3 / 01, \mathrm{Q} 1 / 02, \mathrm{Q} 3 / 02, \mathrm{Q} 1 / 03, \mathrm{Q} 3 / 03, \mathrm{Q} 1 / 04, \mathrm{Q} 3 / 04$

Q1/05, Q3/05, Q1/06, Q3/06, Q1/07, Q3/07, Q1/08, Q3/08,

Q1/09, Q3/09, Q1/10, Q3/10,

Q3/91-Q1/97, Q3/97, Q1/98, Q3/98, Q1/99, Q3/99, Q1/00, Q3/00,

Q1/01, Q3/01, Q1/02, Q3/02, Q1/03, Q3/03, Q1/04, Q3/04,

$\mathrm{Q} 1 / 05, \mathrm{Q} 3 / 05, \mathrm{Q} 1 / 06, \mathrm{Q} 3 / 06, \mathrm{Q} 1 / 07, \mathrm{Q} 3 / 07, \mathrm{Q} 1 / 08, \mathrm{Q} 3 / 08$,

Q1/09, Q3/09, Q1/10, Q3/10

Q1/90-Q1/93

Q1/90-Q1/96, Q2/97-Q3/97, Q1/98, Q3/98, Q1/01, Q3/01, Q4/08, $\mathrm{Q} 2 / 10, \mathrm{Q} 2 / 12$

Q1/90-Q2/96, Q4/96-Q1/98, Q3/98, Q1/99, Q3/99, Q1/00, Q3/00, Q1/01, Q2/06, Q4/08, Q2/10, Q2/12

Q1/90-Q1/96, Q2/04, Q4/04

Q1/90-Q1/96, Q2/04, Q4/04 


\begin{tabular}{|c|c|c|c|c|}
\hline CURRENT & & & & FRACTURES, \\
\hline NAME & T.O.W.R & $\underline{\text { T.O.F.R }}$ & $\underline{\text { AQUIFER }}$ & CAVITIES, and WATER BREAKS \\
\hline GW-612 & 78.00 & 125.00 & B & CAV@ @1-84, 104-119.5 \\
\hline GW-613 & 15.00 & & wB & \\
\hline GW-614 & 7.00 & 50.80 & B & \\
\hline GW-615 & 15.00 & 40.00 & B & CAV@ 33.5-34.5, 36-38.5 \\
\hline GW-616 & 35.00 & 42.00 & B & WB @ 69-70 \\
\hline GW-617 & & & $U$ & \\
\hline GW-618 & 25.00 & 27.00 & $w B / B$ & FRAC @ 32-37 \\
\hline GW-619 & 40.00 & & $\mathrm{U} / \mathrm{wB}$ & \\
\hline GW-620 & 41.00 & 70.00 & $w B / B$ & WB@ @ 50.2, 68 \\
\hline GW-621 & 22.00 & & $\mathrm{U} / \mathrm{wB}$ & \\
\hline GW-622 & 14.20 & & $\mathrm{U} / \mathrm{wB}$ & \\
\hline GW-623 & 16.00 & 38.00 & B & FRAC @ 267, 298 \\
\hline GW-624 & 12.00 & & wB & WB@ 22 \\
\hline GW-625 & 3.00 & 20.00 & B & FRAC @ 274-275 \\
\hline GW-626 & 2.00 & 64.00 & $w B / B$ & WB @ 18 / FRAC @ 73 \\
\hline GW-627 & 3.00 & 43.00 & B & FRAC @ 74, 256 \\
\hline
\end{tabular}

\section{WELL SAMPLE HISTORY}

Q1/90-Q3/92, Q3/96, Q1/01, Q3/01, Q2/04, Q4/04, Q2/06, Q2/07, $\mathrm{Q} 4 / 08, \mathrm{Q} 2 / 13$

Q1/90-Q3/92, Q4/93-Q1/94, Q3/94, Q1/95, Q3/95, Q1/96, Q3/96, $\mathrm{Q} 1 / 97, \mathrm{Q} 3 / 97$

Q1/90-Q3/92, Q4/93-Q1/94, Q3/94, Q1/95, Q3/95, Q1/96, Q3/96, $\mathrm{Q} 1 / 97, \mathrm{Q} 3 / 97$

Q2/90-Q3/92, Q4/99-Q3/00, Q1/04, Q3/04, Q1/06, Q2/07, Q3/08, Q1/09, Q3/10, Q1/11, Q3/12, Q1/13

Q2/90-Q3/92, Q1/01, Q3/01, Q1/05, Q3/05, Q1/06, Q2/07, Q3/08, Q3/09, Q1/10, Q3/11, Q1/12, Q3/13

Q2/90-Q2/96, Q4/96, Q2/97, Q4/97, Q2/06, Q4/08, Q2/13

Q2/90-Q2/96, Q4/96, Q2/97, Q4/97, Q2/98, Q4/98, Q2/99, Q4/99, Q2/00, Q4/00, Q2/01, Q4/01, Q2/02, Q4/02, Q2/03, Q4/03, Q1/05, Q1/06, Q1/07, Q1/08, Q1/09, Q1/10, Q1/11, Q1/12

Q2/90-Q2/96, Q4/96, Q2/98-Q3/98, Q2/06, Q4/08, Q2/10, Q4/12

Q2/90-Q2/96, Q4/96, Q2/97, Q4/97, Q2/98-Q4/98, Q2/99, Q4/99, Q2/00, Q4/00, Q2/01, Q4/01, Q2/02, Q4/02, Q2/03, Q4/03,

Q2/04, Q4/04, Q2/05, Q4/05, Q2/06, Q2/07, Q4/08, Q2/10, Q4/12 Q1/90-Q1/94, Q4/94-Q1/95, Q3/95, Q1/96, Q3/96, Q1/97, Q3/97, Q1/98, Q3/98, Q1/99, Q3/99, Q1/00, Q3/00

$\mathrm{Q} 1 / 90-\mathrm{Q} 3 / 92$

Q2/90, Q1/91-Q3/92, Q1/08, Q3/08, Q1/09, Q3/10, Q1/11, Q3/12, $\mathrm{Q} 1 / 13$

Q2/90-Q2/91, Q2/98-Q3/98, Q2/05, Q4/05

Q1/90-Q4/93, Q2/98-Q3/98, Q1/02, Q3/02, Q2/05, Q4/05-Q1/06, Q3/06, Q1/07, Q3/07, Q1/08-Q4/08, Q1/10, Q3/12

Q1/90-Q1/96, Q3/96, Q1/97, Q3/97, Q1/98-Q3/98, Q1/99, Q3/99, Q1/00, Q3/00, Q1/01, Q3/01, Q1/02, Q3/02, Q1/03, Q3/03,

Q1/04, Q3/04, Q2/05, Q4/05-Q1/06, Q3/06, Q1/07, Q3/07, Q1/08, $\mathrm{Q} 3 / 08, \mathrm{Q} 1 / 09, \mathrm{Q} 3 / 09$, 


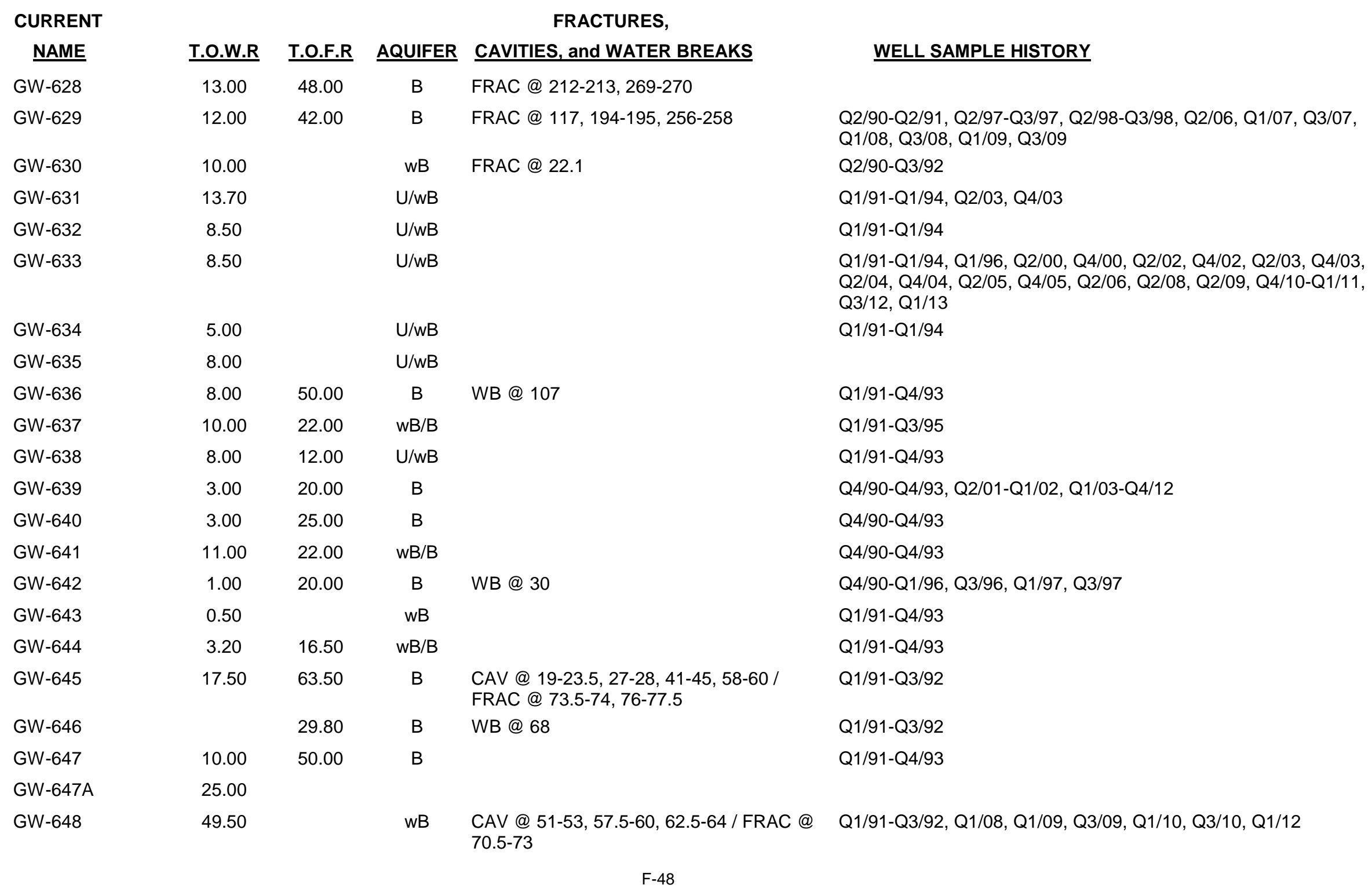




\section{CURRENT \\ NAME}

GW-649

GW-651

GW-652

GW-653

GW-654

GW-655

GW-656

GW-657

GW-658

GW-659

GW-660

GW-661

GW-662

GW-663

GW-664

GW-665

GW-666

GW-667

GW-668

GW-669

GW-670

GW-671
FRACTURES,

T.O.W.R T.O.F.R AQUIFER CAVITIES, and WATER BREAKS

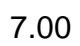

7.50

9.50

3.50

11.00

wB

B

$w B$

FRAC @ 22.5-27

35.00

$w B / B$

$\begin{array}{cccc}4.00 & 7.00 & \text { WB/B } & \text { WB @ } 5 \\ 9.00 & 47.00 & \text { B } & \\ 12.00 & & \text { U/wB } & \text { WB @ 18.4 }\end{array}$

$U$

wB

$w B$

$U$

$U$

U/WB

$U$

U

5.50

WB

U

U

8.00

12.00

$w B / B$

6.30

14.00

8.90

\section{WELL SAMPLE HISTORY}

Q1/91-Q3/92

Q1/91-Q4/93

Q1/91-Q3/95

Q1/91-Q1/96, Q3/96, Q1/97, Q3/97, Q1/98, Q3/98, Q1/99, Q3/99, Q1/00, Q3/00, Q1/01, Q3/01, Q1/02, Q3/02, Q1/03, Q3/03,

$\mathrm{O} 1 / 04, \mathrm{O} 3 / 04, \mathrm{Q} 2 / 05, \mathrm{Q} 4 / 05, \mathrm{Q} 2 / 06, \mathrm{O} 1 / 07, \mathrm{O} 3 / 08, \mathrm{Q} 1 / 09$,

$\mathrm{Q} 3 / 10, \mathrm{Q} 1 / 11, \mathrm{Q} 3 / 12, \mathrm{Q} 1 / 13$

Q1/91-Q4/95

Q1/91-Q4/93

Q1/91-Q4/93, Q2/00, Q4/00, Q2/01, Q4/01, Q4/06, Q4/07, Q4/08, Q2/09, Q4/10, Q3/11, Q1/12, Q3/13

$\mathrm{Q} 1 / 91-\mathrm{Q} 4 / 93$

Q1/91-Q4/93, Q2/98-Q3/98, Q2/02, Q4/02, Q2/03, Q2/04, Q2/05, $\mathrm{Q} 2 / 06, \mathrm{Q} 2 / 07, \mathrm{Q} 2 / 08, \mathrm{Q} 3 / 09, \mathrm{Q} 2 / 10, \mathrm{Q} 2 / 11, \mathrm{Q} 2 / 12$

Q1/91-Q4/93, Q2/98-Q3/98 


\section{CURRENT \\ NAME}

GW-672

GW-673

GW-674

GW-675

GW-676

GW-677

GW-678

GW-679

GW-680

GW-681

GW-682

GW-683

GW-684

GW-685

GW-686

GW-687

GW-688

GW-689

GW-690
FRACTURES,

T.O.W.R T.O.F.R

28.00

15.00

27.00

10.00

53.00

53.00

69.80

40.60

72.00

58.00

22.00

17.00

42.00

84.00

75.00

105.00

73.00

83.00

65.00

26.00

9.50

5.00

15.50

17.00

32.00

17.00

20.00

24.00

\section{AQUIFER CAVITIES, and WATER BREAKS}

U/wB WB @ 11.5-15

U WB @ 5

U/wB WB @ 7

B WB @ 30-30.7, 152

B CAV@ 130

B WB @ 115-132

B WB@ 0, 115 / CAV@ 44-50

B CAV@ 11-83, 175 / WB @ 100, 119, 185

B WB@ 166

B CAV@ 31-32, 149-158 / WB @ 113-118, 123-127

B CAV @ 31-32, 72-73, 122-124 / WB @ $110-115$

B CAV@ 13.7-15, 37-39 / WB @ 64, 110120

U

U

wB WB@ $@ 54$

U

WB WB @ 53

\section{WELL SAMPLE HISTORY}

Q2/04, Q4/04

Q2/04, Q4/04

Q2/91-Q1/94, Q3/94, Q1/95, Q3/95, Q1/96, Q3/96, Q1/97, Q3/97, Q1/98, Q3/98, Q1/99, Q3/99, Q1/00, Q3/00, Q1/01, Q3/01, $\mathrm{Q} 3 / 02, \mathrm{Q} 1 / 03, \mathrm{Q} 3 / 03, \mathrm{Q} 1 / 04, \mathrm{Q} 3 / 04, \mathrm{Q} 1 / 05, \mathrm{Q} 3 / 05, \mathrm{Q} 1 / 06$, $\mathrm{Q} 3 / 06, \mathrm{Q} 1 / 07, \mathrm{Q} 3 / 07, \mathrm{Q} 1 / 08$

Q2/91-Q1/94, Q3/94, Q1/95, Q3/95, Q1/96, Q3/96, Q1/97, Q3/97, Q1/98, Q3/98, Q1/99, Q3/99, Q1/00, Q3/00, Q1/01, Q3/01, $\mathrm{Q} 1 / 02, \mathrm{O} 3 / 02, \mathrm{O} 1 / 03, \mathrm{O} 3 / 03, \mathrm{Q} 1 / 04, \mathrm{Q} 3 / 04, \mathrm{O} 1 / 05, \mathrm{O} 3 / 05$, Q1/06, Q3/06, Q1/07, Q3/07

Q2/91-Q1/94, Q3/94, Q1/95, Q3/95, Q1/96, Q3/96, Q1/97, Q3/97,

Q1/98, Q3/98, Q1/99, Q3/99, Q1/00, Q3/00, Q1/01

Q1/98, Q4/02, Q2/06, Q4/06, Q2/07, Q4/07, Q4/08, Q2/09, $\mathrm{Q} 4 / 10, \mathrm{Q} 2 / 11, \mathrm{Q} 3 / 12, \mathrm{Q} 2 / 13$

Q1/96, Q1/98, Q4/02

Q4/02

Q2/96, Q2/00, Q4/00, Q2/03, Q4/03, Q4/06, Q4/07, Q4/08, Q2/09, Q4/10, Q2/11, Q3/12, Q2/13 


\section{CURRENT \\ NAME}

GW-691

GW-692

GW-693

GW-694

GW-695

GW-696

GW-697

GW-698

GW-699

GW-700

GW-701

GW-702

GW-703

GW-704

GW-705

GW-705A

8.00
23.50

\section{T.O.W.R}

20.00

23.00

11.00

6.00

21.00

18.00

21.00

42.00

13.90

31.00

25.00

21.00

7.00

16.00

23.00

38.00

10.00
FRACTURES,

\section{AQUIFER CAVITIES, and WATER BREAKS}

U

wB WB@ 52

U

B

U

WB@ 11, 22-32

WB@ 10

wB CAV@ 42 / WB @ 75

U WB @ 9

U $\quad$ WB @ 12.5, 24, 30.5

U

$U$

B
WB @ 47.5

WB @ 50-55, 110-115, 255-256

WB @ 10, 79, 120, 180.5

\section{WELL SAMPLE HISTORY}

Q2/96, Q2/04, Q4/04, Q4/06, Q4/07, Q4/08, Q2/09, Q4/09, $\mathrm{Q} 2 / 10, \mathrm{Q} 4 / 10, \mathrm{Q} 2 / 11, \mathrm{Q} 4 / 11-\mathrm{Q} 1 / 12, \mathrm{Q} 3 / 12, \mathrm{Q} 2 / 13$

Q1/96-Q2/96, Q2/04, Q4/04, Q4/06, Q4/07, Q4/08, Q2/09, Q4/10, Q2/11, Q3/12, Q2/13

Q2/91-Q1/94, Q4/94-Q1/95, Q3/95, Q1/96, Q3/96, Q1/98, Q3/98, Q1/02, Q3/02, Q1/05, Q3/05, Q3/06, Q3/07, Q1/08

Q2/91-Q1/94, Q4/94-Q1/95, Q3/95, Q1/96, Q3/96, Q1/97, Q3/97, Q1/98, Q3/98, Q1/99, Q3/99, Q1/00, Q3/00, Q1/01, Q3/01,

Q1/02, Q3/02, Q1/03, Q3/03, Q1/04, Q3/04, Q1/05, Q3/05, Q3/06 Q2/03

Q1/96-Q2/96, Q2/98-Q3/98, Q2/01, Q4/01, Q2/02, Q4/02, Q2/03, Q4/03, Q2/04, Q4/04, Q2/05, Q4/05, Q2/06, Q4/06, Q2/07,

$\mathrm{Q} 4 / 07, \mathrm{Q} 2 / 08, \mathrm{Q} 4 / 08, \mathrm{Q} 2 / 09, \mathrm{Q} 4 / 09, \mathrm{Q} 2 / 10, \mathrm{Q} 4 / 10, \mathrm{Q} 2 / 11, \mathrm{Q} 4 / 11-$ $\mathrm{Q} 1 / 12, \mathrm{Q} 3 / 12, \mathrm{Q} 2 / 13$

Q2/96, Q2/00, Q4/00, Q2/03, Q4/03, Q4/06, Q4/07, Q4/08, $\mathrm{Q} 3 / 09, \mathrm{Q} 1 / 10, \mathrm{Q} 3 / 11, \mathrm{Q} 1 / 12, \mathrm{Q} 3 / 13$

Q2/91-Q1/94, Q4/94-Q1/95, Q3/95, Q1/96, Q3/96, Q1/97, Q3/97, Q1/98, Q3/98, Q1/99, Q3/99, Q1/00, O3/00, O1/01, O3/01, $\mathrm{Q} 1 / 02, \mathrm{Q} 3 / 02, \mathrm{Q} 1 / 03, \mathrm{Q} 3 / 03, \mathrm{Q} 1 / 04, \mathrm{Q} 3 / 04, \mathrm{Q} 1 / 05, \mathrm{Q} 3 / 05$, Q3/06, Q3/07, Q1/08, Q1/09,

Q2/91-Q1/94, Q4/94-Q1/95, Q3/95, Q1/96, Q3/96, Q1/97, Q3/97, Q1/98, Q3/98, Q1/99, Q3/99, Q1/00, Q3/00, Q1/01, Q3/01, Q1/02, Q3/02, Q1/03, Q3/03, Q1/04, Q3/04, Q1/05, Q3/05, $\mathrm{Q} 1 / 06, \mathrm{Q} 3 / 06, \mathrm{Q} 1 / 07, \mathrm{Q} 3 / 07$,

Q2/91-Q4/92 


\section{CURRENT \\ NAME}

GW-705B

GW-706

GW-707

GW-708

GW-709

GW-710

GW-711

GW-712

GW-713

GW-714

GW-715

34.00

GW-721

GW-722

8.00

17.00

20.00

11.00

39.00

15.00

12.00

26.80

27.00

54.00

\section{T.O.W.R}

43.00

3.50

20.50

66.00

63.80

35.00

6.00

\section{B}

WB @ 33(mw), 133-145(mw)

U/wB

CAV @ 37.6-38.6(m), 43.6-44.6(m)

FRACTURES,

\section{AQUIFER CAVITIES, and WATER BREAKS}

FRAC @ 116, 133 / WB @ 171.5, 175.5

U/wB

$\mathrm{U} / \mathrm{wB}$

WB@ 65.6, 74.5-80.6

WB @ 58, 103, 168-173, 479.7-484.7 / FRAC @ 185.3-189.3

WB @ 65, 120.5, 186-191, 246-250, 284287(sulfur), 299-307, 316-320, 412-421, 650-666

WB @ 85-86, 111-114, 155.2-161.2, 180.2181.2, 271-272, 274-276, 311-316, 341.2 $351.2,403.5-405.5$

CAV @ 0, 31.8-34.5, 35.8-40.8, 43.8-48.5, 54.8-61.8

$W B / B$

U/wB/B
CAV @ 58.5-65.5, 100-103.1

\section{WELL SAMPLE HISTORY}

Q2/91-Q1/94, Q4/94-Q1/95, Q3/95, Q1/96, Q3/96, Q1/97, Q3/97, Q1/98-Q3/98, Q1/99, Q3/99, Q1/00, Q3/00, Q1/01, Q3/01, Q1/02, $\mathrm{Q} 3 / 02, \mathrm{Q} 1 / 03, \mathrm{Q} 3 / 03, \mathrm{Q} 1 / 04, \mathrm{Q} 3 / 04, \mathrm{Q} 1 / 05, \mathrm{Q} 3 / 05, \mathrm{Q} 1 / 06$,

Q3/06, Q1/07, Q3/07,

Q1/91-Q4/93, Q1/96

$\mathrm{Q} 1 / 91-\mathrm{Q} 4 / 93$

Q2/91-Q1/95, Q3/95-Q4/95, Q2/96, Q4/96, Q2/97, Q4/97, Q2/98, Q4/98, Q2/99, Q4/99-Q1/00, Q3/00, Q1/01, Q3/01, Q1/02, Q3/02, Q1/03, Q3/03, Q1/04, Q3/04, Q1/05, Q3/05, Q1/06, Q3/06,

Q1/07, Q3/07, Q1/08,

Q4/91-Q1/94, Q3/94, Q1/95, Q3/95, Q1/96, Q3/96, Q1/97, Q3/97, $\mathrm{Q} 1 / 03, \mathrm{Q} 3 / 03$

Q4/91-Q1/94, Q3/94, Q1/95, Q3/95, Q1/96, Q3/96, Q1/97, Q3/97, $\mathrm{Q} 1 / 03, \mathrm{Q} 3 / 03$

Q4/91-Q1/94, Q3/94, Q1/95, Q3/95, Q1/96, Q3/96, Q1/97, Q3/97, Q1/98, Q3/98, Q1/99, Q3/99, Q1/00, Q3/00, Q1/01, Q3/01, Q1/02, Q3/02, Q1/03, Q3/03, Q1/04, Q3/04, Q1/05, Q3/05, $\mathrm{Q} 1 / 06, \mathrm{Q} 3 / 06, \mathrm{Q} 1 / 07, \mathrm{Q} 3 / 07$

Q2/92-Q1/94, Q3/94, Q1/95, Q3/95, Q1/96, Q3/96, Q1/97, Q3/97, $\mathrm{Q} 1 / 98, \mathrm{Q} 3 / 98, \mathrm{Q} 1 / 99, \mathrm{Q} 3 / 99, \mathrm{Q} 1 / 00, \mathrm{Q} 3 / 00, \mathrm{Q} 1 / 01, \mathrm{Q} 3 / 01$, $\mathrm{Q} 1 / 02, \mathrm{Q} 3 / 02, \mathrm{Q} 1 / 03, \mathrm{Q} 3 / 03, \mathrm{Q} 1 / 04, \mathrm{Q} 3 / 04, \mathrm{Q} 1 / 05, \mathrm{Q} 3 / 05$, Q1/06, Q3/06, Q1/07, Q3/07

Q3/92-Q1/94, Q3/94, Q1/95, Q3/95, Q1/96, Q3/96, Q1/97, Q3/97, Q1/98, Q3/98, Q1/99, Q3/99, Q1/00, Q3/00, Q1/01, Q3/01, $\mathrm{Q} 1 / 02, \mathrm{O} 3 / 02, \mathrm{O} 1 / 03, \mathrm{O} 3 / 03, \mathrm{Q} 1 / 04, \mathrm{O} 3 / 04, \mathrm{Q} 1 / 05, \mathrm{O} 3 / 05$, Q1/06, Q3/06, Q1/07, Q3/07

Q3/92-Q1/94, Q3/94, Q1/95, Q3/95, Q1/96, Q3/96, Q1/97, Q3/97, Q1/98, Q3/98, Q1/99, Q3/99, Q1/00, Q3/00, Q1/01, Q3/01, $\mathrm{Q} 1 / 02, \mathrm{Q} 3 / 02, \mathrm{Q} 1 / 03, \mathrm{Q} 3 / 03, \mathrm{Q} 1 / 04$ 


\section{CURRENT \\ NAME}

GW-723

GW-724

GW-725

GW-727

GW-728

GW-729

GW-730

GW-731

GW-732

GW-733

GW-734
14.00

17.50

3.00

25.50

11.50

33.50

40.00

30.00

45.00

95.40

35.60

129.40

85.00

96.00

B

42.50

47.10

35.40

39.40

$w B / B$

U/wB/B

U/wB/B

B

U/wB/B

U/wB/B

B

CAV@ 109.2-129.4, 140-145, 164.5-167.1

Q3/91-Q4/95, Q2/96, Q4/96, Q2/97, Q4/97, Q2/98, Q4/98, Q2/99, $\mathrm{Q} 4 / 99, \mathrm{Q} 2 / 00, \mathrm{Q} 4 / 00, \mathrm{Q} 2 / 01, \mathrm{Q} 4 / 01, \mathrm{Q} 2 / 02, \mathrm{Q} 4 / 02, \mathrm{Q} 2 / 03$,

Q4/03, Q2/04, Q4/04, Q2/05, Q4/05, Q2/06, Q4/06-Q1/07, Q3/08, $\mathrm{Q} 1 / 09, \mathrm{Q} 3 / 10, \mathrm{Q} 1 / 11$,

Q3/91-Q4/95, Q2/96, Q4/96, Q2/97, Q4/97, Q2/98, Q4/98, Q2/99, $\mathrm{Q} 4 / 99, \mathrm{Q} 2 / 00, \mathrm{Q} 4 / 00, \mathrm{Q} 2 / 01, \mathrm{Q} 4 / 01, \mathrm{Q} 2 / 02, \mathrm{Q} 4 / 02, \mathrm{Q} 2 / 03$,

Q4/03, Q2/04, Q4/04, Q2/05, Q4/05, Q2/06, Q4/06-Q1/07, Q3/08, Q1/09, Q3/10, Q1/11,

Q2/92-Q1/97, Q3/97, Q1/98-Q3/98, Q1/99, Q3/99, Q1/00, Q3/00,

Q1/01, Q3/01, Q1/02, Q3/02, Q1/03, Q3/03, Q1/04, O3/04,

Q1/05, Q3/05, Q1/06, Q3/06, Q1/07, Q3/07, Q1/08, Q3/08,

Q1/09, Q3/09, Q1/10, Q3/10, 


\section{CURRENT \\ NAME}

GW-735

GW-736

GW-737

GW-738

GW-739

GW-740

GW-742

GW-743

GW-744

GW-745

GW-746

GW-747

GW-748

GW-749

\section{T.O.W.R}

19.00

T.O.F.R

77.50

7.50

12.00

14.00

12.00

15.10

34.00

42.00

38.10

45.10

68.00

96.00

49.00

87.50

9.60

14.60

9.50

9.00

12.50

11.00

3.85

5.35

0.82

9.00

4.62

11.00

\section{FRACTURES,}

\section{AQUIFER CAVITIES, and WATER BREAKS}

wB/B CAV @ 21-24.1(m), 33.5-35.5(m), 36$39.5(\mathrm{~m}), 42-43$

B

WB @ 4 / FRAC @ 57.5, 94(mW)

FRAC @ 0, 55.6-56(mw) / CAV @ 83-85

CAV @ 41.1-43.1(w), 50.1-51.5(mW), 75.177.1(mW)

B FRAC @ 2, 118.2, 136.2, 172.2, 186.2, 224.5, 243.2

B FRAC @ 71(mw), 98.6-99(mw), 117117.6(mw), 187.5

B

CAV @ 93-96, 180-181 / FRAC @ 189.5, 265, 289.5-29

B CAV@ 63.5-64.5(m), 75-77.5(m), 8888.5(0)

B FRAC @ 0, 25.6(mw)

\section{B FRAC @ 28-29(mw), 32(mw)}

U/wB/B WB @ 9

B WB @ 7(mw), 14.5(mw), 19(w), 27(w), 69.2-79.2(w)

$B$

WB@ 17-19.82(w)

U/wB/B WB @ 6(w)

\section{WELL SAMPLE HISTORY}

Q2/92-Q4/96, Q2/97, Q4/97, Q2/98, Q4/98, Q2/99, Q4/99, Q2/00$\mathrm{Q} 4 / 00, \mathrm{Q} 2 / 01, \mathrm{Q} 4 / 01, \mathrm{Q} 2 / 02, \mathrm{Q} 4 / 02, \mathrm{Q} 2 / 03, \mathrm{Q} 4 / 03, \mathrm{Q} 2 / 04$,

Q4/04, Q2/05, Q4/05, Q1/07, Q3/09, Q3/11, Q1/13

Q2/92-Q1/94, Q4/94-Q1/95, Q3/95, Q1/02, Q3/02, Q1/05, Q3/05

Q2/92-Q1/94, Q4/94-Q1/95, Q3/95, Q1/02, Q3/02, Q1/05, Q3/05

Q2/92-Q1/94, Q4/94-Q1/95, Q3/95, Q1/96, Q3/96, Q1/97, Q3/97, Q1/98, Q3/98, O1/99, O3/99, Q1/00, O3/00, O1/01, Q3/01, Q1/02, Q3/02, Q1/03, Q3/03, Q1/04, Q3/04, Q1/05, Q3/05, Q3/06, Q3/07, Q1/08, Q1/09,

Q2/92-Q1/94, Q4/94-Q1/95, Q3/95, Q1/02, Q3/02, Q1/05, Q3/05

Q2/92-Q1/94, Q4/94-Q1/95, Q3/95, Q1/96, Q3/96, Q1/97, Q3/97, Q1/98, Q3/98, Q1/99, Q3/99, Q1/00, Q3/00, Q1/01, Q3/01 $\mathrm{Q} 1 / 02, \mathrm{Q} 3 / 02, \mathrm{Q} 1 / 03, \mathrm{Q} 3 / 03, \mathrm{Q} 1 / 04, \mathrm{Q} 3 / 04, \mathrm{Q} 1 / 05, \mathrm{Q} 3 / 05$, $\mathrm{Q} 3 / 06, \mathrm{Q} 3 / 07, \mathrm{Q} 1 / 08-\mathrm{Q} 2 / 08$

Q2/92-Q1/96, Q2/04, Q4/04

Q2/92-Q1/96, Q2/04, Q4/04

Q2/92-Q4/96, Q2/97, Q4/97, Q2/98, Q4/98, Q2/99, Q4/99, Q2/00, Q4/00, Q2/01, Q4/01, Q2/02, Q4/02, Q2/03, Q4/03, Q2/04,

Q4/04, Q2/05, Q4/05-Q1/06, Q1/07, Q3/08, Q3/09, Q3/10, Q3/11, $\mathrm{Q} 1 / 12, \mathrm{Q} 3 / 13$

Q2/92-Q4/96, Q2/97, Q4/97

Q2/92-Q4/96, Q2/97, Q4/97

Q3/92-Q4/96, Q2/97, Q4/97, Q2/98, Q4/98, Q2/99, Q4/99, Q2/00, Q4/00, Q2/01, Q4/01, Q2/02, Q4/02, Q2/03, Q4/03, Q2/04, Q4/04, Q2/05, Q4/05-Q1/06, Q1/07, Q3/08, Q3/09, Q3/10, Q3/11, $\mathrm{Q} 1 / 12, \mathrm{Q} 3 / 13$

Q3/92-Q4/96, Q2/97, Q4/97, Q1/06, Q3/08, Q3/10, Q1/12 Q3/92-Q4/96, Q2/97, Q4/97, Q1/06 


\section{CURRENT \\ NAME}

GW-750

GW-751

GW-752

GW-753

GW-754

GW-755

GW-756

GW-757

GW-758

GW-759

GW-760

GW-761

GW-762

GW-763

GW-764

GW-765

GW-766
17.00

\section{T.O.W.R}

18.50

10.00

11.50

1.00

1.00

14.60

13.00

29.50

16.00

14.00

4.00

4.80

12.00

24.90

24.50

9.00

\section{FRACTURES,}

\section{AQUIFER CAVITIES, and WATER BREAKS}

24.80

B

WB @ 6, 64, 64.8-70.8(w) / CAV @ 19. 15.5(m), 20

12.00

12.50

22.50

17.50

22.00

16.00

48.00

19.00

20.00

12.50

11.50

14.50

B

WB @ 9(mw), 12(mw), 50.1-60.1(w)

U/wB/B WB @ 9.5-15.5(mw)

B WB @ 14.2-16(mw), 20.5(mw), 30.2(w)

wB/B WB @ 7.5(mw), 14.1, 24.1(mw)

B WB@5(mw), 50-60(w)

U/wB WB @ 5-16.7(mw)

B CAV @ 67-71(o) / FRAC @ 76-77(m), 9494.5(0), 137-137.5

B WB @ 10(w) / FRAC @ 48-49(mw)

wB/B FRAC @ 25(mw), 28-28.5(mw)

B WB @ 7.5(mw), 27(mw), 45-55(w)

WB/B WB @ 5.3-7(mw), 9-10.5(mw), 14.6(mw)

B WB@7(mw), 48.2-58.2(mw)

U WB @ 11(mw)

33.90

22.00

\section{B}

U/wB

$\mathrm{B}$
WB @ 24.5(mw) / FRAC @ 49(0), 63(mw) WB @ 22-32(mw)

WB @ 6.5-7.5(mw), 14.5-16.5(mw), 18.5(mw), 20(mw)

\section{WELL SAMPLE HISTORY}

Q3/92-Q4/96, Q2/97, Q4/97, Q2/98, Q4/98, Q2/99, Q4/99, Q2/00, $\mathrm{Q} 4 / 00, \mathrm{Q} 2 / 01, \mathrm{Q} 4 / 01, \mathrm{Q} 2 / 02, \mathrm{Q} 4 / 02, \mathrm{Q} 2 / 03, \mathrm{Q} 4 / 03, \mathrm{Q} 2 / 04$,

Q4/04, Q2/05, Q4/05, Q1/07, Q2/09, Q3/11

Q3/92-Q2/96, Q4/96

Q3/92-Q2/96, Q4/96

Q3/92-Q4/93, Q2/97-Q1/98, Q3/98

Q3/92-Q4/93, Q1/96, Q2/97-Q1/98, Q3/98

Q3/92-Q4/93, Q2/97-Q3/97, Q1/98, Q3/98

Q3/92-Q4/93, Q1/96, Q2/97-Q3/97, Q1/98, Q3/98

Q2/92-Q1/95, Q3/95-Q4/95, Q2/96, Q4/96, Q2/97, Q4/97, Q2/98, Q4/98, Q2/99, Q4/99-Q1/00, Q3/00, Q1/01, Q3/01, Q1/02, Q3/02, Q1/03, Q3/03, Q1/04, Q3/04, Q1/05, Q3/05, Q1/06, Q3/06,

Q1/07, Q3/07, Q1/08,

Q3/92-Q4/95

Q3/92-Q4/95

Q3/92-Q4/95, Q2/03, Q4/03, Q2/04

Q3/92-Q4/95, Q2/03, Q4/03

Q3/92-Q2/95, Q1/99, Q3/99, Q2/00-Q3/00, Q1/01, Q3/01, Q1/02,

Q3/02, Q1/03, Q3/03, Q1/04, Q3/04, Q1/05, Q3/05, Q1/06,

Q3/06, Q1/07, Q3/07, Q1/08, Q3/08, Q1/09, Q3/09, Q1/10,

Q3/10, Q1/11, Q3/11, Q1/12,

Q3/92-Q4/96, Q2/97, Q4/97, Q2/98, Q4/98, Q2/99, Q4/99, Q2/00,

$\mathrm{Q} 4 / 00, \mathrm{Q} 2 / 01, \mathrm{Q} 4 / 01, \mathrm{Q} 2 / 02, \mathrm{Q} 4 / 02, \mathrm{Q} 2 / 03, \mathrm{Q} 4 / 03, \mathrm{Q} 2 / 04$,

Q4/04, Q2/05, Q4/05, Q3/06, Q4/07, Q3/08, Q4/09, Q2/10,

$\mathrm{Q} 3 / 11, \mathrm{Q} 1 / 12, \mathrm{Q} 3 / 13$

Q3/92-Q4/95, Q2/03, Q4/03

Q3/92-Q1/96, Q2/03, Q4/03, Q2/04, Q4/07, Q2/09, Q4/11

Q1/93-Q2/96, Q4/96 


\section{CURRENT \\ NAME}

GW-767

GW-768

GW-769

GW-770

GW-771

GW-772

GW-773

GW-774

GW-775

GW-776

GW-777

GW-778

GW-779

GW-780

GW-781

GW-782
T.O.W.R

2.00

28.00

14.20

12.00

17.40

19.00

0.50

0.30

19.00

19.00

16.70

14.50

19.30

20.20

14.00

2.00

7.20

18.20

19.50

0.90

14.00

1.00
FRACTURES,

AQUIFER CAVITIES, and WATER BREAKS

wB WB @ 5.5(mw), 13-13.5(mw)

WB@ 18.8(mw), 54.8-59.8(mw)

WB @ 12(mw), 36.4-61.4(mw)

U/wB/B WB @ 7.8(mw)

B $\quad$ WB @ 4(mw), 7(mw), 11.5(mw) / FRAC @
$\quad 48(\mathrm{mw})$

U WB @ 10.3(mw)

B WB @ 16.8(mw), 23-26.4(mw), 34.4-37, 49.8-54.4(mw)

wB/B WB @ 17-18.5(mw), 24-26.6(mw)

B WB @ 13-16.7(mw), 46.3-56.3(mw)

U/wB/B WB @ 12.7-24(mw)

B WB @ 14(mw), 25-26(mw)

wB/B

B

U

WB @ 4.5-7.2(mw), 13.2-17(mw), 56.1 58(mw), 61.6

WB @ 7(mw)

WB @ 4.5-6(mw), 9-10.7(mw), 27.

30.5(mw), 57.8-68.1

B WB@ 24(mw)

\section{WELL SAMPLE HISTORY}

Q1/93-Q2/96, Q4/96

Q1/93-Q4/95

Q1/93-Q2/96, Q4/96, Q2/97, Q4/97, Q2/98, Q4/98, Q2/99, Q4/99, $\mathrm{Q} 2 / 00, \mathrm{Q} 4 / 00, \mathrm{Q} 2 / 01, \mathrm{Q} 4 / 01, \mathrm{Q} 2 / 02, \mathrm{Q} 4 / 02, \mathrm{Q} 2 / 03, \mathrm{Q} 4 / 03$, $\mathrm{Q} 2 / 04, \mathrm{Q} 4 / 04, \mathrm{Q} 2 / 05, \mathrm{Q} 4 / 05, \mathrm{Q} 2 / 06, \mathrm{Q} 4 / 06, \mathrm{Q} 2 / 07, \mathrm{Q} 4 / 07$,

$\mathrm{Q} 2 / 08, \mathrm{Q} 4 / 08, \mathrm{Q} 2 / 09, \mathrm{Q} 4 / 09$

Q1/93-Q2/96, Q4/96, Q2/97, Q4/97, Q2/98, Q4/98, Q2/99, Q4/99,

$\mathrm{Q} 2 / 00, \mathrm{Q} 4 / 00, \mathrm{Q} 2 / 01, \mathrm{Q} 4 / 01, \mathrm{Q} 2 / 02, \mathrm{Q} 4 / 02, \mathrm{Q} 2 / 03, \mathrm{Q} 4 / 03$,

$\mathrm{Q} 2 / 04, \mathrm{Q} 4 / 04, \mathrm{Q} 2 / 05, \mathrm{Q} 4 / 05, \mathrm{Q} 2 / 06, \mathrm{Q} 4 / 06, \mathrm{Q} 2 / 07, \mathrm{Q} 4 / 07$,

$\mathrm{Q} 2 / 08, \mathrm{Q} 4 / 08, \mathrm{Q} 4 / 09, \mathrm{Q} 2 / 10$

Q1/93-Q4/95, Q2/01, Q4/01

Q1/93-Q4/95, Q2/01, Q4/01

Q1/93-Q4/95

\section{Q1/93-Q4/95}

Q1/93-Q2/96, Q4/96, Q2/97, Q4/97, Q2/98, Q4/98, Q2/99, Q4/99, $\mathrm{Q} 2 / 02, \mathrm{Q} 4 / 02, \mathrm{Q} 2 / 04, \mathrm{Q} 4 / 07, \mathrm{Q} 3 / 09, \mathrm{Q} 1 / 11$

Q1/93-Q2/96, Q4/96, Q2/97, Q4/97, Q2/98, Q4/98, Q2/99, Q4/99, $\mathrm{Q} 2 / 02, \mathrm{Q} 4 / 02, \mathrm{Q} 4 / 07, \mathrm{Q} 3 / 09, \mathrm{Q} 1 / 11$

Q2/94-Q2/95, Q1/13

Q2/94-Q2/95, Q1/13

Q2/94-Q2/96, Q4/96, Q1/07, Q3/07, Q3/09, Q2/11

Q2/94-Q2/96, Q4/96

Q2/94-Q2/96, Q4/96, Q2/97, Q4/97, Q2/98, Q4/98, Q2/99, Q4/99, Q4/06, Q4/07, Q4/08, Q3/09, Q1/10, Q3/11, Q1/12, Q3/13

Q2/94-Q2/96, Q4/96, Q2/97, Q4/97, Q2/98, Q4/98, Q2/99, Q4/99, $\mathrm{Q} 2 / 00, \mathrm{Q} 4 / 00, \mathrm{Q} 2 / 01-\mathrm{Q} 4 / 01, \mathrm{Q} 2 / 02, \mathrm{Q} 4 / 02, \mathrm{Q} 2 / 03, \mathrm{Q} 4 / 03, \mathrm{Q} 2 / 04$, Q4/04, Q2/05, Q4/05, Q4/06, Q4/07, Q4/08, Q3/09, Q1/10,

$\mathrm{Q} 3 / 11, \mathrm{Q} 1 / 12, \mathrm{Q} 3 / 13$ 


\section{CURRENT \\ NAME}

GW-783

GW-784

GW-785

GW-786

GW-787

GW-788

GW-789

GW-790

GW-791

GW-792

GW-793

GW-794

GW-795

GW-796

GW-797

\section{T.O.W.R}

1.00

3.00

4.00

16.00

5.40

17.00

14.70

14.50

11.00

3.50

5.00

102.00

67.10

\section{FRACTURES,}

$8.50 \quad$ WB/B WB @ 13.8(mw)

27.00

25.00

9.00

10.00

20.00

26.00

14.00

13.30

12.00

103.00

89.00

\section{B}

$U$

B

U/wB

B

B

B

B

B

B

WB @ 5(mw), 18.1(mw), 53.2-63.2(mw) WB@ 19.2(mw)

WB @ 51(mw) / FRAC @ 60(mw)

WB@ 4.5(mw), 7(mw), 9.6-14.6(mw)

WB @ 9, 56.8-66.8(mw)

WB@ 10(mw)

CAV @ 0, 121-139.3, 199.3-199.8, 200.9212.5, 219-220

WB @ 3(mw), 5.6(mw), 16.5-18.5(mw), 5969(mw)

WB @ 9.2-10(mw), 13-14.2(mw), 1929.2(mw)

B WB @ 14(mw), 18-20(mw), 28-29(mw)

B WB @ 28-29(mw), 38-39(mw)

wB/B WB @ 12(mw), 14.5(mw)

B WB @ 21(w), 78-79.5(m), 103(mw), 118.7(m)

B WB @ 3(w), 128(mW)

\section{WELL SAMPLE HISTORY}

Q2/94-Q2/96, Q4/96, Q2/97, Q4/97, Q2/98, Q4/98, Q2/99, Q4/99, Q2/00, Q2/04, Q4/06, Q4/07, Q4/08, Q3/09, Q1/10, Q3/11,

$\mathrm{Q} 1 / 12, \mathrm{Q} 3 / 13$

Q2/94-Q2/95, Q2/01, Q4/01

Q2/94-Q2/95, Q2/01, Q4/01

Q2/94-Q2/96, Q4/96, Q2/03, Q4/03

Q2/94-Q2/96, Q4/96, Q2/03, Q4/03

Q2/94-Q2/96, Q4/96, Q2/97, Q4/97, Q2/98, Q4/98, Q2/99, Q4/99

Q2/94-Q2/96, Q4/96, Q2/97, Q4/97, Q2/98, Q4/98, Q2/99, Q4/99, $\mathrm{Q} 2 / 00, \mathrm{Q} 4 / 00, \mathrm{Q} 2 / 01, \mathrm{Q} 4 / 01$

Q2/94-Q2/96, Q4/96, Q2/97, Q4/97, Q2/98, Q4/98, Q2/99, Q4/99, $\mathrm{Q} 2 / 00, \mathrm{Q} 4 / 00, \mathrm{Q} 2 / 01, \mathrm{Q} 4 / 01, \mathrm{Q} 2 / 02, \mathrm{Q} 4 / 02, \mathrm{Q} 2 / 03, \mathrm{Q} 4 / 03$,

Q2/04, Q4/04, Q2/05, Q4/05, Q3/06, Q4/07, Q4/08, Q3/09,

$\mathrm{Q} 1 / 10, \mathrm{Q} 3 / 11, \mathrm{Q} 1 / 12, \mathrm{Q} 3 / 13$

Q2/94-O2/96, Q4/96, O2/97, Q4/97, Q2/98, Q4/98, Q2/99, Q4/99,

$\mathrm{Q} 3 / 06, \mathrm{Q} 4 / 07, \mathrm{Q} 4 / 08, \mathrm{Q} 1 / 10, \mathrm{Q} 1 / 12$

Q4/92-Q1/96, Q3/96

$\mathrm{Q} 4 / 92-\mathrm{Q} 1 / 96, \mathrm{Q} 3 / 96$

Q4/92-Q1/96, Q3/96, Q1/04, Q3/04

Q2/93-Q4/94, Q2/95, Q4/95, Q2/96, Q4/96-Q1/97, Q3/97, Q1/98,

Q3/98, Q1/99, Q3/99, Q1/00, Q3/00, Q1/01, Q3/01, Q1/02, $\mathrm{Q} 3 / 02, \mathrm{Q} 1 / 03, \mathrm{Q} 3 / 03, \mathrm{Q} 1 / 04, \mathrm{Q} 3 / 04, \mathrm{Q} 1 / 05, \mathrm{Q} 3 / 05, \mathrm{Q} 1 / 06$, $\mathrm{Q} 3 / 06, \mathrm{Q} 1 / 07, \mathrm{Q} 3 / 07, \mathrm{Q} 1 / 08$,

Q2/93-Q4/94, Q2/95, Q4/95, Q2/96, Q4/96-Q1/97, Q3/97, Q1/98, Q3/98, Q1/99, Q3/99, Q1/00, Q3/00, Q1/01, Q3/01, Q1/02, Q3/02, Q1/03, Q3/03, Q1/04, Q3/04, Q1/05, Q3/05, Q1/06, Q3/06, Q1/07, Q3/07, Q1/08, 


\section{CURRENT \\ NAME}

GW-798

GW-799

GW-800

GW-801

GW-802

GW-803

GW-804

GW-805

GW-806

GW-807

GW-808

GW-809

GW-810

GW-811

GW-812

GW-813

GW-814

GW-815
FRACTURES,

T.O.W.R T.O.F.R AQUIFER CAVITIES, and WATER BREAKS

$110.22 \quad 111.62 \quad$ B $\quad$ WB @ 76(w), 124.5(mw)

$60.80 \quad 62.80 \quad$ B $\quad$ WB @ 5.3(w), 81.5-83.5(mW)

$10.00 \quad 18.50$

$112.50 \quad 113.40$

WB @ 21(mw), 24.5-25(mw), 26.5-27(mw) 28.5-29(mw), 29.5-30(mw)

B FRAC @ 146.5(mw), 186(mw)

\begin{tabular}{|c|c|c|c|}
\hline 10.00 & 15.00 & wB/B & WB @ 15.5-25.5(w) \\
\hline 2.50 & 15.50 & $w B / B$ & WB @ 21.5(mw) \\
\hline 4.00 & 25.00 & wB & WB @ 13.5-24(w) \\
\hline 11.00 & 13.80 & $U / w B$ & WB @ 7.2-12.2(w) \\
\hline 9.00 & 12.90 & U/wB & WB @ 6.2-11.7(w) \\
\hline 8.00 & 11.30 & $\mathrm{U} / \mathrm{wB}$ & WB@ @.5-10(w) \\
\hline \multirow[t]{3}{*}{4.00} & 6.20 & B & FRAC @ 33.5(mw) \\
\hline & 6.80 & B & FRAC @ 34(mw) \\
\hline & 3.50 & B & FRAC @ 21.5(mw) \\
\hline \multirow[t]{5}{*}{52.20} & 63.80 & $\mathrm{U} / \mathrm{wB} / \mathrm{B}$ & \\
\hline & 44.90 & $\mathrm{U} / \mathrm{wB}$ & \\
\hline & & $U$ & \\
\hline & & $U$ & \\
\hline & & $u$ & \\
\hline
\end{tabular}

\section{WELL SAMPLE HISTORY}

Q2/93-Q4/94, Q2/95, Q4/95, Q2/96, Q4/96-Q1/97, Q3/97, Q1/98, Q3/98, Q1/99, Q3/99, Q1/00, Q3/00-Q1/02, Q3/02, Q1/03, Q3/03, $\mathrm{Q} 1 / 04, \mathrm{Q} 3 / 04, \mathrm{Q} 1 / 05, \mathrm{Q} 3 / 05, \mathrm{Q} 1 / 06, \mathrm{Q} 3 / 06, \mathrm{Q} 1 / 07, \mathrm{Q} 3 / 07$,

Q1/08, Q3/08, Q1/09,

Q2/93-Q4/94, Q2/95, Q4/95, Q2/96, Q4/96-Q1/97, Q3/97, Q1/98,

Q3/98, Q1/99, Q3/99, Q1/00, Q3/00, Q1/01, Q3/01, Q1/02

Q3/02, Q1/03, Q3/03, Q1/04, Q3/04, Q1/05, Q3/05, Q1/06

Q3/06, Q1/07, Q3/07, Q1/08,

Q2/93-Q3/95

Q3/93-Q4/94, Q2/95, Q4/95, Q2/96, Q4/96-Q1/97, Q3/97, Q1/98, Q3/98, Q1/99, Q3/99, Q1/00, Q3/00, Q1/01, Q3/01, Q1/02,

$\mathrm{Q} 3 / 02, \mathrm{Q} 1 / 03, \mathrm{Q} 3 / 03, \mathrm{Q} 1 / 04, \mathrm{Q} 3 / 04, \mathrm{Q} 1 / 05, \mathrm{Q} 3 / 05, \mathrm{Q} 1 / 06$

Q3/06, Q1/07, Q3/07, Q1/08,

Q2/98-Q3/98, Q2/03, Q2/04, Q2/05, Q2/06, Q2/07, Q2/08, Q3/09, Q2/10, Q2/11, Q2/12 


\begin{tabular}{|c|c|c|c|c|}
\hline GW-821 & 19.50 & 43.20 & B & \\
\hline GW-822 & 31.00 & 41.80 & B & \\
\hline GW-823 & 27.00 & 54.00 & B & \\
\hline GW-824 & & 11.00 & $U$ & \\
\hline GW-825 & & & 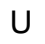 & \\
\hline GW-826 & & & $\mathrm{U}$ & \\
\hline GW-827 & & 40.50 & B & $\begin{array}{l}\text { FRAC @ 49.6(mw), 68.1(mw), 127.6- } \\
\text { 128(mW) / CAV @ 54.5-55.4(mw), } 127.6 \\
\text { 128(mW) }\end{array}$ \\
\hline GW-828 & 7.50 & 38.70 & B & WB @ 10.5, 15.5, 77, 164 \\
\hline GW-829 & 1.30 & 29.00 & B & $\begin{array}{l}\text { WB @ 24-27, 62, 103-115 / CAV @ 54- } \\
55.5\end{array}$ \\
\hline \multicolumn{5}{|l|}{ GW-830 } \\
\hline GW-831 & 134.80 & 140.80 & B & \\
\hline
\end{tabular}

\section{CURRENT \\ NAME}

GW-816

GW-817

GW-818

GW-819

GW-820

FRACTURES,

T.O.W.R T.O.F.R AQUIFER CAVITIES, and WATER BREAKS

U WB @ 7(w)

$11.60 \quad 18.40 \quad$ U/wB/B WB @ 5.5-23$$
\text { . }
$$

(n)

\section{WELL SAMPLE HISTORY}

Q3/94-Q4/96, Q2/97, Q4/97, Q2/98, Q4/98, Q2/99, Q4/99, Q2/00, $\mathrm{Q} 4 / 00, \mathrm{Q} 2 / 01, \mathrm{Q} 4 / 01, \mathrm{O} 2 / 02, \mathrm{O} 4 / 02, \mathrm{Q} 2 / 03, \mathrm{O} 4 / 03, \mathrm{O} 2 / 04$,

Q4/04, Q2/05, Q4/05-Q1/06, Q1/07, Q3/08, Q3/09, Q1/10, Q3/11, $\mathrm{Q} 1 / 12, \mathrm{Q} 3 / 13$

Q3/94-Q4/96, Q2/97, Q4/97

Q1/98, Q1/04

Q1/98

Q1/98, O3/99, O2/00, O4/00, O2/03, O4/03, O2/06, O4/06,

Q2/07, Q4/07, Q2/08, Q4/08, Q2/09, Q4/10, Q2/11, Q4/12, Q2/13
Q2/95, Q4/95, Q2/96, Q4/96, Q2/97, Q4/97, Q2/98, Q4/98, $\mathrm{Q} 2 / 99, \mathrm{Q} 4 / 99-\mathrm{Q} 1 / 00, \mathrm{Q} 3 / 00, \mathrm{Q} 1 / 01, \mathrm{Q} 3 / 01, \mathrm{Q} 1 / 02, \mathrm{Q} 3 / 02, \mathrm{Q} 1 / 03$, Q3/03, Q1/04, Q3/04, Q1/05, Q3/05, Q1/06, Q3/06, Q1/07, Q3/07 Q2/95-Q1/96, Q3/96

Q2/95-Q1/96, Q3/96, Q1/97, Q3/97, Q1/98, Q3/98, Q1/99, Q3/99, $\mathrm{Q} 1 / 00, \mathrm{Q} 3 / 00, \mathrm{Q} 1 / 01, \mathrm{Q} 3 / 01, \mathrm{Q} 1 / 06, \mathrm{Q} 3 / 08, \mathrm{Q} 1 / 10, \mathrm{Q} 3 / 12$ Q3/95

Q3/96-Q1/97, Q3/97, Q1/98, Q3/98, Q1/99, Q3/99, Q1/00, Q3/00, $\mathrm{O} 1 / 01, \mathrm{O} 3 / 01, \mathrm{O} 1 / 02, \mathrm{O} 3 / 02, \mathrm{O} 1 / 03, \mathrm{O} 3 / 03, \mathrm{O} 1 / 04, \mathrm{O} 3 / 04$ Q1/05, Q3/05, Q1/06, Q3/06, Q1/07, Q3/07, Q1/08, Q3/08, Q1/09, Q3/09, Q1/10, Q3/10

Q2/96, Q2/97-Q3/97, Q1/98, Q3/98, Q1/99, Q3/99, Q2/00-Q3/00, $\mathrm{Q} 1 / 01, \mathrm{Q} 3 / 01, \mathrm{Q} 1 / 02, \mathrm{Q} 3 / 02, \mathrm{Q} 1 / 03, \mathrm{Q} 3 / 03, \mathrm{Q} 1 / 04, \mathrm{Q} 3 / 04$, Q1/05, Q3/05, Q1/06, Q3/06, Q1/07, Q3/07, Q1/08, Q3/08, Q1/09, Q3/09, Q1/10, Q3/10, 


\begin{tabular}{|c|c|c|c|c|}
\hline CURRENT & & & & $\begin{array}{c}\text { FRACTURES, } \\
\text { CAVITIES, and WATER BREAKS }\end{array}$ \\
\hline$\underline{\text { NAME }}$ & $\underline{\text { T.O.W.R }}$ & T.O.F.R & $\underline{\text { AQUIFER }}$ & CAVITIES, and WATER BREAKS \\
\hline GW-833 & & & $u$ & \\
\hline GW-834 & 16.80 & & U & \\
\hline GW-835 & 19.20 & & $u$ & \\
\hline GW-836 & 24.40 & & U & \\
\hline GW-837 & 27.90 & & $u$ & \\
\hline GW-838 & 5.00 & 12.00 & B & \\
\hline GW-839 & 6.00 & 60.00 & $w B / B$ & \\
\hline GW-840 & & & $u$ & \\
\hline \multicolumn{5}{|l|}{ GW-841 } \\
\hline GW-842 & 10.00 & & wB & \\
\hline GW-843 & 9.00 & & $w B$ & \\
\hline GW-844 & 15.00 & & B & \\
\hline GW-845 & & 19.80 & B & \\
\hline GW-854 & 12.90 & & $u$ & \\
\hline GW-855 & 17.80 & & $u$ & \\
\hline GW-857 & 14.80 & & $u$ & \\
\hline GW-858 & 17.50 & & $u$ & \\
\hline GW-859 & 14.60 & & $u$ & \\
\hline GW-860 & 20.70 & & U & \\
\hline GW-861 & 16.90 & & $u$ & \\
\hline GW-862 & 14.30 & & $u$ & \\
\hline GW-863 & 23.30 & & U & \\
\hline
\end{tabular}

\section{WELL SAMPLE HISTORY}

Q4/97-Q1/98, Q3/98

Q2/97, Q4/97-Q1/98, Q3/98, Q4/99-Q3/03

Q2/97

Q4/98-Q3/00

Q4/98, Q2/99-Q3/00

Q1/97, Q1/99, Q3/99, Q1/01, Q3/01, Q1/02, Q1/03, Q1/04,

$\mathrm{Q} 1 / 05, \mathrm{Q} 3 / 05, \mathrm{Q} 1 / 06, \mathrm{Q} 3 / 06, \mathrm{Q} 1 / 07, \mathrm{Q} 1 / 08, \mathrm{Q} 1 / 09, \mathrm{Q} 1 / 10, \mathrm{Q} 1 / 12$

Q1/97, Q1/99, Q3/99, Q1/01, Q3/01, Q1/02, Q3/02, Q1/03,

$\mathrm{Q} 3 / 03, \mathrm{Q} 1 / 04, \mathrm{Q} 3 / 04, \mathrm{Q} 1 / 05, \mathrm{Q} 3 / 05, \mathrm{Q} 1 / 06, \mathrm{Q} 3 / 06, \mathrm{Q} 1 / 07$, Q1/08, Q1/09, Q1/10, Q1/11, Q1/12

Q1/97, Q1/99, Q3/99, Q1/01, Q3/01, Q1/02, Q3/02, Q1/03, $\mathrm{Q} 3 / 03, \mathrm{Q} 1 / 04, \mathrm{Q} 3 / 04, \mathrm{Q} 1 / 05, \mathrm{Q} 3 / 05, \mathrm{Q} 1 / 06, \mathrm{Q} 3 / 06$

Q1/97

Q2/98 


\begin{tabular}{|c|c|c|c|c|c|}
\hline \multirow{2}{*}{$\begin{array}{c}\text { CURRENT } \\
\text { NAME }\end{array}$} & \multicolumn{5}{|c|}{ FRACTURES, } \\
\hline & T.O.W.R & $\underline{\text { T.O.F.R }}$ & AQUIFER & CAVITIES, and WATER BREAKS & WELL SAMPLE HISTORY \\
\hline GW-864 & 17.30 & & $u$ & & \\
\hline GW-865 & 12.90 & & U & & \\
\hline GW-866 & 17.40 & & $u$ & & \\
\hline GW-867 & 9.70 & & U & & \\
\hline GW-868 & 17.50 & & $u$ & & \\
\hline GW-869 & 15.30 & & $u$ & & \\
\hline GW-871 & 20.60 & & $u$ & & \\
\hline GW-872 & 11.20 & & $u$ & & \\
\hline GW-873 & 12.30 & & $u$ & & \\
\hline GW-874 & 16.70 & & $u$ & & \\
\hline GW-875 & 19.20 & & $u$ & & \\
\hline GW-876 & 21.10 & & $u$ & & \\
\hline GW-877 & 16.00 & & $u$ & & \\
\hline GW-878 & 15.20 & & $u$ & & \\
\hline GW-879 & 17.10 & & $u$ & & \\
\hline GW-880 & 18.90 & & $u$ & & \\
\hline GW-881 & 20.10 & & $u$ & & \\
\hline GW-882 & 20.10 & & $u$ & & \\
\hline GW-883 & 18.60 & & $u$ & & \\
\hline GW-884 & 9.90 & & $u$ & & \\
\hline GW-885 & 16.10 & & $u$ & & \\
\hline GW-886 & 14.50 & 17.00 & $\mathrm{U} / \mathrm{wB}$ & & \\
\hline GW-887 & 18.00 & 20.50 & $\mathrm{U} / \mathrm{wB}$ & & \\
\hline GW-888 & 19.00 & 21.50 & $\mathrm{U} / \mathrm{wB}$ & & \\
\hline GW-889 & 10.50 & 18.00 & $\mathrm{U} / \mathrm{wB}$ & & \\
\hline
\end{tabular}




\begin{tabular}{|c|c|c|c|}
\hline \multicolumn{4}{|c|}{ CURRENT } \\
\hline NAME & T.O.W.R & T.O.F.R & AQUIFER \\
\hline GW-890 & 5.00 & 11.50 & U/wB \\
\hline GW-902 & 24.50 & 29.50 & \\
\hline \multicolumn{4}{|l|}{ GW-904 } \\
\hline \multicolumn{4}{|l|}{ GW-905 } \\
\hline \multicolumn{4}{|l|}{ GW-906 } \\
\hline \multicolumn{4}{|l|}{ GW-907 } \\
\hline \multicolumn{4}{|l|}{ GW-908 } \\
\hline \multicolumn{4}{|l|}{ GW-909 } \\
\hline \multicolumn{4}{|l|}{ GW-910 } \\
\hline \multicolumn{4}{|l|}{ GW-911 } \\
\hline \multicolumn{4}{|l|}{ GW-912 } \\
\hline \multicolumn{4}{|l|}{ GW-913 } \\
\hline \multicolumn{4}{|l|}{ GW-914 } \\
\hline \multicolumn{4}{|l|}{ GW-915 } \\
\hline GW-916 & 10.00 & 15.00 & $\mathrm{U} / \mathrm{wB}$ \\
\hline GW-917 & 21.00 & 27.00 & $w B / B$ \\
\hline GW-918 & & 30.00 & $w B$ \\
\hline GW-919 & 12.00 & 22.00 & $\mathrm{U} / \mathrm{wB} / \mathrm{B}$ \\
\hline GW-920 & & 12.00 & B \\
\hline GW-921 & & 13.00 & B \\
\hline GW-922 & 10.00 & 13.00 & B \\
\hline GW-923 & & 62.00 & B \\
\hline GW-924 & 21.80 & 22.00 & B \\
\hline GW-925 & & 15.00 & B \\
\hline
\end{tabular}

FRACTURES,

UIFER CAVITIES, and WATER BREAKS

WELL SAMPLE HISTORY

$\mathrm{Q} 4 / 98-\mathrm{Q} 3 / 00$

Q4/98-Q3/00

Q2/01-Q1/02, Q1/03-Q4/12

$\mathrm{Q} 2 / 01-\mathrm{Q} 1 / 02, \mathrm{Q} 1 / 03-\mathrm{Q} 4 / 12$

Q2/01-Q1/02, Q1/03-Q4/12

$\mathrm{Q} 4 / 01-\mathrm{Q} 1 / 02, \mathrm{Q} 1 / 03-\mathrm{Q} 4 / 03$

Q2/01-Q1/02, Q1/03-Q4/12

Q2/01-Q1/02, Q1/03-Q4/12

Q2/01-Q1/02, Q1/03-Q4/12

Q2/01-Q1/02, Q1/03-Q1/04, Q4/04-Q2/05, Q1/06-Q2/06, Q4/06$\mathrm{Q} 2 / 07, \mathrm{Q} 1 / 08-\mathrm{Q} 3 / 08, \mathrm{Q} 1 / 09-\mathrm{Q} 3 / 12$

$\mathrm{Q} 1 / 01-\mathrm{Q} 1 / 02, \mathrm{Q} 1 / 03-\mathrm{Q} 4 / 12$

Q2/01-Q1/02, Q1/03-Q4/12 


\begin{tabular}{|c|c|c|c|c|c|}
\hline \multirow{2}{*}{$\begin{array}{c}\text { CURRENT } \\
\text { NAME }\end{array}$} & \multicolumn{5}{|c|}{ FRACTURES, } \\
\hline & T.O.W.R & $\underline{\text { T.O.F.R }}$ & AQUIFER & CAVITIES, and WATER BREAKS & $\underline{\text { WELL SAMPLE HISTORY }}$ \\
\hline GW-926 & 15.00 & 18.00 & B & & Q2/01-Q1/02, Q1/03-Q4/12 \\
\hline GW-927 & 25.00 & 30.00 & B & & Q2/01-Q1/02, Q1/03-Q4/12 \\
\hline GW-928 & 21.00 & 24.00 & B & & Q1/10-Q4/10, Q2/12 \\
\hline GW-929 & 27.50 & 28.60 & $\mathrm{U} / \mathrm{wB}$ & & Q1/10-Q4/10, Q2/12 \\
\hline GW-930 & 21.00 & 23.00 & B & & Q1/10-Q1/11, Q2/12 \\
\hline GW-931 & 16.00 & 19.80 & $\mathrm{U} / \mathrm{wB}$ & & $\mathrm{Q} 1 / 10-\mathrm{Q} 4 / 10, \mathrm{Q} 2 / 12$ \\
\hline \multicolumn{6}{|l|}{ GW-932 } \\
\hline \multicolumn{6}{|l|}{ GW-933 } \\
\hline GW-934 & 8.50 & 15.60 & B & & \\
\hline GW-935 & & & $u$ & & \\
\hline GW-936 & 17.00 & & $\mathrm{U} / \mathrm{wB}$ & & \\
\hline GW-937 & 2.00 & 20.00 & $w B / B$ & & \\
\hline GW-938 & 26.00 & & $\mathrm{U} / \mathrm{wB}$ & & \\
\hline GW-939 & 1.00 & 20.00 & $w B / B$ & & \\
\hline GW-940 & 13.50 & & $\mathrm{U} / \mathrm{wB}$ & & \\
\hline GW-941 & 13.00 & & $\mathrm{U} / \mathrm{wB}$ & & \\
\hline GW-942 & 4.50 & 13.50 & $\mathrm{U} / \mathrm{wB} / \mathrm{B}$ & & \\
\hline GW-943 & 3.00 & 13.00 & $w B / B$ & & \\
\hline GW-944 & 1.00 & & wB & & \\
\hline GW-945 & & & U & & \\
\hline GW-946 & 32.00 & 53.00 & $w B / B$ & & \\
\hline GW-947 & 10.00 & 35.00 & $w B / B$ & & \\
\hline GW-948 & 3.00 & 25.00 & B & & \\
\hline GW-949 & 48.00 & & $\mathrm{U} / \mathrm{wB}$ & & \\
\hline GW-950 & 16.00 & 35.00 & $w B / B$ & & \\
\hline & & & & F-63 & \\
\hline
\end{tabular}




\begin{tabular}{|c|c|c|c|c|c|}
\hline CURRENT & & & & FRACTURES, & \\
\hline NAME & T.O.W.R & $\underline{\text { T.O.F.R }}$ & $\underline{\text { AQUIFER }}$ & CAVITIES, and WATER BREAKS & WELL SAMPLE HISTORY \\
\hline GW-951 & 39.00 & & $\mathrm{U} / \mathrm{wB}$ & & \\
\hline GW-952 & 30.00 & 33.00 & $\mathrm{U} / \mathrm{wB} / \mathrm{B}$ & & \\
\hline GW-953 & & & $w B$ & & \\
\hline GW-954 & & & UNKN & & \\
\hline GW-955 & & & & & \\
\hline GW-956 & & & UNKN & & \\
\hline GW-959 & & & $\mathrm{U}$ & & Q1/05-Q4/05, Q2/06, Q4/06, Q4/07, Q2/09, Q4/11, Q3/13 \\
\hline GW-960 & 17.00 & 17.20 & & & Q1/07, Q3/07, Q2/08, Q4/08, Q2/09, Q2/11, Q3/13 \\
\hline GW-961 & 1.00 & 9.00 & $w B / B$ & & Q4/09-Q4/12 \\
\hline GW-964 & 4.00 & 30.00 & wB & CAV @ 31-32 & Q3/11-Q4/12 \\
\hline GW-965 & 10.50 & 30.00 & B & & Q3/11-Q4/12 \\
\hline GW-966 & 10.00 & 20.00 & $\mathrm{U} / \mathrm{wB}$ & & Q4/12 \\
\hline GW-967 & & & & & \\
\hline $\mathrm{J}-001$ & & 13.00 & & CAV @ 25.8-27.7 & \\
\hline $\mathrm{J}-002$ & 15.70 & 21.20 & & CAV @ 16.9-21.2(m), 28.3-30.2 & \\
\hline $\mathrm{J}-003$ & & 17.40 & & $\begin{array}{l}\text { CAV @ 4.5-5.8(m), 7-9.3(m), 13.3-14.2(m), } \\
15.3-17.4(\mathrm{~m})\end{array}$ & \\
\hline $\mathrm{J}-004$ & 5.00 & 21.50 & & CAV @ 20.5-21.5(m) & \\
\hline $\mathrm{J}-005$ & 11.00 & 21.20 & & CAV @ 20-21.2(m) & \\
\hline $\mathrm{J}-006$ & 16.60 & 37.60 & & CAV @ 18.3-18.6 & \\
\hline $\mathrm{J}-007$ & 21.70 & 30.10 & & CAV @ 26.9-30.1 & \\
\hline $\mathrm{J}-008$ & 12.00 & 30.80 & & CAV@ 14-22(m), 30-30.8(m) & \\
\hline $\mathrm{J}-009$ & 16.40 & 36.00 & & $\begin{array}{l}\text { CAV @ 20-20.8, 21.3-223.3(m), 25.7-27.5, } \\
35.5-36.1\end{array}$ & \\
\hline $\mathrm{J}-010$ & 8.50 & 28.00 & & & \\
\hline
\end{tabular}




\begin{tabular}{|c|c|c|c|c|}
\hline $\begin{array}{l}\text { CURRENT } \\
\text { NAME }\end{array}$ & $\underline{\text { T.O.W.R }}$ & T.O.F.R & $\underline{\text { AQUIFER }}$ & $\begin{array}{c}\text { FRACTURES, } \\
\text { CAVITIES, and WATER BREAKS }\end{array}$ \\
\hline $\mathrm{J}-011$ & 7.00 & 29.50 & & $\begin{array}{l}\text { CAV @ 8.7-17.8(m), 22.3-24.4(m), 28.9- } \\
29.5(\mathrm{~m})\end{array}$ \\
\hline $\mathrm{J}-012$ & 10.00 & 27.00 & & CAV@ 20.8-27(m) \\
\hline $\mathrm{J}-015$ & & 4.00 & & \\
\hline $\mathrm{J}-017$ & & 19.00 & & CAV@ @ 8.7-11(m), 18-19 \\
\hline $\mathrm{J}-018$ & & 12.00 & & \\
\hline $\mathrm{J}-019$ & 4.30 & 21.00 & & CAV @ 15.5-16(m), 20-21(m) \\
\hline $\mathrm{J}-020$ & 7.00 & 28.80 & & CAV@ 25-28.7(m) \\
\hline $\mathrm{J}-021$ & 6.00 & 19.70 & & \\
\hline $\mathrm{J}-022$ & 5.50 & 10.00 & & CAV @ 9-10(m), 11-13.2(m), 16-19.7(m) \\
\hline $\mathrm{K}-020$ & 7.00 & 30.20 & & $\begin{array}{l}\text { CAV @ 10.2-11.8(m), 21.8-22, 29.8- } \\
30.2(\mathrm{~m})\end{array}$ \\
\hline $\mathrm{K}-021$ & & 12.00 & & \\
\hline $\mathrm{K}-022$ & & 19.80 & & \\
\hline $\mathrm{K}-023$ & & 30.00 & & \\
\hline $\mathrm{K}-024$ & & 31.70 & & \\
\hline K1-015 & 12.70 & & & CAV@ 17.7-36.9(m), 40.25-59 \\
\hline K2-015 & 16.20 & 37.30 & & CAV @ 18.7-37.3(m) \\
\hline LL/HAZ-01 & 19.00 & & $\mathrm{U} / \mathrm{wB}$ & WB@ 39 \\
\hline LL/HAZ-02 & 14.00 & & $\mathrm{U} / \mathrm{wB}$ & WB @ 21 \\
\hline LL/HAZ-03 & 12.00 & & $\mathrm{U} / \mathrm{wB}$ & WB @ 8.5 \\
\hline LL/HAZ-04 & 19.00 & & $\mathrm{U} / \mathrm{wB}$ & WB@ $@ 22$ \\
\hline LL/HAZ-05 & 13.00 & & U/wB & WB @ 17 \\
\hline LL/HAZ-06 & 13.00 & & $\mathrm{U} / \mathrm{wB}$ & WB@ $@ 20$ \\
\hline LL/HAZ-07 & 13.00 & & $\mathrm{U} / \mathrm{wB}$ & WB @ 30 \\
\hline
\end{tabular}




\begin{tabular}{|c|c|c|c|c|c|}
\hline \multirow{2}{*}{$\begin{array}{c}\text { CURRENT } \\
\text { NAME }\end{array}$} & \multicolumn{5}{|c|}{ FRACTURES, } \\
\hline & T.O.W.R & T.O.F.R & $\underline{\text { AQUIFER }}$ & CAVITIES, and WATER BREAKS & WELL SAMPLE HISTORY \\
\hline LL/HAZ-08 & 9.00 & & $\mathrm{U} / \mathrm{wB}$ & WB@ 27 & \\
\hline LL/HAZ-09 & 8.50 & & $U / w B$ & WB@ @46 & \\
\hline LL/HAZ-10 & 8.00 & & $\mathrm{U} / \mathrm{wB}$ & WB @ 36 & \\
\hline LL/HAZ-11 & 16.50 & & $U / w B$ & WB@ @25 & \\
\hline LL/HAZ-12 & 13.50 & & $\mathrm{U} / \mathrm{wB}$ & WB@ 22 & \\
\hline LL/HAZ-13 & 5.00 & 20.00 & $\mathrm{~B}$ & & \\
\hline LL/HAZ-14 & 20.00 & 35.00 & B & & \\
\hline LL/HAZ-15 & 15.00 & 35.00 & B & & \\
\hline LL/HAZ-16 & 15.50 & 24.00 & $\mathrm{U} / \mathrm{wB}$ & WB@ 22 & \\
\hline LL/HAZ-17 & 1.00 & & $\mathrm{U} / \mathrm{wB}$ & WB@ 22 & \\
\hline LL/HAZ-18 & 8.00 & 22.00 & $\mathrm{U} / \mathrm{wB}$ & & \\
\hline LL/HAZ-19 & 14.00 & 23.50 & $\mathrm{U} / \mathrm{wB}$ & & \\
\hline \multicolumn{6}{|l|}{ M-04 } \\
\hline \multicolumn{6}{|l|}{ M-05 } \\
\hline \multicolumn{6}{|l|}{ M-06 } \\
\hline \multicolumn{6}{|l|}{ M-07 } \\
\hline \multicolumn{6}{|l|}{ M-08 } \\
\hline \multicolumn{6}{|l|}{ M-09 } \\
\hline \multicolumn{6}{|l|}{ M-10 } \\
\hline \multicolumn{6}{|l|}{ OR-02 } \\
\hline OR-03 & 5.00 & 20.00 & & & \\
\hline OR-04 & 8.00 & 24.50 & & & \\
\hline OR-05 & 10.00 & 17.00 & & & \\
\hline OR-06 & 10.00 & 31.00 & & & \\
\hline \multirow[t]{2}{*}{ OR-07 } & 10.50 & 17.00 & & & \\
\hline & & & & F-66 & \\
\hline
\end{tabular}




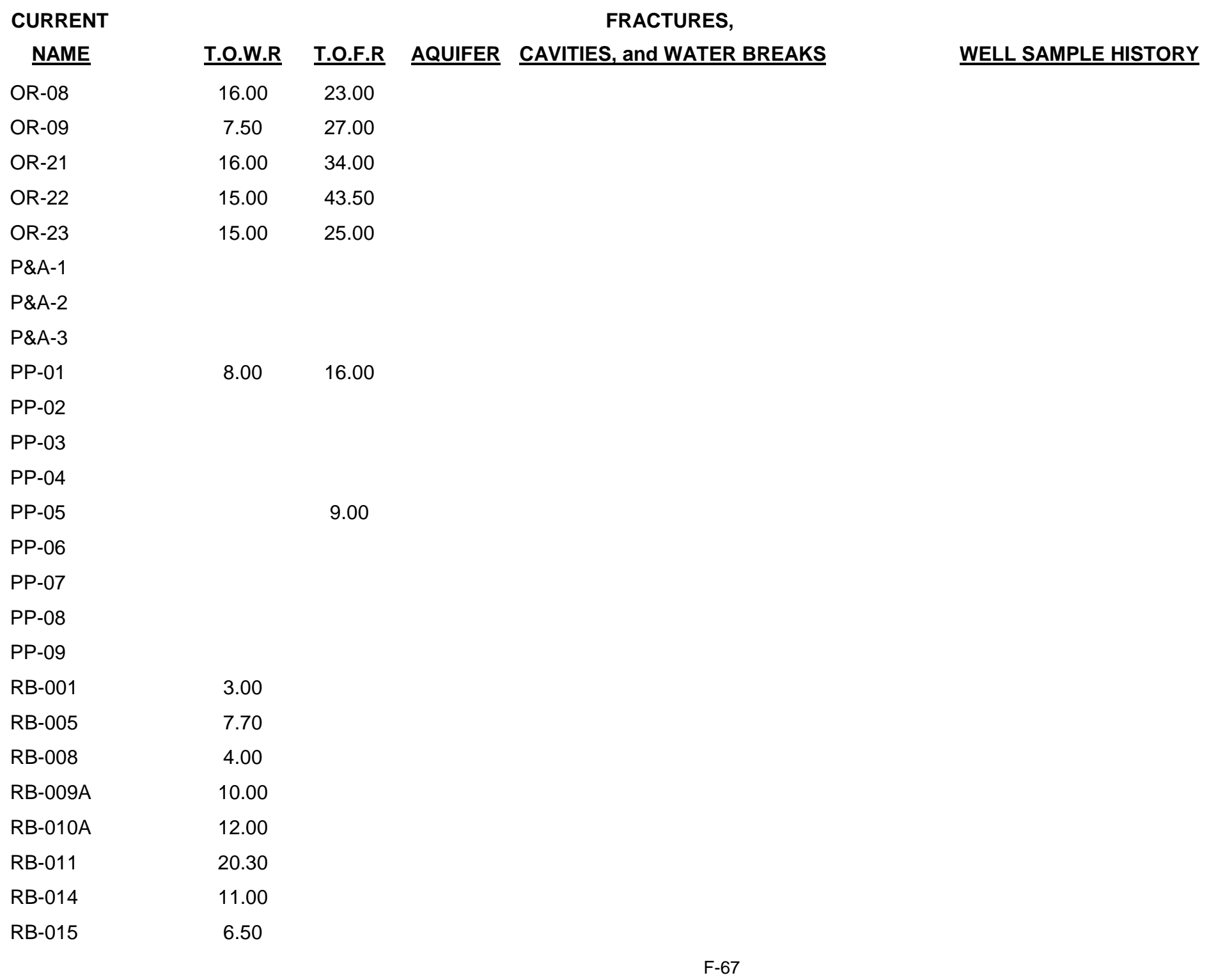




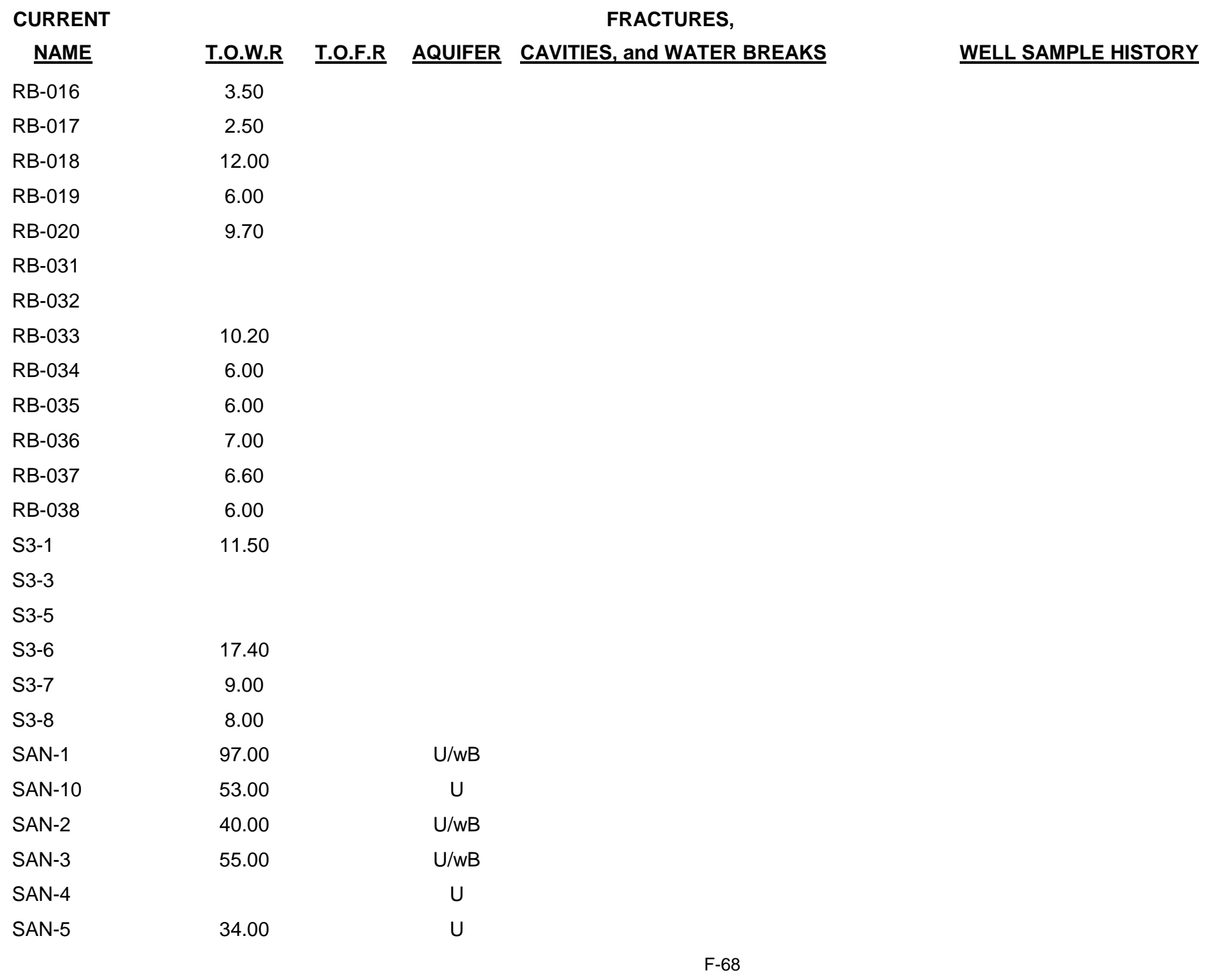




\section{CURRENT}

NAME

SAN-6

SAN-7

SAN-8

SAN-9

UNIDENT A

UNIDENT B

$\mathrm{V}-1$

$V-2$

$\mathrm{V}-3$

$V-4$

$V-5$

$V-6$

$\vee-7$
FRACTURES,

T.O.W.R T.O.F.R AQUIFER CAVITIES, and WATER BREAKS

$U$

$59.50 \quad U$

$39.00 \quad U$

$38.50 \quad U$

U

U
WELL SAMPLE HISTORY 
This Page Intentionally Left Blank 


\section{APPENDIX G}

Borehole and Well Geologic Data Summary 
This Page Intentionally Left Blank 


\section{CURRENT}

NAME

001-L

002-L

003-L

004-L

005-L

006-L

007-L

008-L

009-L

010-L

011-L

012-L

013-L

014-L

015-L

016-L

017-L

018-L

019-L

020-L

021-L

022-L

023-L

024-L

025-L

026-L

\section{ROCK FORMATIONS}

Conasauga/Maryville Limestone

Conasauga/Nolichucky Shale

Conasauga/Maryville Limestone

Conasauga/Nolichucky Shale

Conasauga/Maynardville Limestone

Conasauga/Nolichucky Shale

Conasauga/Nolichucky Shale

Conasauga/Maynardville Limestone

Conasauga/Nolichucky Shale

Conasauga/Nolichucky Shale

Conasauga/Maynardville Limestone

Conasauga/Nolichucky Shale

Conasauga/Nolichucky Shale

Conasauga/Maynardville Limestone

Conasauga/Nolichucky Shale

Conasauga/Nolichucky Shale

Conasauga/Maynardville Limestone

Conasauga/Nolichucky Shale

Conasauga/Nolichucky Shale

Conasauga/Maynardville Limestone

Conasauga/Nolichucky Shale

Conasauga/Nolichucky Shale

Conasauga/Nolichucky Shale

Conasauga/Nolichucky Shale

Conasauga/Nolichucky Shale

Conasauga/Nolichucky Shale

\section{CORE? LOGS? WHICH LOGS?}

yes

yes no

yes no

yes no

yes no

yes no

yes no

yes no

yes no

yes no

yes no

yes no

yes no

yes no

yes no

yes no

yes no

yes no

yes no

yes no

yes no

yes no

yes no

yes no

yes no

yes no 


\section{CURRENT}

NAME

027-L

028-L

029-L

030-L

031-L

032-L

033-L

034-L

035-L

1001

1002

1003

1004

1005

1006

1007

1008

1009

1010

1011

1012

1013

1014

1015

1016

1017
ROCK FORMATIONS

Conasauga/Nolichucky Shale

Conasauga/Maynardville Limestone

Conasauga/Nolichucky Shale

Conasauga/Maynardville Limestone

Conasauga/Nolichucky Shale

Conasauga/Maynardville Limestone

Conasauga/Nolichucky Shale

Conasauga/Maynardville Limestone

Conasauga/Nolichucky Shale

Conasauga/Pumpkin Valley Shale

Conasauga/Maryville Limestone

Conasauga/Nolichucky Shale

Conasauga/Nolichucky Shale, Conasauga/Maynardville Limestone

Conasauga/Maynardville Limestone

Conasauga/Nolichucky Shale

Conasauga/Nolichucky Shale

Conasauga/Nolichucky Shale

Conasauga/Nolichucky Shale

Conasauga/Nolichucky Shale, Conasauga/Maryville Limestone

Conasauga/Nolichucky Shale

Conasauga/Rogersville Shale

Conasauga/Pumpkin Valley Shale

Conasauga/Pumpkin Valley Shale

Conasauga/Pumpkin Valley Shale

Conasauga/Rutledge Limestone

Conasauga/Pumpkin Valley Shale

\section{CORE? LOGS? WHICH LOGS?}

yes

yes no

yes no

yes no

yes no

yes no

yes no

yes no

yes no

no no

no no

no no

no no

no no

no no

no no

no no

no no

no no

no no

no no

no no

no no

no no

no no

no no 


\section{CURRENT}

NAME

1018

1019

1020

1021

1022

1023

1024

1025

1025A

1026

1027

1028

1029

1030

1031

1032

1033

1034

1035

1036

1037

1038

1040

1041

1043

1044

\section{ROCK FORMATIONS}

Conasauga/Rutledge Limestone, Conasauga/Rogersville Shale Conasauga/Rogersville Shale

Conasauga/Rutledge Limestone

Conasauga/Rutledge Limestone

Conasauga/Rutledge Limestone

Conasauga/Pumpkin Valley Shale

Conasauga/Pumpkin Valley Shale

Conasauga/Pumpkin Valley Shale

none

Conasauga/Maryville Limestone

Conasauga/Maryville Limestone

Conasauga/Nolichucky Shale

Conasauga/Nolichucky Shale

Conasauga/Nolichucky Shale

Conasauga/Nolichucky Shale

Conasauga/Maynardville Limestone

Conasauga/Maryville Limestone

Conasauga/Nolichucky Shale

Conasauga/Pumpkin Valley Shale

Conasauga/Maryville Limestone

Conasauga/Nolichucky Shale

Conasauga/Pumpkin Valley Shale

Conasauga/Rutledge Limestone

Conasauga/Maryville Limestone

Conasauga/Maryville Limestone

Conasauga/Maynardville Limestone

\section{CORE? LOGS? WHICH LOGS?}

no no

no no

no no

no no

no no

no no

no no

no no

no no

yes no

no no

no no

no no

no no

no no

no yes

GRL, SP, SPR

GRL, SPR, SP

yes no

no no

no no

no no

no no

no no

no yes

no yes

CL, SP, GRL, SPR

GRL, CL, SPR, SP 


\section{CURRENT}

NAME

1045

1046

1047

1047A

1048

1049

1050

1051

1052

1055

1056

1057

1058

1059

1060

1061

1062

1063

1070

1071

1072

1073

1080

1081

1082

1083

\section{ROCK FORMATIONS}

Conasauga/Maynardville Limestone

Conasauga/Maynardville Limestone

Conasauga/Maynardville Limestone

Conasauga/Nolichucky Shale, Conasauga/Maynardville Limestone

Conasauga/Maryville Limestone

Conasauga/Nolichucky Shale

Conasauga/Maynardville Limestone

Conasauga/Nolichucky Shale

Conasauga/Nolichucky Shale

Conasauga/Nolichucky Shale

Conasauga/Nolichucky Shale

Conasauga/Nolichucky Shale

Conasauga/Nolichucky Shale

Conasauga/Nolichucky Shale

Conasauga/Nolichucky Shale

Conasauga/Nolichucky Shale

Conasauga/Nolichucky Shale

Conasauga/Nolichucky Shale

Conasauga/Nolichucky Shale

Conasauga/Rutledge Limestone

Conasauga/Maryville Limestone

Conasauga/Maynardville Limestone

Knox

Chickamauga

Chickamauga

Chickamauga

\section{CORE? LOGS? WHICH LOGS?}

no yes SPR, GRL

no no

no no

no no

yes no

no no

no no

no no

no no

no no

no no

no no

no no

no no

no no

no no

no no

no no

no no

no no

no no

no no

no no

no no

no yes DHC

no no 


$\begin{array}{ll}\begin{array}{ll}\text { CURRENT } \\ \text { NAME }\end{array} & \\ 1084 & \text { Knox } \\ 1085 & \text { Knox } \\ 1086 & \text { Knox } \\ 1087 & \text { Knox } \\ 1088 & \text { Conasauga/Maynardville Limestone } \\ 1090 & \text { Knox } \\ 1091 & \text { Knox } \\ 1092 & \text { Knox } \\ 1095 & \text { Knox } \\ 1096 & \text { Knox } \\ 1097 & \text { Conasauga/Rogersville Shale } \\ 1100 & \text { Knox } \\ 1101 & \text { Knox } \\ 1102 & \text { Knox } \\ 1103 & \text { Knox } \\ 1104 & \text { Knox } \\ 1105 & \text { Knox } \\ 1106 & \text { Knox } \\ 1107 & \text { Knox } \\ 1108 & \text { Knox } \\ 1109 & \text { Knox } \\ 1110 & \text { Knox } \\ 1111 & \text { Knox } \\ 1112 & \text { Knox } \\ 1130 & \text { Knox } \\ 1131 & \text { Knox } \\ & \end{array}$

\section{CORE? LOGS? WHICH LOGS?}

no yes DHC

no no

no no

no no

yes no

no no

no no

no no

no no

no no

yes no

no no

no no

no no

no no

no no

no no

no no

no no

no no

no no

no no

no no

no no

no no

no no 


\begin{tabular}{|c|c|}
\hline \multicolumn{2}{|c|}{ CURRENT } \\
\hline NAME & ROCK FORMATIONS \\
\hline 1132 & Knox \\
\hline 1133 & Knox \\
\hline 1134 & Knox \\
\hline $2 \mathrm{D}$ & Knox \\
\hline 21 & Knox \\
\hline $2 S$ & Knox \\
\hline $38-D C$ & Conasauga/Maryville Limestone \\
\hline $39-D C$ & Conasauga/Maryville Limestone \\
\hline $3 \mathrm{D}$ & Knox \\
\hline 31 & Knox \\
\hline $3 S$ & Knox \\
\hline 40-DC & Conasauga/Pumpkin Valley Shale \\
\hline 41-DC & Conasauga/Maryville Limestone \\
\hline $42-D C$ & Conasauga/Rogersville Shale \\
\hline 44-DC & Conasauga/Nolichucky Shale \\
\hline $45-D C$ & Conasauga/Nolichucky Shale \\
\hline $4 \mathrm{D}$ & Knox \\
\hline 41 & Knox \\
\hline $4 S$ & Knox \\
\hline $53-1 A$ & Conasauga/Nolichucky Shale \\
\hline $54-1 A$ & Conasauga/Nolichucky Shale \\
\hline $54-2 A$ & Conasauga/Nolichucky Shale \\
\hline $55-1 A$ & Conasauga/Nolichucky Shale \\
\hline $55-1 B$ & Conasauga/Nolichucky Shale \\
\hline $55-1 C$ & Conasauga/Nolichucky Shale \\
\hline $55-2 A$ & Conasauga/Nolichucky Shale \\
\hline
\end{tabular}

\section{CORE? LOGS? WHICH LOGS?}

no no

no no

no no

no no

no no

no no

yes no

yes no

no no

no no

no no

yes no

yes no

yes no

yes no

yes no

no no

no no

no no

no no

no no

no no

no no

no no

no yes CL, CAV, mRL, SPR, SNL, GRL, CDL

no yes DHC 


\section{CURRENT}

NAME

55-2B

$55-2 \mathrm{C}$

$55-3 \mathrm{~A}$

55-3B

55-3C

55-4B

55-4C

55-5A

55-6A

55-7A

55-8A

55-8B

55-9A

56-1A

56-1C

56-2A

56-2B

56-2C

56-3A

56-3B

56-3C

56-4A

56-4B

56-4C

56-5A

\section{ROCK FORMATIONS}

Conasauga/Nolichucky Shale

Conasauga/Nolichucky Shale

Conasauga/Nolichucky Shale

Conasauga/Nolichucky Shale

Conasauga/Nolichucky Shale

Conasauga/Maryville Limestone

Conasauga/Maryville Limestone

Conasauga/Maryville Limestone

Conasauga/Maryville Limestone

Conasauga/Maynardville Limestone

Conasauga/Maynardville Limestone

Conasauga/Maynardville Limestone

Conasauga/Maynardville Limestone

Conasauga/Nolichucky Shale

Conasauga/Nolichucky Shale

Conasauga/Nolichucky Shale

Conasauga/Nolichucky Shale

Conasauga/Nolichucky Shale

Conasauga/Nolichucky Shale

Conasauga/Nolichucky Shale

Conasauga/Nolichucky Shale

Conasauga/Nolichucky Shale

Conasauga/Nolichucky Shale

Conasauga/Nolichucky Shale

Conasauga/Maynardville Limestone

\section{CORE? LOGS? WHICH LOGS?}

yes no

no no

no no

no yes

no

yes

SPR, SNL, CL, GRL, CDL

no

no no

yes no

yes no

no no

no yes

yes yes

no no

no no

yes yes

no no

no no

no yes

no yes

no yes

yes no

no no
$\mathrm{CL}$

GRL

DHC

$\mathrm{mRL}, \mathrm{DHC}, \mathrm{SNL}, \mathrm{GRL}, \mathrm{SPR}, \mathrm{CDL}, \mathrm{CL}$, CAV

DHC, CL, CDL, SPR, mRL, SNL, GRL

CDL, SPR, CL, GRL, CAV, SNL

SNL, CDL, SPR, DHC, CAV, GRL, CL

SNL, GRL, CDL, CAV, SPR, CL 


\section{CURRENT}

NAME

56-5B

$56-5 \mathrm{C}$

56-6A

56-7A

$56-8 \mathrm{~A}$

56-9A

58-1A

58-2A

59-1A

59-1B

59-1C

5D

51

$5 \mathrm{~S}$

60-1A

60-1B

60-1C

60-2A

AN:D-1

AN:D-10

AN:D-11

AN:D-2

AN:D-3

AN:D-4

AN:D-5

AN:D-6

\section{ROCK FORMATIONS}

Conasauga/Maynardville Limestone Conasauga/Maynardville Limestone

Conasauga/Nolichucky Shale

Conasauga/Maynardville Limestone

Conasauga/Maynardville Limestone

Conasauga/Maynardville Limestone

Conasauga/Nolichucky Shale

Conasauga/Maynardville Limestone

Conasauga/Nolichucky Shale

Conasauga/Nolichucky Shale

Conasauga/Nolichucky Shale

Knox

Knox

Knox

Conasauga/Maynardville Limestone

Conasauga/Maynardville Limestone

Conasauga/Maynardville Limestone

Conasauga/Maynardville Limestone

Chickamauga

Chickamauga

Chickamauga

Chickamauga

Chickamauga

Chickamauga

Chickamauga

Chickamauga

\section{CORE? LOGS? WHICH LOGS?}

no

yes no

no yes

no no

no no

no no

no no

no no

no no

no no

yes yes

no no

no no

no no

no no

no no

yes yes

no no

no no

no no

no no

no no

no no

no no

no no

no no
$\mathrm{DHC}$

CAV, CDL, CL, mRL, SNL, SPR, GRL

CAV, CDL, CL, mRL, SNL, SPR, GRL 


\section{CURRENT}

NAME

AN:D-7

AN:D-8

AN:D-9

AN:D-UNKNOWN

B-1

B-3

BC-01

BC-03

BC-06

BC-07

BC-08

BC-09

BC-10

BC-11

BC-14

BC-15

BC-16

BC-17

BC-18

BC-19

BC-21

BC-22

BC-23

BC-25

\section{ROCK FORMATIONS}

Chickamauga

Chickamauga

Chickamauga

hickamauga

Knox

Knox

Conasauga/Nolichucky Shale, Conasauga/Maynardville Limestone

Conasauga/Nolichucky Shale(?-223), Conasauga/Maryville

Limestone(223-?)

Conasauga/Maryville Limestone(?-253), Conasauga/Rogersville Shale(253-?)

Conasauga/Maryville Limestone(?-115), Conasauga/Rogersville Shale(115-237), Conasauga/Rutledge Limestone(237-?)

Conasauga/Rutledge Limestone, Conasauga/Rogersville Shale,

Conasauga/Pumpkin Valley Shale

Conasauga/Pumpkin Valley Shale

Rome, Conasauga/Pumpkin Valley Shale

Rome, Conasauga/Pumpkin Valley Shale

Conasauga/Pumpkin Valley Shale

Conasauga/Pumpkin Valley Shale

Conasauga/Pumpkin Valley Shale

Conasauga/Rutledge Limestone, Conasauga/Rogersville Shale

Conasauga/Rogersville Shale

Conasauga/Rogersville Shale(?-96), Conasauga/Rutledge Limestone(96-?)

Conasauga/Nolichucky Shale

Conasauga/Pumpkin Valley Shale

Conasauga/Pumpkin Valley Shale

Conasauga/Rogersville Shale
CORE? LOGS? WHICH LOGS?

no

no no

no no

no no

no no

no no

yes no

yes no

yes no

yes no

yes no

yes no

yes no

yes no

yes no

yes no

yes no

yes no

yes no

yes no

yes no

yes no

yes no

yes no 


\section{CURRENT \\ NAME}

BC-30

BC-31

BC-32

BC-33

BC-34

BC-35

BC-36

BC-37

$\mathrm{BC}-38$

BC-39

BC-40

BC-41

BC-42

BC-43

BC-45

BC-47

BC-49

BC-50

BC-51

BC-52

BC-53

BC-54

BC-57

BC-59

\section{ROCK FORMATIONS}

Conasauga/Maryville Limestone

Conasauga/Rutledge Limestone(?-84), Conasauga/Pumpkin Valley Shale(84-?)

Conasauga/Rutledge Limestone(?-58), Conasauga/Pumpkin Valley Shale(58-?)

Conasauga/Pumpkin Valley Shale

Conasauga/Pumpkin Valley Shale

Conasauga/Rogersville Shale(?-162), Conasauga/Rutledge Limestone(162-289), Conasauga/Pumpkin Valley Shale(289-?)

Conasauga/Rutledge Limestone(?-70), Conasauga/Pumpkin Valley Shale(70-?)

Conasauga/Pumpkin Valley Shale

Rome, Conasauga/Pumpkin Valley Shale

Rome, Conasauga/Pumpkin Valley Shale

Rome

Rome, Conasauga/Pumpkin Valley Shale

Rome, Conasauga/Pumpkin Valley Shale

Rome, Conasauga/Pumpkin Valley Shale

Rome, Conasauga/Pumpkin Valley Shale

Conasauga/Rutledge Limestone

Conasauga/Maynardville Limestone

Conasauga/Maynardville Limestone

Conasauga/Maynardville Limestone

Conasauga/Nolichucky Shale

Conasauga/Nolichucky Shale

Conasauga/Nolichucky Shale

Conasauga/Maynardville Limestone

Conasauga/Nolichucky Shale

\section{CORE? LOGS? WHICHLOGS?}

yes

yes no

yes no

yes no

yes no

yes no

yes no

yes no

yes no

yes no

yes no

yes no

yes no

yes no

yes no

yes no

yes no

yes no

yes no

yes no

yes no

yes no

yes no

yes no 


\section{CURRENT}

NAME

BC-61

BC-62

BC-63

BC-64

BCU-01

BCU-02

BCU-03

BCU-04

BCU-05

BCU-06

BCU-07

BCU-08

BCU-09

BCU-10

BCU-11

BCU-12

BCU-13

BCU-14

BCU-15

BCU-16

BCU-17

BCU-18

BCU-19

BCU-20

BCU-21

\section{ROCK FORMATIONS}

Conasauga/Rutledge Limestone, Conasauga/Pumpkin Valley Shale

Conasauga/Pumpkin Valley Shale

Conasauga/Maryville Limestone(?-146), Conasauga/Rogersville

Shale(146-272), Conasauga/Rutledge Limestone(272-?)

Rome

none

none

none

none

none

none

none

none

none

none

none

none

none

none

none

none

none

none

none

none

none

\section{CORE? LOGS? WHICH LOGS?}

yes

yes no

yes no

yes no

no no

no no

no no

no no

no no

no no

no no

no no

no no

no no

no no

no no

no no

no no

no no

no no

no no

no no

no no

no no

no no 


$\begin{array}{ll}\begin{array}{l}\text { CURRENT } \\ \text { NAME }\end{array} & \\ \text { BCU-22 } & \text { none } \\ \text { BCU-23 } & \text { none } \\ \text { BCU-24 } & \text { none } \\ \text { BCU-25 } & \text { none } \\ \text { BCU-26 } & \text { none } \\ \text { BCU-27 } & \text { none } \\ \text { BCU-28 } & \text { none } \\ \text { BCU-29 } & \text { none } \\ \text { BY-10 } & \text { none } \\ \text { BY-11 } & \text { none } \\ \text { BY-12 } & \text { none } \\ \text { BY-13 } & \text { none } \\ \text { BY-14 } & \text { none } \\ \text { BY-15 } & \text { none } \\ \text { BY-16 } & \text { none } \\ \text { BY-17 } & \text { none } \\ \text { BY-18 } & \text { none } \\ \text { BY-19 } & \text { none } \\ \text { BY-20 } & \text { none } \\ \text { BY-21 } & \text { none } \\ \text { BY-22 } & \text { none } \\ \text { BY-23 } & \text { none } \\ \text { BY-24 } & \text { none } \\ \text { BY-25 } & \text { none } \\ \text { BY-26 } & \text { none } \\ \text { BY-27 } & \text { none } \\ & \end{array}$

\section{CORE? LOGS? WHICH LOGS?}

no no

no no

no no

no no

no no

no no

no no

no no

no no

no no

no no

no no

no no

no no

no no

no no

no no

no no

no no

no no

no no

no no

no no

no no

no no

no no 


\section{CURRENT}

NAME

BY-28

none

BY-29

BY-30

BY-31

BY-32

BY-33

BY-34

BY-35

BY-36

BY-37

BY-38

BY-39

BY-40

BY-41

BY-43

BY-44

BY-45

BY-46

BY-47

BY-48

BY-49

BY-50

C-1

C-19

C-21

C-25

none

none

none

none

none

none

none

none

none

none

none

none

none

none

none

none

none

none

none

none

none

none

none

none

none
ROCK FORMATIONS

\section{CORE? LOGS? WHICH LOGS?}

no no

no no

no no

no no

no no

no no

no no

no no

no no

no no

no no

no no

no no

no no

no no

no no

no no

no no

no no

no no

no no

no no

no no

no no

no no

no no 


\begin{tabular}{|c|c|}
\hline \multicolumn{2}{|l|}{ CURRENT } \\
\hline NAME & ROCK FORMATIONS \\
\hline C-40 & none \\
\hline C-45 & none \\
\hline C-48 & none \\
\hline C-50 & none \\
\hline C-51 & none \\
\hline $\mathrm{CH}-001$ & Conasauga/Maynardville Limestone \\
\hline $\mathrm{CH}-002$ & Conasauga/Maynardville Limestone \\
\hline $\mathrm{CH}-143$ & Chickamauga(?-177), Knox(177-?) \\
\hline $\mathrm{CH}-157$ & Knox/Copper Ridge Dolomite \\
\hline $\mathrm{CH}-185$ & Chickamauga \\
\hline $\mathrm{CH}-189$ & Chickamauga(?-578), Knox(578-?) \\
\hline $\mathrm{CO}-2$ & none \\
\hline $\mathrm{CO}-4$ & none \\
\hline DC WELL & none \\
\hline ED/Y-12 No. 2 & Conasauga/Maynardville Limestone \\
\hline \multicolumn{2}{|c|}{ EMW-VWUNDRDR none } \\
\hline FP-001 & Rome \\
\hline FP-002 & Rome \\
\hline FP-003 & Rome \\
\hline FP-004 & Rome \\
\hline FP-005 & Rome \\
\hline FP-006 & Rome \\
\hline FP-007 & Rome \\
\hline FP-008A & Rome \\
\hline
\end{tabular}

\section{CORE? LOGS? WHICH LOGS?}

no no

no no

no no

no no

no no

yes no

yes no

yes yes

yes yes

yes yes

yes yes

yes

no no

no no

no no

yes no

no no

yes no

yes no

yes no

yes no

yes no

yes no

yes no

yes no
SPR, CDL, SP, GRL, SNL, CAV, LSN, DEV, TEM, CL

GRL, SPR, DEV, VDL, CL, TEM, LSN, CDL, SP, SNL, BHTV

SNL, LSN, GRL, DEV, CL, CDL, VDL, BHTV, TEM, SPR, SP

GRL, DEV, BHTV, SPR, CL, SNL, TEM, $\mathrm{SP}, \mathrm{CDL}, \mathrm{LSN}, \mathrm{VDL}$ 


\begin{tabular}{ll}
$\begin{array}{cl}\text { CURRENT } \\
\text { NAME }\end{array}$ & \multicolumn{1}{c}{ ROCK FORMATIONS } \\
FP-008B & Rome \\
FP-009 & Rome \\
FP-010 & Rome \\
FP-011 & Rome \\
FT-001 & Knox/Copper Ridge Dolomite \\
FT-002 & Knox/Copper Ridge Dolomite \\
FT-003 & Knox/Copper Ridge Dolomite \\
FW001 & none \\
FW002 & none \\
FW003 & none \\
FW004 & none \\
FW005 & none \\
GW-001 & Conasauga/Maryville Limestone \\
GW-002 & Conasauga/Maryville Limestone \\
GW-003 & Conasauga/Nolichucky Shale \\
GW-004 & Conasauga/Nolichucky Shale \\
GW-005 & Conasauga/Nolichucky Shale \\
GW-006 & Conasauga/Nolichucky Shale \\
GW-007 & Conasauga/Nolichucky Shale \\
GW-008 & Conasauga/Nolichucky Shale \\
GW-009 & Conasauga/Nolichucky Shale \\
GW-010 & Conasauga/Nolichucky Shale \\
GW-011 & Conasauga/Nolichucky Shale \\
GW-012 & Conasauga/Nolichucky Shale \\
GW-013 & Conasauga/Nolichucky Shale \\
GW-014 & Conasauga/Nolichucky Shale \\
&
\end{tabular}

\section{CORE? LOGS? WHICH LOGS?}

yes

yes no

yes no

yes no

yes no

yes no

yes no

no no

no no

no no

no no

no no

yes yes DHC

yes no

yes no

yes no

no no

yes yes

no no

yes yes

yes no

no yes DHC

yes no

no yes DHC

yes no

no no 


\section{CURRENT}

GW-015

Conasauga/Nolichucky Shale

GW-016

GW-017

GW-018

GW-019

GW-020

GW-021

GW-022

GW-023

GW-024

GW-025

GW-025A

GW-026

GW-027

GW-028

GW-029

GW-030

GW-031

GW-032

GW-033

GW-034

GW-035

GW-036

GW-037

GW-038

GW-039

Conasauga/Nolichucky Shale

Conasauga/Nolichucky Shale

Conasauga/Nolichucky Shale

Conasauga/Nolichucky Shale

Conasauga/Nolichucky Shale

Conasauga/Nolichucky Shale

Conasauga/Nolichucky Shale

Conasauga/Nolichucky Shale

Conasauga/Maryville Limestone

Conasauga/Maryville Limestone

Conasauga/Maryville Limestone

Conasauga/Maryville Limestone

Conasauga/Maryville Limestone

Conasauga/Maryville Limestone

Conasauga/Maryville Limestone

Conasauga/Maryville Limestone

Conasauga/Maryville Limestone

Conasauga/Maryville Limestone

Conasauga/Maryville Limestone

Conasauga/Maryville Limestone

Conasauga/Maryville Limestone

Conasauga/Rogersville Shale

Conasauga/Maryville Limestone

Conasauga/Maryville Limestone

Conasauga/Maryville Limestone

no

yes

no

yes

yes

no

yes

yes

yes

yes

no

no

no

no

no

no

yes

yes

no

no

yes

no

yes

no

no no

yes $\mathrm{DHC}$

yes $\mathrm{DHC}$

yes $\mathrm{DHC}$

no

no

no

no

no

no

no

no

no

no

no

no

no

no

no

no

no

no

no

no

no 


\section{CURRENT}

GW-040

GW-041

GW-042

GW-043

GW-044

GW-045

GW-046

GW-047

GW-048

GW-049

GW-050

GW-051

GW-052

GW-053

GW-054

GW-055

GW-056

GW-057

GW-058

GW-059

GW-060

GW-061

GW-062

GW-063

GW-064

GW-065

\section{ROCK FORMATIONS}

Conasauga/Pumpkin Valley Shale Conasauga/Pumpkin Valley Shale

Conasauga/Pumpkin Valley Shale

Conasauga/Maryville Limestone

Conasauga/Maryville Limestone

Conasauga/Maynardville Limestone

Conasauga/Nolichucky Shale

Conasauga/Nolichucky Shale

Conasauga/Maynardville Limestone

Conasauga/Maynardville Limestone

Conasauga/Maynardville Limestone

Conasauga/Maynardville Limestone

Conasauga/Maynardville Limestone

Conasauga/Maynardville Limestone

Conasauga/Maynardville Limestone

Conasauga/Maynardville Limestone

Conasauga/Maynardville Limestone

Conasauga/Maynardville Limestone

Conasauga/Maynardville Limestone

Conasauga/Maynardville Limestone

Conasauga/Maynardville Limestone

Conasauga/Maynardville Limestone

Conasauga/Maynardville Limestone

Conasauga/Maynardville Limestone

Conasauga/Maynardville Limestone

Conasauga/Maynardville Limestone

\section{CORE? LOGS? WHICH LOGS?}

yes $\mathrm{DHC}$

yes yes $\mathrm{DHC}$

no yes DHC

no no

yes no

no yes DHC

no yes DHC

no no

no no

no no

no no

no no

no yes DHC

yes yes DHC

yes yes DHC

yes yes DHC

yes yes DHC

yes yes DHC

yes yes DHC

yes yes DHC

yes no

yes no

yes yes DHC

yes no

yes yes DHC

yes yes DHC 


\section{CURRENT}

NAME

GW-066

GW-067

GW-068

GW-069

GW-070

GW-071

GW-072

GW-073

GW-074

GW-075

GW-076

GW-077

GW-078

GW-079

GW-080

GW-081

GW-082

GW-083

GW-084

GW-085

GW-086

GW-087

GW-088

GW-089

GW-090

GW-091

\section{ROCK FORMATIONS}

Conasauga/Maynardville Limestone Conasauga/Maynardville Limestone

Conasauga/Nolichucky Shale

Conasauga/Nolichucky Shale

Conasauga/Nolichucky Shale

Conasauga/Nolichucky Shale

Conasauga/Nolichucky Shale

Conasauga/Nolichucky Shale

Conasauga/Nolichucky Shale

Conasauga/Nolichucky Shale

Conasauga/Nolichucky Shale

Conasauga/Nolichucky Shale

Conasauga/Nolichucky Shale

Conasauga/Rogersville Shale

Conasauga/Rogersville Shale

Conasauga/Pumpkin Valley Shale

Conasauga/Maryville Limestone

Conasauga/Nolichucky Shale

Conasauga/Maryville Limestone

Conasauga/Nolichucky Shale

Conasauga/Nolichucky Shale

Conasauga/Nolichucky Shale

Conasauga/Maryville Limestone

Conasauga/Rutledge Limestone

Conasauga/Rutledge Limestone

Conasauga/Rogersville Shale

\section{CORE? LOGS? WHICH LOGS?}

yes

no

no no

no yes DHC

no yes DHC

no yes GRL, DHC

no yes GRL

no yes DHC

no no

no yes GRL

no yes DHC

no no

yes no

no yes DHC

no yes DHC

no no

no yes DHC

no yes DHC

yes yes DHC

no yes DHC

yes yes DHC

no yes DHC

no no

no no

no yes DHC

no yes DHC

no yes DHC 


\section{CURRENT}

NAME

GW-092

GW-093

GW-093A

GW-094

GW-095

GW-096

GW-096A

GW-097

GW-097A

GW-098

GW-099

GW-100

GW-101

GW-102

GW-103

GW-104

GW-105

GW-106

GW-107

GW-108

GW-109

GW-110

GW-111

GW-112

GW-113

GW-114

\section{ROCK FORMATIONS}

Conasauga/Maryville Limestone

Conasauga/Nolichucky Shale

Conasauga/Nolichucky Shale

Conasauga/Maynardville Limestone

Conasauga/Maynardville Limestone

Conasauga/Maynardville Limestone

Conasauga/Maynardville Limestone

Conasauga/Nolichucky Shale

Conasauga/Nolichucky Shale

Conasauga/Nolichucky Shale

Knox

Conasauga/Maynardville Limestone

Conasauga/Nolichucky Shale

Conasauga/Nolichucky Shale

Conasauga/Nolichucky Shale

Conasauga/Nolichucky Shale

Conasauga/Nolichucky Shale

Conasauga/Nolichucky Shale

Conasauga/Nolichucky Shale

Conasauga/Nolichucky Shale

Conasauga/Nolichucky Shale

Conasauga/Maynardville Limestone

Conasauga/Maynardville Limestone(?-284.3),

Conasauga/Nolichucky Shale(284.3-300)

Conasauga/Nolichucky Shale, Conasauga/Maynardville Limestone

Conasauga/Maynardville Limestone

Conasauga/Maynardville Limestone

\section{CORE? LOGS? WHICH LOGS?}

no

no no

no no

no yes

no yes

no yes

no

no

no

no

no

yes

no

yes DHC

yes $\mathrm{DHC}$

no

no

yes

yes

yes

yes

yes

no

yes
SPR, SP, GRL, DHC

SP, GRL, SPR

SP, SPR, GRL

FCL, CL, SPR, SP, GRL

$\mathrm{DHC}$

GRL, SPR, SP

$\mathrm{DHC}$

$\mathrm{DHC}$

DHC, GRL, SPR, SP
SPR, CL, FCL, GRL 
CURRENT

NAME

GW-115

GW-116

GW-117

GW-118

GW-119

GW-120

GW-121

GW-122

GW-123

GW-124

GW-125

GW-126

GW-127

GW-128

GW-129

GW-130

GW-131

\section{ROCK FORMATIONS}

Conasauga/Maryville Limestone

Conasauga/Nolichucky Shale

Conasauga/Nolichucky Shale(100-?), Conasauga/Maynardville Limestone(?-100)

Conasauga/Maynardville Limestone(?-155),

Conasauga/Nolichucky Shale(155-?)

Conasauga/Nolichucky Shale(100-?), Conasauga/Maynardville Limestone(?-100)

Conasauga/Nolichucky Shale

Conasauga/Maryville Limestone(?-535), Conasauga/Nolichucky Shale(535-?)

Conasauga/Maynardville Limestone(?-90), Conasauga/Nolichucky Shale(90-?)

Conasauga/Maynardville Limestone(?-90), Conasauga/Nolichucky Shale(90-?)

Conasauga/Maynardville Limestone

Conasauga/Maynardville Limestone(?-200),

Conasauga/Nolichucky Shale(200-?)

Conasauga/Nolichucky Shale

Conasauga/Nolichucky Shale, Conasauga/Maynardville Limestone

Conasauga/Maryville Limestone(?-130), Conasauga/Rogersville Shale(130-303.1), Conasauga/Rutledge Limestone(303.1-463.8) Conasauga/Pumpkin Valley Shal

Conasauga/Nolichucky Shale(?-275.4), Conasauga/Maryville Limestone(275.4-793.6), Conasauga/Rogersville Shale(793.6909.8), Conasauga/Rutledge Limeston

Conasauga/Nolichucky Shale, Conasauga/Maynardville Limestone, Conasauga/Maryville Limestone

Knox(?-588), Conasauga/Maynardville Limestone(588-?)

\section{no}

yes

yes

yes

yes

yes

yes

yes

\section{WHICH LOGS?}

GRL, LSN, SNL, SP, DEV, TEM, VDL, CDL, CL, SPR

TEM, VDL, BHTV, CDL, CL, DEV, GRL, LSN, SNL, SP, SPR

SPR, CL, LSN, CDL, SNL, GRL, TEM, SP, DEV

CL, CDL, GRL, DEV, SNL, LSN, TEM,

SPR, SP

LSN, DEV, CDL, SNL, SP, CL, TEM, SPR, GRL

SNL, GRL, CDL, BHTV, LSN, SP, SPR, DEV, TEM, VDL, CL

GRL, DEV, SPR, SNL, SP, LSN, CL, TEM, CDL

GRL, SP, SNL, LSN, TEM, SPR, VDL, CL, CDL, CAV, BHTV

SNL, LSN, CL, CDL, GRL, SPR, TEM, DEV, SP

SPR, TEM, LSN, SNL, SP, DEV, GRL,

$\mathrm{CDL}, \mathrm{CL}$

SPR, DEV, SP, CDL, SNL, LSN, TEM, CL, GRL

$\mathrm{DHC}$

DEV, SNL, TEM, SPR, SP, CL, CDL, CAV, LSN, GRL

CL, LSN, CDL, SPR, DEV, GRL, SP, SNL, TEM

CL, SP, SNL, TEM, DEV, GRL, SPR CDL, LSN

CDL, LSN, GRL, DEV, SNL, TEM, SPR, SP, VDL, UNL, UAV, CL 
CURRENT

GW-132

GW-133

GW-134

GW-135

GW-136

GW-137

GW-138

GW-139

GW-140

GW-141

GW-142

GW-143

GW-144

GW-145

GW-146

GW-147

GW-148

GW-149

GW-150

\section{ROCK FORMATIONS}

Conasauga/Rogersville Shale(?-102.8), Conasauga/Rutledge Limestone(102.8-258.6), Conasauga/Pumpkin Valley Shale(258.6638.5), Rome(638.5-?)

Conasauga/Rutledge Limestone, Conasauga/Maryville

Limestone(?-449.7), Conasauga/Rogersville Shale(449.7-?)

Conasauga/Maynardville Limestone(?-136.2)

Conasauga/Nolichucky Shale(136.2-709), Conasauga/Maryville Limestone(709-?)

Knox(?-695.4), Conasauga/Maynardville Limestone(695.4-?)

Conasauga/Nolichucky Shale(?-580), Conasauga/Maryville Limestone(580-?)

Conasauga/Nolichucky Shale, Conasauga/Maynardville

Limestone, Conasauga/Maryville Limestone

Knox, Conasauga/Nolichucky Shale, Conasauga/Maynardville

Limestone

Conasauga/Rogersville Shale, Conasauga/Nolichucky Shale, Conasauga/Maryville Limestone

Knox, Conasauga/Nolichucky Shale, Conasauga/Maynardville Limestone

Knox

Knox(?-220), Chickamauga(220-?)

Knox(?-177), Chickamauga(177-?)

Chickamauga, Knox

Knox

Knox

Knox

Conasauga/Maynardville Limestone

Conasauga/Maynardville Limestone

Conasauga/Maynardville Limestone

\section{CORE? LOGS? WHICH LOGS?}

yes

yes

yes
CDL, SNL, LSN, CL, GRL, DEV, TEM, SPR, CAV, SP

UAV, TEM, VDL, DEV, GRL, CDL, SNL, CL, SPR, SP, LSN, UNL

GRL, CDL, CL, UAV, UNL, VDL, SP SPR, TEM, LSN, SNL, DEV

CDL, SP, SNL, LSN, TEM, BHTV, DEV SPR, VDL, CL, GRL

GRL, DEV, SP, SNL, LSN, TEM, SPR, $\mathrm{CL}, \mathrm{CDL}$

CL, SNL, CDL, SP, DEV, LSN, TEM, SPR, GRL

BHTV, GRL, VDL, CDL, CL, SNL, SPR DEV, LSN, TEM, SP

SNL, GRL, SPR, CDL, LSN, TEM, CL, DEV, SP

SP, SPR, SNL, DEV, TEM, CDL, BHTV, LSN, CL, GRL, VDL

SP, SNL, LSN, TEM, SPR, CL, CDL, GRL, DHC, DEV

LSN, CDL, TEM, GRL, SP, SNL, DEV, SPR, CL 


\section{CURRENT}

NAME

GW-151

GW-152

GW-153

GW-154

GW-155

GW-156

GW-157

GW-158

GW-159

GW-160

GW-161

GW-162

GW-163

GW-164

GW-165

GW-166

GW-167

GW-168

GW-169

GW-170

GW-171

GW-172

GW-173

GW-174

GW-175

\section{ROCK FORMATIONS}

Conasauga/Maynardville Limestone

Conasauga/Maynardville Limestone

Conasauga/Maynardville Limestone

\section{Conasauga/Maynardville Limestone}

Knox

Knox

Knox

Knox

Knox

Knox

Knox

Conasauga/Pumpkin Valley Shale

Rome, Conasauga/Pumpkin Valley Shale

Rome, Conasauga/Pumpkin Valley Shale

Knox

Knox

Conasauga/Maynardville Limestone

Conasauga/Nolichucky Shale, Conasauga/Maynardville Limestone

Conasauga/Maynardville Limestone

Conasauga/Maynardville Limestone

Conasauga/Maynardville Limestone

Conasauga/Maynardville Limestone

Knox

Knox

Knox

\section{CORE? LOGS? WHICH LOGS?}

no no

no no

no no

no no

no no

no no

no no

no yes

no no

no no

no no

no no

no no

no yes

no no

no yes

no yes

SPR, TEM, LSN, SNL, CL, DEV, GRL, CAV, CDL, SP

TEM, CDL, SNL, GRL, SP, SPR, DEV LSN, CL

CDL, GRL, DEV, SP, SNL, LSN, SPR, CL,

$$
\text { TEM }
$$

$\mathrm{DHC}$

no no




\section{CURRENT}

NAME

GW-176

GW-177

GW-178

GW-179

GW-180

GW-181

GW-182

GW-183

GW-184

GW-185

GW-186

GW-187

GW-188

GW-189

GW-190

GW-191

GW-192

GW-193

GW-194

GW-195

GW-196

GW-197

GW-198

GW-199

GW-200

GW-201

\section{ROCK FORMATIONS}

Knox

Knox

Knox

Knox

Knox

Knox

Knox

Conasauga

Chickamauga

Chickamauga

Chickamauga

Chickamauga

Chickamauga

Chickamauga

Conasauga

Conasauga/Maryville Limestone

Conasauga/Maryville Limestone

Conasauga

Conasauga/Maryville Limestone

Conasauga/Maryville Limestone

Conasauga/Maryville Limestone

Conasauga/Maryville Limestone

Conasauga/Rutledge Limestone

Conasauga/Rogersville Shale

Conasauga/Rogersville Shale

Conasauga/Rogersville Shale

\section{CORE? LOGS? WHICH LOGS?}

no

no no

no yes DHC

no no

no no

yes no

no no

no no

no no

no no

no no

no no

no no

no no

no no

no yes DHC

no no

no no

no no

no no

no no

no no

no no

no no

no no 


\section{CURRENT}

NAME

GW-202

GW-203

GW-204

GW-205

GW-206

GW-207

GW-208

GW-209

GW-210

GW-211

GW-212

GW-213

GW-214

GW-215

GW-216

GW-217

GW-218

GW-219

GW-220

GW-221

GW-222

GW-223

GW-224

GW-225

GW-226

GW-227

\section{ROCK FORMATIONS}

Conasauga/Rutledge Limestone Knox

Conasauga

Knox

Rome

Rome

Rome

Rome

Rome

Rome

Conasauga/Maynardville Limestone Conasauga/Maynardville Limestone Conasauga/Maynardville Limestone

Conasauga/Maryville Limestone

Conasauga/Maryville Limestone

Knox

Conasauga/Maynardville Limestone

Conasauga/Maynardville Limestone

Conasauga/Maynardville Limestone

Knox

Conasauga/Maynardville Limestone

Conasauga/Maynardville Limestone

Chickamauga

Conasauga/Maynardville Limestone

Conasauga/Maynardville Limestone

Conasauga/Maynardville Limestone

\section{CORE? LOGS? WHICH LOGS?}

no

no no

no no

no no

no yes DHC

no no

no yes

no no

no no

no yes

no no

no no

no yes

no no

no no

no no

no no

no yes

no no

no no

no no

no no

no no

no no

no yes

no no
CL, LSN, SNL, CDL, CAV, GRL

SNL, GRL, CAV, LSN, CDL, CL

GRL, CL, CDL, CAV, SNL, LSN

$\mathrm{DHC}$

$\mathrm{DHC}$ 
CURRENT

NAME

GW-228

GW-229

GW-230

GW-231

GW-232

GW-233

GW-234

GW-235

GW-236

GW-237

GW-238

GW-239

GW-240

GW-241

GW-242

GW-243

GW-244

GW-245

GW-246

GW-247

GW-248

GW-249

GW-250

GW-251

GW-252

GW-253

\section{ROCK FORMATIONS}

Conasauga/Maynardville Limestone Conasauga/Maynardville Limestone

Conasauga/Maynardville Limestone

\section{Knox}

Conasauga/Maynardville Limestone

Knox

Conasauga/Maryville Limestone

Conasauga/Maynardville Limestone

Conasauga/Maynardville Limestone

Conasauga/Maynardville Limestone

Conasauga/Maynardville Limestone

Conasauga/Nolichucky Shale, Conasauga/Maynardville Limestone

Conasauga/Maynardville Limestone

Knox

Conasauga/Pumpkin Valley Shale

Conasauga/Nolichucky Shale

Conasauga/Nolichucky Shale

Conasauga/Nolichucky Shale

Conasauga/Nolichucky Shale

Conasauga/Nolichucky Shale

Conasauga/Maryville Limestone

Conasauga/Maryville Limestone

Conasauga/Maryville Limestone

Conasauga/Maynardville Limestone

Conasauga/Maynardville Limestone

Conasauga/Maynardville Limestone

\section{CORE? LOGS? WHICH LOGS?}

no

no yes DHC

no yes GRL

no no

no yes

CL, CDL, CAV, SNL, LSN, GRL

no no

no no

no no

no no

no yes

no no

no yes

no no

no no

no yes

no no

no no

no no

no yes

no no

no no

no no

no no

no no

no no

no no

\section{DHC}

LSN, CAV, GRL, SNL, CL, CDL

DHC

DHC 
CURRENT

NAME

GW-254

GW-255

GW-256

GW-257

GW-258

GW-259

GW-260

GW-261

GW-262

GW-263

GW-264

GW-265

GW-266

GW-267

GW-268

GW-269

GW-270

GW-271

GW-272

GW-273

GW-274

GW-275

GW-276

GW-277

GW-278

GW-279

\section{ROCK FORMATIONS}

Conasauga/Maynardville Limestone

Conasauga/Maynardville Limestone

Conasauga/Maynardville Limestone

Conasauga/Maryville Limestone

Conasauga/Nolichucky Shale, Conasauga/Maryville Limestone

Conasauga/Nolichucky Shale, Conasauga/Maryville Limestone

Conasauga/Maynardville Limestone

Conasauga/Maryville Limestone

Conasauga/Maryville Limestone

Conasauga/Maryville Limestone

Conasauga/Maryville Limestone

Conasauga/Maryville Limestone

Conasauga/Maryville Limestone

Conasauga/Maryville Limestone

Conasauga/Maryville Limestone

Conasauga/Maryville Limestone

Conasauga/Nolichucky Shale

Conasauga/Nolichucky Shale

Conasauga/Nolichucky Shale

Conasauga/Nolichucky Shale

Conasauga/Nolichucky Shale

Conasauga/Nolichucky Shale

Conasauga/Nolichucky Shale

Conasauga/Nolichucky Shale

Conasauga/Maynardville Limestone

Conasauga/Maynardville Limestone

\section{CORE? LOGS? WHICH LOGS?}

no

no

no no

no no

no no

no no

no no

no no

no no

no no

no no

no no

no no

no no

no no

no no

no no

no no

no no

no no

no no

no no

no no

no no

no no

no no

no no 


\section{CURRENT}

NAME

GW-280

GW-281

GW-282

GW-283

GW-284

GW-285

GW-286

GW-287

GW-288

GW-289

GW-290

GW-291

GW-292

GW-293

GW-294

GW-295

GW-296

GW-297

GW-298

GW-299

GW-300

GW-301

GW-302

GW-303

GW-304

GW-305

\section{ROCK FORMATIONS}

Conasauga/Maynardville Limestone

Conasauga/Nolichucky Shale

Conasauga/Nolichucky Shale

Conasauga/Nolichucky Shale

Conasauga/Nolichucky Shale

Conasauga/Nolichucky Shale

Conasauga/Nolichucky Shale, Conasauga/Maryville Limestone

Conasauga/Nolichucky Shale, Conasauga/Maryville Limestone

Conasauga/Nolichucky Shale, Conasauga/Maryville Limestone

Conasauga/Nolichucky Shale, Conasauga/Maryville Limestone

Conasauga/Maryville Limestone

Conasauga/Maryville Limestone

Knox

Knox

Knox

Knox

Knox

Knox

Knox

Knox

Knox

Knox

Knox

Knox

Knox

Knox

\section{CORE? LOGS? WHICH LOGS?}

no no

no no

no no

no no

no no

no no

no no

no yes DHC

no no

no no

no no

no no

no no

no no

no no

no no

no no

no no

no no

no no

no no

no no

no no

no no

no no

no no 


\section{CURRENT}

NAME

GW-306

GW-307

GW-308

GW-309

GW-310

GW-311

GW-312

GW-313

GW-314

GW-315

GW-316

GW-317

GW-318

GW-319

GW-320

GW-321

GW-322

GW-323

GW-324

GW-325

GW-326

GW-327

GW-328

GW-329

GW-330

GW-331

\section{ROCK FORMATIONS}

Conasauga/Maynardville Limestone

Conasauga/Maynardville Limestone

Conasauga/Maynardville Limestone

Conasauga/Maynardville Limestone

Conasauga/Maynardville Limestone

Conasauga/Maynardville Limestone

Conasauga/Maynardville Limestone

Conasauga/Maynardville Limestone

Conasauga/Maynardville Limestone

Conasauga/Maynardville Limestone

Knox, Conasauga/Maynardville Limestone

Conasauga/Maynardville Limestone(?-65), Knox(65-?)

Chickamauga

Chickamauga

Knox

Knox

Knox

Knox

Conasauga/Nolichucky Shale

Conasauga/Nolichucky Shale

Conasauga/Maryville Limestone

Conasauga/Maryville Limestone

Conasauga/Maryville Limestone

Conasauga/Nolichucky Shale

Conasauga/Nolichucky Shale

Conasauga/Nolichucky Shale

\section{CORE? LOGS? WHICH LOGS?}

no

no

no no

no no

no no

no no

no no

no no

no no

no no

no no

no no

no no

no no

no no

no no

no no

no no

no no

no no

no no

no no

no no

no no

no no

no no

no no 


\section{CURRENT}

\section{NAME}

GW-332

GW-333

GW-334

GW-335

GW-336

GW-337

GW-338

GW-339

GW-340

GW-341

GW-342

GW-343

GW-344

GW-345

GW-346

GW-347

GW-348

GW-349

GW-350

GW-363

GW-364

GW-365

GW-366

GW-367

GW-368

GW-369

\section{ROCK FORMATIONS}

Conasauga/Nolichucky Shale

Conasauga/Nolichucky Shale

Conasauga/Nolichucky Shale

Conasauga/Nolichucky Shale

Conasauga/Nolichucky Shale

Conasauga/Nolichucky Shale

Conasauga/Nolichucky Shale

Knox

Conasauga/Nolichucky Shale

Conasauga/Nolichucky Shale

Conasauga/Pumpkin Valley Shale

Conasauga/Pumpkin Valley Shale

Conasauga/Pumpkin Valley Shale

Conasauga/Nolichucky Shale

Conasauga/Nolichucky Shale

Conasauga/Maynardville Limestone

Conasauga/Maynardville Limestone

Conasauga/Maynardville Limestone

Conasauga/Maynardville Limestone

Conasauga/Nolichucky Shale

Conasauga/Maynardville Limestone

Conasauga/Maynardville Limestone

Knox, Conasauga/Maynardville Limestone

Knox, Conasauga/Maynardville Limestone

Knox, Conasauga/Maynardville Limestone

Knox, Conasauga/Maynardville Limestone

\section{CORE? LOGS? WHICH LOGS?}

no

no no

no no

no no

no no

no no

no no

no yes

$\mathrm{DHC}$ 
CURRENT

NAME

GW-370

GW-371

GW-372

GW-373

GW-374

GW-375

GW-376

GW-380

GW-381

GW-382

GW-383

GW-384

GW-385

GW-400

GW-401

GW-402

GW-403

GW-404

GW-405

GW-406

GW-407

GW-408

GW-409

GW-410

\section{ROCK FORMATIONS}

Conasauga/Maryville Limestone

Conasauga/Maryville Limestone

Conasauga/Maryville Limestone

Conasauga/Maryville Limestone

Conasauga/Nolichucky Shale

Conasauga/Maynardville Limestone

Conasauga/Maryville Limestone

Conasauga/Maynardville Limestone

Conasauga/Maynardville Limestone

Conasauga/Maynardville Limestone

Conasauga/Nolichucky Shale

Conasauga/Nolichucky Shale

Conasauga/Nolichucky Shale

Rome(1056-?), Conasauga/Maryville Limestone(?-465.2),

Conasauga/Rogersville Shale(465.2-558), Conasauga/Rutledge Limestone(558-680), Conasauga/Pumpkin

Conasauga/Nolichucky Shale(?-451), Conasauga/Maryville Limestone(451-?)

Conasauga/Maynardville Limestone(?-110),

Conasauga/Nolichucky Shale(110-?)

Conasauga/Nolichucky Shale(?-312), Conasauga/Maryville

Limestone(312-?)

Conasauga/Nolichucky Shale, Conasauga/Maryville Limestone

none

none

none

none

none

none

\section{CORE? LOGS? WHICH LOGS?}

no

no

no no

no no

no no

no no

no no

no no

no yes

DHC 


$\begin{array}{cc}\text { CURRENT } & \\ \text { NAME } & \\ \text { GW-411 } & \text { none } \\ \text { GW-412 } & \text { none } \\ \text { GW-413 } & \text { none } \\ \text { GW-414 } & \text { none } \\ \text { GW-415 } & \text { none } \\ \text { GW-416 } & \text { none } \\ \text { GW-417 } & \text { none } \\ \text { GW-418 } & \text { none } \\ \text { GW-419 } & \text { none } \\ \text { GW-420 } & \text { none } \\ \text { GW-421 } & \text { none } \\ \text { GW-422 } & \text { none } \\ \text { GW-423 } & \text { none } \\ \text { GW-424 } & \text { none } \\ \text { GW-425 } & \text { none } \\ \text { GW-426 } & \text { none } \\ \text { GW-427 } & \text { none } \\ \text { GW-428 } & \text { none } \\ \text { GW-429 } & \text { none } \\ \text { GW-430 } & \text { none } \\ \text { GW-431 } & \text { none } \\ \text { GW-432 } & \text { none } \\ \text { GW-433 } & \text { none } \\ \text { GW-434 } & \text { none } \\ \text { GW-435 } & \text { none } \\ \text { GW-436 } & \text { none } \\ \end{array}$

\section{CORE? LOGS? WHICH LOGS?}

no no

no no

no no

no no

no no

no no

no no

no no

no no

no no

no no

no no

no no

no no

no no

no no

no no

no no

no no

no no

no no

no no

no no

no no

no no

no no 


\section{CURRENT}

GW-437

GW-438

GW-439

GW-440

GW-441

GW-442

GW-443

GW-445

GW-447

GW-448

GW-449

GW-450

GW-451

GW-452

GW-453

GW-455

none

none

none

none

none

none

Conasauga/Maynardville Limestone

Conasauga/Nolichucky Shale

none

none

none

Conasauga/Pumpkin Valley Shale(?-250), Rome(250-?)

none

Conasauga/Rutledge Limestone(221-347), Conasauga/Pumpkin Valley Shale(347-745), Conasauga/Maryville Limestone(?-70), Conasauga/Rogersville Shale(70-22

Conasauga/Nolichucky Shale(?-109), Conasauga/Maryville

\section{no}

no

Conasauga/Nolichucky Shale

Conasauga/Nolichucky Shale

Conasauga/Nolichucky Shale

Conasauga/Nolichucky Shale

Conasauga/Nolichucky Shale

Conasauga/Nolichucky Shale

Conasauga/Nolichucky Shale

Conasauga/Maynardville Limestone

Conasauga/Maynardville Limestone no

no

no

no

no

no

no

no

no

no

no

no

yes

yes

no

no

no

no

no

no

no

no

no

no

no

no

no

no

no

no no

no no

no no

no no

no no

no no

no no

GW-463

GW-464 
GW-465

GW-466

GW-467

GW-468

GW-469

GW-470

GW-471

GW-472

GW-473

GW-474

GW-475A

GW-475B

GW-475C

GW-476A

GW-476B

GW-476C

GW-477A

GW-477B

GW-477C

GW-478A

GW-478B

GW-478C

GW-479

GW-480A

GW-480B

Conasauga/Maynardville Limestone

Conasauga/Maynardville Limestone

Conasauga/Maynardville Limestone

Conasauga/Nolichucky Shale, Conasauga/Maynardville Limestone

Conasauga/Nolichucky Shale(?-450), Conasauga/Maryville Limestone(450-?)

Conasauga/Maynardville Limestone(?-105),

Conasauga/Nolichucky Shale(105-?)

Conasauga/Nolichucky Shale(?-20), Conasauga/Maryville

Limestone(20-?)

Conasauga/Nolichucky Shale

Conasauga/Maryville Limestone

Conasauga/Maryville Limestone

Conasauga/Maryville Limestone

Conasauga/Maryville Limestone

Conasauga/Maryville Limestone

Conasauga/Maryville Limestone

Conasauga/Maryville Limestone

Conasauga/Maryville Limestone

Conasauga/Maryville Limestone

Conasauga/Maryville Limestone

Conasauga/Maryville Limestone

Conasauga/Maryville Limestone

Conasauga/Maryville Limestone

Conasauga/Maryville Limestone

Conasauga/Maryville Limestone

Conasauga/Maryville Limestone

Conasauga/Maryville Limestone no

no

no

no no

yes no

no no

no no

yes no

no no

no no

no no

no no

no no

no no

no no

no no

no no

no no

no no

no no

no no

no no

no no

no no

no no

no no 


\section{CURRENT}

NAME

GW-480C

GW-481A

GW-481B

GW-481C

GW-482A

GW-482B

GW-482C

GW-483

GW-484

GW-485

GW-486

GW-487

GW-488

GW-489

GW-490

GW-491

GW-492

GW-493

GW-494

GW-495

GW-496

GW-497

GW-498

GW-499

GW-499A

GW-499AA

\section{ROCK FORMATIONS}

Conasauga/Maryville Limestone

Conasauga/Maryville Limestone

Conasauga/Maryville Limestone

Conasauga/Maryville Limestone

Conasauga/Maryville Limestone

Conasauga/Maryville Limestone

Conasauga/Maryville Limestone

Conasauga/Maryville Limestone

Conasauga/Maryville Limestone

Conasauga/Maryville Limestone

Conasauga/Maryville Limestone

Conasauga/Maryville Limestone

Conasauga/Maryville Limestone

Conasauga/Maryville Limestone

Conasauga/Maryville Limestone

Conasauga/Maryville Limestone

Conasauga/Maryville Limestone

Conasauga/Maryville Limestone

Conasauga/Maryville Limestone

Conasauga/Maryville Limestone

Conasauga/Maryville Limestone

Conasauga/Maryville Limestone

Conasauga/Maryville Limestone

Conasauga/Nolichucky Shale, Conasauga/Maryville Limestone

Conasauga/Nolichucky Shale, Conasauga/Maryville Limestone

Conasauga/Nolichucky Shale, Conasauga/Maryville Limestone

\section{CORE? LOGS? WHICH LOGS?}

no no

no no

no no

no no

no no

no no

no no

no no

no no

no no

no no

no no

no no

no no

no no

no no

no no

no no

no no

no no

no no

no no

no no

no no

no no 
GW-499AB

Conasauga/Nolichucky Shale, Conasauga/Maryville Limestone

GW-499AC

GW-499AD

GW-499AE

GW-499AF

GW-499B

GW-499C

GW-499D

GW-499E

GW-499F

GW-499G

GW-499H

GW-499I

GW-499J

GW-499K

GW-499L

GW-499M

GW-499N

GW-4990

GW-499P

GW-499Q

GW-499R

GW-499S

GW-499T

GW-499U

GW-499V Conasauga/Nolichucky Shale, Conasauga/Maryville Limestone Conasauga/Nolichucky Shale, Conasauga/Maryville Limestone Conasauga/Nolichucky Shale, Conasauga/Maryville Limestone Conasauga/Nolichucky Shale, Conasauga/Maryville Limestone Conasauga/Nolichucky Shale, Conasauga/Maryville Limestone Conasauga/Nolichucky Shale, Conasauga/Maryville Limestone Conasauga/Nolichucky Shale, Conasauga/Maryville Limestone Conasauga/Nolichucky Shale, Conasauga/Maryville Limestone Conasauga/Nolichucky Shale, Conasauga/Maryville Limestone Conasauga/Nolichucky Shale, Conasauga/Maryville Limestone Conasauga/Nolichucky Shale, Conasauga/Maryville Limestone Conasauga/Nolichucky Shale, Conasauga/Maryville Limestone Conasauga/Nolichucky Shale, Conasauga/Maryville Limestone Conasauga/Nolichucky Shale, Conasauga/Maryville Limestone Conasauga/Nolichucky Shale, Conasauga/Maryville Limestone Conasauga/Nolichucky Shale, Conasauga/Maryville Limestone Conasauga/Nolichucky Shale, Conasauga/Maryville Limestone Conasauga/Nolichucky Shale, Conasauga/Maryville Limestone Conasauga/Nolichucky Shale, Conasauga/Maryville Limestone Conasauga/Nolichucky Shale, Conasauga/Maryville Limestone Conasauga/Nolichucky Shale, Conasauga/Maryville Limestone Conasauga/Nolichucky Shale, Conasauga/Maryville Limestone Conasauga/Nolichucky Shale, Conasauga/Maryville Limestone Conasauga/Nolichucky Shale, Conasauga/Maryville Limestone Conasauga/Nolichucky Shale, Conasauga/Maryville Limestone

\section{no}

no

no

no

no

no

no

no

no

no

no

no

no

no

no

no

no

no

no

no

no

no

no

no no

no

no

no

no

no

no

no

no

no

no

no

no

no

no

no

no

no

no

no

no

no 


\section{CURRENT}

\section{NAME}

GW-499W

GW-499X

GW-499Y

GW-499Z

GW-502

GW-505

GW-506

GW-507

GW-508

GW-509

GW-511

GW-512

GW-513

GW-514

GW-520

GW-521

GW-522

GW-526

GW-527

GW-528

GW-531

GW-532

GW-533

GW-534

GW-535

GW-536

\section{ROCK FORMATIONS}

Conasauga/Nolichucky Shale, Conasauga/Maryville Limestone Conasauga/Nolichucky Shale, Conasauga/Maryville Limestone Conasauga/Nolichucky Shale, Conasauga/Maryville Limestone Conasauga/Nolichucky Shale, Conasauga/Maryville Limestone

Conasauga/Nolichucky Shale

Conasauga/Nolichucky Shale

Conasauga/Nolichucky Shale

Conasauga/Nolichucky Shale

Conasauga/Nolichucky Shale

Conasauga/Nolichucky Shale

Knox

Knox

Knox

Knox

Knox, Conasauga/Maynardville Limestone

Knox

Knox

Conasauga/Nolichucky Shale

Conasauga/Maryville Limestone

Conasauga/Maryville Limestone

Conasauga/Maryville Limestone

Conasauga/Maryville Limestone

Conasauga

Conasauga

Conasauga/Nolichucky Shale

Conasauga/Nolichucky Shale

\section{CORE? LOGS? WHICH LOGS?}

no no

no no

no no

no no

no yes

DHC

no

no

no

no no

no no

no no

no no

no no

no no

no no

no no

no no

no no

no no

no yes

DHC 


\begin{tabular}{ll}
$\begin{aligned} \text { CURRENT } \\
\text { NAME }\end{aligned}$ & \multicolumn{1}{c}{ ROCK FORMATIONS } \\
GW-537 & Conasauga/Nolichucky Shale \\
GW-538 & Conasauga \\
GW-539 & Knox \\
GW-540 & Knox \\
GW-541 & Knox \\
GW-542 & Knox \\
GW-543 & Knox \\
GW-544 & Knox \\
GW-545 & Knox \\
GW-546 & Knox \\
GW-551 & Knox \\
GW-552 & Knox \\
GW-553 & Knox \\
GW-554 & Knox \\
GW-555 & Knox \\
GW-556 & Knox \\
GW-557 & Knox \\
GW-558 & Knox \\
GW-559 & Knox \\
GW-560 & Knox \\
GW-561 & Knox \\
GW-562 & Knox \\
GW-563 & Knox \\
GW-564 & Knox \\
GW-565 & Knox \\
GW-566 & Knox \\
&
\end{tabular}

\section{CORE? LOGS? WHICH LOGS?}

$\begin{array}{ll}\text { no } & \text { no } \\ \text { no } & \text { no } \\ \text { no } & \text { no } \\ \text { no } & \text { no } \\ \text { no } & \text { no } \\ \text { no } & \text { no } \\ \text { no } & \text { no } \\ \text { no } & \text { no } \\ \text { no } & \text { no } \\ \text { yes } & \text { no } \\ \text { yes } & \text { no } \\ \text { yes } & \text { no } \\ \text { yes } & \text { no } \\ \text { yes } & \text { no } \\ \text { yes } & \text { no } \\ \text { no } & \text { no } \\ \text { no } & \text { no } \\ \text { no } & \text { no } \\ \text { no } & \text { no } \\ \text { no } & \text { no } \\ \text { no } & \text { no } \\ \text { no } & \text { no } \\ \text { no } & \text { no } \\ \text { no } & \text { no } \\ \text { no } & \text { no }\end{array}$




\section{CURRENT}

NAME

GW-567

GW-568

GW-569

GW-570

GW-571

GW-572

GW-573D

GW-573S

GW-576

GW-580

GW-601

GW-602

GW-603

GW-604

GW-605

GW-606

GW-607

GW-608

GW-608A

GW-609

GW-610

GW-611

GW-612

GW-613

GW-614

GW-615

\section{ROCK FORMATIONS}

Knox

Knox

Knox

Knox

Knox

Knox

Knox

Knox

Knox

Knox

Knox, Conasauga/Maynardville Limestone Knox, Conasauga/Maynardville Limestone

Conasauga/Maynardville Limestone

Conasauga/Maynardville Limestone

Conasauga/Maynardville Limestone

Conasauga/Maynardville Limestone

\section{Knox}

Knox

Knox

Knox

Knox

Knox

Knox

Conasauga/Nolichucky Shale

Conasauga/Nolichucky Shale

Conasauga/Nolichucky Shale
CORE? LOGS? WHICH LOGS?

no no

no no

no no

no no

no no

no no

no no

no no

no no

no no

no yes DHC

no yes DHC

no no

no no

no no

no no

no no

no no

no no

no no

no no

no no

no no

no no

no no

no yes DHC 


\section{CURRENT}

\section{NAME}

GW-616

GW-617

GW-618

GW-619

GW-620

GW-621

GW-622

GW-623

GW-624

GW-625

GW-626

GW-627

GW-628

GW-629

GW-630

GW-631

GW-632

GW-633

GW-634

GW-635

GW-636

GW-637

GW-638

GW-639

GW-640

GW-641

\section{ROCK FORMATIONS}

Conasauga/Nolichucky Shale, Conasauga/Maynardville Limestone Conasauga/Maynardville Limestone

Conasauga/Maynardville Limestone

Conasauga/Maynardville Limestone

Conasauga/Maynardville Limestone

Conasauga/Maynardville Limestone

Conasauga/Nolichucky Shale

Conasauga/Nolichucky Shale

Conasauga/Nolichucky Shale

Conasauga/Nolichucky Shale

Conasauga/Nolichucky Shale

Conasauga/Nolichucky Shale

Conasauga/Nolichucky Shale

Conasauga/Nolichucky Shale

Conasauga

Conasauga/Nolichucky Shale

Conasauga/Nolichucky Shale

Conasauga/Nolichucky Shale

Conasauga/Nolichucky Shale

Conasauga/Nolichucky Shale

Conasauga/Nolichucky Shale

Conasauga/Nolichucky Shale

Conasauga/Nolichucky Shale

Conasauga/Nolichucky Shale

Conasauga/Nolichucky Shale

Conasauga/Nolichucky Shale

\section{CORE? LOGS? WHICH LOGS?}

no

no no

no no

no yes

no no

no no

no yes

no yes

$\mathrm{DHC}, \mathrm{DHC}$

DHC

no no

no no

no yes

$\mathrm{DHC}$ 


\section{CURRENT}

GW-642

GW-643

GW-644

GW-645

GW-646

GW-647

GW-647A

GW-648

GW-649

GW-651

GW-652

GW-653

GW-654

GW-655

GW-656

GW-657

GW-658

GW-659

GW-660

GW-661

GW-662

GW-663

GW-664

GW-665

GW-666

GW-667

\section{ROCK FORMATIONS}

Conasauga/Rogersville Shale

Conasauga/Maryville Limestone

Conasauga/Nolichucky Shale

Conasauga/Maynardville Limestone

Conasauga/Maynardville Limestone

Knox/Copper Ridge Dolomite

Conasauga/Maynardville Limestone

Conasauga/Maynardville Limestone

Conasauga/Nolichucky Shale

Conasauga/Maynardville Limestone

Conasauga/Maynardville Limestone

Conasauga/Nolichucky Shale

Conasauga/Maryville Limestone

Conasauga/Pumpkin Valley Shale

Conasauga/Nolichucky Shale

Conasauga/Maynardville Limestone

Conasauga/Nolichucky Shale

Conasauga/Nolichucky Shale

Rome

Rome

Rome

Rome

Rome

Rome

Rome

Rome

\section{CORE? LOGS? WHICH LOGS?}

no no

no no

no no

no no

no no

no no

no no

no no

no no

no no

no no

no no

no no

no no

no yes DHC

no no

no no

no no

no no

no no

no no

no no

no no

no no

no no

no no 


\section{CURRENT}

NAME

GW-668

GW-669

GW-670

GW-671

GW-672

GW-673

GW-674

GW-675

GW-676

GW-677

GW-678

GW-679

GW-680

GW-681

GW-682

GW-683

GW-684

GW-685

GW-686

GW-687

GW-688

GW-689

GW-690

GW-691

GW-692

GW-693

\section{ROCK FORMATIONS}

Rome

Rome

Rome

Rome

Knox

Knox

Knox

Chickamauga

Knox

Knox

Knox

Knox

Knox

Knox

Knox

Knox

Knox, Conasauga/Maynardville Limestone

Conasauga/Maynardville Limestone

Conasauga/Maynardville Limestone

Conasauga/Maynardville Limestone

Conasauga/Maynardville Limestone

Conasauga/Maynardville Limestone

Conasauga/Maynardville Limestone

Conasauga/Maynardville Limestone

Conasauga/Maynardville Limestone

Conasauga/Maynardville Limestone

\section{CORE? LOGS? WHICH LOGS?}

no no

no no

no no

no no

no no

no no

no no

no no

no no

no no

no no

no no

no yes DHC

no yes DHC

no yes DHC

no yes GRL

no no

no yes DHC

no no

no no

no no

no no

no no

no no

no no

no no 
CURRENT

NAME

GW-694

GW-695

GW-696

GW-697

GW-698

GW-699

GW-700

GW-701

GW-702

GW-703

GW-704

GW-705

GW-705A

GW-705B

GW-706

GW-707

GW-708

GW-709

GW-710

GW-711

GW-712

GW-713

GW-714

GW-715

\section{ROCK FORMATIONS}

Conasauga/Maynardville Limestone

Knox

Conasauga/Maynardville Limestone

Conasauga/Maynardville Limestone

Conasauga/Maynardville Limestone

Conasauga/Maynardville Limestone

Conasauga/Maynardville Limestone

Conasauga/Maynardville Limestone

Conasauga/Maynardville Limestone

Knox(?-35), Conasauga/Maynardville Limestone(35-?)

Conasauga/Maynardville Limestone

Conasauga/Maynardville Limestone(?-236),

Conasauga/Nolichucky Shale(236-?)

Knox

Knox

Conasauga/Maynardville Limestone

Conasauga/Maynardville Limestone

Conasauga/Maynardville Limestone

Knox

Knox, Conasauga/Maynardville Limestone

Knox, Conasauga/Maynardville Limestone

Knox, Conasauga/Maynardville Limestone

Knox, Conasauga/Maynardville Limestone

Conasauga/Maynardville Limestone

Conasauga/Maynardville Limestone

\section{CORE? LOGS? WHICH LOGS?}

no

DHC

no yes

no no

no no

no no

no no

no no

no no

no no

no yes

DHC

no no

no yes

GRL

no no

no no

no no

no no

no no

no no

no yes

IFL, UNL, GRL, FRL, TEM, VDL, BHTV, CDL, SPR, SP, CL, LSN, SNL

no yes

IFL, GRL, FRL, CL, CDL, VDL, TEM, SP, LSN, SNL

no yes

SNL, SP, TEM, VDL, CDL, CL, FRL, GRL, IFL, LSN

no no

no no

no yes DHC 
CURRENT

NAME

GW-721

GW-722

GW-723

GW-724

GW-725

GW-726

GW-727

GW-728

GW-729

GW-730

GW-731

GW-732

GW-733

GW-734

GW-735

GW-736

GW-737

GW-738

GW-739

GW-740

\section{ROCK FORMATIONS}

Rome

Conasauga/Maynardville Limestone(?-584),

Conasauga/Nolichucky Shale(584-?)

Knox(?-139), Conasauga/Maynardville Limestone(139-?)

Conasauga/Maynardville Limestone

Conasauga/Maynardville Limestone

Conasauga/Nolichucky Shale

Conasauga/Maynardville Limestone(?-232),

Conasauga/Nolichucky Shale(232-?)

Conasauga/Maynardville Limestone(?-280),

Conasauga/Nolichucky Shale(280-?)

Conasauga/Maynardville Limestone(?-595),

Conasauga/Nolichucky Shale(595-?)

Conasauga/Rogersville Shale(1406-?), Conasauga/Maynardville

Limestone(?-412), Conasauga/Nolichucky Shale(412-995),

Conasauga/Maryville Limestone(995-1

Knox

Knox

Knox(?-73), Conasauga/Maynardville Limestone(73-?)

Conasauga/Maynardville Limestone

Conasauga/Maynardville Limestone(?-75.5),

Conasauga/Nolichucky Shale(75.5-?)

Conasauga/Maynardville Limestone

Conasauga/Maynardville Limestone

Conasauga/Maynardville Limestone

Knox(?-125), Conasauga/Maynardville Limestone(125-?)

Knox(?-110), Conasauga/Maynardville Limestone(110-?)

\section{CORE? LOGS? WHICH LOGS?}

no

yes

yes

yes

no

yes

yes

no

no

no

no

no

no

no

no

no yes

yes

SPR, UNL

SP, SPR, LSN, CDL, CL, TEM, VDL, UNL, GRL

GRL, SPR, VDL, UNL, TEM, LSN, CL, SP, $\mathrm{CDL}$

VDL, UNL, TEM, SPR, SP, CDL, LSN, GRL, CL

SP, FWS, GRL, DEV, TEM, FCL, LSN,

$\mathrm{FRL}, \mathrm{CL}$

TEM, DEV, LSN, CL, SP, GRL, SPR

no

GRL, SP, CL, LSN, TEM, FRL, DEV, FWS

yes

SP, SPR, TEM, CL, LSN, FWS, GRL

no

GRL

no

yes

$\mathrm{DHC}$ 


\section{CURRENT}

GW-742

Knox

GW-743

Knox

GW-744

Conasauga/Pumpkin Valley Shale

Conasauga/Pumpkin Valley Shale

no no

GW-745

GW-746

Conasauga/Pumpkin Valley Shale

no no

GW-747

GW-748

GW-749

GW-750

Conasauga/Maryville Limestone

Conasauga/Maryville Limestone

Conasauga/Maryville Limestone

Conasauga/Maynardville Limestone(?-45), Conasauga/Nolichucky Shale(45-?)

GW-751

GW-752

Conasauga/Nolichucky Shale

\section{Conasauga/Nolichucky Shale}

Conasauga/Maryville Limestone(?-61), Conasauga/Nolichucky Shale(61-?)

GW-754

GW-755

GW-756

GW-757

GW-758

GW-759

GW-760

GW-761

GW-762

GW-763

GW-764

GW-765

GW-766

Conasauga/Nolichucky Shale

Conasauga/Rogersville Shale, Conasauga/Maryville Limestone

Conasauga/Maryville Limestone

Knox

Conasauga/Rutledge Limestone

Conasauga/Rutledge Limestone

Conasauga/Maryville Limestone

Conasauga/Maryville Limestone

Conasauga/Nolichucky Shale

Conasauga/Nolichucky Shale

Conasauga/Rogersville Shale

Conasauga/Rogersville Shale

Conasauga/Nolichucky Shale, Conasauga/Maryville Limestone

no no




\section{CURRENT}

NAME

GW-767

GW-768

GW-769

GW-770

GW-771

GW-772

GW-773

GW-774

GW-775

GW-776

GW-777

GW-778

GW-779

GW-780

GW-781

GW-782

GW-783

GW-784

GW-785

GW-786

GW-787

GW-788

GW-789

GW-790

GW-791

GW-792

\section{ROCK FORMATIONS}

Conasauga/Maryville Limestone

Conasauga/Rogersville Shale

Conasauga/Nolichucky Shale

Conasauga/Nolichucky Shale

Conasauga/Rogersville Shale

Conasauga/Rogersville Shale

Conasauga/Maryville Limestone

Conasauga/Maryville Limestone

Conasauga/Nolichucky Shale

Conasauga/Nolichucky Shale

Conasauga/Maryville Limestone

Conasauga/Maryville Limestone

Conasauga/Maryville Limestone

Conasauga/Nolichucky Shale

Conasauga/Nolichucky Shale

Conasauga/Nolichucky Shale

Conasauga/Nolichucky Shale

Conasauga/Rogersville Shale

Conasauga/Maryville Limestone

Conasauga/Maryville Limestone

Conasauga/Maryville Limestone

Conasauga/Nolichucky Shale

Conasauga/Nolichucky Shale

Conasauga/Maynardville Limestone(?-294.8)

Conasauga/Nolichucky Shale(294.8-?)

Conasauga/Maryville Limestone

Conasauga/Maryville Limestone

\section{CORE? LOGS? WHICH LOGS?}

no

no no

no no

no no

no no

no no

no no

no no

no no

no no

no no

no no

no yes

$\mathrm{DHC}$

no

yes DHC

no yes

no no

no yes

no no

no no

no no

no no

no no

no no

yes no

no no

no no 


\section{CURRENT}

NAME

GW-793

GW-794

GW-795

GW-796

GW-797

GW-798

GW-799

GW-800

GW-801

GW-802

GW-803

GW-804

GW-805

GW-806

GW-807

GW-808

GW-809

GW-810

GW-811

GW-812

GW-813

GW-814

GW-815

GW-816

GW-817

GW-818

\section{ROCK FORMATIONS}

Conasauga/Nolichucky Shale

Conasauga/Nolichucky Shale, Conasauga/Maynardville Limestone

Conasauga/Nolichucky Shale, Conasauga/Maynardville Limestone

Knox

Knox

Knox

Knox

Knox

Knox

Conasauga/Nolichucky Shale

Conasauga/Nolichucky Shale

Conasauga/Nolichucky Shale

Conasauga/Maynardville Limestone

Conasauga/Maynardville Limestone

Conasauga/Maynardville Limestone

Conasauga/Maynardville Limestone

Conasauga/Maynardville Limestone

Conasauga/Maynardville Limestone

Conasauga/Maynardville Limestone

Conasauga/Maynardville Limestone

Conasauga/Maynardville Limestone

Conasauga/Maynardville Limestone

Conasauga/Maynardville Limestone

Rome

Conasauga/Nolichucky Shale

none
CORE? LOGS? WHICH LOGS?

no

no no

no no

no no

no no

no no

no no

no no

no no

no no

no no

no no

no no

no no

no no

no no

no no

no no

no no

no no

no no

no no

no no

no no

no no

no no 


\begin{tabular}{ll}
$\begin{aligned} \text { CURRENT } \\
\text { NAME }\end{aligned}$ & \multicolumn{1}{c}{ ROCK FORMATIONS } \\
GW-819 & none \\
GW-820 & none \\
GW-821 & Conasauga/Nolichucky Shale \\
GW-822 & Conasauga/Nolichucky Shale \\
GW-823 & Conasauga/Nolichucky Shale \\
GW-824 & Conasauga/Nolichucky Shale \\
GW-825 & Knox \\
GW-826 & Knox \\
GW-827 & Knox \\
GW-828 & Conasauga/Nolichucky Shale \\
GW-829 & Conasauga/Nolichucky Shale \\
GW-830 & none \\
GW-831 & Knox \\
GW-832 & Conasauga/Maynardville Limestone \\
GW-833 & Conasauga \\
GW-834 & none \\
GW-835 & none \\
GW-836 & none \\
GW-837 & none \\
GW-838 & none \\
GW-839 & none \\
GW-840 & none \\
GW-841 & none \\
GW-842 & Chickamauga \\
GW-843 & Chickamauga \\
GW-844 & none \\
&
\end{tabular}

\section{CORE? LOGS? WHICH LOGS?}

no no

no no

yes no

yes no

yes no

no no

no no

no no

no no

no no

no yes DHC

no no

no no

no no

yes no

no no

no no

no no

no no

no no

no no

no no

no no

no no

no no

no no 


$\begin{array}{cc}\text { CURRENT } & \\ \text { NAME } & \\ \text { GW-845 } & \text { none } \\ \text { GW-854 } & \text { none } \\ \text { GW-855 } & \text { none } \\ \text { GW-857 } & \text { none } \\ \text { GW-858 } & \text { none } \\ \text { GW-859 } & \text { none } \\ \text { GW-860 } & \text { none } \\ \text { GW-861 } & \text { none } \\ \text { GW-862 } & \text { none } \\ \text { GW-863 } & \text { none } \\ \text { GW-864 } & \text { none } \\ \text { GW-865 } & \text { none } \\ \text { GW-866 } & \text { none } \\ \text { GW-867 } & \text { none } \\ \text { GW-868 } & \text { none } \\ \text { GW-869 } & \text { none } \\ \text { GW-871 } & \text { none } \\ \text { GW-872 } & \text { none } \\ \text { GW-873 } & \text { none } \\ \text { GW-874 } & \text { none } \\ \text { GW-875 } & \text { none } \\ \text { GW-876 } & \text { none } \\ \text { GW-877 } & \text { none } \\ \text { GW-878 } & \text { none } \\ \text { GW-879 } & \text { none } \\ \text { GW-880 } & \text { none } \\ \end{array}$

\section{CORE? LOGS? WHICH LOGS?}

no no

no no

no no

no no

no no

no no

no no

no no

no no

no no

no no

no no

no no

no no

no no

no no

no no

no no

no no

no no

no no

no no

no no

no no

no no

no no 


\begin{tabular}{|c|c|c|}
\hline \multicolumn{3}{|c|}{ CURRENT } \\
\hline NAME & & ROCK FORMATIONS \\
\hline GW-881 & none & \\
\hline GW-882 & none & \\
\hline GW-883 & none & \\
\hline GW-884 & none & \\
\hline GW-885 & none & \\
\hline GW-886 & none & \\
\hline GW-887 & none & \\
\hline GW-888 & none & \\
\hline GW-889 & none & \\
\hline GW-890 & none & \\
\hline GW-902 & none & \\
\hline GW-904 & none & \\
\hline GW-905 & none & \\
\hline GW-906 & none & \\
\hline GW-907 & none & \\
\hline GW-908 & none & \\
\hline GW-909 & none & \\
\hline GW-910 & none & \\
\hline GW-911 & none & \\
\hline GW-912 & none & \\
\hline GW-913 & none & \\
\hline GW-914 & none & \\
\hline GW-915 & none & \\
\hline GW-916 & none & \\
\hline GW-917 & none & \\
\hline GW-918 & none & \\
\hline
\end{tabular}

\section{CORE? LOGS? WHICH LOGS?}

no no

no no

no no

no no

no no

no no

no no

no no

no no

no no

no no

no no

no no

no no

no no

no no

no no

no no

no no

no no

no no

no no

no no

no no

no no

no no 


\section{CURRENT}

NAME

GW-919

GW-920

GW-921

GW-922

GW-923

GW-924

GW-925

GW-926

GW-927

GW-928

GW-929

GW-930

GW-931

GW-932

GW-933

GW-934

GW-935

GW-936

GW-937

GW-938

GW-939

GW-940

GW-941

GW-942

GW-943

GW-944

\section{ROCK FORMATIONS}

none

Conasauga/Nolichucky Shale

Conasauga/Nolichucky Shale

Conasauga/Nolichucky Shale

Conasauga/Maryville Limestone

Conasauga/Nolichucky Shale

Conasauga/Maryville Limestone

Conasauga/Nolichucky Shale

Conasauga/Maryville Limestone

Conasauga/Pumpkin Valley Shale(21-44)

Conasauga/Pumpkin Valley Shale(27.5-28.6)

Conasauga/Rutledge Limestone(21-41.5)

Conasauga/Rutledge Limestone(16-19.8)

none

none

none

none

none

none

none

none

none

none

none

none

none

\section{CORE? LOGS? WHICH LOGS?}

no no

no no

no no

no no

no yes DHC

no no

no no

no no

no no

no yes DHC

no yes DHC

no yes DHC

no yes DHC

no no

no no

no no

no no

no no

no no

no no

no no

no no

no no

no no

no no

no no 


\section{CURRENT}

GW-945

none

GW-946

none

GW-947

none

GW-948

none

GW-949 none

GW-950 none

GW-951

GW-952

none

none

GW-953

none

GW-954

none

GW-955

none

GW-956

none

GW-959

Conasauga/Maynardville Limestone(?-9)

none

GW-960

Unknown(1-27)

GW-964

none

GW-965

none

GW-966

none

GW-967

none

$\mathrm{J}-001$

$\mathrm{J}-002$

$\mathrm{J}-003$

J-004

J-005

J-006

$\mathrm{J}-007$

\section{Conasauga/Maynardville Limestone}

Conasauga/Maynardville Limestone

Conasauga/Maynardville Limestone

Conasauga/Maynardville Limestone

Conasauga/Nolichucky Shale, Conasauga/Maynardville Limestone

Conasauga/Nolichucky Shale

Conasauga/Maynardville Limestone

no no

no no

no no

no no

no no

no no

no no

no no

no no

no no

no no

no no

no no

no no

no no

no no

no no

no no

no no

yes no

yes no

yes no

yes no

yes no

yes no

yes no 


\section{CURRENT \\ NAME}

J-008

J-009

$\mathrm{J}-010$

$\mathrm{J}-011$

J-012

J-015

$\mathrm{J}-017$

$\mathrm{J}-018$

$\mathrm{J}-019$

$\mathrm{J}-020$

$\mathrm{J}-021$

$\mathrm{J}-022$

K-020

$\mathrm{K}-021$

$\mathrm{K}-022$

$\mathrm{K}-023$

$\mathrm{K}-024$

K1-015

K2-015

LL/HAZ-01

LL/HAZ-02

LL/HAZ-03

LL/HAZ-04

LL/HAZ-05

LL/HAZ-06

LL/HAZ-07

\section{ROCK FORMATIONS}

Conasauga/Maynardville Limestone

Conasauga/Maynardville Limestone

Conasauga/Nolichucky Shale

Conasauga/Maynardville Limestone

Conasauga/Maynardville Limestone

Conasauga/Maynardville Limestone

Conasauga/Maynardville Limestone

Conasauga/Nolichucky Shale

Conasauga/Maynardville Limestone

Conasauga/Maynardville Limestone

Conasauga/Nolichucky Shale

Conasauga/Nolichucky Shale

Conasauga/Maynardville Limestone

Conasauga/Maynardville Limestone

Conasauga/Maynardville Limestone

Conasauga/Maynardville Limestone

Conasauga/Maynardville Limestone

Conasauga/Maynardville Limestone

Conasauga/Maynardville Limestone

Conasauga/Pumpkin Valley Shale

Conasauga/Pumpkin Valley Shale

Conasauga/Nolichucky Shale, Conasauga/Maryville Limestone

Conasauga/Nolichucky Shale

Conasauga/Nolichucky Shale

Conasauga/Nolichucky Shale

Conasauga/Pumpkin Valley Shale

\section{CORE? LOGS? WHICH LOGS?}

yes

yes no

yes no

yes no

yes no

yes no

yes no

yes no

yes no

yes no

yes no

yes no

yes no

yes no

yes no

yes no

yes no

yes no

yes no

no no

no no

no no

no no

no no

no no

no no 


\section{CURRENT}

NAME

LL/HAZ-08

LL/HAZ-09

LL/HAZ-10

LL/HAZ-11

LL/HAZ-12

LL/HAZ-13

LL/HAZ-14

LL/HAZ-15

LL/HAZ-16

LL/HAZ-17

LL/HAZ-18

LL/HAZ-19

$\mathrm{M}-04$

M-05

M-06

M-07

M-08

M-09

$\mathrm{M}-10$

OR-02

OR-03

OR-04

OR-05

OR-06

OR-07

\section{ROCK FORMATIONS}

Conasauga/Rutledge Limestone

Conasauga/Maryville Limestone

Conasauga/Rogersville Shale

Conasauga/Maryville Limestone

Conasauga/Maryville Limestone

Conasauga/Rutledge Limestone(?-106), Conasauga/Pumpkin

Valley Shale(106-?)

Conasauga/Maryville Limestone(?-204.5), Conasauga/Rogersville Shale(204.5-?)

Conasauga/Nolichucky Shale(?-94), Conasauga/Maryville

Limestone(94-?)

Conasauga

Conasauga

Conasauga

Conasauga

Conasauga/Maryville Limestone

Conasauga/Nolichucky Shale

Conasauga/Nolichucky Shale

Conasauga/Maynardville Limestone

Conasauga/Maryville Limestone

Conasauga/Maryville Limestone

Conasauga/Pumpkin Valley Shale

Rome

Conasauga/Maryville Limestone

Conasauga/Nolichucky Shale

Conasauga/Maryville Limestone

Conasauga/Maryville Limestone

Conasauga/Pumpkin Valley Shale

\section{CORE? LOGS? WHICH LOGS?}

no

no no

no no

no no

no no

yes no

yes no

yes no

no no

no no

no no

no no

no no

no no

no no

no no

no no

no no

no no

yes no

yes no

yes no

yes no

yes no

yes no 


\section{CURRENT \\ NAME}

OR-08

OR-09

OR-21

OR-22

OR-23

P\&A-1

P\&A-2

$P \& A-3$

PP-01

PP-02

PP-03

PP-04

PP-05

PP-06

PP-07

PP-08

PP-09

RB-001

RB-005

RB-008

RB-009A

RB-010A

RB-011

RB-014

RB-015

RB-016

\section{ROCK FORMATIONS}

Conasauga/Pumpkin Valley Shale

Conasauga/Rutledge Limestone, Conasauga/Rogersville Shale

Conasauga/Pumpkin Valley Shale

Conasauga/Pumpkin Valley Shale

Conasauga/Rutledge Limestone, Conasauga/Rogersville Shale

none

none

none

none

none

none

none

none

none

none

none

none

Conasauga/Nolichucky Shale

Conasauga/Nolichucky Shale

Conasauga/Nolichucky Shale

Conasauga/Maynardville Limestone

Conasauga/Maynardville Limestone

Conasauga/Nolichucky Shale

Conasauga/Maynardville Limestone

Conasauga/Nolichucky Shale

Conasauga/Maynardville Limestone

\section{CORE? LOGS? WHICH LOGS?}

yes

yes no

yes no

yes no

yes no

no no

no no

no no

no no

no no

no no

no no

no no

no no

no no

no no

no no

yes no

yes no

yes no

yes no

yes no

yes no

yes no

yes no

yes no 


\begin{tabular}{ll}
$\begin{aligned} \text { CURRENT } \\
\text { NAME }\end{aligned}$ & \multicolumn{1}{c}{ ROCK FORMATIONS } \\
RB-017 & Conasauga/Maynardville Limestone \\
RB-018 & Conasauga/Maynardville Limestone \\
RB-019 & Conasauga/Nolichucky Shale \\
RB-020 & Conasauga/Nolichucky Shale \\
RB-031 & Conasauga/Nolichucky Shale \\
RB-032 & Conasauga/Nolichucky Shale \\
RB-033 & Conasauga/Maynardville Limestone \\
RB-034 & Conasauga/Nolichucky Shale \\
RB-035 & Conasauga/Maynardville Limestone \\
RB-036 & Conasauga/Maynardville Limestone \\
RB-037 & Conasauga/Maynardville Limestone \\
RB-038 & Conasauga/Maynardville Limestone \\
S3-1 & none \\
S3-3 & none \\
S3-5 & none \\
S3-6 & none \\
S3-7 & none \\
S3-8 & none \\
SAN-1 & Knox \\
SAN-10 & Knox \\
SAN-2 & Knox \\
SAN-3 & Knox \\
SAN-4 & Knox \\
SAN-5 & Knox \\
SAN-6 & Knox \\
SAN-7 & Knox \\
&
\end{tabular}

\section{CORE? LOGS? WHICH LOGS?}

yes

yes no

yes no

yes no

yes no

yes no

yes no

yes no

yes no

yes no

yes no

no no

no no

no no

no no

no no

no no

no no

no no

no no

no no

no no

no no

no no

no no 


\section{CURRENT}

NAME

SAN-8

SAN-9

UNIDENT A

UNIDENT B

$\mathrm{V}-1$

$\mathrm{V}-2$

$\mathrm{V}-3$

$\mathrm{V}-4$

V-5

V-6

V-7
ROCK FORMATIONS

Knox

Knox

Knox

Knox

none

none

none

none

none

none

none
CORE? LOGS? WHICH LOGS?

no no

no no

no no

no no

no no

no no

no no

no no

no no

no no

no no 
APPENDIX H

Multiport Instrumented Corehole Data Summary 
This Page Intentionally Left Blank 


\begin{tabular}{|c|c|c|c|c|c|c|}
\hline \multirow{3}{*}{$\begin{array}{c}\text { CURRENT } \\
\text { NAME }\end{array}$} & \multirow{3}{*}{$\begin{array}{l}\text { OTHER } \\
\text { NAME }\end{array}$} & & & \multirow{2}{*}{$\begin{array}{l}\text { MEAS } \\
\text { PORT }\end{array}$} & \multirow{2}{*}{$\begin{array}{l}\text { PUMP } \\
\text { PORT }\end{array}$} & \multirow[b]{3}{*}{ REFERENCE } \\
\hline & & & & & & \\
\hline & & PACKER & I INTERVAL & DEPTH & DEPTH & \\
\hline GW-131-01 & GW-131 & 1064.00 & to 1089.00 & 1075.00 & 1065.00 & $\begin{array}{l}\text { King, H. L., and C. S. } \\
\text { Haase(1987), Martin Marietta } \\
\text { Energy Systems(1995) }\end{array}$ \\
\hline GW-131-02 & GW-131 & 1049.00 & to 1061.00 & 1060.00 & & $\begin{array}{l}\text { King, H. L., and C. S. } \\
\text { Haase(1987), Martin Marietta } \\
\text { Energy Systems(1995) }\end{array}$ \\
\hline GW-131-03 & GW-131 & 1014.00 & to 1046.00 & 1025.00 & & $\begin{array}{l}\text { King, H. L., and C. S. } \\
\text { Haase(1987), Martin Marietta } \\
\text { Energy Systems(1995) }\end{array}$ \\
\hline GW-131-04 & GW-131 & 992.00 & to 1011.00 & 1003.00 & 1010.00 & $\begin{array}{l}\text { King, H. L., and C. S. } \\
\text { Haase(1987), Martin Marietta } \\
\text { Energy Systems(1995) }\end{array}$ \\
\hline GW-131-05 & GW-131 & 977.00 & to 989.00 & 988.00 & & $\begin{array}{l}\text { King, H. L., and C. S. } \\
\text { Haase(1987), Martin Marietta } \\
\text { Energy Systems(1995) }\end{array}$ \\
\hline GW-131-06 & GW-131 & 948.00 & to 974.00 & 963.00 & & $\begin{array}{l}\text { King, H. L., and C. S. } \\
\text { Haase(1987), Martin Marietta } \\
\text { Energy Systems(1995) }\end{array}$ \\
\hline GW-131-07 & GW-131 & 933.00 & to 945.00 & 944.00 & & $\begin{array}{l}\text { King, H. L., and C. S. } \\
\text { Haase(1987), Martin Marietta } \\
\text { Energy Systems(1995) }\end{array}$ \\
\hline GW-131-08 & GW-131 & 903.00 & to 930.00 & 914.00 & 929.00 & $\begin{array}{l}\text { King, H. L., and C. S. } \\
\text { Haase(1987), Martin Marietta } \\
\text { Energy Systems(1995) }\end{array}$ \\
\hline GW-131-09 & GW-131 & 888.00 & to 900.00 & 899.00 & & $\begin{array}{l}\text { King, H. L., and C. S. } \\
\text { Haase(1987), Martin Marietta } \\
\text { Energy Systems(1995) }\end{array}$ \\
\hline GW-131-10 & GW-131 & 839.00 & to 885.00 & 860.00 & & $\begin{array}{l}\text { King, H. L., and C. S. } \\
\text { Haase(1987), Martin Marietta } \\
\text { Energy Systems(1995) }\end{array}$ \\
\hline GW-131-11 & GW-131 & 794.00 & to 836.00 & 815.00 & & $\begin{array}{l}\text { King, H. L., and C. S. } \\
\text { Haase(1987), Martin Marietta } \\
\text { Energy Systems(1995) }\end{array}$ \\
\hline
\end{tabular}

\section{COMMENTS}

Westbay well GW-131, Zone 1 measurement port @ 1075 ft, pumping port @ 1065 ft bgs.

Westbay well GW-131, Zone 2 measurement port @ 1060 ft bgs.

Westbay well GW-131, Zone 3 measurement port @ 1025 ft bgs.

Westbay well GW-131, Zone 4 measurement port @ 1003 ft, pumping port @ 1010 ft bgs.

Westbay well GW-131, Zone 5 measurement port @ 988 ft bgs.

Westbay well GW-131, Zone 6 measurement port @ 963 ft bgs.

Westbay well GW-131, Zone 7 measurement port @ 944 ft bgs.

Westbay well GW-131, Zone 8 measurement port @ 914 ft, pumping port @ 929 ft bgs.

Westbay well GW-131, Zone 9 measurement port @ 899 ft bgs.

Westbay well GW-131, Zone 10 measurement port @ 860 ft bgs.

Westbay well GW-131, Zone 11 measurement port @ 815 ft bgs. 


\begin{tabular}{|c|c|c|c|c|c|c|}
\hline $\begin{array}{c}\text { CURRENT } \\
\text { NAME }\end{array}$ & $\begin{array}{l}\text { OTHER } \\
\text { NAME }\end{array}$ & PACKEF & R INTERVAL & $\begin{array}{c}\text { MEAS } \\
\text { PORT } \\
\text { DEPTH }\end{array}$ & $\begin{array}{c}\text { PUMP } \\
\text { PORT } \\
\text { DEPTH }\end{array}$ & REFERENCE \\
\hline GW-131-12 & GW-131 & 769.00 & to 791.00 & 780.00 & 790.00 & $\begin{array}{l}\text { King, H. L., and C. S. } \\
\text { Haase(1987), Martin Marietta } \\
\text { Energy Systems(1995) }\end{array}$ \\
\hline GW-131-13 & GW-131 & 754.00 & to 766.00 & 765.00 & & $\begin{array}{l}\text { King, H. L., and C. S. } \\
\text { Haase(1987), Martin Marietta } \\
\text { Energy Systems(1995) }\end{array}$ \\
\hline GW-131-14 & GW-131 & 709.00 & to 751.00 & 730.00 & & $\begin{array}{l}\text { King, H. L., and C. S. } \\
\text { Haase(1987), Martin Marietta } \\
\text { Energy Systems(1995) }\end{array}$ \\
\hline GW-131-15 & GW-131 & 667.00 & to 691.00 & 678.00 & 690.00 & $\begin{array}{l}\text { King, H. L., and C. S. } \\
\text { Haase(1987), Martin Marietta } \\
\text { Energy Systems(1995) }\end{array}$ \\
\hline GW-131-16 & GW-131 & 652.00 & to 664.00 & 663.00 & & $\begin{array}{l}\text { King, H. L., and C. S. } \\
\text { Haase(1987), Martin Marietta } \\
\text { Energy Systems(1995) }\end{array}$ \\
\hline GW-131-17 & GW-131 & 607.00 & to 649.00 & 628.00 & & $\begin{array}{l}\text { King, H. L., and C. S. } \\
\text { Haase(1987), Martin Marietta } \\
\text { Energy Systems(1995) }\end{array}$ \\
\hline GW-131-18 & GW-131 & 562.00 & to 604.00 & 583.00 & & $\begin{array}{l}\text { King, H. L., and C. S. } \\
\text { Haase(1987), Martin Marietta } \\
\text { Energy Systems(1995) }\end{array}$ \\
\hline GW-131-19 & GW-131 & 537.00 & to 559.00 & 548.00 & & $\begin{array}{l}\text { King, H. L., and C. S. } \\
\text { Haase(1987), Martin Marietta } \\
\text { Energy Systems(1995) }\end{array}$ \\
\hline GW-131-20 & GW-131 & 522.00 & to 534.00 & 533.00 & & $\begin{array}{l}\text { King, H. L., and C. S. } \\
\text { Haase(1987), Martin Marietta } \\
\text { Energy Systems(1995) }\end{array}$ \\
\hline GW-131-21 & GW-131 & 497.00 & to 519.00 & 498.00 & 508.00 & $\begin{array}{l}\text { King, H. L., and C. S. } \\
\text { Haase(1987), Martin Marietta } \\
\text { Energy Systems(1995) }\end{array}$ \\
\hline GW-131-22 & GW-131 & 482.00 & to 494.00 & 493.00 & & $\begin{array}{l}\text { King, H. L., and C. S. } \\
\text { Haase(1987), Martin Marietta } \\
\text { Energy Systems(1995) }\end{array}$ \\
\hline
\end{tabular}

\section{COMMENTS}

Westbay well GW-131, Zone 12 measurement port @ 780 ft, pumping port @ 790 ft bgs.

Westbay well GW-131, Zone 13 measurement port @ $765 \mathrm{ft}$ bgs.

Westbay well GW-131, Zone 14 measurement port @ 730 ft bgs.

Westbay well GW-131, Zone 15 measurement port @ $678 \mathrm{ft}$, pumping port @ $690 \mathrm{ft}$ bgs.

Westbay well GW-131, Zone 16 measurement port @ $663 \mathrm{ft}$ bgs.

Westbay well GW-131, Zone 17 measurement port @ $628 \mathrm{ft}$ bgs.

Westbay well GW-131, Zone 18 measurement port @ $583 \mathrm{ft}$ bgs.

Westbay well GW-131, Zone 19 measurement port @ $548 \mathrm{ft}$ bgs.

Westbay well GW-131, Zone 20 measurement port @ $533 \mathrm{ft}$ bgs.

Westbay well GW-131, Zone 21 measurement port @ 498 ft, pumping port @ 508 ft bgs.

Westbay well GW-131, Zone 22 measurement port @ $493 \mathrm{ft}$ bgs. 


\begin{tabular}{|c|c|c|c|c|c|c|}
\hline $\begin{array}{c}\text { CURRENT } \\
\text { NAME }\end{array}$ & $\begin{array}{l}\text { OTHER } \\
\text { NAME }\end{array}$ & PACKE & R INTERVAL & $\begin{array}{c}\text { MEAS } \\
\text { PORT } \\
\text { DEPTH }\end{array}$ & $\begin{array}{c}\text { PUMP } \\
\text { PORT } \\
\text { DEPTH }\end{array}$ & REFERENCE \\
\hline GW-131-23 & GW-131 & 462.00 & to 479.00 & 478.00 & & $\begin{array}{l}\text { King, H. L., and C. S. } \\
\text { Haase(1987), Martin Marietta } \\
\text { Energy Systems(1995) }\end{array}$ \\
\hline GW-131-24 & GW-131 & 437.00 & to 459.00 & 458.00 & 448.00 & $\begin{array}{l}\text { King, H. L., and C. S. } \\
\text { Haase(1987), Martin Marietta } \\
\text { Energy Systems(1995) }\end{array}$ \\
\hline GW-131-25 & GW-131 & 422.00 & to 434.00 & 433.00 & & $\begin{array}{l}\text { King, H. L., and C. S. } \\
\text { Haase(1987), Martin Marietta } \\
\text { Energy Systems(1995) }\end{array}$ \\
\hline GW-131-26 & GW-131 & 405.00 & to 419.00 & 418.00 & & $\begin{array}{l}\text { King, H. L., and C. S. } \\
\text { Haase(1987), Martin Marietta } \\
\text { Energy Systems(1995) }\end{array}$ \\
\hline GW-131-27 & GW-131 & 390.00 & to 402.00 & 401.00 & & $\begin{array}{l}\text { King, H. L., and C. S. } \\
\text { Haase(1987), Martin Marietta } \\
\text { Energy Systems(1995) }\end{array}$ \\
\hline GW-131-28 & GW-131 & 365.00 & to 387.00 & 376.00 & 386.00 & $\begin{array}{l}\text { King, H. L., and C. S. } \\
\text { Haase(1987), Martin Marietta } \\
\text { Energy Systems(1995) }\end{array}$ \\
\hline GW-131-29 & GW-131 & 350.00 & to 362.00 & 361.00 & & $\begin{array}{l}\text { King, H. L., and C. S. } \\
\text { Haase(1987), Martin Marietta } \\
\text { Energy Systems(1995) }\end{array}$ \\
\hline GW-131-30 & GW-131 & 283.00 & to 347.00 & 326.00 & & $\begin{array}{l}\text { King, H. L., and C. S. } \\
\text { Haase(1987), Martin Marietta } \\
\text { Energy Systems(1995) }\end{array}$ \\
\hline GW-131-31 & GW-131 & 268.00 & to 280.00 & 279.00 & & $\begin{array}{l}\text { King, H. L., and C. S. } \\
\text { Haase(1987), Martin Marietta } \\
\text { Energy Systems(1995) }\end{array}$ \\
\hline GW-131-32 & GW-131 & 238.00 & to 265.00 & 259.00 & 249.00 & $\begin{array}{l}\text { King, H. L., and C. S. } \\
\text { Haase(1987), Martin Marietta } \\
\text { Energy Systems(1995) }\end{array}$ \\
\hline GW-131-33 & GW-131 & 223.00 & to 235.00 & 234.00 & & $\begin{array}{l}\text { King, H. L., and C. S. } \\
\text { Haase(1987), Martin Marietta } \\
\text { Energy Systems(1995) }\end{array}$ \\
\hline
\end{tabular}

\section{COMMENTS}

Westbay well GW-131, Zone 23 measurement port @ $478 \mathrm{ft}$ bgs.

Westbay well GW-131, Zone 24 measurement port @ $458 \mathrm{ft}$, pumping port @ $448 \mathrm{ft}$ bgs.

Westbay well GW-131, Zone 25 measurement port @ 433 ft bgs.

Westbay well GW-131, Zone 26 measurement port @ $418 \mathrm{ft}$ bgs.

Westbay well GW-131, Zone 27 measurement port @ $401 \mathrm{ft}$ bgs.

Westbay well GW-131, Zone 28 measurement port @ $376 \mathrm{ft}$, pumping port @ $386 \mathrm{ft}$ bgs.

Westbay well GW-131, Zone 29 measurement port @ $361 \mathrm{ft}$ bgs.

Westbay well GW-131, Zone 30 measurement port @ 326 ft bgs.

Westbay well GW-131, Zone 31 measurement port @ $279 \mathrm{ft}$ bgs.

Westbay well GW-131, Zone 32 measurement port @ $259 \mathrm{ft}$ bgs, pumping port @ $249 \mathrm{ft}$ bgs.

Westbay well GW-131, Zone 33 measurement port @ $234 \mathrm{ft}$ bgs. 


\begin{tabular}{|c|c|c|c|c|c|c|}
\hline $\begin{array}{c}\text { CURRENT } \\
\text { NAME }\end{array}$ & $\begin{array}{l}\text { OTHER } \\
\text { NAME }\end{array}$ & PACKE & R INTERVAL & $\begin{array}{c}\text { MEAS } \\
\text { PORT } \\
\text { DEPTH }\end{array}$ & $\begin{array}{c}\text { PUMP } \\
\text { PORT } \\
\text { DEPTH }\end{array}$ & REFERENCE \\
\hline GW-131-34 & GW-131 & 193.00 & to 220.00 & 209.00 & & $\begin{array}{l}\text { King, H. L., and C. S. } \\
\text { Haase(1987), Martin Marietta } \\
\text { Energy Systems(1995) }\end{array}$ \\
\hline GW-131-35 & GW-131 & 178.00 & to 190.00 & 189.00 & & $\begin{array}{l}\text { King, H. L., and C. S. } \\
\text { Haase(1987), Martin Marietta } \\
\text { Energy Systems(1995) }\end{array}$ \\
\hline GW-131-36 & GW-131 & 153.00 & to 175.00 & 164.00 & 174.00 & $\begin{array}{l}\text { King, H. L., and C. S. } \\
\text { Haase(1987), Martin Marietta } \\
\text { Energy Systems(1995) }\end{array}$ \\
\hline GW-131-37 & GW-131 & 138.00 & to 150.00 & 149.00 & & $\begin{array}{l}\text { King, H. L., and C. S. } \\
\text { Haase(1987), Martin Marietta } \\
\text { Energy Systems(1995) }\end{array}$ \\
\hline GW-131-38 & GW-131 & 43.00 & to 135.00 & 134.00 & & $\begin{array}{l}\text { King, H. L., and C. S. } \\
\text { Haase(1987), Martin Marietta } \\
\text { Energy Systems(1995) }\end{array}$ \\
\hline GW-132-01 & GW-132 & 732.00 & to 759.50 & 738.00 & 743.00 & $\begin{array}{l}\text { King, H. L., and C. S. } \\
\text { Haase(1987), Martin Marietta } \\
\text { Energy Systems(1995) }\end{array}$ \\
\hline GW-132-02 & GW-132 & 717.00 & to 729.00 & 728.00 & & $\begin{array}{l}\text { King, H. L., and C. S. } \\
\text { Haase(1987), Martin Marietta } \\
\text { Energy Systems(1995) }\end{array}$ \\
\hline GW-132-03 & GW-132 & 685.00 & to 714.00 & 696.00 & & $\begin{array}{l}\text { King, H. L., and C. S. } \\
\text { Haase(1987), Martin Marietta } \\
\text { Energy Systems(1995) }\end{array}$ \\
\hline GW-132-04 & GW-132 & 670.00 & to 682.00 & 681.00 & & $\begin{array}{l}\text { King, H. L., and C. S. } \\
\text { Haase(1987), Martin Marietta } \\
\text { Energy Systems(1995) }\end{array}$ \\
\hline GW-132-05 & GW-132 & 640.00 & to 667.00 & 646.00 & 656.00 & $\begin{array}{l}\text { King, H. L., and C. S. } \\
\text { Haase(1987), Martin Marietta } \\
\text { Energy Systems(1995) }\end{array}$ \\
\hline GW-132-06 & GW-132 & 625.00 & to 637.00 & 636.00 & & $\begin{array}{l}\text { King, H. L., and C. S. } \\
\text { Haase(1987), Martin Marietta } \\
\text { Energy Systems(1995) }\end{array}$ \\
\hline
\end{tabular}

\section{COMMENTS}

Westbay well GW-131, Zone 34 measurement port @ $209 \mathrm{ft}$ bgs.

Westbay well GW-131, Zone 35 measurement port @ $189 \mathrm{ft}$ bgs.

Westbay well GW-131, Zone 36 measurement port @ $164 \mathrm{ft}$ bgs, pumping port @ $174 \mathrm{ft}$ bgs.

Westbay well GW-131, Zone 37 measurement port @ 149 ft bgs.

Westbay well GW-131, Zone 38 measurement port @ $134 \mathrm{ft}$ bgs.

Westbay well GW-132, Zone 1 measurement port @ 738 ft, pumping port @ $743 \mathrm{ft}$ bgs.

Westbay well GW-132, Zone 2 measurement port @ $728 \mathrm{ft}$ bgs.

Westbay well GW-132, Zone 3 measurement port@ $696 \mathrm{ft}$ bgs.

Westbay well GW-132, Zone 4 measurement port @ $681 \mathrm{ft}$ bgs.

Westbay well GW-132, Zone 5 measurement port @ $646 \mathrm{ft}$, pumping port @ $656 \mathrm{ft}$ bgs.

Westbay well GW-132, Zone 6 measurement port @ $636 \mathrm{ft}$ bgs. 


\begin{tabular}{|c|c|c|c|c|c|c|}
\hline $\begin{array}{c}\text { CURRENT } \\
\text { NAME }\end{array}$ & $\begin{array}{l}\text { OTHER } \\
\text { NAME }\end{array}$ & PACKE & R INTERVAL & $\begin{array}{c}\text { MEAS } \\
\text { PORT } \\
\text { DEPTH }\end{array}$ & $\begin{array}{c}\text { PUMP } \\
\text { PORT } \\
\text { DEPTH }\end{array}$ & REFERENCE \\
\hline GW-132-07 & GW-132 & 580.00 & to 622.00 & 601.00 & & $\begin{array}{l}\text { King, H. L., and C. S. } \\
\text { Haase(1987), Martin Marietta } \\
\text { Energy Systems(1995) }\end{array}$ \\
\hline GW-132-08 & GW-132 & 565.00 & to 577.00 & 576.00 & & $\begin{array}{l}\text { King, H. L., and C. S. } \\
\text { Haase(1987), Martin Marietta } \\
\text { Energy Systems(1995) }\end{array}$ \\
\hline GW-132-09 & GW-132 & 535.00 & to 562.00 & 541.00 & 551.00 & $\begin{array}{l}\text { King, H. L., and C. S. } \\
\text { Haase(1987), Martin Marietta } \\
\text { Energy Systems(1995) }\end{array}$ \\
\hline GW-132-10 & GW-132 & 520.00 & to 532.00 & 531.00 & & $\begin{array}{l}\text { King, H. L., and C. S. } \\
\text { Haase(1987), Martin Marietta } \\
\text { Energy Systems(1995) }\end{array}$ \\
\hline GW-132-11 & GW-132 & 490.00 & to 517.00 & 501.00 & & $\begin{array}{l}\text { King, H. L., and C. S. } \\
\text { Haase(1987), Martin Marietta } \\
\text { Energy Systems(1995) }\end{array}$ \\
\hline GW-132-12 & GW-132 & 475.00 & to 487.00 & 486.00 & & $\begin{array}{l}\text { King, H. L., and C. S. } \\
\text { Haase(1987), Martin Marietta } \\
\text { Energy Systems(1995) }\end{array}$ \\
\hline GW-132-13 & GW-132 & 450.00 & to 472.00 & 461.00 & 471.00 & $\begin{array}{l}\text { King, H. L., and C. S. } \\
\text { Haase(1987), Martin Marietta } \\
\text { Energy Systems(1995) }\end{array}$ \\
\hline GW-132-14 & GW-132 & 435.00 & to 447.00 & 446.00 & & $\begin{array}{l}\text { King, H. L., and C. S. } \\
\text { Haase(1987), Martin Marietta } \\
\text { Energy Systems(1995) }\end{array}$ \\
\hline GW-132-15 & GW-132 & 388.00 & to 432.00 & 409.00 & & $\begin{array}{l}\text { King, H. L., and C. S. } \\
\text { Haase(1987), Martin Marietta } \\
\text { Energy Systems(1995) }\end{array}$ \\
\hline GW-132-16 & GW-132 & 373.00 & to 385.00 & 384.00 & & $\begin{array}{l}\text { King, H. L., and C. S. } \\
\text { Haase(1987), Martin Marietta } \\
\text { Energy Systems(1995) }\end{array}$ \\
\hline GW-132-17 & GW-132 & 348.00 & to 370.00 & 359.00 & 369.00 & $\begin{array}{l}\text { King, H. L., and C. S. } \\
\text { Haase(1987), Martin Marietta } \\
\text { Energy Systems(1995) }\end{array}$ \\
\hline
\end{tabular}

\section{COMMENTS}

Westbay well GW-132, Zone 7 measurement port @ $601 \mathrm{ft}$ bgs.

Westbay well GW-132, Zone 8 measurement port @ $576 \mathrm{ft}$ bgs.

Westbay well GW-132, Zone 9 measurement port @ $541 \mathrm{ft}$, pumping port @ $551 \mathrm{ft}$ bgs.

Westbay well GW-132, Zone 10 measurement port @ 531 ft bgs.

Westbay well GW-132, Zone 11 measurement port @ $501 \mathrm{ft}$ bgs.

Westbay well GW-132, Zone 12 measurement port @ $486 \mathrm{ft}$ bgs.

Westbay well GW-132, Zone 13 measurement port @ $461 \mathrm{ft}$, pumping port @ $471 \mathrm{ft}$ bgs.

Westbay well GW-132, Zone 14 measurement port @ 446 ft bgs.

Westbay well GW-132, Zone 15 measurement port @ $409 \mathrm{ft}$ bgs.

Westbay well GW-132, Zone 16 measurement port @ $384 \mathrm{ft}$ bgs.

Westbay well GW-132, Zone 17 measurement port @ $359 \mathrm{ft}$ bgs, pumping port @ $369 \mathrm{ft}$ bgs. 


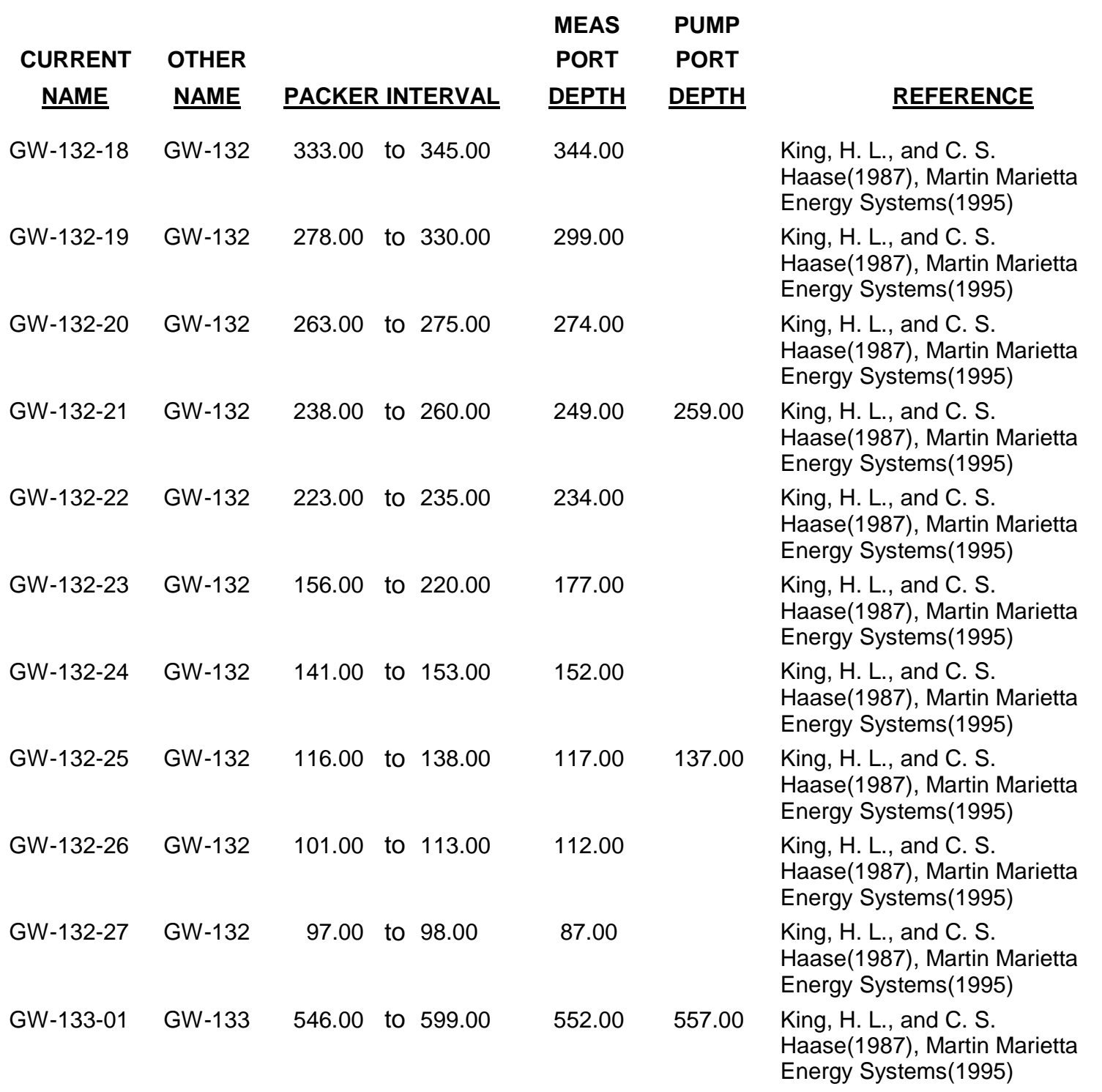

\section{COMMENTS}

Westbay well GW-132, Zone 18 measurement port @ $344 \mathrm{ft}$ bgs.

Westbay well GW-132, Zone 19 measurement port @ $299 \mathrm{ft}$ bgs.

Westbay well GW-132, Zone 20 measurement port @ $274 \mathrm{ft}$ bgs.

Westbay well GW-132, Zone 21 measurement port @ $249 \mathrm{ft}$ bgs, pumping port @ $259 \mathrm{ft}$ bgs.

Westbay well GW-132, Zone 22 measurement port @ $234 \mathrm{ft}$ bgs.

Westbay well GW-132, Zone 23 measurement port @ $177 \mathrm{ft}$ bgs.

Westbay well GW-132, Zone 24 measurement port @ $152 \mathrm{ft}$ bgs.

Westbay well GW-132, Zone 25 measurement port @ $117 \mathrm{ft}$ bgs, pumping port @ $137 \mathrm{ft}$ bgs.

Westbay well GW-132, Zone 26 measurement port @ $112 \mathrm{ft}$ bgs.

Westbay well GW-132, Zone 27 measurement port @ $87 \mathrm{ft}$ bgs.

Westbay well GW-133, Zone 1 measurement port @ $552 \mathrm{ft}$ bgs, pumping port @ $557 \mathrm{ft}$ bgs. 


\begin{tabular}{|c|c|c|c|c|c|c|}
\hline $\begin{array}{c}\text { CURRENT } \\
\text { NAME }\end{array}$ & $\begin{array}{l}\text { OTHER } \\
\text { NAME }\end{array}$ & PACKE & R INTERVAL & $\begin{array}{c}\text { MEAS } \\
\text { PORT } \\
\text { DEPTH }\end{array}$ & $\begin{array}{c}\text { PUMP } \\
\text { PORT } \\
\text { DEPTH }\end{array}$ & REFERENCE \\
\hline GW-133-02 & GW-133 & 531.00 & to 543.00 & 542.00 & & $\begin{array}{l}\text { King, H. L., and C. S. } \\
\text { Haase(1987), Martin Marietta } \\
\text { Energy Systems(1995) }\end{array}$ \\
\hline GW-133-03 & GW-133 & 516.00 & to 528.00 & 527.00 & & $\begin{array}{l}\text { King, H. L., and C. S. } \\
\text { Haase(1987), Martin Marietta } \\
\text { Energy Systems(1995) }\end{array}$ \\
\hline GW-133-04 & GW-133 & 501.00 & to 513.00 & 512.00 & & $\begin{array}{l}\text { King, H. L., and C. S. } \\
\text { Haase(1987), Martin Marietta } \\
\text { Energy Systems(1995) }\end{array}$ \\
\hline GW-133-05 & GW-133 & 476.00 & to 498.00 & 487.00 & 497.00 & $\begin{array}{l}\text { King, H. L., and C. S. } \\
\text { Haase(1987), Martin Marietta } \\
\text { Energy Systems(1995) }\end{array}$ \\
\hline GW-133-06 & GW-133 & 461.00 & to 473.00 & 472.00 & & $\begin{array}{l}\text { King, H. L., and C. S. } \\
\text { Haase(1987), Martin Marietta } \\
\text { Energy Systems(1995) }\end{array}$ \\
\hline GW-133-07 & GW-133 & 451.00 & to 458.00 & 457.00 & & $\begin{array}{l}\text { King, H. L., and C. S. } \\
\text { Haase(1987), Martin Marietta } \\
\text { Energy Systems(1995) }\end{array}$ \\
\hline GW-133-08 & GW-133 & 426.00 & to 448.00 & 427.00 & 447.00 & $\begin{array}{l}\text { King, H. L., and C. S. } \\
\text { Haase(1987), Martin Marietta } \\
\text { Energy Systems(1995) }\end{array}$ \\
\hline GW-133-09 & GW-133 & 414.00 & to 423.00 & 422.00 & & $\begin{array}{l}\text { King, H. L., and C. S. } \\
\text { Haase(1987), Martin Marietta } \\
\text { Energy Systems(1995) }\end{array}$ \\
\hline GW-133-10 & GW-133 & 384.00 & to 411.00 & 405.00 & 410.00 & $\begin{array}{l}\text { King, H. L., and C. S. } \\
\text { Haase(1987), Martin Marietta } \\
\text { Energy Systems(1995) }\end{array}$ \\
\hline GW-133-11 & GW-133 & 369.00 & to 381.00 & 380.00 & & $\begin{array}{l}\text { King, H. L., and C. S. } \\
\text { Haase(1987), Martin Marietta } \\
\text { Energy Systems(1995) }\end{array}$ \\
\hline GW-133-12 & GW-133 & 349.00 & to 366.00 & 365.00 & & $\begin{array}{l}\text { King, H. L., and C. S. } \\
\text { Haase(1987), Martin Marietta } \\
\text { Energy Systems(1995) }\end{array}$ \\
\hline
\end{tabular}

\section{COMMENTS}

Westbay well GW-133, Zone 2 measurement port @ 542 ft bgs.

Westbay well GW-133, Zone 3 measurement port @ 527 ft bgs.

Westbay well GW-133, Zone 4 measurement port @ 512 ft bgs.

Westbay well GW-133, Zone 5 measurement port @ 487 ft bgs, pumping port @ $497 \mathrm{ft}$ bgs.

Westbay well GW-133, Zone 6 measurement port @ 472 ft bgs.

Westbay well GW-133, Zone 7 measurement port @ 457 ft bgs.

Westbay well GW-133, Zone 8 measurement port @ $427 \mathrm{ft}$ bgs, pumping port @ $447 \mathrm{ft}$ bgs.

Westbay well GW-133, Zone 9 measurement port @ 422 ft bgs.

Westbay well GW-133, Zone 10 measurement port @ $405 \mathrm{ft}$ bgs, duplicate measurement port @ $395 \mathrm{ft}$ bgs, pumping port @ $410 \mathrm{ft}$ bgs.

Westbay well GW-133, Zone 11 measurement port @ 380 ft bgs.

Westbay well GW-133, Zone 12 measurement port @ 365 ft bgs. 


\begin{tabular}{|c|c|c|c|c|c|c|}
\hline $\begin{array}{c}\text { CURRENT } \\
\text { NAME }\end{array}$ & $\begin{array}{l}\text { OTHER } \\
\text { NAME }\end{array}$ & PACKE & R INTERVAL & $\begin{array}{c}\text { MEAS } \\
\text { PORT } \\
\text { DEPTH }\end{array}$ & $\begin{array}{c}\text { PUMP } \\
\text { PORT } \\
\text { DEPTH }\end{array}$ & REFERENCE \\
\hline GW-133-13 & GW-133 & 334.00 & to 346.00 & 345.00 & & $\begin{array}{l}\text { King, H. L., and C. S. } \\
\text { Haase(1987), Martin Marietta } \\
\text { Energy Systems(1995) }\end{array}$ \\
\hline GW-133-14 & GW-133 & 307.00 & to 331.00 & 313.00 & 330.00 & $\begin{array}{l}\text { King, H. L., and C. S. } \\
\text { Haase(1987), Martin Marietta } \\
\text { Energy Systems(1995) }\end{array}$ \\
\hline GW-133-15 & GW-133 & 292.00 & to 304.00 & 303.00 & & $\begin{array}{l}\text { King, H. L., and C. S. } \\
\text { Haase(1987), Martin Marietta } \\
\text { Energy Systems(1995) }\end{array}$ \\
\hline GW-133-16 & GW-133 & 272.00 & to 289.00 & 288.00 & & $\begin{array}{l}\text { King, H. L., and C. S. } \\
\text { Haase(1987), Martin Marietta } \\
\text { Energy Systems(1995) }\end{array}$ \\
\hline GW-133-17 & GW-133 & 247.00 & to 269.00 & 253.00 & 268.00 & $\begin{array}{l}\text { King, H. L., and C. S. } \\
\text { Haase(1987), Martin Marietta } \\
\text { Energy Systems(1995) }\end{array}$ \\
\hline GW-133-18 & GW-133 & 232.00 & to 244.00 & 243.00 & & $\begin{array}{l}\text { King, H. L., and C. S. } \\
\text { Haase(1987), Martin Marietta } \\
\text { Energy Systems(1995) }\end{array}$ \\
\hline GW-133-19 & GW-133 & 187.00 & to 229.00 & 218.00 & & $\begin{array}{l}\text { King, H. L., and C. S. } \\
\text { Haase(1987), Martin Marietta } \\
\text { Energy Systems(1995) }\end{array}$ \\
\hline GW-133-20 & GW-133 & 172.00 & to 184.00 & 183.00 & & $\begin{array}{l}\text { King, H. L., and C. S. } \\
\text { Haase(1987), Martin Marietta } \\
\text { Energy Systems(1995) }\end{array}$ \\
\hline GW-133-21 & GW-133 & 147.00 & to 169.00 & 158.00 & 168.00 & $\begin{array}{l}\text { King, H. L., and C. S. } \\
\text { Haase(1987), Martin Marietta } \\
\text { Energy Systems(1995) }\end{array}$ \\
\hline GW-133-22 & GW-133 & 132.00 & to 144.00 & 143.00 & & $\begin{array}{l}\text { King, H. L., and C. S. } \\
\text { Haase(1987), Martin Marietta } \\
\text { Energy Systems(1995) }\end{array}$ \\
\hline GW-133-23 & GW-133 & 112.00 & to 129.00 & 128.00 & & $\begin{array}{l}\text { King, H. L., and C. S. } \\
\text { Haase(1987), Martin Marietta } \\
\text { Energy Systems(1995) }\end{array}$ \\
\hline
\end{tabular}

\section{COMMENTS}

Westbay well GW-133, Zone 13 measurement port @ $345 \mathrm{ft}$ bgs.

Westbay well GW-133, Zone 14 measurement port @ $313 \mathrm{ft}$ bgs, pumping port @ $330 \mathrm{ft}$ bgs.

Westbay well GW-133, Zone 15 measurement port @ 303 ft bgs.

Westbay well GW-133, Zone 16 measurement port @ $288 \mathrm{ft}$ bgs.

Westbay well GW-133, Zone 17 measurement port @ $253 \mathrm{ft}$ bgs, pumping port @ $268 \mathrm{ft}$ bgs.

Westbay well GW-133, Zone 18 measurement port @ $243 \mathrm{ft}$ bgs.

Westbay well GW-133, Zone 19 measurement port @ $218 \mathrm{ft}$ bgs.

Westbay well GW-133, Zone 20 measurement port @ $183 \mathrm{ft}$ bgs.

Westbay well GW-133, Zone 21 measurement port @ $158 \mathrm{ft}$ bgs, pumping port @ $168 \mathrm{ft}$ bgs.

Westbay well GW-133, Zone 22 measurement port @ $143 \mathrm{ft}$ bgs.

Westbay well GW-133, Zone 23 measurement port @ $128 \mathrm{ft}$ bgs. 


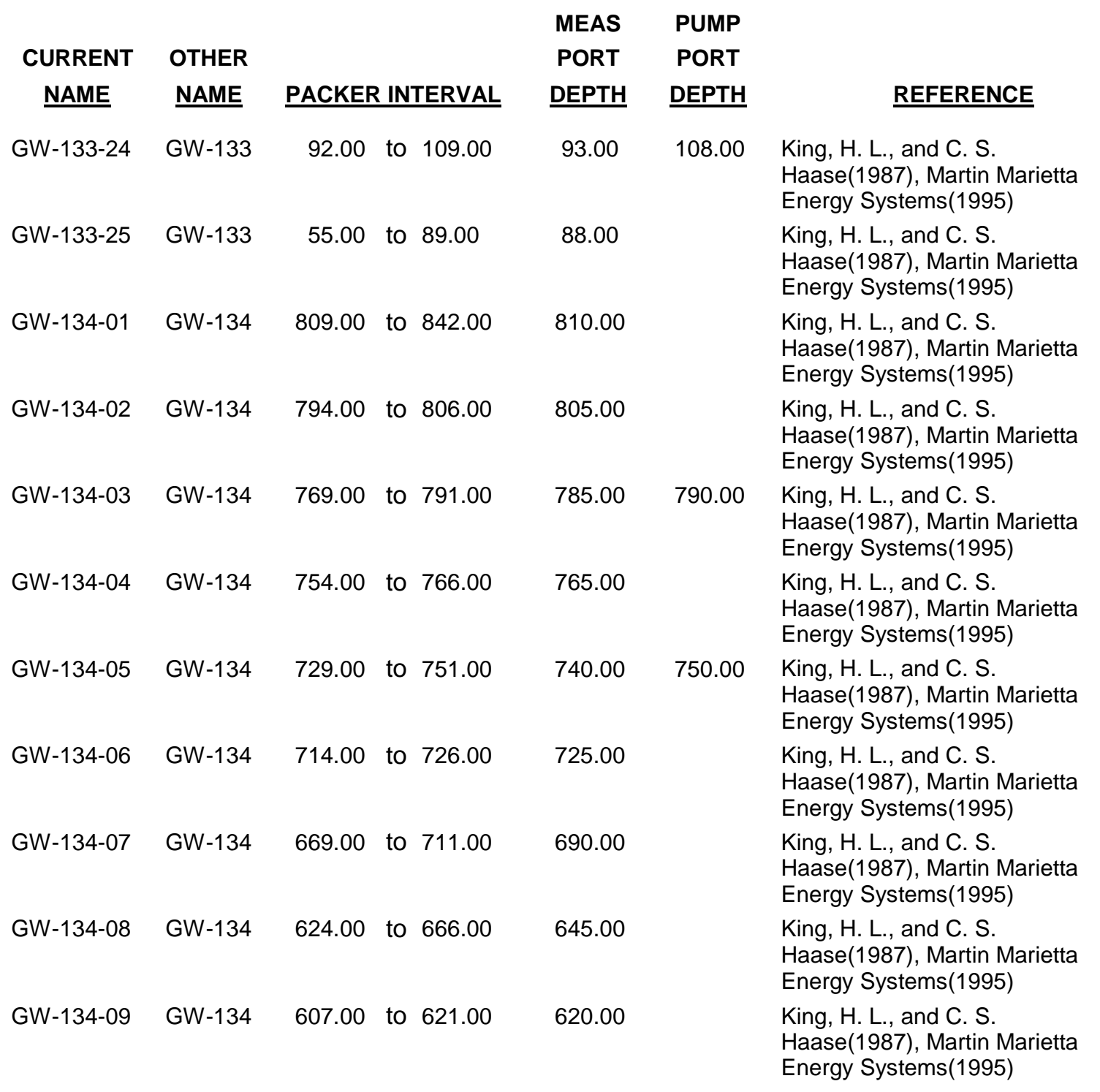

\section{COMMENTS}

Westbay well GW-133, Zone 24 measurement port @ $93 \mathrm{ft}$ bgs, pumping port @ $108 \mathrm{ft}$ bgs.

Westbay well GW-133, Zone 25 measurement port @ $88 \mathrm{ft}$ bgs.

Westbay well GW-134, Zone 1 measurement port @ $810 \mathrm{ft}$ bgs.

Westbay well GW-134, Zone 2 measurement port @ $805 \mathrm{ft}$ bgs.

Westbay well GW-134, Zone 3 measurement port @ $785 \mathrm{ft}$ bgs, pumping port @ $790 \mathrm{ft}$ bgs.

Westbay well GW-134, Zone 4 measurement port @ $765 \mathrm{ft}$ bgs.

Westbay well GW-134, Zone 5 measurement port @ $740 \mathrm{ft}$ bgs, pumping port @ $750 \mathrm{ft}$ bgs.

Westbay well GW-134, Zone 6 measurement port @ 725 ft bgs.

Westbay well GW-134, Zone 7 measurement port @ $690 \mathrm{ft}$ bgs.

Westbay well GW-134, Zone 8 measurement port @ $645 \mathrm{ft}$ bgs.

Westbay well GW-134, Zone 9 measurement port @ $620 \mathrm{ft}$ bgs. 


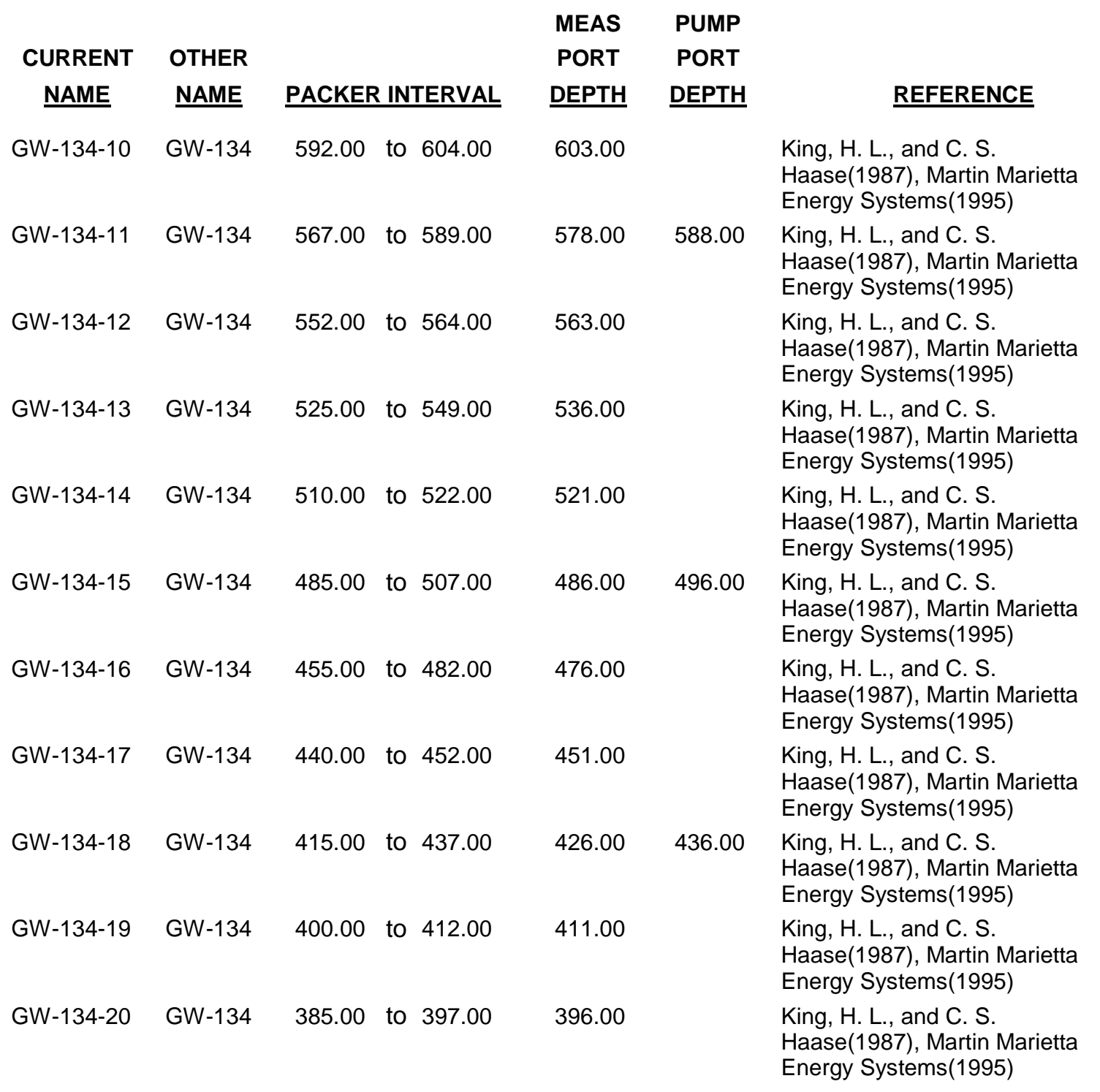

\section{COMMENTS}

Westbay well GW-134, Zone 10 measurement port @ $603 \mathrm{ft}$ bgs.

Westbay well GW-134, Zone 11 measurement port @ $578 \mathrm{ft}$ bgs, pumping port @ 588 ft bgs.

Westbay well GW-134, Zone 12 measurement port @ 563 ft bgs.

Westbay well GW-134, Zone 13 measurement port @ $536 \mathrm{ft}$ bgs.

Westbay well GW-134, Zone 14 measurement port @ $521 \mathrm{ft}$ bgs.

Westbay well GW-134, Zone 15 measurement port @ $486 \mathrm{ft}$ bgs, pumping port @ $496 \mathrm{ft}$ bgs.

Westbay well GW-134, Zone 16 measurement port @ 476 ft bgs.

Westbay well GW-134, Zone 17 measurement port @ 451 ft bgs.

Westbay well GW-134, Zone 18 measurement port @ $426 \mathrm{ft}$ bgs, pumping port @ $436 \mathrm{ft}$ bgs.

Westbay well GW-134, Zone 19 measurement port @ $411 \mathrm{ft}$ bgs.

Westbay well GW-134, Zone 20 measurement port @ $396 \mathrm{ft}$ bgs. 


\begin{tabular}{|c|c|c|c|c|}
\hline \multirow{3}{*}{$\begin{array}{c}\text { CURRENT } \\
\text { NAME }\end{array}$} & \multirow{3}{*}{$\begin{array}{l}\text { OTHER } \\
\text { NAME }\end{array}$} & & & \multirow{2}{*}{$\begin{array}{l}\text { MEAS } \\
\text { PORT }\end{array}$} \\
\hline & & & & \\
\hline & & PACKER & INTERVAL & DEPTH \\
\hline GW-134-21 & GW-134 & 360.00 & to 382.00 & 371.00 \\
\hline GW-134-22 & GW-134 & 345.00 & to 357.00 & 356.00 \\
\hline GW-134-23 & GW-134 & 315.00 & to 342.00 & 331.00 \\
\hline GW-134-24 & GW-134 & 300.00 & to 312.00 & 311.00 \\
\hline GW-134-25 & GW-134 & 275.00 & to 297.00 & 286.00 \\
\hline GW-134-26 & GW-134 & 260.00 & to 272.00 & 271.00 \\
\hline GW-134-27 & GW-134 & 215.00 & to 257.00 & 236.00 \\
\hline GW-134-28 & GW-134 & 200.00 & to 212.00 & 211.00 \\
\hline GW-134-29 & GW-134 & 170.00 & to 197.00 & 171.00 \\
\hline GW-134-30 & GW-134 & 155.00 & to 167.00 & 166.00 \\
\hline GW-134-31 & GW-134 & 135.00 & to 152.00 & 146.00 \\
\hline
\end{tabular}

\section{PUMP}

PORT

DEPTH

381.00 King, H. L., and C. S. Haase(1987), Martin Marietta Energy Systems(1995)

King, H. L., and C. S. Haase(1987), Martin Marietta Energy Systems(1995)

King, H. L., and C. S. Haase(1987), Martin Marietta Energy Systems(1995)

King, H. L., and C. S.

Haase(1987), Martin Marietta Energy Systems(1995)

296.00 King, H. L., and C. S.

Haase(1987), Martin Marietta Energy Systems(1995)

King, H. L., and C. S.

Haase(1987), Martin Marietta Energy Systems(1995)

King, $\mathrm{H}$. L., and C. S. Haase(1987), Martin Marietta Energy Systems(1995)

King, H. L., and C. S.

Haase(1987), Martin Marietta Energy Systems(1995)

186.00

King, H. L., and C. S.

Haase(1987), Martin Marietta Energy Systems(1995)

King, H. L., and C. S.

Haase(1987), Martin Marietta

Energy Systems(1995)

King, H. L., and C. S.

Haase(1987), Martin Marietta

Energy Systems(1995)

\section{COMMENTS}

Westbay well GW-134, Zone 21 measurement port @ 371 ft bgs, pumping port @ 381 ft bgs.

Westbay well GW-134, Zone 22 measurement port @ 356 ft bgs.

Westbay well GW-134, Zone 23 measurement port @ 331 ft bgs.

Westbay well GW-134, Zone 24 measurement port @ 311 ft bgs.

Westbay well GW-134, Zone 25 measurement port @ 286 ft bgs, pumping port @ 296 ft bgs.

Westbay well GW-134, Zone 26 measurement port @ 271 ft bgs.

Westbay well GW-134, Zone 27 measurement port @ 236 ft bgs.

Westbay well GW-134, Zone 28 measurement port @ 211 ft bgs.

Westbay well GW-134, Zone 29 measurement port @ 171 ft bgs, pumping port @ 186 ft bgs.

Westbay well GW-134, Zone 30 measurement port @ 166 ft bgs.

Westbay well GW-134, Zone 31 measurement port @ 146 ft bgs. 


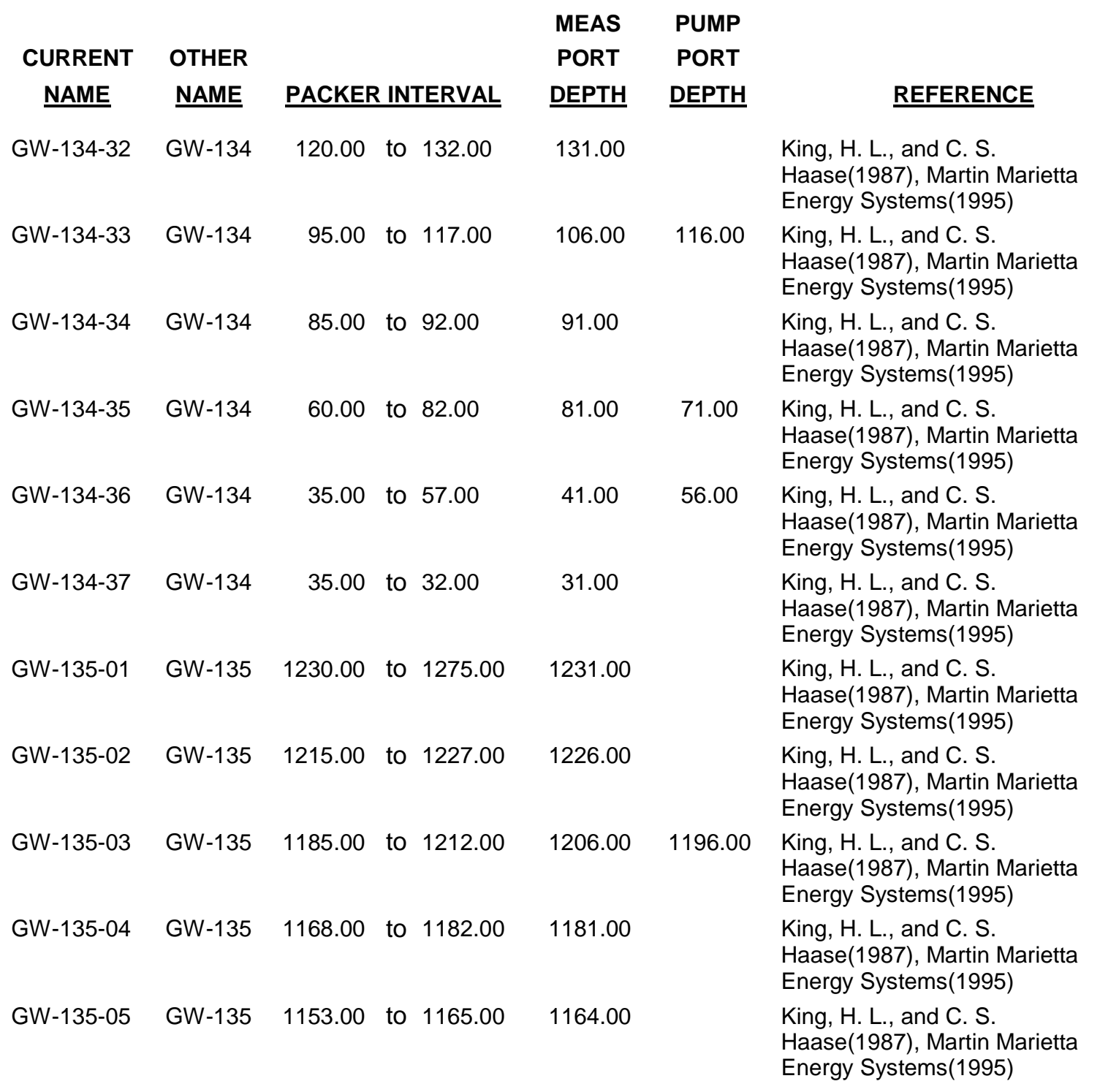

\section{COMMENTS}

Westbay well GW-134, Zone 32 measurement port @ 131 ft bgs.

Westbay well GW-134, Zone 33 measurement port @ 106 ft bgs, pumping port @ 116 ft bgs.

Westbay well GW-134, Zone 34 measurement port @ 91 ft bgs.

Westbay well GW-134, Zone 35 measurement port @ 81 ft bgs, pumping port @ 71 ft bgs.

Westbay well GW-134, Zone 36 measurement port @ 41 ft bgs, pumping port @ 56 ft bgs.

Westbay well GW-134, Zone 37 measurement port @ $31 \mathrm{ft}$ bgs.

Westbay well GW-135, Zone 1 measurement port @ 1231 ft bgs.

Westbay well GW-135, Zone 2 measurement port @ 1226 ft bgs.

Westbay well GW-135, Zone 3 measurement port @ 1206 ft bgs, pumping port @ $1196 \mathrm{ft}$ bgs.

Westbay well GW-135, Zone 4 measurement port @ 1181 ft bgs.

Westbay well GW-135, Zone 5 measurement port @ 1164 ft bgs. 


\begin{tabular}{|c|c|c|c|c|c|c|}
\hline $\begin{array}{c}\text { CURRENT } \\
\text { NAME }\end{array}$ & $\begin{array}{l}\text { OTHER } \\
\text { NAME }\end{array}$ & PACKE & INTERVAL & $\begin{array}{l}\text { MEAS } \\
\text { PORT } \\
\text { DEPTH }\end{array}$ & $\begin{array}{c}\text { PUMP } \\
\text { PORT } \\
\text { DEPTH }\end{array}$ & REFERENCE \\
\hline GW-135-06 & GW-135 & 1124.00 & to 1150.00 & 1142.00 & 1149.00 & $\begin{array}{l}\text { King, H. L., and C. S. } \\
\text { Haase(1987), Martin Marietta } \\
\text { Energy Systems(1995) }\end{array}$ \\
\hline GW-135-07 & GW-135 & 1109.00 & to 1121.00 & 1120.00 & & $\begin{array}{l}\text { King, H. L., and C. S. } \\
\text { Haase(1987), Martin Marietta } \\
\text { Energy Systems(1995) }\end{array}$ \\
\hline GW-135-08 & GW-135 & 1064.00 & to 1106.00 & 1085.00 & & $\begin{array}{l}\text { King, H. L., and C. S. } \\
\text { Haase(1987), Martin Marietta } \\
\text { Energy Systems(1995) }\end{array}$ \\
\hline GW-135-09 & GW-135 & 1004.00 & to 1061.00 & 1030.00 & & $\begin{array}{l}\text { King, H. L., and C. S. } \\
\text { Haase(1987), Martin Marietta } \\
\text { Energy Systems(1995) }\end{array}$ \\
\hline GW-135-10 & GW-135 & 989.00 & to 1001.00 & 990.00 & & $\begin{array}{l}\text { King, H. L., and C. S. } \\
\text { Haase(1987), Martin Marietta } \\
\text { Energy Systems(1995) }\end{array}$ \\
\hline GW-135-11 & GW-135 & 959.00 & to 986.00 & 965.00 & 975.00 & $\begin{array}{l}\text { King, H. L., and C. S. } \\
\text { Haase(1987), Martin Marietta } \\
\text { Energy Systems(1995) }\end{array}$ \\
\hline GW-135-12 & GW-135 & 944.00 & to 956.00 & 955.00 & & $\begin{array}{l}\text { King, H. L., and C. S. } \\
\text { Haase(1987), Martin Marietta } \\
\text { Energy Systems(1995) }\end{array}$ \\
\hline GW-135-13 & GW-135 & 870.00 & to 941.00 & 901.00 & & $\begin{array}{l}\text { King, H. L., and C. S. } \\
\text { Haase(1987), Martin Marietta } \\
\text { Energy Systems(1995) }\end{array}$ \\
\hline GW-135-14 & GW-135 & 855.00 & to 867.00 & 866.00 & & $\begin{array}{l}\text { King, H. L., and C. S. } \\
\text { Haase(1987), Martin Marietta } \\
\text { Energy Systems(1995) }\end{array}$ \\
\hline GW-135-15 & GW-135 & 825.00 & to 852.00 & 836.00 & 846.00 & $\begin{array}{l}\text { King, H. L., and C. S. } \\
\text { Haase(1987), Martin Marietta } \\
\text { Energy Systems(1995) }\end{array}$ \\
\hline GW-135-16 & GW-135 & 810.00 & to 822.00 & 821.00 & & $\begin{array}{l}\text { King, H. L., and C. S. } \\
\text { Haase(1987), Martin Marietta } \\
\text { Energy Systems(1995) }\end{array}$ \\
\hline
\end{tabular}

\section{COMMENTS}

Westbay well GW-135, Zone 6 measurement port @ 1142 ft bgs, pumping port @ 1149 ft bgs.

Westbay well GW-135, Zone 7 measurement port @ $1120 \mathrm{ft}$ bgs.

Westbay well GW-135, Zone 8 measurement port @ 1085 ft bgs.

Westbay well GW-135, Zone 9 measurement port @ 1030 ft bgs.

Westbay well GW-135, Zone 10 measurement port @ 990 ft bgs.

Westbay well GW-135, Zone 11 measurement port @ 965 ft bgs, pumping port @ 975 ft bgs.

Westbay well GW-135, Zone 12 measurement port @ 955 ft bgs.

Westbay well GW-135, Zone 13 measurement port @ 901 ft bgs.

Westbay well GW-135, Zone 14 measurement port @ 866 ft bgs.

Westbay well GW-135, Zone 15 measurement port @ 836 ft bgs, pumping port @ 846 ft bgs.

Westbay well GW-135, Zone 16 measurement port @ 821 ft bgs. 


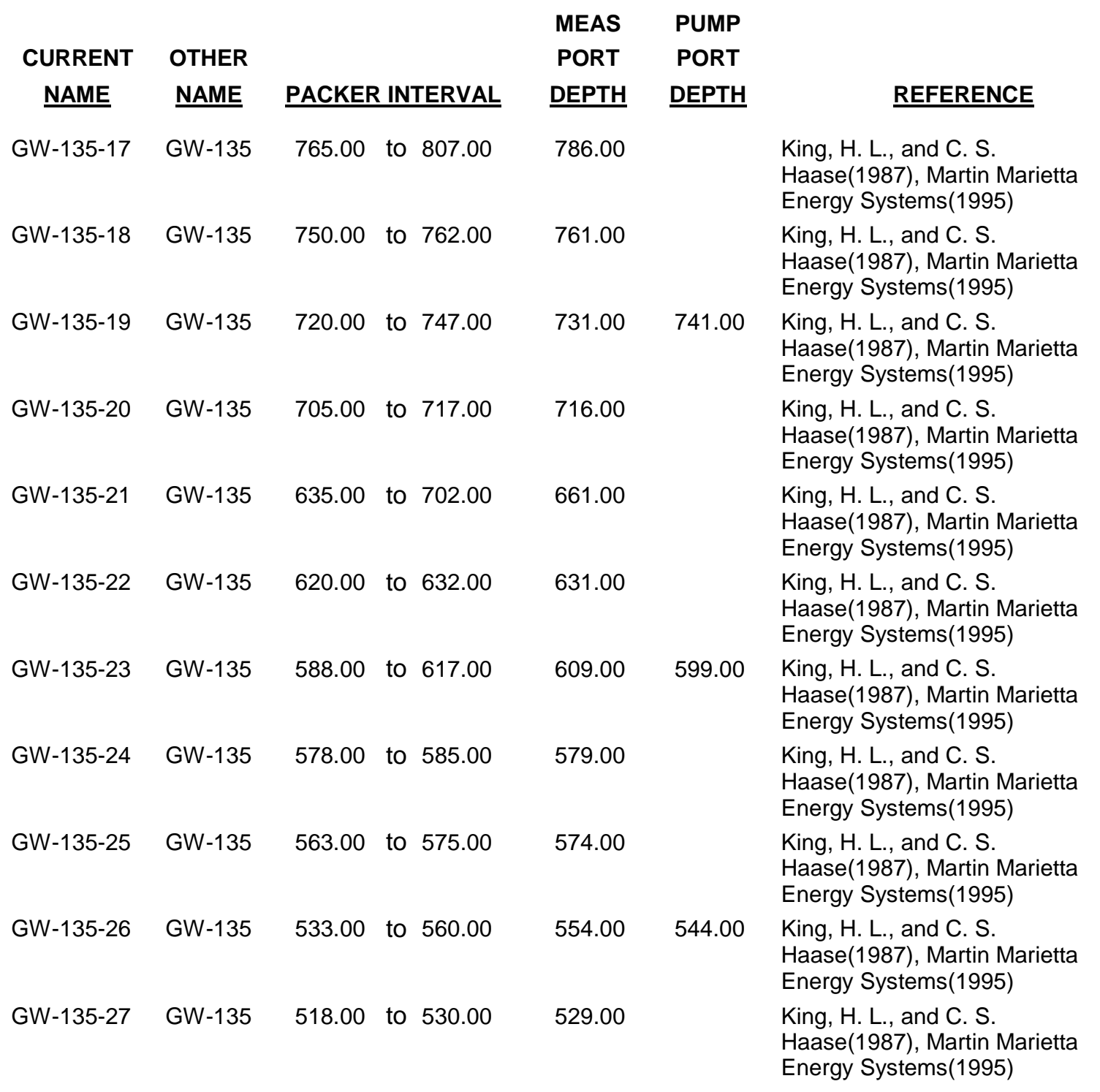

\section{COMMENTS}

Westbay well GW-135, Zone 17 measurement port @ $786 \mathrm{ft}$ bgs.

Westbay well GW-135, Zone 18 measurement port @ $761 \mathrm{ft}$ bgs.

Westbay well GW-135, Zone 19 measurement port @ $731 \mathrm{ft}$ bgs, pumping port @ $741 \mathrm{ft}$ bgs.

Westbay well GW-135, Zone 20 measurement port @ 716 ft bgs.

Westbay well GW-135, Zone 21 measurement port @ $661 \mathrm{ft}$ bgs.

Westbay well GW-135, Zone 22 measurement port @ $631 \mathrm{ft}$ bgs.

Westbay well GW-135, Zone 23 measurement port @ $609 \mathrm{ft}$ bgs, pumping port @ $599 \mathrm{ft}$ bgs.

Westbay well GW-135, Zone 24 measurement port @ 579 ft bgs.

Westbay well GW-135, Zone 25 measurement port @ $574 \mathrm{ft}$ bgs.

Westbay well GW-135, Zone 26 measurement port @ $554 \mathrm{ft}$ bgs, pumping port @ $544 \mathrm{ft}$ bgs.

Westbay well GW-135, Zone 27 measurement port @ $529 \mathrm{ft}$ bgs. 


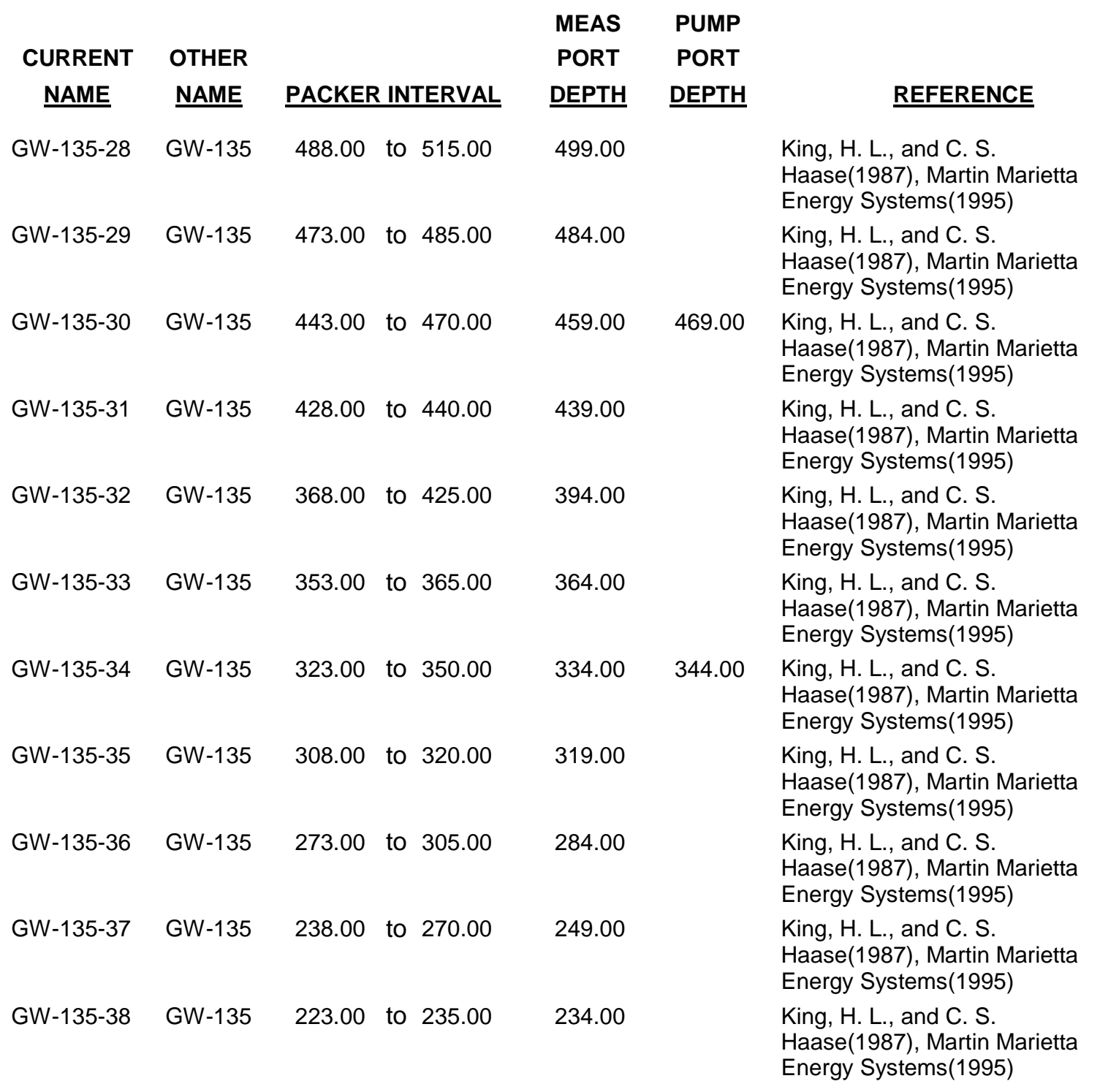

\section{COMMENTS}

Westbay well GW-135, Zone 28 measurement port @ $499 \mathrm{ft}$ bgs.

Westbay well GW-135, Zone 29 measurement port @ $484 \mathrm{ft}$ bgs.

Westbay well GW-135, Zone 30 measurement port @ $459 \mathrm{ft}$ bgs, pumping port @ $469 \mathrm{ft}$ bgs.

Westbay well GW-135, Zone 31 measurement port @ 439 ft bgs.

Westbay well GW-135, Zone 32 measurement port @ $394 \mathrm{ft}$ bgs.

Westbay well GW-135, Zone 33 measurement port @ $364 \mathrm{ft}$ bgs.

Westbay well GW-135, Zone 34 measurement port @ $334 \mathrm{ft}$ bgs, pumping port @ $344 \mathrm{ft}$ bgs.

Westbay well GW-135, Zone 35 measurement port @ 319 ft bgs.

Westbay well GW-135, Zone 36 measurement port @ $284 \mathrm{ft}$ bgs.

Westbay well GW-135, Zone 37 measurement port @ $249 \mathrm{ft}$ bgs.

Westbay well GW-135, Zone 38 measurement port @ $234 \mathrm{ft}$ bgs. 


\begin{tabular}{|c|c|c|c|c|c|c|c|}
\hline $\begin{array}{l}\text { CURRENT } \\
\text { NAME }\end{array}$ & $\begin{array}{l}\text { OTHER } \\
\text { NAME }\end{array}$ & PACKEF & 2 INTERVAL & $\begin{array}{l}\text { MEAS } \\
\text { PORT } \\
\text { DEPTH }\end{array}$ & $\begin{array}{l}\text { PUMP } \\
\text { PORT } \\
\text { DEPTH }\end{array}$ & REFERENCE & COMMENTS \\
\hline GW-135-39 & GW-135 & 193.00 & to 220.00 & 214.00 & 204.00 & $\begin{array}{l}\text { King, H. L., and C. S. } \\
\text { Haase(1987), Martin Marietta } \\
\text { Energy Systems(1995) }\end{array}$ & $\begin{array}{l}\text { Westbay well GW-135, Zone } 39 \text { measurement } \\
\text { port @ } 214 \mathrm{ft} \text { bgs, pumping port @ } 204 \mathrm{ft} \text { bgs. }\end{array}$ \\
\hline GW-135-40 & GW-135 & 178.00 & to 190.00 & 189.00 & & $\begin{array}{l}\text { King, H. L., and C. S. } \\
\text { Haase(1987), Martin Marietta } \\
\text { Energy Systems(1995) }\end{array}$ & $\begin{array}{l}\text { Westbay well GW-135, Zone } 40 \text { measurement } \\
\text { port @ } 189 \mathrm{ft} \text { bgs. }\end{array}$ \\
\hline GW-135-41 & GW-135 & 80.00 & to 175.00 & 164.00 & & $\begin{array}{l}\text { King, H. L., and C. S. } \\
\text { Haase(1987), Martin Marietta } \\
\text { Energy Systems(1995) }\end{array}$ & $\begin{array}{l}\text { Westbay well GW-135, Zone } 41 \text { measurement } \\
\text { port @ } 164 \mathrm{ft} \text { bgs. }\end{array}$ \\
\hline GW-722-01 & GW-722 & 629.00 & to 644.00 & 630.00 & & $\begin{array}{l}\text { SAIC(1991a), Shevenell, L. A., R. } \\
\text { B. Dreier, and W. K. Jago(1992), } \\
\text { Martin Marietta Energy } \\
\text { Systems(1995) }\end{array}$ & $\begin{array}{l}\text { Westbay well GW-722, Zone } 1 \text { measurement } \\
\text { port @ } 630 \mathrm{ft} \text { bgs. }\end{array}$ \\
\hline GW-722-02 & GW-722 & 614.00 & to 626.00 & 620.00 & 625.00 & $\begin{array}{l}\text { SAIC(1991a), Shevenell, L. A., R. } \\
\text { B. Dreier, and W. K. Jago(1992), } \\
\text { Martin Marietta Energy } \\
\text { Systems(1995) }\end{array}$ & $\begin{array}{l}\text { Westbay well GW-722, Zone } 2 \text { measurement } \\
\text { port @ } 630 \mathrm{ft} \text { bgs, pumping port @ } 625 \mathrm{ft} \text { bgs. }\end{array}$ \\
\hline GW-722-03 & GW-722 & 604.00 & to 611.00 & 605.00 & & $\begin{array}{l}\text { SAIC(1991a), Shevenell, L. A., R. } \\
\text { B. Dreier, and W. K. Jago(1992), } \\
\text { Martin Marietta Energy } \\
\text { Systems(1995) }\end{array}$ & $\begin{array}{l}\text { Westbay well GW-722, Zone } 3 \text { measurement } \\
\text { port @ } 605 \mathrm{ft} \text { bgs. }\end{array}$ \\
\hline GW-722-04 & GW-722 & 584.00 & to 601.00 & 585.00 & & $\begin{array}{l}\text { SAIC(1991a), Shevenell, L. A., R. } \\
\text { B. Dreier, and W. K. Jago(1992), } \\
\text { Martin Marietta Energy } \\
\text { Systems(1995) }\end{array}$ & $\begin{array}{l}\text { Westbay well GW-722, Zone } 4 \text { measurement } \\
\text { port @ } 585 \mathrm{ft} \text { bgs. }\end{array}$ \\
\hline GW-722-05 & GW-722 & 569.00 & to 581.00 & 570.00 & & $\begin{array}{l}\text { SAIC(1991a), Shevenell, L. A., R. } \\
\text { B. Dreier, and W. K. Jago(1992), } \\
\text { Martin Marietta Energy } \\
\text { Systems(1995) }\end{array}$ & $\begin{array}{l}\text { Westbay well GW- } 722 \text {, Zone } 5 \text { measurement } \\
\text { port @ } 570 \mathrm{ft} \text { bgs. }\end{array}$ \\
\hline GW-722-06 & GW-722 & 554.00 & to 566.00 & 560.00 & 565.00 & $\begin{array}{l}\text { SAIC(1991a), Shevenell, L. A., R. } \\
\text { B. Dreier, and W. K. Jago(1992), } \\
\text { Martin Marietta Energy } \\
\text { Systems(1995) }\end{array}$ & $\begin{array}{l}\text { Westbay well GW- } 722 \text {, Zone } 6 \text { measurement } \\
\text { port @ } 560 \mathrm{ft} \text { bgs, pumping port @ } 565 \mathrm{ft} \text { bgs. }\end{array}$ \\
\hline
\end{tabular}




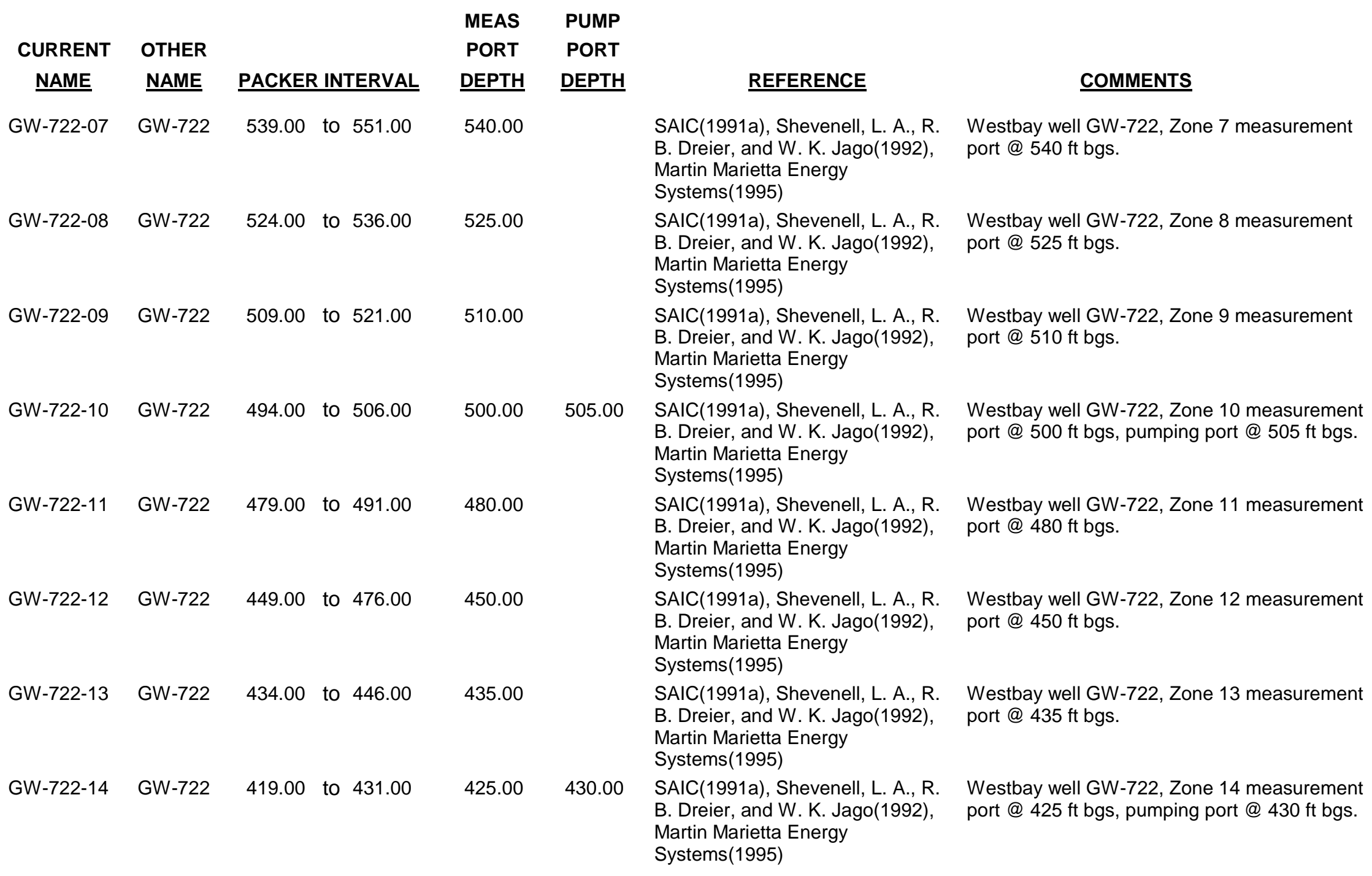




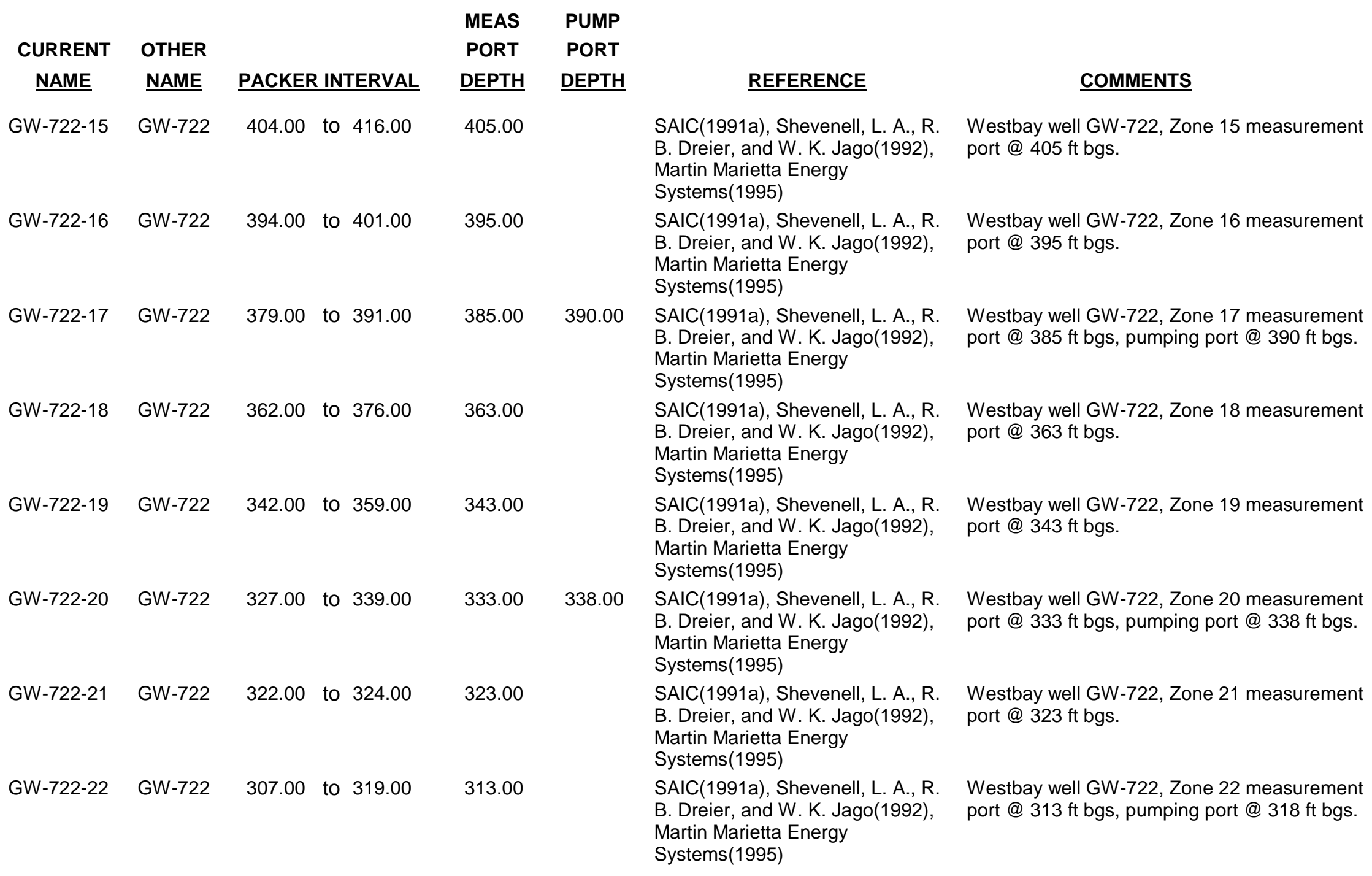




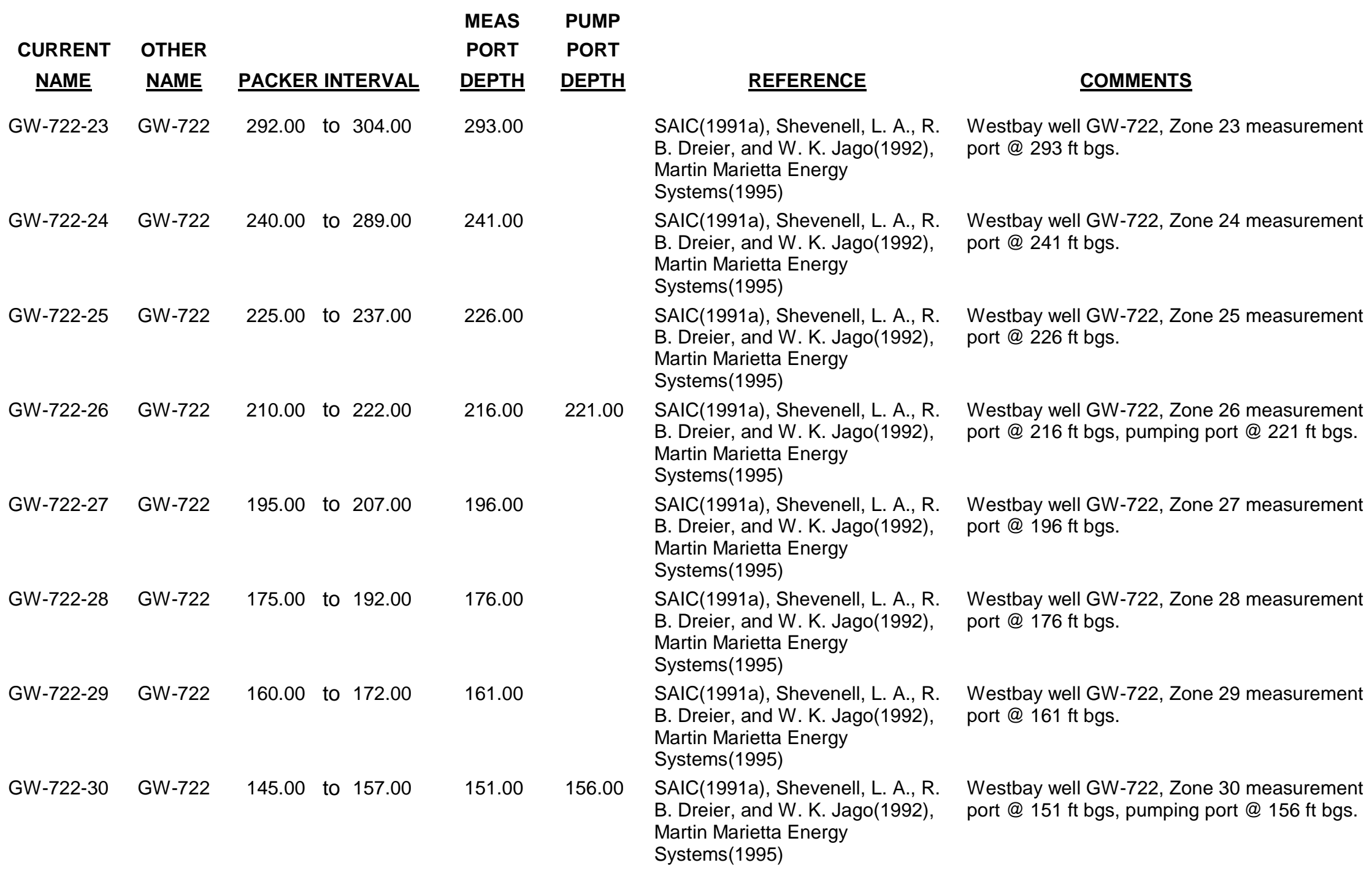




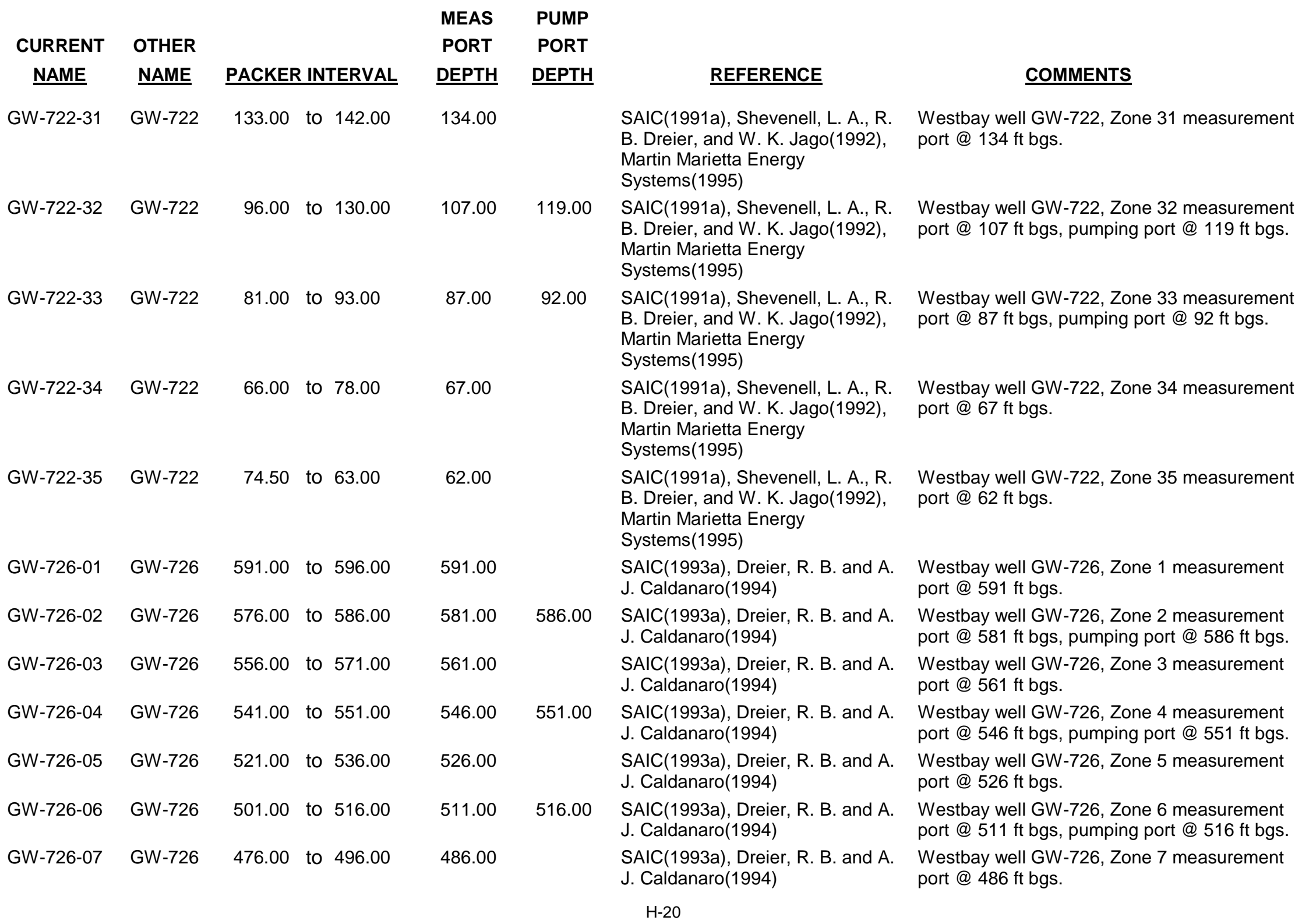




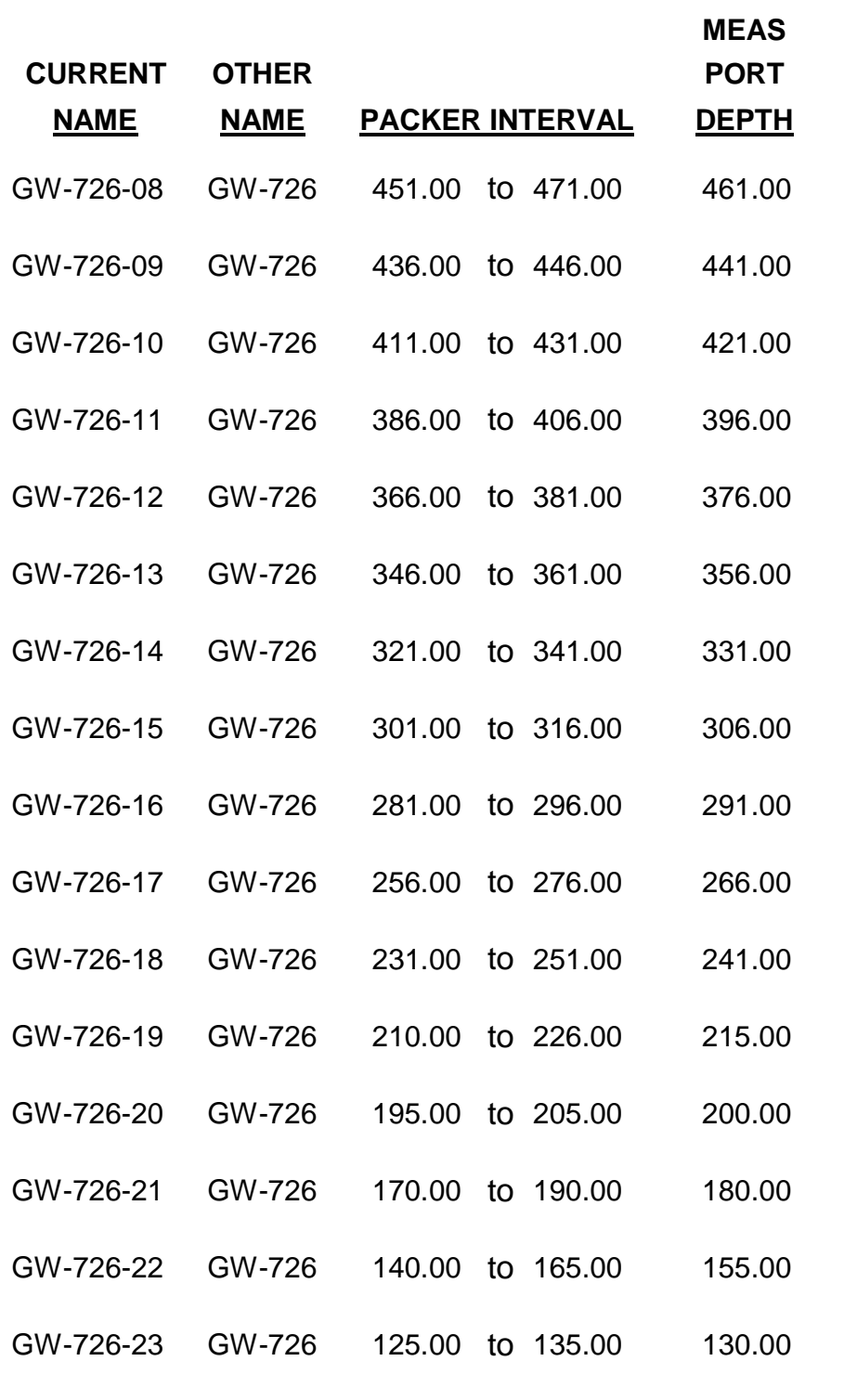

PUMP

PORT

DEPTH

SAIC(1993a), Dreier, R. B. and A. Westbay well GW-726, Zone 8 measurement J. Caldanaro(1994) port @ $461 \mathrm{ft}$ bgs.

446.00 SAIC(1993a), Dreier, R. B. and A. Westbay well GW-726, Zone 9 measurement J. Caldanaro(1994) port @ $441 \mathrm{ft}$ bgs, pumping port @ $446 \mathrm{ft}$ bgs.

SAIC(1993a), Dreier, R. B. and A. Westbay well GW-726, Zone 10 measurement J. Caldanaro(1994) port @ $421 \mathrm{ft}$ bgs.

SAIC(1993a), Dreier, R. B. and A. Westbay well GW-726, Zone 11 measurement J. Caldanaro(1994) port @ $396 \mathrm{ft}$ bgs.

381.00 SAIC(1993a), Dreier, R. B. and A. Westbay well GW-726, Zone 12 measurement J. Caldanaro(1994) port @ $376 \mathrm{ft}$ bgs, pumping port @ $381 \mathrm{ft}$ bgs.

SAIC(1993a), Dreier, R. B. and A. Westbay well GW-726, Zone 13 measurement J. Caldanaro(1994) port @ $356 \mathrm{ft}$ bgs.

SAIC(1993a), Dreier, R. B. and A. Westbay well GW-726, Zone 14 measurement J. Caldanaro(1994) port @ $331 \mathrm{ft}$ bgs.

SAIC(1993a), Dreier, R. B. and A. Westbay well GW-726, Zone 15 measurement J. Caldanaro(1994) port @ $306 \mathrm{ft}$ bgs.

296.00 SAIC(1993a), Dreier, R. B. and A. Westbay well GW-726, Zone 16 measurement J. Caldanaro(1994) port @ $291 \mathrm{ft}$ bgs, pumping port @ $296 \mathrm{ft}$ bgs.

SAIC(1993a), Dreier, R. B. and A. Westbay well GW-726, Zone 17 measurement J. Caldanaro(1994) port @ $266 \mathrm{ft}$ bgs.

SAIC(1993a), Dreier, R. B. and A. Westbay well GW-726, Zone 18 measurement J. Caldanaro(1994) port @ $241 \mathrm{ft}$ bgs.

SAIC(1993a), Dreier, R. B. and A. Westbay well GW-726, Zone 19 measurement J. Caldanaro(1994) port @ $215 \mathrm{ft}$ bgs.

205.00 SAIC(1993a), Dreier, R. B. and A. Westbay well GW-726, Zone 20 measurement J. Caldanaro(1994) port @ $200 \mathrm{ft}$ bgs, pumping port @ $205 \mathrm{ft}$ bgs.

SAIC(1993a), Dreier, R. B. and A. Westbay well GW-726, Zone 21 measurement J. Caldanaro(1994) port @ $180 \mathrm{ft}$ bgs.

SAIC(1993a), Dreier, R. B. and A. Westbay well GW-726, Zone 22 measurement J. Caldanaro(1994) port @ $155 \mathrm{ft}$ bgs.

135.00 SAIC(1993a), Dreier, R. B. and A. Westbay well GW-726, Zone 23 measurement J. Caldanaro(1994) port @ 130 ft bgs, pumping port @ 135 ft bgs. 


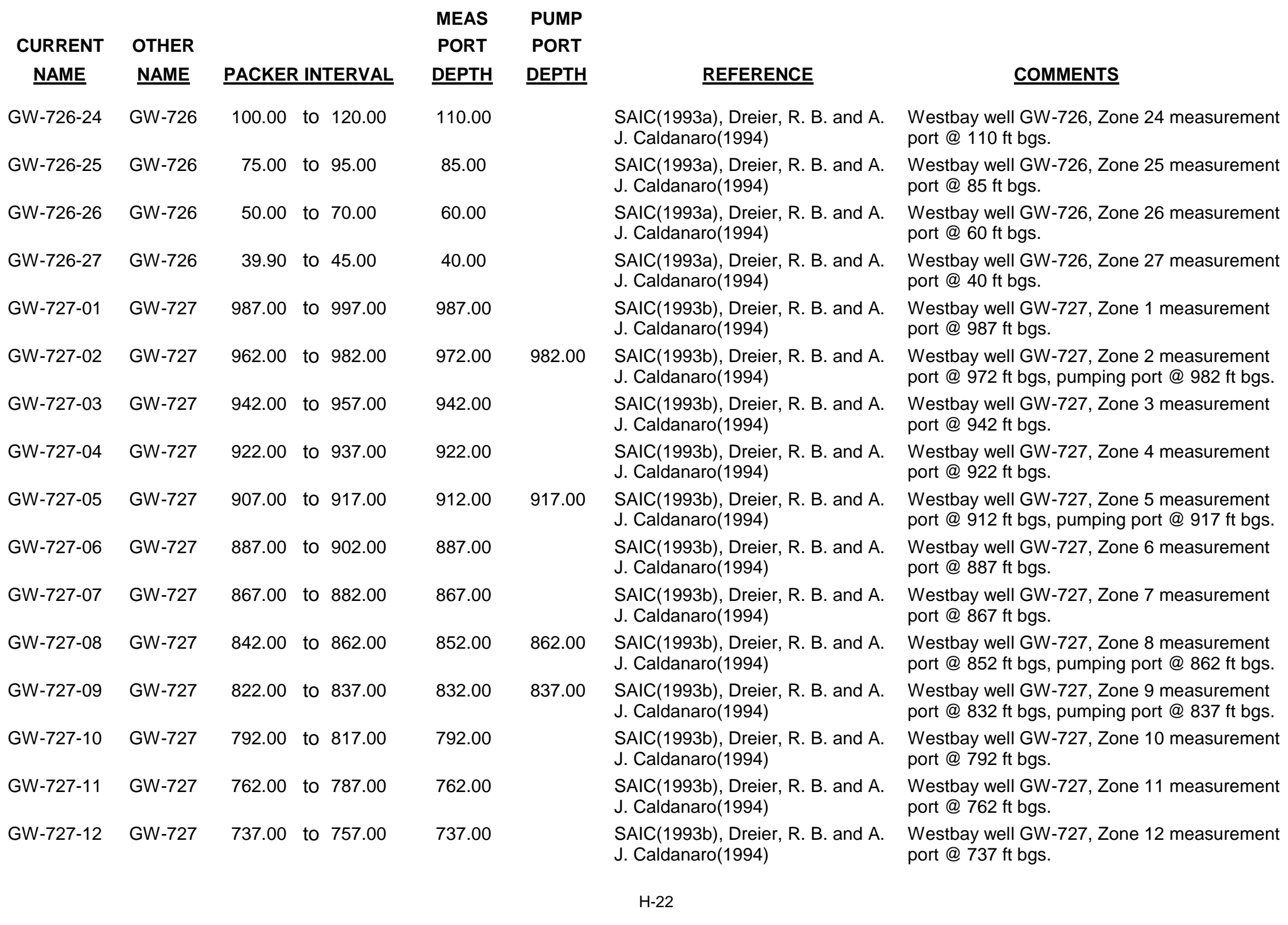




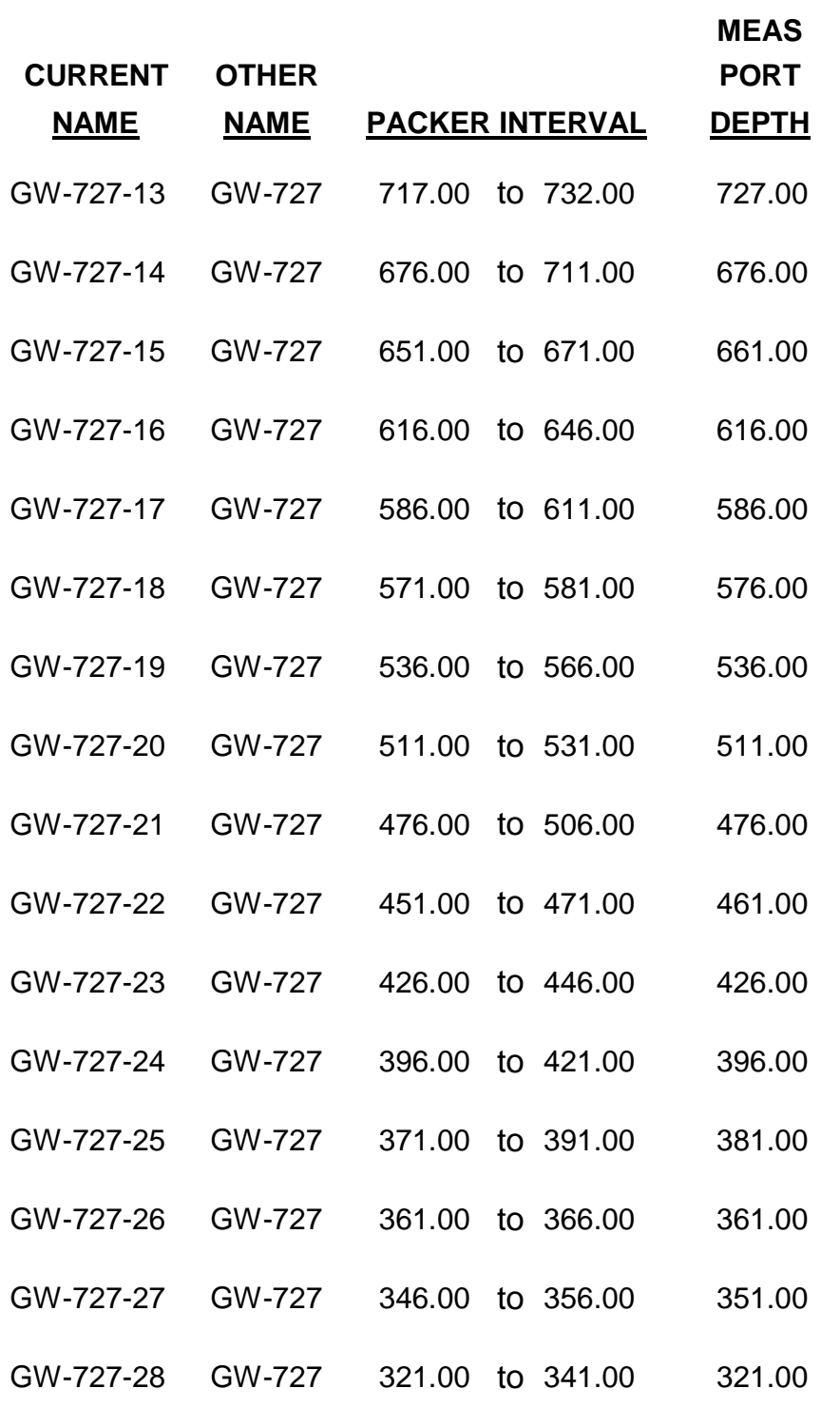

\section{PUMP}

PORT

DEPTH

732.00 SAIC(1993b), Dreier, R. B. and A. J. Caldanaro(1994)

SAIC(1993b), Dreier, R. B. and A. Westbay well GW-727, Zone 14 measurement J. Caldanaro(1994)

671.00 SAIC(1993b), Dreier, R. B. and A. Westbay well GW-727, Zone 15 measurement J. Caldanaro(1994) port @ 661 ft bgs, pumping port @ 671 ft bgs.

SAIC(1993b), Dreier, R. B. and A. Westbay well GW-727, Zone 16 measurement J. Caldanaro(1994) port @ 616 ft bgs.

SAIC(1993b), Dreier, R. B. and A. Westbay well GW-727, Zone 17 measurement J. Caldanaro(1994) port @ 586 ft bgs.

581.00 SAIC(1993b), Dreier, R. B. and A. Westbay well GW-727, Zone 18 measurement J. Caldanaro(1994) port @ $576 \mathrm{ft}$ bgs, pumping port @ 581 ft bgs.

SAIC(1993b), Dreier, R. B. and A. Westbay well GW-727, Zone 19 measurement J. Caldanaro(1994) port @ 536 ft bgs.

SAIC(1993b), Dreier, R. B. and A. Westbay well GW-727, Zone 20 measurement J. Caldanaro(1994) port @ 511 ft bgs.

SAIC(1993b), Dreier, R. B. and A. Westbay well GW-727, Zone 21 measurement J. Caldanaro(1994) port @ $476 \mathrm{ft}$ bgs.

471.00 SAIC(1993b), Dreier, R. B. and A. Westbay well GW-727, Zone 22 measurement J. Caldanaro(1994) port @ $461 \mathrm{ft}$ bgs, pumping port @ $471 \mathrm{ft}$ bgs.

SAIC(1993b), Dreier, R. B. and A. Westbay well GW-727, Zone 23 measurement J. Caldanaro(1994) port @ $426 \mathrm{ft}$ bgs.

SAIC(1993b), Dreier, R. B. and A. Westbay well GW-727, Zone 24 measurement J. Caldanaro(1994) port @ 396 ft bgs.

391.00 SAIC(1993b), Dreier, R. B. and A. Westbay well GW-727, Zone 25 measurement J. Caldanaro(1994) port @ 381 ft bgs, pumping port @ 391 ft bgs.

SAIC(1993b), Dreier, R. B. and A. Westbay well GW-727, Zone 26 measurement J. Caldanaro(1994) port @ 361 ft bgs.

356.00 SAIC(1993b), Dreier, R. B. and A. Westbay well GW-727, Zone 27 measurement J. Caldanaro(1994)

SAIC(1993b), Dreier, R. B and A Westbay well GW-727, Zone 28 measurement J. Caldanaro(1994) port @ 351 ft bgs, pumping port @ 356 ft bgs. 


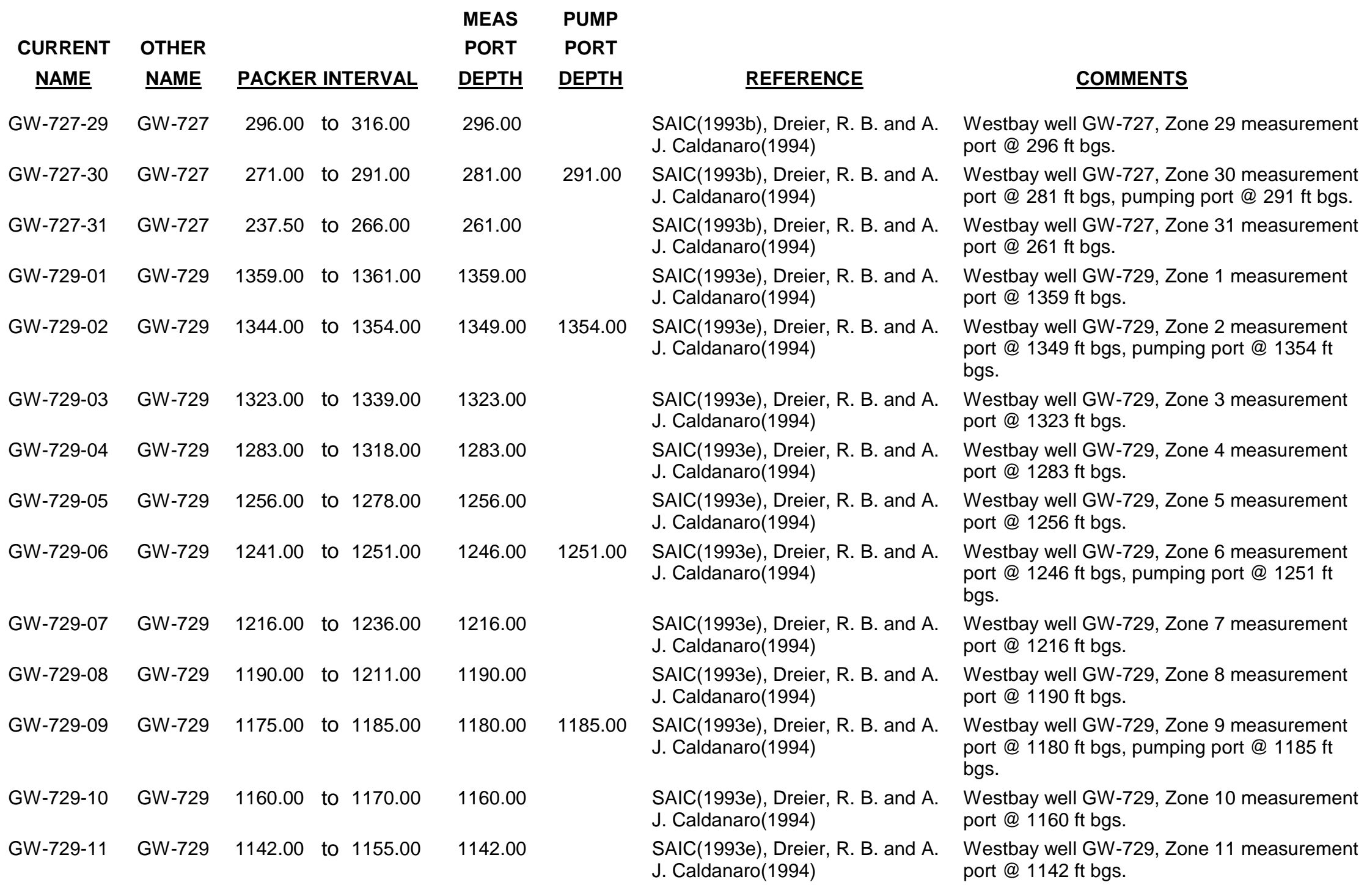




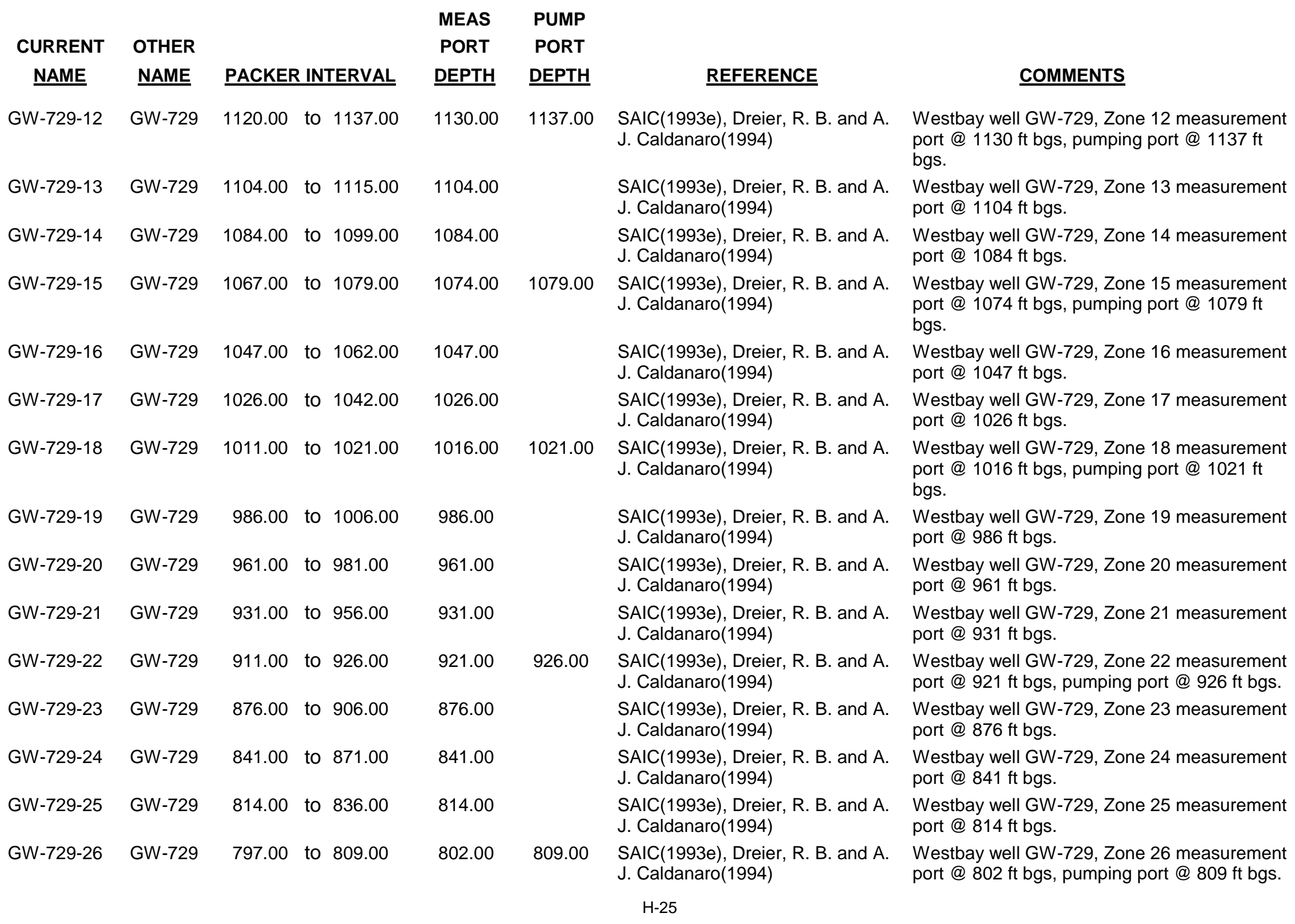




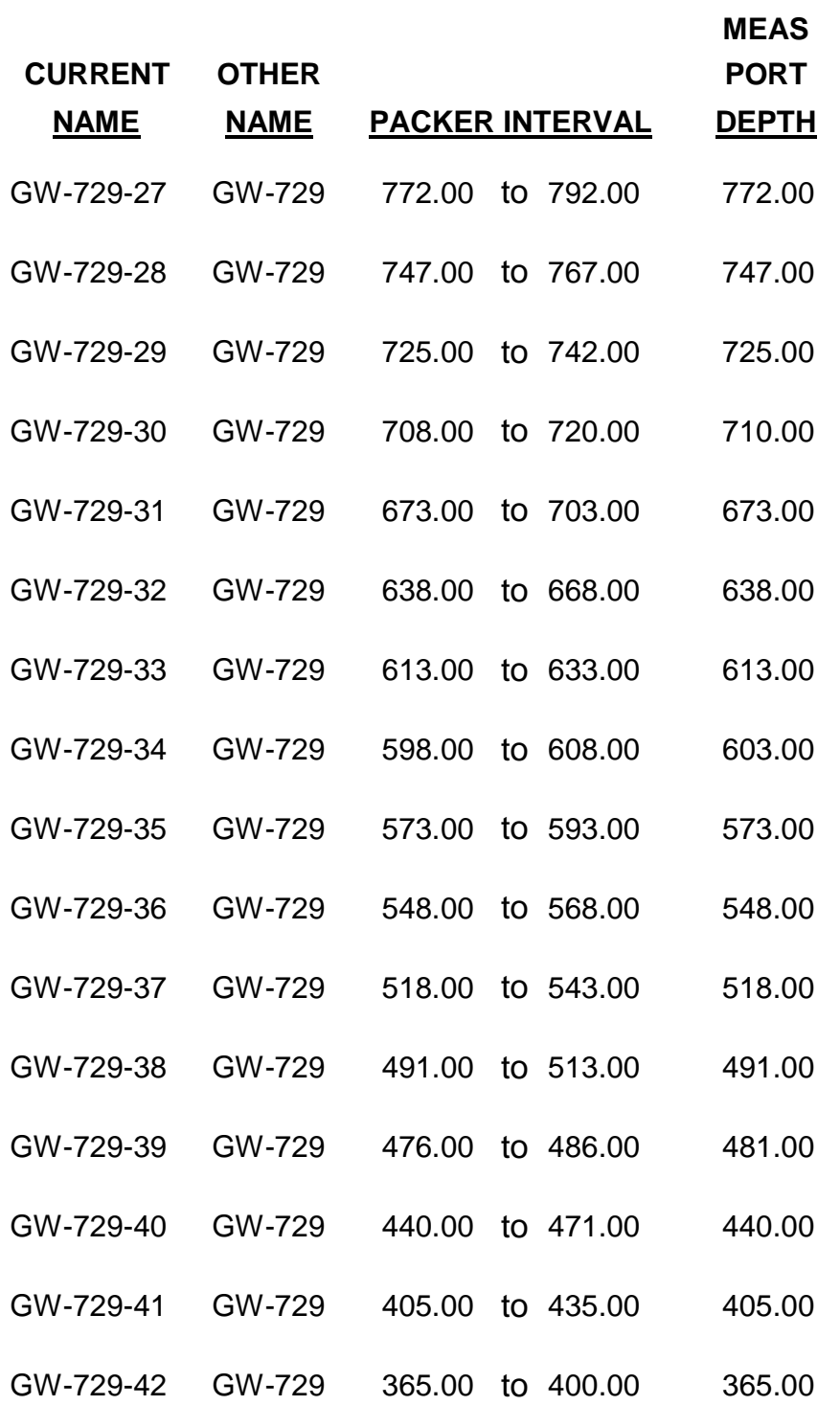

\section{PUMP}

PORT

DEPTH

SAIC(1993e), Dreier, R. B. and A. J. Caldanaro(1994)

SAIC(1993e), Dreier, R. B. and A. Westbay well GW-729, Zone 28 measurement

J. Caldanaro(1994)

SAIC(1993e), Dreier, R. B. and A. Westbay well GW-729, Zone 29 measurement

J. Caldanaro(1994)

720.00 SAIC(1993e), Dreier, R. B. and A.

J. Caldanaro(1994)

Westbay well GW-729, Zone 30 measurement port @ $710 \mathrm{ft}$ bgs, pumping port @ 720 ft bgs.

SAIC(1993e), Dreier, R. B. and A. Westbay well GW-729, Zone 31 measurement

J. Caldanaro(1994) port @ $673 \mathrm{ft}$ bgs.

SAIC(1993e), Dreier, R. B. and A. Westbay well GW-729, Zone 32 measurement

J. Caldanaro(1994) port @ 638 ft bgs.

SAIC(1993e), Dreier, R. B. and A. Westbay well GW-729, Zone 33 measurement J. Caldanaro(1994) port @ $613 \mathrm{ft}$ bgs.

608.00 SAIC(1993e), Dreier, R. B. and A. J. Caldanaro(1994)

Westbay well GW-729, Zone 34 measurement port @ $603 \mathrm{ft}$ bgs, pumping port @ $608 \mathrm{ft}$ bgs.

SAIC(1993e), Dreier, R. B. and A. Westbay well GW-729, Zone 35 measurement

J. Caldanaro(1994) port @ $573 \mathrm{ft}$ bgs.

SAIC(1993e), Dreier, R. B. and A. Westbay well GW-729, Zone 36 measurement J. Caldanaro(1994) port @ 548 ft bgs.

SAIC(1993e), Dreier, R. B. and A. Westbay well GW-729, Zone 37 measurement J. Caldanaro(1994) port @ 518 ft bgs.

SAIC(1993e), Dreier, R. B. and A. Westbay well GW-729, Zone 38 measurement J. Caldanaro(1994) port @ 491 ft bgs.

SAIC(1993e), Dreier, R. B. and A. Westbay well GW-729, Zone 39 measurement J. Caldanaro(1994) port @ 481 ft bgs.

SAIC(1993e), Dreier, R. B. and A. Westbay well GW-729, Zone 40 measurement J. Caldanaro(1994) port @ 440 ft bgs.

SAIC(1993e), Dreier, R. B. and A. Westbay well GW-729, Zone 41 measurement J. Caldanaro(1994) port @ 405 ft bgs.

SAIC(1993e), Dreier, R. B. and A. Westbay well GW-729, Zone 42 measurement J. Caldanaro(1994) 


\begin{tabular}{|c|c|c|c|c|c|c|c|}
\hline \multirow{3}{*}{$\begin{array}{l}\text { CURRENT } \\
\text { NAME }\end{array}$} & \multirow[b]{2}{*}{ OTHER } & & & \multirow{2}{*}{$\begin{array}{l}\text { MEAS } \\
\text { PORT }\end{array}$} & PUMP & \multirow[b]{3}{*}{ REFERENCE } & \multirow[b]{3}{*}{ COMMENTS } \\
\hline & & & & & PORT & & \\
\hline & NAME & PACKEF & INTERVAL & DEPTH & DEPTH & & \\
\hline GW-729-43 & GW-729 & 330.00 & to 360.00 & 330.00 & & $\begin{array}{l}\text { SAIC(1993e), Dreier, R. B. and A. } \\
\text { J. Caldanaro(1994) }\end{array}$ & $\begin{array}{l}\text { Westbay well GW-729, Zone } 43 \text { measurement } \\
\text { port @ } 330 \mathrm{ft} \text { bgs. }\end{array}$ \\
\hline GW-729-44 & GW-729 & 315.00 & to 325.00 & 320.00 & 325.00 & $\begin{array}{l}\text { SAIC(1993e), Dreier, R. B. and A. } \\
\text { J. Caldanaro(1994) }\end{array}$ & $\begin{array}{l}\text { Westbay well GW- } 729 \text {, Zone } 44 \text { measurement } \\
\text { port @ } 320 \mathrm{ft} \text { bgs, pumping port @ } 325 \mathrm{ft} \text { bgs. }\end{array}$ \\
\hline GW-729-45 & GW-729 & 281.50 & to 310.00 & 300.00 & & $\begin{array}{l}\text { SAIC(1993e), Dreier, R. B. and A. } \\
\text { J. Caldanaro(1994) }\end{array}$ & $\begin{array}{l}\text { Westbay well GW-729, Zone } 45 \text { measurement } \\
\text { port @ } 300 \mathrm{ft} \text { bgs. }\end{array}$ \\
\hline GW-730-01 & GW-730 & 1374.00 & to 1384.00 & 1374.00 & & $\begin{array}{l}\text { SAIC(1993C), Dreier, R. B. and A. } \\
\text { J. Caldanaro(1994) }\end{array}$ & $\begin{array}{l}\text { Westbay well GW-730, Zone } 1 \text { measurement } \\
\text { port @ } 1374 \mathrm{ft} \text { bgs. }\end{array}$ \\
\hline GW-730-02 & GW-730 & 1359.00 & to 1369.00 & 1364.00 & 1369.00 & $\begin{array}{l}\text { SAIC(1993C), Dreier, R. B. and A. } \\
\text { J. Caldanaro(1994) }\end{array}$ & $\begin{array}{l}\text { Westbay well GW-730, Zone } 2 \text { measurement } \\
\text { port @ } 1364 \mathrm{ft} \text { bgs, pumping port @ } 1369 \mathrm{ft} \\
\text { bgs. }\end{array}$ \\
\hline GW-730-03 & GW-730 & 1344.00 & to 1354.00 & 1344.00 & & $\begin{array}{l}\text { SAIC(1993C), Dreier, R. B. and A. } \\
\text { J. Caldanaro(1994) }\end{array}$ & $\begin{array}{l}\text { Westbay well GW-730, Zone } 3 \text { measurement } \\
\text { port @ } 1344 \mathrm{ft} \text { bgs. }\end{array}$ \\
\hline GW-730-04 & GW-730 & 1298.00 & to 1339.00 & 1298.00 & & $\begin{array}{l}\text { SAIC(1993C), Dreier, R. B. and A. } \\
\text { J. Caldanaro(1994) }\end{array}$ & $\begin{array}{l}\text { Westbay well GW-730, Zone } 4 \text { measurement } \\
\text { port @ } 1298 \mathrm{ft} \text { bgs. }\end{array}$ \\
\hline GW-730-05 & GW-730 & 1263.00 & to 1293.00 & 1263.00 & & $\begin{array}{l}\text { SAIC(1993C), Dreier, R. B. and A. } \\
\text { J. Caldanaro(1994) }\end{array}$ & $\begin{array}{l}\text { Westbay well GW-730, Zone } 5 \text { measurement } \\
\text { port @ } 1263 \mathrm{ft} \text { bgs. }\end{array}$ \\
\hline GW-730-06 & GW-730 & 1253.00 & to 1258.00 & 1253.00 & & $\begin{array}{l}\text { SAIC(1993C), Dreier, R. B. and A. } \\
\text { J. Caldanaro(1994) }\end{array}$ & $\begin{array}{l}\text { Westbay well GW-730, Zone } 6 \text { measurement } \\
\text { port @ } 1253 \mathrm{ft} \text { bgs. }\end{array}$ \\
\hline GW-730-07 & GW-730 & 1233.00 & to 1248.00 & 1243.00 & 1248.00 & $\begin{array}{l}\text { SAIC(1993C), Dreier, R. B. and A. } \\
\text { J. Caldanaro(1994) }\end{array}$ & $\begin{array}{l}\text { Westbay well GW-730, Zone } 7 \text { measurement } \\
\text { port @ } 1243 \mathrm{ft} \text { bgs, pumping port @ } 1248 \mathrm{ft} \\
\text { bgs. }\end{array}$ \\
\hline GW-730-08 & GW-730 & 1223.00 & to 1228.00 & 1223.00 & & $\begin{array}{l}\text { SAIC(1993C), Dreier, R. B. and A. } \\
\text { J. Caldanaro(1994) }\end{array}$ & $\begin{array}{l}\text { Westbay well GW-730, Zone } 8 \text { measurement } \\
\text { port @ } 1223 \mathrm{ft} \text { bgs. }\end{array}$ \\
\hline GW-730-09 & GW-730 & 1168.00 & to 1218.00 & 1182.00 & & $\begin{array}{l}\text { SAIC(1993C), Dreier, R. B. and A. } \\
\text { J. Caldanaro(1994) }\end{array}$ & $\begin{array}{l}\text { Westbay well GW-730, Zone } 9 \text { measurement } \\
\text { port @ } 1182 \mathrm{ft} \text { bgs. }\end{array}$ \\
\hline GW-730-10 & GW-730 & 1168.00 & to 1178.00 & 1168.00 & & $\begin{array}{l}\text { SAIC(1993C), Dreier, R. B. and A. } \\
\text { J. Caldanaro(1994) }\end{array}$ & $\begin{array}{l}\text { Westbay well GW-730, Zone } 10 \text { measurement } \\
\text { port @ } 1168 \mathrm{ft} \text { bgs. }\end{array}$ \\
\hline GW-730-11 & GW-730 & 1153.00 & to 1163.00 & 1158.00 & 1163.00 & $\begin{array}{l}\text { SAIC(1993C), Dreier, R. B. and A. } \\
\text { J. Caldanaro(1994) }\end{array}$ & $\begin{array}{l}\text { Westbay well GW-730, Zone } 11 \text { measurement } \\
\text { port @ } 1158 \mathrm{ft} \text { bgs, pumping port @ } 1163 \mathrm{ft} \\
\text { bgs. }\end{array}$ \\
\hline GW-730-12 & GW-730 & 1143.00 & to 1148.00 & 1143.00 & & $\begin{array}{l}\text { SAIC(1993C), Dreier, R. B. and A. } \\
\text { J. Caldanaro(1994) }\end{array}$ & $\begin{array}{l}\text { Westbay well GW-730, Zone } 12 \text { measurement } \\
\text { port @ } 1143 \mathrm{ft} \text { bgs. }\end{array}$ \\
\hline & & & & & & $\mathrm{H}-27$ & \\
\hline
\end{tabular}




\begin{tabular}{|c|c|c|c|c|}
\hline & & & & MEAS \\
\hline CURRENT & OTHER & & & PORT \\
\hline NAME & NAME & PACKEF & 2 INTERVAL & DEPTH \\
\hline GW-730-13 & GW-730 & 1108.00 & to 1138.00 & 1108.00 \\
\hline GW-730-14 & GW-730 & 1098.00 & to 1103.00 & 1098.00 \\
\hline GW-730-15 & GW-730 & 1078.00 & to 1093.00 & 1088.00 \\
\hline GW-730-16 & GW-730 & 1048.00 & to 1073.00 & 1048.00 \\
\hline GW-730-17 & GW-730 & 1018.00 & to 1043.00 & 1018.00 \\
\hline GW-730-18 & GW-730 & 1003.00 & to 1013.00 & 1008.00 \\
\hline GW-730-19 & GW-730 & 968.00 & to 998.00 & 968.00 \\
\hline GW-730-20 & GW-730 & 942.00 & to 963.00 & 942.00 \\
\hline GW-730-21 & GW-730 & 912.00 & to 937.00 & 922.00 \\
\hline GW-730-22 & GW-730 & 887.00 & to 907.00 & 887.00 \\
\hline GW-730-23 & GW-730 & 862.00 & to 882.00 & 862.00 \\
\hline GW-730-24 & GW-730 & 847.00 & to 857.00 & 847.00 \\
\hline GW-730-25 & GW-730 & 832.00 & to 842.00 & 837.00 \\
\hline GW-730-26 & GW-730 & 792.00 & to 827.00 & 792.00 \\
\hline GW-730-27 & GW-730 & 767.00 & to 787.00 & 767.0 \\
\hline
\end{tabular}

\section{PUMP \\ PORT \\ DEPTH}

SAIC(1993c), Dreier, R. B. and A. J. Caldanaro(1994)

SAIC(1993c), Dreier, R. B. and A. Westbay well GW-730, Zone 14 measurement J. Caldanaro(1994)

1093.00 SAIC(1993c), Dreier, R. B. and A.

J. Caldanaro(1994)

SAIC(1993c), Dreier, R. B. and A.

J. Caldanaro(1994)

SAIC(1993C), Dreier, R. B. and A.

J. Caldanaro(1994)

1013.00 SAIC(1993c), Dreier, R. B. and A.

J. Caldanaro(1994)

SAIC(1993c), Dreier, R. B. and A.

J. Caldanaro(1994)

SAIC(1993c), Dreier, R. B. and A.

J. Caldanaro(1994)

932.00 SAIC(1993C), Dreier, R. B. and A.

J. Caldanaro(1994)

SAIC(1993c), Dreier, R. B. and A.

J. Caldanaro(1994)

SAIC(1993c), Dreier, R. B. and A.

J. Caldanaro(1994)

SAIC(1993c), Dreier, R. B. and A.

J. Caldanaro(1994)

842.00 SAIC(1993c), Dreier, R. B. and A.

J. Caldanaro(1994)

SAIC(1993c), Dreier, R. B. and A.

J. Caldanaro(1994)

SAIC(1993c), Dreier, R. B. and A.

J. Caldanaro(1994)

\section{COMMENTS}

Westbay well GW-730, Zone 13 measurement ort @ 1108 ft bgs.

port @ 1098 ft bgs.

Westbay well GW-730, Zone 15 measurement port @ 1088 ft bgs, pumping port @ $1093 \mathrm{ft}$ bgs.

Westbay well GW-730, Zone 16 measurement port @ 1048 ft bgs.

Westbay well GW-730, Zone 17 measurement port @ $1088 \mathrm{ft}$ bgs.

Westbay well GW-730, Zone 18 measurement port @ 1008 ft bgs, pumping port @ $1013 \mathrm{ft}$ bgs.

Westbay well GW-730, Zone 19 measurement port @ 968 ft bgs.

Westbay well GW-730, Zone 20 measurement port @ 942 ft bgs.

Westbay well GW-730, Zone 21 measurement port @ 922 ft bgs, pumping port @ 932 ft bgs.

Westbay well GW-730, Zone 22 measurement port @ 887 ft bgs.

Westbay well GW-730, Zone 23 measurement port @ 862 ft bgs.

Westbay well GW-730, Zone 24 measurement port @ 847 ft bgs.

Westbay well GW-730, Zone 25 measurement port @ $837 \mathrm{ft}$ bgs, pumping port @ $842 \mathrm{ft}$ bgs.

Westbay well GW-730, Zone 26 measurement port @ $792 \mathrm{ft}$ bgs.

Westbay well GW-730, Zone 27 measurement port @ 767 ft bgs. 


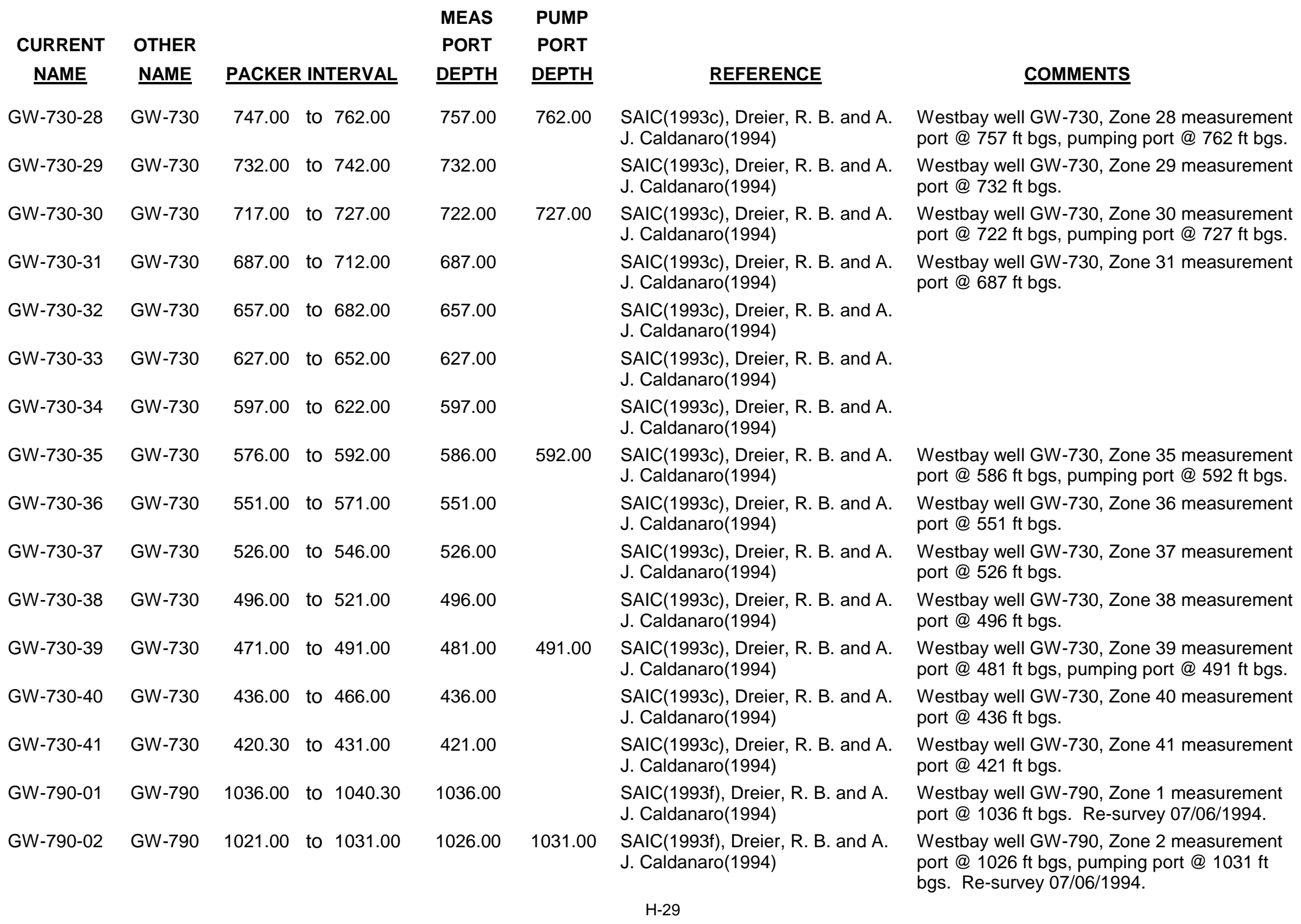




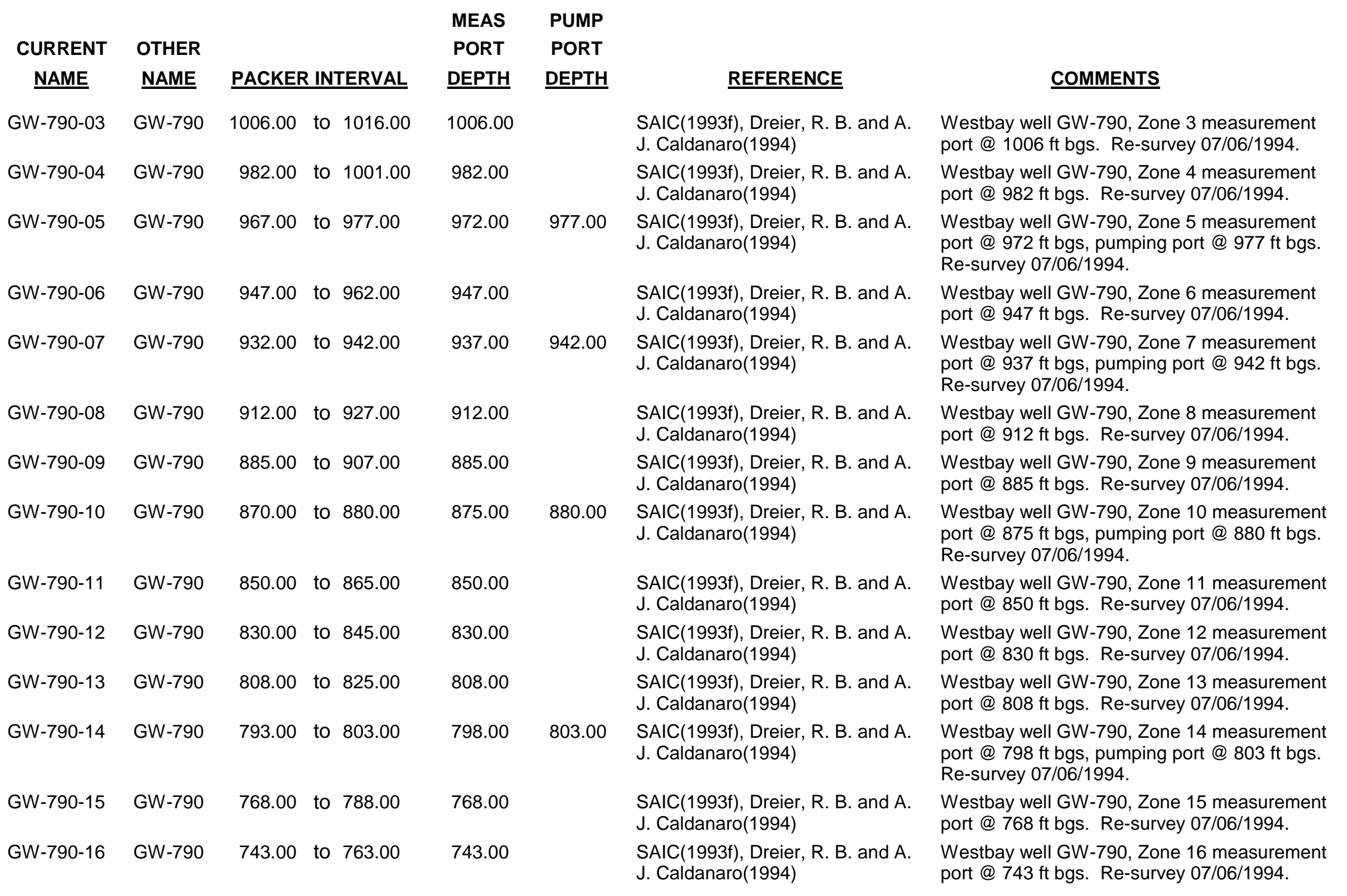




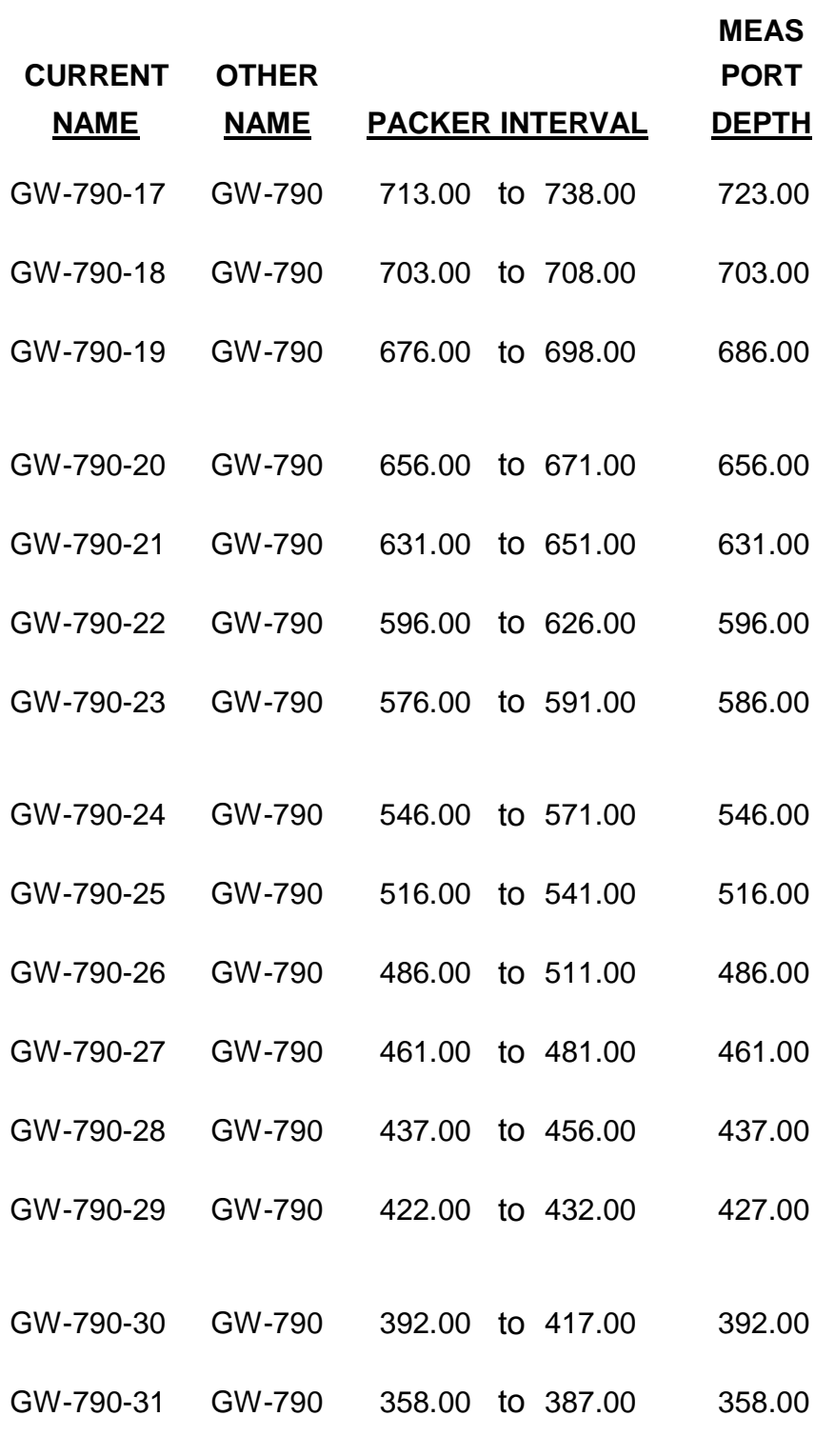

PUMP

PORT

DEPTH

REFERENCE

SAIC(1993f), Dreier, R. B. and A. J. Caldanaro(1994)

SAIC(1993f), Dreier, R. B. and A.

J. Caldanaro(1994)

698.00 SAIC(1993f), Dreier, R. B. and A. J. Caldanaro(1994)

SAIC(1993f), Dreier, R. B. and A. J. Caldanaro(1994)

SAIC(1993f), Dreier, R. B. and A.

J. Caldanaro(1994)

SAIC(1993f), Dreier, R. B. and A.

J. Caldanaro(1994)

591.00 SAIC(1993f), Dreier, R. B. and A. J. Caldanaro(1994)

SAIC(1993f), Dreier, R. B. and A. J. Caldanaro(1994)

SAIC(1993f), Dreier, R. B. and A.

J. Caldanaro(1994)

SAIC(1993f), Dreier, R. B. and A.

J. Caldanaro(1994)

SAIC(1993f), Dreier, R. B. and A.

J. Caldanaro(1994)

SAIC(1993f), Dreier, R. B. and A.

J. Caldanaro(1994)

432.00 SAIC(1993f), Dreier, R. B. and A.

J. Caldanaro(1994)

SAIC(1993f), Dreier, R. B. and A. J. Caldanaro(1994)

SAIC(1993f), Dreier, R. B. and A.

J. Caldanaro(1994)

\section{COMMENTS}

Westbay well GW-790, Zone 17 measurement port @ 723 ft bgs. Re-survey 07/06/1994.

Westbay well GW-790, Zone 18 measurement port @ 703 ft bgs. Re-survey 07/06/1994.

Westbay well GW-790, Zone 19 measurement port @ $686 \mathrm{ft}$ bgs, pumping port @ $698 \mathrm{ft}$ bgs. Re-survey 07/06/1994.

Westbay well GW-790, Zone 20 measurement port @ 656 ft bgs. Re-survey 07/06/1994.

Westbay well GW-790, Zone 21 measurement port @ 631 ft bgs. Re-survey 07/06/1994.

Westbay well GW-790, Zone 22 measurement port @ 596 ft bgs. Re-survey 07/06/1994.

Westbay well GW-790, Zone 23 measurement port @ $586 \mathrm{ft}$ bgs, pumping port @ $591 \mathrm{ft}$ bgs. Re-survey 07/06/1994.

Westbay well GW-790, Zone 24 measurement port @ 546 ft bgs. Re-survey 07/06/1994.

Westbay well GW-790, Zone 25 measurement port @ 516 ft bgs. Re-survey 07/06/1994.

Westbay well GW-790, Zone 26 measurement port @ 486 ft bgs. Re-survey 07/06/1994.

Westbay well GW-790, Zone 27 measurement port @ 461 ft bgs. Re-survey 07/06/1994.

Westbay well GW-790, Zone 28 measurement port @ $437 \mathrm{ft}$ bgs. Re-survey 07/06/1994.

Westbay well GW-790, Zone 29 measurement port @ $427 \mathrm{ft}$ bgs, pumping port @ $432 \mathrm{ft}$ bgs. Re-survey 07/06/1994.

Westbay well GW-790, Zone 30 measurement port @ $392 \mathrm{ft}$ bgs. Re-survey 07/06/1994.

Westbay well GW-790, Zone 31 measurement port @ 358 ft bgs. Re-survey 07/06/1994. 


\begin{tabular}{|c|c|c|c|c|c|c|c|}
\hline & & & & MEAS & PUMP & & \\
\hline CURRENT & OTHER & & & PORT & PORT & & \\
\hline NAME & NAME & PACKEF & 2 INTERVAL & DEPTH & DEPTH & REFERENCE & COMMENTS \\
\hline GW-790-32 & GW-790 & 343.00 & to 353.00 & 348.00 & 353.00 & $\begin{array}{l}\text { SAIC(1993f), Dreier, R. B. and A. } \\
\text { J. Caldanaro(1994) }\end{array}$ & $\begin{array}{l}\text { Westbay well GW-790, Zone } 32 \text { measurement } \\
\text { port @ } 348 \mathrm{ft} \text { bgs, pumping port @ } 353 \mathrm{ft} \text { bgs. } \\
\text { Re-survey 07/06/1994. }\end{array}$ \\
\hline GW-790-33 & GW-790 & 318.00 & to 338.00 & 318.00 & & $\begin{array}{l}\text { SAIC(1993f), Dreier, R. B. and A. } \\
\text { J. Caldanaro(1994) }\end{array}$ & $\begin{array}{l}\text { Westbay well GW-790, Zone } 33 \text { measurement } \\
\text { port @ } 318 \mathrm{ft} \text { bgs. Re-survey 07/06/1994. }\end{array}$ \\
\hline GW-790-34 & GW-790 & 292.00 & to 313.00 & 292.00 & & $\begin{array}{l}\text { SAIC(1993f), Dreier, R. B. and A. } \\
\text { J. Caldanaro(1994) }\end{array}$ & $\begin{array}{l}\text { Westbay well GW-790, Zone } 34 \text { measurement } \\
\text { port @ } 292 \mathrm{ft} \text { bgs. Re-survey 07/06/1994. }\end{array}$ \\
\hline GW-790-35 & GW-790 & 277.00 & to 287.00 & 282.00 & 287.00 & $\begin{array}{l}\text { SAIC(1993f), Dreier, R. B. and A. } \\
\text { J. Caldanaro(1994) }\end{array}$ & $\begin{array}{l}\text { Westbay well GW-790, Zone } 35 \text { measurement } \\
\text { port @ } 282 \mathrm{ft} \text { bgs, pumping port @ } 287 \mathrm{ft} \text { bgs } \\
\text { Re-survey 07/06/1994.. }\end{array}$ \\
\hline GW-790-36 & GW-790 & 260.00 & to 272.00 & 260.00 & & $\begin{array}{l}\text { SAIC(1993f), Dreier, R. B. and A. } \\
\text { J. Caldanaro(1994) }\end{array}$ & $\begin{array}{l}\text { Westbay well GW-790, Zone } 36 \text { measurement } \\
\text { port @ } 260 \mathrm{ft} \text { bgs. Re-survey 07/06/1994. }\end{array}$ \\
\hline GW-790-37 & GW-790 & 235.00 & to 255.00 & 235.00 & & $\begin{array}{l}\text { SAIC(1993f), Dreier, R. B. and A. } \\
\text { J. Caldanaro(1994) }\end{array}$ & $\begin{array}{l}\text { Westbay well GW-790, Zone } 37 \text { measurement } \\
\text { port @ } 235 \mathrm{ft} \text { bgs. Re-survey 07/06/1994. }\end{array}$ \\
\hline GW-790-38 & GW-790 & 220.00 & to 230.00 & 220.00 & & $\begin{array}{l}\text { SAIC(1993f), Dreier, R. B. and A. } \\
\text { J. Caldanaro(1994) }\end{array}$ & $\begin{array}{l}\text { Westbay well GW-790, Zone } 38 \text { measurement } \\
\text { port @ } 220 \mathrm{ft} \text { bgs. Re-survey 07/06/1994. }\end{array}$ \\
\hline GW-790-39 & GW-790 & 212.50 & to 215.00 & 203.00 & 215.00 & $\begin{array}{l}\text { SAIC(1993f), Dreier, R. B. and A. } \\
\text { J. Caldanaro(1994) }\end{array}$ & $\begin{array}{l}\text { Westbay well GW-790, Zone } 39 \text { measurement } \\
\text { port @ } 203 \mathrm{ft} \text { bgs, pumping port @ } 215 \mathrm{ft} \text { bgs. } \\
\text { Re-survey 07/06/1994. }\end{array}$ \\
\hline GW-821-01 & GW-821 & 43.00 & to 50.50 & 43.25 & & $\begin{array}{l}\text { Moline, G. R. and M. E. } \\
\text { Schreiber(1996) }\end{array}$ & \\
\hline GW-821-02 & GW-821 & 38.00 & to 39.75 & 38.75 & & $\begin{array}{l}\text { Moline, G. R. and M. E. } \\
\text { Schreiber(1996) }\end{array}$ & \\
\hline GW-821-03 & GW-821 & 33.50 & to 35.00 & 34.25 & & $\begin{array}{l}\text { Moline, G. R. and M. E. } \\
\text { Schreiber(1996) }\end{array}$ & \\
\hline GW-821-04 & GW-821 & 25.00 & to 26.50 & 25.75 & & $\begin{array}{l}\text { Moline, G. R. and M. E. } \\
\text { Schreiber(1996) }\end{array}$ & \\
\hline GW-821-05 & GW-821 & 19.25 & to 21.50 & 20.25 & & $\begin{array}{l}\text { Moline, G. R. and M. E. } \\
\text { Schreiber(1996) }\end{array}$ & \\
\hline GW-822-01 & GW-822 & 62.00 & to 63.60 & 62.75 & & $\begin{array}{l}\text { Moline, G. R. and M. E. } \\
\text { Schreiber(1996) }\end{array}$ & \\
\hline \multirow[t]{2}{*}{ GW-822-02 } & GW-822 & 53.50 & to 55.00 & 54.25 & & $\begin{array}{l}\text { Moline, G. R. and M. E. } \\
\text { Schreiber(1996) }\end{array}$ & \\
\hline & & & & & & $\mathrm{H}-32$ & \\
\hline
\end{tabular}




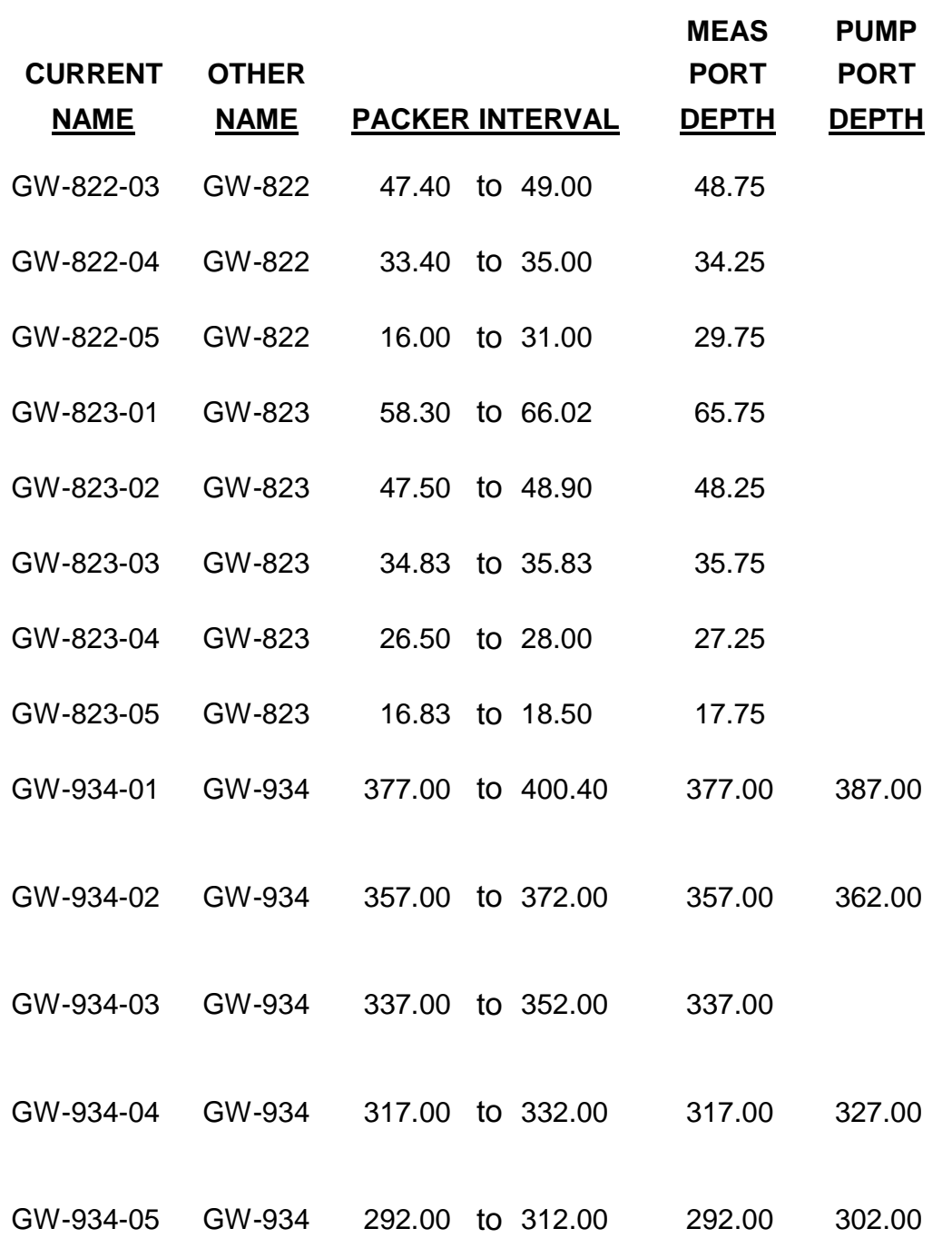

\section{REFERENCE}

Moline, G. R. and M. E. Schreiber(1996)

Moline, G. R. and M. E. Schreiber(1996)

Moline, G. R. and M. E. Schreiber(1996)

Moline, G. R. and M. E. Schreiber(1996)

Moline, G. R. and M. E. Schreiber(1996)

Moline, G. R. and M. E. Schreiber(1996)

Moline, G. R. and M. E. Schreiber(1996)

Moline, G. R. and M. E. Schreiber(1996)

Westbay well GW-934, Zone 1 measurement port @ 377 ft bgs, pumping port @ 387 ft bgs Survey info updated 07/08/2008.

Westbay well GW-934, Zone 2 measurement port @ 357 ft bgs, pumping port @ 362 ft bgs Survey info updated 07/08/2008.

Westbay well GW-934, Zone 3 measurement port @ 337 ft bgs. Survey info updated 07/08/2008.

Westbay well GW-934, Zone 4 measurement port @ $317 \mathrm{ft}$ bgs, pumping port @ 327 ft bgs. Survey info updated 07/08/2008.

Westbay well GW-934, Zone 5 measurement port @ $292 \mathrm{ft}$ bgs, pumping port @ $302 \mathrm{ft}$ bgs. Survey info updated 07/08/2008. 


\begin{tabular}{|c|c|c|c|c|c|}
\hline & & & & MEAS & PUMP \\
\hline CURRENT & OTHER & & & PORT & PORT \\
\hline NAME & NAME & PACKER & ? INTERVAL & DEPTH & DEPTH \\
\hline GW-934-06 & GW-934 & 277.00 & to 287.00 & 277.00 & \\
\hline GW-934-07 & GW-934 & 257.00 & to 272.00 & 257.00 & 262.00 \\
\hline GW-934-08 & GW-934 & 247.00 & to 252.00 & 247.00 & \\
\hline GW-934-09 & GW-934 & 227.00 & to 242.00 & 227.00 & 232.00 \\
\hline GW-934-10 & GW-934 & 212.00 & to 222.00 & 212.00 & \\
\hline GW-934-11 & GW-934 & 192.00 & to 207.00 & 192.00 & 197.00 \\
\hline GW-934-12 & GW-934 & 70.00 & to 187.00 & 70.00 & 75.00 \\
\hline GW-954-1 & GW-954 & 78.00 & to 104.00 & 90.00 & \\
\hline GW-954-2 & GW-954 & 52.00 & to 68.00 & 58.00 & \\
\hline GW-954-3 & GW-954 & 28.00 & to 38.00 & 31.00 & \\
\hline GW-956-1 & GW-956 & 108.00 & to 124.00 & 111.50 & \\
\hline GW-956-2 & GW-956 & 78.00 & to 98.00 & 86.00 & \\
\hline GW-956-3 & GW-956 & 50.00 & to 62.00 & 54.00 & \\
\hline GW-956-4 & GW-956 & 18.00 & to 36.00 & 25.00 & \\
\hline
\end{tabular}

REFERENCE

Westbay well GW-934, Zone 6 measurement port @ $277 \mathrm{ft}$ bgs. Survey info updated 07/08/2008.

Westbay well GW-934, Zone 7 measurement port @ 257 ft bgs, pumping port @ 262 ft bgs. Survey info updated 07/08/2008.

Westbay well GW-934, Zone 8 measurement port @ $247 \mathrm{ft}$ bgs. Survey info updated 07/08/2008.

Westbay well GW-934, Zone 9 measurement port @ $227 \mathrm{ft}$ bgs, pumping port @ 232 Ft bgs. Survey info updated 07/08/2008.

Westbay well GW-934, Zone 10 measurement port @ $212 \mathrm{ft}$ bgs. Survey info updated 07/08/2008.

Westbay well GW-934, Zone 11 measurement port @ 192 ft bgs, pumping port @ 197 Ft bgs. Survey info updated 07/08/2008.

Westbay well GW-934, Zone 12 measurement port @ 70 ft bgs, pumping port @ 75 Ft bgs. Survey info updated 07/08/2008. 
APPENDIX I

Surface Water Location Summary 
This Page Intentionally Left Blank 


\begin{tabular}{|c|c|c|c|c|c|c|c|c|}
\hline CURRENT & & LOC & & & & & & \\
\hline NAME & OTHER NAME & TYPE & FUNCTIONAL AREA & EASTING & NORTHING & SYSTEM & MAP & SAMPLING HISTORY \\
\hline 304 & & $\mathrm{RV}$ & Unknown & 27571.23 & 29128.64 & $\mathrm{Y}-12$ & 2 & \\
\hline 9201-1 K-22 SU & & SU & Y-12 Plant Site & 59380.00 & 29425.00 & Admin & $5 \& 6$ & Q2/04, Q4/04 \\
\hline $\begin{array}{l}\text { 9201-2 Sump } \\
\text { E22 }\end{array}$ & $\begin{array}{l}\text { Bldg. 9201-2 Sump } \\
\text { E-22 }\end{array}$ & SU & Exit Pathway Spring/Surface Water & 60134.00 & 29236.00 & Y-12 & $5 \& 6$ & Q1/97, Q2/98-Q3/98 \\
\hline 9201-2 Sump G & Bldg. 9201-2 Sump G & SU & Exit Pathway Spring/Surface Water & 59903.00 & 29313.00 & $\mathrm{Y}-12$ & $5 \& 6$ & Q3/97 \\
\hline 9201-3C-4SP & & SP & Y-12 Plant Site & 60528.59 & 29132.89 & Y-12 & $5 \& 6$ & $\mathrm{Q} 2 / 04, \mathrm{Q} 4 / 04$ \\
\hline 9201-4 Sump B & Bldg. 9201-4 Sump B & SU & Exit Pathway Spring/Surface Water & 56686.00 & 30216.00 & $\mathrm{Y}-12$ & 5 & Q2/98-Q3/98 \\
\hline 9201-4 Sump G & Bldg. 9201-4 Sump G & SU & Exit Pathway Spring/Surface Water & 56203.00 & 30066.00 & $\mathrm{Y}-12$ & 5 & Q1/97, Q3/97, Q2/98-Q3/98 \\
\hline 9201-4 Sump H & Bldg. 9201-4 Sump H & SU & Exit Pathway Spring/Surface Water & 56224.00 & 30260.00 & Y-12 & 5 & Q2/98-Q3/98 \\
\hline 9201-5 Sump A & A5-SU A & SU & Y-12 Plant Site & 55791.04 & 30103.50 & Y-12 & 5 & $\mathrm{Q} 4 / 12$ \\
\hline 9201-5 Sump E & Bldg. 9201-5 Sump E & SU & Exit Pathway Spring/Surface Water & 55559.67 & 30138.13 & $\mathrm{Y}-12$ & 5 & Q1/97, Q3/97, Q2/98-Q3/98, Q4/12 \\
\hline $9201-5 N-J 4$ & & SU & Y-12 Plant Site & 55109.13 & 30534.76 & $\mathrm{Y}-12$ & 5 & Q1/99 \\
\hline $\begin{array}{l}\text { 9204-1 Sump } \\
\text { E24 }\end{array}$ & $\begin{array}{l}\text { Bldg. 9204-1 Sump } \\
\text { E-24 }\end{array}$ & su & Exit Pathway Spring/Surface Water & 58021.00 & 29401.00 & Y-12 & 5 & Q2/98-Q3/98 \\
\hline 9204-2E_NW & & SU & Y-12 Plant Site & 56949.53 & 30355.79 & $\mathrm{Y}-12$ & 5 & Q1/99 \\
\hline 9204-2-NE & & SU & Y-12 Plant Site & 57200.00 & 30100.00 & Y-12 & 5 & Q1/99 \\
\hline 9204-2NW & & SU & Y-12 Plant Site & 57027.91 & 30153.32 & $\mathrm{Y}-12$ & 5 & Q1/99 \\
\hline 9204-4ELSU & & SU & Y-12 Plant Site & 54600.00 & 30200.00 & $\mathrm{Y}-12$ & 5 & Q3/97, Q2/98-Q3/98, Q1/99 \\
\hline 9212-W-2-BSTM & 9212-W2 & SU & Y-12 Plant Site & 57475.02 & 31151.40 & Y-12 & 5 & Q1/00 \\
\hline 9215-STACK 11 & & SU & Y-12 Plant Site & 57085.27 & 30473.84 & $\mathrm{Y}-12$ & 5 & $\mathrm{Q} 4 / 00-\mathrm{Q} 2 / 01$ \\
\hline $\begin{array}{l}\text { 9402-1 Sump } \\
\text { E24 }\end{array}$ & $\begin{array}{l}\text { Bldg. 9402-1 Sump } \\
\text { E-24 }\end{array}$ & su & Exit Pathway Spring/Surface Water & 58021.00 & 29401.00 & $\mathrm{Y}-12$ & 5 & Q3/97 \\
\hline 9422-15 & & PP & Exit Pathway Spring/Surface Water & 55077.96 & 30092.47 & ADMIN & 5 & \\
\hline
\end{tabular}




\section{CURRENT NAME}

9422-16

94223

BCK 11.54

BCK 11.59

BCK 11.84

BCK 12.34

BCK 12.38

BCK 12.47

BCK 3.3

BCK 4.6

BCK 9.12

BCK 9.47
LOC

OTHER NAME

9422-3,STATION 8

PP Exit Pathway Spring/Surface Water

RV Exit Pathway Spring/Surface Water

RV Unknown

RV Exit Pathway Spring/Surface Water

RV Unknown

RV Bear Creek Exit Pathway

RV Exit Pathway Spring/Surface Wate

RV Exit Pathway Spring/Surface Water

BCK $3.3, B C K$ 03.3,BCK-03.3,BCK-

3.3

\section{EASTING NORTHING SYSTEM MAP}

SAMPLING HISTORY

55122.2930078 .08 ADMIN 5

$59621.42 \quad 29139.39$

Y-12 5 \& 6 Q1/96-Q3/98, Q1/99-Q3/99, Q1/00Q1/04, Q3/04, Q1/05, Q3/05, Q1/06, Q3/06-Q1/10, Q3/10, Q1/11-Q3/11, Q1/12, Q3/12

$47560.00 \quad 29200.00 \quad Y-12$

4 Q3/01-Q3/03, Q1/04, Q3/04-Q1/05, Q3/05, Q1/06, Q3/06, Q4/08-Q1/09, Q3/09-Q3/10, Q1/11, Q3/11-Q4/11, Q2/12, Q4/12

$\begin{array}{llll}47871.50 & 29327.50 \quad \text { Admin } 4 \text { Q4/00-Q3/01 }\end{array}$

$48710.00 \quad 29480.00 \quad Y-12$

$4 \mathrm{Q} 3 / 01-\mathrm{Q} 2 / 02, \mathrm{Q} 4 / 02-\mathrm{Q} 3 / 03, \mathrm{Q} 1 / 04$, Q3/04-Q1/05, Q3/05, Q1/06, Q3/06, Q4/09-Q3/10

$50246.00 \quad 29648.00$

$50490.00 \quad 29657.00$

$\mathrm{Y}-124$ \& 5 Q2/99-Q3/06, Q4/07-Q4/12

$\mathrm{Y}-124$ \& 5 Q1/98, Q3/98

$50966.00 \quad 29573.00$

$\mathrm{Y}-124$ \& 5 Q3/97-Q1/98, Q3/98, Q2/99-Q3/99, Q1/00-Q3/06

$26304.42 \quad 32636.46 \quad$ Admin

$2 \mathrm{Q} 1 / 01, \mathrm{Q} 3 / 01, \mathrm{Q} 1 / 02, \mathrm{Q} 3 / 02, \mathrm{Q} 1 / 03$, Q3/03, Q1/04, Q3/04, Q1/05, Q3/05, $\mathrm{Q} 1 / 06, \mathrm{Q} 3 / 06, \mathrm{Q} 4 / 09-\mathrm{Q} 3 / 10, \mathrm{Q} 4 / 11$, Q2/12, Q4/12

$27676.5329213 .26 \quad \mathrm{Y}-12 \quad 2$ Q1/10, Q3/10, Q1/11, Q3/11-Q4/11, $\mathrm{Q} 2 / 12, \mathrm{Q} 4 / 12$

$40556.00 \quad 28636.00 \quad \mathrm{Y}-12 \quad 3 \& 4$ Q1/99

$41380.0028800 .00 \quad \mathrm{Y}-12 \quad 4 \quad \mathrm{Q} 3 / 99, \mathrm{Q} 1 / 00, \mathrm{Q} 3 / 00, \mathrm{Q} 1 / 01, \mathrm{Q} / 01$ $\mathrm{Q} 2 / 02, \mathrm{Q} 4 / 02-\mathrm{Q} 3 / 03, \mathrm{Q} 1 / 04, \mathrm{Q} 3 / 04-$ Q3/05, Q1/06, Q3/06 


\section{CURRENT \\ NAME}

BCK-00.63

BCK-03.87

BCK-04.55

BCK 4.55,OF-304

RV Exit Pathway Spring/Surface Water

RV Exit Pathway Spring/Surface Water

$\begin{array}{ll}\text { BCK-05.95 } & \text { SS-5.95KM } \\ \text { BCK-06.60 } & \text { SS-6.6,SS- } \\ & 6.60 \mathrm{KM}, \text { SS-6.6K }\end{array}$

BCK-07.75

BCK-07.87

BCK-09.40

BCK 9.4

LOC

\section{TYPE FUNCTIONAL AREA}

RV Exit Pathway Spring/Surface Water

RV Exit Pathway Spring/Surface Water

RV Exit Pathway Spring/Surface Water

RV Exit Pathway Spring/Surface Water

RV Exit Pathway Spring/Surface Water

RV Exit Pathway Spring/Surface Water
BCK-09.47

$36500.00 \quad 29000.00$

\section{EASTING NORTHING SYSTEM MAP SAMPLING HISTORY}

$22929.0036586 .00 \quad \mathrm{Y}-12 \quad 7$ Q1/92-Q4/92, Q3/96, Q1/97, Q3/97, Q1/98, Q3/98, Q1/99, Q3/99, Q1/00, Q3/00, Q1/01, Q3/01, Q1/02, Q3/02, Q1/03, Q3/03, Q1/04, Q3/04, Q1/05, Q3/05

$41208.90 \quad 28651.98$

Y-12

$27570.00 \quad 30790.00 \quad Y-12$

$27613.00 \quad 28965.00 \quad Y-12$

$29389.00 \quad 28544.00$

$30365.00 \quad 28554.00$

$36644.06 \quad 28838.37$

Y-12

$\mathrm{Y}-12$

$\mathrm{Y}-12$

$\mathrm{Y}-12$

\section{Q}

Q1/01, Q3/01, Q1/02, Q3/02, Q1/03, Q3/03, Q1/04, Q3/04, Q1/05, Q3/05, Q1/06, Q3/06, Q4/09-Q3/10, Q1/12, Q3/12

4 Q3/90-Q1/94, Q3/94, Q1/95, Q3/95, Q1/96, Q3/96, Q1/97-Q3/97, Q1/98, Q3/98, Q1/99, Q3/99, Q1/00, Q3/00, Q1/01, Q3/01, Q1/02, Q3/02, Q1/03, Q3/03, Q2/08, Q3/09, Q1/10, Q3/11, Q1/12, Q3/13 


\begin{tabular}{|c|c|c|c|}
\hline CURRENT & & LOC & \\
\hline NAME & OTHER NAME & TYPE & FUNCTIONAL AREA \\
\hline BCK-10.60 & & RV & Exit Pathway Spring/Surface Water \\
\hline BCK-11.54 & & RV & Exit Pathway Spring/Surface Water \\
\hline BCK-11.84 & & RV & Exit Pathway Spring/Surface Water \\
\hline BCK-11.97 & BCK 11.97 & RV & Exit Pathway Spring/Surface Water \\
\hline BCK9.2 & BCK 9.2,BCK-09.20 & RV & Bear Creek Exit Pathway \\
\hline CB-E & & RV & Construction/Demolition Landfill VII \\
\hline CB-W & & RV & Industrial Landfill V \\
\hline CWESTTOE & & PP & Bear Creek Burial Grounds WMA \\
\hline $\begin{array}{l}\text { EEVOC- } \\
\text { INFLUENT }\end{array}$ & $\begin{array}{l}9422-22 \text { TF INF,GW- } \\
845\end{array}$ & PP & Y-12 Plant Site \\
\hline EFK 24.1 & LRBP-1 & RV & Exit Pathway Spring/Surface Water \\
\hline EMWNT-03 & & RV & Unknown \\
\hline EMWNT-03A & & RV & Unknown \\
\hline EMWNT-05 & & RV & Unknown \\
\hline EMW-VWEIR & & RV & Unknown \\
\hline ET-4 & $\begin{array}{l}\text { White Wing Scrap } \\
\text { Yard }\end{array}$ & RV & Exit Pathway Spring/Surface Water \\
\hline GCS1 & & SP & Grassy Creek \\
\hline
\end{tabular}

\section{EASTING NORTHING SYSTEM MAP SAMPLING HISTORY}

$45300.00 \quad 29400.00 \quad$ Admin

4 Q1/96, Q3/96, Q1/97, Q3/97, Q1/98, Q3/98, Q1/99

$\begin{array}{llll}47560.00 & 29200.00 \quad Y-12 \quad 4\end{array}$

$\begin{array}{llll}48710.00 & 29480.00 \quad Y-12 & 4\end{array}$

$49188.00 \quad 29481.00 \quad \mathrm{Y}-12 \quad 4$ \& 5 Q3/90-Q4/92, Q1/94, Q3/94, Q1/95,

Q3/95, Q1/96, Q3/96, Q1/97, Q3/97,

Q1/98, Q3/98, Q1/99, Q3/99, Q1/00,

$\mathrm{Q} 3 / 00, \mathrm{Q} 1 / 01, \mathrm{Q} 3 / 01, \mathrm{Q} 1 / 02, \mathrm{Q} 3 / 02$,

Q1/03, Q3/03, Q2/08, Q3/09, Q1/10,

Q3/11, Q1/12, Q3/13

$39972.9428638 .95 \quad \mathrm{Y}-12 \quad 3$ \& 4 Q2/97-Q3/97, Q1/98, Q3/98, Q1/01,

$\mathrm{Q} 3 / 01-\mathrm{Q} 3 / 03, \mathrm{Q} 1 / 04, \mathrm{Q} 3 / 04-\mathrm{Q} 1 / 05$,

Q3/05-Q1/06, Q3/06, Q4/08-Q4/12

$61564.04 \quad 25974.26$ Admin 6 Q1/12

$59718.33 \quad 25550.45$ Admin 5 \& 6 Q1/12

$42328.00 \quad 30208.00 \quad \mathrm{Y}-12 \quad 4 \quad$ Q2/97-Q3/97, Q2/98-Q3/98

$\begin{array}{lllll}64392.00 & 28601.00 & \mathrm{Y}-12 & 6 & \mathrm{Q} 3 / 00-\mathrm{Q} 4 / 12\end{array}$

$64123.3129197 .52 \quad \mathrm{Y}-12 \quad 6 \quad \mathrm{Q} 4 / 11-\mathrm{Q} 4 / 12$

$\begin{array}{lllll}48500.00 & 30610.00 \quad \mathrm{Y}-12 & 4 & \mathrm{Q} 1 / 03\end{array}$

48405.4730533 .17 Admin 4 Q3/03-Q2/06, Q4/06-Q4/12

$\begin{array}{lllll}45802.92 & 30189.33 \quad \mathrm{Y}-12 \quad 4 \quad \mathrm{Q} 1 / 03-\mathrm{Q} 4 / 12\end{array}$

$45708.9529617 .18 \quad \mathrm{Y}-12 \quad 4 \quad \mathrm{Q} 1 / 03-\mathrm{Q} 3 / 03, \mathrm{Q} 1 / 04, \mathrm{Q} 4 / 04-\mathrm{Q} 4 / 12$

$26280.00 \quad 35070.00 \quad \mathrm{Y}-12 \quad 7 \quad \mathrm{Q} 1 / 01, \mathrm{Q} 1 / 03$

$\begin{array}{lllll}14900.00 & 27900.00 & \text { Y-12 } & 1 & \text { Q2/95, Q4/95, Q1/13 }\end{array}$ 


\section{CURRENT \\ NAME}

GHK2.51ESW

GHK2.51WSW

KERRHOLLOW

POND

KHB-01

KHB-02

KHB-03

KHB-04

KHB-05

KHB-06

KHB-07

KHB-08

KHB-09

KHB-10

KHB-11

KHB-11A

KHB-12

KHB-13

KHB-14
LOC

OTHER NAME

\section{TYPE FUNCTIONAL AREA}

RV Exit Pathway Spring/Surface Water

RV Exit Pathway Spring/Surface Water

LK Kerr Hollow Quarry

RV Exit Pathway Spring/Surface Water

RV Exit Pathway Spring/Surface Water

RV Exit Pathway Spring/Surface Water

RV Exit Pathway Spring/Surface Water

RV Exit Pathway Spring/Surface Water

RV Exit Pathway Spring/Surface Water

RV Exit Pathway Spring/Surface Water

RV Exit Pathway Spring/Surface Water

RV Exit Pathway Spring/Surface Water

RV Exit Pathway Spring/Surface Water

RV Exit Pathway Spring/Surface Water

RV Exit Pathway Spring/Surface Water

RV Exit Pathway Spring/Surface Water

RV Exit Pathway Spring/Surface Water

RV Exit Pathway Spring/Surface Water

\section{EASTING NORTHING SYSTEM MAP SAMPLING HISTORY}

$39870.0034751 .00 \quad \mathrm{Y}-12 \quad 7$ Q3/99, Q2/00, Q4/00, Q2/01, Q2/02, $\mathrm{Q} 4 / 02, \mathrm{Q} 2 / 03, \mathrm{Q} 4 / 03, \mathrm{Q} 2 / 04, \mathrm{Q} 4 / 04$, Q2/05, Q4/05

$39820.00 \quad 34751.00 \quad$ Y-12 7

$\mathrm{O} 3 / 99, \mathrm{Q} 2 / 00, \mathrm{O} 4 / 00, \mathrm{O} 2 / 01, \mathrm{Q} 4 / 01$ $\mathrm{Q} 2 / 02, \mathrm{Q} 4 / 02, \mathrm{Q} 2 / 03, \mathrm{Q} 4 / 03, \mathrm{Q} 2 / 04$ Q4/04, Q2/05, Q4/05, Q2/06, Q4/07, Q4/08-Q1/09, Q3/10, Q1/11, Q3/12,

Q1/13

$\begin{array}{lllll}63472.35 & 24626.69 & \text { Admin } & 6 & \mathrm{Q} 1 / 12\end{array}$

$63547.00 \quad 22998.00 \quad$ Admin 6

$63495.00 \quad 22963.00 \quad$ Admin 6

$63500.00 \quad 22981.00 \quad$ Admin 6

$63309.00 \quad 23037.00 \quad$ Admin 6

$63213.00 \quad 23212.00 \quad$ Admin 6

$63132.00 \quad 23403.00 \quad$ Admin 6

$63027.00 \quad 23606.00 \quad$ Admin 6

$62877.00 \quad 23722.00 \quad$ Admin 6

$62865.00 \quad 23932.00 \quad$ Admin 6

$62916.00 \quad 24127.00 \quad$ Admin 6

$63051.00 \quad 24273.00 \quad$ Admin 6

$63103.00 \quad 24365.00 \quad$ Admin 6

$\begin{array}{lllll}63149.06 & 24403.83 & \text { Admin } 6 & \text { Q1/12 }\end{array}$

$63214.00 \quad 24958.00 \quad$ Admin 6

$63303.00 \quad 24770.00 \quad$ Admin 6

\section{Q4/11-Q4/12}




\section{CURRENT NAME}

KHB-15

KHB-16

KHB-17

KHB-18

KHB-19

KHB-20

KHB-21

KHQ1

LRS

LRSPW

MCK 1.4

MCK 2.0

MCK 2.05

MS1

\section{OTHER NAME}

LOC

\section{TYPE FUNCTIONAL AREA}

RV Exit Pathway Spring/Surface Water

RV Exit Pathway Spring/Surface Water

RV Exit Pathway Spring/Surface Water

RV Exit Pathway Spring/Surface Water

RV Exit Pathway Spring/Surface Water

RV Exit Pathway Spring/Surface Water

RV Exit Pathway Spring/Surface Water

LK Kerr Hollow Quarry

SU New Hope Pond

SP Exit Pathway Spring/Surface Water

SL Exit Pathway Spring/Surface Water

SL Unknown

SL Unknown

MS-1

\section{EASTING NORTHING SYSTEM MAP}

SAMPLING HISTORY

63445.8424928 .39 Admin 6

$63557.5625103 .36 \quad$ Admin 6

63690.0625251 .00 Admin 6 Q1/12

$63633.20 \quad 25456.88 \quad$ Admin 6

$63570.60 \quad 25649.06 \quad$ Admin 6

$63559.88 \quad 25854.40 \quad$ Admin 6

$\begin{array}{lllll}63527.10 & 26048.32 & \text { Admin } 6 & \text { Q1/12 }\end{array}$

$63359.00 \quad 24596.00 \quad$ Admin 6

$64117.0029420 .00 \quad \mathrm{Y}-12 \quad 6 \quad$ Q3/94-Q4/94, Q1/96, Q3/12

$63960.0029694 .00 \quad \mathrm{Y}-12 \quad 6 \quad$ Q3/94-Q1/95, Q3/95, Q1/96, Q3/96, Q2/97, Q4/97, Q2/98, Q4/98, Q2/99, Q4/99, Q2/00, Q4/00, Q2/01, Q4/01, Q2/02, Q4/02

$56383.00 \quad 24044.00 \quad Y-12$

$57090.00 \quad 26210.00 \quad Y-12$

5 Q2/97-Q3/97, Q1/09, Q3/09, Q1/10, Q3/10, Q1/11, Q3/11, Q1/12, Q3/12

$5 \mathrm{Q} 1 / 98, \mathrm{Q} 3 / 98, \mathrm{Q} 1 / 99, \mathrm{Q} 3 / 99, \mathrm{Q} 1 / 00$ $\mathrm{Q} 3 / 00, \mathrm{Q} 1 / 01, \mathrm{Q} 3 / 01, \mathrm{Q} 1 / 02, \mathrm{Q} 3 / 02$ Q1/03, Q3/03, Q1/04, Q3/04, Q1/05, Q3/05, Q1/06, Q3/06, Q1/07, Q3/07, Q1/08, Q3/08, Q1/09, Q3/09, Q1/10, Q3/10, Q1/11, Q3/11, Q1/1

$57105.00 \quad 26280.00 \quad Y-12$

$5 \mathrm{Q} 1 / 98, \mathrm{Q} 3 / 98, \mathrm{Q} 1 / 99, \mathrm{Q} 3 / 99, \mathrm{Q} 1 / 00$, Q3/00, Q1/01, Q3/01, Q1/02, Q3/02, Q1/03, Q3/03, Q1/04, Q3/04, Q1/05, $\mathrm{Q} 3 / 05, \mathrm{Q} 1 / 06, \mathrm{Q} 3 / 06, \mathrm{Q} 1 / 07, \mathrm{Q} 3 / 07$, Q1/08, Q3/08, Q1/09, Q3/09, Q1/10, Q3/10, Q1/11, Q3/11, Q1/1

$\begin{array}{llll}51000.00 \quad 29700.00 \quad Y-12 \quad 4 \& 5 & Q 2 / 95\end{array}$ 


\section{CURRENT NAME \\ NHPCEMSP \\ NPR07.0SW \\ NPR10.0SW \\ NPR12.0SW}

NPR23.0SW

N-S PIPE

NT-01

NT-02

NT-04

NT-05

NT-06
LOC

OTHER NAME

\section{TYPE FUNCTIONAL AREA}

SP Exit Pathway Spring/Surface Water

RV Exit Pathway Spring/Surface Water

RV Exit Pathway Spring/Surface Water

RV Exit Pathway Spring/Surface Water

RV Exit Pathway Spring/Surface Water

OF-200 N/S PIPE

NT-1

RV Exit Pathway Spring/Surface Water

RV Exit Pathway Spring/Surface Water

RV Unknown

NT-5 $\mathrm{H}$ flume

RV Unknown

RV Exit Pathway Spring/Surface Water

\section{EASTING NORTHING SYSTEM MAP SAMPLING HISTORY}

$\begin{array}{lllll}63129.30 & 30098.97 & \mathrm{Y}-12 & 6 & \mathrm{Q} 1 / 96, \mathrm{Q} 3 / 96\end{array}$

$59897.3432518 .75 \quad \mathrm{Y}-12 \quad 5 \& 6$ Q3/99, Q2/00, Q4/00, Q2/01, Q4/01, $\mathrm{Q} 2 / 02, \mathrm{Q} 4 / 02, \mathrm{Q} 2 / 03, \mathrm{Q} 4 / 03, \mathrm{Q} 2 / 04$ Q4/04, Q2/05, Q4/05, Q2/06, Q4/07

$\begin{array}{lllll}58156.79 & 32502.39 & \mathrm{Y}-12 & 5 & \mathrm{Q} 2 / 00\end{array}$

$57202.90 \quad 32566.40 \quad Y-12$

$5 \mathrm{Q} 3 / 99, \mathrm{Q} 2 / 00, \mathrm{Q} 4 / 00, \mathrm{Q} 2 / 01, \mathrm{Q} 4 / 01$, $\mathrm{Q} 2 / 02, \mathrm{Q} 4 / 02, \mathrm{Q} 2 / 03, \mathrm{Q} 4 / 03, \mathrm{Q} 2 / 04$, $\mathrm{Q} 4 / 04, \mathrm{O} 2 / 05, \mathrm{O} 4 / 05, \mathrm{Q} 2 / 06, \mathrm{Q} 4 / 07$ $\mathrm{Q} 4 / 08-\mathrm{Q} 1 / 09, \mathrm{Q} 3 / 10-\mathrm{Q} 1 / 11, \mathrm{Q} 3 / 12$, $\mathrm{Q} 1 / 13$

$49980.89 \quad 34378.71 \quad Y-12$

7 Q2/01, Q4/01, Q2/02, Q4/02, Q2/03, $\mathrm{Q} 4 / 03, \mathrm{Q} 2 / 04, \mathrm{Q} 4 / 04, \mathrm{Q} 2 / 05, \mathrm{Q} 4 / 05$ Q2/06, Q4/07, Q4/08-Q1/09, Q3/10Q1/11, Q3/12, Q1/13

$57700.00 \quad 29400.00 \quad Y-12$

5 Q1/96-Q4/96, Q3/97-Q3/98, Q3/99Q3/00, Q3/02, Q1/03-Q1/04, Q2/05, Q3/07

$50585.00 \quad 29720.00$

Y-12

4 \& 5 Q1/93-Q1/94, Q3/94, Q1/95, Q3/95, Q1/96, Q3/96, Q1/97-Q2/97, Q4/97Q1/98, Q3/98, Q1/99, Q3/99, Q1/00 $\mathrm{Q} 3 / 00-\mathrm{Q} 3 / 06, \mathrm{Q} 3 / 07, \mathrm{Q} 1 / 08, \mathrm{Q} 2 / 09-$ $\mathrm{Q} 4 / 12, \mathrm{Q} 3 / 13$

$49414.0029582 .00 \quad \mathrm{Y}-12 \quad 4$ \& 5 Q1/95, Q3/95, Q2/97-Q3/97, Q1/98, $\mathrm{Q} 3 / 98, \mathrm{Q} 1 / 99$

$\begin{array}{lllll}46785.93 & 29419.79 & \mathrm{Y}-12 & 4 & \mathrm{Q} 1 / 03-\mathrm{Q} 4 / 12\end{array}$

$45551.7529413 .36 \quad \mathrm{Y}-12 \quad 4 \quad \mathrm{Q} 4 / 06-\mathrm{Q} 1 / 10, \mathrm{Q} 4 / 11-\mathrm{Q} 4 / 12$

$44327.00 \quad 29678.00 \quad \mathrm{Y}-12 \quad 4 \quad$ Q2/97-Q3/97, Q1/98, Q1/99 


\section{CURRENT NAME}

NT-07

NT-08

NT-08W

NT-13

NT-1N

NT-1S

NT-3

NT-8-E

NT-8-W

OF 51

OF 002

OF 004

OF 017

OF 109
LOC

OTHER NAME

BC-NT7,NT-7

BC-NT8,NT-8

RV Bear Creek Burial Grounds WMA

RV Bear Creek Burial Grounds WMA

RV Exit Pathway Spring/Surface Water

RV Exit Pathway Spring/Surface Water

RV Exit Pathway Spring/Surface Water

RV Exit Pathway Spring/Surface Water

RV Bear Creek Burial Grounds WMA

RV Bear Creek Burial Grounds WMA

OF 051, OF 51 ,OF051 ,OF-

51,OF051, OF51

Exit Pathway Spring/Surface Water

PP Exit Pathway Spring/Surface Water

PP Exit Pathway Spring/Surface Water

PP Exit Pathway Spring/Surface Water

\section{EASTING NORTHING SYSTEM MAP SAMPLING HISTORY}

$42914.0029070 .00 \quad \mathrm{Y}-12 \quad 4$ Q2/97-Q3/97, Q1/98, Q3/98, Q1/99, Q3/99, Q1/00, Q3/00, Q1/01, Q3/01, Q1/02, Q1/03, Q3/03, Q1/04, Q3/04, $\mathrm{Q} 1 / 05, \mathrm{Q} 1 / 06, \mathrm{Q} 3 / 06, \mathrm{Q} 1 / 07-\mathrm{Q} 2 / 07$, Q4/07-Q2/08, Q4/08-Q4/12

$41426.00 \quad 28779.00 \quad Y-12$

$41636.00 \quad 29935.00$

$34341.00 \quad 29021.00$

$50848.00 \quad 30065.00$

$50675.00 \quad 29708.00$

$48136.00 \quad 29338.00$

$42154.00 \quad 29798.00$

$41636.00 \quad 29935.00$

60102.7929144 .31

$63937.00 \quad 30176.00$

$63712.00 \quad 29405.00$

$61900.00 \quad 28672.00$

$58838.05 \quad 29271.43$
4 Q2/97-Q3/97, Q1/98, Q3/98, Q1/99, Q3/99, Q1/00, Q3/00, Q1/01, Q3/01, $\mathrm{Q} 1 / 02, \mathrm{Q} 1 / 03, \mathrm{Q} 3 / 03, \mathrm{Q} 1 / 04, \mathrm{Q} 3 / 04$ Q1/05, Q3/05, Q1/06, Q3/06, Q1/07Q3/07, Q1/08-Q1/09, Q3/09-Q4/12

$\mathrm{Y}-12 \quad 4$ Q1/02, Q1/03, Q3/03

$\mathrm{Y}-12 \quad 3$ Q3/90-Q1/94, Q3/94

$\mathrm{Y}-12 \quad 4$ \& 5 Q2/95

$\mathrm{Y}-12 \quad 4 \& 5$ Q2/95, Q3/98

4 Q2/98, Q2/99-Q3/99, Q1/00-Q2/02, Q4/02-Q3/10, Q1/11, Q3/11-Q4/11, Q2/12, Q4/12

4

5 \& 6 Q1/96-Q4/96, Q3/97-Q3/98, Q1/99,

Q3/99-Q3/01, Q1/02, Q3/02, Q1/03Q3/11, Q1/12-Q4/12

6 Q1/97

$6 \mathrm{Q} 1 / 96$

$6 \mathrm{Q} 1 / 97, \mathrm{Q} 4 / 04-\mathrm{Q} / 05$

5 Q1/97, Q4/97, Q2/98, Q4/00-Q4/01, Q4/02 


\begin{tabular}{|c|c|c|c|c|c|c|c|c|}
\hline CURRENT & & LOC & & & & & & \\
\hline NAME & OTHER NAME & $\underline{\text { TYPE }}$ & FUNCTIONAL AREA & EASTING & NORTHING & SYSTEM & MAP & SAMPLING HISTORY \\
\hline OF 135 & & PP & Exit Pathway Spring/Surface Water & 57698.37 & 29405.63 & ADMIN & 5 & Q4/96-Q3/98, Q4/00-Q4/01, Q4/02 \\
\hline OF 141 & & PP & Exit Pathway Spring/Surface Water & 57609.00 & 29335.00 & $\mathrm{Y}-12$ & 5 & Q4/96 \\
\hline OF 150 & & PP & Exit Pathway Spring/Surface Water & 56936.12 & 29474.28 & ADMIN & 5 & $\begin{array}{l}\text { Q4/00-Q4/02, Q2/03, Q4/03, Q1/11- } \\
\text { Q4/12 }\end{array}$ \\
\hline OF 160 & OF-160,Outfall 160 & PP & Y-12 Plant Site & 56334.10 & 29526.65 & Admin & 5 & Q1/11-Q4/12 \\
\hline OF 163 & OF-163,Outfall 163 & PP & Y-12 Plant Site & 55961.31 & 29560.42 & Admin & 5 & Q1/11-Q4/12 \\
\hline OF 169 & OF-169,Outfall 169 & PP & Y-12 Plant Site & 55393.45 & 29629.96 & Admin & 5 & Q1/11-Q4/12 \\
\hline OF $200 A$ & & PP & Exit Pathway Spring/Surface Water & 57537.75 & 29416.83 & Admin & 5 & Q4/96-Q2/98, Q4/00 \\
\hline OF 201 & & RV & Exit Pathway Spring/Surface Water & 57787.00 & 29316.00 & $Y-12$ & 5 & Q1/96 \\
\hline OF 221 & OUTFALL 221 & PP & Y-12 Plant Site & 53777.00 & 30005.00 & $Y-12$ & 5 & Q2/99-Q3/99, Q1/00, Q3/00 \\
\hline OF 225 & OF-225 & SL & Exit Pathway Spring/Surface Water & 53303.76 & 30139.20 & Admin & 5 & $\begin{array}{l}\text { Q2/97, Q2/98-Q3/98, Q2/99-Q3/99, } \\
\text { Q1/00, Q3/00 }\end{array}$ \\
\hline OF 301 & OUTFALL301 & PP & Kerr Hollow Quarry & 63298.00 & 24589.00 & Admin & 6 & $\begin{array}{l}\text { Q1/96, Q3/96, Q2/97, Q4/98-Q1/99, } \\
\text { Q3/99-Q4/99, Q2/00, Q4/00, Q2/01, } \\
\text { Q4/01 }\end{array}$ \\
\hline OF-196 & & SL & Exit Pathway Spring/Surface Water & 53707.00 & 29925.00 & $\mathrm{Y}-12$ & 5 & Q2/99 \\
\hline OF-3304 & & SL & Exit Pathway Spring/Surface Water & 54061.00 & 29749.00 & $\mathrm{Y}-12$ & 5 & Q2/97, Q2/98 \\
\hline Outfall 195 & & SL & Exit Pathway Spring/Surface Water & 53865.00 & 29862.00 & $Y-12$ & 5 & Q2/99-Q3/99 \\
\hline RGQWWSP & & SP & Union Valley - Exit Pathway & 76600.00 & 28500.00 & $\mathrm{Y}-12$ & 7 & Q2/96, Q2/97 \\
\hline RT4-UEFPC & $\begin{array}{l}\text { EPK 24.8,UEFPC } \\
24.8\end{array}$ & RV & Y-12 Plant Site & 60710.00 & 28901.00 & $Y-12$ & $5 \& 6$ & $\begin{array}{l}\text { Q4/00, Q2/01, Q4/01, Q2/02, Q4/02, } \\
\text { Q2/03, Q4/03, Q2/04, Q4/04, Q2/06, } \\
\text { Q4/06, Q2/07, Q4/07 }\end{array}$ \\
\hline S01 & & RV & S-3 Site & 52147.69 & 29424.84 & $Y-12$ & 5 & \\
\hline S02 & & RV & Unknown & 52424.72 & 29928.55 & Y-12 & 5 & \\
\hline
\end{tabular}




\begin{tabular}{|c|c|c|c|c|c|c|c|c|}
\hline CURRENT & & LOC & & & & & & \\
\hline NAME & OTHER NAME & $\underline{\text { TYPE }}$ & FUNCTIONAL AREA & EASTING & NORTHING & SYSTEM & MAP & SAMPLING HISTORY \\
\hline S03 & & PP & Unknown & 50575.41 & 29726.33 & Y-12 & $4 \& 5$ & \\
\hline S04 & & PP & Unknown & 53863.75 & 34709.98 & $\mathrm{Y}-12$ & 7 & \\
\hline S05 & & $\mathrm{RV}$ & Unknown & 50575.41 & 29726.33 & $\mathrm{Y}-12$ & $4 \& 5$ & \\
\hline S06 & & $\mathrm{RV}$ & Unknown & 50465.29 & 29668.85 & $\mathrm{Y}-12$ & $4 \& 5$ & $\begin{array}{l}\text { Q2/07, Q4/07-Q1/11, Q3/11-Q4/11, } \\
\text { Q2/12-Q3/12 }\end{array}$ \\
\hline S07 & & $\mathrm{RV}$ & Exit Pathway Spring/Surface Water & 49453.22 & 29385.23 & Y-12 & $4 \& 5$ & $\begin{array}{l}\text { Q3/01-Q3/03, Q1/04, Q3/04-Q1/05, } \\
\text { Q1/06, Q3/06, Q4/09-Q3/10, Q3/12 }\end{array}$ \\
\hline S08 & & $\mathrm{RV}$ & Unknown & 48061.09 & 29226.16 & Y-12 & 4 & \\
\hline S09 & & $\mathrm{RV}$ & Unknown & 46648.00 & 29300.80 & $\mathrm{Y}-12$ & 4 & \\
\hline S10 & & $\mathrm{RV}$ & Unknown & 45500.61 & 29240.24 & $\mathrm{Y}-12$ & 4 & \\
\hline S11 & & $\mathrm{RV}$ & Unknown & 44332.86 & 29149.54 & $\mathrm{Y}-12$ & 4 & \\
\hline $\mathrm{S} 12$ & & $\mathrm{RV}$ & Unknown & 42952.60 & 29114.38 & $\mathrm{Y}-12$ & 4 & \\
\hline S13 & & $\mathrm{RV}$ & Unknown & 42891.53 & 29023.83 & $\mathrm{Y}-12$ & 4 & \\
\hline S14 & & $\mathrm{RV}$ & Unknown & 41434.00 & 28821.28 & $\mathrm{Y}-12$ & 4 & \\
\hline S17 & & $\mathrm{RV}$ & Chestnut Ridge & 62907.85 & 23844.24 & $\mathrm{Y}-12$ & 6 & $\begin{array}{l}\text { Q1/03, Q3/03, Q1/04, Q3/04, Q1/05, } \\
\text { Q3/05, Q2/06, Q2/07, Q2/08, Q4/09, } \\
\text { Q2/10, Q3/11-Q4/12, Q2/13 }\end{array}$ \\
\hline S18 & & $\mathrm{RV}$ & Unknown & 61092.29 & 25317.26 & $\mathrm{Y}-12$ & 6 & Q4/07, Q4/10, Q2/12 \\
\hline S19 & & $\mathrm{RV}$ & Chestnut Ridge & 56393.00 & 23479.00 & Y-12 & 5 & $\begin{array}{l}\text { Q1/03, Q4/03, Q2/05, Q2/07, Q1/08, } \\
\text { Q4/09, Q4/10 }\end{array}$ \\
\hline $\mathrm{S} 20$ & & $\mathrm{PP}$ & Unknown & 53263.25 & 27519.44 & $\mathrm{Y}-12$ & 5 & \\
\hline S24 & & $\mathrm{RV}$ & Unknown & 41328.03 & 28717.11 & $\mathrm{Y}-12$ & 4 & Q4/06-Q4/11, Q2/12 \\
\hline SCFSEEP & & SE & South Campus Facility, Bethel Valley & 66100.00 & 23000.00 & Y-12 & 6 & Q1/97 \\
\hline
\end{tabular}




\section{CURRENT NAME}

SCF-WS1

SCF-WS2

SCR1.25SP

SCR 1.25

SCR1.4SP

SCR 1.4

SCR1.5SW

SCR2.1SP

SCR 2.1

SCR2.2SP

SCR 2.2SP

SCR2.2SW

SCR3.4SP
LOC

\section{TYPE FUNCTIONAL AREA}

SL Unknown

SL Unknown

SP Exit Pathway Spring/Surface Water

SP Exit Pathway Spring/Surface Water

RV Exit Pathway Spring/Surface Water

SP Exit Pathway Spring/Surface Water

SP Exit Pathway Spring/Surface Water

RV Exit Pathway Spring/Surface Water

SP Exit Pathway Spring/Surface Water

$56770.38 \quad 25354.92$
$52462.00 \quad 24126.00 \quad \mathrm{Y}-12$

$52149.58 \quad 23251.03 \quad \mathrm{Y}-12$

$53941.00 \quad 25051.00$

$53869.00 \quad 23472.00$

\section{EASTING NORTHING SYSTEM MAP}

SAMPLING HISTORY

$66297.59 \quad 22719.23 \quad Y-12$

$66297.59 \quad 22719.23 \quad Y-12$

$52779.73 \quad 25074.12 \quad Y-12$

54990.55
Y-12

Y-12

7 Q1/04, Q1/05, Q1/07, Q1/08, Q1/09, Q1/10, Q1/11, Q1/12

7 Q1/04, Q1/05, Q1/08, Q1/09, Q1/10, Q1/11, Q1/12

5 Q2/97-Q3/97, Q1/98, Q3/98, Q1/99, Q3/99, Q1/00, Q3/00, Q1/01, Q3/01, $\mathrm{Q} 1 / 02, \mathrm{Q} 3 / 02, \mathrm{Q} 1 / 03, \mathrm{Q} 3 / 03, \mathrm{Q} 1 / 04$, Q3/04, Q1/05, Q3/05, Q1/06, Q3/06 Q1/07, Q3/07, Q1/08, Q3/08, Q1/09, Q3/09, Q1/10, Q3/10, Q1/11

5 Q2/97-Q3/97, Q1/98, Q3/98

$5 \mathrm{Q} 1 / 01, \mathrm{Q} 3 / 01, \mathrm{Q} 1 / 02, \mathrm{Q} 3 / 02, \mathrm{Q} 1 / 03$ $\mathrm{Q} 3 / 03, \mathrm{Q} 1 / 04, \mathrm{Q} 3 / 04, \mathrm{Q} 1 / 05, \mathrm{Q} 3 / 05$, $\mathrm{Q} 2 / 06, \mathrm{Q} 2 / 07, \mathrm{Q} 2 / 08, \mathrm{Q} 4 / 09, \mathrm{Q} 2 / 10$,

5 Q2/97-Q3/97, Q1/98, Q3/98, Q1/99, Q3/99, Q1/00, Q3/00, Q1/01, Q3/01, $\mathrm{Q} 1 / 03, \mathrm{Q} 3 / 03, \mathrm{Q} 1 / 04, \mathrm{Q} 3 / 04, \mathrm{Q} 1 / 05$, $\mathrm{Q} 3 / 05, \mathrm{Q} 2 / 06, \mathrm{Q} 2 / 07, \mathrm{Q} 2 / 08, \mathrm{Q} 2 / 09$, Q4/09, Q2/10, Q3/11, Q2/12, Q2/13

5 Q1/95, Q1/97, Q3/97, Q1/98, Q3/98, Q1/99, Q3/99, Q1/00, Q3/00, Q1/01, $\mathrm{Q} 3 / 01, \mathrm{Q} 1 / 03, \mathrm{Q} 3 / 03, \mathrm{Q} 1 / 04, \mathrm{Q} / 04$, $\mathrm{Q} 1 / 05, \mathrm{Q} 3 / 05, \mathrm{Q} 2 / 06, \mathrm{Q} 2 / 07, \mathrm{Q} 2 / 08$, Q4/09, Q2/10, Q3/11, Q2/12, Q2/13

$\mathrm{Y}-12$

5 Q1/01, Q1/02, Q3/02

Admin Q3/11, Q2/12, Q2/13
5 Q2/97-Q3/97, Q1/98, Q3/98, Q1/99, Q3/99, Q1/00, Q3/00, Q1/01 


\section{CURRENT NAME}

SCR3.5SP

SCR4.3SP

CBS-1,Green Barn Spring

SCR4.4SW

SCR5.1ESP

SCR5.1SP

SCR5.2SP

SCR5.2SW

SCR5.4SP

SCR 5.4

SCR7.10SP

SCR7.12SW

SCR7.14SP

SCR7.16SP

OTHER NAME

SCR 3.5

SCR 5.1

SCR 7.10

LOC

RV Chestnut Ridge

SP Industrial Landfill V

\section{TYPE}

SP
Exit Pathway Spring/Surface Water

\section{EASTING NORTHING SYSTEM MAP}

$\mathrm{Y}-12$

$56618.00 \quad 24138.00$

$61216.00 \quad 24690.00$

Y-12

RV Exit Pathway Spring/Surface Water

SP Exit Pathway Spring/Surface Water

SP Exit Pathway Spring/Surface Water

SP Exit Pathway Spring/Surface Water

RV Exit Pathway Spring/Surface Water

SP Exit Pathway Spring/Surface Water

SP Exit Pathway Spring/Surface Water

RV Union Valley - Exit Pathway

SP Union Valley - Exit Pathway

SP Union Valley - Exit Pathway
$61970.00 \quad 23950.00 \quad$ Admin 6

$63483.93 \quad 26121.67 \quad$ Admin

$63023.00 \quad 24296.00 \quad$ Admin

$63250.00 \quad 24450.00 \quad Y-12$

$63192.00 \quad 23301.00 \quad Y-12$

$68225.00 \quad 27115.00 \quad Y-12$

$68100.00 \quad 28200.00 \quad Y-12$

$68900.00 \quad 29000.00 \quad Y-12$

$68800.00 \quad 29000.00 \quad Y-12$

6

\section{SAMPLING HISTORY}

5 Q2/97-Q3/97, Q1/98, Q3/98, Q1/99, Q3/99, Q1/00, Q3/00, Q1/01, Q3/01, Q1/02, Q3/02, Q1/03, Q3/03, Q1/04, Q3/04, Q3/05, Q1/06, Q3/06, Q1/07, Q3/07, Q1/08, Q3/08, Q1/09, Q3/09, Q1/10, Q3/10, Q1/11, Q3/11

$5 \mathrm{Q} 3 / 03, \mathrm{Q} 1 / 04, \mathrm{Q} 3 / 04, \mathrm{Q} 1 / 05, \mathrm{Q} 3 / 05$, Q2/06, Q2/07, Q2/08, Q4/09, Q2/10, Q3/11, Q2/12, Q2/13

$6 \mathrm{Q} 1 / 94, \mathrm{Q} 3 / 94, \mathrm{Q} 2 / 95, \mathrm{Q} 4 / 95, \mathrm{Q} 2 / 96$, Q4/96-Q1/97, Q3/97, Q1/98, Q3/98, Q1/99, Q3/99, Q1/00, Q3/00, Q1/01. $\mathrm{Q} 3 / 01, \mathrm{Q} 1 / 02, \mathrm{Q} 3 / 02, \mathrm{Q} 1 / 03, \mathrm{Q} 3 / 03$, Q1/04, Q3/04, Q1/05, Q3/05, Q1/06, Q3/06, Q1/07, Q3/07, Q1/08

Q1/01, Q3/01, Q1/02, Q3/02

6 Q2/97-Q3/97, Q1/98, Q3/98, Q1/99, Q3/99, Q1/00, Q3/00, Q1/01, Q3/01, Q4/11-Q4/12

$6 \mathrm{Q} 1 / 01, \mathrm{Q} 3 / 01, \mathrm{Q} 1 / 02, \mathrm{Q} 3 / 02, \mathrm{Q} 4 / 11-$ Q4/12

6 Q2/97-Q3/97, Q1/98, Q3/98, Q1/99, Q3/99, Q1/00, Q3/00, Q1/01, Q3/01

6 Q2/97-Q3/97, Q1/98, Q3/98

$6 \mathrm{Q} 1 / 96$

6 Q1/96

$6 \mathrm{Q} 1 / 96$ 


\section{CURRENT NAME}

SCR7.18SP

SCR7.1SP

7UV7.1SP,UV7.1SP

$\begin{array}{ll}\text { SCR7.4SP } & \text { SCR } 7.4 \\ \text { SCR7.6SP } & \text { SCR } 7.6 \\ \text { SCR7.79SW } & \begin{array}{l}\text { Scarboro Creek } \\ \text { downstream of SCR }\end{array} \\ & 7.8 \\ \text { SCR7.7SP } & \text { SCR } 7.7 \\ \text { SCR7.8SP } & \text { SCR } 7.8\end{array}$

SCR7.8SSP

SCR7.9SW

SEEP1

SEEP2

SEEPS 3+4 BG- Seeps 3\&4 BG-C CW

West

SP-17

SP17

SPR14.0SP
SP Union Valley - Exit Pathway

LOC

\section{TYPE FUNCTIONAL AREA}

SP Union Valley - Exit Pathway

SP Exit Pathway Spring/Surface Water

SP Exit Pathway Spring/Surface Water

SL Exit Pathway Spring/Surface Water

SP Exit Pathway Spring/Surface Water

SP Exit Pathway Spring/Surface Water

SP Union Valley - Exit Pathway

RV Union Valley - Exit Pathway

SE Bear Creek Burial Grounds WMA

SE Bear Creek Burial Grounds WMA

SL Exit Pathway Spring/Surface Water

SP Y-12 Plant Site

SP Pine Ridge

\section{EASTING NORTHING SYSTEM MAP SAMPLING HISTORY}

$68107.4328384 .93 \quad \mathrm{Y}-12 \quad 6$ Q1/96-Q2/96, Q2/97-Q3/97, Q1/98, Q3/98, Q1/99, Q3/99, Q1/00-Q2/00

$67970.00 \quad 28440.00 \quad Y-12$

6 Q4/95-Q2/96, Q2/97-Q3/97, Q1/98, $\mathrm{Q} 3 / 98, \mathrm{Q} 1 / 99, \mathrm{Q} 3 / 99, \mathrm{Q} 1 / 00-\mathrm{Q} 3 / 00$, Q1/01, Q3/01, Q1/02, Q3/02, Q1/03, Q3/03, Q1/04, Q3/04, Q1/05, Q3/05, Q1/06, Q1/07, Q1/08, Q1/09, Q1/10, $\mathrm{Q} 1 / 11, \mathrm{Q} 1 / 12$

$68245.00 \quad 25631.00 \quad \mathrm{Y}-12 \quad 6 \quad \mathrm{Q} 2 / 97-\mathrm{Q} 3 / 97, \mathrm{Q} 1 / 98, \mathrm{Q} 3 / 98$

$68233.00 \quad 26563.00 \quad \mathrm{Y}-12 \quad 6 \quad \mathrm{Q} 2 / 97-\mathrm{Q} 3 / 97, \mathrm{Q} 1 / 98, \mathrm{Q} 3 / 98$

$68232.00 \quad 27292.00 \quad \mathrm{Y}-12 \quad 6 \quad \mathrm{Q} 2 / 97-\mathrm{Q} 3 / 97, \mathrm{Q} 1 / 98, \mathrm{Q} 3 / 98$

$68237.00 \quad 26814.00 \quad \mathrm{Y}-12 \quad 6 \quad \mathrm{Q} 2 / 97-\mathrm{Q} 3 / 97, \mathrm{Q} 1 / 98, \mathrm{Q} 3 / 98$

$68300.00 \quad 27915.00 \quad Y-12$

6 Q2/97-Q3/97, Q1/98, Q3/98, Q1/99, $\mathrm{Q} 3 / 99, \mathrm{Q} 1 / 00-\mathrm{Q} 3 / 00, \mathrm{Q} 1 / 01, \mathrm{Q} 3 / 01$,

$\mathrm{Q} 1 / 02, \mathrm{Q} 3 / 02, \mathrm{Q} 1 / 03, \mathrm{Q} 3 / 03, \mathrm{Q} 1 / 04$ Q3/04, Q1/05, Q3/05, Q1/06, Q1/07, Q1/08, Q1/09, Q1/10, Q1/11, Q1/12

$\begin{array}{lllll}68200.00 & 28000.00 & \mathrm{Y}-12 & 6 & \mathrm{Q} 1 / 96-\mathrm{Q} 2 / 96\end{array}$

$\begin{array}{lllll}68300.00 & 24000.00 & \mathrm{Y}-12 & 6 & \mathrm{Q} 1 / 96\end{array}$

$\begin{array}{lllll}43629.00 & 30474.00 & \mathrm{Y}-12 & 4 & \mathrm{Q} 2 / 97-\mathrm{Q} 3 / 97, \mathrm{Q} 2 / 98-\mathrm{Q} 3 / 98\end{array}$

$\begin{array}{lllll}42831.00 & 30740.00 & \mathrm{Y}-12 & 4 & \mathrm{Q} 2 / 97-\mathrm{Q} 3 / 97, \mathrm{Q} 2 / 98-\mathrm{Q} 3 / 98\end{array}$

$42344.00 \quad 30210.00 \quad \mathrm{Y}-12 \quad 4 \quad$ Q2/97-Q3/97, Q2/98-Q3/98

$62000.00 \quad 28605.00 \quad \mathrm{Y}-12 \quad 6 \quad \mathrm{Q} 2 / 06, \mathrm{Q} 3 / 09, \mathrm{Q} 1 / 11, \mathrm{Q} 3 / 13$

$\begin{array}{lllll}54168.52 & 31638.58 & \mathrm{Y}-12 & 5 & \mathrm{Q} 1 / 04\end{array}$ 


\section{CURRENT NAME}

SS-1

SS-4

SS-5

SS6.6

SS-6E

SS-6W
LOC

OTHER NAME

\section{TYPE}

SP Exit Pathway Spring/Surface Water

SP Exit Pathway Spring/Surface Water

SP Exit Pathway Spring/Surface Water

Red Tail Hawk

Spring

SS-6,SS-6 EAST

SP Maynardville exit pathway

SP Exit Pathway Spring/Surface Water
28676.64

\section{$\frac{\text { EASTING }}{50481.36} \frac{\text { NORTHING }}{29564.83}$}

$44213.00 \quad 28831.00$

$41208.00 \quad 28650.00$

35666.55

$\sqrt{2}+n^{2}$

$726.00 \quad 28747.00$

\section{YSTEM}

$\mathrm{Y}-12$ Q1/96, Q3/96, Q1/97-Q2/97, Q4/97Q1/98, Q3/98, Q1/99, Q3/99, Q1/00, Q3/00, Q1/01, Q3/01, Q1/02, Q3/02, Q1/03, Q3/03, Q1/04, Q3/04, Q1/05, Q3/05, Q2/06

4 Q3/90-Q1/94, Q3/94, Q1/95, Q3/95, Q1/96, Q3/96, Q1/97, Q3/97, Q1/98, Q3/98, Q1/99, Q3/99, Q1/00, Q3/00, Q1/01, Q3/01, Q1/02, Q3/02, Q1/03, Q3/03, Q1/04, Q3/04, Q1/05, Q3/05, Q2/06-Q3/06, Q3/07, Q1/08,

4 Q3/90-Q1/94, Q3/94, Q1/95, Q3/95, Q1/96, Q3/96, Q1/97, Q3/97, Q1/98, Q3/98, Q1/99, Q3/99, Q1/00, Q3/00, Q1/01, Q3/01-Q3/03, Q1/04, Q3/04Q3/05, Q1/06-Q3/06, Q3/07, Q1/08, Q4/08-Q1/09, Q3/09-Q3/10, Q1/

3 Q1/05, Q3/05, Q1/06, Q3/06, Q4/09Q3/10, Q1/12, Q3/12

3 Q3/90-Q4/93, Q1/95, Q3/95, Q2/97Q3/97, Q1/98, Q3/98, Q1/99, Q3/99, Q1/00, Q3/00, Q1/01, Q3/01, Q1/02, Q1/05, Q3/05, Q1/06, Q3/06, Q1/07, Q3/07, Q1/08, Q3/08, Q1/09, Q3/09, Q1/10, Q3/10, Q1/11, Q3/11,

3 Q2/95, Q4/95, Q2/97-Q3/97, Q1/98, Q3/98, Q1/99, Q3/99, Q1/00, Q3/00, Q1/03 


\section{CURRENT NAME}

SS-7

SS-8

\section{OTHER NAME}

LOC

TYPE

SP Exit Pathway Spring/Surface Water

STATION $17 \quad 9422-1$

TS

UEFPC-SP17

SP 17, SP-17,SP17

UNC SW-1

UPPER POND 1

UV8.2SW

UV8.5SP

UV8.6SP

UV8.7SP
SP Exit Pathway Spring/Surface Water

RV Exit Pathway Spring/Surface Water

SE Exit Pathway Spring/Surface Water

SP Exit Pathway Spring/Surface Water

SP United Nuclear Corporation Site

LK Exit Pathway Spring/Surface Water

RV Union Valley - Exit Pathway

SP Union Valley - Exit Pathway

SP Union Valley - Exit Pathway

SP Union Valley - Exit Pathway

\section{EASTING NORTHING SYSTEM MAP SAMPLING HISTORY}

$27918.0028760 .00 \quad$ Y-12 2 Q2/95, Q4/95, Q2/97-Q3/97, Q1/98, $\mathrm{Q} 3 / 98, \mathrm{Q} 1 / 99, \mathrm{Q} 3 / 99, \mathrm{Q} 1 / 00, \mathrm{Q} 3 / 00$, Q1/01, Q3/01, Q1/02, Q1/03, Q3/03, Q3/05, Q1/06, Q3/06, Q4/09-Q3/10, $\mathrm{Q} 1 / 12, \mathrm{Q} 3 / 12$

$27552.0028887 .00 \quad \mathrm{Y}-12 \quad 2 \quad$ Q3/90-Q4/93, Q2/97-Q3/97, Q1/98, Q3/98, Q1/99, Q3/99, Q1/00, Q3/00 $\mathrm{Q} 1 / 01, \mathrm{Q} 3 / 01, \mathrm{Q} 1 / 02, \mathrm{Q} 3 / 02, \mathrm{Q} 1 / 03$ Q3/03, Q1/05, Q3/05, Q1/06, Q3/06, $\mathrm{Q} 4 / 09-\mathrm{Q} 3 / 10, \mathrm{Q} 1 / 12, \mathrm{Q} 3 / 12$

$64100.00 \quad 30500.00 \quad \mathrm{Y}-12 \quad 6 \quad \mathrm{Q} 1 / 96-\mathrm{Q} 3 / 98, \mathrm{Q} 1 / 99-\mathrm{Q} 4 / 12$

$50762.00 \quad 29791.00 \quad \mathrm{Y}-12 \quad 4 \& 5$ Q2/95

$61834.0028725 .00 \quad \mathrm{Y}-12 \quad 6 \quad \mathrm{Q} 1 / 96-\mathrm{Q} 3 / 98, \mathrm{Q} 1 / 99, \mathrm{Q} 3 / 99-\mathrm{Q} 3 / 00$ $\mathrm{Q} 3 / 02, \mathrm{Q} 1 / 03-\mathrm{Q} 3 / 04$

53521.8327861 .10 ADMIN 5 Q1/08, Q3/08, Q1/09, Q3/09, Q1/10, $\mathrm{Q} 3 / 10, \mathrm{Q} 1 / 11, \mathrm{Q} 3 / 11, \mathrm{Q} 1 / 12$

$62920.18 \quad 25091.89 \quad$ Admin 6

$\begin{array}{lllll}72800.00 & 28600.00 & \mathrm{Y}-12 & 7 & \mathrm{Q} 1 / 96\end{array}$

$\begin{array}{lllll}79300.00 & 28500.00 & \mathrm{Y}-12 & 7 & \mathrm{Q} 2 / 96-\mathrm{Q} 3 / 96\end{array}$

$\begin{array}{lllll}74700.00 & 28700.00 & Y-12 & 7 & \text { Q3/96 }\end{array}$

$\begin{array}{lllll}75000.00 & 28500.00 & Y-12 & 7 & \text { Q3/96 }\end{array}$ 
This Page Intentionally Left Blank 
APPENDIX J

Data Correction Form 
This Page Intentionally Left Blank 


\section{Y-12 GWPP SUBSURFACE DATA BASE $\sim$ CORRECTIONS}

DATE:

$\begin{aligned} \text { ADDRESS: } & \\ \text { PHONE: } & \longrightarrow\end{aligned}$

NATURE OF CORRECTION:

LOCATION OF ERROR IN DOCUMENT (Page number and/or map number):

Mail corrections to: $\quad \mathrm{Y}-12$ Groundwater Protection Program Manager B\&W Y-12 L.L.C.

Building 9108, MS-8087

Oak Ridge, Tennessee 37831-8219

ATTN: Subsurface Data Base Corrections 
This Page Intentionally Left Blank 


\section{APPENDIX K}

Borehole Location Maps - Existing Boreholes, Wells, and Surface Locations 
This Page Intentionally Left Blank 

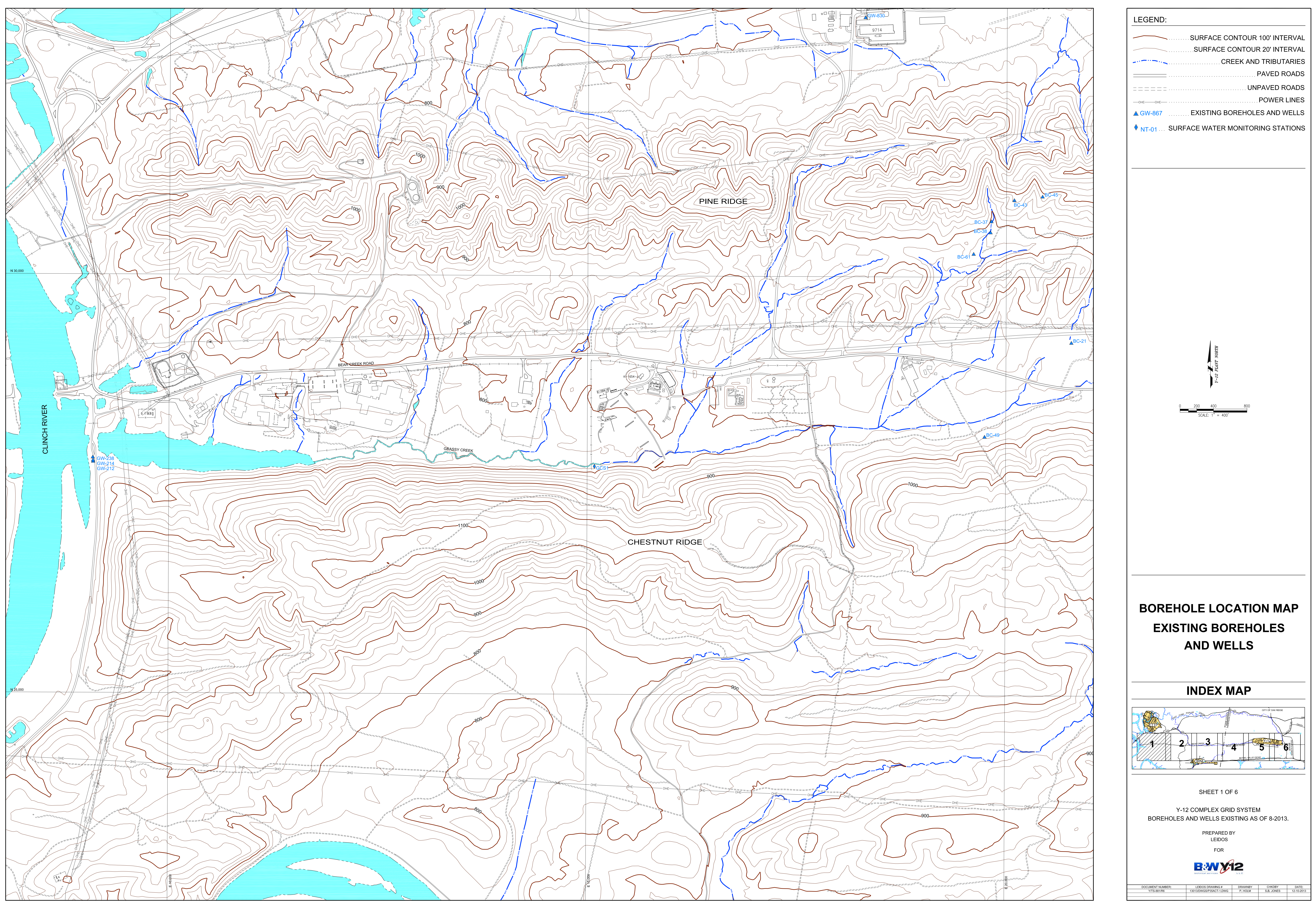

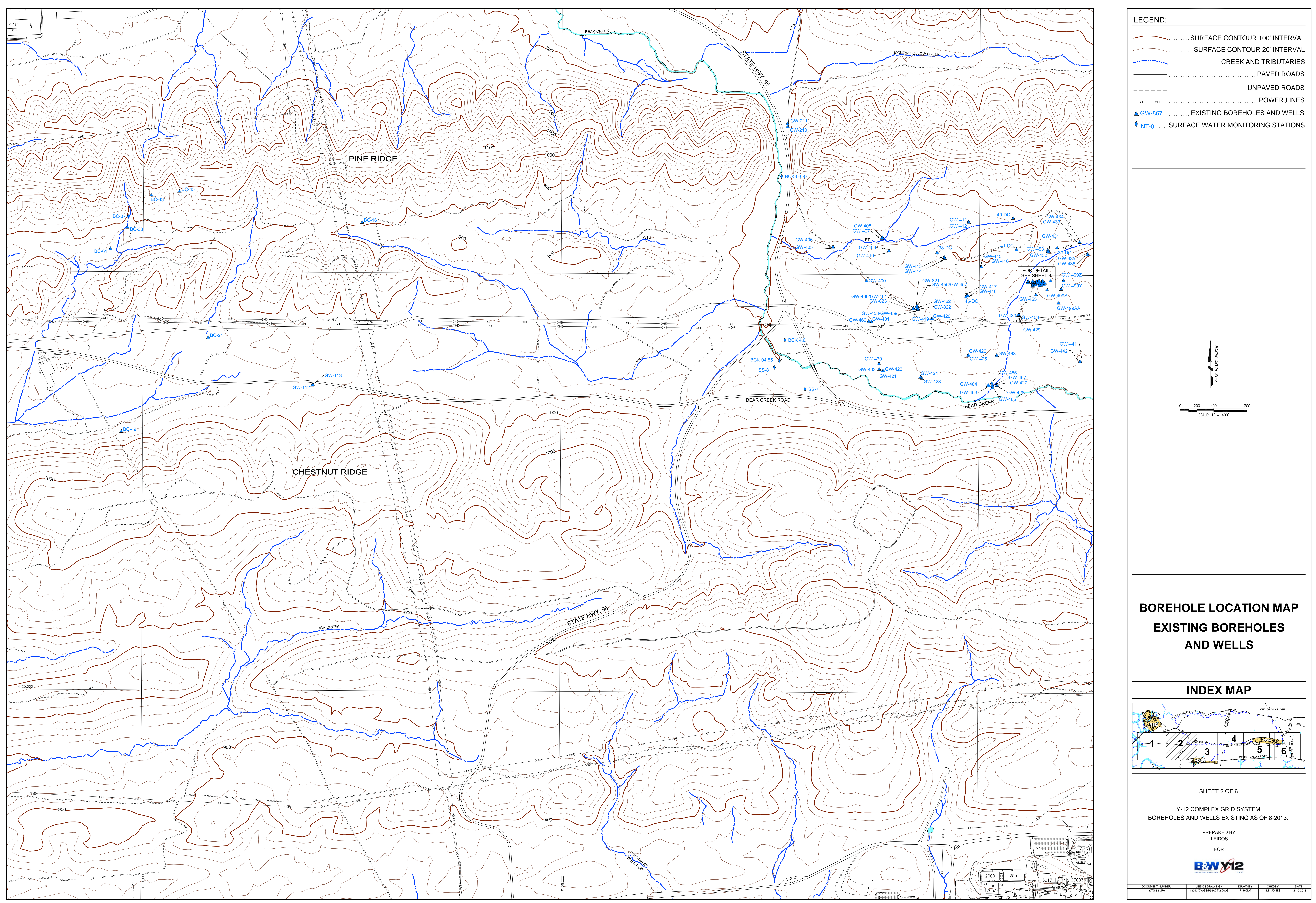

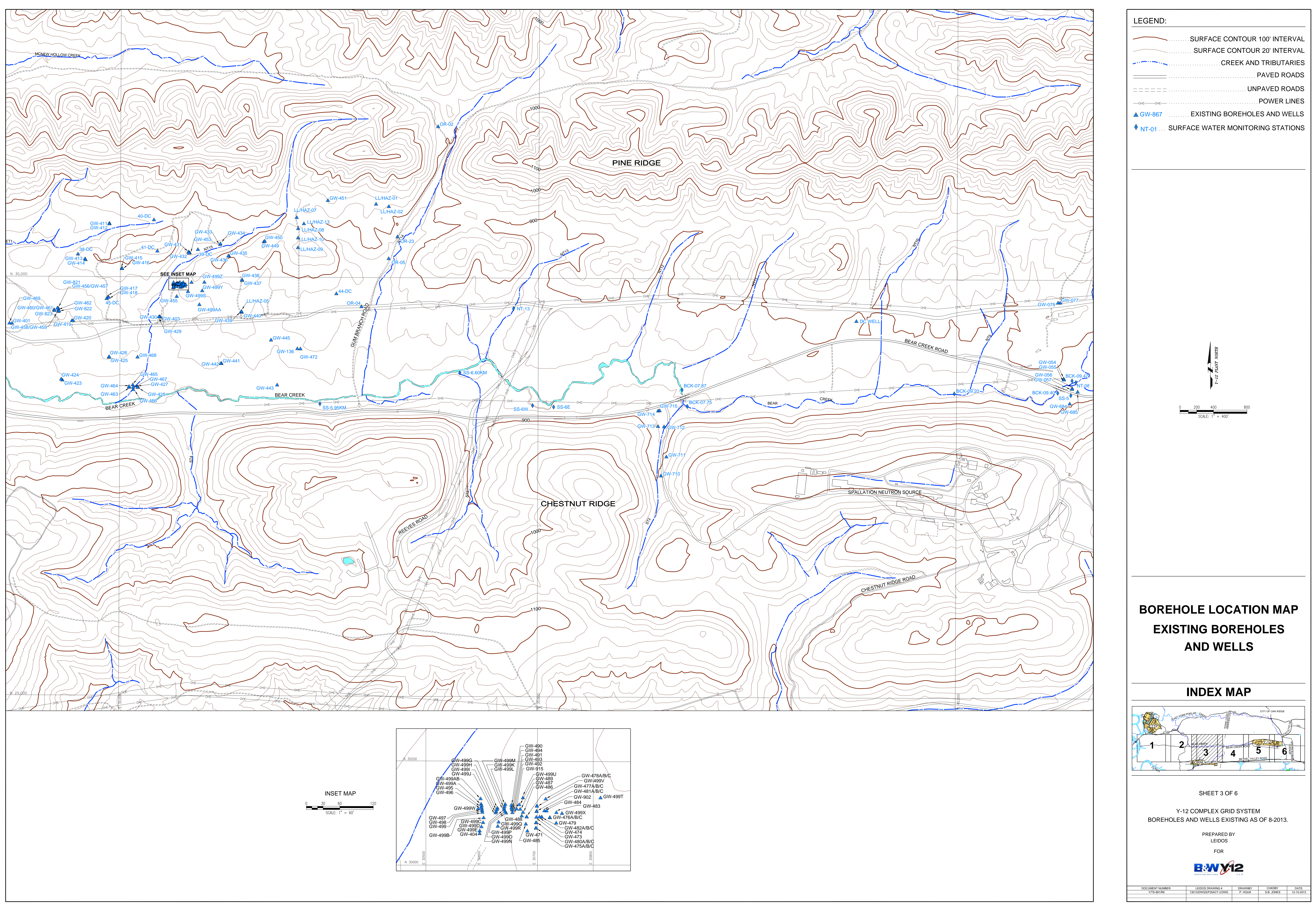

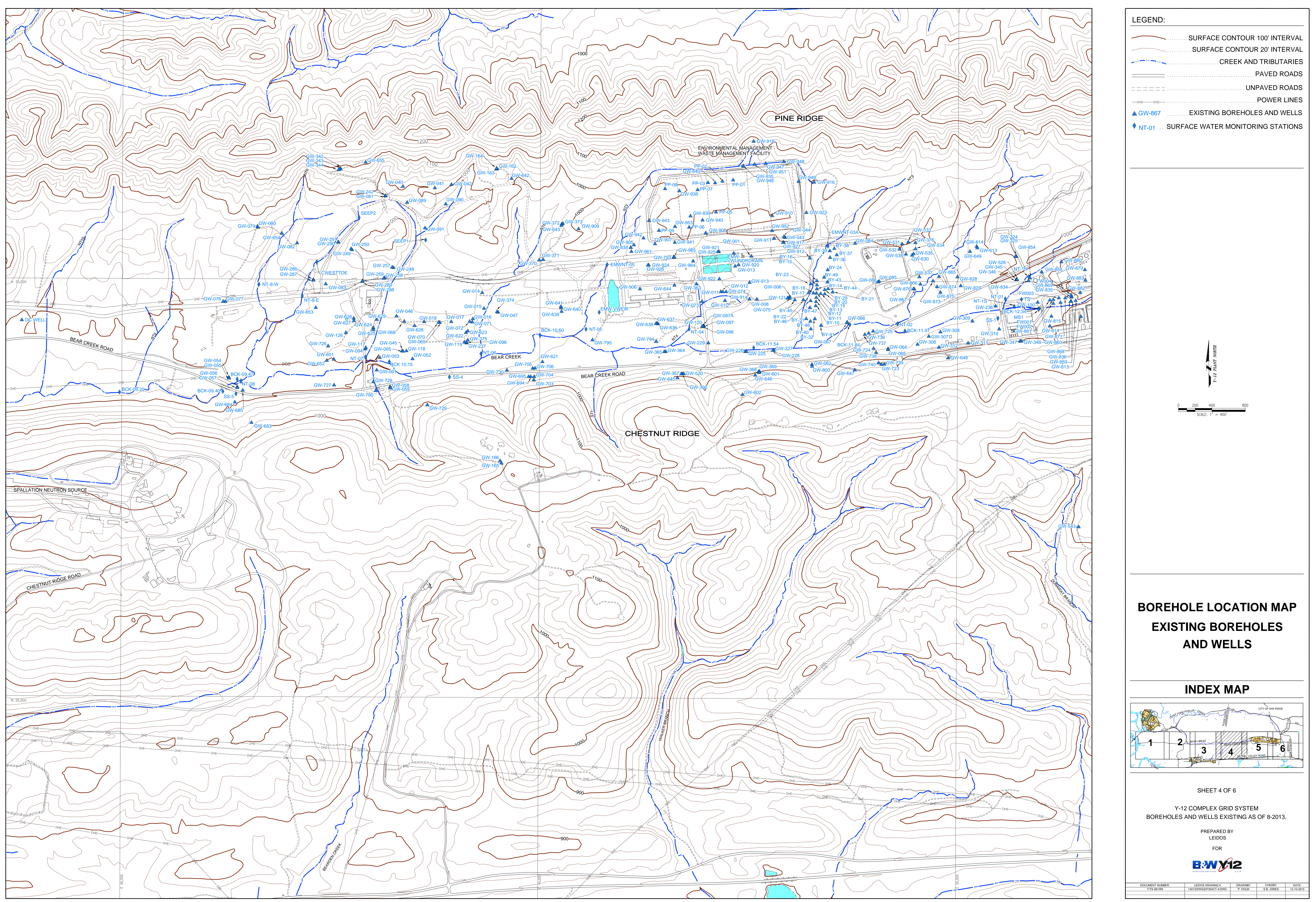

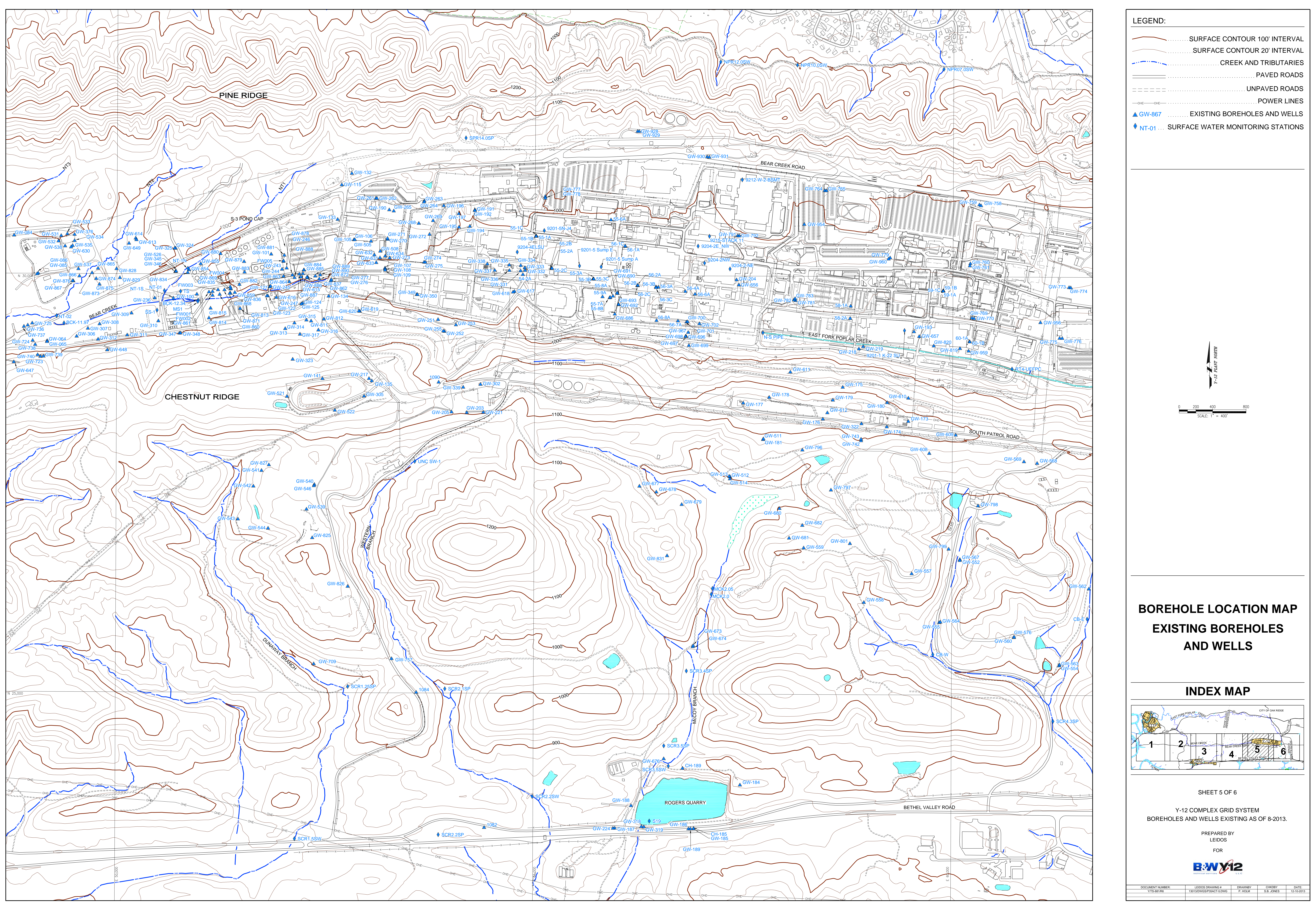

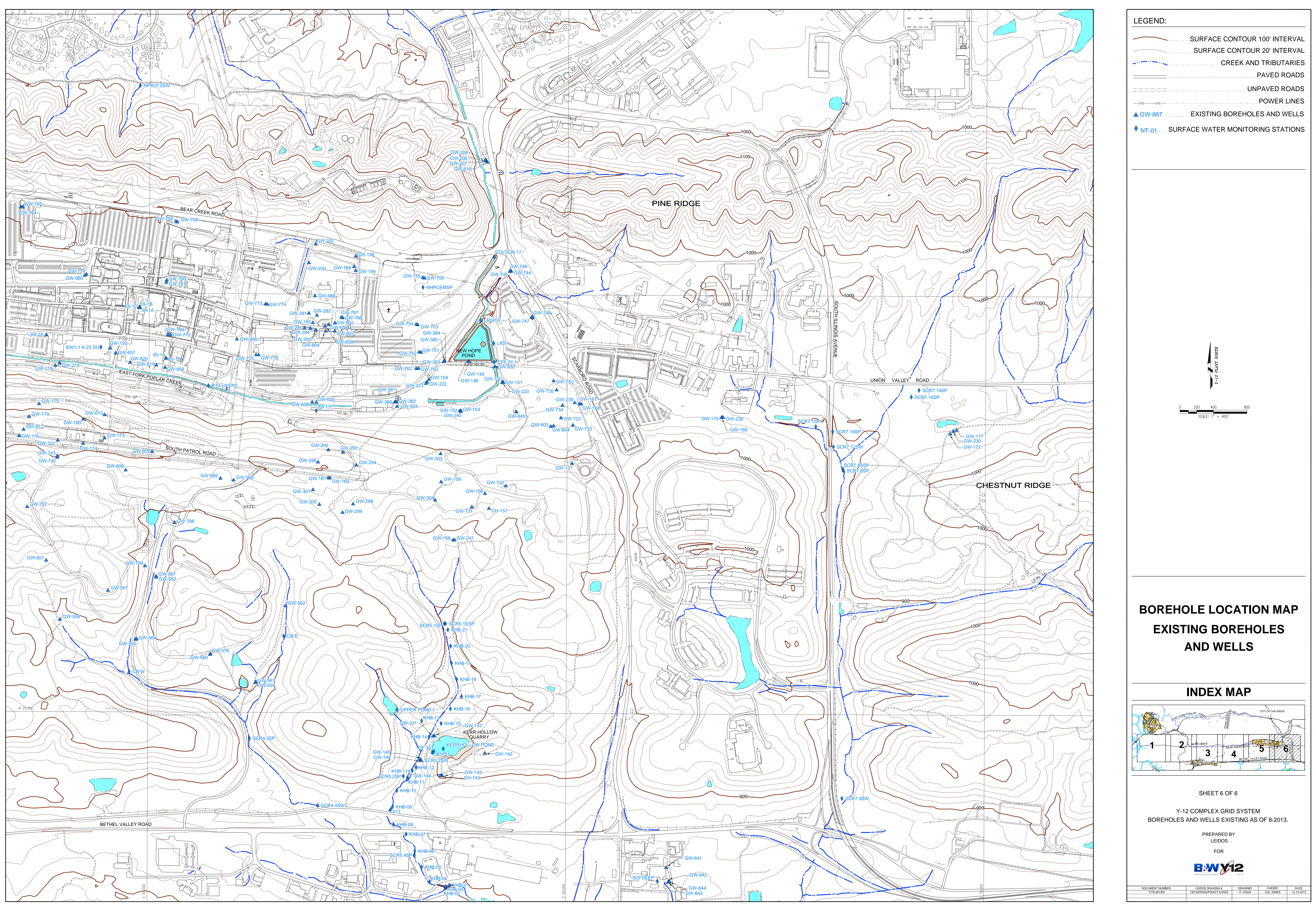


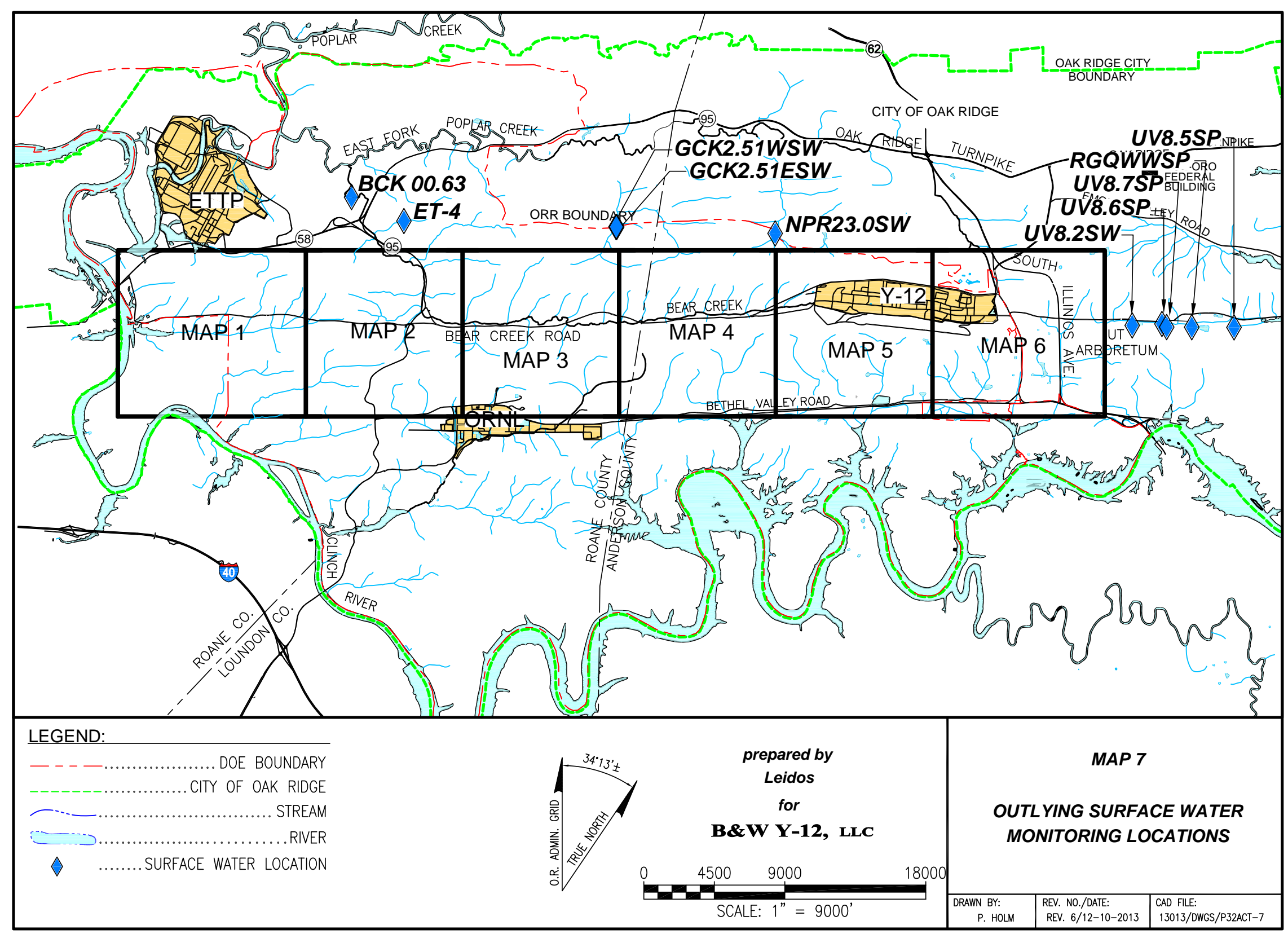


APPENDIX L

Borehole Location Maps - Inactive Boreholes and Wells 
This Page Intentionally Left Blank 

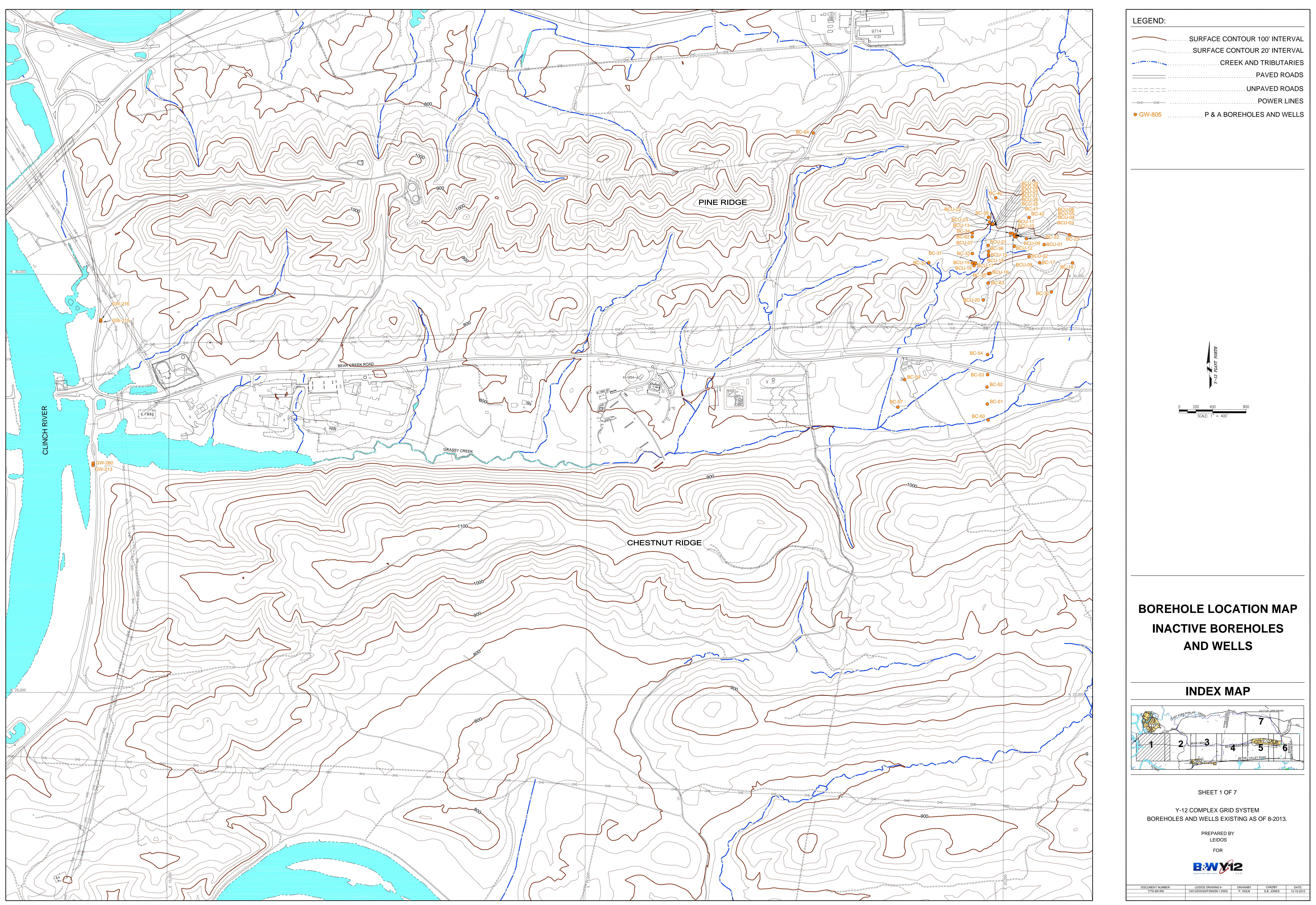

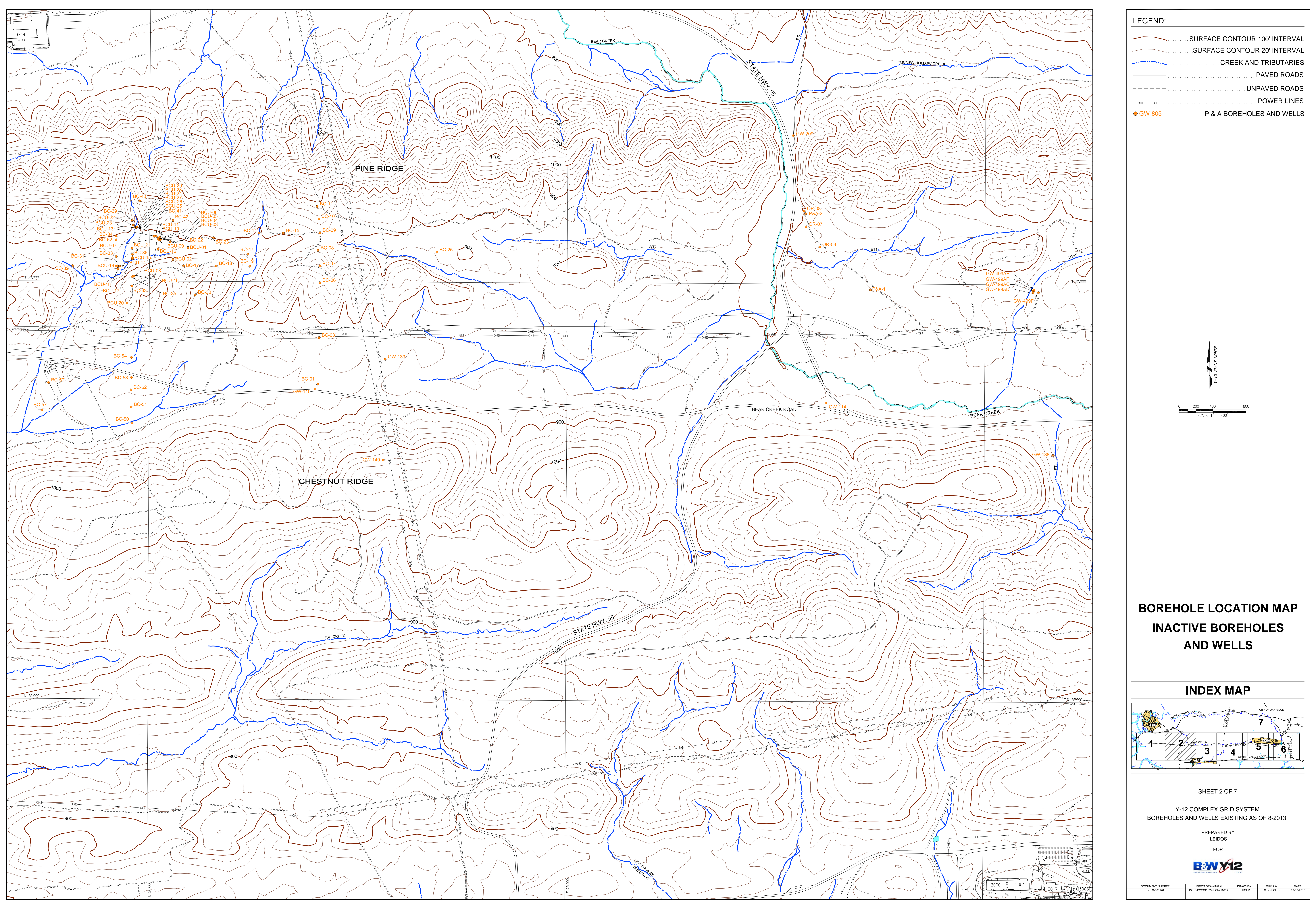

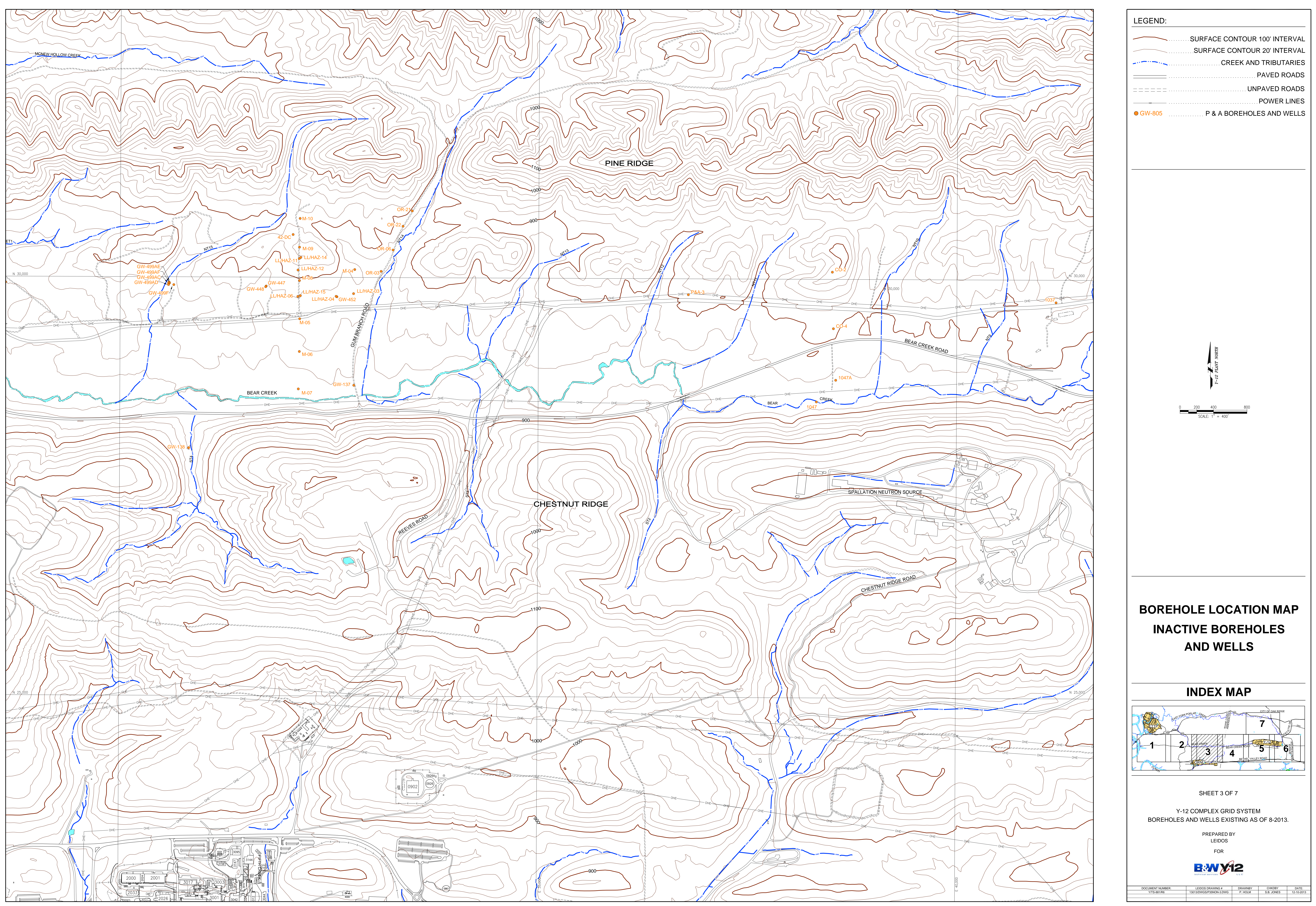

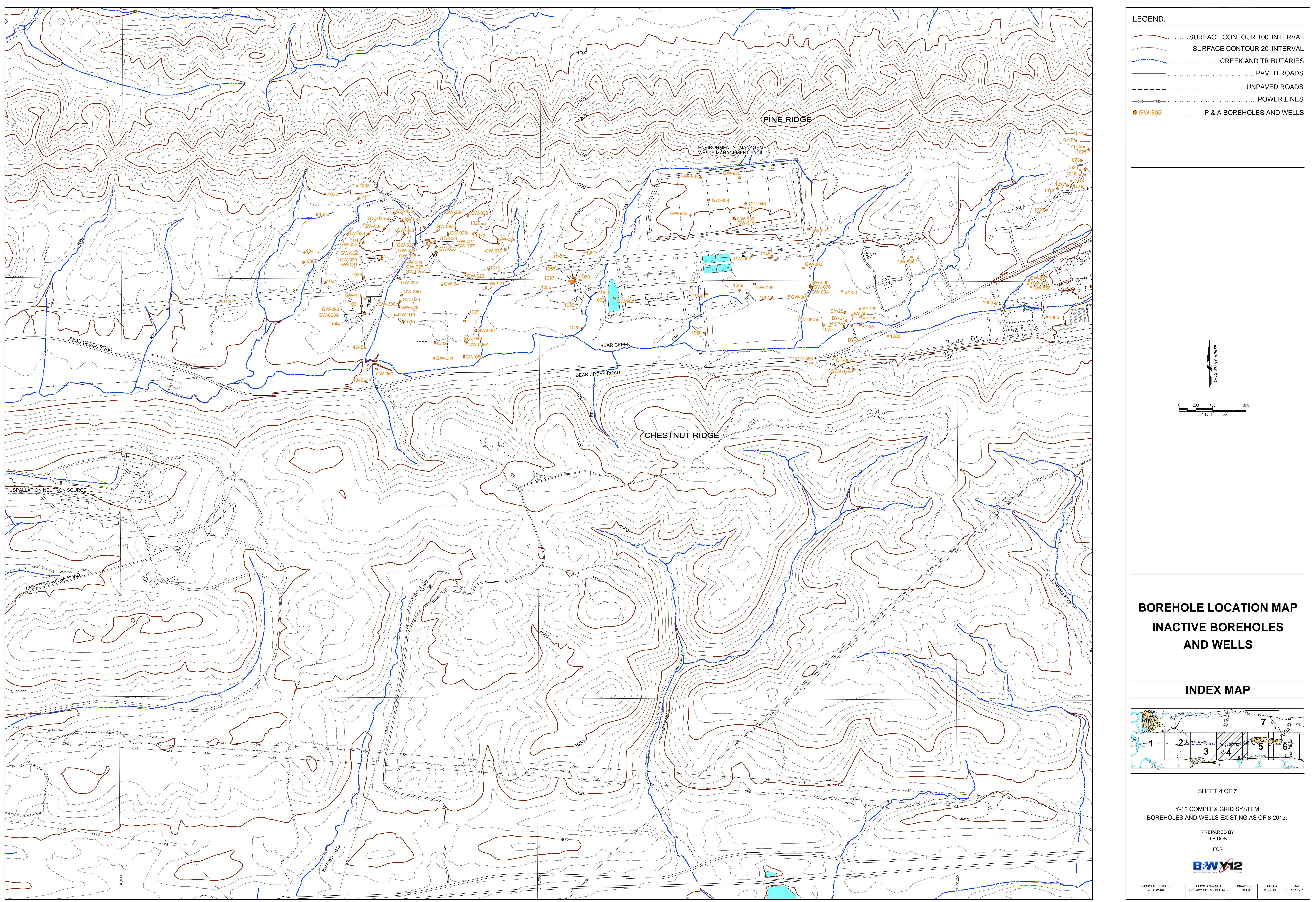

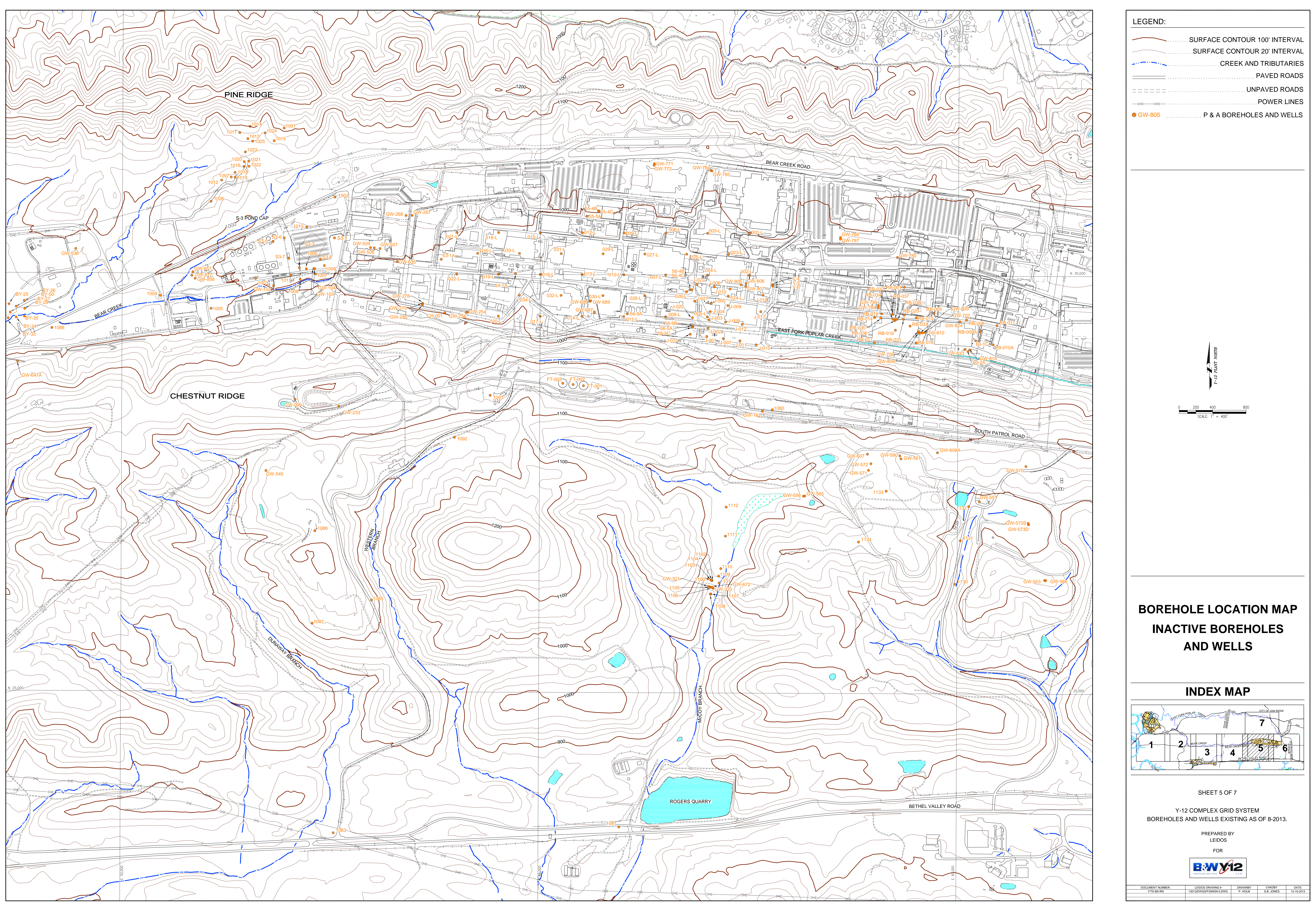

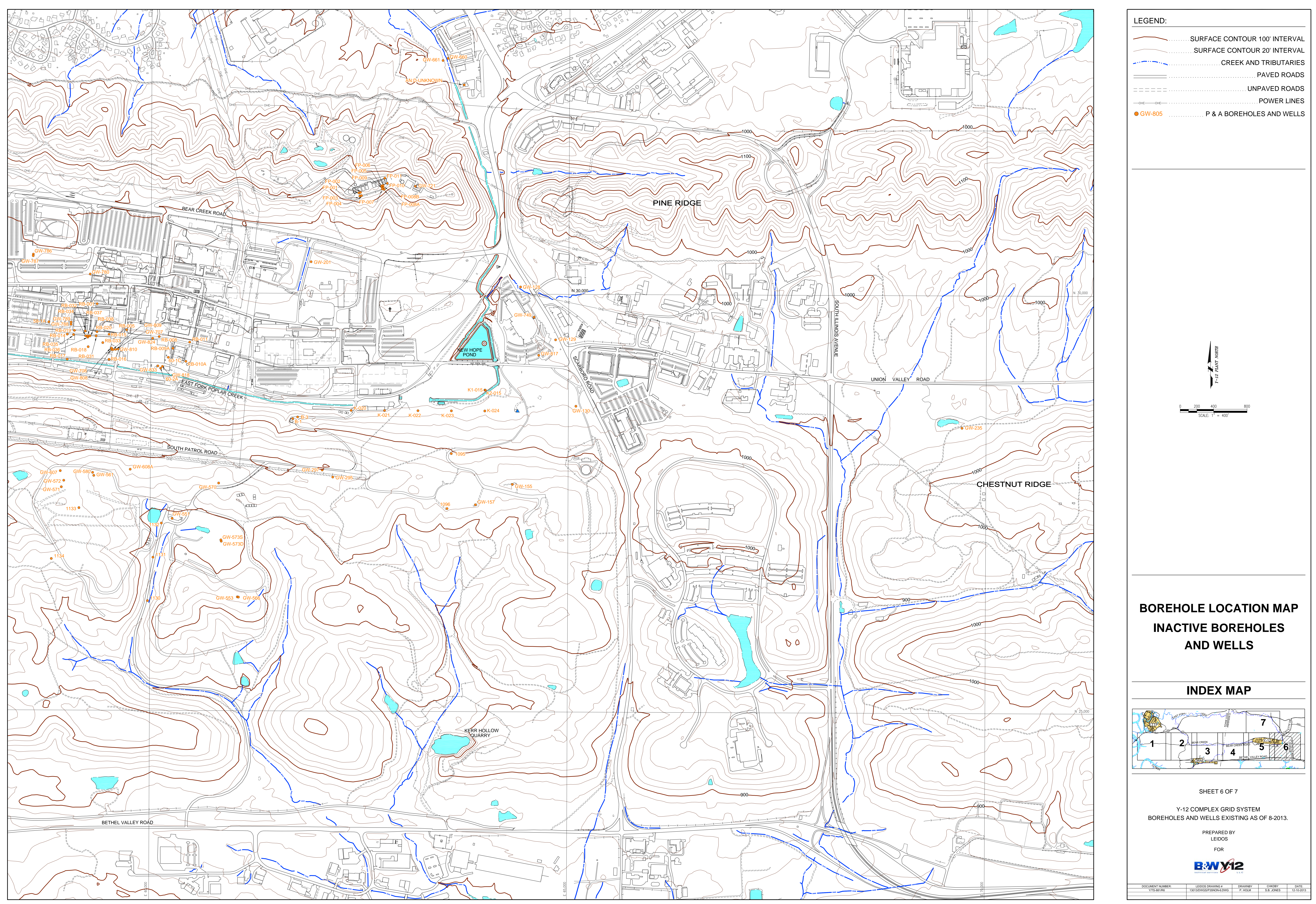

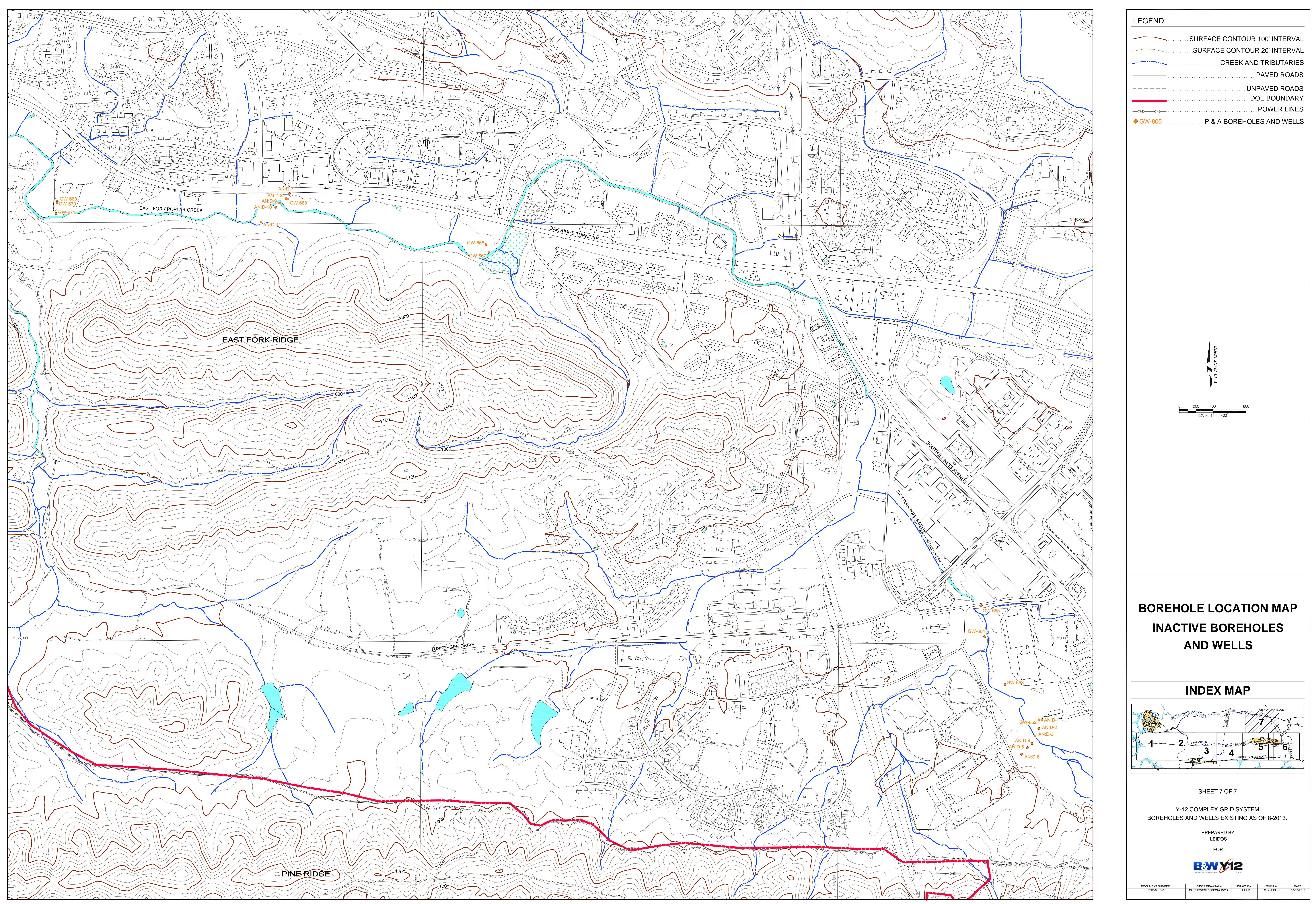


\section{DISTRIBUTION}

DEPARTMENT OF ENERGY

J. D. Darby

J. P. Donnelly

TENNESSEE DEPARTMENT OF ENVIRONMENT AND CONSERVATION, DOE OVERSIGHT

J. A. Owsley

J. E. Sebastian

B\&W Y-12, LLC PROGRAM MANAGEMENT

D. R. McDaniel

B\&W Y-12, LLC ENVIRONMENTAL COMPLIANCE DEPARTMENT

V. J. Brumback

W. P. Carlton

T. K. Cothron

S. M. Field

C. C. Hill

S. B. Jones

D. P. McDonald

S. E. McNamara

E. R. Schultz

L. O. Vaughan

J. T. Williams

B\&W Y-12, LLC ENGINEERING

\section{ORGANIZATION}

S. B. Ahmed

V. A. Oldham

R. T. Scott

S. A. Shults

B\&W Y-12, LLC INFORMATION TECHNOLOGY DEPARTMENT

S. W. King

9114DMC-01971865.6651-RC

Y-12 Records ServicesYDCC-RC

YDCC-RC
URS/CH2M OAK RIDGE, LLC

B. A. Brucken

M. E. Cleveland

C. S. Haase (RSI)

H. K. Haase (RSI)

S. A. Kautz

R. H. Ketelle (RSI)

J. W. Kubarewicz

S. M. Selecman (RSI)

L. M. Sims (RSI)

D. J. Watson (RSI)

File-EMEF-DMC

File-Y-12 Project PDCC

$\underline{\text { LEIDOS, INC }}$

T. J. Coffey

P. A. Holm

W. K. Jago

ELVADO ENVIRONMENTAL, LLC

T. R. Harrison

J. R. Walker

UNIVERISTY OF TENNESSEE-BATTELLE, $\underline{\text { LLC }}$

D. B. Watson 
This Page Intentionally Left Blank 